\title{
Expanded High-Level Waste Glass Property Data Development: Phase I
}
MJ Schweiger ${ }^{(a)}$
BJ Riley ${ }^{(a)}$
JV Crum ${ }^{(a)}$
PR Hrma ${ }^{(a)}$
CP Rodriguez ${ }^{(a)}$
BM Arrigoni $^{(a)}$

\author{
JB Lang ${ }^{(a)}$ \\ DS Kim ${ }^{(a)}$ \\ JD Vienna ${ }^{(a)}$ \\ FC Raszewski ${ }^{(b)}$ \\ DK Peeler ${ }^{(b)}$ \\ TB Edwards ${ }^{(b)}$
}

DR Best ${ }^{(b)}$

IA Reamer ${ }^{(b)}$

WT Riley ${ }^{(b)}$

PT Simmons ${ }^{(b)}$

RJ Workman ${ }^{(\mathrm{b})}$

(a) Pacific Northwest National Laboratory

(b) Savannah River National Laboratory

January 2011

Pacific Northwest

NATIONAL LABORATORY

Proudly Operated by Battelle Since 1965 


\title{
DISCLAIMER
}

This report was prepared as an account of work sponsored by an agency of the United States Government. Neither the United States Government nor any agency thereof, nor Battelle Memorial Institute, nor any of their employees, makes any warranty, express or implied, or assumes any legal liability or responsibility for the accuracy, completeness, or usefulness of any information, apparatus, product, or process disclosed, or represents that its use would not infringe privately owned rights. Reference herein to any specific commercial product, process, or service by trade name, trademark, manufacturer, or otherwise does not necessarily constitute or imply its endorsement, recommendation, or favoring by the United States Government or any agency thereof, or Battelle Memorial Institute. The views and opinions of authors expressed herein do not necessarily state or reflect those of the United States Government or any agency thereof.

\author{
PACIFIC NORTHWEST NATIONAL LABORATORY \\ operated by \\ BATTELLE \\ for the \\ UNITED STATES DEPARTMENT OF ENERGY \\ under Contract DE-AC05-76RL01830 \\ Printed in the United States of America \\ Available to DOE and DOE contractors from the \\ Office of Scientific and Technical Information, \\ P.O. Box 62, Oak Ridge, TN 37831-0062; \\ ph: (865) 576-8401 \\ fax: (865) 576-5728 \\ email: reports@adonis.osti.gov

\begin{abstract}
Available to the public from the National Technical Information Service, U.S. Department of Commerce, 5285 Port Royal Rd., Springfield, VA 22161 ph: (800) 553-6847 fax: (703) 605-6900

email: orders@ntis.fedworld.gov

online ordering: http://www.ntis.gov/ordering.htm
\end{abstract}

This document was printed on recycled paper.

(9/2003) 


\section{Expanded High-Level Waste Glass Property Data Development: Phase I}

\begin{tabular}{|c|c|c|}
\hline MJ Schweiger ${ }^{(a)}$ & JB Lang $^{(a)}$ & DR Best ${ }^{(b)}$ \\
\hline BJ Riley ${ }^{(a)}$ & $\operatorname{DS~Kim}^{(\mathrm{a})}$ & IA Reamer ${ }^{(b)}$ \\
\hline JV Crum $^{(\mathrm{a})}$ & JD Vienna ${ }^{(a)}$ & WT Riley $^{(b)}$ \\
\hline PR Hrma $^{(a)}$ & FC Raszewski ${ }^{(b)}$ & PT Simmons ${ }^{(b)}$ \\
\hline CP Rodriguez ${ }^{(a)}$ & DK Peeler ${ }^{(b)}$ & RJ Workman ${ }^{(b)}$ \\
\hline BM Arrigoni $^{(a)}$ & TB Edwards ${ }^{(b)}$ & \\
\hline
\end{tabular}

(a) Pacific Northwest National Laboratory

(b) Savannah River National Laboratory

January 2011

Prepared for the U.S. Department of Energy under Contract DE-AC05-76RL01830

Pacific Northwest National Laboratory

Richland, Washington 99354 


\section{Summary}

This report summarizes and analyzes the data collected on a first matrix of glasses intended to expand the composition region over which high-level waste glass property models are valid. These glasses consisted of a one-component-at-a-time variation around a baseline glass where the following components were varied: $\mathrm{Al}_{2} \mathrm{O}_{3}, \mathrm{~B}_{2} \mathrm{O}_{3}, \mathrm{Bi}_{2} \mathrm{O}_{3}, \mathrm{CaO}, \mathrm{Cr}_{2} \mathrm{O}_{3}, \mathrm{~F}, \mathrm{Fe}_{2} \mathrm{O}_{3}, \mathrm{~K}, \mathrm{Li}_{2} \mathrm{O}, \mathrm{MnO}, \mathrm{Na}_{2} \mathrm{O}, \mathrm{NiO}, \mathrm{P}_{2} \mathrm{O}_{5}$, $\mathrm{SiO}_{2}, \mathrm{ZrO}_{2}$, and noble metal oxides (i.e., $\mathrm{PdO}+\mathrm{RuO}_{2}+\mathrm{Rh}_{2} \mathrm{O}_{3}$ ). The analysis performed on these glasses include chemical composition (for target compositional verification), viscosity, electrical conductivity, liquidus temperature, equilibrium crystal fraction, canister centerline cooling with crystal identification, product consistency test, and toxicity characteristic leach procedure.

This report also includes data obtained for Hanford High Alumina (HAL) glasses formulated and tested as a part of DOE EM-31 Office of Waste Processing International Glass Program to develop high waste-loaded glasses for Hanford high-alumina high-level wastes. 



\section{Contents}

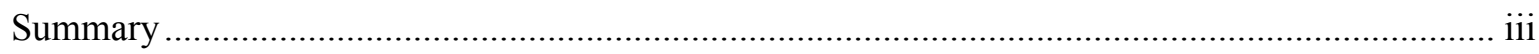

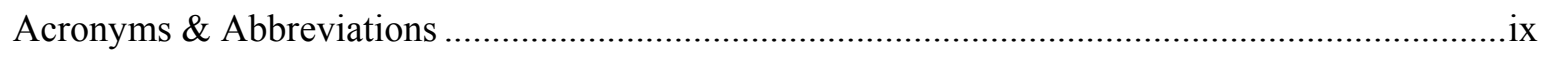

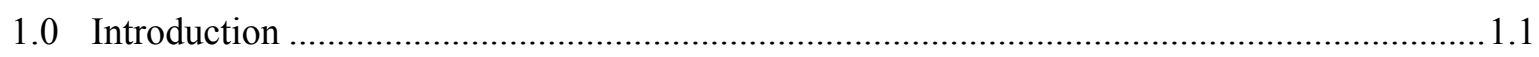

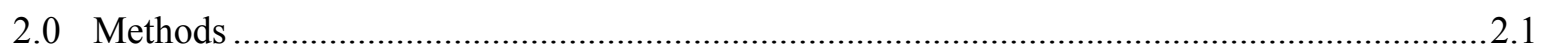

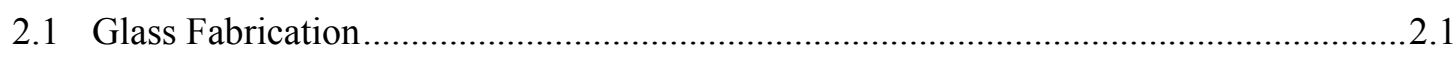

2.2 Chemical Analysis of Glass Composition ...............................................................2.2

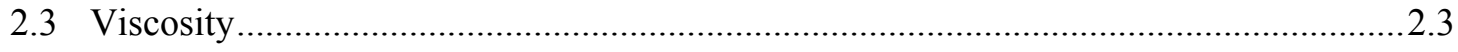

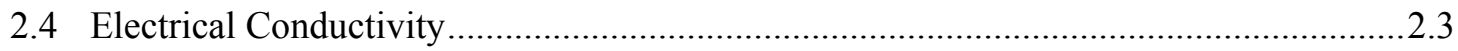

2.5 Liquidus Temperature and Equilibrium Crystal Fraction ..........................................2.4

2.6 Canister Centerline Cooling (CCC) and Crystal Identification....................................... 2.4

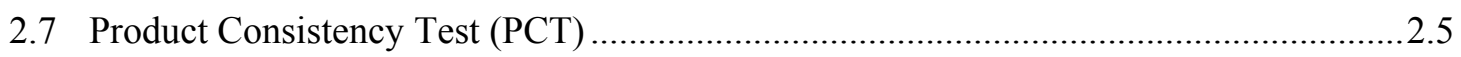

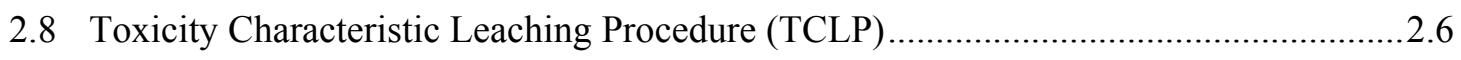

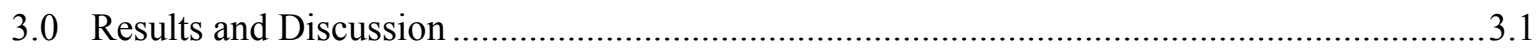

3.1 Chemical Analysis of Glass Composition .................................................................... 3.1

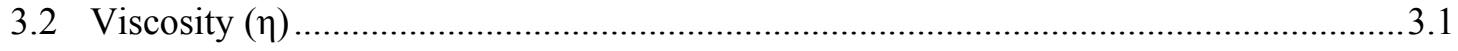

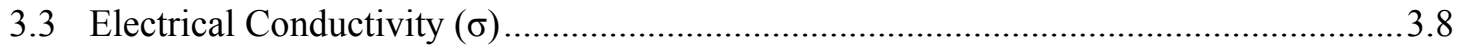

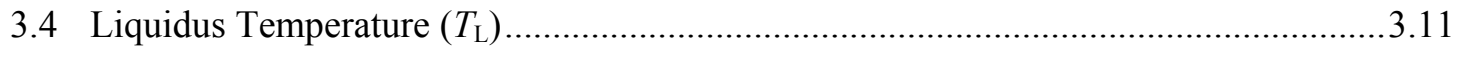

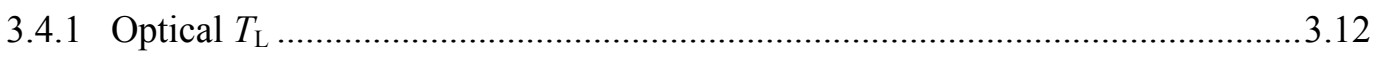

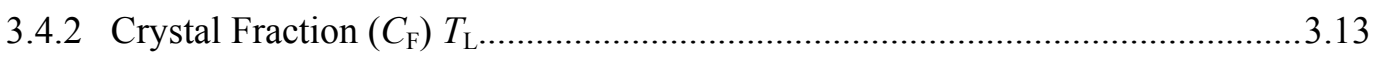

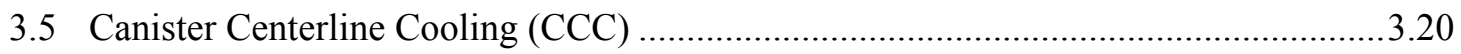

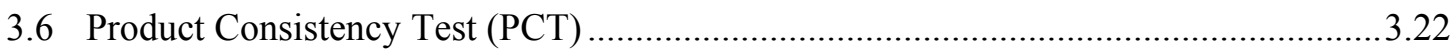

3.7 Toxicity Characteristic Leach Procedure (TCLP) ........................................................2.24

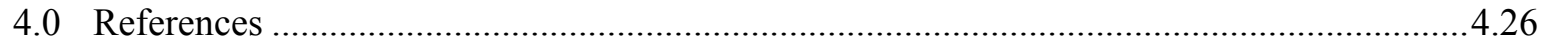

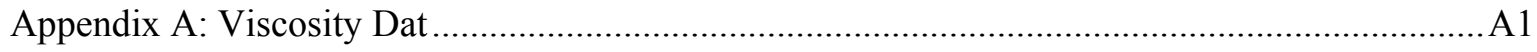

Appendix B: XRD of Canister Centerline Cooling (CCC) Treated Glasses................................. B1

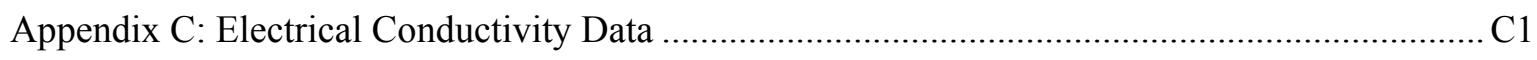

Appendix D: Optical TL Experiments and Observations ....................................................... D1

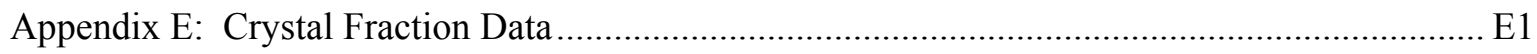

Appendix F: Toxicity Characteristic Leach Procedure (TCLP) Results ......................................F1 


\section{Figures}

Figure 2.1. Plot of Temperature Schedule during CCC Treatment.............................................2.5

Figure 3.1. Spider Plot of Viscosity at $1150^{\circ} \mathrm{C}$ versus Component Mass Fraction ........................3.3

Figure 3.2. Viscosity versus Component Mass Fraction and Temperature ...................................6

Figure 3.3. Viscosity Data, Calculated, Using Equation (1) with Coefficients Listed in Table 3.2, Versus Measured (outliers are identified in legend) ..................................................................... 3.7

Figure 3.4. Calculated EC Versus Measured Using Model 1 ..................................................... 3.9

Figure 3.5. Calculated EC versus Measured Using Model 2 ...................................................10

Figure 3.6. Spider Plot for EC Data as a Function of Compositional Deviation from the Baseline ( $\Delta x_{i}$ in mass fraction) 3.11

Figure 3.7. Spider Plot of Optical $T_{\mathrm{L}}$ as Determined by Optical Method vs. Change in Composition from the Baseline (designated by element of oxide components). This spider plot shows the location of additional glasses made for verifying data gaps from the original study. Yellow circles points show data points of new glasses that were measured by the optical method (EM07-B-125, B-175, Li-00, Li-05, Na-225, P-02, P-03, and Si-335) and red circles show data points of glasses that could not be measured by the optical method but were measured by the crystal fraction method (EM07-A1-225, Cr-0145, and Cr-02).

Figure 3.8. Spider Plot of Crystal Fraction $T_{\mathrm{L}}$, Mass $\%$ as Determined by XRD Crystal Fraction Method vs. Change in Composition from the Baseline as $\Delta g_{\mathrm{i}}$. 3.16

Figure 3.9. Spider Plot of $T_{1 \%}$ for EM07 Glasses as Determined by XRD Crystal Fraction Method vs. Change in Composition from the Baseline as $\Delta g_{\mathrm{i}}$. 3.17

Figure 3.10. Comparison of $T_{\mathrm{L}}$ Data Obtained Using XRD (mass\%) Methods against Data Obtained Using Optical Methods for the 36 Glasses for Which Data was Collected Using Both Methods. Trend line presented runs through all data points presented. 3.17

Figure 3.11. Calculated vs. Measured Data for the Different Data Sets. 3.20

\section{Tables}

Table 1.1. Description of Matrix Testing Responsibilities ...... 1.1

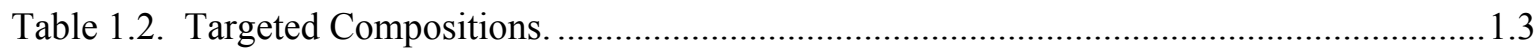

Table 2.1. Melting Temperatures and Times Used in Fabricating the EM07 Glasses...................2.1

Table 2.2. Temperature Schedule during CCC Treatment... .2 .4

Table 3.1. Measured $\log (\eta / \mathrm{Pa} \cdot \mathrm{s})$ Values Versus Temperature (in the sequence of measurement) for Glasses Tested; EM07-BL-1 and EM07-BL-2 Are Centroid Glass Duplicates (note that EM07-Bi05 glass also has a duplicate). 3.2

Table 3.2. The $B_{\mathrm{i}}$ Values from Fitting Equation (1) to Data. 3.7 
Table 3.3. EC Model 1 Coefficients. In this model, $A=8.55 \ln (\mathrm{S} / \mathrm{m}), R^{2}=0.967, R^{2}(\operatorname{adj})=0.965$, and $p$ (number of model parameters) $=18$ 3.8

Table 3.4. EC Model 2 Coefficients. In this model, $R^{2}=0.971, R^{2}(\operatorname{adj})=0.968$, and $p$ (number of model parameters) $=34$

Table 3.5. Summary of Liquidus Temperature Results. Tabulated below are the results for $T_{\mathrm{L}}$ determined using optical observations (" $T_{\mathrm{c}}$ ", " $T_{\mathrm{a}}$ ", and " $T_{\mathrm{L}}$, Optical"), crystal fraction results for both mass $\%$ (" $T_{\mathrm{L}}$ Mass\%") and volume\% (" $T_{\mathrm{L}} \mathrm{Vol} \%$ ") data, and $T_{1 \%}$. Additional data are included such as the \# of temperatures used to calculate crystal fraction ("\# Pts"), the slope of the line used to calculate the crystal fraction (temperature vs. crystal fraction, "Slope"), as well as the furnace used to make the measurements for the data presented ("Furnace Used"). Glasses that precipitated micron-sized crystals upon cooling are listed with Glass ID in bold and italics. The primary phase in all glasses was spinel. Crystal fraction and optical $T_{\mathrm{L}}$ were not determined for all of the glasses. ......3.14

Table 3.6. First-Order Regression Coefficients for the Calculated vs. Measured $T_{\mathrm{L}}$ data. ${ }^{*}$ The data presented for "Others" refers to the minor components in the glasses (i.e., $\mathrm{BaO}, \mathrm{CdO}, \mathrm{MgO}, \mathrm{PbO}$, $\mathrm{PdO}, \mathrm{Rh}_{2} \mathrm{O}_{3}, \mathrm{RuO}_{2}, \mathrm{SO}_{3}, \mathrm{SrO}, \mathrm{Ce}_{2} \mathrm{O}_{3}, \mathrm{La}_{2} \mathrm{O}_{3}, \mathrm{Nd}_{2} \mathrm{O}_{3}, \mathrm{TiO}_{2}$, and $\mathrm{ZnO}$ ). 3.18

Table 3.7. Calculated Data for the Three Different Regression Data Fits Presented in Table 3.6. Values were calculated even for glasses where data could not be measured (see Table 3.5)..........3.18

Table 3.8. Determination of Weight Percent Crystallinity and Identification of Crystals of CCC Heat Treated Glasses by XRD 3.21

Table 3.9. PCT Results (in ppm) taken from Raszewski et al. 2008b 3.22

Table 3.10. EPA TCLP Limits for Characteristically Hazardous Designation (ppm) 3.24

Table 3.11. Pb and Cd Metals Detected above the Reporting Detection Limit of the ICP of $0.1 \mathrm{ppm}$ for $\mathrm{Pb}$ and $0.05 \mathrm{ppm}$ for $\mathrm{Cd}$ 3.25 



\section{Acronyms \& Abbreviations}

\begin{tabular}{|c|c|}
\hline AES & atomic emission spectroscopy \\
\hline APEL & Applied Process Engineering Laboratory \\
\hline APEL-PAD-V & operation manual for APEL XRD \\
\hline ARM & Approved Reference Material \\
\hline Ave. & average \\
\hline $\mathrm{bc}$ & bias-corrected \\
\hline $\mathrm{CCC}$ & canister centerline cooling heat treatment \\
\hline$C_{\mathrm{F}}$ & crystal fraction \\
\hline EA & Environmental Assessment \\
\hline $\mathrm{EC}$ & electrical conductivity \\
\hline EPA & U.S. Environmental Protection Agency \\
\hline$\eta$ & viscosity \\
\hline GDL-ECC & Electrical Conductivity Calibration Procedure for Molten Glass \\
\hline GDL-ELC & Electrical Conductivity Measurement Procedure \\
\hline GDL-GBM & Glass Batching and Melting Procedure \\
\hline GDL-HTR & Heat Treatment of Glass Procedure \\
\hline GDL-LQT & $\begin{array}{l}\text { Standard Test Methods for Determining Liquidus Temperature of Waste } \\
\text { Glasses and Simulant Waste Glasses }\end{array}$ \\
\hline GDL-VIS & $\begin{array}{l}\text { Glass Development Laboratory Viscosity Measurement Procedure for } \\
\text { Vitrified Nuclear Waste }\end{array}$ \\
\hline GDL-VSC & Standard Viscosity Calibration Procedure \\
\hline GDL-XRD & Glass Development Laboratory XRD Semiquantitative Analysis Procedure \\
\hline HLW & high-level waste \\
\hline $\mathrm{Hz}$ & hertz (electrical frequency unit) \\
\hline IA & image analysis \\
\hline ICP-AES & inductively coupled plasma - atomic emissions spectroscopy \\
\hline LM & lithium metaborate $\left(\mathrm{LiBO}_{2}\right)$ \\
\hline LRM & low-activity waste reference material \\
\hline NM & noble metals \\
\hline $\mathrm{OM}$ & optical microscopy \\
\hline PCT & product consistency test \\
\hline $\mathrm{PF}$ & peroxide fusion \\
\hline $\mathrm{PH}$ & potassium hydroxide \\
\hline PNNL & Pacific Northwest National Laboratory \\
\hline RCRA & Resource Conservation and Recovery Act \\
\hline
\end{tabular}




\begin{tabular}{|c|c|}
\hline RDL & reporting detection limits \\
\hline $\mathrm{rpm}$ & revolutions per minute \\
\hline SEM & scanning electron microscope or scanning electron microscopy \\
\hline$\sigma$ & electrical conductivity \\
\hline SRNL & Savannah River National Laboratory \\
\hline SRS & Savannah River Site \\
\hline St. Dev. & standard deviation \\
\hline$T_{\mathrm{a}}$ & upper bound of liquidus temperature (amorphous specimen) \\
\hline$T_{\mathrm{c}}$ & lower bound of liquidus temperature (crystallized specimen) \\
\hline TCLP & toxicity characteristic leaching procedure \\
\hline$T_{\mathrm{L}}$ & liquidus temperature \\
\hline UTS & Universal Treatment Standard \\
\hline WCP & waste compliance plan \\
\hline XRD & X-ray diffraction \\
\hline
\end{tabular}




\subsection{Introduction}

Two separate test matrices were developed as part of a U.S. Department of Energy (DOE), Office of Environmental Management (EM), Office of Engineering and Technology (EM-21, now Office of Technology Development \& Innovation, EM-31) study to expand the range of waste glass property models to allow for prediction of properties for higher waste loaded glasses to potentially be processed at the Savannah River Site (SRS) and Hanford. This initial study included the development and testing of glasses in two matrices. The first matrix, developed using a single component-at-a-time design method and covering glasses of interest primarily to Hanford, is addressed in this report. The second matrix which was developed using a statistical design and covering glasses of interest primarily to SRS, was documented at Savannah River National Laboratory (SRNL) (Raszewski et al. 2008a). Test activities were performed jointly at the Pacific Northwest National Laboratory (PNNL) and the SRNL as described in Table 1.1.

Table 1.1. Description of Matrix Testing Responsibilities

\begin{tabular}{|l|l|l|}
\hline Activity & Matrix I & Matrix II \\
\hline Matrix design & PNNL & SRNL \\
\hline Glass fabrication & PNNL & SRNL \\
\hline Canister centerline cooling $(C C C)$ & PNNL & SRNL \\
\hline Crystal identification after canister centerline heat treatment & PNNL & PNNL \\
\hline Liquidus temperature $\left(T_{\mathrm{L}}\right)$ & PNNL & PNNL \\
\hline Crystal fraction $\left(C_{\mathrm{F}}\right)$ vs temperature & PNNL & PNNL* \\
\hline Viscosity $(\eta)$ vs temperature & PNNL & SRNL \\
\hline Electrical conductivity $(\sigma)$ vs temperature & PNNL & N/A \\
\hline Chemical composition analyses & SRNL & SRNL \\
\hline Product consistency test $($ PCT) analyses & SRNL & SRNL \\
\hline Toxicity characteristic leaching procedure $(T C L P)$ & SRNL & N/A \\
\hline Matrix glass number & 47 & 22 \\
\hline
\end{tabular}

*this analysis was performed on 3 of the glasses

N/A: not applicable

This report summarizes the experimental methods to fabricate, heat treat, and test 48 Matrix I glasses prepared at PNNL. Originally, 38 glasses (including a duplicated baseline glass [EM07-BL-1 and BL-2]) were fabricated in 2007 and due to some gaps in the data, 10 extra glasses were fabricated in 2009 to complete the study. One of the glasses from the original set (EM07-P-025) was removed from this data package due to experimental difficulties. Therefore, 47 total glasses are reported. Measured properties related to glass performance and melter processing are described in this report and Appendices.

Additionally, Appendix G summarizes the target glass composition and the results of crystal identification and product consistency test (PCT) of the 25 high alumina (HAL) glasses developed for the DOE Office of Waste Processing International Glass Program to develop high waste-loaded glasses for Hanford high-alumina high-level wastes (HLW). Two glass compositions were selected from this study to test in Russian research scale melters (see Appendix G for details). 
The data acquired in this study was used to generate preliminary models for some of the properties (viscosity, liquidus temperature and electrical conductivity) to confirm testing results and were included in a revised glass property model by Vienna et al. (2009). The targeted glass compositions (in mass fractions) are listed in Table 1.2. 
Table 1.2. Targeted Compositions.

\begin{tabular}{|c|c|c|c|c|c|c|c|c|c|c|c|c|c|c|}
\hline $\begin{array}{l}\text { Glass } \\
\text { ID }\end{array}$ & $\begin{array}{l}\text { BL-1 / } \\
\text { BL-2 }\end{array}$ & Al-06 & Al-15 & Al-20 & Al-225 & B-05 & B-125 & B-15 & B-175 & B-20 & Bi-025 & Bi-05 & Ca-035 & Ca-07 \\
\hline $\mathrm{B}_{2} \mathrm{O}_{3}$ & 0.10000 & 0.10444 & 0.09444 & 0.08889 & 0.08611 & 0.05000 & 0.12500 & 0.15000 & 0.17500 & 0.20000 & 0.09750 & 0.09500 & 0.09650 & 0.09300 \\
\hline $\mathrm{Bi}_{2} \mathrm{O}_{3}$ & 0.00000 & 0.00000 & 0.00000 & 0.00000 & 0.00000 & 0.00000 & 0.00000 & 0.00000 & 0.00000 & 0.00000 & 0.02500 & $\mathbf{0 . 0 5 0 0 0}$ & 0.00000 & 0.00000 \\
\hline $\mathrm{CaO}$ & 0.00000 & 0.00000 & 0.00000 & 0.00000 & 0.00000 & 0.00000 & 0.00000 & 0.00000 & 0.00000 & 0.00000 & 0.00000 & 0.00000 & 0.03500 & 0.07000 \\
\hline $\mathrm{CdO}$ & 0.00140 & 0.00146 & 0.00132 & 0.00124 & 0.00121 & 0.00148 & 0.00136 & 0.00132 & 0.00128 & 0.00124 & 0.00137 & 0.00133 & 0.00135 & 0.00130 \\
\hline $\mathrm{F}$ & 0.00100 & 0.00104 & 0.00094 & 0.00089 & 0.00086 & 0.00106 & 0.00097 & 0.00094 & 0.00092 & 0.00089 & 0.00098 & 0.00095 & 0.00097 & 0.00093 \\
\hline $\mathrm{Fe}_{2} \mathrm{O}_{3}$ & 0.10000 & 0.10444 & 0.09444 & 0.08889 & 0.08611 & 0.10556 & 0.09722 & 0.09444 & 0.09167 & 0.08889 & 0.09750 & 0.09500 & 0.09650 & 0.09300 \\
\hline $\mathrm{K}_{2} \mathrm{O}$ & 0.00000 & 0.00000 & 0.00000 & 0.00000 & 0.00000 & 0.00000 & 0.00000 & 0.00000 & 0.00000 & 0.00000 & 0.00000 & 0.00000 & 0.00000 & 0.00000 \\
\hline $\mathrm{La}_{2} \mathrm{O}_{3}$ & 0.00070 & 0.00073 & 0.00066 & 0.00062 & 0.00060 & 0.00074 & 0.00068 & 0.00066 & 0.00064 & 0.00062 & 0.00068 & 0.00067 & 0.00068 & 0.00065 \\
\hline $\mathrm{Li}_{2} \mathrm{O}$ & 0.02750 & 0.02872 & 0.02597 & 0.02444 & 0.02368 & 0.02903 & 0.02674 & 0.02597 & 0.02521 & 0.02444 & 0.02681 & 0.02613 & 0.02654 & 0.02558 \\
\hline $\mathrm{MgO}$ & 0.00150 & 0.00157 & 0.00142 & 0.00133 & 0.00129 & 0.00158 & 0.00146 & 0.00142 & 0.00138 & 0.00133 & 0.00146 & 0.00143 & 0.00145 & 0.00140 \\
\hline $\mathrm{P}_{2} \mathrm{O}_{5}$ & 0.01250 & 0.01306 & 0.01181 & 0.01111 & 0.01076 & 0.01319 & 0.01215 & 0.01181 & 0.01146 & 0.01111 & 0.01219 & 0.01188 & 0.01206 & 0.01163 \\
\hline $\mathrm{PbO}$ & 0.00370 & 0.00386 & 0.00349 & 0.00329 & 0.00319 & 0.00391 & 0.00360 & 0.00349 & 0.00339 & 0.00329 & 0.00361 & 0.00352 & 0.00357 & 0.00344 \\
\hline $\mathrm{PdO}$ & 0.00009 & 0.00009 & 0.00009 & 0.00008 & 0.00008 & 0.00010 & 0.00009 & 0.00009 & 0.00008 & 0.00008 & 0.00009 & 0.00009 & 0.00009 & 0.00008 \\
\hline $\mathrm{Rh}_{2} \mathrm{O}_{3}$ & 0.00003 & 0.00003 & 0.00003 & 0.00003 & 0.00003 & 0.00003 & 0.00003 & 0.00003 & 0.00003 & 0.00003 & 0.00003 & 0.00003 & 0.00003 & 0.00003 \\
\hline $\mathrm{RuO}_{2}$ & 0.00018 & 0.00019 & 0.00017 & 0.00016 & 0.00016 & 0.00019 & 0.00018 & 0.00017 & 0.00017 & 0.00016 & 0.00018 & 0.00017 & 0.00017 & 0.00017 \\
\hline $\mathrm{SiO}_{2}$ & 0.43330 & 0.45256 & 0.40923 & 0.38516 & 0.37312 & 0.45737 & 0.42126 & 0.40923 & 0.39719 & 0.38516 & 0.42247 & 0.41164 & 0.41813 & 0.40297 \\
\hline $\mathrm{SO}_{3}$ & 0.00300 & 0.00313 & 0.00283 & 0.00267 & 0.00258 & 0.00317 & 0.00292 & 0.00283 & 0.00275 & 0.00267 & 0.00293 & 0.00285 & 0.00290 & 0.00279 \\
\hline $\mathrm{SrO}$ & 0.00240 & 0.00251 & 0.00227 & 0.00213 & 0.00207 & 0.00253 & 0.00233 & 0.00227 & 0.00220 & 0.00213 & 0.00234 & 0.00228 & 0.00232 & 0.00223 \\
\hline $\mathrm{TiO}_{2}$ & 0.00040 & 0.00042 & 0.00038 & 0.00036 & 0.00034 & 0.00042 & 0.00039 & 0.00038 & 0.00037 & 0.00036 & 0.00039 & 0.00038 & 0.00039 & 0.00037 \\
\hline $\mathrm{ZnO}$ & 0.00060 & 0.00063 & 0.00057 & 0.00053 & 0.00052 & 0.00063 & 0.00058 & 0.00057 & 0.00055 & 0.00053 & 0.00059 & 0.00057 & 0.00058 & 0.00056 \\
\hline $\mathrm{ZrO}_{2}$ & 0.02500 & 0.02611 & 0.02361 & 0.02222 & 0.02153 & 0.02639 & 0.02431 & 0.02361 & 0.02292 & 0.02222 & 0.02438 & 0.02375 & 0.02413 & 0.02325 \\
\hline
\end{tabular}


Table 1.2 (contd)

\begin{tabular}{|c|c|c|c|c|c|c|c|c|c|c|c|c|c|c|}
\hline $\begin{array}{l}\text { Glass } \\
\text { ID }\end{array}$ & Cr-001 & Cr-012 & Cr-0145 & Cr-02 & F-02 & $\mathrm{Fe}-05$ & Fe-15 & Fe-20 & K-03 & K-06 & Li-00 & Li-015 & Li-04 & Li-05 \\
\hline $\mathrm{Al}_{2} \mathrm{O}_{3}$ & 0040 & 09930 & 99905 & 69849 & .09810 & 10556 & 09444 & .08889 & 09700 & .09400 & 10283 & 10129 & .09871 & 0.097 \\
\hline $\mathrm{B}_{2} \mathrm{O}_{3}$ & 0040 & 9930 & 9905 & 9849 & 09810 & 0556 & 09444 & 0.08889 & 09700 & .09400 & 10283 & 10129 & .09871 & .09769 \\
\hline $\mathrm{BaO}$ & 0050 & 0.00050 & 0.00050 & 00049 & 0.00049 & .00053 & 0.00047 & 0.00044 & .00049 & 0.00047 & 0.00051 & 0.00051 & 0.00049 & 0.00049 \\
\hline $\mathrm{Bi}_{2} \mathrm{O}_{3}$ & 0000 & 0.00000 & 0.00000 & 0.00000 & 0.00000 & .00000 & 0.00000 & 0.00000 & 0.00000 & 0.00000 & 0.00000 & 00000 & 0.00000 & 0.00000 \\
\hline $\mathrm{CaO}$ & 0000 & 0.00000 & 0.00000 & 00000 & 0.00000 & 00000 & 0.00000 & 0.00000 & 0.00000 & 0.00000 & 0.00000 & .00000 & 0.00000 & 0.00000 \\
\hline $\mathrm{CdO}$ & 141 & 0.00139 & 0.00139 & 138 & 0.00137 & 148 & 0.00132 & 0.00124 & 0136 & 0.0 & 144 & 42 & 138 & 0.00137 \\
\hline $\mathrm{Ce}_{2} \mathrm{O}_{3}$ & 0050 & 0.00050 & 0.00050 & 0.00049 & 0.00049 & 0.00053 & 0.00047 & 0.00044 & 0.00049 & 0.00047 & 0.00051 & 0.00051 & 0.00049 & 0.00049 \\
\hline $\mathrm{Cr}_{2} \mathrm{O}_{3}$ & 0100 & 0.01200 & 0.01450 & 0.02000 & 0.00490 & 0.00528 & 0.00472 & 0.00444 & 0.00485 & 0.00470 & 0.00514 & 0.00506 & 0.00494 & 0.00488 \\
\hline $\mathrm{F}$ & 0100 & 0.00099 & 0.00099 & 0.00098 & 0.02000 & 0.00106 & 0.00094 & 0.00089 & 0.00097 & 0.00094 & 0.00103 & 0.00101 & 0.00099 & 0.00098 \\
\hline $\mathrm{Fe}_{2} \mathrm{O}_{3}$ & 0040 & 0.09930 & 0.09905 & 0.09849 & 0.09810 & 0.05000 & 0.15000 & 0.20000 & 0.09700 & 0.09400 & 0.10283 & 0.10129 & 0.09871 & 0.09769 \\
\hline $\mathrm{K}_{2} \mathrm{O}$ & 0.00000 & 0.00000 & 0.00000 & 0.00000 & 0.00000 & 0.00000 & 0.00000 & 0.00000 & 0.03000 & 0.06000 & 0.00000 & 0.00000 & 0.00000 & 0.00000 \\
\hline $\mathrm{La}_{2} \mathrm{O}_{3}$ & 0070 & 0.00070 & 0.00069 & 0.00069 & 0.00069 & 0.00074 & 0.00066 & 0.00062 & 0.00068 & 0.00066 & 0.00072 & 0.00071 & 0.00069 & 0.00068 \\
\hline $\mathrm{Li}_{2} \mathrm{O}$ & 0.02761 & 0.02731 & 0.02724 & 0.02709 & 0.02698 & 0.02903 & 0.02597 & 0.02444 & 0.02668 & 0.02585 & 0.00000 & 0.01500 & 0.04000 & 0.05000 \\
\hline $\mathrm{MgO}$ & 0.00151 & 0.00149 & 0.00149 & 0.00148 & 0.00147 & 0.00158 & 0.00142 & 0.00133 & 0.00146 & 0.00141 & 0.00154 & 0.00152 & 0.00148 & 0.00147 \\
\hline $\mathrm{MnO}$ & 02008 & 0.01986 & 0.01981 & 0.01970 & 0.01962 & 0.02111 & 0.01889 & 0.01778 & 0.01940 & 0.01880 & 0.02057 & 0.02 & 0.0 & 0.01954 \\
\hline $\mathrm{Na}_{2} \mathrm{O}$ & 0.15060 & 0.14894 & 0.14857 & 0.14774 & 0.14715 & 0.15833 & 0.14167 & 0.13333 & 0.14550 & 0.14100 & 0.15424 & 0.15193 & 0.14807 & 0.14653 \\
\hline $\mathrm{Nd}_{2} \mathrm{O}_{3}$ & 0.00070 & 0.00070 & 0.00069 & 0.00069 & 0.00069 & 0.00074 & 0.00066 & 0.00062 & 0.00068 & 0.00066 & 0.00072 & 0.00071 & 0.00069 & 0.00068 \\
\hline $\mathrm{NiO}$ & 0.01004 & 0.00993 & 0.00990 & 0.00985 & 0.00981 & 0.01056 & 0.00944 & 0.00889 & 0.00970 & 0.00940 & 0.01028 & 0.01013 & 0.00987 & 0.00977 \\
\hline $\mathrm{P}_{2} \mathrm{O}_{5}$ & 0.01255 & 0.01241 & 0.01238 & 0.01231 & 0.01226 & 0.01319 & 0.01181 & 0.01111 & 0.01213 & 0.01175 & 0.01285 & 0.01266 & 0.01234 & 0.01221 \\
\hline $\mathrm{PbO}$ & 0.00371 & 0.00367 & 0.00366 & 0.00364 & 0.00363 & 0.00391 & 0.00349 & 0.00329 & 0.00359 & 0.00348 & 0.00380 & 0.00375 & 0.00365 & 0.00361 \\
\hline $\mathrm{PdO}$ & 0.00009 & 0.00009 & 0.00009 & 0.00009 & 0.00009 & 0.00010 & 0.00009 & 0.00008 & 0.00009 & 0.00008 & 0.00009 & 0.00009 & 0.00009 & 0.00009 \\
\hline $\mathrm{Rh}_{2} \mathrm{O}_{3}$ & 0.00003 & 0.00003 & 0.00003 & 0.00003 & 0.00003 & 0.00003 & 0.00003 & 0.00003 & 0.00003 & 0.00003 & 0.00003 & 0.00003 & 0.00003 & 0.00003 \\
\hline $\mathrm{RuO}_{2}$ & 0.00018 & 0.00018 & 0.00018 & 0.00018 & 0.00018 & 0.00019 & 0.00017 & 0.00016 & 0.00017 & 0.00017 & 0.00019 & 0.00018 & 0.00018 & 0.00018 \\
\hline $\mathrm{SiO}_{2}$ & 0.43504 & 0.43025 & 0.42916 & 0.42677 & 0.42506 & 0.45737 & 0.40923 & 0.38516 & 0.42030 & 0.40730 & 0.44555 & 0.43887 & 0.42773 & 0.42328 \\
\hline $\mathrm{SO}_{3}$ & 0.00301 & 0.00298 & 0.00297 & 0.00295 & 0.00294 & 0.00317 & 0.00283 & 0.00267 & 0.00291 & 0.00282 & 0.00308 & 0.00304 & 0.00296 & 0.00293 \\
\hline $\mathrm{SrO}$ & 0.00241 & 0.00238 & 0.00238 & 0.00236 & 0.00235 & 0.00253 & 0.00227 & 0.00213 & 0.00233 & 0.00226 & 0.00247 & 0.00243 & 0.00237 & 0.00234 \\
\hline $\mathrm{TiO}_{2}$ & 0.00040 & 0.00040 & 0.00040 & 0.00039 & 0.00039 & 0.00042 & 0.00038 & 0.00036 & 0.00039 & 0.00038 & 0.00041 & 0.00041 & 0.00039 & 0.00039 \\
\hline $\mathrm{ZnO}$ & 0.00060 & 0.00060 & 0.00059 & 0.00059 & 0.00059 & 0.00063 & 0.00057 & 0.00053 & 0.00058 & 0.00056 & 0.00062 & 0.00061 & 0.00059 & 0.00059 \\
\hline $\mathrm{ZrO}_{2}$ & 0.02510 & 0.02482 & 0.02476 & 0.02462 & 0.02452 & 0.02639 & 0.02361 & 0.02222 & 0.02425 & 0.02350 & 0.02571 & 0.02532 & 0.02468 & 0.02442 \\
\hline
\end{tabular}


Table 1.2 (contd)

\begin{tabular}{|c|c|c|c|c|c|c|c|c|c|c|c|c|c|c|}
\hline $\begin{array}{l}\text { Glass } \\
\text { ID } \\
\end{array}$ & Mn-01 & Mn-04 & $\mathrm{Na}-05$ & Na-10 & $\mathrm{Na}-20$ & $\mathrm{Na}-225$ & Ni-001 & $\mathrm{Ni}-02$ & P-0 & P-02 & P-03 & Si-30 & Si-335 & Si-37 \\
\hline $\mathrm{Al}_{2} \mathrm{O}_{3}$ & 0194 & 0.09796 & 11176 & 0.10588 & 0.09412 & 09118 & 0.10091 & 0.09899 & 0.10127 & 0.09924 & 0.09823 & 0.12352 & 0.11735 & 0.11117 \\
\hline$\overline{\mathrm{B}_{2} \mathrm{O}_{3}}$ & 0.10194 & 0.09796 & 0.11176 & 0.10588 & 0.09412 & 0.09118 & 0.10091 & 0.09899 & 0.10127 & 0.09924 & 0.09823 & 0.12352 & 0.11735 & 0.11117 \\
\hline $\mathrm{BaO}$ & 0.00051 & 0.00049 & 0.00056 & 0.00053 & 0.00047 & 0.00046 & 0.00050 & 0.00049 & 0.00051 & 0.00050 & 0.00049 & 0.00062 & 0.00059 & 0.00056 \\
\hline $\mathrm{Bi}_{2} \mathrm{O}_{3}$ & 0.00000 & 0.00000 & 0.00000 & 0.00000 & 0.00000 & 0.00000 & 0.00000 & 0.00000 & 0.00000 & 0.00000 & 0.00000 & 0.00000 & 0.00000 & 0.00000 \\
\hline $\mathrm{CaO}$ & 0.00000 & 0.00000 & 0.00000 & 0.00000 & 0.00000 & 0.00000 & 0.00000 & 0.00000 & 0.00000 & 0.00000 & 0.00000 & 0.00000 & 0.00000 & 0.00000 \\
\hline $\mathrm{CdO}$ & 0.00143 & 0.00137 & 0.00156 & 0.00148 & 0.00132 & 0.00128 & 0.00141 & 0.00139 & 0.00142 & 0.00139 & 0.00138 & 0.00173 & 0.00164 & 0.00156 \\
\hline $\mathrm{Ce}_{2} \mathrm{O}_{3}$ & 0.00051 & 0.00049 & 0.00056 & 0.00053 & 0.00047 & 0.00046 & 0.00050 & 0.00049 & 0.00051 & 0.00050 & 0.00049 & 0.00062 & 0.00059 & 0.00056 \\
\hline $\mathrm{Cr}_{2} \mathrm{O}_{3}$ & 0.00510 & 0.00490 & 0.00559 & 0.00529 & 0.00471 & 0.00456 & 0.00505 & 0.00495 & 0.00506 & 0.00496 & 0.00491 & 0.00618 & 0.00587 & 0.00556 \\
\hline $\mathrm{F}$ & 0.00102 & 0.00098 & 0.00112 & 0.00106 & 0.00094 & 0.00091 & 0.00101 & 0.00099 & 0.00101 & 0.00099 & 0.00098 & 0.00124 & 0.00117 & 0.00111 \\
\hline $\mathrm{Fe}_{2} \mathrm{O}_{3}$ & 0.10194 & 0.09796 & 0.11176 & 0.10588 & 0.09412 & 0.09118 & 0.10091 & 0.09899 & 0.10127 & 0.09924 & 0.09823 & 0.12352 & 0.11735 & 0.11117 \\
\hline $\mathrm{K}_{2} \mathrm{O}$ & 0.00000 & 0.00000 & 0.00000 & 0.00000 & 0.00000 & 0.00000 & 0.00000 & 0.00000 & 0.00000 & 0.00000 & 0.00000 & 0.00000 & 0.00000 & 0.00000 \\
\hline $\mathrm{La}_{2} \mathrm{O}_{3}$ & 0.00071 & 0.00069 & 0.00078 & 0.00074 & 0.00066 & 0.00064 & 0.00071 & 0.00069 & 0.00071 & 0.00069 & 0.00069 & 0.00086 & 0.00082 & 0.00078 \\
\hline $\mathrm{Li}_{2} \mathrm{O}$ & 0.02803 & 0.02694 & 0.03074 & 0.02912 & 0.02588 & 0.02507 & 0.02775 & 0.02722 & 0.02785 & 0.02729 & 0.02701 & 0.03397 & 0.03227 & 0.03057 \\
\hline $\mathrm{MgO}$ & 0.00153 & 0.00147 & 0.00168 & 0.00159 & 0.00141 & 0.00137 & 0.00151 & 0.00148 & 0.00152 & 0.00149 & 0.00147 & 0.00185 & 0.00176 & 0.00167 \\
\hline $\mathrm{MnO}$ & 0.00100 & 0.04000 & 0.02235 & 0.02118 & 0.01882 & 0.01824 & 0.02018 & 0.01980 & 0.02025 & 0.01985 & 0.01965 & 0.02470 & 0.02347 & 0.02223 \\
\hline $\mathrm{Na}_{2} \mathrm{O}$ & 0.15291 & 0.14694 & 0.05000 & 0.10000 & 0.20000 & 0.22500 & 0.15136 & 0.14848 & 0.15190 & 0.14886 & 0.14734 & 0.18528 & 0.17602 & 0.16675 \\
\hline $\mathrm{Nd}_{2} \mathrm{O}_{3}$ & 0.00071 & 0.00069 & 0.00078 & 0.00074 & 0.00066 & 0.00064 & 0.00071 & 0.00069 & 0.00071 & 0.00069 & 0.00069 & 0.00086 & 0.00082 & 0.00078 \\
\hline $\mathrm{NiO}$ & 0.01019 & 0.00980 & 0.01118 & 0.01059 & 0.00941 & 0.00912 & 0.00100 & 0.02000 & 0.01013 & 0.00992 & 0.00982 & 0.01235 & 0.01173 & 0.01112 \\
\hline $\mathrm{P}_{2} \mathrm{O}_{5}$ & 0.01274 & 0.01224 & 0.01397 & 0.01324 & 0.01176 & 0.01140 & 0.01261 & 0.01237 & 0.00000 & 0.02000 & 0.03000 & 0.01544 & 0.01467 & 0.01390 \\
\hline $\mathrm{PbO}$ & 0.00377 & 0.00362 & 0.00414 & 0.00392 & 0.00348 & 0.00337 & 0.00373 & 0.00366 & 0.00375 & 0.00367 & 0.00363 & 0.00457 & 0.00434 & 0.00411 \\
\hline $\mathrm{PdO}$ & 0.00009 & 0.00009 & 0.00010 & 0.00010 & 0.00008 & 0.00008 & 0.00009 & 0.00009 & 0.00009 & 0.00009 & 0.00009 & 0.00011 & 0.00011 & 0.00010 \\
\hline $\mathrm{Rh}_{2} \mathrm{O}_{3}$ & 0.00003 & 0.00003 & 0.00003 & 0.00003 & 0.00003 & 0.00003 & 0.00003 & 0.00003 & 0.00003 & 0.00003 & 0.00003 & 0.00004 & 0.00004 & 0.00003 \\
\hline $\mathrm{RuO}_{2}$ & 0.00018 & 0.00018 & 0.00020 & 0.00019 & 0.00017 & 0.00016 & 0.00018 & 0.00018 & 0.00018 & 0.00018 & 0.00018 & 0.00022 & 0.00021 & 0.00020 \\
\hline $\mathrm{SiO}_{2}$ & 0.44170 & 0.42446 & 0.48428 & 0.45879 & 0.40781 & 0.39507 & 0.43724 & 0.42892 & 0.43878 & 0.43001 & 0.42562 & 0.30000 & 0.33500 & 0.37000 \\
\hline $\mathrm{SO}_{3}$ & 0.00306 & 0.00294 & 0.00335 & 0.00318 & 0.00282 & 0.00274 & 0.00303 & 0.00297 & 0.00304 & 0.00298 & 0.00295 & 0.00371 & 0.00352 & 0.00334 \\
\hline $\mathrm{SrO}$ & 0.00245 & 0.00235 & 0.00268 & 0.00254 & 0.00226 & 0.00219 & 0.00242 & 0.00238 & 0.00243 & 0.00238 & 0.00236 & 0.00296 & 0.00282 & 0.00267 \\
\hline $\mathrm{TiO}_{2}$ & 0.00041 & 0.00039 & 0.00045 & 0.00042 & 0.00038 & 0.00036 & 0.00040 & 0.00040 & 0.00041 & 0.00040 & 0.00039 & 0.00049 & 0.00047 & 0.00044 \\
\hline $\mathrm{ZnO}$ & 0.00061 & 0.00059 & 0.00067 & 0.00064 & 0.00056 & 0.00055 & 0.00061 & 0.00059 & 0.00061 & 0.00060 & 0.00059 & 0.00074 & 0.00070 & 0.00067 \\
\hline $\mathrm{ZrO}_{2}$ & 0.02548 & 0.02449 & 0.02794 & 0.02647 & 0.02353 & 0.02279 & 0.02523 & 0.02475 & 0.02532 & 0.02481 & 0.02456 & 0.03088 & 0.02934 & 0.02779 \\
\hline
\end{tabular}


PNNL-17950, Rev 0

Table 1.2 (contd)

\begin{tabular}{|c|c|c|c|c|}
\hline Glass ID & Si-50 & Zr-001 & Zr-05 & NM-0025 \\
\hline $\mathrm{Al}_{2} \mathrm{O}_{3}$ & 0.08823 & 0.10246 & 0.09744 & 0.09979 \\
\hline $\mathrm{B}_{2} \mathrm{O}_{3}$ & 0.08823 & 0.10246 & 0.09744 & 0.09979 \\
\hline $\mathrm{BaO}$ & 0.00044 & 0.00051 & 0.00049 & 0.00050 \\
\hline $\mathrm{Bi}_{2} \mathrm{O}_{3}$ & 0.00000 & 0.00000 & 0.00000 & 0.00000 \\
\hline $\mathrm{CaO}$ & 0.00000 & 0.00000 & 0.00000 & 0.00000 \\
\hline $\mathrm{CdO}$ & 0.00124 & 0.00143 & 0.00136 & 0.00140 \\
\hline $\mathrm{Ce}_{2} \mathrm{O}_{3}$ & 0.00044 & 0.00051 & 0.00049 & 0.00050 \\
\hline $\mathrm{Cr}_{2} \mathrm{O}_{3}$ & 0.00441 & 0.00512 & 0.00487 & 0.00499 \\
\hline$F$ & 0.00088 & 0.00102 & 0.00097 & 0.00100 \\
\hline $\mathrm{Fe}_{2} \mathrm{O}_{3}$ & 0.08823 & 0.10246 & 0.09744 & 0.09979 \\
\hline $\mathrm{K}_{2} \mathrm{O}$ & 0.00000 & 0.00000 & 0.00000 & 0.00000 \\
\hline $\mathrm{La}_{2} \mathrm{O}_{3}$ & 0.00062 & 0.00072 & 0.00068 & 0.00070 \\
\hline $\mathrm{Li}_{2} \mathrm{O}$ & 0.02426 & 0.02818 & 0.02679 & 0.02744 \\
\hline $\mathrm{MgO}$ & 0.00132 & 0.00154 & 0.00146 & 0.00150 \\
\hline $\mathrm{MnO}$ & 0.01765 & 0.02049 & 0.01949 & 0.01996 \\
\hline $\mathrm{Na}_{2} \mathrm{O}$ & 0.13235 & 0.15369 & 0.14615 & 0.14968 \\
\hline $\mathrm{Nd}_{2} \mathrm{O}_{3}$ & 0.00062 & 0.00072 & 0.00068 & 0.00070 \\
\hline $\mathrm{NiO}$ & 0.00882 & 0.01025 & 0.00974 & 0.00998 \\
\hline $\mathrm{P}_{2} \mathrm{O}_{5}$ & 0.01103 & 0.01281 & 0.01218 & 0.01247 \\
\hline $\mathrm{PbO}$ & 0.00326 & 0.00379 & 0.00361 & 0.00369 \\
\hline $\mathrm{PdO}$ & 0.00008 & 0.00009 & 0.00009 & 0.00072 \\
\hline $\mathrm{Rh}_{2} \mathrm{O}_{3}$ & 0.00003 & 0.00003 & 0.00003 & 0.00024 \\
\hline $\mathrm{RuO}_{2}$ & 0.00016 & 0.00018 & 0.00018 & 0.00144 \\
\hline $\mathrm{SiO}_{2}$ & 0.50000 & 0.44397 & 0.42219 & 0.43239 \\
\hline $\mathrm{SO}_{3}$ & 0.00265 & 0.00307 & 0.00292 & 0.00299 \\
\hline $\mathrm{SrO}$ & 0.00212 & 0.00246 & 0.00234 & 0.00239 \\
\hline $\mathrm{TiO}_{2}$ & 0.00035 & 0.00041 & 0.00039 & 0.00040 \\
\hline $\mathrm{ZnO}$ & 0.00053 & 0.00061 & 0.00058 & 0.00060 \\
\hline $\mathrm{ZrO}_{2}$ & 0.02206 & 0.00100 & 0.05000 & 0.02495 \\
\hline
\end{tabular}

NM: noble metals 
PNNL-17950, Rev 0

\subsection{Methods}

\subsection{Glass Fabrication}

Glass fabrication was performed according to the PNNL procedure GDL-GBM for glass batching and melting with the exception of the addition of $\mathrm{PdO}$ and $\mathrm{RuO}_{2}$. For $\mathrm{PdO}$ and $\mathrm{RuO}_{2}$, nitrate solutions were carefully weighed and added, drop by drop, to $100 \mathrm{~g}$ of $\mathrm{SiO}_{2}$ dispersed on a Petri dish to absorb the liquid. The dish was placed in a $105^{\circ} \mathrm{C}$ oven for about 1 hour and cooled. The dried $\mathrm{SiO}_{2}$ cake was incorporated into the rest of the chemical batch and mixed in an agate milling chamber.

Each glass was prepared in Pt-alloy crucibles using a two step melt process. The first melt was of raw materials after mechanically mixing in an agate milling chamber. Melting was performed nominally at $1200{ }^{\circ} \mathrm{C}$ for 2 hours. A second melt of the glass was accomplished after the quenched glass was ground to a fine powder. Generally, the second melt was at 25 to $50{ }^{\circ} \mathrm{C}$ lower than the initial melt and between 45 to 60 minute in duration (See Table 2.1 for specific melt times and temperatures). A few of the matrix glasses required temperatures above $1200^{\circ} \mathrm{C}$ to adequately melt the batch.

Table 2.1. Melting Temperatures and Times Used in Fabricating the EM07 Glasses

\begin{tabular}{|l|l|l|l|l|}
\hline & \multicolumn{2}{|c|}{ First Melt } & \multicolumn{2}{c|}{ Second Melt } \\
\hline GLASS ID & Temp. $\left({ }^{\circ}\right.$ C) & Time (hrs) & Temp. $\left({ }^{\circ}\right.$ C) & Time (hrs) \\
\hline EM07-BL-1 & 1200 & 2 & 1150 & 1 \\
\hline EM07-BL-2 & 1200 & 2 & 1150 & 1 \\
\hline EM07-Al-06 & 1200 & 2 & 1150 & 0.75 \\
\hline EM07-Al-15 & 1250 & 2.25 & 1250 & 0.75 \\
\hline EM07-A1-20 & 1300 & 2 & 1300 & 0.75 \\
\hline EM07-A1-225 & 1225 & 1 & $1225^{*}$ & 1 \\
\hline EM07-B-05 & 1200 & 2 & 1175 & 0.75 \\
\hline EM07-B-125 & 1150 & 0.75 & 1150 & 1 \\
\hline EM07-B-15 & 1200 & 2 & 1150 & 1 \\
\hline EM07-B-175 & 1150 & 1 & 1150 & 1 \\
\hline EM07-B-20 & 1200 & 2 & 1175 & 0.75 \\
\hline EM07-Bi-025 & 1200 & 2 & 1175 & 0.75 \\
\hline EM07-Bi-05 & 1200 & 2 & 1175 & 0.75 \\
\hline EM07-Ca-035 & 1200 & 2 & 1175 & 0.75 \\
\hline EM07-Ca-07 & 1200 & 2 & 1175 & 0.75 \\
\hline EM07-Cr-001 & 1200 & 2 & 1150 & 0.75 \\
\hline EM07-Cr-012 & 1200 & 2 & 1200 & 0.75 \\
\hline EM07-Cr-0145 & 1250 & 1 & 1200 & 1 \\
\hline EM07-Cr-02 & 1225 & 2 & 1225 & 1 \\
\hline EM07-F02 & 1200 & 2 & 1150 & 1 \\
\hline EM07-Fe-05 & 1200 & 2 & 1150 & 1 \\
\hline EM07-Fe-15 & 1200 & 2 & 1150 & 1 \\
\hline
\end{tabular}


PNNL-17950, Rev 0

\begin{tabular}{|l|l|l|l|l|}
\hline & \multicolumn{2}{|c|}{ First Melt } & \multicolumn{2}{c|}{ Second Melt } \\
\hline GLASS ID & Temp. $\left({ }^{\circ}\right.$ C) & Time (hrs) & Temp. $\left({ }^{\circ}\right.$ C) & Time (hrs) \\
\hline EM07-Fe-20 & 1200 & 2 & 1150 & 1 \\
\hline EM07-K-03 & 1200 & 2 & 1175 & 0.75 \\
\hline EM07-K-06 & 1200 & 2 & 1175 & 0.75 \\
\hline EM07-Li-00 & 1200 & 1 & 1150 & 1 \\
\hline EM07-Li-015 & 1200 & 2 & 1175 & 0.75 \\
\hline EM07-Li-04 & 1200 & 2 & 1175 & 0.75 \\
\hline EM07-Li-05 & 1125 & 1 & 1100 & 1 \\
\hline EM07-Mn-01 & 1200 & 2 & 1150 & 1 \\
\hline EM07-Mn-04 & 1200 & 2 & 1150 & 1 \\
\hline EM07-Na-05 & 1200 & 2 & 1175 & 0.75 \\
\hline EM07-Na-10 & $1200 \& 1225^{+}$ & $1.15 \& 0.75$ & 1175 & 0.75 \\
\hline EM07-Na-20 & 1200 & 2 & 1150 & 0.75 \\
\hline EM07-Na-225 & 1150 & 1.25 & 1150 & 1 \\
\hline EM07-Ni-001 & 1200 & 2 & 1175 & 1 \\
\hline EM07-Ni-02 & 1200 & 2 & 1175 & 0.75 \\
\hline EM07-P-0 & 1200 & 2 & 1175 & 0.75 \\
\hline EM07-P-02 & 1075 & 1 & 1100 & 1 \\
\hline EM07-P-03 & 1050 & 1 & 1075 & 1 \\
\hline EM07-Si-30 & 1150 & 2 & 1150 & 0.75 \\
\hline EM07-Si-335 & 1200 & 1 & 1150 & 1 \\
\hline EM07-Si-37 & 1200 & 2 & 1150 & 1 \\
\hline EM07-Si-50 & 1250 & 2 & 1175 & 0.75 \\
\hline EM07-Zr-001 & 1200 & 2 & 1175 & 0.75 \\
\hline EM07-Zr-05 & 1200 & 2 & 1175 & 0.75 \\
\hline EM07-NM-0025 & 1200 & 2 & 1175 & 0.75 \\
\hline & & & \\
\hline
\end{tabular}

$* 3^{\text {rd }}$ melt: $1225^{\circ} \mathrm{C}$ for 0.5 hours

${ }^{+}$The $1^{\text {st }}$ melt was heated for 70 minutes at $1200^{\circ} \mathrm{C}$ then at $1225^{\circ} \mathrm{C}$ for 45 minutes. The $2^{\text {nd }}$ melt was heated 45 minutes at $1175^{\circ} \mathrm{C}$.

\subsection{Chemical Analysis of Glass Composition}

To confirm that the "as-fabricated" glasses correspond to the defined target compositions, a representative sample of each glass was chemically analyzed at the SRNL Process Science Analytical Laboratory (PSAL). Three dissolution methods were utilized in measuring these chemical compositions: samples prepared by lithium metaborate $\left(\mathrm{LM}=\mathrm{LiBO}_{2}\right)$ fusion followed by $\mathrm{HNO}_{3}$ dissolution were used to measure elemental concentrations of $\mathrm{Al}, \mathrm{Ba}, \mathrm{Bi}, \mathrm{Ca}, \mathrm{Cd}, \mathrm{Ce}, \mathrm{Cr}, \mathrm{Fe}, \mathrm{K}, \mathrm{La}, \mathrm{Mg}, \mathrm{Mn}, \mathrm{Na}, \mathrm{Nd}, \mathrm{Ni}, \mathrm{P}, \mathrm{Pb}, \mathrm{S}$, $\mathrm{Ti}, \mathrm{Zn}$, and $\mathrm{Zr}$, while samples from glasses prepared by sodium peroxide fusion $\left(\mathrm{PF}-\mathrm{Na}_{2} \mathrm{O}_{2}\right)$ and $\mathrm{HNO}_{3}$ dissolution were used to measure elemental concentrations of boron (B), lithium (Li), and silicon ( $\mathrm{Si}$ ) and a sample from a select glass was prepared by potassium hydroxide $(\mathrm{PH}-\mathrm{KOH})$ method to conduct a fluorine (F) measurement. For each glass, measurements were obtained from samples prepared in duplicate by each of these fusion/dissolution methods. All of the prepared samples were analyzed (twice 
for each element of interest) by inductively coupled plasma - atomic emission spectroscopy (ICP-AES). The instrument was re-calibrated between the duplicate analyses.

Samples of two glass standards were included in the analytical plans to provide an opportunity for checking the performance of the instrumentation over the course of the analyses and for potential bias correction. Specifically, several samples of the Defense Waste Processing Facility (DWPF) waste compliance plan (WCP) batch 1 were included in the LM and PF portions of both analytical plans. The low-activity reference material (LRM) was included in the PH portion of the analytical plan. This standard contains fluorine, which was of interest for the PH-prepared samples of one of the glasses.

A detailed statistical analysis of the chemical compositions is published elsewhere (Raszewski et al. 2008b). A short summary of these results is included below in the results section.

\subsection{Viscosity}

The viscosity of glasses was measured as a function of temperature following PNNL procedure GDLVIS using a Brookfield rotating spindle digital viscometer (DV-III) staged above a high temperature Deltech ${ }^{\circledR}$ furnace and equipped with a $\mathrm{Pt} / \mathrm{Rh}$ spindle which fitted through a hole in the top of the furnace. A $50 \mathrm{~mL}$ glass sample, measured by liquid displacement, was added into a $\mathrm{Pt} / \mathrm{Rh}$ crucible and placed into the furnace set at $1150{ }^{\circ} \mathrm{C}$. The spindle was immersed into the molten glass in the center of the crucible with its lower end of the rod at $5.1 \mathrm{~mm}$ above the bottom. A thermocouple was located directly under the bottom, center of the crucible. The furnace was set to the required ramp/soak schedule and digital data collection of spindle torque and temperature commenced. The temperature sequence was 1150, 1100, $1050,1000,950,1050,1150,1200$, and then $1150^{\circ} \mathrm{C}$. The soak time was $30 \mathrm{~min}$ at each temperature except the second soak at $1150{ }^{\circ} \mathrm{C}$ for $45 \mathrm{~min}$. The hysteresis approach allows for the potential impacts of crystallization (at lower temperatures) to be assessed (via reproducibility) with duplicate measurements being taken in the range at which the melter is anticipated to be operating and volatilization (at higher temperatures) minimized by measuring viscosity at temperatures above $1150{ }^{\circ} \mathrm{C}$ as the final viscosity measurement. The viscometer was calibrated with a standard glass (DWPF Start-up Frit) at specified intervals following PNNL procedure GDL-VSC.

\subsection{Electrical Conductivity}

The electrical conductivities of molten glasses were measured as a function of temperature using a probe with two platinum-10\% rhodium blades according to the PNNL procedures GDL-ELC (for measurement) and GDL-ECC (for calibration). The $50 \mathrm{~mL}$ of glass used for viscosity measurements was added back into a $\mathrm{Pt} / \mathrm{Rh}$ crucible and placed into the furnace at $1100{ }^{\circ} \mathrm{C}$. The probe was then lowered through a hole in the top of the furnace and into the melt, making sure that the probe was in the center of the crucible. Using the automated Solartron Analytical 1455 Cell Test System which was connected to the probe, the probe was lowered into the glass precisely $1.27 \mathrm{~cm}$. The glass soaked at 1200, 1100, 1000, and $900{ }^{\circ} \mathrm{C}$ for 45 minutes at each temperature, allowing the program to collect impedance data at frequencies of 10000, 1000, 100 and $63 \mathrm{~Hz}$ at five minutes apart at the end of each temperature setting when the sample was at thermal equilibrium. Only the $1000 \mathrm{~Hz}$ frequency data were used in the conductivity calculation since these were the closest values to real impedance. Measured data was exported into Microsoft Excel, where the impedance data was converted into conductance. 
The electrical conductivity system was checked at specified intervals in 0.1 and $1 \mathrm{M}$ solutions of $\mathrm{KCl}$ at room temperature to determine a cell constant. Two measurements were taken at intervals of $\sim 5$ minutes for each solution. The cell constant was then used to calculate the conductivity of each glass melt.

\subsection{Liquidus Temperature and Equilibrium Crystal Fraction}

The liquidus temperature and equilibrium crystal fraction as a function of temperature were measured in Pt-alloy crucibles and boats with tight fitting lids (to minimize volatility) according the PNNL procedure GDL-LQT. The heat treatment times were $48 \pm 2$ hours for heat treatment temperatures $<850$ ${ }^{\circ} \mathrm{C}, 24 \pm 2$ hours for $850-1300{ }^{\circ} \mathrm{C}$, and $\sim 4$ hours for $>1300{ }^{\circ} \mathrm{C}$ to ensure equilibrium was achieved without excessive volatility. Samples were quenched and analyzed to determine the type and quantity of crystal fractions (quantitative analyses) according to the PNNL procedure GDL-XRD. Heat treatment temperatures were varied so that the temperature at $0 \%$ crystals can be narrowed to within $10{ }^{\circ} \mathrm{C}$ for the liquidus temperature. For the equilibrium crystal fraction as a function of temperature, heat treatment was performed for the temperature range from the liquidus temperature down to the approximate temperature of maximum crystallization. Notes were taken on the location of crystals within the crucible to distinguish between surface and bulk crystals (see Appendix D).

\subsection{Canister Centerline Cooling (CCC) and Crystal Identification}

Each glass was subjected to slow cooling heat treatments (roughly 150 g glass sample) according to the simulated CCC profile the glass in the canister centerline experiences following the PNNL procedure GDL-HTR. Table 2.2 and Figure 2.1 show the temperature schedule of CCC heat treatment for Hanford HLW glasses used at WTP. ${ }^{1}$

Table 2.2. Temperature Schedule during CCC Treatment

\begin{tabular}{|l|c|c|c|}
\hline Segment & Time (min) & Start Temp. $\left({ }^{\circ} \mathbf{C}\right)$ & Rate $\left({ }^{\circ} \mathbf{C} / \mathbf{m i n}\right)$ \\
\hline 1 & $0-45$ & 1050 & -1.556 \\
\hline 2 & $45-107$ & 980 & -0.806 \\
\hline 3 & $107-200$ & 930 & -0.591 \\
\hline 4 & $200-329$ & 875 & -0.388 \\
\hline 5 & $329-527$ & 825 & -0.253 \\
\hline 6 & $527-707$ & 775 & -0.278 \\
\hline 7 & $707-1776$ & 725 & -0.304 \\
\hline
\end{tabular}

\footnotetext{
${ }^{1}$ Memorandum, Canister Centerline Cooling Data, Revision 1, CCN: 074851, RPP-WTP, October 29, 2003.
} 
PNNL-17950, Rev 0

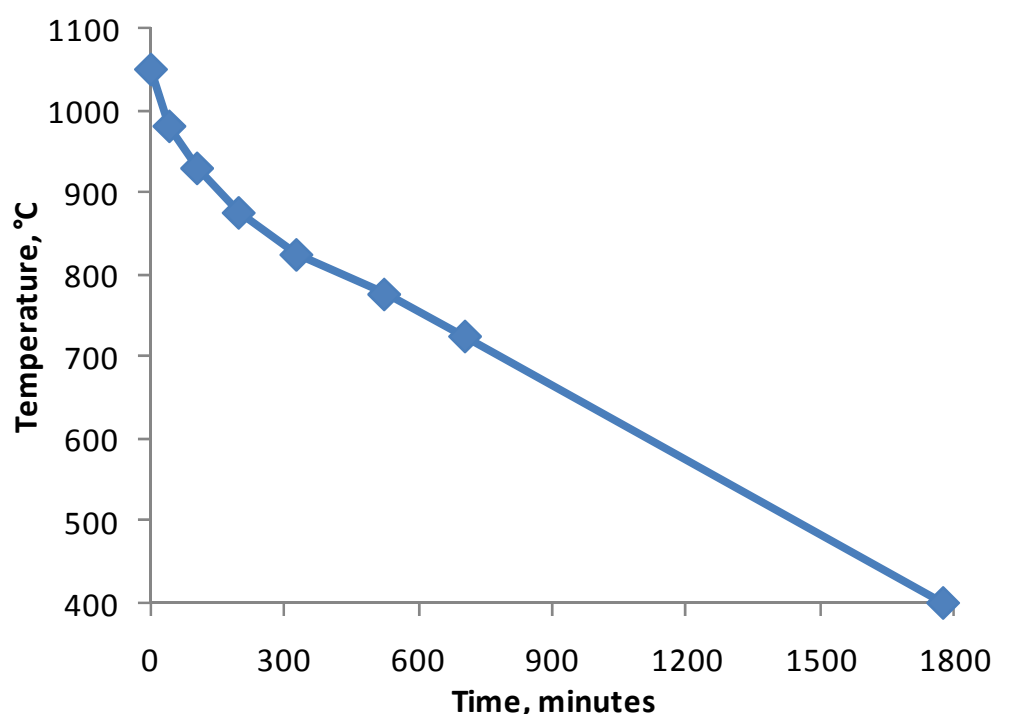

Figure 2.1. Plot of Temperature Schedule during CCC Treatment

The amount and type of crystalline phases that formed during $\mathrm{CCC}$ heat treatment were analyzed by X-ray diffraction (XRD) according to the PNNL procedures APEL-PAD-V and GDL-XRD. Powdered samples were prepared using five weight percent of $\mathrm{CaF}_{2}$ as an internal standard phase with about 1.5 to $2.5 \mathrm{~g}$ of glass. Glass and $\mathrm{CaF}_{2}$ were milled together for 2 minutes in a $10 \mathrm{~cm}^{3}$ tungsten carbide disc mill to a fine powder. The powder samples were loaded XRD sample holders and scanned at a $0.04^{\circ} 2 \theta$ step size, 4 seconds dwell time, from 10 to $70^{\circ} 2 \theta$. Spectra were analyzed with Jade ${ }^{\circledR} 6.0$ Software (MDI, Inc.) for phase identification. Full-pattern Rietveld refinement using RIQAS ${ }^{\circledR} 4$ (MDI Inc.) was performed to quantify the amounts of crystal phases on some samples with the high crystalline content.

Optical microscopy-image analysis (OM-IA) was also used to examine the crystalline phases found in some CCC glass samples. Scanning electron microscopy (SEM) was not used for this study.

\subsection{Product Consistency Test (PCT)}

Product consistency test (PCT) responses were measured at SRNL in triplicate on each of the glasses including quenched and CCC samples using Method A of the procedure (ASTM 2008). Also included in the experimental test matrix and tested in triplicate was the Environmental Assessment (EA) (Jantzen et al. 1993) glass, the Approved Reference Material (ARM) (Mellinger and Daniel 1984) glass, and blanks from the sample cleaning batch. Glass samples were ground, washed, and prepared according to the standard PCT procedure. The resulting solutions were sampled (filtered and acidified) and analyzed by PSAL under the auspices of an analytical plan (SRNL-SCS-2007-00059). Samples of a multi-element, standard solution were also included in the analytical plan (as a check on the accuracy of the ICP-AES). Normalized release rates were calculated based on target, measured, and bias-corrected (bc) compositions using the average of the logs of the leachate concentrations. Results from the PCT are published elsewhere (Raszewski et al. 2008b) and short summary of these results is included below in the results section. 


\subsection{Toxicity Characteristic Leaching Procedure (TCLP)}

The toxicity characteristic leaching procedure (TCLP) was performed on both quenched and CCC glass samples at Davis and Floyd Laboratory under an SRNL subcontract. The extraction and analyses were performed according to SW 846 Method 1311 (U.S. Environmental Protection Agency, or EPA, 1992) and SW-846 quality assurance/quality control requirements were followed. Crushed glass pieces that passed through a $9.5 \mathrm{~mm}(0.4 \mathrm{inch})$ sieve and $\geq 100 \mathrm{~g}$ in mass, are placed in dilute acetic acid $(\mathrm{pH}$ value of $4.98 \pm 0.05$ ) and agitated at $30 \pm 2 \mathrm{rpm}$ for $18 \pm 2$ hours at room temperature. The concentrations of hazardous metals in solution were then measured by ICP and analyzed values checked against the values specified by the Resource Conservation and Recovery Act (RCRA) and by the Universal Treatment Standard (UTS) put forth by the EPA (40 CFR 268.48) for certain waste categories defined in 40 CFR 268.48 in May 1998. 


\subsection{Results and Discussion}

\subsection{Chemical Analysis of Glass Composition}

Since the results from chemical analysis are published elsewhere (Raszewski et al. 2008b), only select observations will be made here. Target composition when compared to analyzed composition had excellent agreement except for some general observations discussed below.

Phosphorus content in 37 glasses (EM07-P-0 had no P) was low. For most glasses there was a 20 to 30 percent difference between the target composition and the analyzed $\mathrm{P}$ level, but for a few glasses the $\mathrm{P}$ was much lower. For example: glass EM07-P-025 (target $\mathrm{P}_{2} \mathrm{O}_{5}=2.5$ mass\%) had analyzed $\mathrm{P}$ content about the same as the baseline glass (target $\mathrm{P}_{2} \mathrm{O}_{5}=1.25$ mass\%) even though the correct level of $\mathrm{NaPO}_{3}$ was added to the chemical batch. This is of concern because property measurements, such as $T_{L}$, viscosity and TCLP, would likely be affected by this magnitude of composition change. This glass also had the lowest analyzed total oxides of the 38 glasses (about 96 mass \% with the next lowest glass at about 97.5 mass\%). The cause of discrepancies in $\mathrm{P}_{2} \mathrm{O}_{5}$ contents between target and analyzed glass compositions could be caused by 1) the $\mathrm{NaPO}_{3}$ source containing less phosphorous than stoichiometric, 2) the phosphorous was separated from the glass during fabrication (e.g., volatilizing), or 3) the analyses had a systematic bias. Although not certain, it appears based on previous studies showing little impurities in $\mathrm{NaPO}_{3}$ and relatively low crucible melt volatility of phosphorous that the third option is the most likely.

Zirconium was another metal with the analyzed concentrations, on average, about 20 mass\% lower than the targeted in all 38 glasses. The source of this difference is unknown, but one possibility is that there may have been difficulty in dissolving all $\mathrm{Zr}$ into solution while preparing the samples for ICP analysis, which has often occurred with glass analyses in the past.

The other noticeable observation from the chemical analyses is that EM07-Zr-001 had roughly double the target amount of $\mathrm{Nd}$ in glass. As this was not a systematic difference it is most likely caused by a batching error. The small amount of excess Nd in the glass is not likely to affect the chemical and property measurements made in this study. This glass will be given a reported composition containing double the targeted $\mathrm{Nd}_{2} \mathrm{O}_{3}$.

\subsection{Viscosity ( $n)$}

The results of viscosity measurements are listed in Appendix A and summarized in Table 3.1. Note that viscosity was measured three times at $1150^{\circ} \mathrm{C}$, at the beginning, in the middle, and at the end of the testing sequence. The centroid (EM07-BL-1), EM07-Bi-025 and Bi-05 glasses were batched and tested twice. Low-temperature viscosities were not measured for a number of glasses (EM07-A1-15, Al-20, BL1, Fe-20, Li-015, Na-05, Na-10, and Si-50) because the viscosity had increased beyond the $\sim 1000 \mathrm{P}$ upper limit for the viscometer. The viscosity data as a function of temperature are presented in Table 3.1.

All data are graphically displayed for each component varied in this study as a spider plot (Figure 3.1) and individually (Figure 3.2). Note that most of the data points of repeated measurements are indistinguishable even for glasses with high concentrations of components that may produce crystallinity $\left(\mathrm{Cr}_{2} \mathrm{O}_{3}, \mathrm{NiO}\right.$, and $\left.\mathrm{Fe}_{2} \mathrm{O}_{3}\right)$. Oxides that change their valence state with increasing temperature [Fe(III) to $\mathrm{Fe}(\mathrm{II})$ and $\mathrm{Cr}(\mathrm{VI})$ to $\mathrm{Cr}(\mathrm{III})]$ exhibited slightly irregular responses, such as an increase of the $\ln (\eta)$ versus 
$x_{i}$ slope at $x_{\mathrm{Fe}_{2} \mathrm{O}_{3}}>0.15$ or the different form of the $\ln (\eta)$ versus $x_{\mathrm{Cr}_{2} \mathrm{O}_{3}}$ function at $1200{ }^{\circ} \mathrm{C}$ and $950{ }^{\circ} \mathrm{C}$. Also, the $\ln (\eta)$ versus $x_{i}$ slope sharply increased at $x_{\mathrm{Na}_{2} \mathrm{O}}<0.1$.

Table 3.1. Measured $\log (\eta / \mathrm{Pa} \cdot \mathrm{s})$ Values Versus Temperature (in the sequence of measurement) for Glasses Tested; EM07-BL-1 and EM07-BL-2 Are Centroid Glass Duplicates (note that EM07-Bi-05 glass also has a duplicate)

\begin{tabular}{|c|c|c|c|c|c|c|c|c|c|}
\hline$T,{ }^{\circ} \mathrm{C}$ & 150 & 1100 & 1050 & 1000 & 950 & 1050 & 1150 & 1200 & 1150 \\
\hline Glass ID & \multicolumn{9}{|c|}{$\log (\eta / \mathbf{P a} \cdot \mathbf{s})$} \\
\hline EM07-BL-1 & 0.859 & 1.065 & 1.299 & 1.552 & 1.848 & 1.308 & 0.864 & 0.669 & 0.857 \\
\hline EM07-BL-2 & 0.850 & 1.063 & 1.295 & 1.557 & 1.853 & 1.302 & 0.864 & 0.667 & 0.860 \\
\hline EM07-Al-06 & 0.616 & 0.806 & 1.019 & 1.254 & 1.529 & 1.025 & 0.626 & 0.453 & 0.623 \\
\hline EM07-A1-15 & 1.209 & 1.457 & 1.703 & 1.988 & - & 1.710 & 1.227 & 0.996 & 1.223 \\
\hline EM07-Al-20 & 1.673 & 1.964 & - & - & - & - & - & 1.372 & 1.630 \\
\hline EM07-B-05 & 1.166 & 1.406 & 1.664 & 1.949 & - & 1.668 & 1.180 & 0.956 & 1.167 \\
\hline EM07-B-15 & 0.641 & 0.844 & 1.065 & 1.311 & 1.599 & 1.064 & 0.650 & 0.469 & 0.642 \\
\hline EM07-B-20 & 0.487 & 0.673 & 0.889 & 1.130 & 1.408 & 0.896 & 0.484 & 0.314 & 0.478 \\
\hline EM07-Bi-025 & 0.809 & 1.015 & 1.246 & 1.507 & 1.802 & 1.253 & 0.827 & - & - \\
\hline EM07-Bi-05a & 0.780 & 0.990 & 1.220 & 1.476 & 1.765 & 1.228 & 0.788 & 0.593 & 0.775 \\
\hline EM07-Bi-05b & 0.775 & 0.979 & 1.207 & 1.461 & 1.751 & 1.220 & 0.782 & 0.592 & 0.774 \\
\hline EM07-Ca-035 & 0.689 & 0.898 & 1.128 & 1.386 & 1.679 & 1.142 & 0.691 & 0.496 & 0.677 \\
\hline EM07-Ca-07 & 0.533 & 0.742 & 0.974 & 1.233 & 1.534 & 0.992 & 0.542 & 0.353 & 0.528 \\
\hline EM07-Cr-001 & 0.869 & 1.059 & 1.254 & 1.500 & 1.786 & 1.283 & 0.857 & 0.682 & 0.854 \\
\hline EM07-Cr-012 & 0.921 & 1.134 & 1.366 & 1.623 & 1.920 & 1.358 & 0.926 & 0.732 & 0.926 \\
\hline EM07-Cr-02 & 1.092 & 1.313 & 1.555 & 1.781 & 2.085 & 1.535 & 1.093 & 0.899 & 1.078 \\
\hline EM07-F-02 & 0.747 & 0.951 & 1.180 & 1.443 & 1.721 & 1.200 & 0.766 & 0.585 & 0.751 \\
\hline EM07-Fe-05 & 0.875 & 1.067 & 1.283 & 1.530 & 1.807 & 1.293 & 0.878 & 0.696 & 0.873 \\
\hline EM07-Fe-15 & 0.861 & 1.102 & 1.359 & 1.627 & 1.930 & 1.351 & 0.867 & 0.652 & 0.867 \\
\hline EM07-Fe-20 & 1.023 & 1.330 & 1.613 & 1.903 & - & 1.574 & 1.006 & 0.745 & 1.028 \\
\hline EM07-K-03 & 0.749 & 0.943 & 1.155 & 1.392 & 1.664 & 1.163 & 0.757 & 0.571 & 0.750 \\
\hline EM07-K-06 & 0.677 & 0.869 & 1.075 & 1.308 & 1.568 & 1.089 & 0.686 & 0.520 & 0.693 \\
\hline EM07-Li-015 & 1.154 & 1.386 & 1.636 & 1.927 & - & 1.638 & 1.156 & 0.941 & 1.143 \\
\hline EM07-Li2-04 & 0.648 & 0.852 & 1.072 & 1.312 & 1.592 & 1.074 & 0.662 & 0.474 & 0.649 \\
\hline EM07-Mn-01 & 0.933 & 1.143 & 1.381 & 1.623 & 1.916 & 1.383 & 0.939 & 0.747 & 0.935 \\
\hline EM07-Mn-04 & 0.823 & 1.039 & 1.283 & 1.546 & 1.821 & 1.283 & 0.836 & 0.620 & 0.808 \\
\hline EM07-Na-05 & 2.414 & - & - & - & - & - & - & 1.964 & 2.366 \\
\hline EM07-Na-10 & 1.451 & 1.708 & 1.978 & - & - & 1.983 & 1.473 & 1.197 & 1.440 \\
\hline EM07-Na-20 & 0.496 & 0.678 & 0.877 & 1.095 & 1.340 & 0.880 & 0.504 & 0.340 & 0.503 \\
\hline EM07-Ni-001 & 0.861 & 1.064 & 1.286 & 1.536 & 1.818 & 1.291 & 0.873 & - & - \\
\hline EM07-Ni-02 & 0.834 & 1.063 & 1.315 & 1.599 & 1.902 & 1.300 & 0.853 & 0.646 & 0.851 \\
\hline EM07-P-0 & 0.884 & 1.095 & 1.331 & 1.590 & 1.895 & 1.333 & 0.890 & 0.693 & 0.886 \\
\hline EM07-P-025 & 0.845 & 1.051 & 1.281 & 1.539 & 1.825 & 1.286 & 0.851 & 0.656 & 0.846 \\
\hline EM07-Si-30 & 0.214 & 0.425 & 0.642 & 0.875 & 1.139 & 0.624 & 0.208 & -0.013 & 0.184 \\
\hline
\end{tabular}


PNNL-17950, Rev 0

\begin{tabular}{|l|c|c|c|c|c|c|c|c|c|}
\hline EM07-Si-37 & 0.526 & 0.739 & 0.969 & 1.216 & 1.489 & 0.965 & 0.535 & 0.339 & 0.528 \\
\hline EM07-Si-50 & 1.257 & 1.484 & 1.731 & 2.025 & - & 1.733 & 1.264 & 1.062 & 1.258 \\
\hline EM07-Zr-001 & 0.860 & 1.054 & 1.273 & 1.515 & 1.816 & 1.280 & 0.868 & 0.681 & 0.867 \\
\hline EM07-Zr-05 & 0.930 & 1.153 & 1.405 & 1.689 & 2.014 & 1.416 & 0.934 & 0.716 & 0.927 \\
\hline
\end{tabular}

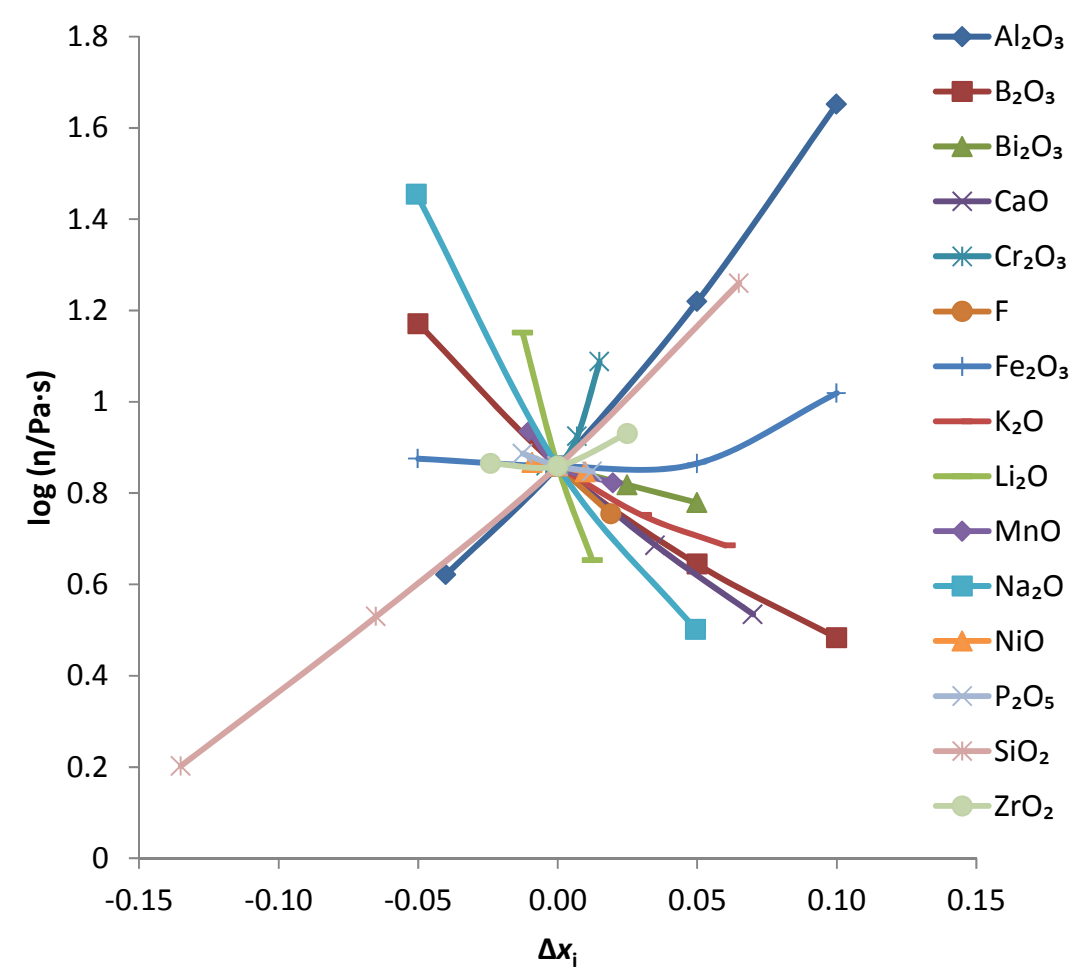

Figure 3.1. Spider Plot of Viscosity at $1150^{\circ} \mathrm{C}$ versus Component Mass Fraction
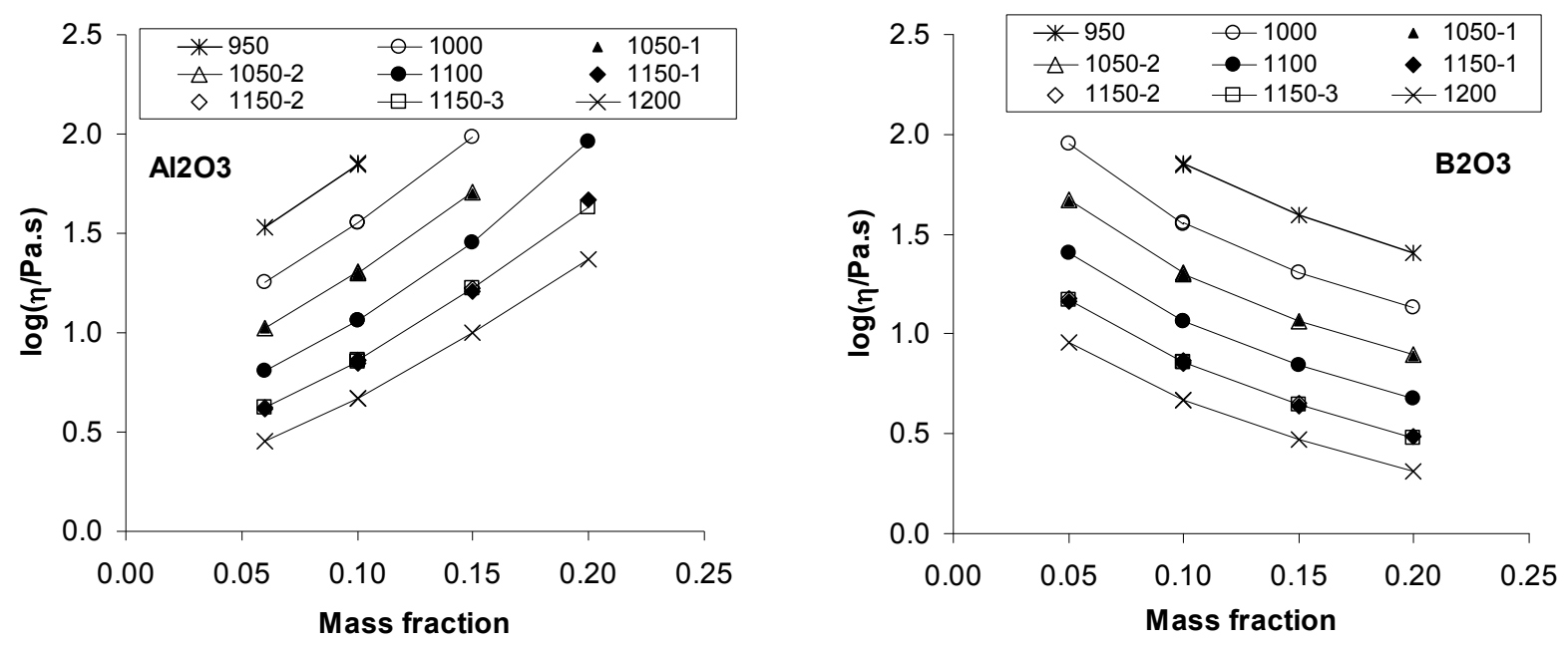
PNNL-17950, Rev 0
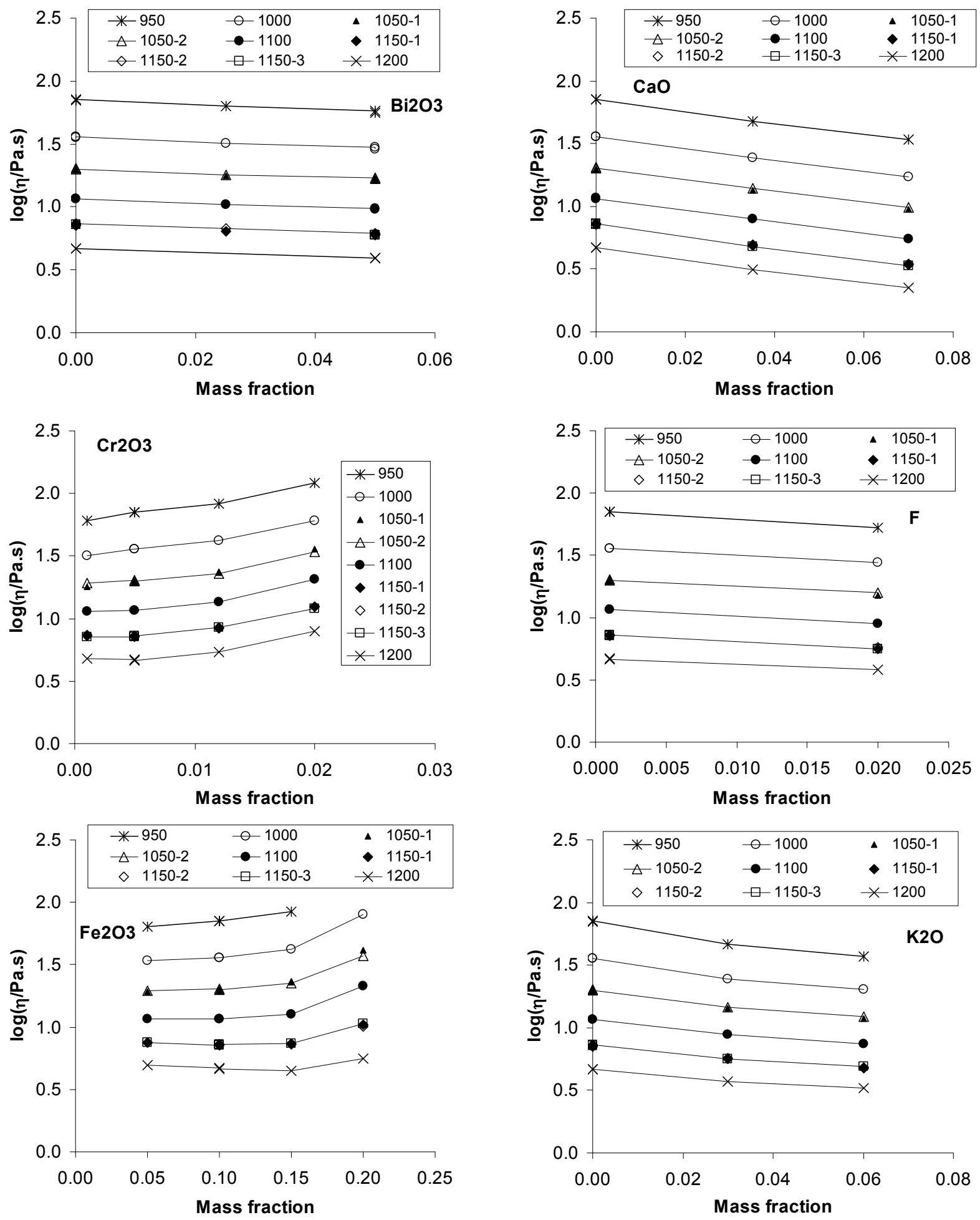
PNNL-17950, Rev 0
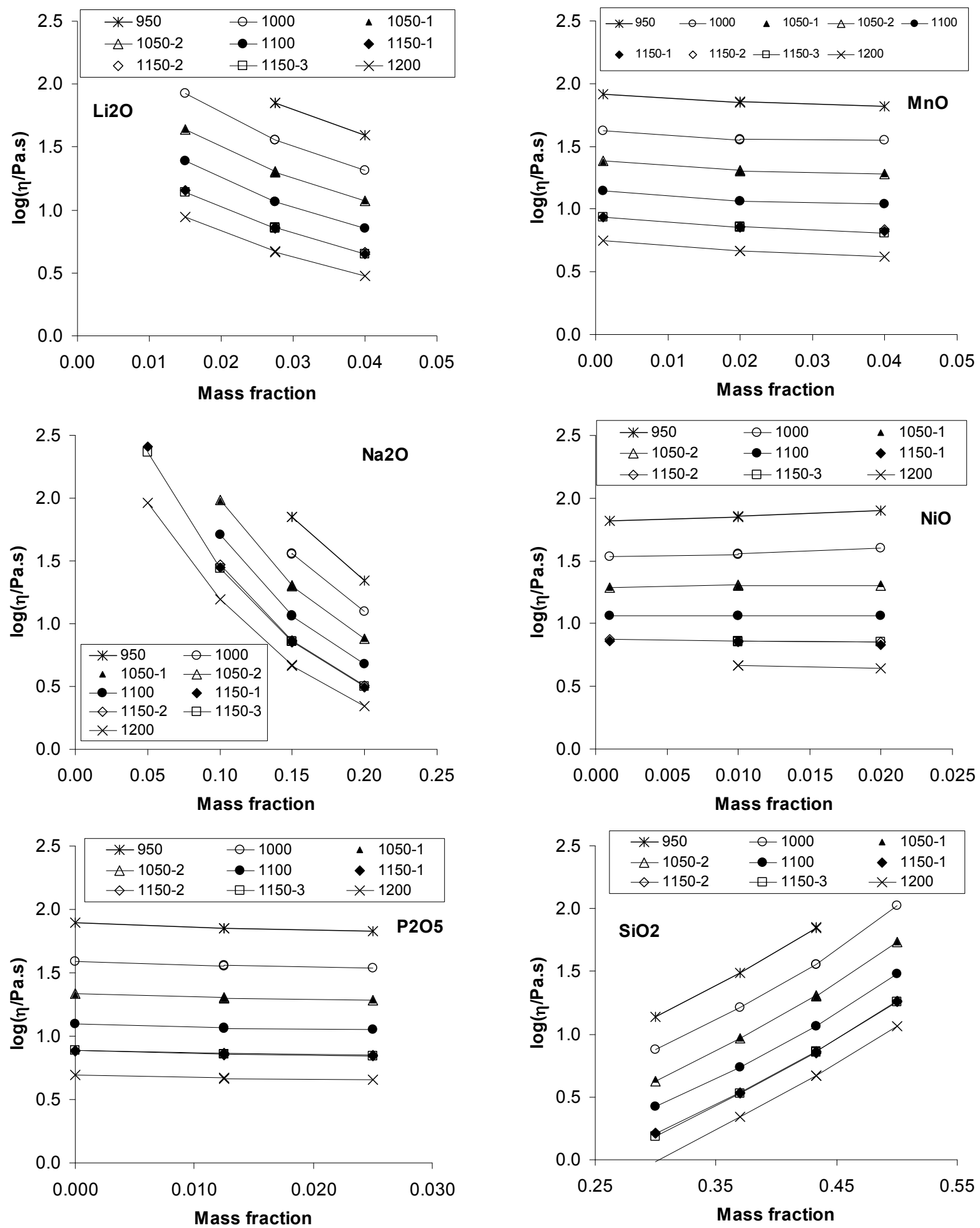
PNNL-17950, Rev 0

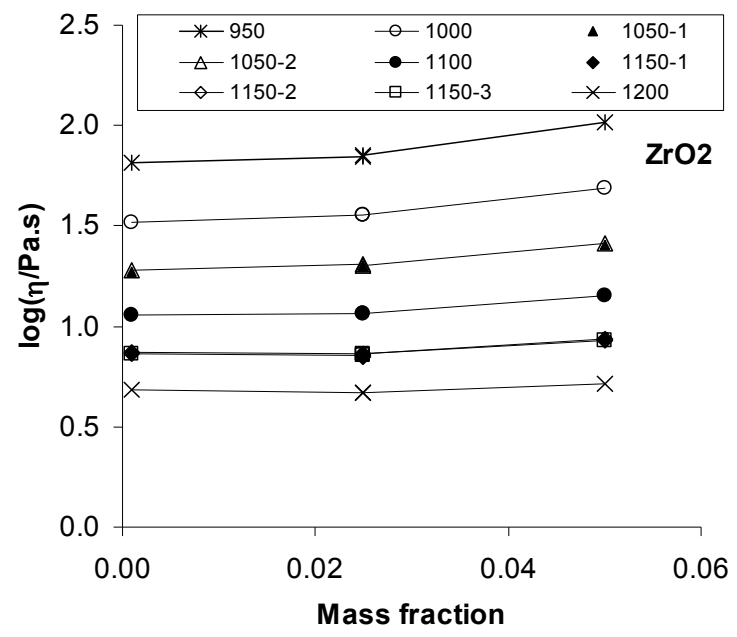

Figure 3.2. Viscosity versus Component Mass Fraction and Temperature

To represent viscosity as a function of temperature and composition, Equation (1) was fitted to data. Not included were all EM07-Na-05 glass data and low-temperature data of EM07-Fe-20 glass because viscosity measurements were affected by crystallization which caused these values to substantially deviated from linearity (See Figure 3.2) as defined by Equation (1). Also excluded were three data points for which $\eta>100 \mathrm{~Pa} \cdot \mathrm{s}$ because of their slight but systematic deviations from Arrhenius behavior. The resulting values were $A=-11.59(\ln [\mathrm{Pa} \cdot \mathrm{s}])$ and the $B$ coefficient listed in Table 3.2. Note that $A$ appears to be independent of composition. Figure 3.3 compares the model-calculated and measured viscosities.

$$
\ln (\eta)=A+\left(10^{4} / T\right) \sum_{i=1}^{N} B_{i} x_{i}
$$

where $x_{i}$ is the mass fraction of the $i$-th oxide component, $A$ is a constant $(\ln [\mathrm{Pa} \cdot \mathrm{s}]), T$ is the absolute temperature $(\mathrm{K}), B_{i}$ is the coefficient for an Arrhenius constant $B$ for the $i$-th component $\left(10^{-4} \mathrm{~K}\right)$, and $N$ is the number of model components. The model forms for viscosity in this section and for electrical conductivity in Section 3.3 are based on the Arrhenius equation for their temperature dependence, $\ln (\eta$ or $\sigma)=A+B / T$ where $A$ and $B$ are independent of temperature.

The viscosity model was developed for the narrow composition range described in Table 3.2. This range is narrower than the original range described in Table 1.2 because it excludes EM07-Na-05 and only marginally predicted EM07-Fe-20 for which the $950^{\circ} \mathrm{C}$ data is excluded. Note in Figure 3.3 how EM07-Na-05 data are clear outliers whereas EM07-Fe-20 data are hard to distinguish as outliers.

Model uncertainty will likely be high if the model is used beyond the range in Table 3.2. The experimental design within this study did not test component interactions which may be influential in this glass composition region and will affect the use of the model if used in predicting outside the narrow scope of this study. 
Table 3.2. The $B_{\mathrm{i}}$ Values from Fitting Equation (1) to Data $\mathrm{R}^{2}=0.989, \mathrm{R}_{\text {adj }}^{2}=0.988$, standard deviation is 0.102

\begin{tabular}{|l|c|c|c|c|}
\hline \multirow{2}{*}{ Oxide } & \multicolumn{2}{|c|}{$x_{i}$ ranges } & \multirow{2}{*}{$x_{i}^{\text {centroid }}$} & $B_{i}$ \\
\cline { 2 - 3 } \cline { 5 - 5 } & Min & max & & $10^{-4} \mathrm{~K}$ \\
\hline $\mathrm{Al}_{2} \mathrm{O}_{3}$ & 0.060 & 0.200 & 0.100 & 4.102 \\
\hline $\mathrm{B}_{2} \mathrm{O}_{3}$ & 0.050 & 0.200 & 0.100 & 0.637 \\
\hline $\mathrm{Bi}_{2} \mathrm{O}_{3}$ & 0.000 & 0.050 & 0.000 & 1.153 \\
\hline $\mathrm{CaO}$ & 0.000 & 0.070 & 0.000 & 0.362 \\
\hline $\mathrm{Cr}_{2} \mathrm{O}_{3}$ & 0.001 & 0.020 & 0.005 & 5.766 \\
\hline $\mathrm{F}$ & 0.001 & 0.020 & 0.001 & -0.246 \\
\hline $\mathrm{Fe}_{2} \mathrm{O}_{3}$ & 0.050 & 0.200 & 0.100 & 2.159 \\
\hline $\mathrm{K}_{2} \mathrm{O}$ & 0.000 & 0.060 & 0.000 & 0.654 \\
\hline $\mathrm{Li}_{2} \mathrm{O}$ & 0.015 & 0.040 & 0.028 & -4.465 \\
\hline $\mathrm{MnO}^{n}$ & 0.001 & 0.040 & 0.020 & 1.153 \\
\hline $\mathrm{Na}_{2} \mathrm{O}$ & 0.100 & 0.200 & 0.150 & -0.352 \\
\hline $\mathrm{NiO}^{2} \mathrm{O}$ & 0.001 & 0.020 & 0.010 & 2.126 \\
\hline $\mathrm{P}_{2} \mathrm{O}_{5}$ & 0.000 & 0.025 & 0.013 & 1.396 \\
\hline $\mathrm{SiO}$ & 0.300 & 0.500 & 0.433 & 2.894 \\
\hline $\mathrm{ZrO}_{2}$ & 0.001 & 0.050 & 0.025 & 2.568 \\
\hline $\mathrm{Others}_{2}$ & 0.014 & 0.019 & 0.016 & 1.071 \\
\hline
\end{tabular}

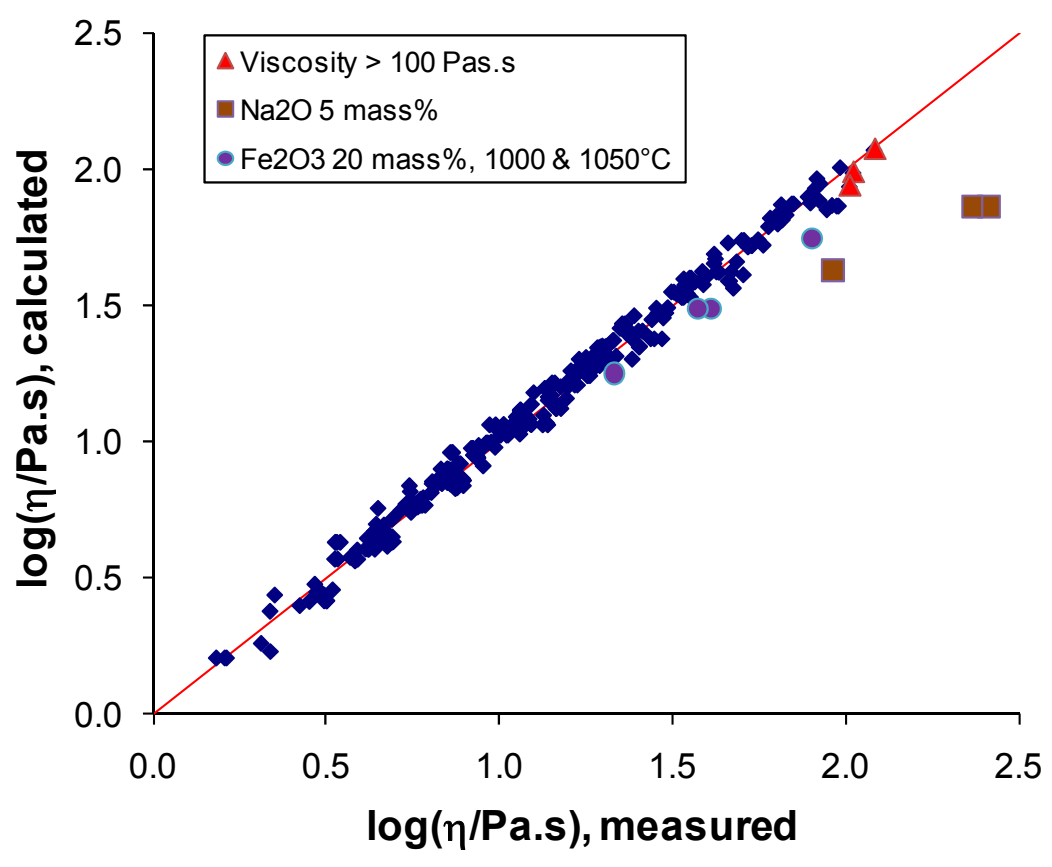

Figure 3.3. Viscosity Data, Calculated, Using Equation (1) with Coefficients Listed in Table 3.2, Versus Measured (outliers are identified in legend) 


\subsection{Electrical Conductivity $(\sigma)$}

See Appendix $\mathrm{C}$ for the data obtained from the EC experiments. Two model forms were used to fit the EC data. Model 1 used the coefficients presented in Table 3.3 and Equation (2)

$$
\ln (\sigma)=A+\left(10^{4} / T\right) \sum_{i=1}^{N} B_{i} x_{i}
$$

where $x_{i}$ is the mass fraction of the $i$-th oxide component, $A$ is a constant (with respect to composition and temperature) $(\ln [\mathrm{S} / \mathrm{m}]), T$ is the absolute temperature $(\mathrm{K}), B_{i}$ is the coefficient for Arrhenius constant $\mathrm{B}$ for the $i$-th component $\left(10^{-4} \mathrm{~K}\right)$, and $N$ is the number of model components. The resulting calculated EC values were plotted against the measured values in Figure 3.4. Model 2 utilized the coefficients presented in Table 3.4 and Equation (3). The resulting calculated values from Model 2 were plotted against the measured values in Figure 3.5.

$$
\ln (\sigma)=\sum_{i=1}^{N} A_{i} x_{i}+\left(10^{4} / T\right) \sum_{i=1}^{N} B_{i} x_{i}
$$

where $A_{i}$ is the coefficient for Arrhenius constant A for the $i$-th component $(\ln [\mathrm{S} / \mathrm{m}])$.

Table 3.3. EC Model 1 Coefficients. In this model, $A=8.55 \ln (\mathrm{S} / \mathrm{m}), R^{2}=0.967, R^{2}(\operatorname{adj})=0.965$, and $p$ (number of model parameters) $=18$

\begin{tabular}{|l|c|}
\hline Oxide & $B_{i}, \ln (\mathrm{S} / \mathrm{m}) \mathrm{K}$ \\
\hline $\mathrm{Al}_{2} \mathrm{O}_{3}$ & -0.92 \\
\hline $\mathrm{B}_{2} \mathrm{O}_{3}$ & -0.98 \\
\hline $\mathrm{Bi}_{2} \mathrm{O}_{3}$ & -0.62 \\
\hline $\mathrm{CaO}$ & -1.22 \\
\hline $\mathrm{Cr}_{2} \mathrm{O}_{3}$ & -1.26 \\
\hline $\mathrm{F}$ & 1.67 \\
\hline $\mathrm{Fe}_{2} \mathrm{O}_{3}$ & -0.79 \\
\hline $\mathrm{K}_{2} \mathrm{O}$ & -0.82 \\
\hline $\mathrm{Li}_{2} \mathrm{O}$ & 0.56 \\
\hline $\mathrm{MnO}$ & -1.31 \\
\hline $\mathrm{Na}_{2} \mathrm{O}$ & 0.45 \\
\hline $\mathrm{NiO}_{2}$ & -2.50 \\
\hline $\mathrm{P}_{2} \mathrm{O}_{5}$ & 0.84 \\
\hline $\mathrm{SiO}$ & -0.91 \\
\hline $\mathrm{ZrO}_{2}$ & -0.81 \\
\hline $\mathrm{NM}_{2}$ & -30.48 \\
\hline Others & -2.55 \\
\hline
\end{tabular}


PNNL-17950, Rev 0

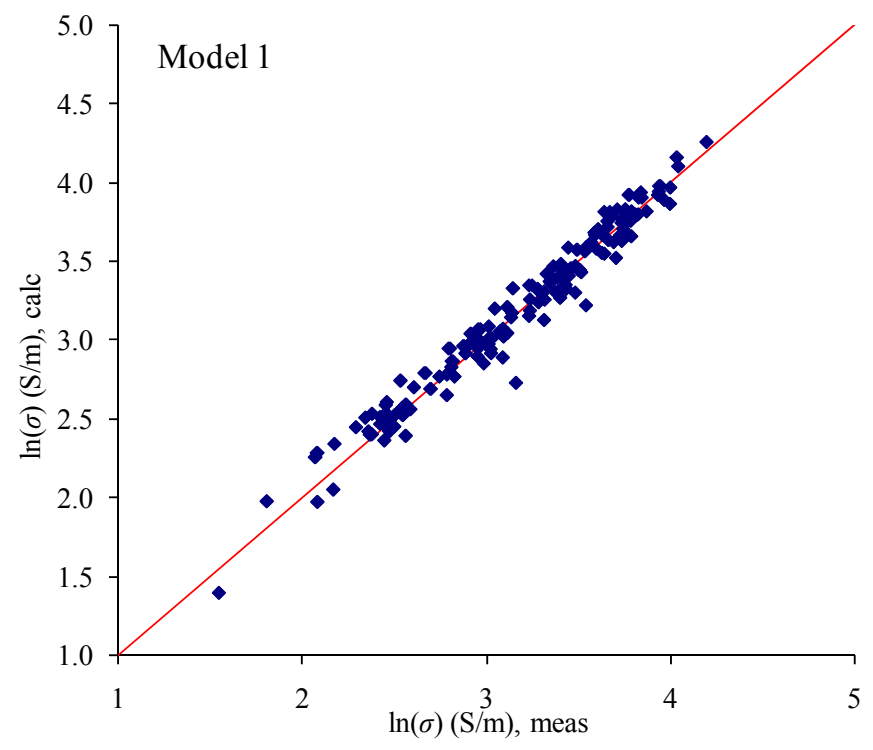

Figure 3.4. Calculated EC Versus Measured Using Model 1

Table 3.4. EC Model 2 Coefficients. In this model, $R^{2}=0.971, R^{2}(\operatorname{adj})=0.968$, and $p$ (number of model parameters) $=34$

\begin{tabular}{|c|r|r|}
\hline Oxide & $A_{i}, \ln (\mathrm{S} / \mathrm{m})$ & $B_{i}, \ln (\mathrm{S} / \mathrm{m}) \mathrm{K}$ \\
\hline $\mathrm{Al}_{2} \mathrm{O}_{3}$ & 10.69 & -1.20 \\
\hline $\mathrm{B}_{2} \mathrm{O}_{3}$ & 12.00 & -1.42 \\
\hline $\mathrm{Bi}_{2} \mathrm{O}_{3}$ & 9.32 & -0.72 \\
\hline $\mathrm{CaO}$ & 24.81 & -3.31 \\
\hline $\mathrm{Cr}_{2} \mathrm{O}_{3}$ & 15.15 & -2.11 \\
\hline $\mathrm{F}$ & -1.99 & 3.03 \\
\hline $\mathrm{Fe}_{2} \mathrm{O}_{3}$ & 7.79 & -0.69 \\
\hline $\mathrm{K}_{2} \mathrm{O}$ & 16.82 & -1.89 \\
\hline $\mathrm{Li}_{2} \mathrm{O}$ & 21.44 & -1.09 \\
\hline $\mathrm{MnO}$ & 2.36 & -0.51 \\
\hline $\mathrm{Na}_{2} \mathrm{O}$ & 12.81 & -0.10 \\
\hline $\mathrm{NiO}_{2}$ & -7.66 & -0.36 \\
\hline $\mathrm{P}_{2} \mathrm{O}_{5}$ & -83.30 & 12.66 \\
\hline $\mathrm{SiO}_{2}$ & 10.03 & -1.10 \\
\hline $\mathrm{ZrO}_{2}$ & 15.04 & -1.64 \\
\hline $\mathrm{NM}^{2}$ & -119.44 & -14.01 \\
\hline Others & -47.67 & 4.65 \\
\hline
\end{tabular}




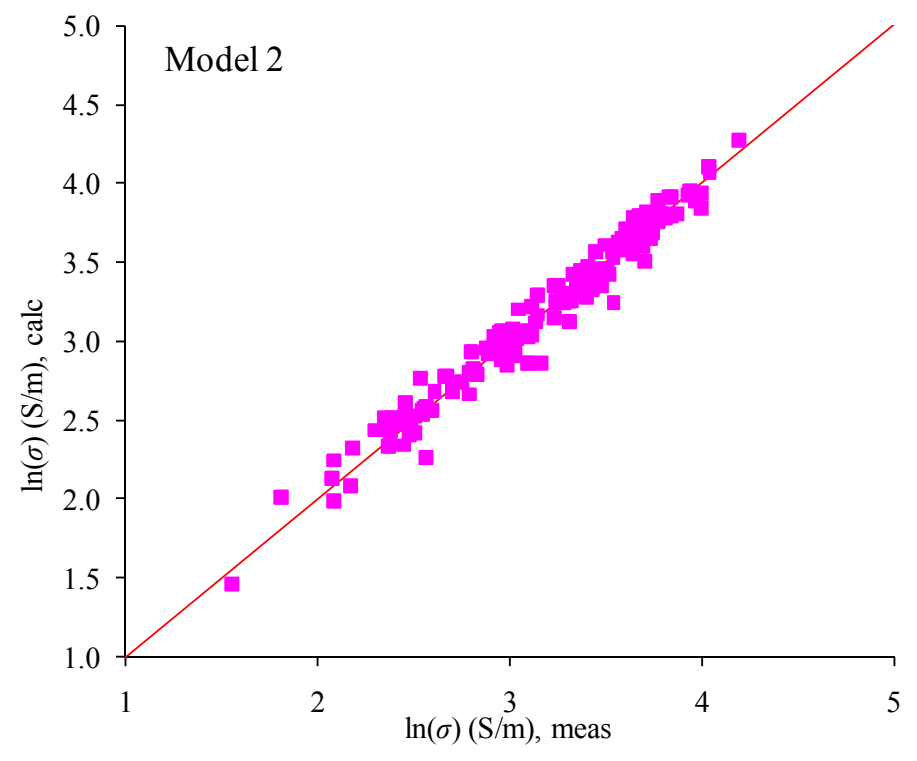

Figure 3.5. Calculated EC versus Measured Using Model 2

It is interesting to note that the EC model that assumes a composition independent $A$ model, Model 1, yields an $R^{2}$ that is $0.39 \%$ lower than that of the composition dependent $A$ model, Model 2, and contains roughly half of the coefficients (18 vs. 34). This suggests that like in the case of viscosity, the preexponential is composition independent. Electrical conductivity values at $1150^{\circ} \mathrm{C}$ for each of the 38 glasses in the Matrix 1 test were plotted against the composition variation from the baseline glass (see Figure 3.6). 


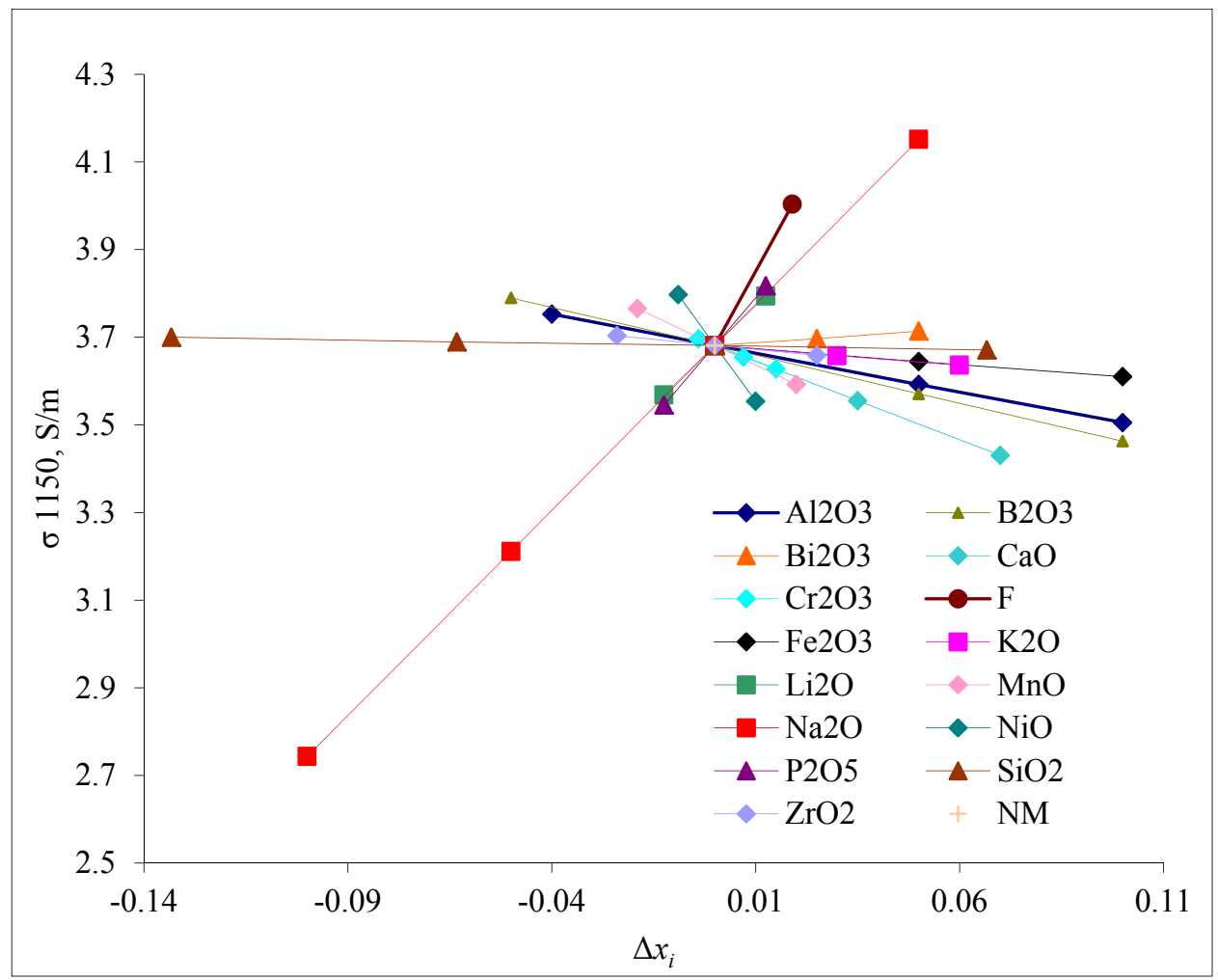

Figure 3.6. Spider Plot for EC Data as a Function of Compositional Deviation from the Baseline ( $\Delta x_{i}$ in mass fraction)

\subsection{Liquidus Temperature $\left(T_{\mathrm{L}}\right)$}

Liquidus temperature $\left(T_{\mathrm{L}}\right)$ was measured on all of these glasses using both optical and by crystal fraction as a function of temperature (semiquantitative analysis by XRD method) though some data points were not achieved due to difficulty with the measurements at elevated temperatures. The data was analyzed and models were generated from the data. Some gaps were observed in the variation of $T_{\mathrm{L}}$ as a function of each component variation from the baseline composition.

Eight additional glasses were added to the original experimental plan to verify the gaps in the optical $T_{\mathrm{L}}$ data and these glasses include: EM07-Al-225, EM07-B-125, EM07-B-175, EM07-Cr-0145, EM07-Li00, EM07-Li-05, EM07-Na-225, EM07-P-02, EM07-P-03, and EM07-Si-335. Optical $T_{\mathrm{L}}$ measurements for EM07-Al-225 and EM07-Cr-0145 were abandoned because not enough glass was available to complete the analysis. EM07-B-125 and EM07-B-175 were omitted from the data processing after careful consideration due to problems with the data. There was a significant difference from the baseline for these to glasses as they showed an enormous inconsistency with the other data points for boron variations. EM07-Na-225 was significantly different from the other sodium variant glasses in the matrix and was included on the spider plot (Figure 3.7) for optical $T_{\mathrm{L}}$ but was omitted from the data used to generate the $1^{\text {st }}$ order model as an outlier. 
A spider plot was constructed to observe the variations in $T_{\mathrm{L}}$ of glasses in comparison to the mass fraction change in composition from the baseline (EM07-BL-1) of the $i$-th oxide component, $\Delta g_{i}$, and is presented in Figure 3.7. The component with the most dramatic positive effect on optical $T_{L}$ is $\mathrm{Cr}_{2} \mathrm{O}_{3}$ (slope of $\left.258^{\circ} \mathrm{C} / \mathrm{mass} \%\right)$ and the $2^{\text {nd }}$ most impactful component was $\mathrm{NiO}\left(83^{\circ} \mathrm{C} / \mathrm{mass} \%\right)$. The largest negative effect was caused by the change in $\mathrm{Li}_{2} \mathrm{O}, \mathrm{Na}_{2} \mathrm{O}$ and $\mathrm{K}_{2} \mathrm{O}\left(-46,-34\right.$ and $-30{ }^{\circ} \mathrm{C} / \mathrm{mass} \%$, respectively).

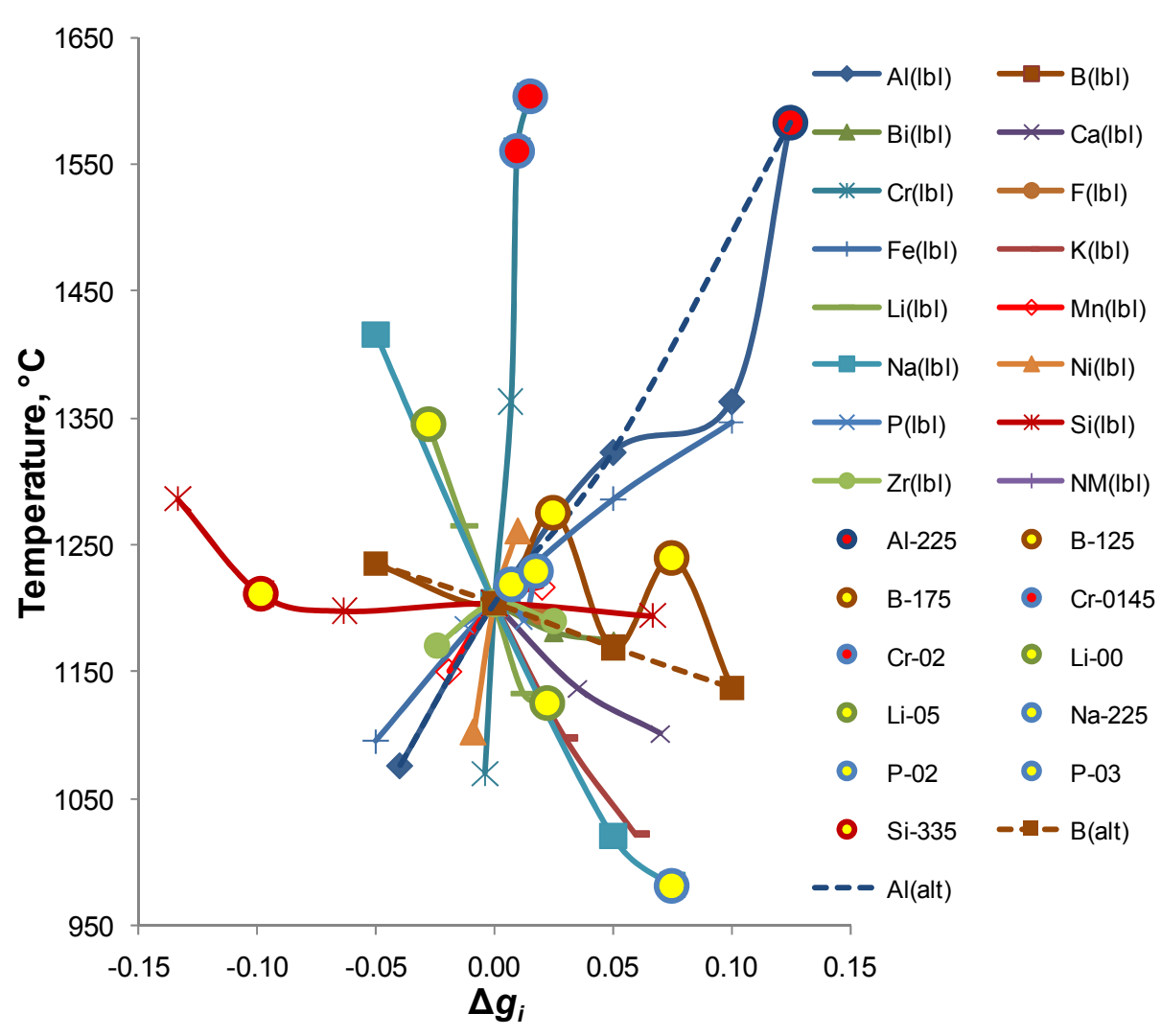

Figure 3.7. Spider Plot of Optical $T_{\mathrm{L}}$ as Determined by Optical Method vs. Change in Composition from the Baseline (designated by element of oxide components). This spider plot shows the location of additional glasses made for verifying data gaps from the original study. Yellow circles points show data points of new glasses that were measured by the optical method (EM07-B-125, B-175, Li-00, Li-05, Na-225, P-02, P-03, and Si-335) and red circles show data points of glasses that could not be measured by the optical method but were measured by the crystal fraction method (EM07-Al-225, Cr-0145, and Cr-02).

\subsubsection{Optical $T_{L}$}

A log of the heat treatments and optical observations for the Matrix I and HWL (Matrix II by SRNL) glasses is documented in Appendix D. For each sample, $T_{\mathrm{c}}$ and $T_{\mathrm{a}}$ values were first obtained, where $T_{\mathrm{c}}$ is the lower bound of liquidus temperature (highest temperature heat treated specimen containing crystals) and $T_{\mathrm{a}}$ is the upper bound of liquidus temperature (lowest temperature heat treated specimen not containing crystals), and then a $T_{\mathrm{L}}$ was determined from these $T_{\mathrm{c}}$ and $T_{\mathrm{a}}$ values where the cognizant scientist extrapolated the $T_{\mathrm{L}}$ as a value between $T_{\mathrm{c}}$ and $T_{\mathrm{a}}$ though not necessarily the average of $T_{\mathrm{c}}$ and $T_{\mathrm{a}}$. 
The $T_{\mathrm{c}}, T_{\mathrm{a}}$, and $T_{\mathrm{L}}$ data for the EM07 glasses are summarized in Table 3.5. Glasses EM07-Cr-02 and EM07-Na-05 were found to have such high liquidus temperatures that they were abandoned (optically) due to their tendency to crystallize severely upon cooling and listed as having a $T_{\mathrm{L}}$ values higher than $1500^{\circ} \mathrm{C}$ and $1361^{\circ} \mathrm{C}$, respectively.

In the glasses with measured $T_{\mathrm{L}} \sim 1250^{\circ} \mathrm{C}$ and above, a $\mathrm{Fe}_{2} \mathrm{O}_{3}$ to $\mathrm{FeO}$ reduction was observed at high resulting in a very dark glassy matrix where visible spinels could only be seen optically by the Fedepleted halo surrounding the opaque spinel crystals. In order to optically observe the spinels in the high temperature heat treatments $\left(T \geq 1250^{\circ} \mathrm{C}\right)$ for the high $T_{\mathrm{L}}$ glasses, the thin sections made of heat treatment specimens had to be polished extremely thin.

\subsubsection{Crystal Fraction $\left(C_{\mathrm{F}}\right) T_{\mathrm{L}}$}

For each glass composition, several heat treatment specimens were prepared to cover a large $\Delta T$ to make the crystal fraction extrapolation method ( or $C_{\mathrm{F}}$ ) more efficient and reduce error in the calculations. Heat treated specimens were doped with $5 \mathrm{mass} \% \mathrm{CaF}_{2}$, milled to powderize the glass and adequately mix, and then analyzed using XRD. The $\mathrm{CaF}_{2}$ doped into the specimen was used as a standard for semiquantitative analysis to determine the mass fraction of crystal phase(s) in a specimen. For the equilibrium $C_{\mathrm{F}}$ as a function of temperature, heat treatments were run from $T_{\mathrm{L}}$ down towards at temperature of $\sim 2-3$ mass $\%$ crystallinity (more in some glasses).

The mass $\%$ of each crystalline phase was plotted versus temperature and the crystal mass fraction was extrapolated to zero mass\% yielding the " $T_{\mathrm{L}}$ Mass\%" value for each glass (see Table 3.5). Once $C_{\mathrm{F}}$ data were calculated for each heat treatment, the remaining mass of glass could be calculated as a difference. The mass fraction of glass, $g_{\mathrm{g}}$, is given by

$$
g_{g}=g_{t}-\sum_{i=1}^{N} g_{c, i}
$$

where $g_{\mathrm{t}}$ is the total mass (in this case, the value is normalized to 1 and thus component values are mass fractions) and $g_{\mathrm{c}}$ is the mass fraction of a given crystalline phase. By converting the $g_{\mathrm{i}}$ values into mole fractions, $M_{\mathrm{i}}$, the density of glass, $\rho_{\mathrm{g}}$, can be computed using Equation (5)

$$
\rho_{g}=\sum_{i=1}^{N} M_{i} M W_{i} / \sum_{i=1}^{N} M_{i} V_{M, i}
$$

where $M_{\mathrm{i}}$ is the mole fraction of the $\mathrm{i}$-th component additive, $M W_{\mathrm{i}}$ is the molecular weight of the $\mathrm{i}$-th oxide, and $V_{\mathrm{M}, \mathrm{i}}$ is the molar volume of the $\mathrm{i}$-th component additive explained elsewhere (Vienna, 2002). The total volume of each heat treatment, $V_{\mathrm{HT}}$, was calculated using Equation (6)

$$
V_{H T}=g_{g} / \rho_{g}+\sum_{i=1}^{N} g_{i} / \rho_{i}
$$


where $g_{\mathrm{i}}$ is the mass fraction of component $\mathrm{i}$ of $N$ total phases in the heat treated specimen and $\rho_{\mathrm{i}}$ is the density of component $\mathrm{i}$; the value used for spinel, $\rho_{\text {trevorite }}=5.33 \mathrm{~g} / \mathrm{cm}^{3}$ since trevorite was the primary spinel phase determined by XRD for all glasses (Lide 2007-2008). The volume \% of the i-th component, $V_{\mathrm{i}}$, in the heat treated specimen is denoted by

$$
V_{i}=100 \cdot \frac{g_{i}}{\rho_{i} \cdot V_{H T}}
$$

The values of $V_{\mathrm{i}}$ were plotted as a function of temperature and a linear correlation fit to the data using $V_{\mathrm{i}}=m T+b$ where $T_{1 \%}=\left(V_{\mathrm{i}}-b\right) / m$ when $V_{\mathrm{i}}=1$ and the temperature at which each glass would contain $1 \mathrm{vol} \%, T_{1 \%}$, of trevorite was calculated. This data is presented in Table 3.5. In general, " $T_{\mathrm{L}}$ Optical" data aligns reasonably well with the crystal fraction $T_{\mathrm{L}}$ (" $T_{\mathrm{L}}$ Mass\%" and $T_{\mathrm{L}} \mathrm{Vol} \%$ " data.

Table 3.5. Summary of Liquidus Temperature Results. Tabulated below are the results for $T_{\mathrm{L}}$ determined using optical observations (" $T_{\mathrm{c}}$ ", " $T_{\mathrm{a}}$ ", and " $T_{\mathrm{L}}$, Optical"), crystal fraction results for both mass\% (" $T_{\mathrm{L}}$ Mass\%") and volume\% (" $T_{\mathrm{L}}$ Vol\%") data, and $T_{1 \%}$. Additional data are included such as the \# of temperatures used to calculate crystal fraction ("\# Pts"), the slope of the line used to calculate the crystal fraction (temperature vs. crystal fraction, "Slope"), as well as the furnace used to make the measurements for the data presented ("Furnace Used"). Glasses that precipitated micron-sized crystals upon cooling are listed with Glass ID in bold and italics. The primary phase in all glasses was spinel. Crystal fraction and optical $T_{\mathrm{L}}$ were not determined for all of the glasses.

\begin{tabular}{|c|c|c|c|c|c|c|c|c|c|}
\hline & \multicolumn{3}{|c|}{ Optical Values } & \multicolumn{3}{|c|}{ Mass\% Values } & \multicolumn{2}{|c|}{ Vol\% Values } & \multirow[b]{2}{*}{$\begin{array}{c}\text { Furnace } \\
\text { Used }\end{array}$} \\
\hline Glass ID & $\begin{array}{c}T_{\mathrm{c}} \\
\left({ }^{\circ} \mathbf{C}\right)\end{array}$ & $\begin{array}{c}T_{\mathrm{a}} \\
\left({ }^{\circ} \mathrm{C}\right)\end{array}$ & $\begin{array}{c}T_{\mathrm{L}} \\
\text { Optical } \\
\left({ }^{\circ} \mathrm{C}\right) \\
\end{array}$ & \# Pts & Slope & $\begin{array}{c}T_{\mathrm{L}} \\
\operatorname{Mass} \% \\
\left({ }^{\circ} \mathrm{C}\right)\end{array}$ & $\begin{array}{c}T_{\mathrm{L}} \\
\mathrm{Vol} \% \\
\left({ }^{\circ} \mathrm{C}\right) \\
\end{array}$ & $\begin{array}{l}T_{1 \%} \\
\left({ }^{\circ} \mathbf{C}\right)\end{array}$ & \\
\hline EM07-BL-1 & 1200 & 1208 & 1204 & 4 & -116.2 & 1181 & 1180 & 952.9 & 10 \\
\hline EM07-BL-2 & 1201 & 1208 & 1204 & 3 & -96.92 & 1182 & 1182 & 992.6 & 10 \\
\hline EM07-Al-06 & 1074 & 1077 & 1076 & 4 & -84.62 & 1086 & 1086 & 921.3 & 10 \\
\hline EM07-Al-15 & 1318 & 1327 & 1323 & 3 & -135.1 & 1297 & 1296 & 1030 & 5 \\
\hline EM07-Al-20 & 1360 & 1364 & 1363 & 3 & -101.0 & 1380 & 1377 & 1178 & 5 \\
\hline EM07-Al-225 & \multicolumn{3}{|c|}{ Not measured for this glass } & 3 & -73.58 & 1581 & 1575 & 1432 & 5 \\
\hline EM07-B-05 & 1230 & 1239 & 1235 & 4 & -107.7 & 1228 & 1227 & 1018 & 10 \\
\hline EM07-B-125 & 1276 & 1281 & 1271 & \multicolumn{5}{|c|}{ Not measured for this glass } & 8 \\
\hline EM07-B-15 & 1165 & 1173 & 1169 & 3 & -66.13 & 1107 & 1106 & 976.6 & 10 \\
\hline EM07-B-175 & 1234 & 1244 & 1239 & \multicolumn{5}{|c|}{ Not measured for this glass } & 5 \\
\hline EM07-B-20 & 1134 & 1140 & 1137 & 4 & -172.1 & 1143 & 1143 & 798.3 & 8 \\
\hline EM07-Bi-025 & 1177 & 1184 & 1181 & 4 & -121.4 & 1173 & 1173 & 939.7 & 8 \\
\hline EM07-Bi-05 & 1170 & 1177 & 1174 & 4 & -146.3 & 1174 & 1174 & 897.8 & 10 \\
\hline EM07-Ca-035 & 1134 & 1141 & 1137 & 5 & -92.52 & 1126 & 1125 & 946.2 & 8 \\
\hline EM07-Ca-07 & 1099 & 1102 & 1101 & 4 & -104.4 & 1112 & 1111 & 910.6 & 5 \\
\hline EM07-Cr-001 & 1065 & 1074 & 1070 & 5 & -86.68 & 1067 & 1067 & 896.6 & 8 \\
\hline
\end{tabular}


PNNL-17950, Rev 0

\begin{tabular}{|c|c|c|c|c|c|c|c|c|c|}
\hline & \multicolumn{3}{|c|}{ Optical Values } & \multicolumn{3}{|c|}{ Mass\% Values } & \multicolumn{2}{|c|}{ Vol\% Values } & \multirow[b]{2}{*}{$\begin{array}{c}\text { Furnace } \\
\text { Used }\end{array}$} \\
\hline Glass ID & $\begin{array}{c}T_{\mathrm{c}} \\
\left({ }^{\circ} \mathrm{C}\right)\end{array}$ & $\begin{array}{c}T_{\mathrm{a}} \\
\left({ }^{\circ} \mathrm{C}\right)\end{array}$ & $\begin{array}{c}T_{\mathrm{L}} \\
\text { Optical } \\
\left({ }^{\circ} \mathrm{C}\right)\end{array}$ & \# Pts & Slope & $\begin{array}{c}T_{\mathrm{L}} \\
\operatorname{Mass} \% \\
\left({ }^{\circ} \mathrm{C}\right)\end{array}$ & $\begin{array}{c}T_{\mathrm{L}} \\
\mathrm{Vol} \% \\
\left({ }^{\circ} \mathrm{C}\right) \\
\end{array}$ & $\begin{array}{l}T_{1 \%} \\
\left({ }^{\circ} \mathrm{C}\right)\end{array}$ & \\
\hline EM07-Cr-012 & 1360 & 1364 & 1363 & 3 & -125.5 & 1320 & 1319 & 1075 & 5 \\
\hline EM07-Cr-0145 & \multicolumn{3}{|c|}{ Not measured for this glass } & 3 & -166.0 & 1561 & 1558 & 1237 & 5 \\
\hline EM07-Cr-02 & \multicolumn{3}{|c|}{ Not measured for this glass } & 4 & -189.3 & 1603 & 1599 & 1235 & N/A \\
\hline EM07-F-02 & 1191 & 1198 & 1195 & 4 & -158.3 & 1182 & 1181 & 870.5 & 8 \\
\hline EM07-Fe-05 & 1090 & 1099 & 1095 & 5 & -154.6 & 1065 & 1065 & 755.5 & 5 \\
\hline EM07-Fe-15 & 1281 & 1292 & 1286 & 4 & -110.0 & 1270 & 1269 & 1058 & 5 \\
\hline EM07-Fe-20 & 1343 & 1350 & 1347 & 3 & -88.21 & 1398 & 1394 & 1232 & 5 \\
\hline EM07-K-03 & 1093 & 1099 & 1098 & 4 & -101.1 & 1105 & 1105 & 906.4 & 5 \\
\hline EM07-K-06 & 1018 & 1024 & 1022 & 3 & -112.9 & 1048 & 1047 & 825.8 & 8 \\
\hline EM07-Li-00 & 1340 & 1349 & 1345 & \multicolumn{5}{|c|}{ Not measured for this glass } & 5 \\
\hline EM07-Li-015 & 1262 & 1268 & 1265 & 3 & -165.0 & 1283 & 1283 & 958.9 & 5 \\
\hline EM07-Li-04 & 1129 & 1134 & 1133 & 5 & -60.13 & 1075 & 1074 & 957.1 & 8 \\
\hline EM07-Li-05 & 1121 & 1131 & 1126 & \multicolumn{5}{|c|}{ Not measured for this glass } & 5 \\
\hline EM07-Mn-01 & 1148 & 1151 & 1150 & 3 & -113.1 & 1134 & 1133 & 910.3 & 8 \\
\hline EM07-Mn-04 & 1213 & 1220 & 1217 & 4 & -91.87 & 1143 & 1143 & 965.6 & 5 \\
\hline EM07-Na-05 & \multicolumn{3}{|c|}{ Not measured for this glass } & 2 & -100.0 & 1598 & 1581 & 1393 & $\mathrm{~N} / \mathrm{A}$ \\
\hline EM07-Na-10 & 1410 & 1418 & 1416 & 3 & -150.0 & 1424 & 1423 & 1129 & 5 \\
\hline EM07-Na-20 & 1018 & 1024 & 1021 & 4 & -90.95 & 1006 & 1005 & 828.7 & 8 \\
\hline EM07-Na-225 & 972 & 992 & 982 & 3 & -127.8 & 978 & 978 & 729.8 & 8 \\
\hline EM07-Ni-001 & 1099 & 1105 & 1102 & 5 & -156.5 & 1076 & 1076 & 766.4 & 5 \\
\hline EM07-Ni-02 & 1257 & 1264 & 1261 & 4 & -115.0 & 1264 & 1263 & 1040 & 8 \\
\hline EM07-P-0 & 1184 & 1191 & 1187 & 5 & -104.9 & 1174 & 1173 & 968.8 & 8 \\
\hline EM07-P-02 & 1215 & 1224 & 1220 & \multicolumn{5}{|c|}{ Not measured for this glass } & 5 \\
\hline EM07-P-03 & 1224 & 1234 & 1229 & \multicolumn{5}{|c|}{ Not measured for this glass } & 5 \\
\hline EM07-Si-30 & 1280 & 1288 & 1286 & 3 & -101.9 & 1284 & 1284 & 1091 & 8 \\
\hline EM07-Si-335 & 1208 & 1215 & 1212 & \multicolumn{5}{|c|}{ Not measured for this glass } & 5 \\
\hline EM07-Si-37 & 1194 & 1201 & 1198 & 4 & -73.17 & 1198 & 1197 & 1057 & 10 \\
\hline EM07-Si-50 & 1190 & 1197 & 1194 & 4 & -147.3 & 1184 & 1183 & 890.0 & 10 \\
\hline EM07-Zr-001 & 1166 & 1176 & 1171 & 4 & -93.50 & 1160 & 1160 & 974.8 & 8 \\
\hline EM07-Zr-05 & 1184 & 1191 & 1190 & 3 & -108.2 & 1170 & 1170 & 960.7 & 8 \\
\hline EM07-NM-0025 & 1203 & 1214 & 1206 & 3 & -129.8 & 1220 & 1220 & 965.5 & 10 \\
\hline
\end{tabular}

Since the data for $T_{\mathrm{L}}$, Mass $\%$ and $T_{\mathrm{L}}, \mathrm{Vol} \%$ were so similar, only a spider plot for $T_{\mathrm{L}}$, Mass $\%$ was assembled for the extrapolated $T_{\mathrm{L}}$ obtained using crystal fraction data for each glass in Table 3.5 with crystal fraction data as a function of the concentration change from EM07-BL-1 of the i-th oxide 
component, $\Delta g_{i}$, and can be found in Figure 3.8. The effect of component concentration change on the $T_{1 \%}$ is presented in Figure 3.9 for those glasses in Table 3.5 with crystal fraction data. Figure 3.10 compares the $T_{\mathrm{L}}$ Mass $\%$ data with the optical $T_{\mathrm{L}}$ data.

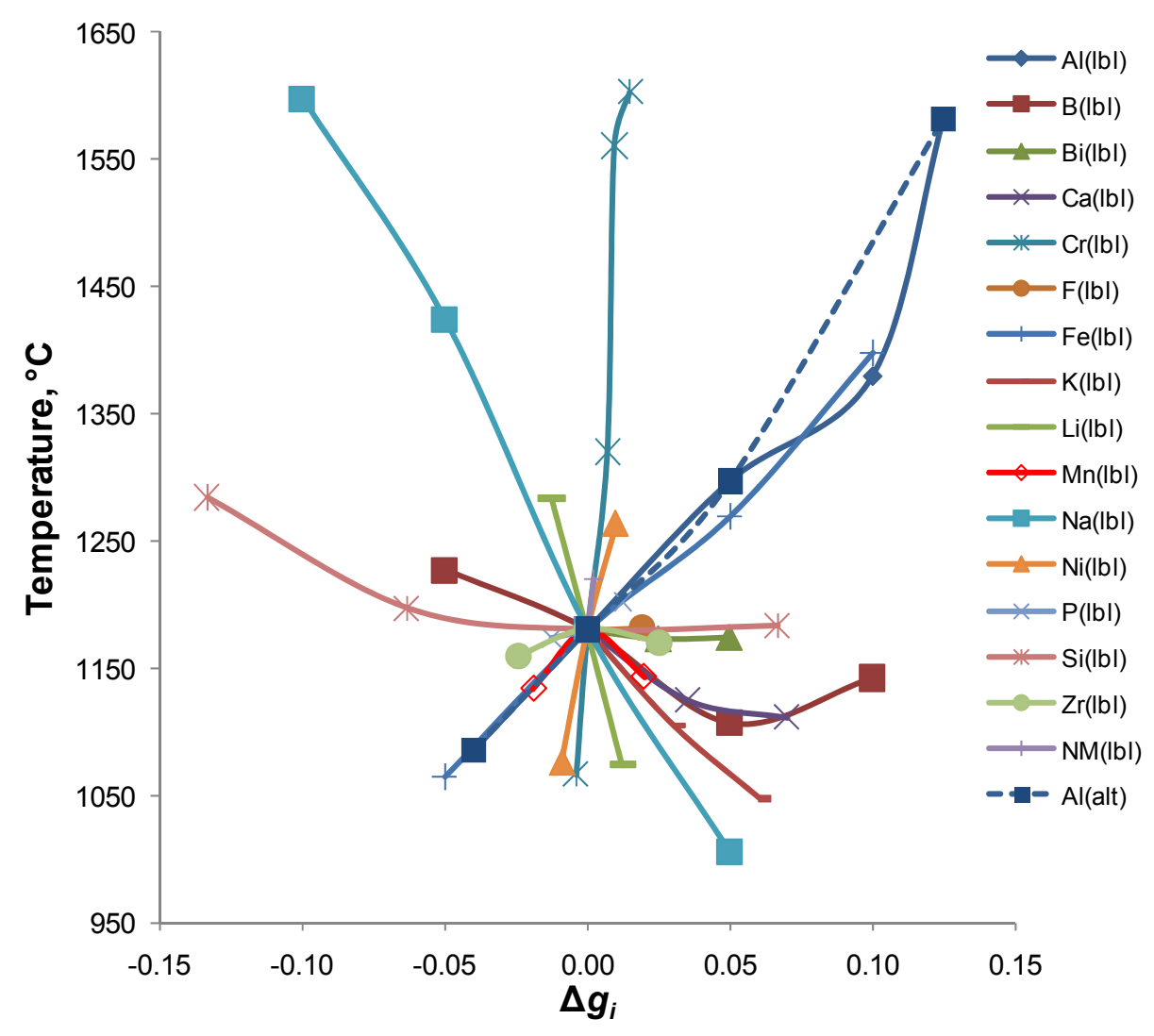

Figure 3.8. Spider Plot of Crystal Fraction $T_{\mathrm{L}}$, Mass $\%$ as Determined by XRD Crystal Fraction Method vs. Change in Composition from the Baseline as $\Delta g_{\mathrm{i}}$. 
PNNL-17950, Rev 0

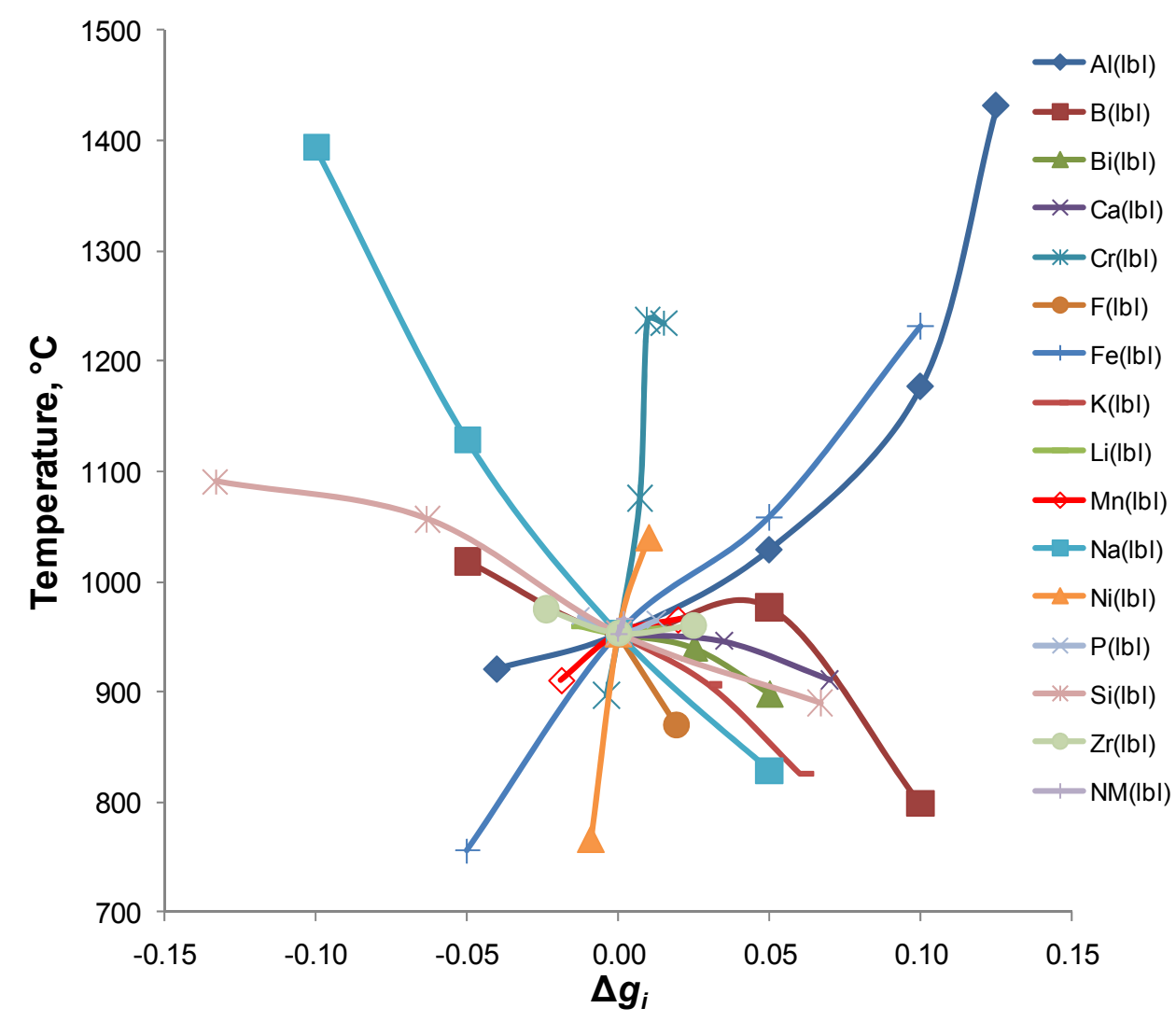

Figure 3.9. Spider Plot of $T_{1 \%}$ for EM07 Glasses as Determined by XRD Crystal Fraction Method vs. Change in Composition from the Baseline as $\Delta g_{\mathrm{i}}$.

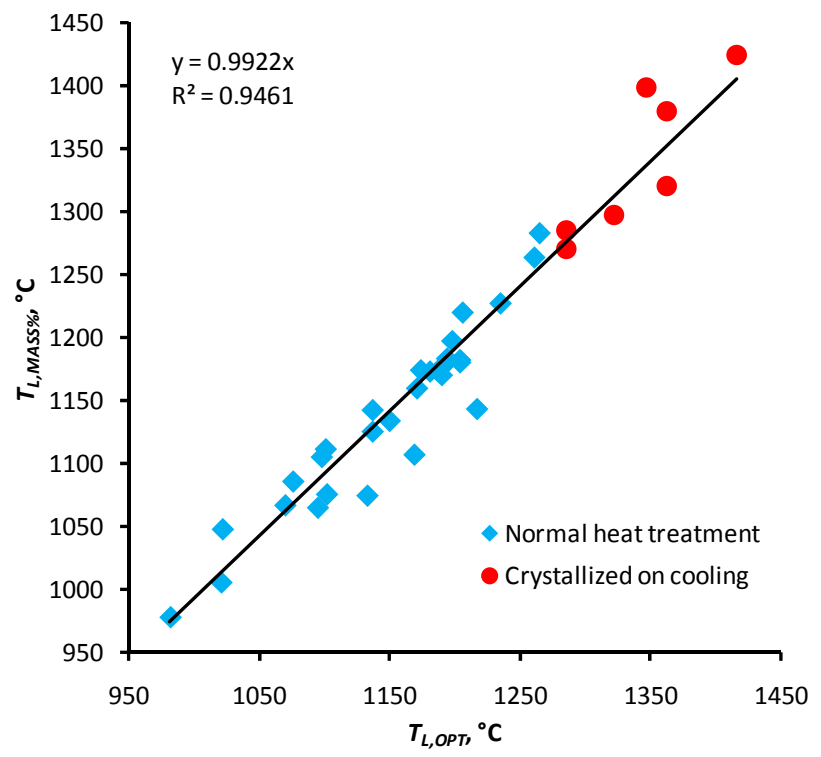

Figure 3.10. Comparison of $T_{\mathrm{L}}$ Data Obtained Using XRD (mass\%) Methods against Data Obtained Using Optical Methods for the 36 Glasses for Which Data was Collected Using Both Methods. Trend line presented runs through all data points presented. 
First-order, standard least squares models were fit to the data for Optical $T_{\mathrm{L}}, T_{\mathrm{L}}$ Mass $\%$, and $T_{1 \%}$ (using volume \%) using JMP ${ }^{\circledR} 6.0$ software and the coefficients are presented in Table 3.6.

Table 3.6. First-Order Regression Coefficients for the Calculated vs. Measured $T_{\mathrm{L}}$ data. ${ }^{*}$ The data presented for "Others" refers to the minor components in the glasses (i.e., $\mathrm{BaO}, \mathrm{CdO}, \mathrm{MgO}$, $\mathrm{PbO}, \mathrm{PdO}, \mathrm{Rh}_{2} \mathrm{O}_{3}, \mathrm{RuO}_{2}, \mathrm{SO}_{3}, \mathrm{SrO}, \mathrm{Ce}_{2} \mathrm{O}_{3}, \mathrm{La}_{2} \mathrm{O}_{3}, \mathrm{Nd}_{2} \mathrm{O}_{3}, \mathrm{TiO}_{2}$, and $\mathrm{ZnO}$ ).

\begin{tabular}{|l|c|c|c|}
\hline & Optical $\boldsymbol{T}_{\boldsymbol{L}}$ & $\boldsymbol{T}_{\boldsymbol{L}}$ Mass\% & $\boldsymbol{T}_{\mathbf{1}} \%$ \\
\hline $\mathrm{Al}_{2} \mathrm{O}_{3}$ & 2975 & 3572 & 3550 \\
\hline $\mathrm{B}_{2} \mathrm{O}_{3}$ & 645.7 & 573.4 & -138.9 \\
\hline $\mathrm{Bi}_{2} \mathrm{O}_{3}$ & 719.0 & 965.7 & -231.4 \\
\hline $\mathrm{CaO}$ & -230.4 & 37.42 & 292.6 \\
\hline $\mathrm{Cr}_{2} \mathrm{O}_{3}$ & 26835 & 30792 & 21307 \\
\hline $\mathrm{F}$ & 1134 & 1108 & -3823 \\
\hline $\mathrm{Fe}_{2} \mathrm{O}_{3}$ & 2677 & 3083 & 3494 \\
\hline $\mathrm{K}_{2} \mathrm{O}$ & -1782 & -1138 & -1215 \\
\hline $\mathrm{Li}_{2} \mathrm{O}$ & -3316 & -7027 & 958.1 \\
\hline $\mathrm{MnO}$ & 2875 & 1336 & 2342 \\
\hline $\mathrm{Na}_{2} \mathrm{O}$ & -1664 & -2029 & -2148 \\
\hline $\mathrm{NiO}_{2}$ & 9405 & 10837 & 14908 \\
\hline $\mathrm{P}_{2} \mathrm{O}_{5}$ & 2859 & 1822 & 443.0 \\
\hline $\mathrm{SiO}_{2}$ & 980.6 & 866.1 & 360.3 \\
\hline $\mathrm{ZrO}_{2}$ & 1572 & 1356 & 701.3 \\
\hline $\mathrm{Others}_{2}$ & 7670 & 15014 & 5670 \\
\hline
\end{tabular}

Using the coefficients listed in Table 3.6 and Equation (8) $T_{\mathrm{L}}$ values were calculated for each glass for Optical $T_{\mathrm{L}}, T_{\mathrm{L}}$ Mass\%, and $T_{1 \%}$ methods. In Equations (8) $J$ is the method used for calculating $T_{\mathrm{L}}, N$ is the number of applicable glasses $\left(N=41\right.$ for Optical $T_{L}$ and $N=40$ for $T_{\mathrm{L}}$ Mass $\%$ and $\left.T_{1 \%}\right), g_{\mathrm{i}}$ is the i-th oxide component addition to the glass (in mass fraction), and $b_{\mathrm{i}}$ is the $\mathrm{i}$-th oxide additive component coefficient (see Table 3.6). These values are presented in Table 3.7 along with the measured values. For all cases, the calculated values align well with the measured values and the correlations are plotted in Figure 3.11.

$$
T_{L, J}=\sum_{i=1}^{N} g_{i} b_{i}
$$

Table 3.7. Calculated Data for the Three Different Regression Data Fits Presented in Table 3.6. Values were calculated even for glasses where data could not be measured (see Table 3.5).

\begin{tabular}{|l|c|c|c|}
\hline Glass ID & $\boldsymbol{T}_{\mathrm{L}, \mathbf{O P T}}\left({ }^{\circ} \mathbf{C}\right)$ & $\boldsymbol{T}_{\mathrm{L}, \mathbf{M A S S}}\left({ }^{\circ} \mathbf{C}\right)$ & $\boldsymbol{T}_{\mathbf{1} \%}\left({ }^{\circ} \mathbf{C}\right)$ \\
\hline EM07-BL-1 & 1196 & 1183 & 961 \\
\hline EM07-BL-2 & 1196 & 1183 & 961 \\
\hline EM07-Al-06 & 1117 & 1077 & 846 \\
\hline EM07-Al-15 & 1295 & 1316 & 1105 \\
\hline
\end{tabular}


PNNL-17950, Rev 0

\begin{tabular}{|l|c|c|c|}
\hline Glass ID & $\boldsymbol{T}_{\mathbf{L}, \mathbf{O P T}}\left({ }^{\circ} \mathbf{C}\right)$ & $\boldsymbol{T}_{\mathbf{L}, \mathbf{M A S S}}{ }^{\left({ }^{\circ} \mathbf{C}\right)}$ & $\left.\boldsymbol{T}_{\mathbf{1} \%}{ }^{\circ} \mathbf{C}\right)$ \\
\hline EM07-Al-20 & 1394 & 1449 & 1249 \\
\hline EM07-Al-225 & 1443 & 1515 & 1321 \\
\hline EM07-B-05 & 1227 & 1217 & 1023 \\
\hline EM07-B-15 & 1166 & 1149 & 900 \\
\hline EM07-B-20 & 1135 & 1115 & 839 \\
\hline EM07-Bi-025 & 1184 & 1178 & 932 \\
\hline EM07-Bi-05 & 1172 & 1172 & 902 \\
\hline EM07-Ca-035 & 1146 & 1143 & 938 \\
\hline EM07-Ca-07 & 1096 & 1103 & 915 \\
\hline EM07-Cr-001 & 1093 & 1064 & 880 \\
\hline EM07-Cr-012 & 1377 & 1391 & 1105 \\
\hline EM07-Cr-0145 & 1441 & 1466 & 1156 \\
\hline EM07-Cr-02 & 1583 & 1629 & 1268 \\
\hline EM07-F-02 & 1195 & 1182 & 870 \\
\hline EM07-Fe-05 & 1114 & 1078 & 821 \\
\hline EM07-Fe-15 & 1278 & 1289 & 1102 \\
\hline EM07-Fe-20 & 1361 & 1394 & 1243 \\
\hline EM07-K-03 & 1107 & 1113 & 896 \\
\hline EM07-K-06 & 1018 & 1044 & 831 \\
\hline EM07-Li-00 & 1324 & 1415 & 962 \\
\hline EM07-Li-015 & 1254 & 1289 & 962 \\
\hline EM07-Li-04 & 1138 & 1078 & 961 \\
\hline EM07-Li-05 & 1092 & 993 & 961 \\
\hline EM07-Mn-01 & 1164 & 1180 & 935 \\
\hline EM07-Mn-04 & 1230 & 1186 & 990 \\
\hline EM07-Na-05 & 1533 & 1561 & 1327 \\
\hline EM07-Na-10 & 1364 & 1372 & 1144 \\
\hline EM07-Na-20 & 1028 & 994 & 779 \\
\hline EM07-Na-225 & 944 & 900 & 687 \\
\hline EM07-Ni-001 & 1122 & 1095 & 835 \\
\hline EM07-Ni-02 & 1279 & 1281 & 1102 \\
\hline EM07-P-0 & 1175 & 1175 & 968 \\
\hline EM07-P-02 & 1209 & 1188 & 958 \\
\hline EM07-P-03 & 1226 & 1194 & 952 \\
\hline EM07-Si-30 & 1247 & 1258 & 1103 \\
\hline EM07-Si-335 & 1234 & 1238 & 1066 \\
\hline EM07-Si-37 & 1220 & 1218 & 1029 \\
\hline EM07-Si-50 & 1171 & 1146 & 891 \\
\hline EM07-Zr-001 & 1187 & 1179 & 968 \\
\hline EM07-Zr-05 & 1206 & 1188 & 955 \\
\hline EM07-NM-0025 & 1210 & 1212 & 971 \\
\hline
\end{tabular}



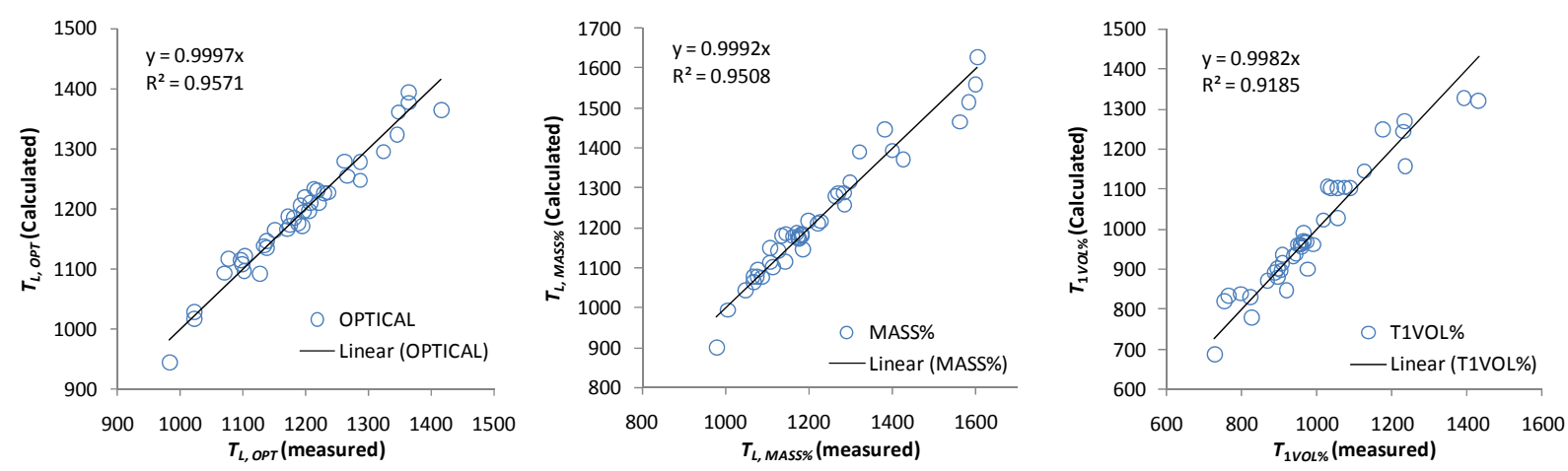

Figure 3.11. Calculated vs. Measured Data for the Different Data Sets.

These models are not valid through the compositional range listed in Table 1.2 but the validity ranges for each $T_{\mathrm{L}}$ measurement method are dictated by the component range for which data was measured successfully (Table 3.5). For example, EM07-Al-225 was not successfully measured with $T_{\mathrm{L}, \mathrm{OPT}}$ and thus, the upper validity limit for $\mathrm{Al}_{2} \mathrm{O}_{3}$ additions to the baseline composition is 20 mass $\% \mathrm{Al}_{2} \mathrm{O}_{3}$ (EM07-Al-20), the next highest $\mathrm{Al}_{2} \mathrm{O}_{3}$-loaded glass tested in this study.

\subsection{Canister Centerline Cooling (CCC)}

See Appendix B for XRD data obtained from CCC heat treatments. XRD scans of CCC samples identified spinel crystals in all 38 Matrix I glass samples. Crystal content in the matrix glasses ranged from a minimum of 2.2 mass \% observed in EM07-Fe-05 and a maximum of 37 mass\% present in EM07Si-30. Nineteen samples were evaluated by quantitative analysis using RIQAS and are in Table 3.8 as values without an approximation $(\sim)$ symbol. All other weight percent determinations of crystal content are estimations.

Thirty two samples (84\%) have crystal content between 2.2 and about 6 mass\%. Four samples have crystal content between approximately 7 and 8.3 mass\% while two samples, EM07-Na-05 and Si-30, have greater than 10 mass $\%$ crystallinity. These last two samples contain zircon and nepheline respectively in addition to spinel. 
PNNL-17950, Rev 0

Table 3.8. Determination of Weight Percent Crystallinity and Identification of Crystals of CCC Heat Treated Glasses by XRD

\begin{tabular}{|c|c|c|}
\hline Glass-ID & $\begin{array}{l}\text { Mass\% } \\
\text { Crystallinity }\end{array}$ & $\begin{array}{l}\text { Crystal Phase } \\
\text { Identification }\end{array}$ \\
\hline EM07-BL-1 & $\overline{\sim 5}$ & Trevorite \\
\hline EM07-BL-2 & $\sim 5$ & Trevorite \\
\hline EM07-Al-06 & $\sim 5$ & Trevorite \\
\hline EM07-Al-15 & $\sim 7$ & Chromite \\
\hline EM07-Al-20 & $7-8$ & Magnetite \\
\hline EM09-Al-22.5 & $\begin{array}{l}6.6 \\
14.4\end{array}$ & $\begin{array}{l}\text { Magnetite } \\
\text { Nepheline }\end{array}$ \\
\hline EM07-B-05 & $\sim 4$ & Trevorite \\
\hline EM09-B-125 & 3.9 & Magnetite \\
\hline EM07-B-15 & $\sim 4$ & Trevorite \\
\hline EM09-B-175 & 0.8 & Magnetite \\
\hline EM07-B-20 & $5-6$ & Magnetite \\
\hline EM07-Bi-025 & $\sim 4$ & Trevorite \\
\hline EM07-Bi-05 & 4.2 & Trevorite \\
\hline EM07-Ca-035 & $\sim 4$ & Chromite \\
\hline EM07-Ca-07 & 2.9 & Trevorite \\
\hline EM07-Cr-001 & $\sim 4$ & Trevorite \\
\hline EM07-Cr-012 & $\sim 5$ & Trevorite \\
\hline EM09-Cr-0145 & 3.7 & Magnetite \\
\hline EM07-Cr-02 & $\sim 6$ & Trevorite \\
\hline EM07-F-02 & $\sim 3.5$ & Chromite \\
\hline EM07-Fe-05 & 2.2 & Trevorite \\
\hline EM07-Fe-15 & 7.4 & Chromite \\
\hline EM07-Fe-20 & 8.3 & Chromite \\
\hline EM07-K-03 & $\sim 4$ & Trevorite \\
\hline EM07-K-06 & 2.8 & Magnetite \\
\hline EM09-Li-0 & 4.1 & Magnetite \\
\hline EM07-Li-015 & $\sim 4$ & Magnetite \\
\hline EM07-Li-04 & $\sim 4$ & Trevorite \\
\hline EM09-Li-05 & $\begin{array}{l}2.5 \\
1.4\end{array}$ & $\begin{array}{l}\text { Magnetite } \\
\text { Corundum }\end{array}$ \\
\hline EM07-Mn-01 & $\sim 4$ & Trevorite \\
\hline EM07-Mn-04 & $\sim 4$ & Chromite \\
\hline EM07-Na-05 & $\begin{array}{l}8.0 \\
2.3 \\
\end{array}$ & $\begin{array}{l}\text { Magnetite } \\
\text { Zircon }\end{array}$ \\
\hline EM07-Na-10 & $\sim 6$ & Chromite \\
\hline EM07-Na-20 & $\sim 3$ & Trevorite \\
\hline EM09-Na-225 & 1.8 & Magnetite \\
\hline EM07-Ni-001 & $\sim 3$ & Trevorite \\
\hline EM07-Ni-02 & $\sim 6$ & Trevorite \\
\hline EM07-P-0 & $\sim 4$ & Trevorite \\
\hline EM09-P-02 & 4.5 & Magnetite \\
\hline EM09-P-03 & $\begin{array}{l}3.7 \\
2.6 \\
3.1\end{array}$ & $\begin{array}{l}\text { Magnetite } \\
\text { Corundum } \\
\text { Lithium Phosphate }\end{array}$ \\
\hline EM07-Si-30 & $\begin{array}{l}27.7 \\
9.2\end{array}$ & $\begin{array}{l}\text { Nepheline } \\
\text { Magnesium iron oxide }\end{array}$ \\
\hline EM09-Si-335 & $\begin{array}{l}5.4 \\
7.5 \\
3.0\end{array}$ & $\begin{array}{l}\text { Magnetite } \\
\text { Nepheline } \\
\text { Corundum }\end{array}$ \\
\hline EM07-Si-37 & 5.9 & Trevorite \\
\hline
\end{tabular}


PNNL-17950, Rev 0

\begin{tabular}{|l|l|l|}
\hline EM07-Si-50 & $\sim 3$ & Trevorite \\
\hline EM07-Zr-001 & $\sim 5$ & Trevorite \\
\hline EM07-Zr-05 & $\sim 6$ & Chromite \\
\hline EM07-NM-0025 & 4.8 & Magnetite \\
\hline
\end{tabular}

\subsection{Product Consistency Test (PCT)}

The results of PCT are published elsewhere (Raszewski et al. 2008b) but are summarized in Table 3.9. Most glasses have a release rate for $\mathrm{Na}$ an order of magnitude below the EA glass and about the same release rate as the ARM-1 glass used as a reference glass in the test. Exceptions to this are EM07Si-30 which had high levels of nepheline crystals in the CCC heat treated glass and had Na release rates (3,600 to 3,800 ppm) over twice that of the EA glass (1,600 to 1,700 ppm). The quenched Si-30 glass was one of the glasses that had elevated $\mathrm{Na}$ release rates $(300 \mathrm{ppm})$ above most EM07 glasses. Other glasses that had similar release rates as quenched Si-30 were EM07-Na-20, both quenched (150 ppm) and CCC (175 ppm) samples and EM07-K-06 CCC samples (150 to $175 \mathrm{ppm}$ ). All other samples were below these levels except for EM07-B-20 which had Na release rates at about 400 and $650 \mathrm{ppm}$ for quenched and CCC samples respectively, the highest release rate except the Si-30 CCC samples.

Table 3.9. PCT Results (in ppm) taken from Raszewski et al. 2008b

\begin{tabular}{|c|c|c|c|c|c|c|c|c|c|}
\hline \multirow{2}{*}{ Glass ID } & \multirow{2}{*}{ Type } & \multicolumn{2}{|r|}{ B } & \multicolumn{2}{|r|}{$\mathrm{Li}$} & \multicolumn{2}{|r|}{$\mathrm{Na}$} & \multicolumn{2}{|r|}{$\mathrm{Si}$} \\
\hline & & Ave. & St. Dev. & Ave. & St. Dev. & Ave. & St. Dev. & Ave. & St. Dev. \\
\hline \multirow{2}{*}{ EM07-BL-1 } & Quenched & 8.82 & 0.55 & 3.63 & 0.13 & 32.8 & 0.36 & 36.7 & 1.55 \\
\hline & $\mathrm{CCC}$ & 8.37 & 0.42 & 3.59 & 0.09 & 30.5 & 0.91 & 38.8 & 1.04 \\
\hline \multirow{2}{*}{ EM07-BL-2 } & Quenched & 8.87 & 0.16 & 3.64 & 0.09 & 31.9 & 0.35 & 35.7 & 0.64 \\
\hline & $\mathrm{CCC}$ & 8.75 & 0.27 & 3.51 & 0.07 & 29.7 & 0.67 & 36.7 & 0.30 \\
\hline \multirow{2}{*}{ EM07-Al-06 } & Quenched & 13.4 & 0.40 & 4.64 & 0.14 & 44.7 & 0.21 & 41.6 & 1.37 \\
\hline & $\mathrm{CCC}$ & 11.5 & 0.25 & 4.15 & 0.09 & 35.7 & 0.71 & 31.8 & 0.40 \\
\hline \multirow{2}{*}{ EM07-Al-15 } & Quenched & 7.86 & 0.21 & 3.90 & 0.07 & 28.0 & 3.93 & 34.7 & 0.23 \\
\hline & $\mathrm{CCC}$ & 7.09 & 0.10 & 3.47 & 0.08 & 25.0 & 0.06 & 37.0 & 0.47 \\
\hline \multirow{2}{*}{ EM07-Al-20 } & Quenched & 8.77 & 2.65 & 5.33 & 1.64 & 27.7 & 8.98 & 44.8 & 13.8 \\
\hline & $\mathrm{CCC}$ & 8.04 & 0.26 & 4.39 & 0.08 & 22.8 & 0.71 & 37.6 & 0.23 \\
\hline \multirow{2}{*}{ EM07-B-05 } & Quenched & 5.38 & 2.32 & 3.84 & 0.10 & 41.5 & 1.00 & 41.3 & 0.72 \\
\hline & $\mathrm{CCC}$ & 5.47 & 0.88 & 3.88 & 0.13 & 39.2 & 0.26 & 42.8 & 0.35 \\
\hline \multirow{2}{*}{ EM07-B-15 } & Quenched & 38.1 & 2.82 & 8.36 & 0.13 & 55.8 & 1.06 & 28.8 & 0.51 \\
\hline & $\mathrm{CCC}$ & 49.0 & 5.39 & 10.3 & 0.20 & 64.5 & 1.59 & 31.1 & 0.45 \\
\hline \multirow{2}{*}{ EM07-B-20 } & Quenched & 248 & 6.24 & 39.2 & 0.44 & 221 & 5.86 & 20.4 & 0.17 \\
\hline & $\mathrm{CCC}$ & 371 & 11.8 & 58.1 & 1.19 & 323 & 6.56 & 20.2 & 0.55 \\
\hline \multirow{2}{*}{ EM07-Bi-025 } & Quenched & 9.74 & 1.09 & 3.98 & 0.11 & 34.1 & 1.01 & 35.0 & 1.16 \\
\hline & $\mathrm{CCC}$ & 10.2 & 3.75 & 3.84 & 0.08 & 30.8 & 0.36 & 36.1 & 1.05 \\
\hline \multirow{2}{*}{ EM07-Bi-05 } & Quenched & 8.24 & 0.55 & 3.87 & 0.13 & 33.0 & 0.56 & 33.5 & 1.01 \\
\hline & $\mathrm{CCC}$ & 8.77 & 0.19 & 3.90 & 0.13 & 31.6 & 0.60 & 35.1 & 1.25 \\
\hline \multirow{2}{*}{ EM07-Ca-035 } & Quenched & 6.59 & 5.36 & 3.83 & - & 37.3 & - & 30.6 & - \\
\hline & $\mathrm{CCC}$ & 8.41 & 0.38 & 3.61 & 0.14 & 32.6 & 1.01 & 29.5 & 0.65 \\
\hline \multirow{2}{*}{ EM07-Ca-07 } & Quenched & 12.1 & 0.31 & 4.95 & 0.09 & 47.8 & 0.85 & 29.0 & 0.38 \\
\hline & $\mathrm{CCC}$ & 8.56 & 0.15 & 4.19 & 0.04 & 37.5 & 0.55 & 24.8 & 0.75 \\
\hline
\end{tabular}




\begin{tabular}{|c|c|c|c|c|c|c|c|c|c|}
\hline \multirow{2}{*}{ Glass ID } & \multirow{2}{*}{ Type } & \multicolumn{2}{|r|}{$\mathrm{B}$} & \multicolumn{2}{|r|}{$\mathrm{Li}$} & \multicolumn{2}{|c|}{$\mathrm{Na}$} & \multicolumn{2}{|r|}{$\mathrm{Si}$} \\
\hline & & Ave. & St. Dev. & Ave. & St. Dev. & Ave. & St. Dev. & Ave. & St. De \\
\hline \multirow{2}{*}{ EM07-Cr-001 } & Quenched & 9.27 & 0.20 & 3.87 & 0.06 & 32.9 & 0.32 & 37.8 & 0.12 \\
\hline & $\overline{\mathrm{CCC}}$ & 9.67 & 0.20 & 4.05 & 0.08 & 32.2 & 0.36 & 41.6 & 0.26 \\
\hline \multirow{2}{*}{ EM07-Cr-012 } & Quenched & 8.78 & 0.44 & 3.73 & 0.13 & 32.3 & 1.04 & 36.5 & 1.48 \\
\hline & $\mathrm{CCC}$ & 9.10 & 0.54 & 3.89 & 0.14 & 32.9 & 0.83 & 40.7 & 1.47 \\
\hline \multirow{2}{*}{ EM07-Cr-02 } & Quenched & 8.88 & 0.55 & 3.75 & 0.10 & 32.7 & 0.35 & 37.8 & 1.31 \\
\hline & $\mathrm{CCC}$ & 8.31 & 0.37 & 3.51 & 0.10 & 29.3 & 0.30 & 38.7 & 0.98 \\
\hline \multirow{2}{*}{ EM07-F-02 } & Quenched & 7.97 & 0.69 & 3.58 & 0.11 & 31.1 & 1.30 & 32.4 & 1.01 \\
\hline & $\overline{\mathrm{CCC}}$ & 7.31 & 0.29 & 3.21 & 0.02 & 28.2 & 0.42 & 33.1 & 0.45 \\
\hline \multirow{2}{*}{ EM07-Fe-05 } & Quenched & 9.45 & 0.21 & 3.52 & 0.09 & 34.4 & 0.72 & 36.5 & 0.65 \\
\hline & $\mathrm{CCC}$ & 8.93 & 0.24 & 3.63 & 0.10 & 32.3 & 0.46 & 38.7 & 0.60 \\
\hline \multirow{2}{*}{ EM07-Fe-15 } & Quenched & 9.08 & 0.17 & 3.98 & 0.09 & 31.7 & 0.55 & 35.4 & 0.85 \\
\hline & $\overline{\mathrm{CCC}}$ & 8.97 & 0.59 & 3.69 & 0.27 & 30.8 & 1.91 & 40.5 & 2.55 \\
\hline \multirow{2}{*}{ EM07-Fe-20 } & Quenched & 9.22 & 0.26 & 4.15 & 0.08 & 30.4 & 1.21 & 35.0 & 0.44 \\
\hline & $\mathrm{CCC}$ & 7.90 & 0.14 & 3.13 & 0.05 & 28.6 & 0.72 & 37.9 & 0.26 \\
\hline \multirow{2}{*}{ EM07-K-03 } & Quenched & 9.49 & 0.45 & 3.55 & 0.10 & \begin{tabular}{|l|}
34.8 \\
\end{tabular} & 0.70 & 32.6 & 1.10 \\
\hline & $\overline{\mathrm{CCC}}$ & 11.0 & 0.32 & 4.11 & 0.14 & 36.8 & 1.97 & 36.2 & 0.23 \\
\hline \multirow{2}{*}{ EM07-K-06 } & Quenched & 16.5 & 1.08 & 5.99 & 0.34 & 51.7 & 1.53 & 35.9 & 1.10 \\
\hline & $\overline{\mathrm{CCC}}$ & 22.6 & 4.56 & 8.12 & 1.80 & 68.3 & 13.9 & 46.4 & 15.8 \\
\hline \multirow{2}{*}{ EM07-Li-015 } & Quenched & 7.48 & 0.34 & 1.95 & 0.02 & 29.8 & 1.80 & 31.0 & 0.60 \\
\hline & $\mathrm{CCC}$ & 7.36 & 0.48 & 2.01 & 0.10 & 29.0 & 1.13 & 34.3 & 1.61 \\
\hline \multirow{2}{*}{ EM07-Li-04 } & Quenched & 9.47 & 0.30 & 5.71 & 0.07 & \begin{tabular}{|l|}
37.3 \\
\end{tabular} & 0.60 & 37.0 & 0.87 \\
\hline & $\mathrm{CCC}$ & 9.22 & 0.18 & 5.74 & 0.05 & 35.5 & 0.12 & 39.5 & 0.35 \\
\hline \multirow{2}{*}{ EM07-Mn-01 } & Quenched & 8.44 & 0.41 & 3.61 & 0.08 & 32.6 & 0.70 & 37.0 & 0.64 \\
\hline & $\mathrm{CCC}$ & 8.36 & 0.33 & 3.63 & 0.10 & 31.2 & 0.40 & 38.4 & 1.16 \\
\hline \multirow{2}{*}{ EM07-Mn-04 } & Quenched & 9.51 & 0.21 & 3.88 & 0.04 & 34.4 & 1.25 & 37.7 & 1.39 \\
\hline & $\mathrm{CCC}$ & 8.70 & 0.33 & 3.67 & 0.07 & 31.9 & 0.21 & 37.4 & 1.19 \\
\hline \multirow{2}{*}{ EM07-Na-05 } & Quenched & 6.32 & 0.12 & 5.26 & 0.26 & 1.22 & - & 34.6 & 2.35 \\
\hline & $\mathrm{CCC}$ & 6.07 & 0.32 & 5.33 & 0.19 & 0.59 & 0.39 & 33.7 & 1.31 \\
\hline \multirow{2}{*}{ EM07-Na-10 } & Quenched & 7.31 & 0.27 & 5.45 & 0.05 & 10.4 & 0.38 & 35.0 & 0.30 \\
\hline & $\mathrm{CCC}$ & 6.48 & 0.22 & 4.56 & 0.06 & 10.6 & 0.12 & 36.3 & 0.49 \\
\hline FMO07 Na 20 & Quenched & 15.5 & 0.30 & 3.54 & 0.02 & 87.3 & 2.29 & 42.6 & 0.57 \\
\hline EIVIO $/-1 \mathrm{Na}-20$ & $\mathrm{CCC}$ & 17.7 & 0.21 & 4.59 & 0.09 & 89.7 & 0.61 & 45.2 & 1.15 \\
\hline $\mathrm{FN}$ & Quenched & 7.90 & 0.59 & 3.59 & 0.03 & 31.3 & 0.67 & 33.1 & 0.46 \\
\hline 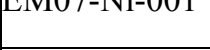 & $\mathrm{CCC}$ & 7.73 & 0.27 & 3.57 & 0.05 & 30.3 & 0.91 & 35.8 & 0.61 \\
\hline $\mathrm{FN}$ & Quenched & 8.21 & 0.26 & 3.67 & 0.11 & 33.6 & 1.98 & 34.3 & 1.16 \\
\hline LIVIO $1-101-02$ & $\mathrm{CCC}$ & 8.07 & 0.20 & 3.64 & 0.05 & 32.0 & 1.71 & 35.9 & 0.40 \\
\hline $\mathrm{FN}$ & Quenched & 10.4 & 1.00 & 3.75 & 0.11 & 32.6 & 0.59 & 34.8 & 0.68 \\
\hline EN10/-1-0 & $\mathrm{CCC}$ & 8.98 & 1.89 & 3.74 & 0.08 & 31.4 & 0.29 & 37.2 & 1.12 \\
\hline 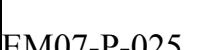 & Quenched & 10.4 & 0.81 & 3.93 & 0.11 & 32.8 & 0.97 & 34.9 & 1.33 \\
\hline 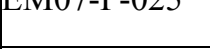 & $\mathrm{CCC}$ & 8.67 & 0.30 & 7.74 & 0.09 & 38.1 & 0.25 & 38.5 & 0.55 \\
\hline $\mathrm{FN}$ & Quenched & 64.7 & 1.45 & 16.6 & 0.21 & 154 & 1.00 & 35.1 & 0.85 \\
\hline LIVIUt-DI-50 & $\mathrm{CCC}$ & 1300 & 10.0 & 294 & 21.1 & 2197 & 103 & 91.9 & 0.97 \\
\hline FN $>>+2$ & Quenched & 17.4 & 2.82 & 5.00 & 0.09 & 52.1 & 0.35 & 31.5 & 0.57 \\
\hline Liviti-Mi-2t & $\mathrm{CCC}$ & 24.5 & 0.72 & 7.27 & 0.01 & 66.8 & 0.06 & 36.3 & 0.78 \\
\hline
\end{tabular}




\begin{tabular}{|l|l|l|l|l|l|l|l|l|c|}
\hline \multirow{2}{*}{ Glass ID } & \multirow{2}{*}{ Type } & \multicolumn{2}{|c|}{ B } & \multicolumn{2}{|c|}{ Li } & \multicolumn{2}{|c|}{ Na } & \multicolumn{2}{|c|}{ Si } \\
\cline { 3 - 10 } & & Ave. & St. Dev. & Ave. & St. Dev. & Ave. & St. Dev. & Ave. & St. Dev. \\
\hline \multirow{2}{*}{ EM07-Si-50 } & Quenched & 6.56 & 0.72 & 3.53 & 0.08 & 20.8 & 0.82 & 36.7 & 0.80 \\
\cline { 2 - 10 } & CCC & 6.75 & 0.52 & 3.39 & 0.07 & 20.2 & 0.60 & 39.5 & 0.65 \\
\hline \multirow{2}{*}{ EM07-Zr-001 } & Quenched & 9.14 & 0.15 & 3.73 & 0.05 & 35.3 & 0.81 & 39.7 & 0.87 \\
\cline { 2 - 10 } & CCC & 9.12 & 0.37 & 3.83 & 0.06 & 34.2 & 1.31 & 42.5 & 0.31 \\
\hline \multirow{2}{*}{ EM07-Zr-05 } & Quenched & 7.34 & 0.34 & 3.55 & 0.08 & 29.2 & 1.19 & 29.5 & 0.79 \\
\cline { 2 - 10 } & CCC & 6.88 & 0.04 & 3.39 & 0.03 & 27.3 & 0.93 & 30.3 & 0.15 \\
\hline \multirow{2}{*}{ EM07-NM-0025 } & Quenched & 9.18 & 0.35 & 3.72 & 0.15 & 31.9 & 0.67 & 35.7 & 1.10 \\
\cline { 2 - 10 } & CCC & 8.52 & 0.36 & 3.55 & 0.08 & 30.4 & 0.50 & 39.0 & 0.40 \\
\hline
\end{tabular}

*Standard deviation (St. Dev.) was not calculated for entries where three measured values were not acquired (sometimes values were below detection limit) and thus a "_" is presented for these entries.

\subsection{Toxicity Characteristic Leach Procedure (TCLP)}

See Appendix F for the data obtained from the TCLP measurements (quenched glass results page F.1 through F.23 and CCC from F.24 through F.39). Note, only the 2007 Matrix I glasses (38 glasses) were tested by TCLP. Results of TCLP tests of hazardous metals above Reporting Detection Limits (RDL) are found in Table 3.11. Note that only $\mathrm{Pb}$ and $\mathrm{Cd}$ were above $\mathrm{RDL}$ and their concentrations were well below the EPA's limits listed in Table 3.10.

Table 3.10. EPA TCLP Limits for Characteristically Hazardous Designation (ppm)

\begin{tabular}{|l|l|l|l|l|l|l|}
\hline As & Ba & Cd & Cr & Pb & Se & Ag \\
\hline 5 & 100 & 1 & 5 & 5 & 1 & 5 \\
\hline
\end{tabular}


Table 3.11. $\mathrm{Pb}$ and $\mathrm{Cd}$ Metals Detected above the Reporting Detection Limit of the ICP of $0.1 \mathrm{ppm}$ for $\mathrm{Pb}$ and $0.05 \mathrm{ppm}$ for $\mathrm{Cd}$.

\begin{tabular}{|l|c|c|c|c|}
\hline & \multicolumn{2}{|c|}{ Quenched } & \multicolumn{2}{c|}{ CCC } \\
\hline Glass & Pb (ppm) & Cd (ppm) & Pb (ppm) & Cd (ppm) \\
\hline EM07-BL-1 & 0.20 & - & 0.12 & - \\
\hline EM07-Al-20 & 0.15 & - & - & - \\
\hline EM07-B-05 & 0.18 & - & - & - \\
\hline EM07-B-15 & 0.13 & - & 0.12 & - \\
\hline EM07-B-20 & 0.13 & 0.072 & 0.20 & 0.10 \\
\hline EM07-Bi-025 & 0.12 & - & 0.10 & - \\
\hline EM07-Ca-035 & - & - & 0.10 & - \\
\hline EM07-Ca-07 & 0.13 & 0.079 & 0.12 & 0.53 \\
\hline EM07-F-02 & - & - & - & 0.051 \\
\hline EM07-K-03 & - & - & 0.12 & - \\
\hline EM07-K-06 & 0.12 & 0.065 & 0.11 & - \\
\hline EM07-Na-05 & - & - & - & 0.11 \\
\hline EM07-Na-20 & - & 0.057 & 0.16 & 0.053 \\
\hline EM07-Ni-001 & - & - & 0.11 & - \\
\hline EM07-P-0 & - & - & 0.14 & - \\
\hline EM07-Si-30 & 0.41 & 0.20 & 0.10 & 0.25 \\
\hline EM07-Si-37 & 0.15 & 0.07 & 0.15 & 0.093 \\
\hline EM07-Si-50 & - & - & 0.37 & - \\
\hline
\end{tabular}


PNNL-17950, Rev 0

\subsection{References}

ASTM-American Society of Testing and Materials. 2008. Standard Test Methods for Determining Chemical Durability of Nuclear, Hazardous, and Mixed Waste Glasses and Multiphase Glass Ceramics: The Product Consistency Test (PCT). ASTM C 1285-02.

Jantzen CM, NE Bibler, DC Beam, CL Crawford, and MA Pickett. 1993. Characterization of the Defense Waste Processing Facility (DWPF) Environmental Assessment (EA) Glass Standard Reference Material (U). WSRC-TR-92-346. Savannah River Site, Aiken, South Carolina.

Kim D-S and JD Vienna. 2002. Model for TCLP Releases from Waste Glasses. PNNL-14061, Pacific Northwest National Laboratory, Richland, Washington.

Lide, D. R., Editor, CRC Handbook of Chemistry and Physics, (2007-2008).

Mellinger GB and JL Daniel. 1984. Approved Reference and Testing Materials for Use in Nuclear Waste Management Research and Development Programs. PNL-49552, Pacific Northwest Laboratory, Richland, Washington.

Raszewski FC, TB Edwards, and DK Peeler. 2008a. Enhanced DOE High-Level Waste Melter Throughput Studies: SRNL Glass Selection Strategy. WSRC-STI-2007-00652, Savannah River National Laboratory, Aiken, South Carolina.

Raszewski FC, TB Edwards, and DK Peeler. 2008b. Matrix 1 Results of the FY07 Enhanced DOE HighLevel Waste Melter Throughput Studies at SRNL. SRNS-STI-2008-00056, Savannah River National Laboratory, Aiken, South Carolina.

Vienna, JD, D-S Kim and P Hrma, Database and Interim Glass Property Models for Hanford HLW and LAW Glasses, PNNL-14060, Pacific Northwest National Laboratory, 2002. 


\section{Appendix A: Viscosity Data}

\section{A.1 EM07-BL-1 Viscosity Data}

Table A.1. Viscosity data for EM07-BL-1

EM07-BL-1

\begin{tabular}{|c|c|}
\hline speed & speed $^{2}$ \\
\hline
\end{tabular}

Data collected

\begin{tabular}{rrrrrrrr}
\hline \multicolumn{2}{r}{ Setpoint, C } & Temperature, Speed, rpm Torque, \% S.F. & Viscosity, Pa's & $1 / \mathrm{T} \times 10000, \mathrm{~K}$ & $\operatorname{Ln}(\eta)$, Pa's \\
\hline 1150.0 & 1145 & 58.85 & 40.66 & 10.47 & 7.232 & 7.053 & 1.978 \\
1100.0 & 1097 & 36.53 & 41.25 & 10.29 & 11.616 & 7.301 & 2.452 \\
1050.0 & 1047 & 21.35 & 41.91 & 10.15 & 19.932 & 7.577 & 2.992 \\
1000.0 & 997 & 11.53 & 40.92 & 10.06 & 35.708 & 7.874 & 3.575 \\
950.0 & 947 & 9.80 & 68.86 & 10.04 & 70.580 & 8.195 & 4.257 \\
1050.0 & 1042 & 21.48 & 43.03 & 10.15 & 20.345 & 7.602 & 3.013 \\
1150.0 & 1144 & 60.51 & 42.27 & 10.48 & 7.322 & 7.056 & 1.991 \\
1200.0 & 1194 & 70.00 & 30.96 & 10.55 & 4.667 & 6.814 & 1.540 \\
1150.0 & 1146 & 61.62 & 42.28 & 10.49 & 7.197 & 7.044 & 1.974
\end{tabular}

EM07-BL-1

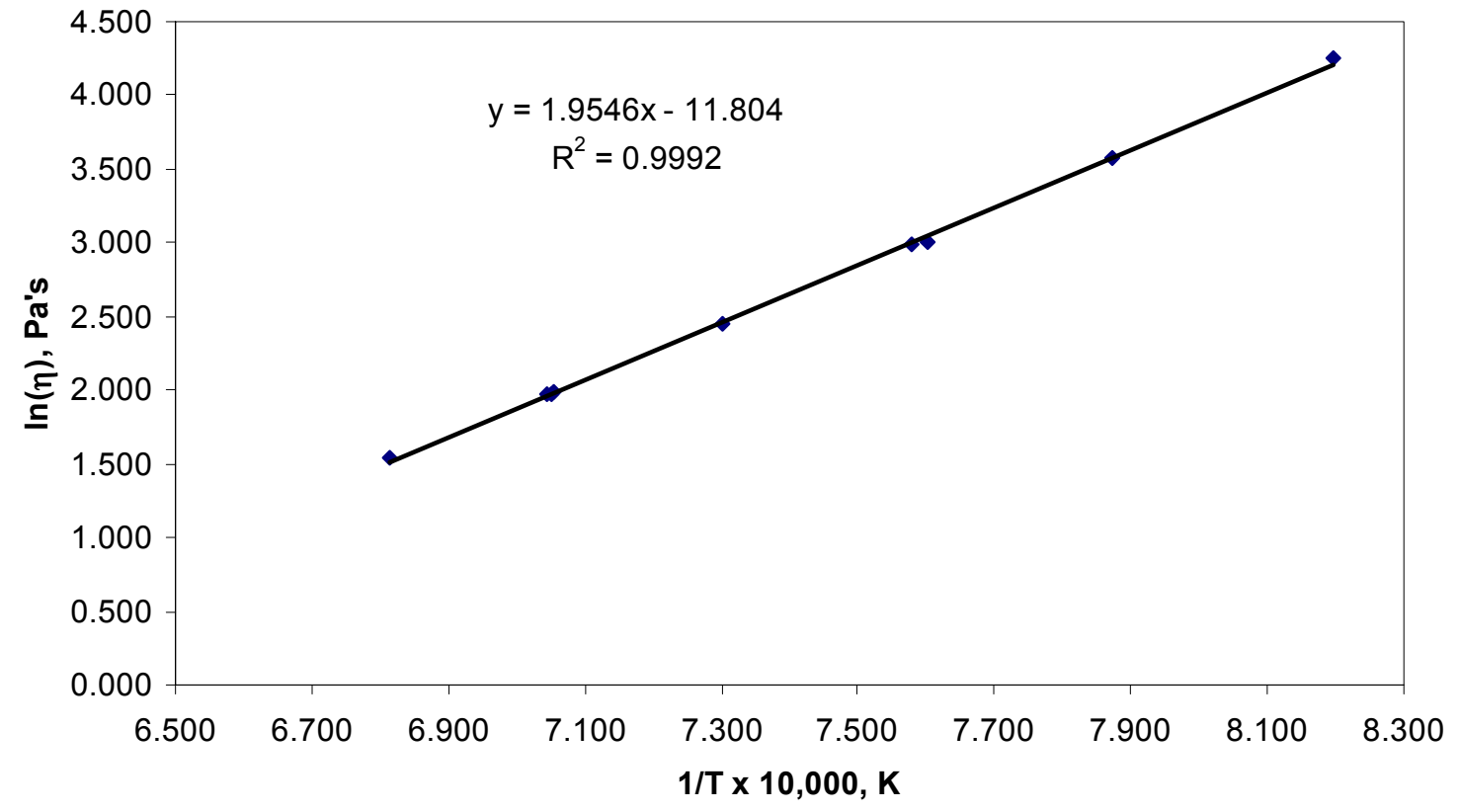

Figure A.1. Viscosity trend for EM07-BL-1 


\section{A.2 EM07-BL-2 Viscosity Data}

Table A.2. Viscosity data for EM07-BL-2

\section{EM07-BL-2}

\begin{tabular}{|r|r|r|}
\hline speed & speed $^{2}$ & \multicolumn{2}{c|}{ intercept } \\
\hline 0.01 & -0.00002 & 9.9485 \\
\hline
\end{tabular}

Data collected

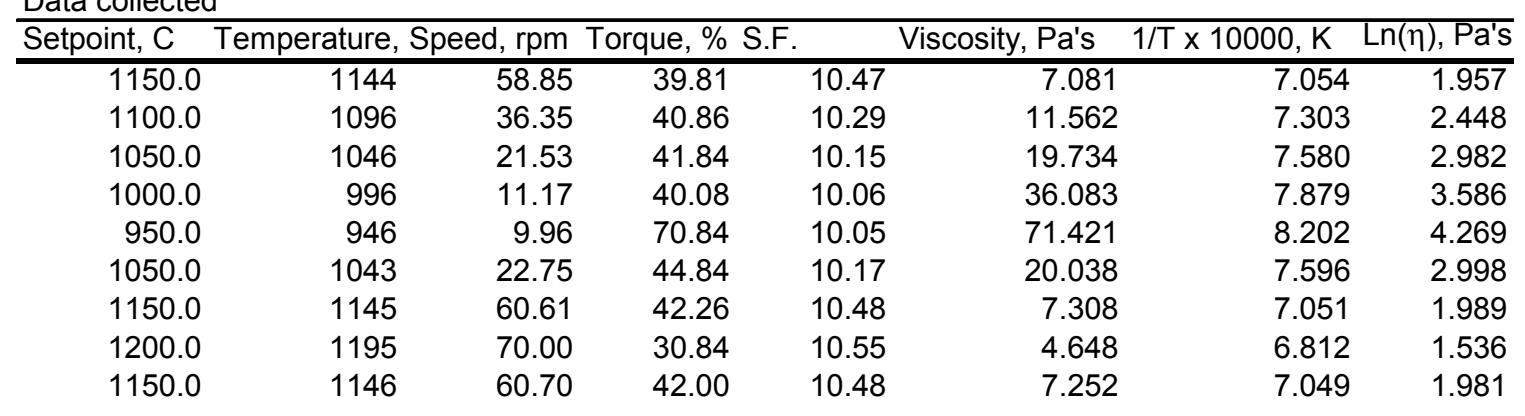

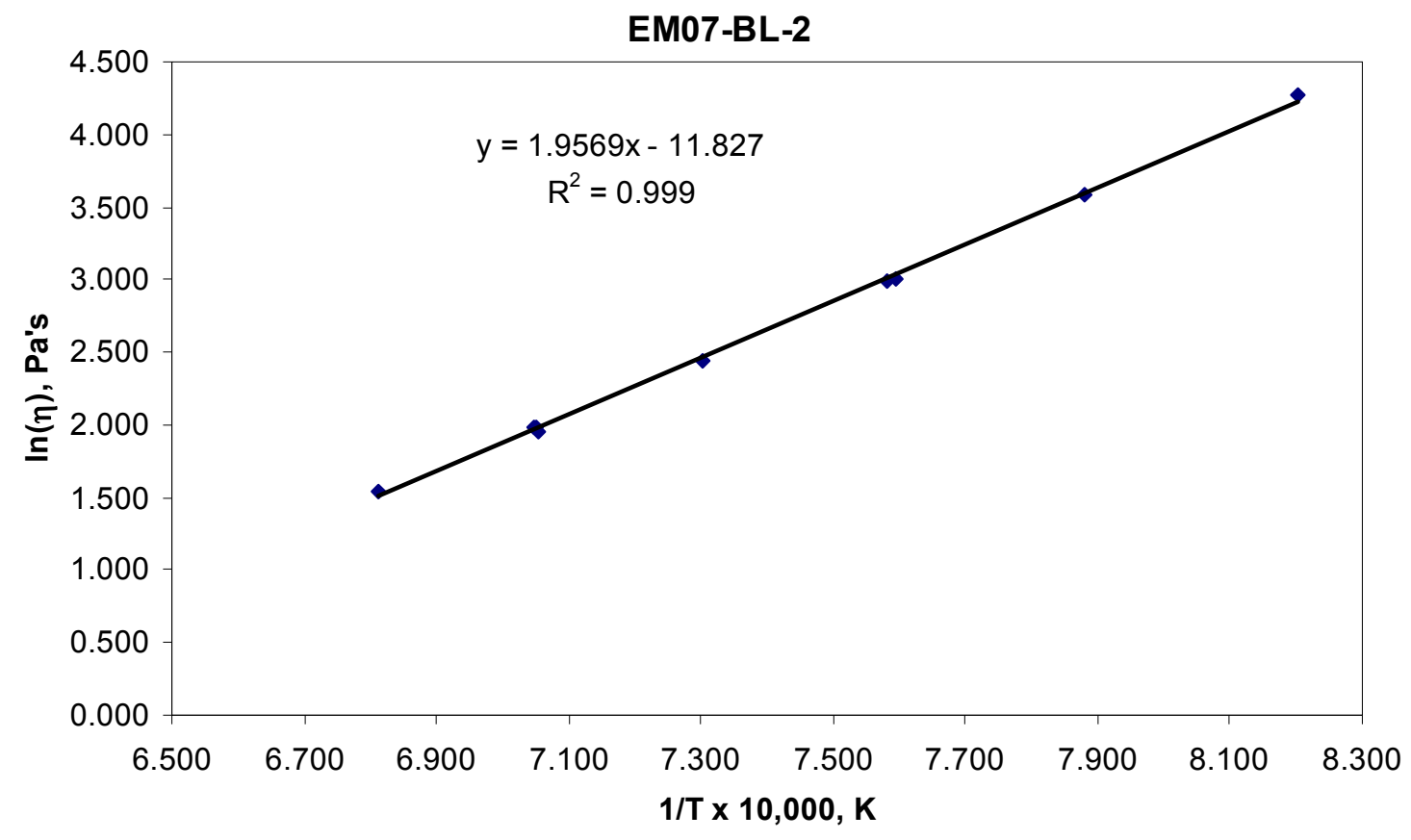

Figure A.2. Viscosity Trend for EM07-BL-2 


\section{A.3 EM07-Al-06 Viscosity Data}

Table A.3. Viscosity data for EM07-Al-06

\section{EM07-AI-06}

\begin{tabular}{|c|c|c|}
\hline speed & speed $^{2}$ & intercept \\
\hline 0.01 & -0.00002 & 9.9485 \\
\hline
\end{tabular}

Data collected

Setpoint, C Temperature, Speed, rpm Torque, \% S.F.

1150.0

1100.0

1050.0

1000.0

950.0

1050.0

1150.0

1200.0

1150.0

$1144 \quad 63.85$

1095

1046

996

946

1043

1143

1194

1145
41.17

23.85

13.85

11.10

28.12

66.45

70.00

65.86

$25.10 \quad 10.51$

$25.50 \quad 10.33$

$24.51 \quad 10.18$

$24.69 \quad 10.08$

$37.30 \quad 10.06$

$29.14 \quad 10.21$

$26.67 \quad 10.52$

$18.84 \quad 10.55$

$26.29 \quad 10.52$
Viscosity, Pa's

$\begin{array}{rll}4.130 & 7.058 & 1.418 \\ 6.397 & 7.308 & 1.856 \\ 10.458 & 7.582 & 2.347 \\ 17.973 & 7.878 & 2.889 \\ 33.798 & 8.201 & 3.520 \\ 10.586 & 7.596 & 2.360 \\ 4.224 & 7.060 & 1.441 \\ 2.840 & 6.818 & 1.044 \\ 4.200 & 7.053 & 1.435\end{array}$

EM07-Al-06

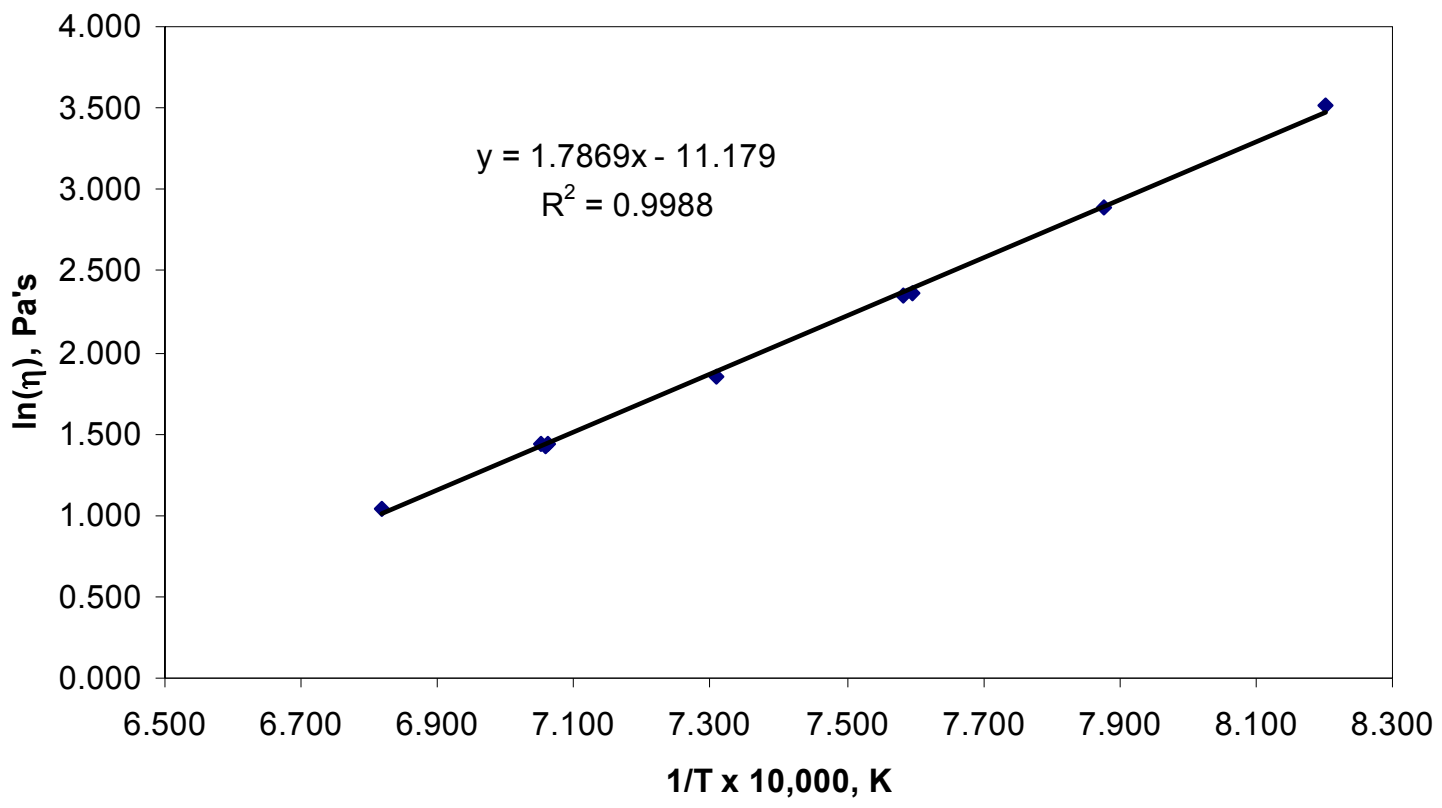

Figure A.3. Viscosity Trend for EM07-Al-06 


\section{A.4 EM07-Al-15 Viscosity Data}

Table A.4. Viscosity data for EM07-Al-15

\section{EM07-AI-15}

\begin{tabular}{|c|c|c|}
\hline speed & speed $^{2}$ & intercept \\
\hline 0.01 & -0.00002 & 9.9485 \\
\hline
\end{tabular}

Data collected

\begin{tabular}{rrrrrrrr}
\multicolumn{2}{l}{ Setpoint, C } & Temperature, Speed, rpm & Torque, \% S.F. & Viscosity, Pa's & $1 / T \times 10000, \mathrm{~K}$ & $\mathrm{Ln}(\eta), \mathrm{Pa}$ 's \\
\hline 1150.0 & 1146 & 25.10 & 39.92 & 10.19 & 16.201 & 7.046 & 2.785 \\
1100.0 & 1097 & 13.85 & 39.32 & 10.08 & 28.630 & 7.299 & 3.354 \\
1050.0 & 1047 & 11.10 & 55.70 & 10.06 & 50.469 & 7.575 & 3.921 \\
1000.0 & 996 & 7.78 & 75.48 & 10.03 & 97.291 & 7.876 & 4.578 \\
950.0 & 949 & 2.64 & 61.47 & 9.97 & 232.266 & 8.180 & 5.448 \\
1050.0 & 1045 & 11.53 & 58.78 & 10.06 & 51.299 & 7.587 & 3.938 \\
1150.0 & 1143 & 28.28 & 46.70 & 10.22 & 16.867 & 7.063 & 2.825 \\
1200.0 & 1195 & 45.41 & 43.47 & 10.36 & 9.919 & 6.811 & 2.294 \\
1150.0 & 1146 & 28.57 & 46.73 & 10.22 & 16.716 & 7.046 & 2.816
\end{tabular}

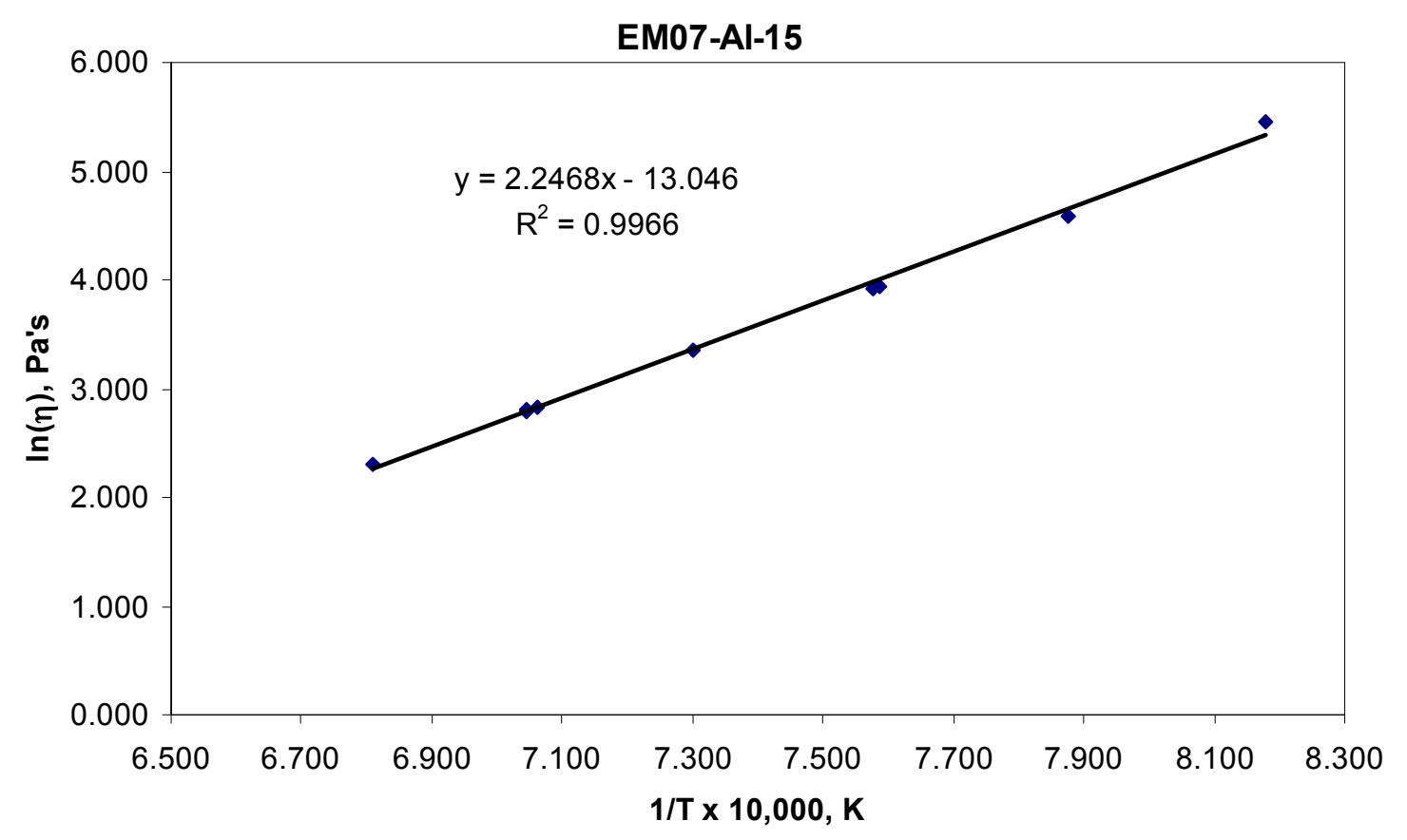

Figure A.4. Viscosity Trend for EM07-Al-15 


\section{A.5 EM07-Al-20 Viscosity Data}

Table A.5. Viscosity data for EM07-Al-20

\section{EM07-AI-20}

\begin{tabular}{|r|r|r|}
\hline speed & \multicolumn{1}{|c|}{ speed $^{2}$} & intercept \\
\hline 0.01 & -0.00002 & 9.9485 \\
\hline
\end{tabular}

Data collected

\begin{tabular}{|c|c|c|c|c|c|c|c|}
\hline Setpoint, C & Temperature, & Speed, rpm & Torque, $\%$ & & Viscosity, Pa's & $1 / \mathrm{T} \times 10000, \mathrm{~K}$ & $\mathrm{Ln}(\eta), \mathrm{Pa}$ 's \\
\hline 1150.0 & 1146 & 11.10 & 51.98 & 10.06 & 47.100 & 7.048 & 3.852 \\
\hline 1100.0 & 1096 & 7.57 & 69.64 & 10.02 & 92.159 & 7.303 & 4.524 \\
\hline 1050.0 & & & & & & & \\
\hline 1000.0 & & & & & & & \\
\hline 950.0 & & & & & & & \\
\hline 1050.0 & & & & & & & \\
\hline 1150.0 & & & & & & & \\
\hline 1200.0 & 1195 & 18.29 & 42.55 & 10.12 & 23.552 & 6.813 & 3.159 \\
\hline 1150.0 & 1146 & 12.49 & 52.95 & 10.07 & 42.693 & 7.046 & 3.754 \\
\hline
\end{tabular}

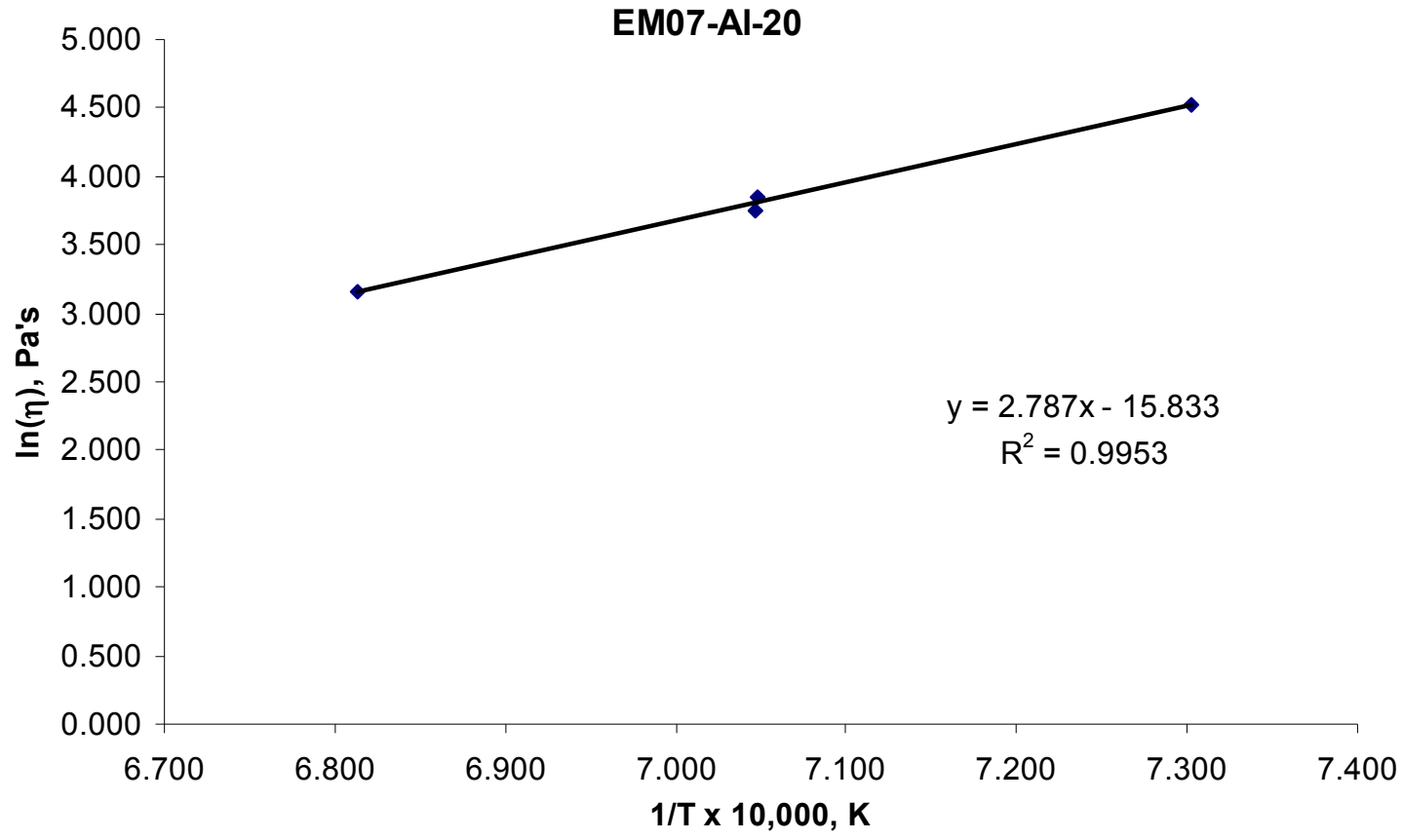

Figure A.5. Viscosity Trend for EM07-Al-20 


\section{A.6 EM07-B-05 Viscosity Data}

Table A.6. Viscosity Data for EM07-B-05

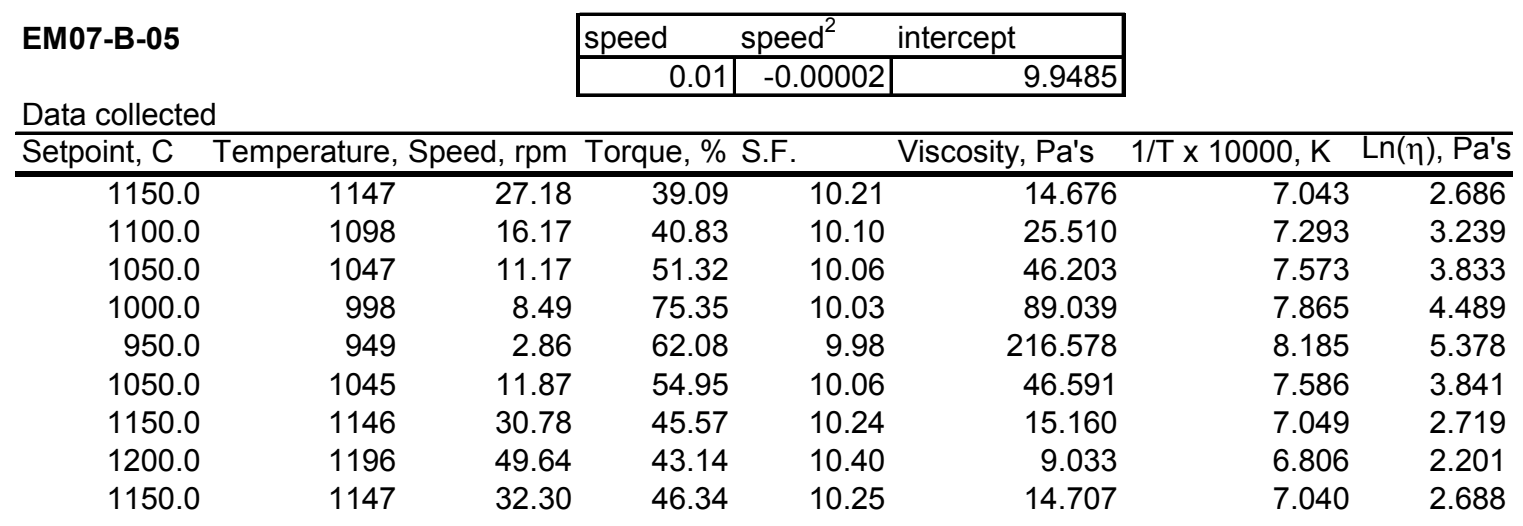

\section{EM07-B-05}

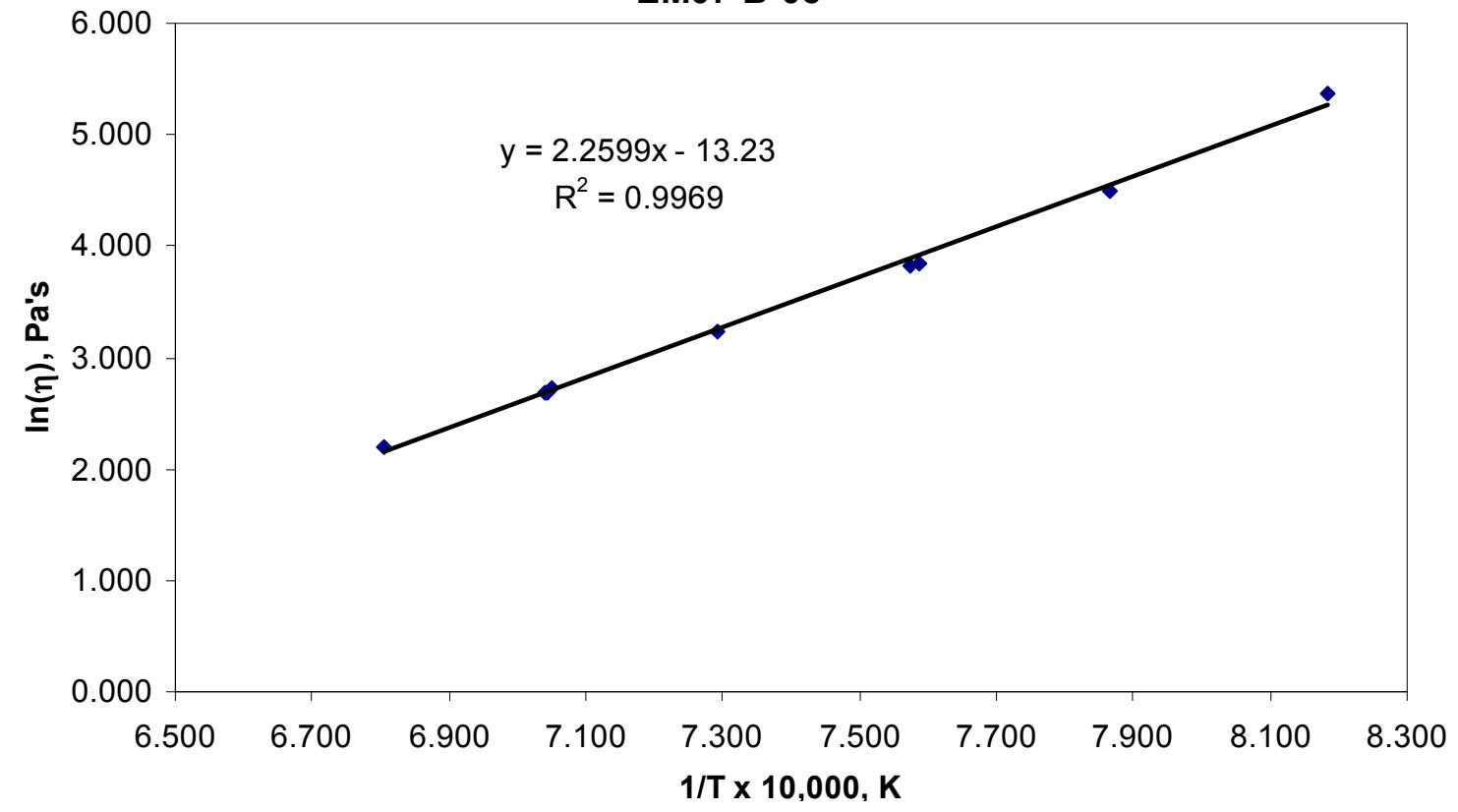

Figure A.6. Viscosity Trend for EM07-B-05 


\section{A.7 EM07-B-15 Viscosity Data}

Table A.7. Viscosity Data for EM07-B-15

\section{EM07-B-15}

\begin{tabular}{|r|r|r|}
\hline speed & speed^2 & intercept \\
\hline 0.01 & -0.00002 & 9.9485 \\
\hline
\end{tabular}

Data collected

\begin{tabular}{rrrrrrrr}
\hline Setpoint, C & Temperature, Speed, rpm Torque, \% S.F. & Viscosity, Pa's & $1 / \mathrm{T} \times 10000, \mathrm{~K}$ & $\mathrm{Ln}(\eta), \mathrm{Pa}$ 's \\
\hline 1150.0 & 1140 & 75.00 & 31.03 & 10.59 & 4.379 & 7.074 & 1.477 \\
1100.0 & 1092 & 63.33 & 42.13 & 10.50 & 6.987 & 7.326 & 1.944 \\
1050.0 & 1041 & 39.46 & 44.46 & 10.31 & 11.617 & 7.609 & 2.452 \\
1000.0 & 992 & 21.36 & 43.04 & 10.15 & 20.463 & 7.905 & 3.019 \\
950.0 & 942 & 12.09 & 47.73 & 10.07 & 39.740 & 8.227 & 3.682 \\
1050.0 & 1038 & 39.61 & 44.56 & 10.31 & 11.602 & 7.627 & 2.451 \\
1150.0 & 1137 & 75.00 & 31.63 & 10.59 & 4.465 & 7.091 & 1.496 \\
1200.0 & 1188 & 75.00 & 20.85 & 10.59 & 2.944 & 6.842 & 1.080 \\
1150.0 & 1141 & 75.00 & 31.09 & 10.59 & 4.389 & 7.069 & 1.479
\end{tabular}

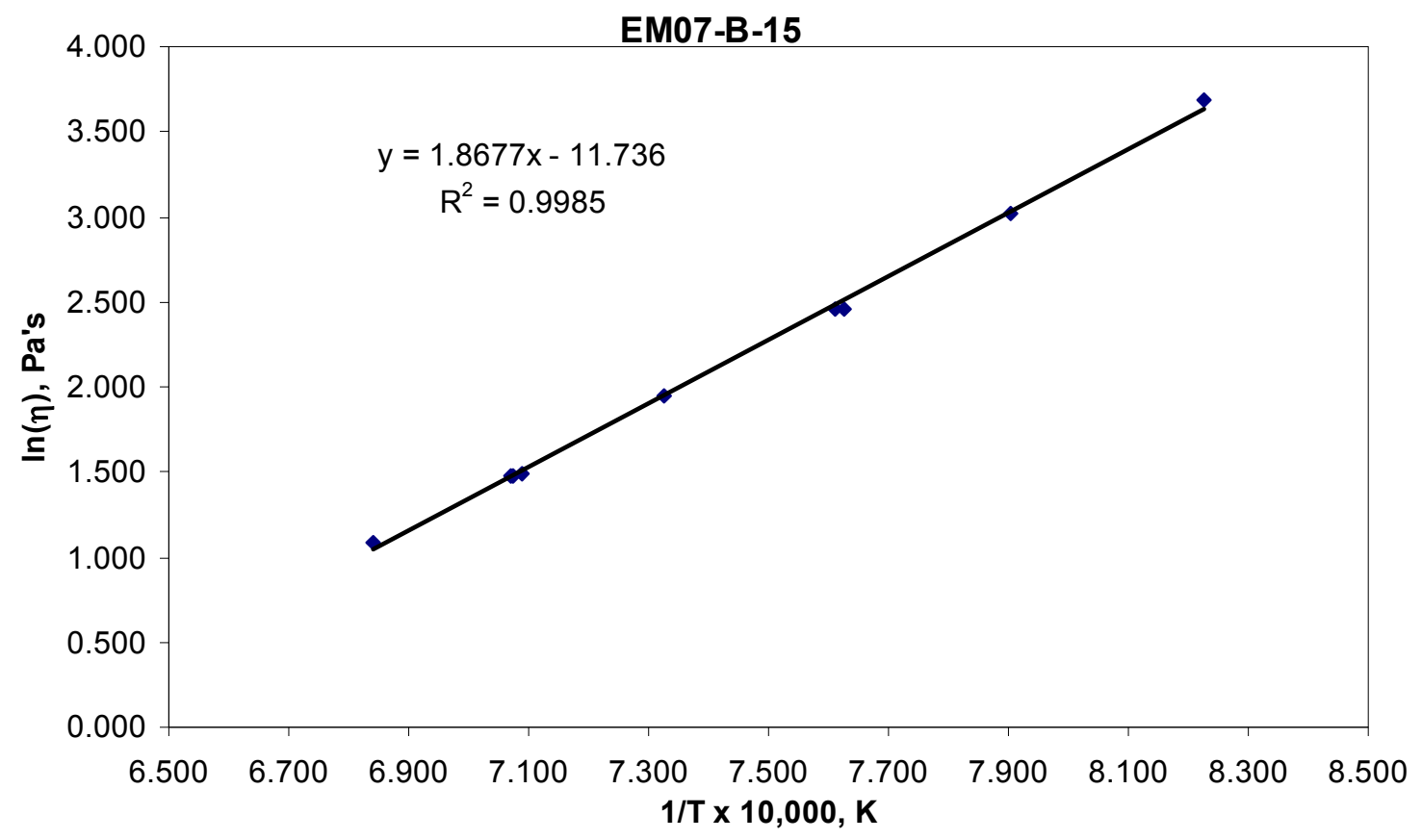

Figure A.7. Viscosity Trend for EM07-B-15 


\section{A.8 EM07-B-20 Viscosity Data}

Table A.8. Viscosity Data for EM07-B-20

\begin{tabular}{|c|c|c|c|c|c|c|c|}
\hline \multirow{2}{*}{\multicolumn{3}{|c|}{ EM07-B-20 }} & \multicolumn{3}{|c|}{$\begin{array}{lll}\text { speed } & \text { speed }^{2} & \text { intercept }\end{array}$} & \\
\hline & & & 0.01 & -0.00002 & 9.9485 & & \\
\hline \multicolumn{8}{|l|}{ Data collected } \\
\hline Setpoint, C & Temperature, & $\mathrm{d}, \mathrm{rpm}$ & Torque, $\%$ & S.F. & Viscosity, Pa's & $1 / \mathrm{T} \times 10000, \mathrm{~K}$ & 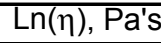 \\
\hline 1150.0 & 1142 & 70.00 & 20.36 & 10.55 & 3.068 & 7.065 & 1.121 \\
\hline 1100.0 & 1096 & 70.00 & 31.27 & 10.55 & 4.713 & 7.303 & 1.550 \\
\hline 1050.0 & 1046 & 57.41 & 42.52 & 10.46 & 7.745 & 7.581 & 2.047 \\
\hline 1000.0 & 996 & 34.29 & 45.12 & 10.27 & 13.510 & 7.878 & 2.603 \\
\hline 950.0 & 948 & 17.14 & 43.37 & 10.11 & 25.595 & 8.190 & 3.242 \\
\hline 1050.0 & 1043 & 56.83 & 42.79 & 10.45 & 7.871 & 7.596 & 2.063 \\
\hline 1150.0 & 1145 & 70.00 & 20.22 & 10.55 & 3.048 & 7.051 & 1.115 \\
\hline 1200.0 & 1194 & 70.00 & 13.67 & 10.55 & 2.060 & 6.815 & 0.723 \\
\hline 1150.0 & 1146 & 70.00 & 19.96 & 10.55 & 3.009 & 7.047 & 1.102 \\
\hline
\end{tabular}

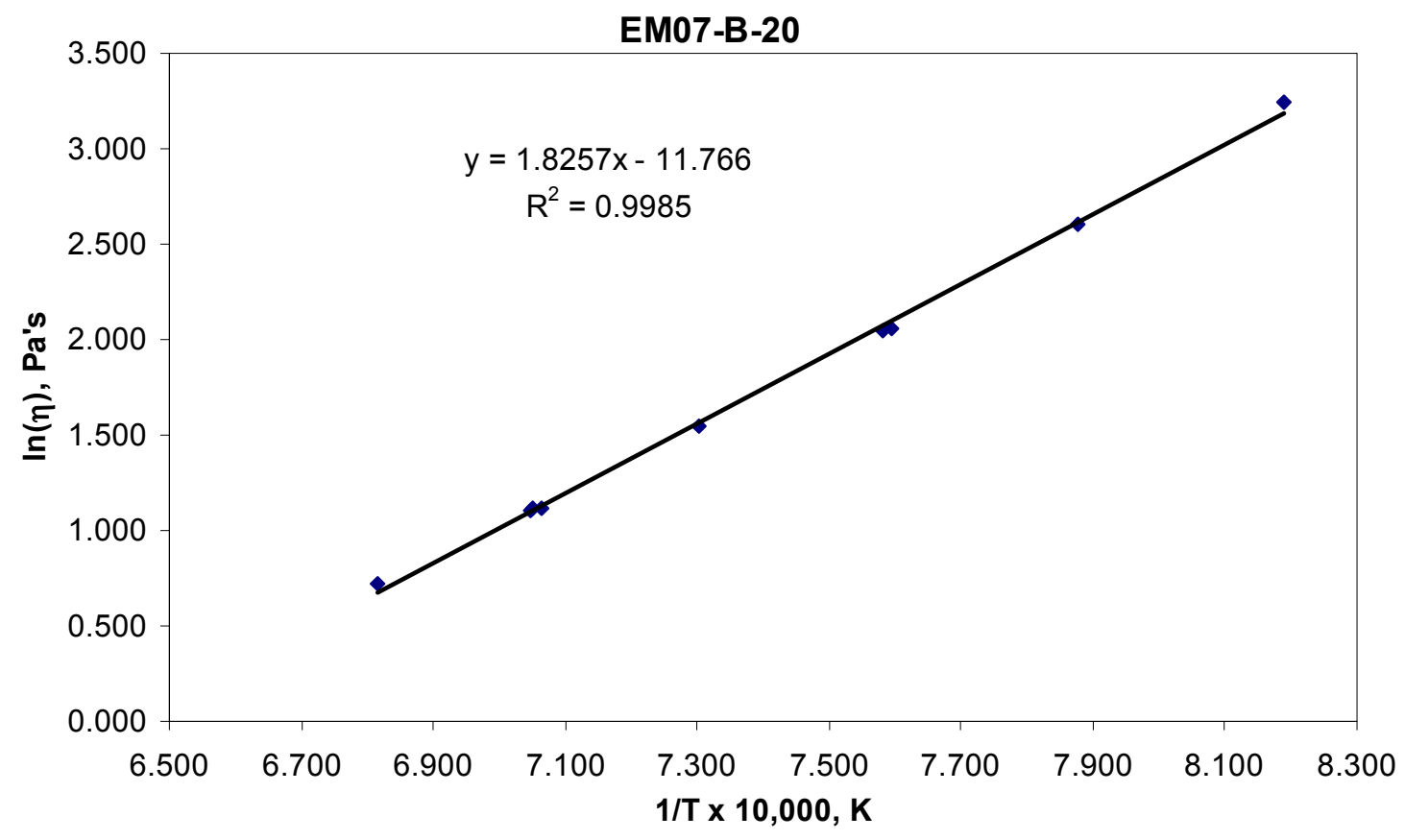

Figure A.8. Viscosity Trend for EM07-B-20 


\section{A.9 EM07-Bi-025 Viscosity Data}

Table A.9. Viscosity Data for EM07-Bi-025

\begin{tabular}{|c|c|c|c|c|c|c|c|}
\hline \multirow{2}{*}{\multicolumn{3}{|c|}{ EM07-Bi-025 }} & \multirow{2}{*}{\multicolumn{3}{|c|}{ speed $\quad$ speed $^{2} \quad$ intercept }} & \\
\hline & & & & & & & \\
\hline Data collectec & & & 0.01 & & & & \\
\hline Setpoint, C & Temperature, & Speed, rpm & Torque, $\%$ & S.F. & Viscosity, Pa's & $1 / \mathrm{T} \times 10000, \mathrm{~K}$ & 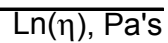 \\
\hline 1150.0 & 1140 & 75.00 & 23.03 & 10.59 & 3.251 & 7.076 & 1.179 \\
\hline 1100.0 & 1091 & 75.00 & 35.03 & 10.59 & 4.945 & 7.331 & 1.598 \\
\hline 1050.0 & 1041 & 56.99 & 42.50 & 10.45 & 7.795 & 7.608 & 2.054 \\
\hline 1000.0 & 991 & 35.18 & 44.17 & 10.28 & 12.900 & 7.910 & 2.557 \\
\hline 950.0 & 945 & 19.01 & 42.51 & 10.13 & 22.655 & 8.212 & 3.120 \\
\hline 1050.0 & 1040 & 56.26 & 42.60 & 10.45 & 7.912 & 7.615 & 2.068 \\
\hline 1150.0 & 1139 & 75.00 & 23.63 & 10.59 & 3.336 & 7.083 & 1.205 \\
\hline 1200.0 & 1190 & 75.00 & 16.27 & 10.59 & 2.296 & 6.835 & 0.831 \\
\hline 1150.0 & 1142 & 75.00 & 23.65 & 10.59 & 3.338 & 7.068 & 1.205 \\
\hline
\end{tabular}

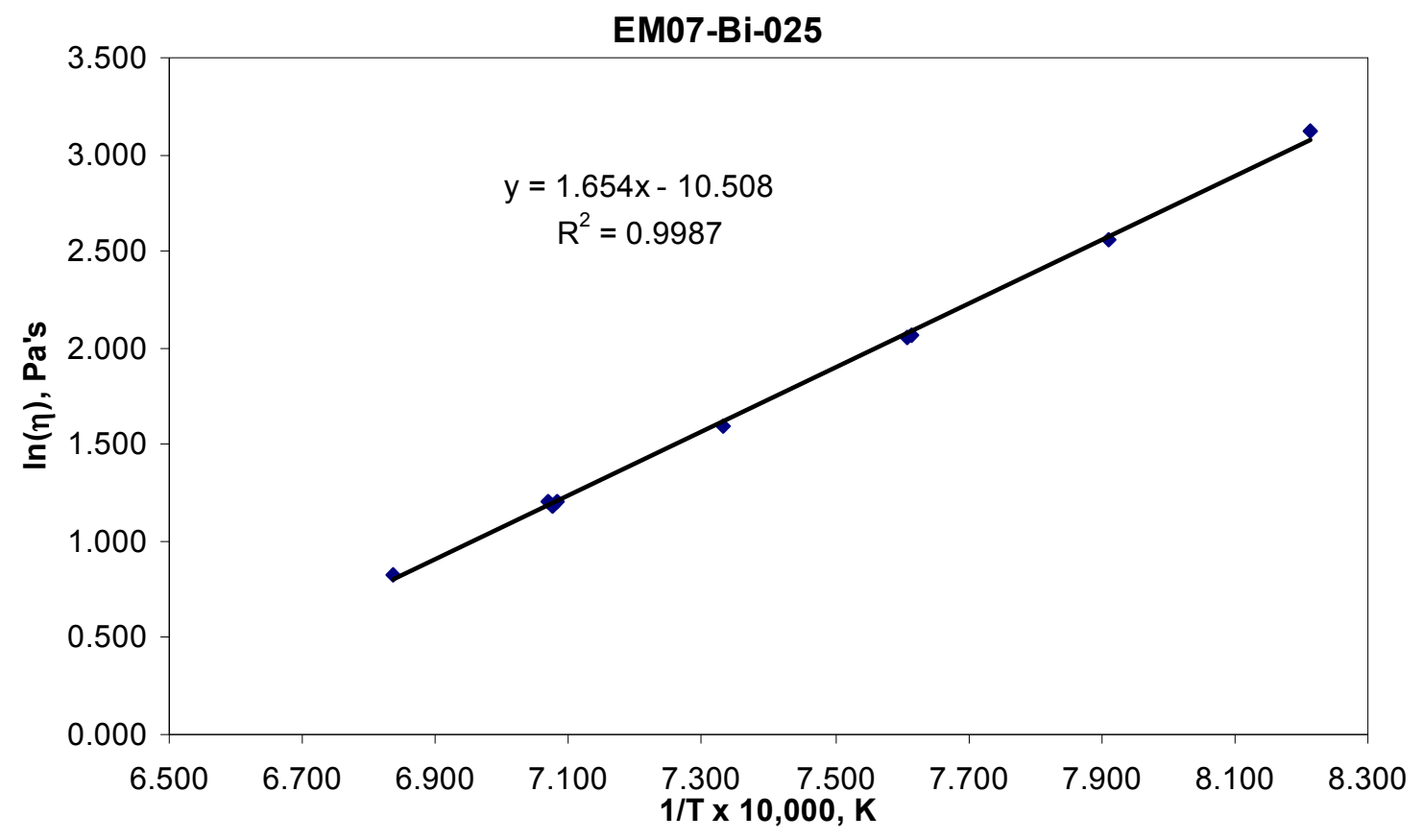

Figure A.9. Viscosity Trend for EM07-Bi-025 


\section{A.10 EM07-Bi-025 Duplicate Viscosity Data}

Table A.10. Viscosity Data for EM07-Bi-025 Duplicate

\begin{tabular}{|c|c|c|c|c|c|c|c|}
\hline \multirow{2}{*}{\multicolumn{3}{|c|}{ EM07-Bi-025 Duplicate }} & \multicolumn{3}{|c|}{\begin{tabular}{|lll} 
speed & speed $^{2}$ & intercept
\end{tabular}} & & \\
\hline & & & 0.01 & \begin{tabular}{|l|}
-0.00002 \\
\end{tabular} & 9.9485 & & \\
\hline Data collectec & & & & & & & \\
\hline Setpoint, C & Temperature, & Speed, rpm & Torque, $\%$ & S.F. & Viscosity, Pa's & $1 / \mathrm{T} \times 10000, \mathrm{~K}$ & 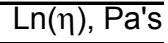 \\
\hline 1150.0 & 1145 & 66.35 & 40.60 & 10.52 & 6.439 & 7.053 & $\overline{1.862}$ \\
\hline 1100.0 & 1096 & 38.71 & 38.91 & 10.31 & 10.358 & 7.303 & 2.338 \\
\hline 1050.0 & 1047 & 23.71 & 41.12 & 10.17 & 17.645 & 7.573 & 2.870 \\
\hline 1000.0 & 996 & 12.24 & 39.12 & 10.07 & 32.168 & 7.881 & 3.471 \\
\hline 950.0 & 945 & 11.35 & 71.55 & 10.06 & 63.413 & 8.211 & 4.150 \\
\hline 1050.0 & 1043 & 26.42 & 46.43 & 10.20 & 17.924 & 7.595 & 2.886 \\
\hline 1150.0 & 1142 & 65.54 & 41.83 & 10.52 & 6.713 & 7.065 & 1.904 \\
\hline
\end{tabular}

\section{EM07-Bi-025 ReBatch}

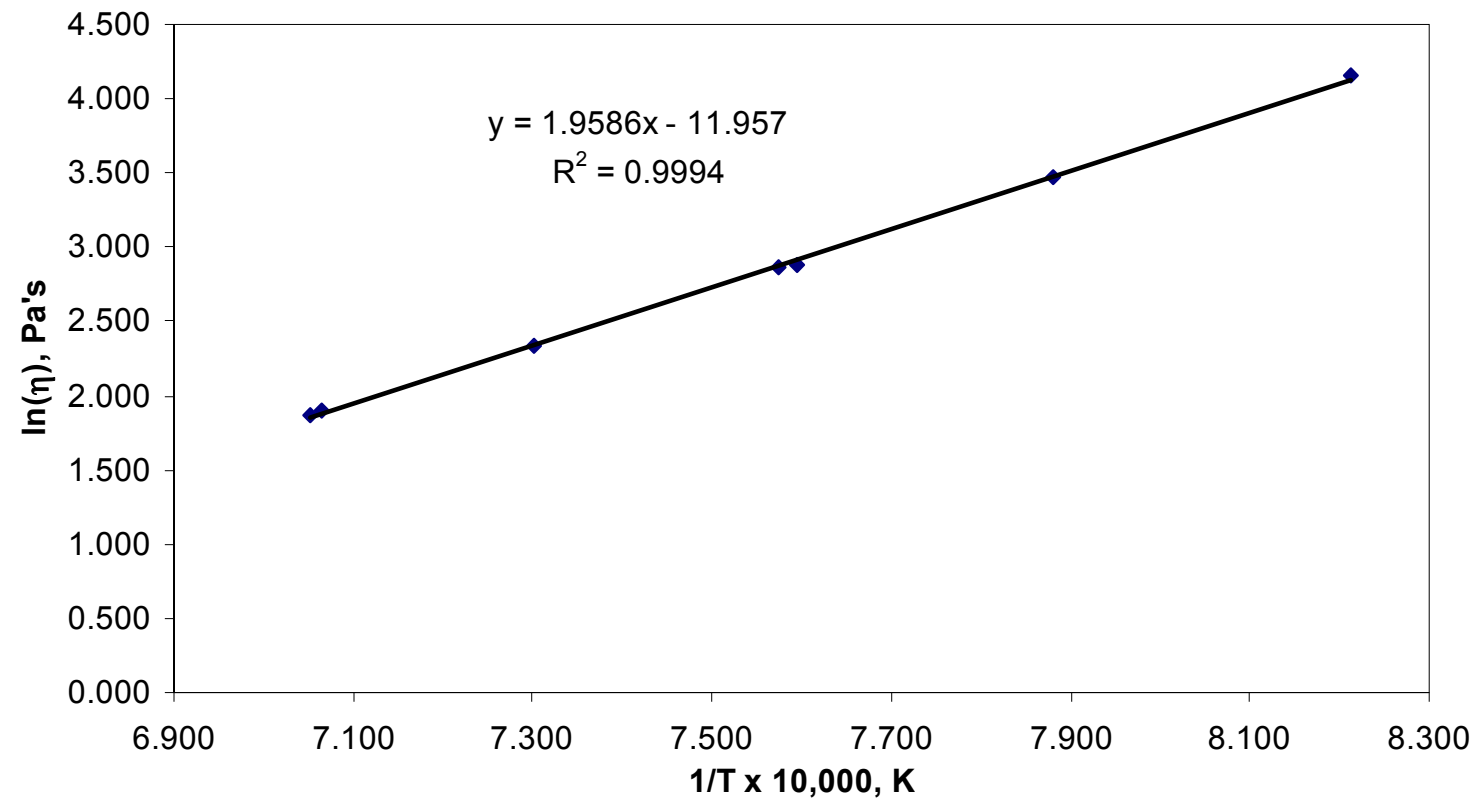

Figure A.11. Viscosity Trend for EM07-Bi-025 Duplicate 


\section{A.12 EM07-Bi-05 Viscosity Data}

Table A.12. Viscosity Data for EM07-Bi-05

EM07-Bi-05

\begin{tabular}{|r|r|r|}
\hline speed & \multicolumn{1}{|c|}{ speed $^{2}$} & intercept \\
\hline 0.01 & -0.00002 & 9.9485 \\
\hline
\end{tabular}

Data collected

\begin{tabular}{rrrrrrrr}
\hline Setpoint, C & Temperature, Speed, rpm Torque, \% S.F. & Viscosity, Pa's & $1 / T \times 10000, \mathrm{~K}$ & $\operatorname{Ln}(\eta), P a ' s$ \\
\hline 1150.0 & 1146 & 70.00 & 39.97 & 10.55 & 6.024 & 7.049 & 1.796 \\
1100.0 & 1096 & 46.36 & 43.73 & 10.37 & 9.780 & 7.303 & 2.280 \\
1050.0 & 1046 & 28.69 & 46.67 & 10.22 & 16.621 & 7.579 & 2.811 \\
1000.0 & 997 & 14.83 & 44.05 & 10.09 & 29.971 & 7.875 & 3.400 \\
950.0 & 945 & 12.11 & 70.14 & 10.07 & 58.303 & 8.206 & 4.066 \\
1050.0 & 1042 & 28.02 & 46.43 & 10.21 & 16.925 & 7.604 & 2.829 \\
1150.0 & 1143 & 70.00 & 40.74 & 10.55 & 6.141 & 7.059 & 1.815 \\
1200.0 & 1194 & 70.00 & 26.00 & 10.55 & 3.919 & 6.814 & 1.366 \\
1150.0 & 1147 & 70.00 & 39.57 & 10.55 & 5.964 & 7.042 & 1.786
\end{tabular}

EM07-Bi-05

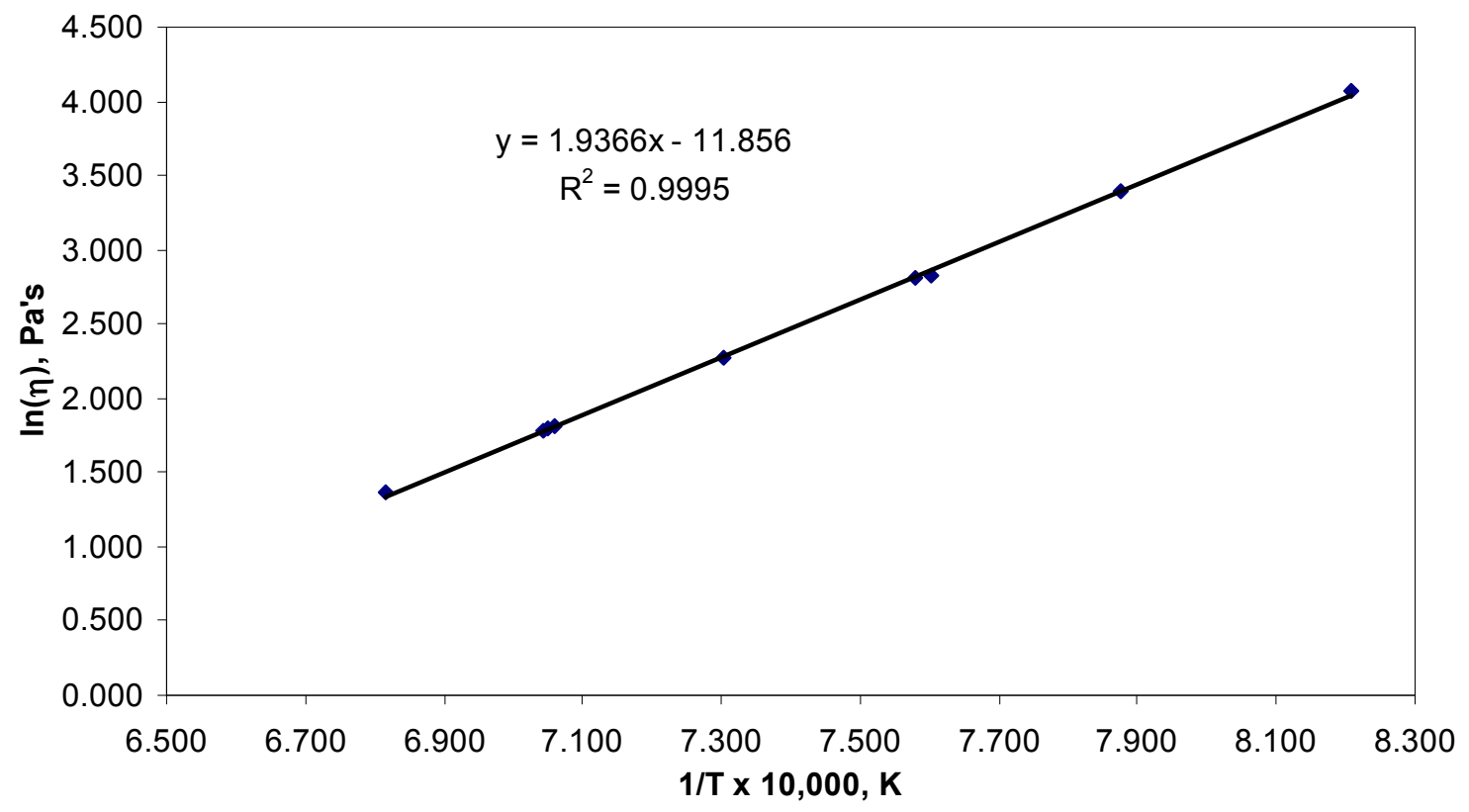

Figure A.13. Viscosity Trend for EM07-Bi-05 


\section{A.14 EM07-Bi-05 Duplicate Viscosity Data}

Table A.14. Viscosity Data for EM07-Bi-05 Duplicate

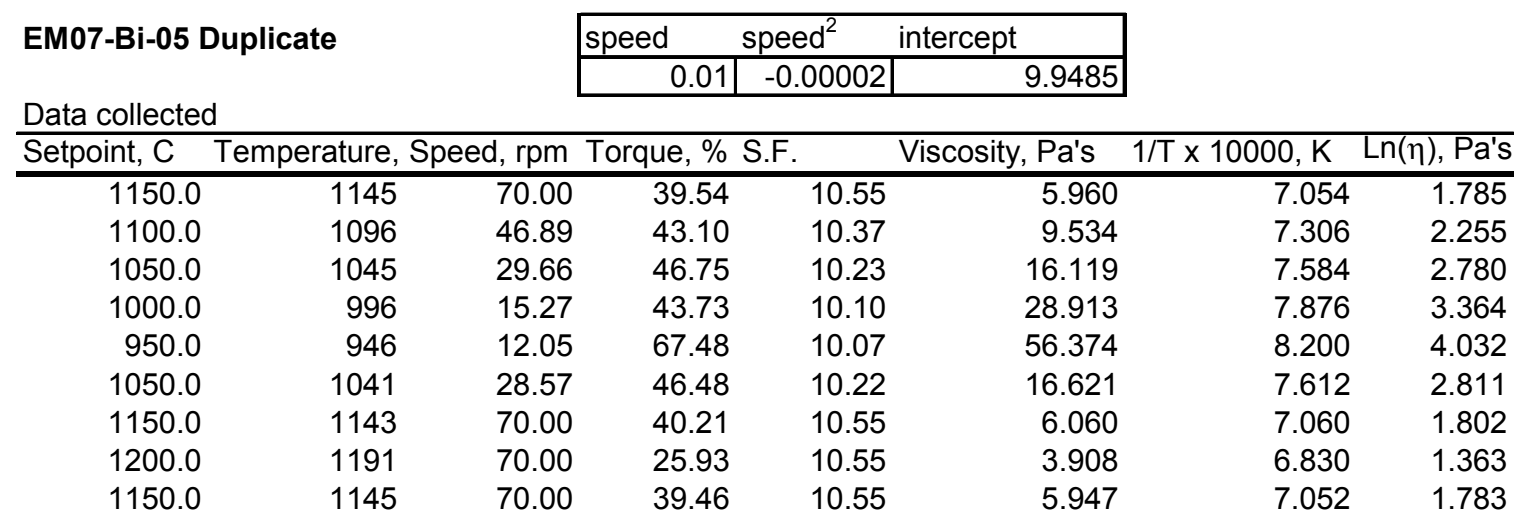

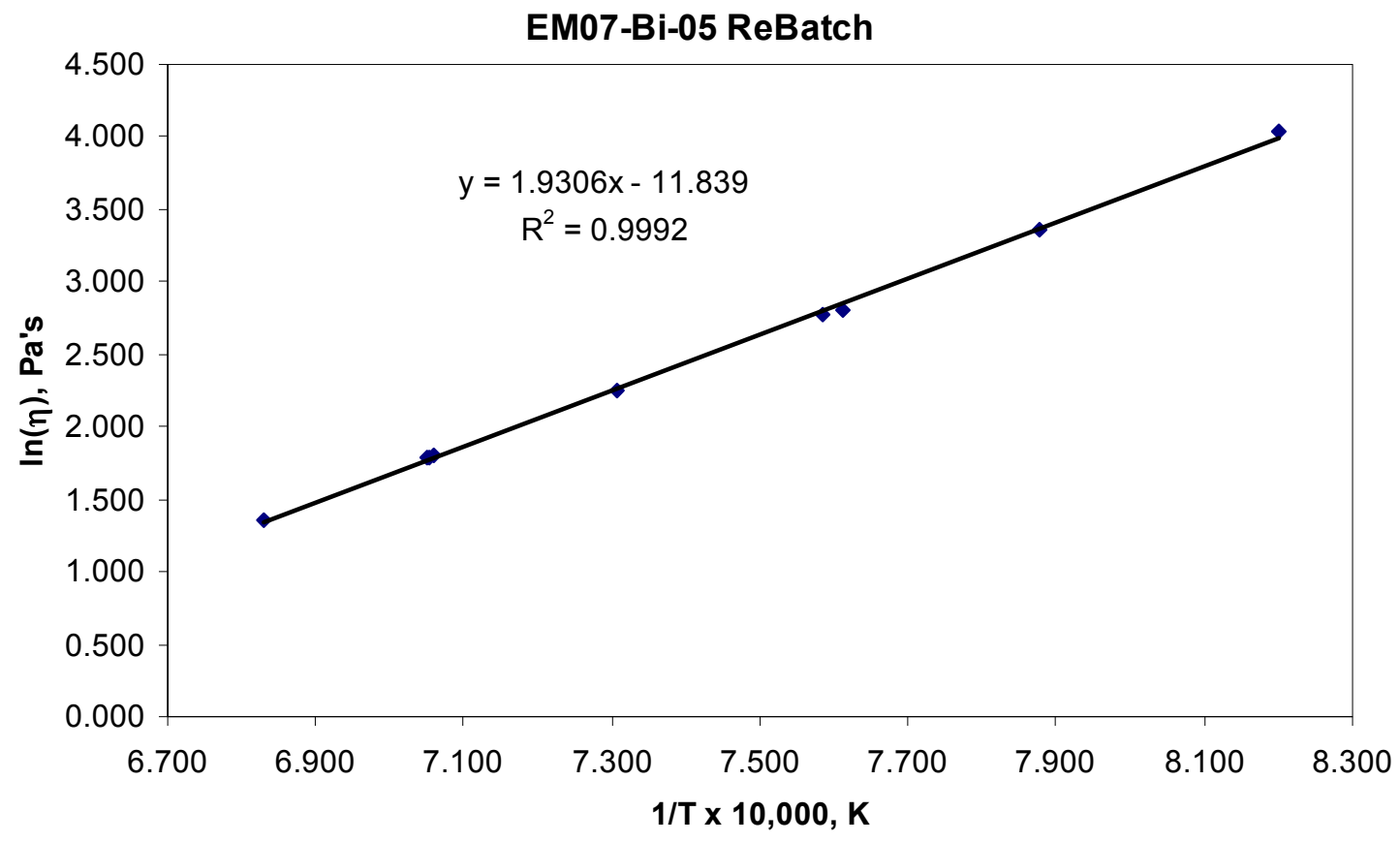

Figure A.15. Viscosity Trend for EM07-Bi-05 Duplicate 


\section{A.16 EM07-Ca-035 Viscosity Data}

Table A.16. Viscosity Data for EM07-Ca-035

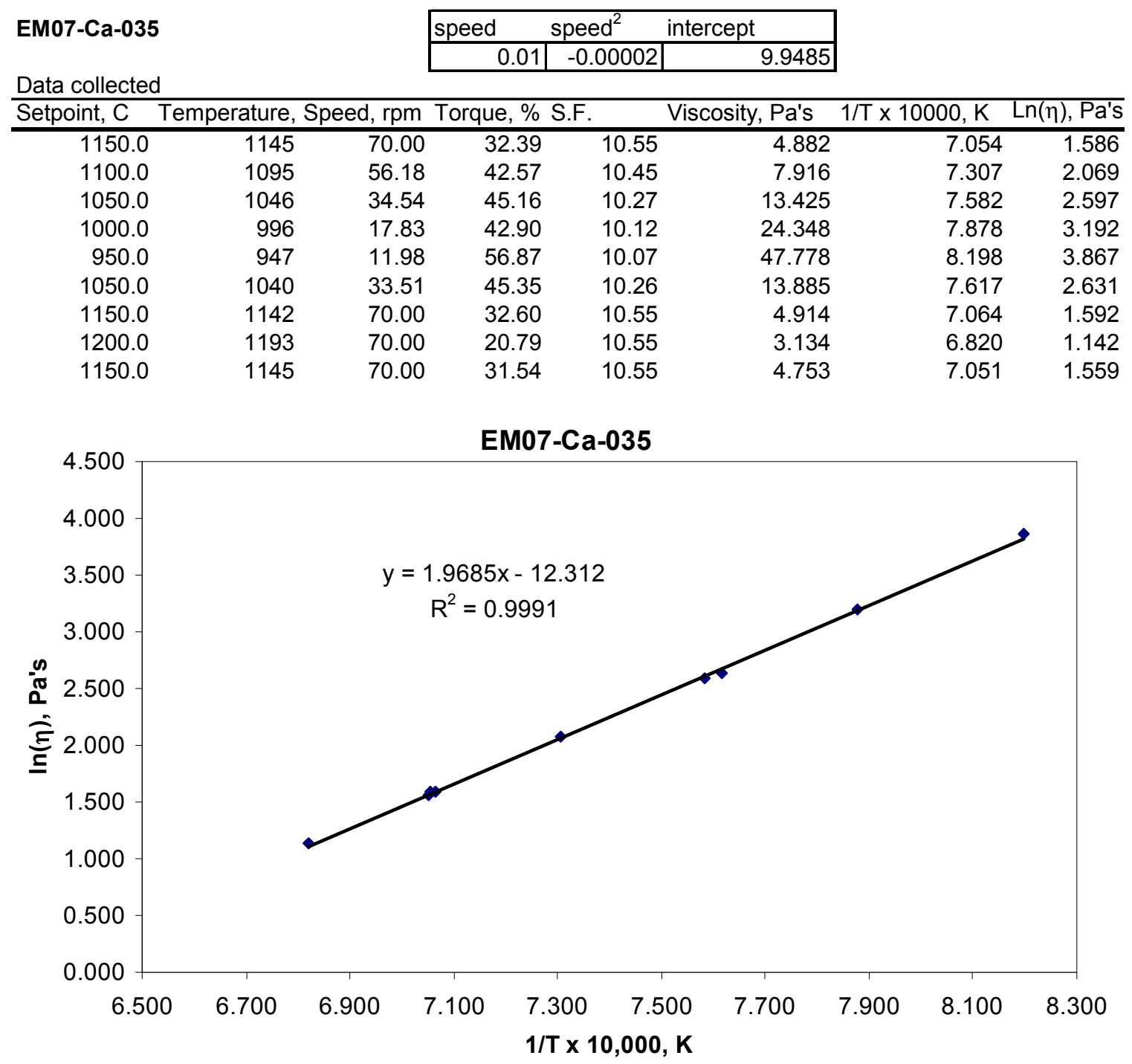

Figure A.16. Viscosity Trend for EM07-Ca-035 


\section{A.17 EM07-Ca-07 Viscosity Data}

Table A.17. Viscosity Data for EM07-Ca-07

EM07-Ca-07

\begin{tabular}{|r|r|r|}
\hline speed & \multicolumn{1}{|c|}{ speed $^{2}$} & intercept \\
\hline 0.01 & -0.00002 & 9.9485 \\
\hline
\end{tabular}

Data collected

\begin{tabular}{rrrrrrrr}
\hline Setpoint, C & Temperature, Speed, rpm Torque, \% S.F. & \multicolumn{3}{c}{ Viscosity, Pa's } & $1 / \mathrm{T} \times 10000, \mathrm{~K}$ & $\mathrm{Ln}(\eta)$, Pa's \\
\hline 1150.0 & 1145 & 70.00 & 22.64 & 10.55 & 3.412 & 7.050 & 1.227 \\
1100.0 & 1096 & 70.00 & 36.66 & 10.55 & 5.526 & 7.304 & 1.709 \\
1050.0 & 1046 & 47.77 & 43.40 & 10.38 & 9.430 & 7.584 & 2.244 \\
1000.0 & 997 & 28.09 & 47.06 & 10.21 & 17.111 & 7.875 & 2.840 \\
950.0 & 947 & 12.48 & 42.37 & 10.07 & 34.187 & 8.198 & 3.532 \\
1050.0 & 1042 & 46.35 & 43.87 & 10.37 & 9.814 & 7.603 & 2.284 \\
1150.0 & 1144 & 70.00 & 23.13 & 10.55 & 3.486 & 7.059 & 1.249 \\
1200.0 & 1194 & 70.00 & 14.96 & 10.55 & 2.254 & 6.815 & 0.813 \\
1150.0 & 1147 & 70.00 & 22.38 & 10.55 & 3.374 & 7.044 & 1.216
\end{tabular}

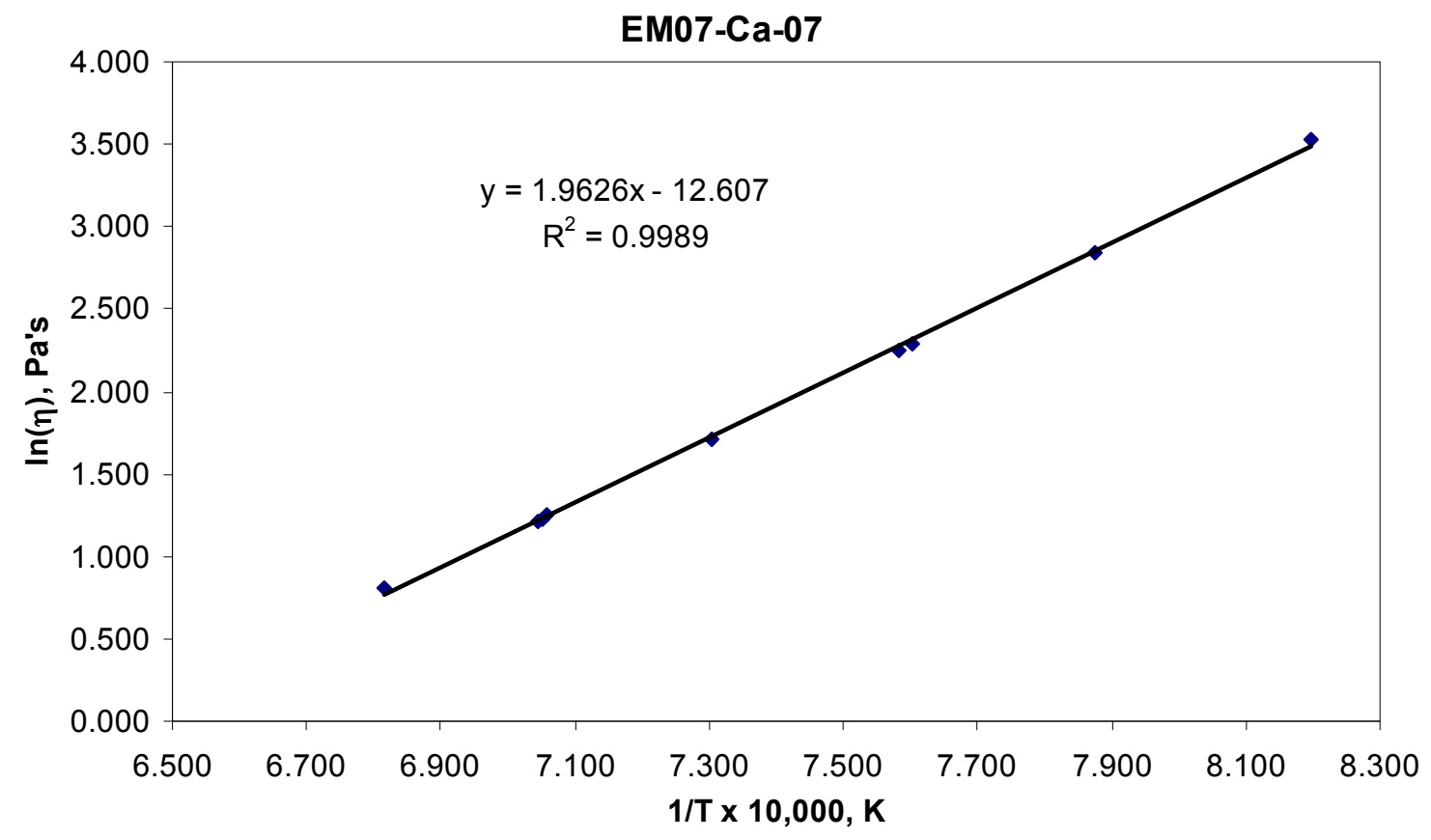

Figure A.17. Viscosity Trend for EM07-Ca-07 


\section{A.18 EM07-Cr-001 Viscosity Data}

Table A.18. Viscosity Data for EM07-Cr-001

EM07-Cr-001

Data collected

\begin{tabular}{cccc}
\hline Setpoint, C & Temperature, Speed, rpm & Torque, \% S.F. \\
\hline 1150.0 & 1145 & 56.35 & 39.90
\end{tabular}

1100.0

1050.0

1000.0

950.0

1050.0

1150.0

1200.0

1150.0
1096

1046

996

947

1042

1144

1194

1145

36.17

22.60

13.16

11.35

26.08

61.52

70.00

62.26

40.32

40.32

41.29

68.91

49.09

42.24

31.94

42.37

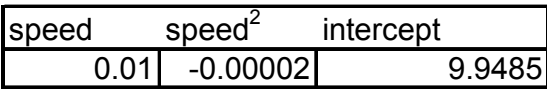

10.45

10.28

10.16

10.08

10.06

10.20

10.49

10.55

10.49

\begin{tabular}{rrr} 
Viscosity, Pa's & $1 / \mathrm{T} \times 10000, \mathrm{~K}$ & $\mathrm{Ln}(\eta), \mathrm{Pa}$ 's \\
\hline 7.399 & 7.051 & 2.001 \\
11.462 & 7.305 & 2.439 \\
17.963 & 7.579 & 2.888 \\
31.628 & 7.877 & 3.454 \\
61.071 & 8.197 & 4.112 \\
19.191 & 7.603 & 2.954 \\
7.201 & 7.058 & 1.974 \\
4.815 & 6.815 & 1.572 \\
7.140 & 7.050 & 1.966
\end{tabular}

\section{EM07-Cr-001}

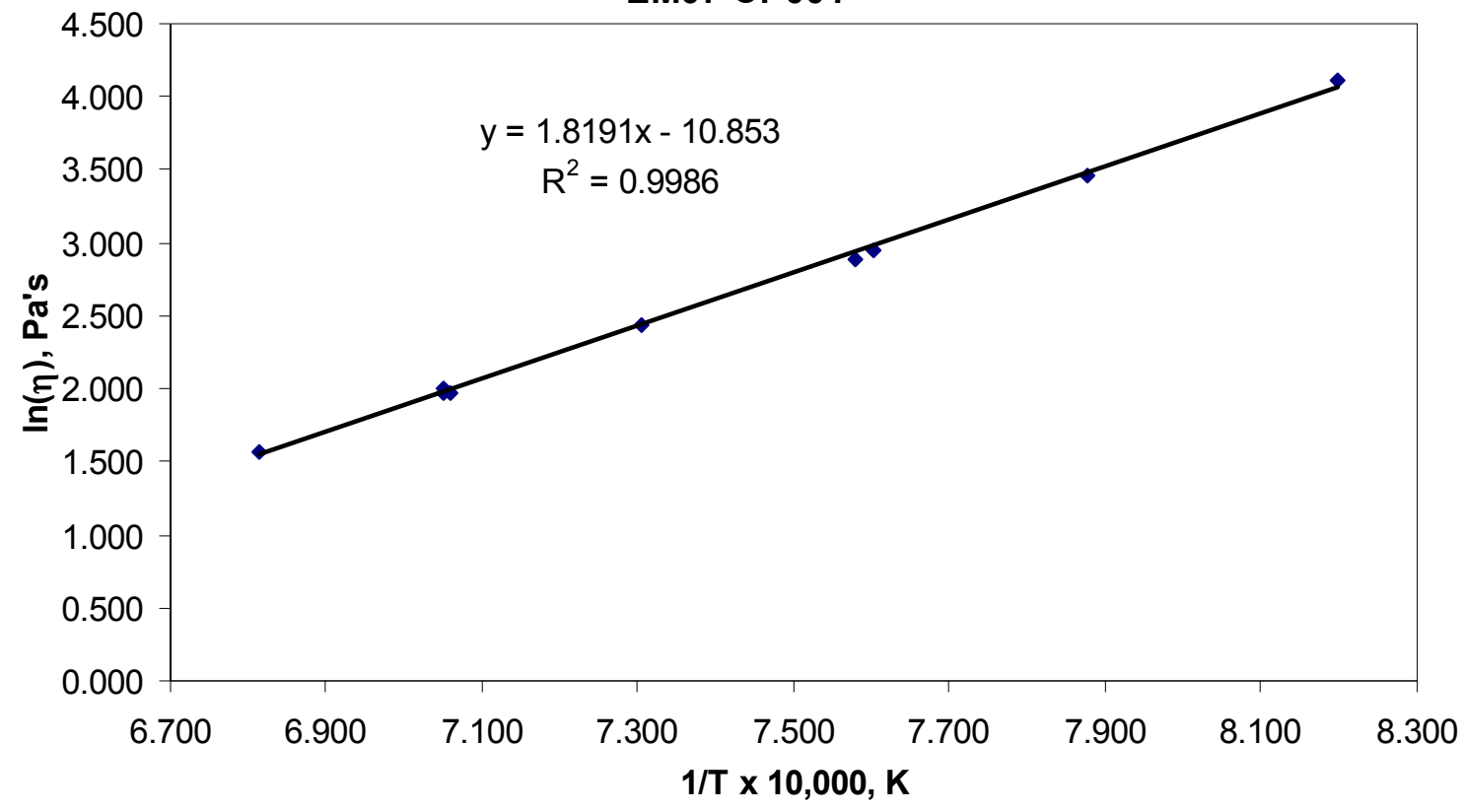

Figure A.18. Viscosity Trend for EM07-Cr-001 


\section{A.19 EM07-Cr-012 Viscosity Data}

Table A.19. Viscosity Data for EM07-Cr-012

EM07-Cr-012

\begin{tabular}{|r|r|r|}
\hline speed & \multicolumn{1}{|c|}{ speed $^{2}$} & intercept \\
\hline 0.01 & -0.00002 & 9.9485 \\
\hline
\end{tabular}

Data collected

\begin{tabular}{rrrrrrrr}
\hline Setpoint, C & Temperature, Speed, rpm Torque, \% S.F. & Viscosity, Pa's & $1 / \mathrm{T} \times 10000, \mathrm{~K}$ & $\mathrm{Ln}(\eta), \mathrm{Pa}$ 's \\
\hline 1150.0 & 1146 & 49.03 & 39.36 & 10.39 & 8.342 & 7.049 & 2.121 \\
1100.0 & 1096 & 29.68 & 39.52 & 10.23 & 13.618 & 7.304 & 2.611 \\
1050.0 & 1045 & 17.24 & 39.62 & 10.11 & 23.243 & 7.585 & 3.146 \\
1000.0 & 995 & 11.69 & 48.86 & 10.06 & 42.052 & 7.887 & 3.739 \\
950.0 & 945 & 8.76 & 72.72 & 10.03 & 83.304 & 8.207 & 4.422 \\
1050.0 & 1042 & 19.29 & 43.42 & 10.13 & 22.813 & 7.601 & 3.127 \\
1150.0 & 1142 & 52.97 & 42.83 & 10.42 & 8.428 & 7.066 & 2.132 \\
1200.0 & 1194 & 70.00 & 35.83 & 10.55 & 5.401 & 6.815 & 1.687 \\
1150.0 & 1146 & 52.67 & 42.64 & 10.42 & 8.437 & 7.045 & 2.133
\end{tabular}

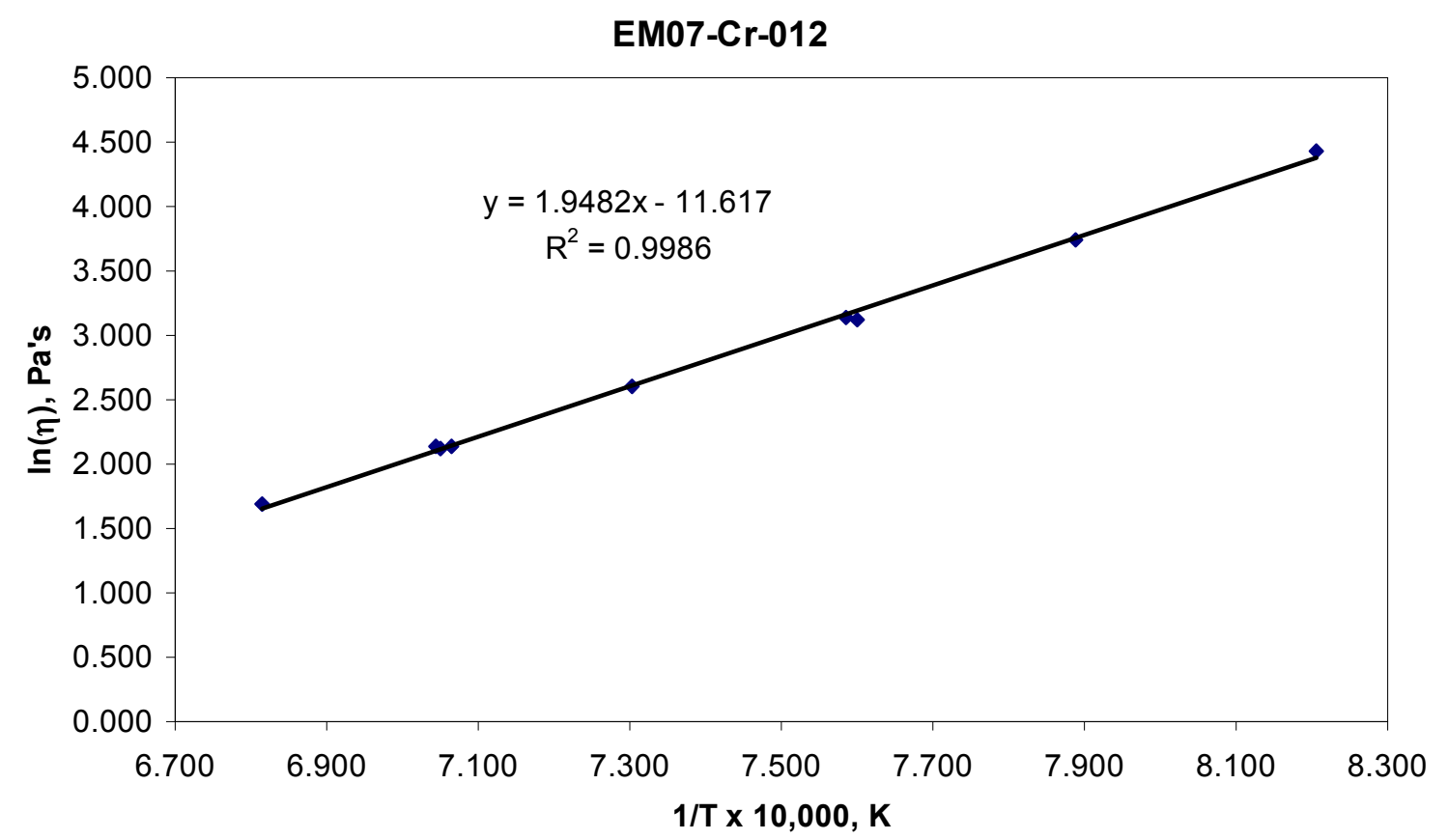

Figure A.19. Viscosity Trend for EM07-Cr-012 


\section{A.20 EM07-Cr-02 Viscosity Data}

Table A.20. Viscosity Data for EM07-Cr-02

\begin{tabular}{|c|c|c|c|c|c|c|c|}
\hline \multirow{2}{*}{\multicolumn{3}{|c|}{ EM07-Cr-02 }} & \multicolumn{3}{|c|}{ speed $\quad$ speed $^{2} \quad$ intercept } & & \\
\hline & & & 0.01 & \begin{tabular}{|l|}
-0.00002 \\
\end{tabular} & 9.9485 & & \\
\hline \multicolumn{8}{|l|}{ Data collected } \\
\hline Setpoint, C & Temperature, & Speed, rpm & Torque, $\%$ & S.F. & Viscosity, Pa's & $1 / \mathrm{T} \times 10000, \mathrm{~K}$ & Ln( $(\eta), P a ' s$ \\
\hline 1150.0 & 1145 & 33.67 & 40.53 & 10.26 & 12.352 & 7.054 & 2.514 \\
\hline 1100.0 & 1097 & 18.67 & 37.94 & 10.13 & 20.581 & 7.301 & 3.024 \\
\hline 1050.0 & 1047 & 11.17 & 39.90 & 10.06 & 35.926 & 7.576 & 3.581 \\
\hline 1000.0 & 997 & 11.35 & 68.12 & 10.06 & 60.373 & 7.871 & 4.101 \\
\hline 950.0 & 947 & 5.72 & 69.59 & 10.01 & 121.786 & 8.194 & 4.802 \\
\hline 1050.0 & 1043 & 12.31 & 41.93 & 10.07 & 34.298 & 7.599 & 3.535 \\
\hline 1150.0 & 1144 & 36.89 & 44.41 & 10.29 & 12.386 & 7.054 & 2.517 \\
\hline 1200.0 & 1195 & 56.03 & 42.55 & 10.45 & 7.933 & 6.811 & 2.071 \\
\hline 1150.0 & 1149 & 38.75 & 44.97 & 10.31 & 11.960 & 7.034 & 2.482 \\
\hline
\end{tabular}

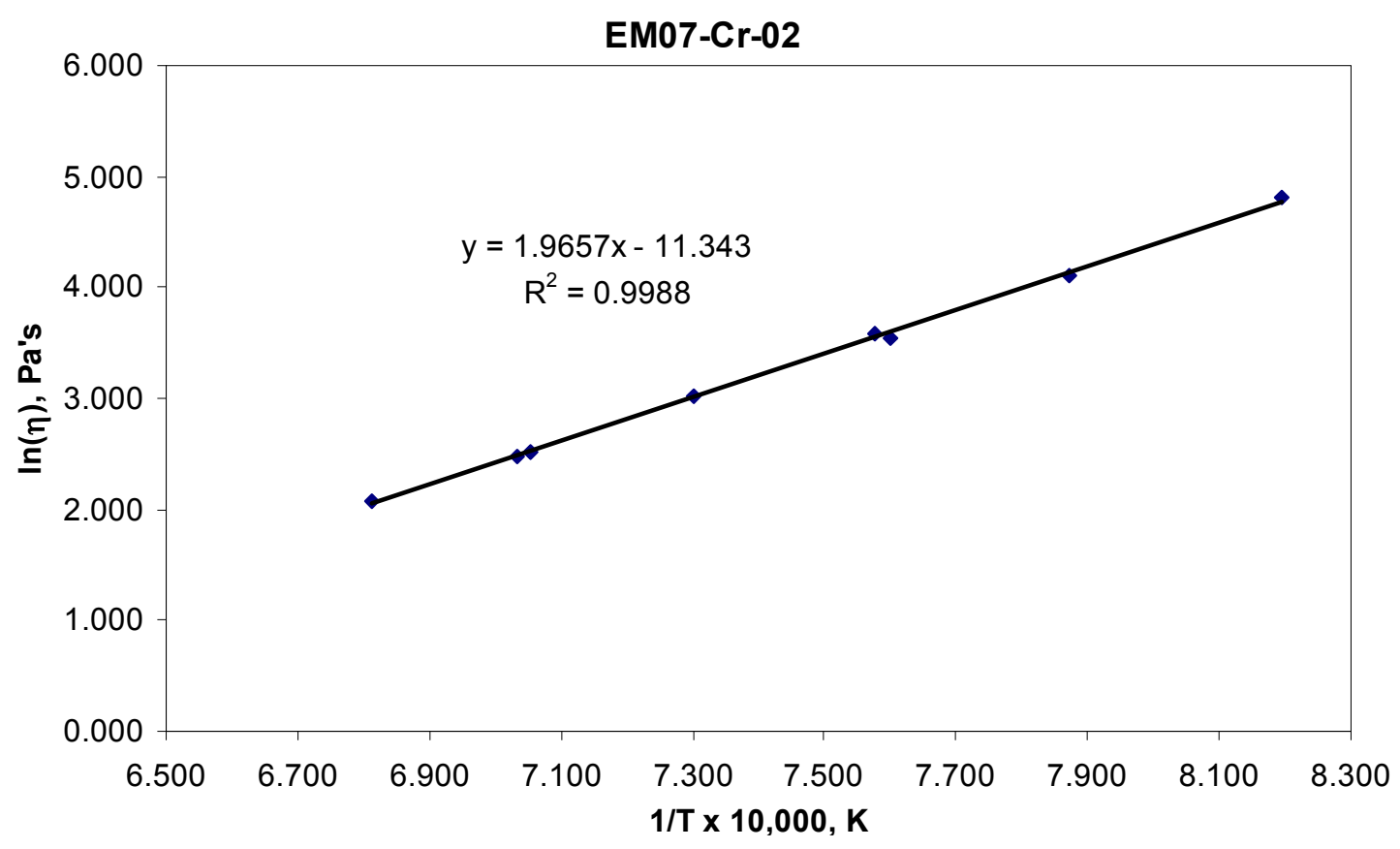

Figure A.20. Viscosity Trend for EM07-Cr-02 


\section{A.21 EM07-F-02 Viscosity Data}

Table A.21. Viscosity Data for EM07-F-02

\section{EM07-F-02}

Data collected

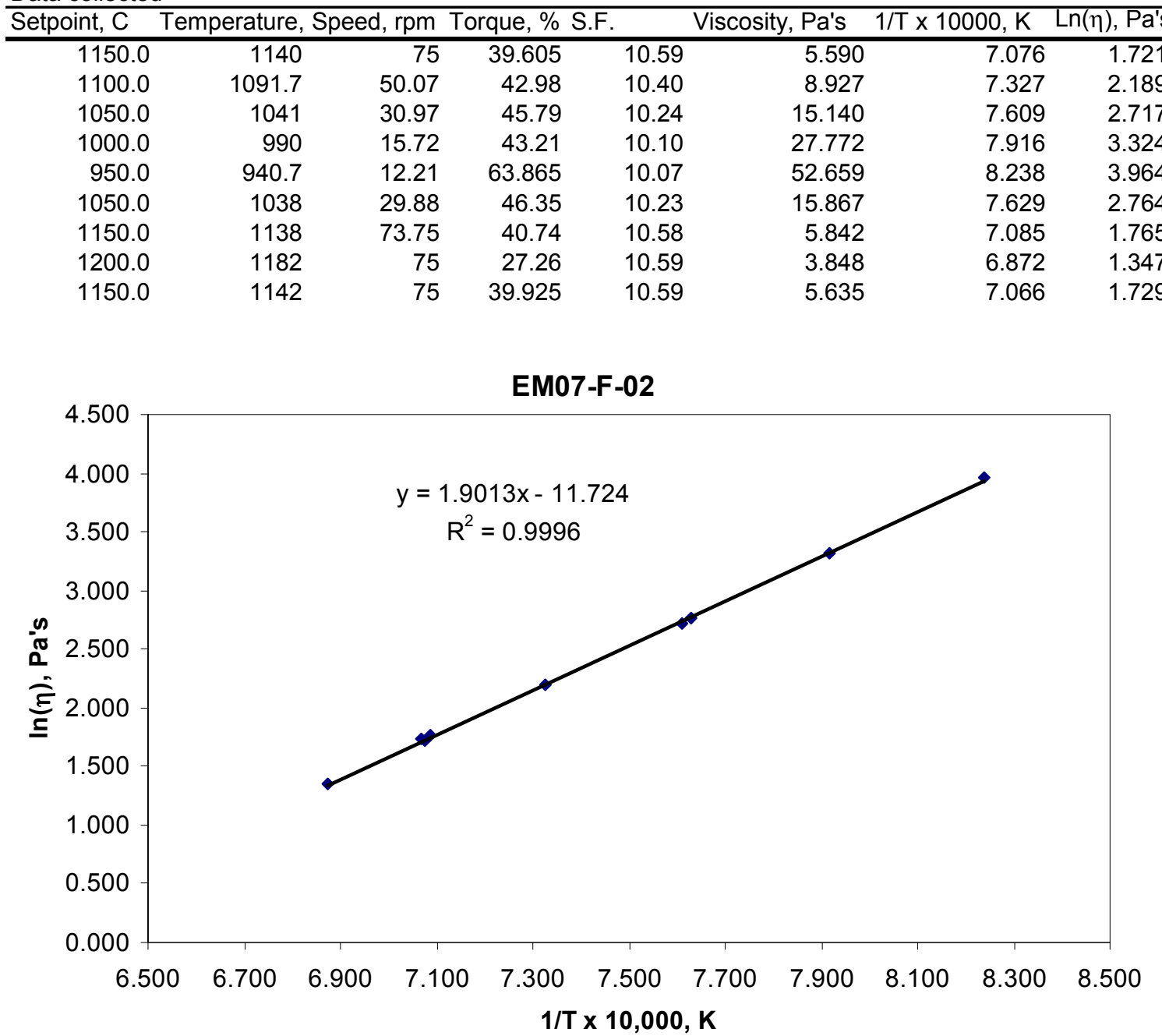

Figure A.21. Viscosity Trend for EM07-F-02

\begin{tabular}{|r|r|r|}
\hline speed & speed $^{2}$ & intercept \\
\hline 0.01 & -0.00002 & 9.9485 \\
\hline
\end{tabular}

\section{EM07-F-02} a's 


\section{A.22 EM07-Fe-05 Viscosity Data}

Table A.22. Viscosity Data for EM07-Fe-05

EM07-Fe-05

\begin{tabular}{|r|r|r|}
\hline speed & \multicolumn{1}{|c|}{ speed $^{2}$} & intercept \\
\hline 0.01 & -0.00002 & 9.9485 \\
\hline
\end{tabular}

Data collected

\begin{tabular}{rrrrrrrr}
\hline Setpoint, C & Temperature, Speed, rpm Torque, \% S.F. & Viscosity, Pa's & $1 / \mathrm{T} \times 10000, \mathrm{~K}$ & $\mathrm{Ln}(\eta), \mathrm{Pa}$ 's \\
\hline 1150.0 & 1146 & 56.53 & 40.53 & 10.45 & 7.493 & 7.046 & 2.014 \\
1100.0 & 1097 & 35.10 & 39.89 & 10.27 & 11.676 & 7.301 & 2.458 \\
1050.0 & 1047 & 21.35 & 40.41 & 10.15 & 19.216 & 7.575 & 2.956 \\
1000.0 & 997 & 11.17 & 37.64 & 10.06 & 33.890 & 7.873 & 3.523 \\
950.0 & 947 & 11.53 & 73.60 & 10.06 & 64.229 & 8.194 & 4.162 \\
1050.0 & 1043 & 22.06 & 42.62 & 10.16 & 19.633 & 7.598 & 2.977 \\
1150.0 & 1144 & 59.11 & 42.64 & 10.47 & 7.554 & 7.054 & 2.022 \\
1200.0 & 1195 & 70.00 & 32.94 & 10.55 & 4.965 & 6.812 & 1.602 \\
1150.0 & 1146 & 59.26 & 42.27 & 10.47 & 7.469 & 7.046 & 2.011
\end{tabular}

\section{EM07-Fe-05}

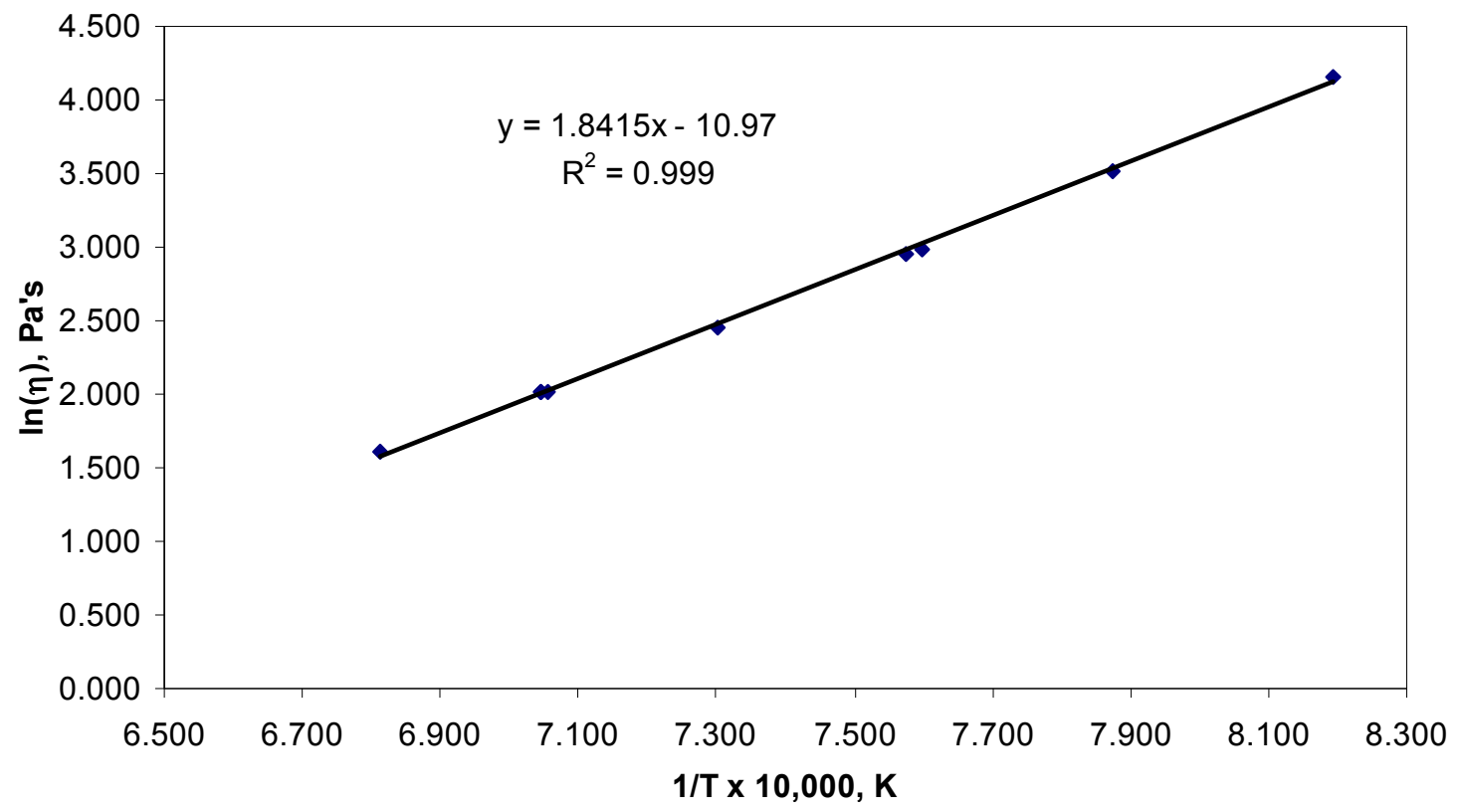

Figure A.22. Viscosity Trend for EM07-Fe-05 


\section{A.23 EM07-Fe-15 Viscosity Data}

Table A.23. Viscosity Data for EM07-Fe-15

\section{EM07-Fe-15}

\begin{tabular}{|r|r|r|}
\hline speed & \multicolumn{1}{c|}{ speed $^{2}$} & \multicolumn{2}{c|}{ intercept } \\
\hline 0.01 & -0.00002 & 9.9485 \\
\hline
\end{tabular}

Data collected

\begin{tabular}{rrrrrrrr}
\hline Setpoint, C & Temperature, Speed, rpm Torque, \% S.F. & Viscosity, Pa's & $1 / \mathrm{T} \times 10000, \mathrm{~K}$ & $\operatorname{Ln}(\eta)$, Pa's \\
\hline 1150.0 & 1146 & 57.24 & 39.76 & 10.46 & 7.263 & 7.046 & 1.983 \\
1100.0 & 1096 & 31.98 & 39.46 & 10.25 & 12.645 & 7.302 & 2.537 \\
1050.0 & 1046 & 17.24 & 38.97 & 10.11 & 22.858 & 7.578 & 3.129 \\
1000.0 & 996 & 11.86 & 49.98 & 10.06 & 42.410 & 7.876 & 3.747 \\
950.0 & 946 & 8.20 & 69.64 & 10.03 & 85.133 & 8.200 & 4.444 \\
1050.0 & 1044 & 19.13 & 42.42 & 10.13 & 22.471 & 7.594 & 3.112 \\
1150.0 & 1145 & 60.21 & 42.32 & 10.48 & 7.364 & 7.050 & 1.997 \\
1200.0 & 1195 & 70.00 & 29.76 & 10.55 & 4.485 & 6.812 & 1.501 \\
1150.0 & 1146 & 60.27 & 42.34 & 10.48 & 7.361 & 7.045 & 1.996
\end{tabular}

\section{EM07-Fe-15}

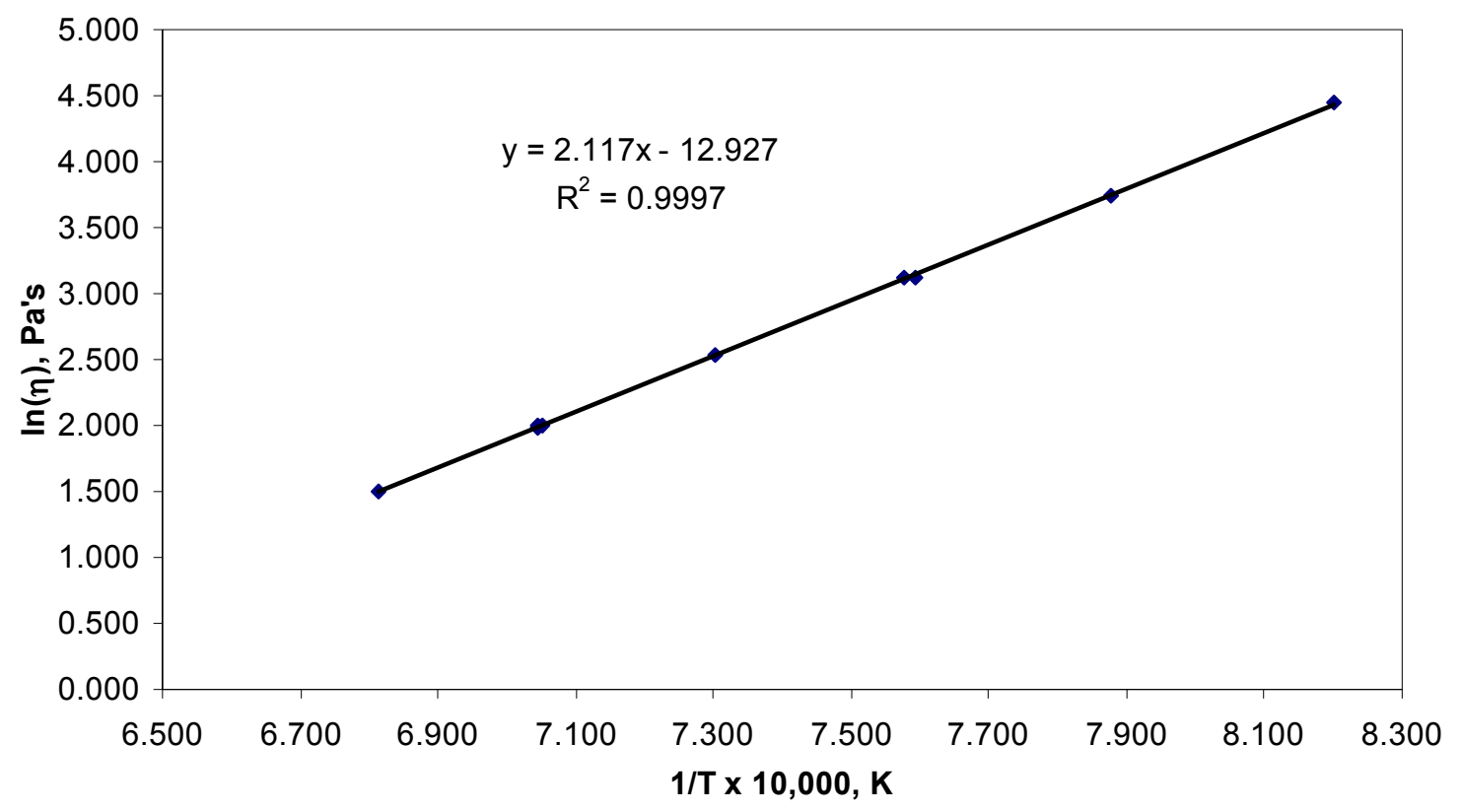

Figure A.23. Viscosity Trend for EM07-Fe-15 


\section{A.24 EM07-Fe-20 Viscosity Data}

Table A.24. Viscosity Data for EM07-Fe-20

EM07-Fe-20

Data collected

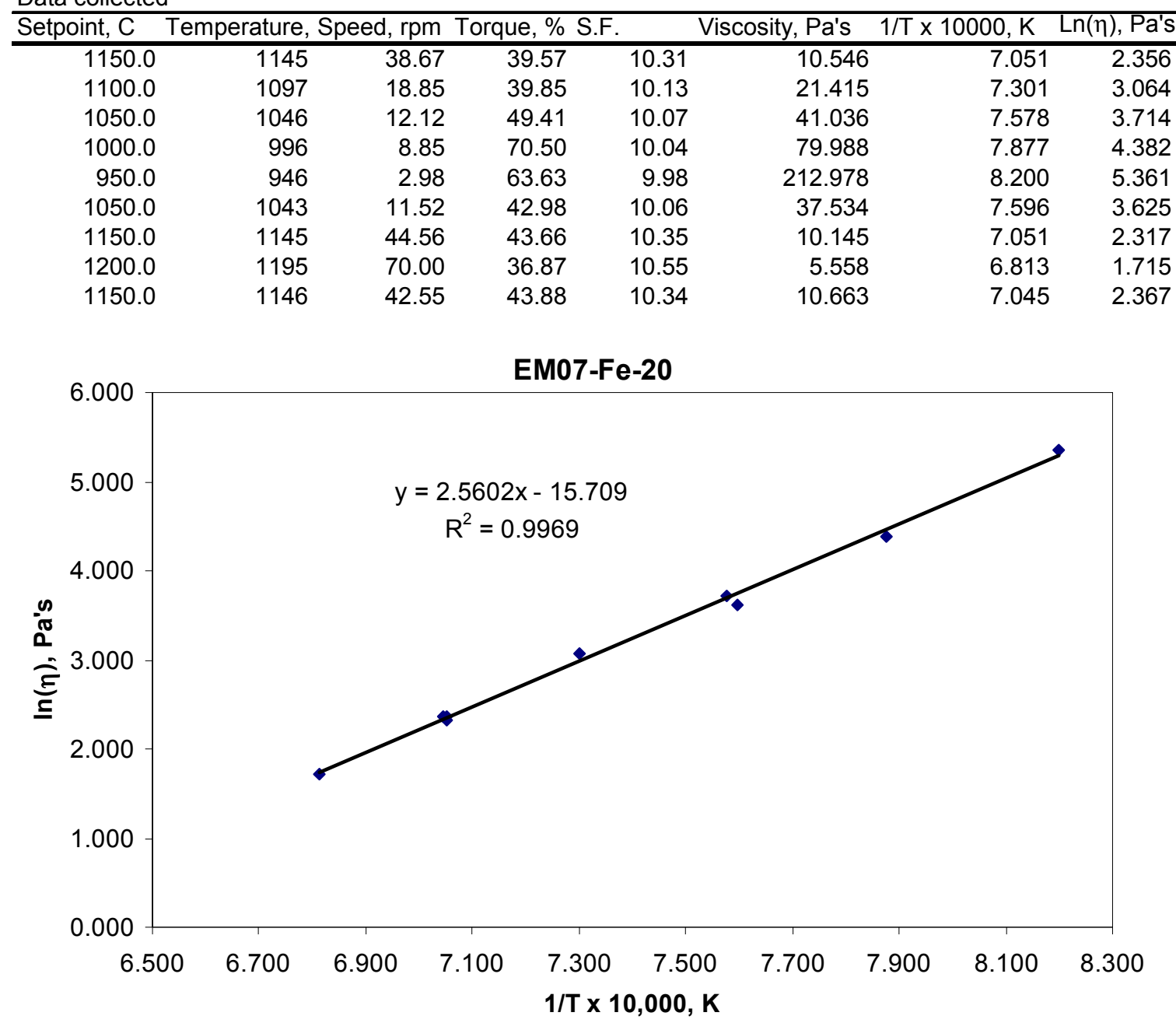

Figure A.24. Viscosity Trend for EM07-Fe-20

\begin{tabular}{|c|c|}
\hline speed & speed $^{2}$ \\
\hline 0.01 & -0.00002 \\
\hline
\end{tabular}

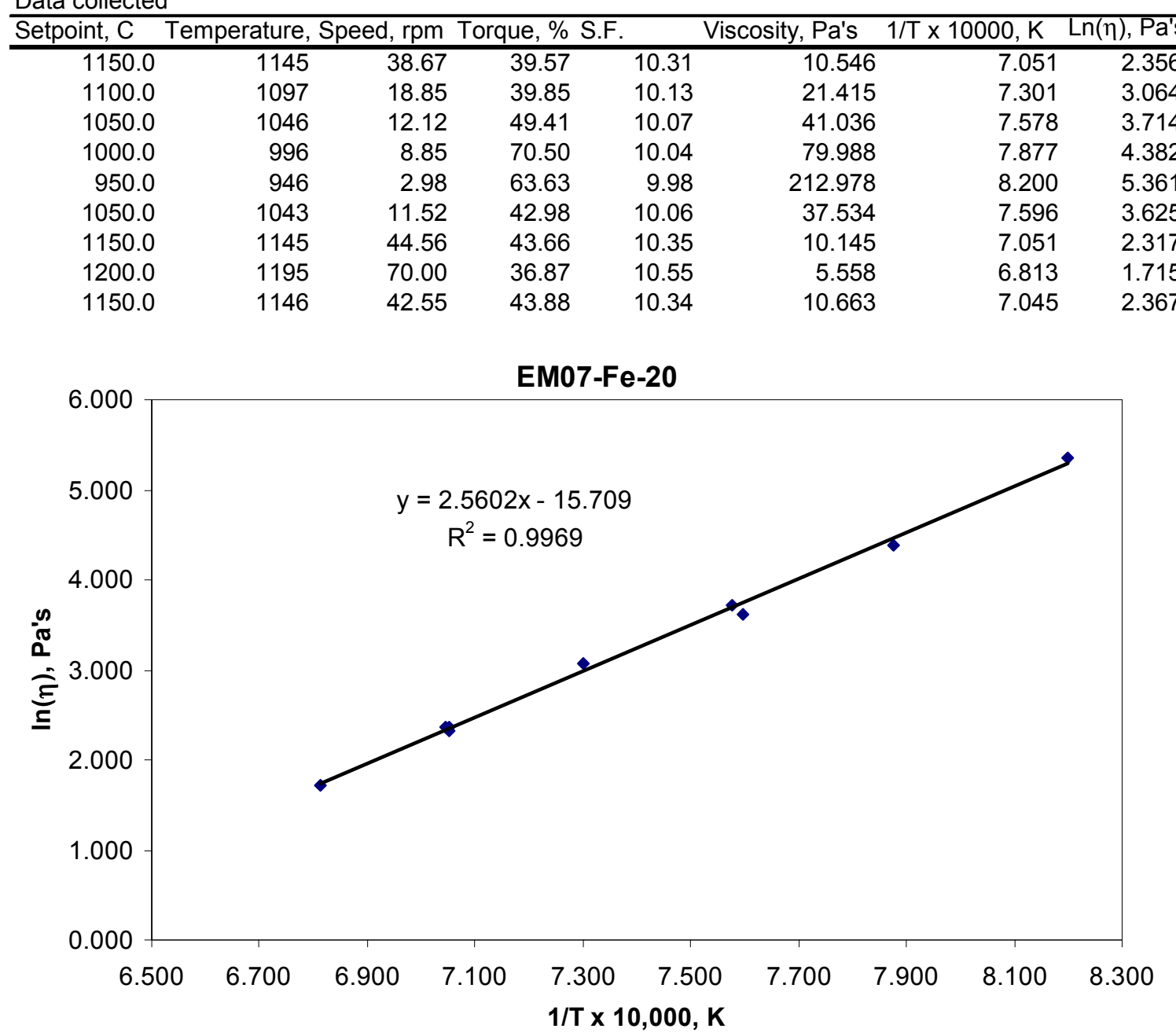




\section{A.25 EM07-K-03 Viscosity Data}

Table A.25. Viscosity Data for EM07-K-03

\begin{tabular}{|c|c|c|c|c|c|c|c|}
\hline \multirow{2}{*}{\multicolumn{3}{|c|}{ EM07-K-03 }} & \multicolumn{3}{|c|}{\begin{tabular}{|lll} 
speed & speed $^{2}$ & intercept
\end{tabular}} & & \\
\hline & & & 0.01 & -0.00002 & 9.9485 & & \\
\hline \multicolumn{8}{|l|}{ Data collected } \\
\hline Setpoint, C & Temperature, & Speed, rpm & Torque, $\%$ & S.F. & Viscosity, Pa's & $1 / \mathrm{T} \times 10000, \mathrm{~K}$ & Ln( $(\eta), P a ' s$ \\
\hline 1150.0 & 1142 & 70.00 & 37.25 & 10.55 & 5.614 & 7.065 & 1.725 \\
\hline 1100.0 & 1095.1 & 50.99 & 42.95 & 10.41 & 8.767 & 7.309 & 2.171 \\
\hline 1050.0 & 1046 & 33.02 & 46.03 & 10.26 & 14.298 & 7.580 & 2.660 \\
\hline 1000.0 & 996 & 17.57 & 42.88 & 10.12 & 24.694 & 7.878 & 3.207 \\
\hline 950.0 & 946 & 12.49 & 57.21 & 10.07 & 46.125 & 8.199 & 3.831 \\
\hline 1050.0 & 1043 & 32.36 & 45.96 & 10.25 & 14.559 & 7.598 & 2.678 \\
\hline 1150.0 & 1143 & 70.00 & 37.91 & 10.55 & 5.715 & 7.060 & 1.743 \\
\hline 1200.0 & 1193 & 70.00 & 24.73 & 10.55 & 3.728 & 6.819 & 1.316 \\
\hline 1150.0 & 1145 & 70.00 & 37.32 & 10.55 & 5.624 & 7.053 & 1.727 \\
\hline
\end{tabular}

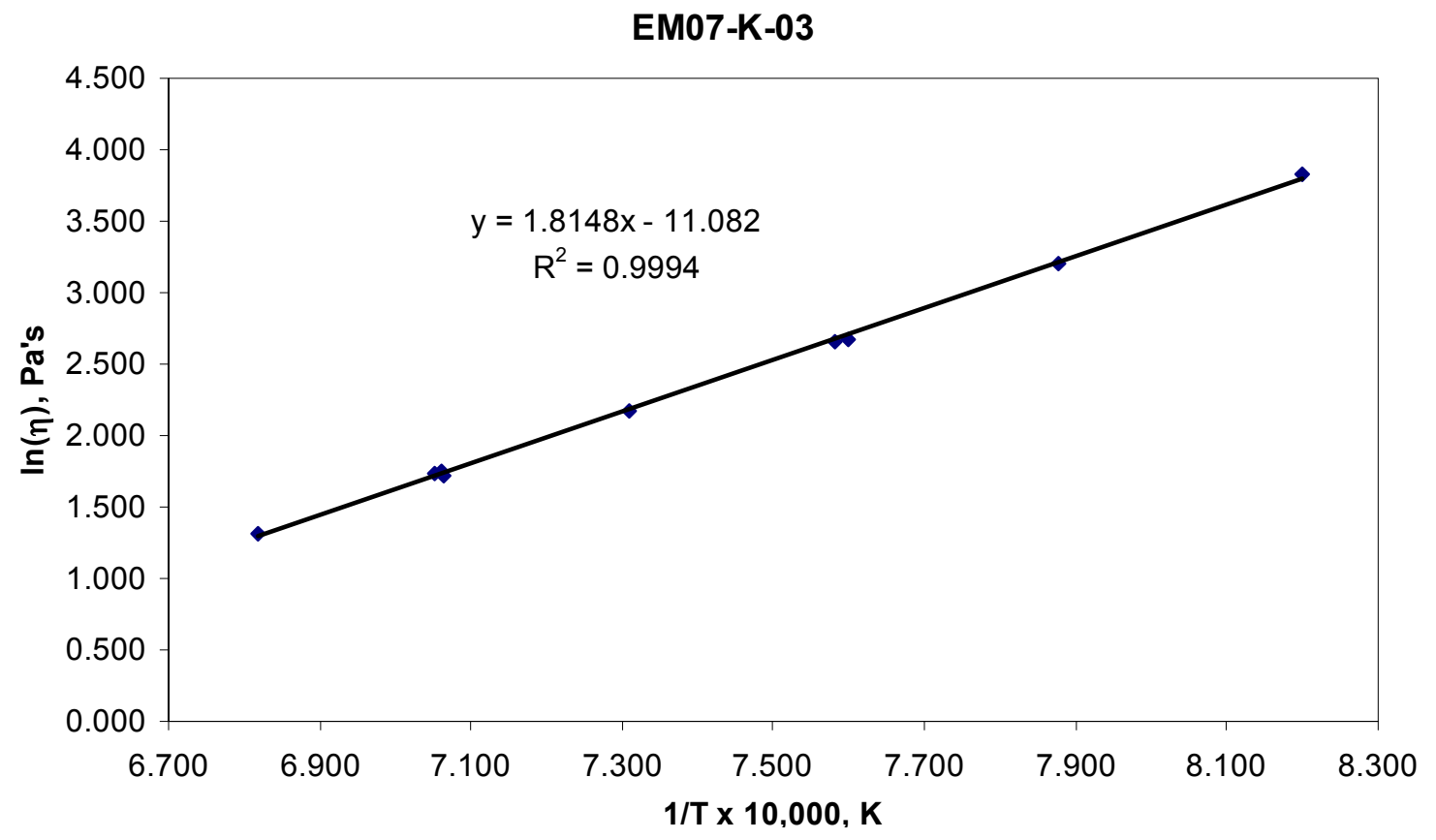

Figure A.25. Viscosity Trend for EM07-K-03 


\section{A.26 EM07-K-06 Viscosity Data}

Table A.26. Viscosity Data for EM07-K-06

\section{EM07-K-06}

\begin{tabular}{|c|c|c|}
\hline speed & speed $^{2}$ & intercept \\
\hline$\overline{0.0}$ & $11-0.00002$ & 9.9485 \\
\hline
\end{tabular}

Data collected

\begin{tabular}{rrrrrrrr}
\hline Setpoint, C & Temperature, Speed, rpm Torque, \% S.F. & Viscosity, Pa's & $1 / \mathrm{T} \times 10000, \mathrm{~K}$ & $\operatorname{Ln}(\eta)$, Pa's \\
\hline 1150.0 & 1146 & 70.00 & 31.53 & 10.55 & 4.752 & 7.049 & 1.558 \\
1100.0 & 1096 & 59.73 & 42.20 & 10.47 & 7.400 & 7.302 & 2.002 \\
1050.0 & 1047 & 38.52 & 44.42 & 10.30 & 11.881 & 7.576 & 2.475 \\
1000.0 & 997 & 21.35 & 42.78 & 10.15 & 20.347 & 7.874 & 3.013 \\
950.0 & 947 & 12.48 & 45.82 & 10.07 & 36.970 & 8.194 & 3.610 \\
1050.0 & 1042 & 37.47 & 44.66 & 10.30 & 12.270 & 7.603 & 2.507 \\
1150.0 & 1144 & 70.00 & 32.17 & 10.55 & 4.849 & 7.059 & 1.579 \\
1200.0 & 1194 & 70.00 & 21.99 & 10.55 & 3.315 & 6.815 & 1.198 \\
1150.0 & 1145 & 70.00 & 32.73 & 10.55 & 4.933 & 7.050 & 1.596
\end{tabular}

\section{EM07-K-06}

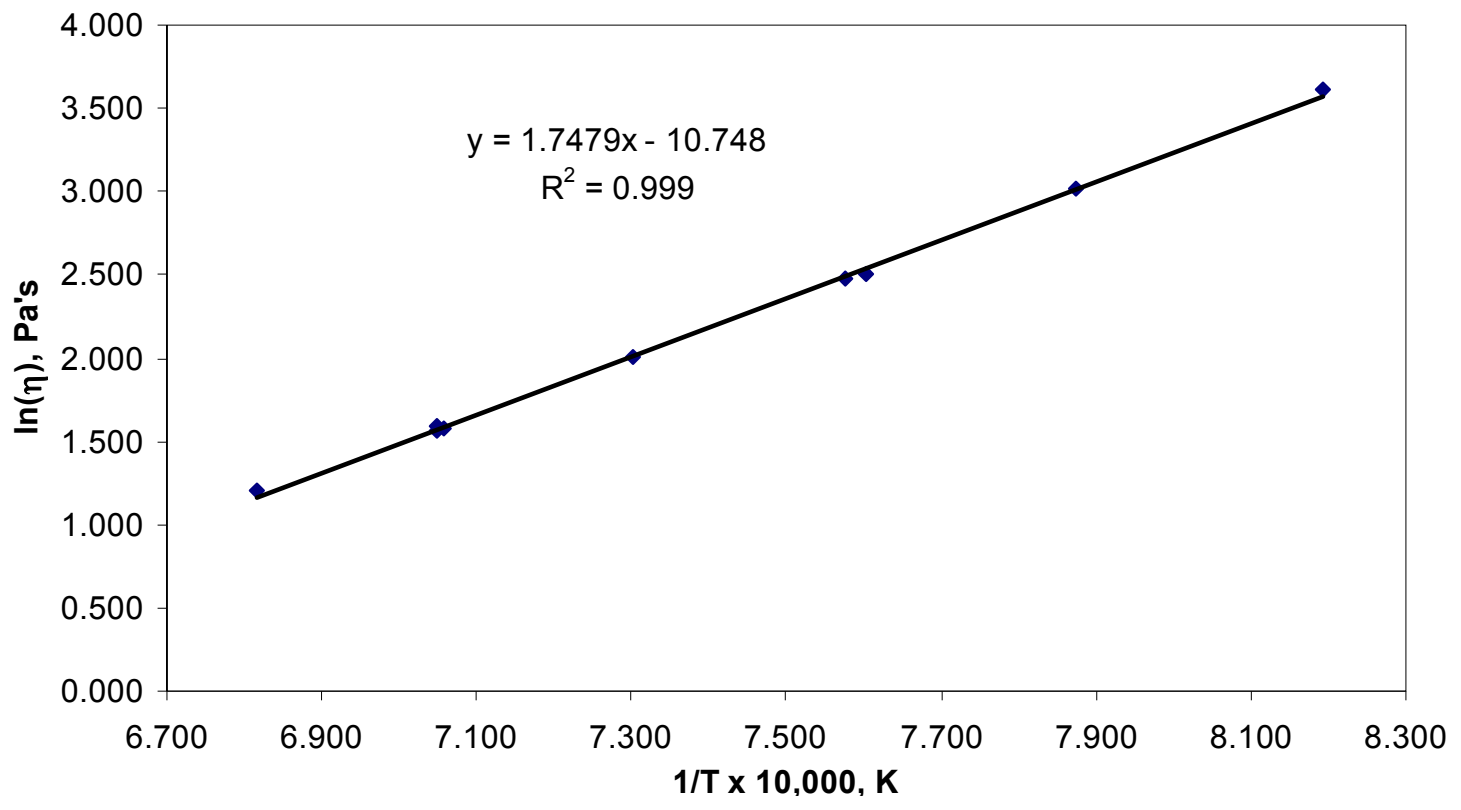

Figure A.26. Viscosity Trend for EM07-K-06 


\section{A.27 EM07-Li-015 Viscosity Data}

Table A.27. Viscosity Data for EM07-Li-015

\begin{tabular}{|c|c|c|c|c|c|c|c|}
\hline \multirow{3}{*}{\multicolumn{3}{|c|}{ EMC }} & \multirow{2}{*}{\multicolumn{3}{|c|}{ speed $^{2} \quad$ intercept }} & & \\
\hline & & & & & & & \\
\hline & & & 0.01 & -0.00002 & 9.9485 & & \\
\hline ollected & & & & & & & \\
\hline Setpoint, C & Temperature, & Speed, rpm & Torque, $\%$ & S.F. & Viscosity, Pa's & $1 / \mathrm{T} \times 10000, \mathrm{~K}$ & $\operatorname{Ln}(\eta), \mathrm{Pa}^{\prime} \mathrm{s}$ \\
\hline 1150.0 & 1138 & 28.67 & 40.01 & 10.22 & 14.260 & 7.084 & 2.657 \\
\hline 1100.0 & 1091 & 16.35 & 39.40 & 10.11 & 24.352 & 7.329 & 3.193 \\
\hline 1050.0 & 1042 & 11.35 & 48.88 & 10.06 & 43.321 & 7.606 & 3.769 \\
\hline 1000.0 & 990 & 8.57 & 72.29 & 10.03 & 84.596 & 7.914 & 4.438 \\
\hline 950.0 & 942 & 3.26 & 64.46 & 9.98 & 197.254 & 8.228 & 5.284 \\
\hline 1050.0 & 1038 & 12.23 & 52.87 & 10.07 & 43.524 & 7.627 & 3.773 \\
\hline 1150.0 & 1138 & 32.56 & 45.52 & 10.25 & 14.335 & 7.086 & 2.663 \\
\hline 1200.0 & 1188 & 50.75 & 42.55 & 10.40 & 8.724 & 6.843 & 2.166 \\
\hline 1150.0 & 1141 & 33.50 & 45.43 & 10.26 & 13.913 & 7.071 & 2.633 \\
\hline
\end{tabular}

\section{EM07-Li-015}

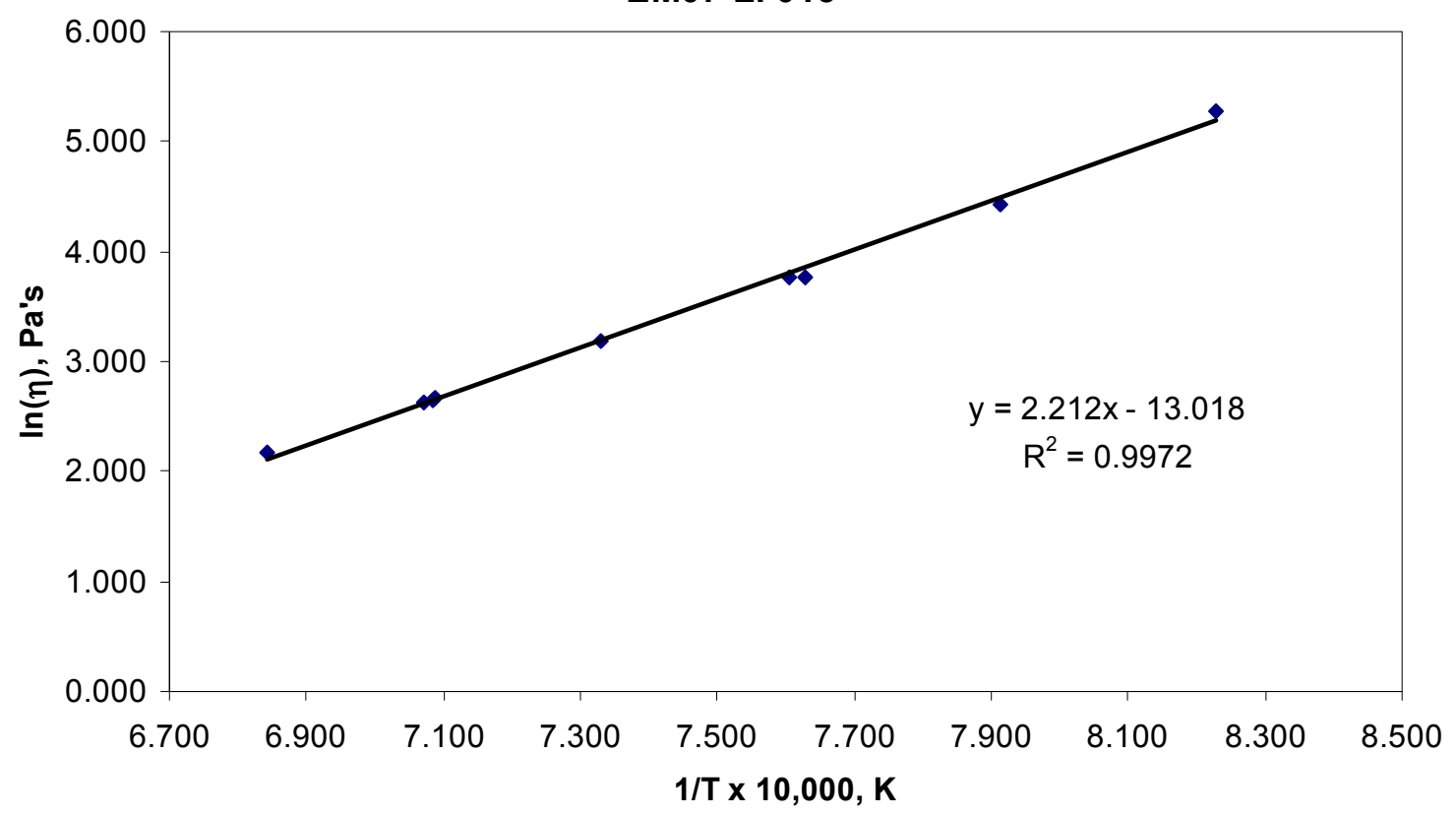

Figure A.27. Viscosity Trend for EM07-Li-015 


\section{A.28 EM07-Li-04 Viscosity Data}

Table A.28. Viscosity Data for EM07-Li-04

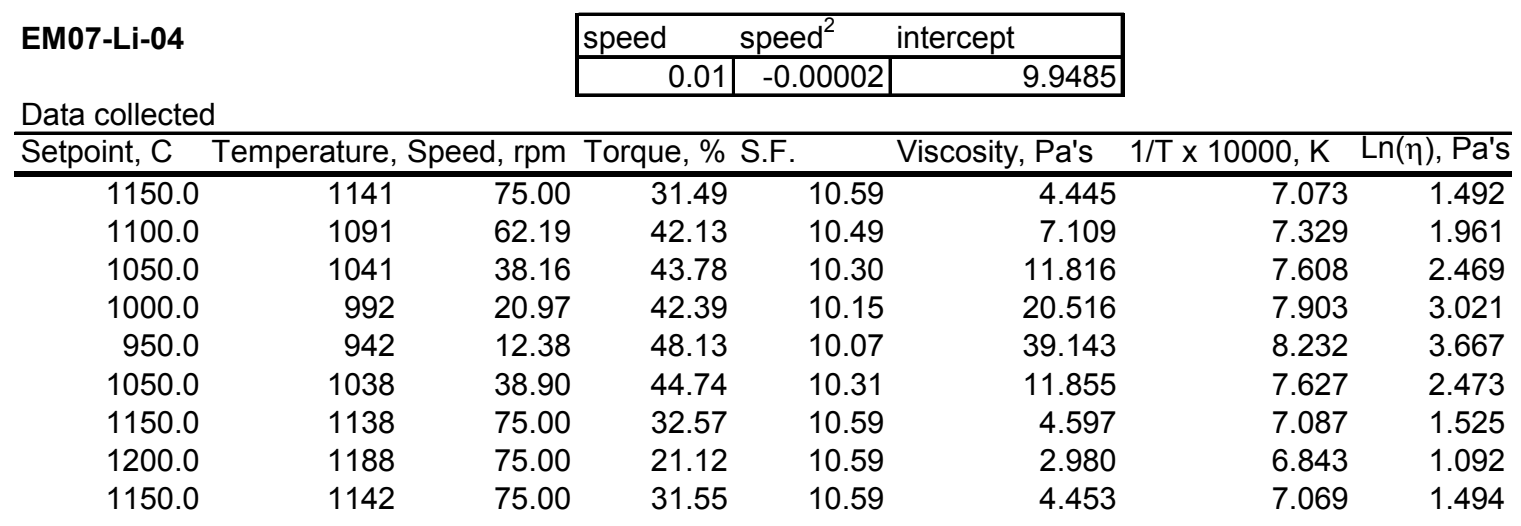

\section{EM07-Li-04}

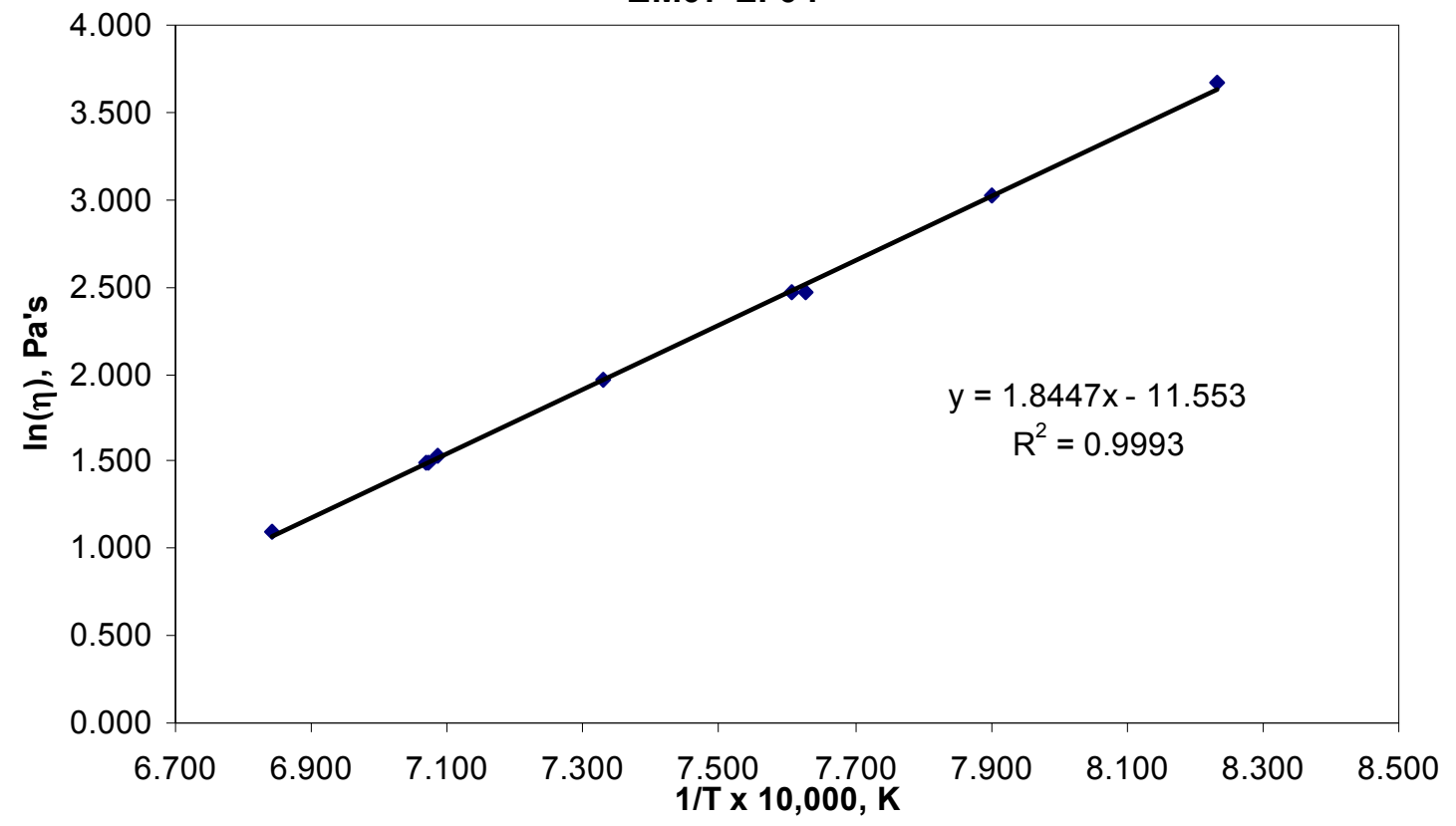

Figure A.28. Viscosity Trend for EM07-Li-04 


\section{A.29 EM07-Mn-01 Viscosity Data}

Table A.29. Viscosity Data for EM07-Mn-01

\begin{tabular}{|c|c|c|c|c|c|c|c|}
\hline \multirow{3}{*}{\multicolumn{3}{|c|}{1}} & \multirow{2}{*}{\multicolumn{3}{|c|}{ speed $^{2} \quad$ intercept }} & & \\
\hline & & & & & & & \\
\hline & & & 0.01 & -0.00002 & 9.9485 & & \\
\hline Data collected & & & & & & & \\
\hline Setpoint, C & Temperature, & ed, rpm & Torque, $\%$ & S.F. & Viscosity, Pa's & $1 / \mathrm{T} \times 10000, \mathrm{~K}$ & $\mathrm{Ln}(\eta), \mathrm{Pa} \mathrm{s}^{\prime}$ \\
\hline 1150.0 & 1146 & 48.85 & 40.30 & 10.39 & 8.570 & 7.049 & 2.148 \\
\hline 1100.0 & 1096 & 28.85 & 39.29 & 10.22 & 13.920 & 7.303 & 2.633 \\
\hline 1050.0 & 1047 & 16.35 & 38.88 & 10.11 & 24.031 & 7.577 & 3.179 \\
\hline 1000.0 & 997 & 11.85 & 49.49 & 10.06 & 42.033 & 7.875 & 3.738 \\
\hline 950.0 & 947 & 8.81 & 72.34 & 10.04 & 82.424 & 8.194 & 4.412 \\
\hline 1050.0 & 1042 & 18.26 & 43.60 & 10.12 & 24.175 & 7.604 & 3.185 \\
\hline 1150.0 & 1144 & 51.41 & 42.89 & 10.41 & 8.685 & 7.059 & 2.162 \\
\hline 1200.0 & 1194 & 70.00 & 37.08 & 10.55 & 5.589 & 6.815 & 1.721 \\
\hline 1150.0 & 1145 & 51.98 & 42.98 & 10.41 & 8.610 & 7.050 & 2.153 \\
\hline
\end{tabular}

\section{EM07-Mn-01}

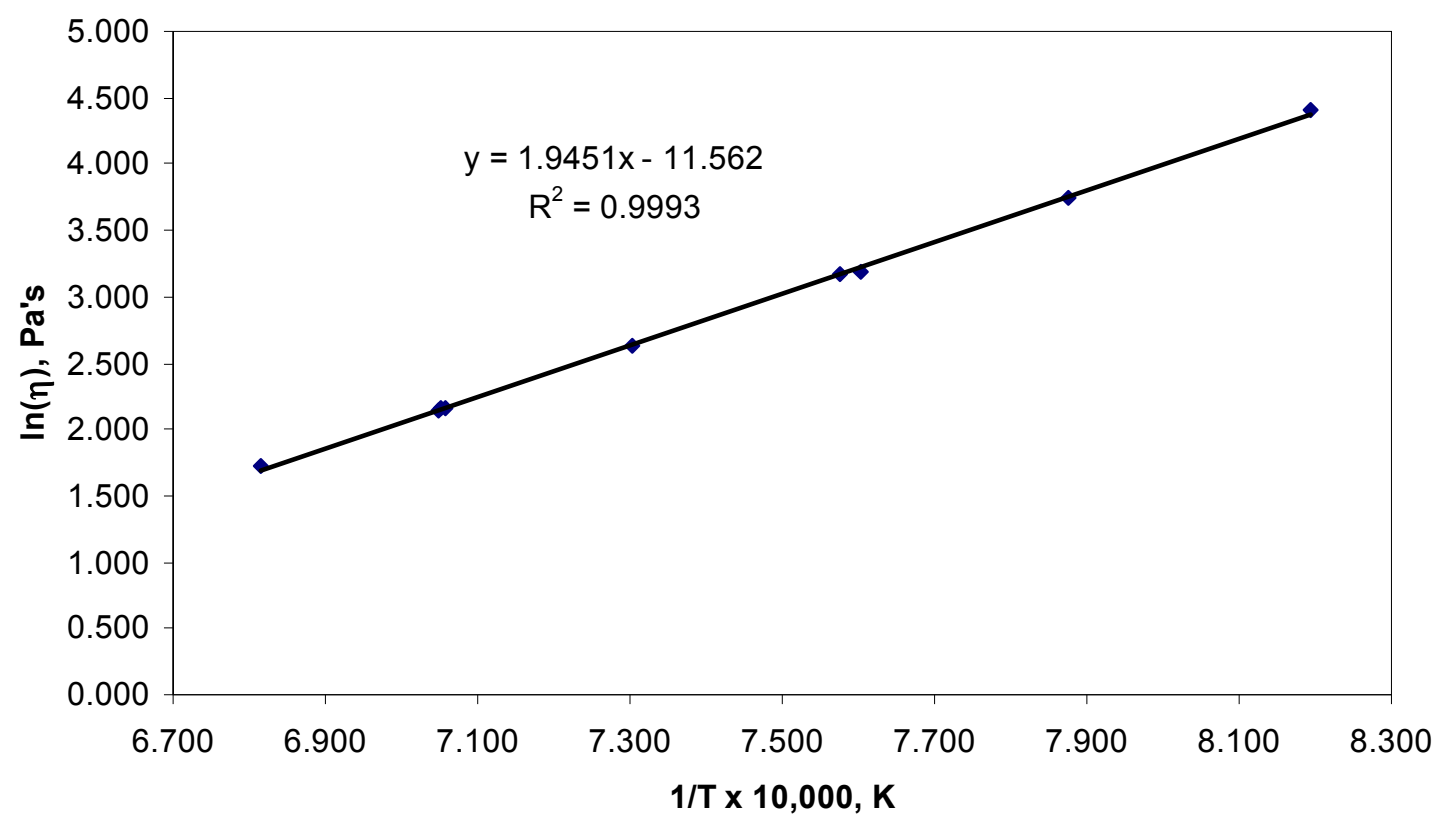

Figure A.29. Viscosity Trend for EM07-Mn-01 


\section{A.30 EM07-Mn-04 Viscosity Data}

Table A.30. Viscosity Data for EM07-Mn-04

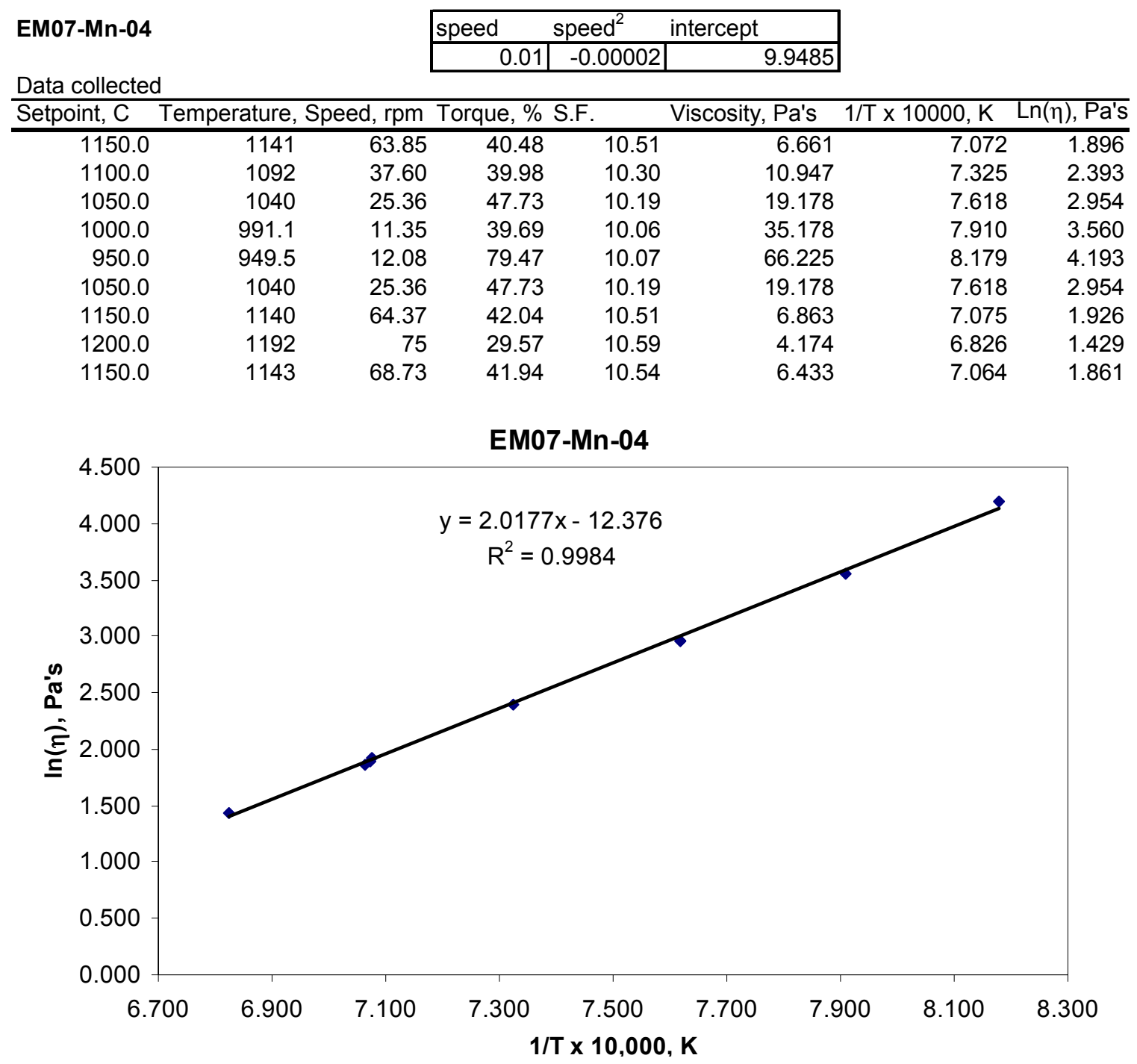

Figure A.30. Viscosity Trend for EM07-Mn-04 


\section{A.31 EM07-Na-05 Viscosity Data}

Table A.31. Viscosity Data for EM07-Na-05

\section{EM07-Na-05}

\begin{tabular}{|r|r|r|}
\hline speed & \multicolumn{1}{|c|}{ speed $^{2}$} & intercept \\
\hline 0.01 & -0.00002 & 9.9485 \\
\hline
\end{tabular}

Data collected

\begin{tabular}{rrrrrrrr}
\hline Setpoint, C & Temperature, Speed, rpm & Torque, \% S.F. & & Viscosity, Pa's & $1 / T \times 10000, \mathrm{~K}$ & $\operatorname{Ln}(\eta)$, Pa's \\
\hline 1150.0 & 1139 & 2.36 & 61.46 & 9.97 & 259.462 & 7.082 & 5.559 \\
1200.0 & 1190 & 7.93 & 72.77 & 10.03 & 92.025 & 6.832 & 4.522 \\
1150.0 & 1141 & 2.79 & 65.03 & 9.98 & 232.544 & 7.070 & 5.449
\end{tabular}

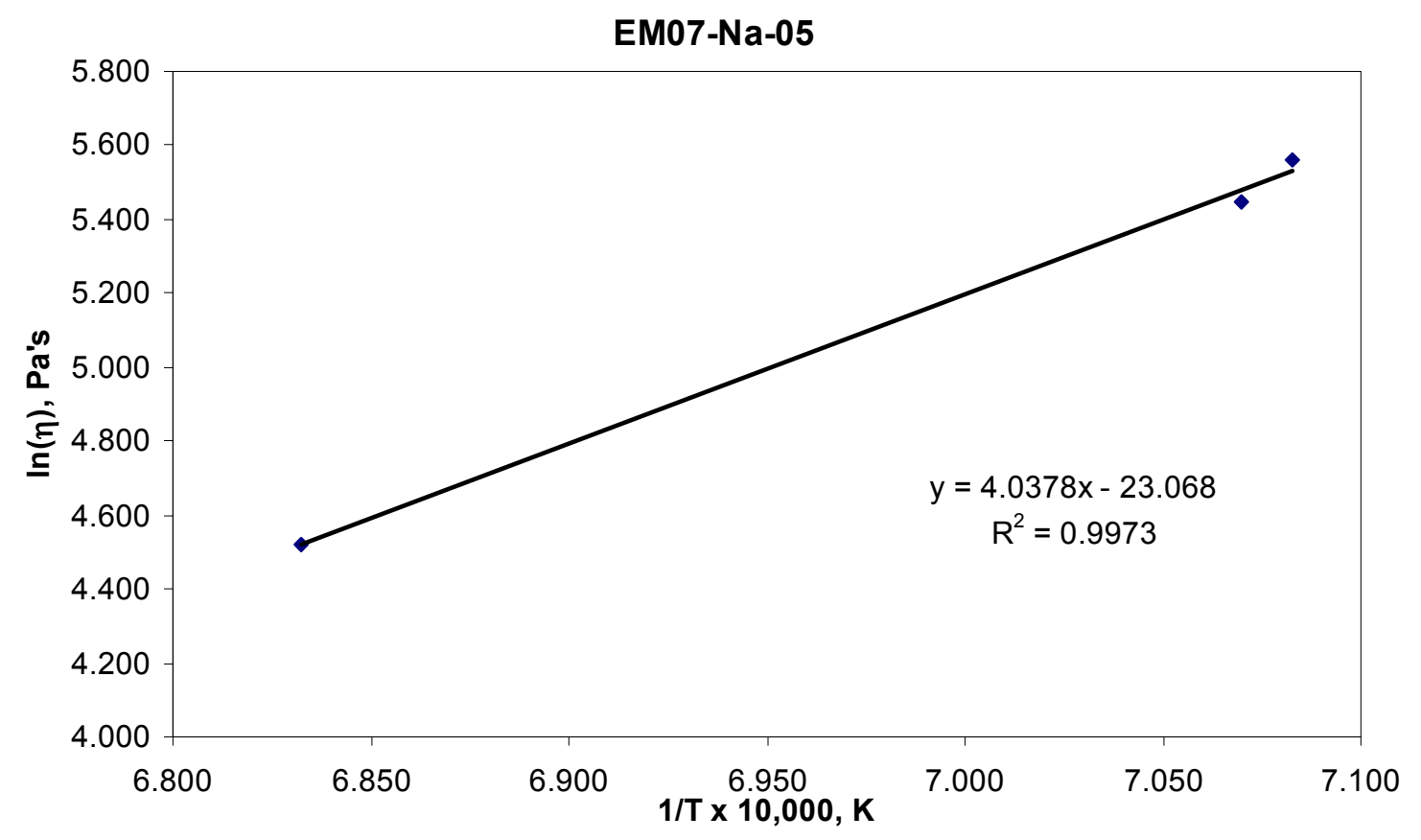

Figure A.31. Viscosity Trend for EM07-Na-05 


\section{A.32 EM07-Na-10 Viscosity Data}

Table A.32. Viscosity Data for EM07-Na-10

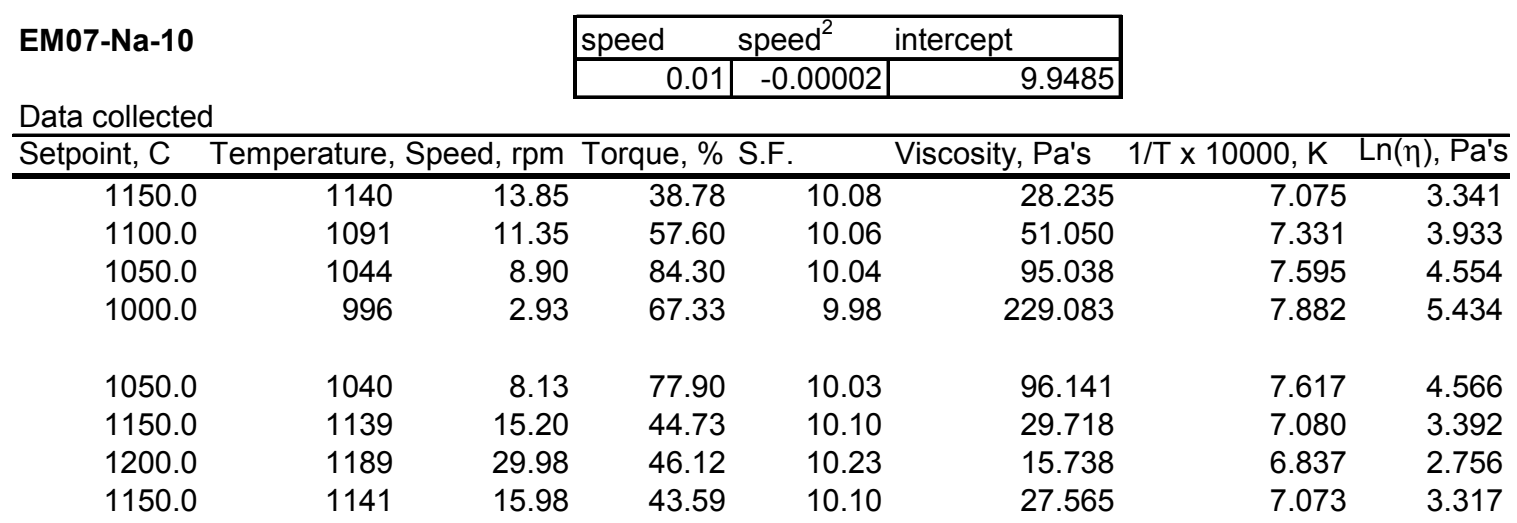

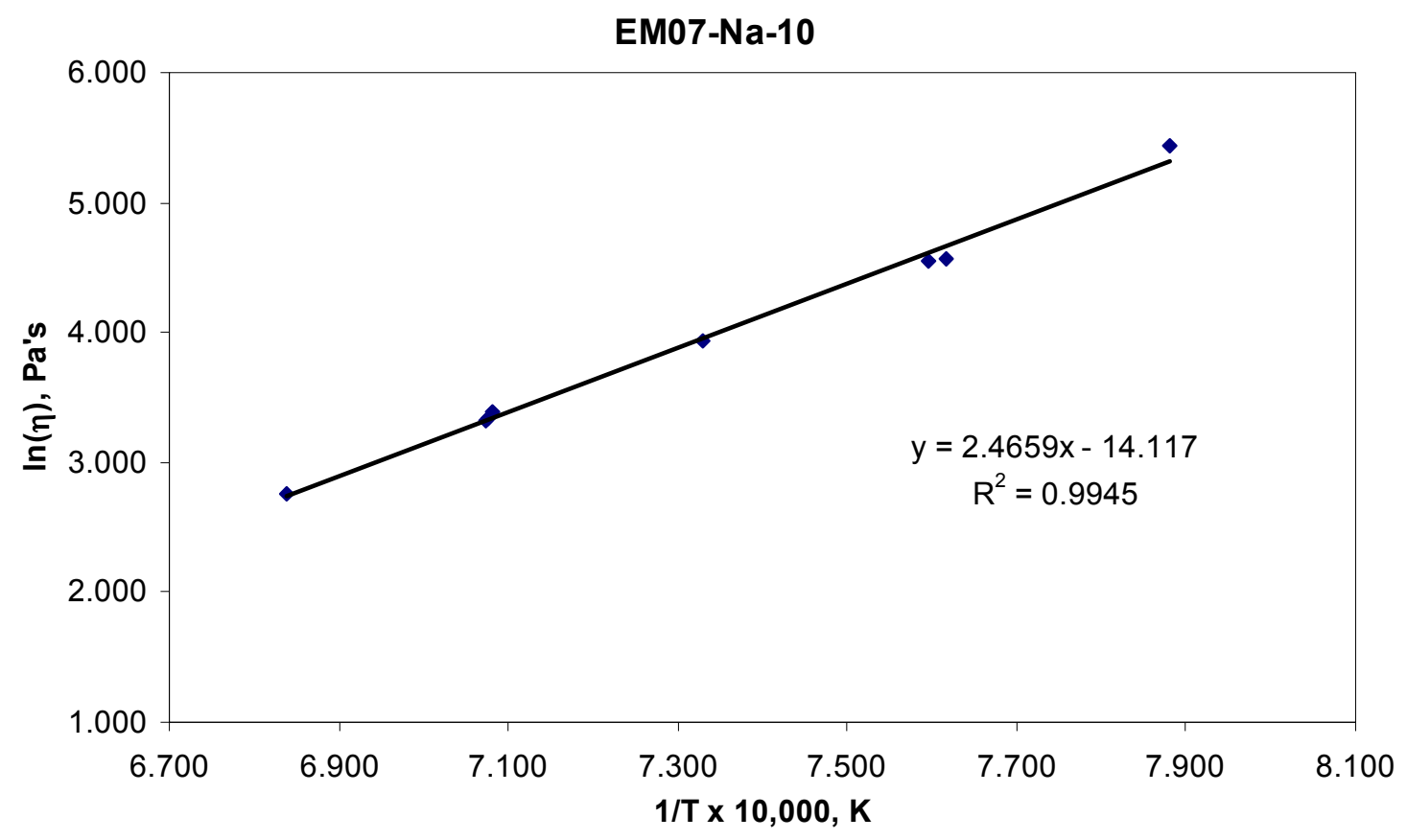

Figure A.32. Viscosity Trend for EM07-Na-10 


\section{A.33 EM07-Na-20 Viscosity Data}

Table A.33. Viscosity Data for EM07-Na-20

\begin{tabular}{|c|c|c|c|c|c|c|c|}
\hline \multirow{3}{*}{\multicolumn{3}{|c|}{ E }} & \multirow{2}{*}{\multicolumn{3}{|c|}{ speed $^{2} \quad$ intercept }} & & \\
\hline & & & & & & & \\
\hline & & & 0.01 & -0.00002 & 9.9485 & & \\
\hline Data collected & & & & & & & \\
\hline Setpoint, C & Temperature, & $\mathrm{d}, \mathrm{rpm}$ & Torque, $\%$ & S.F. & Viscosity, Pa's & $1 / \mathrm{T} \times 10000, \mathrm{~K}$ & $\mathrm{Ln}(\eta), \mathrm{Pa} \mathrm{s}^{\prime}$ \\
\hline 1150.0 & 1145 & 70.00 & 20.79 & 10.55 & 3.133 & 7.050 & 1.142 \\
\hline 1100.0 & 1096 & 70.00 & 31.65 & 10.55 & 4.770 & 7.301 & 1.562 \\
\hline 1050.0 & 1046 & 58.85 & 42.40 & 10.47 & 7.542 & 7.579 & 2.020 \\
\hline 1000.0 & 996 & 37.34 & 45.14 & 10.29 & 12.443 & 7.878 & 2.521 \\
\hline 950.0 & 946 & 19.83 & 42.83 & 10.14 & 21.898 & 8.204 & 3.086 \\
\hline 1050.0 & 1043 & 58.48 & 42.45 & 10.46 & 7.597 & 7.597 & 2.028 \\
\hline 1150.0 & 1140 & 70.00 & 21.18 & 10.55 & 3.192 & 7.079 & 1.161 \\
\hline 1200.0 & 1195 & 70.00 & 14.53 & 10.55 & 2.190 & 6.813 & 0.784 \\
\hline 1150.0 & 1146 & 70.00 & 21.11 & 10.55 & 3.182 & 7.045 & 1.158 \\
\hline
\end{tabular}

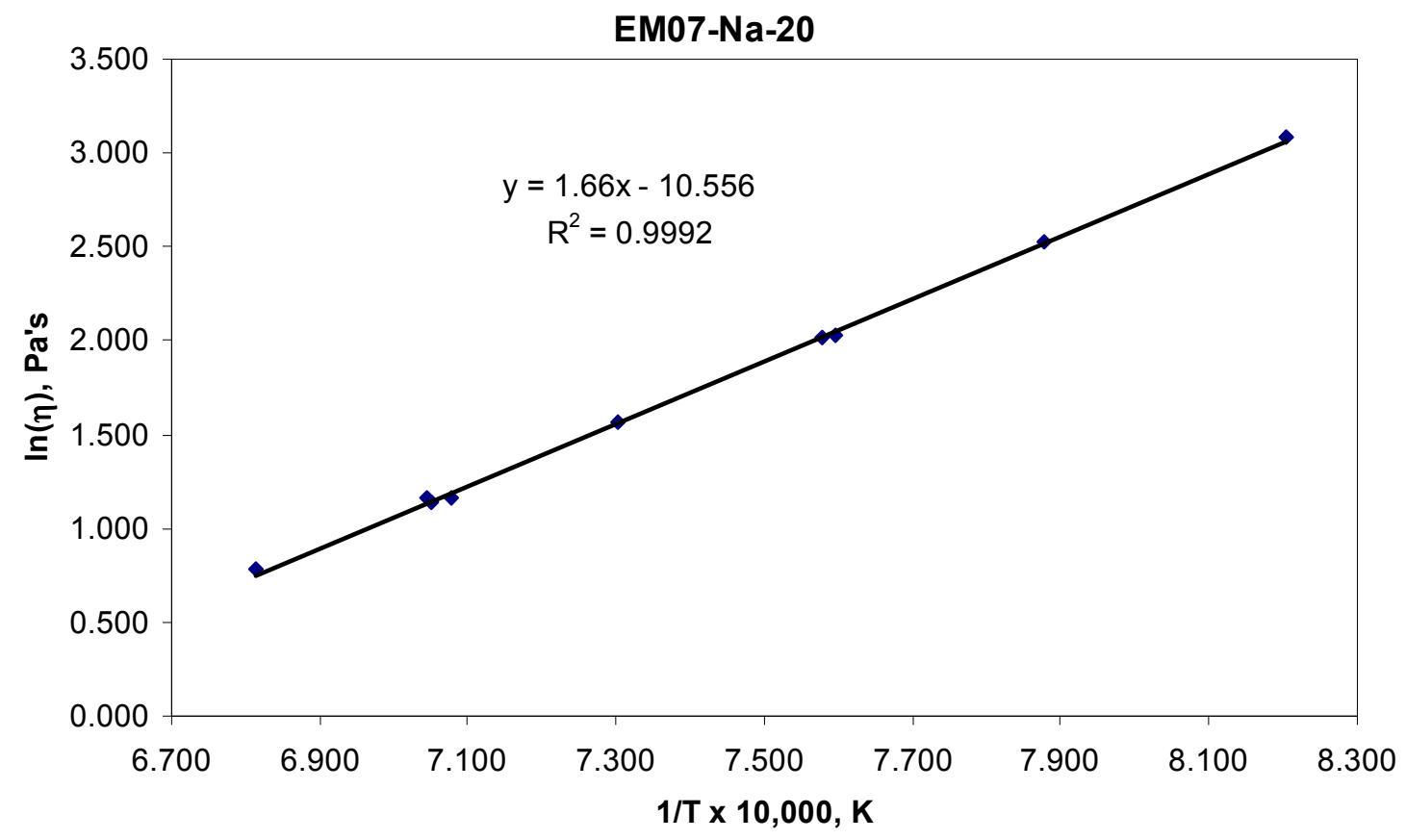

Figure A.33. Viscosity Trend for EM07-Na-20 


\section{A.34 EM07-Ni-001 Viscosity Data}

Table A.34. Viscosity Data for EM07-Ni-001

\section{EM07-Ni-001}

\begin{tabular}{|r|r|r|}
\hline speed & speed $^{2}$ & intercept \\
\hline 0.01 & -0.00002 & 9.9485 \\
\hline
\end{tabular}

Data collected

\begin{tabular}{rrrrrrrr}
\hline Setpoint, C & Temperature, Speed, rpm Torque, \% S.F. & Viscosity, Pa's & $1 / \mathrm{T} \times 10000, \mathrm{~K}$ & $\operatorname{Ln}(\eta), \mathrm{Pa}$ 's \\
\hline 1150.0 & 1144 & 57.60 & 39.99 & 10.46 & 7.260 & 7.055 & 1.982 \\
1100.0 & 1095 & 36.35 & 40.95 & 10.29 & 11.586 & 7.308 & 2.450 \\
1050.0 & 1046 & 21.21 & 40.38 & 10.15 & 19.327 & 7.583 & 2.961 \\
1000.0 & 996 & 11.17 & 38.15 & 10.06 & 34.344 & 7.881 & 3.536 \\
950.0 & 945 & 12.10 & 79.03 & 10.07 & 65.746 & 8.211 & 4.186 \\
1050.0 & 1041 & 25.40 & 48.74 & 10.19 & 19.551 & 7.609 & 2.973 \\
1150.0 & 1141 & 59.54 & 42.40 & 10.47 & 7.459 & 7.070 & 2.009
\end{tabular}

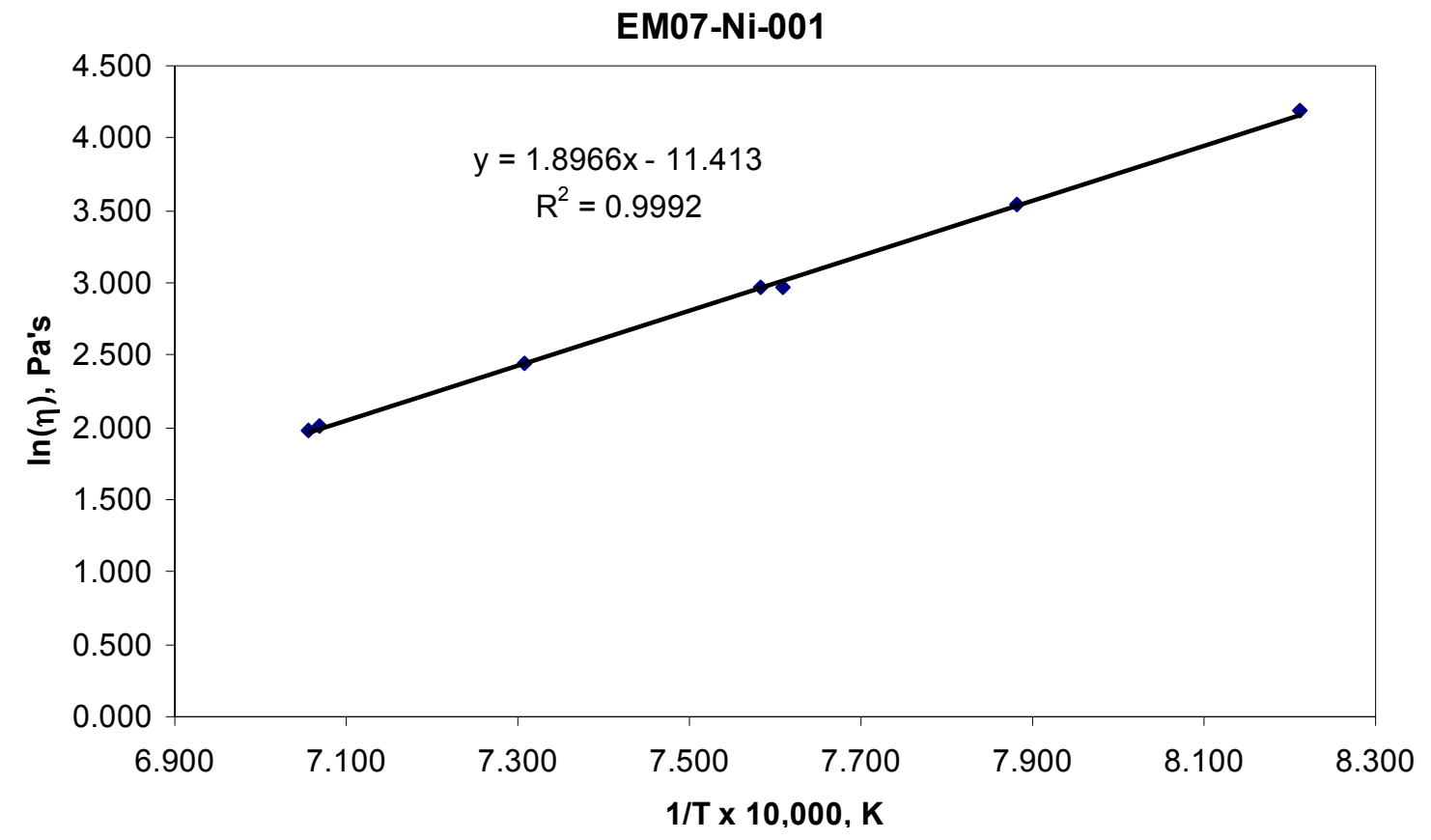

Figure A.34. Viscosity Trend for EM07-Ni-001 


\section{A.35 EM07-Ni-02 Viscosity Data}

Table A.35. Viscosity Data for EM07-Ni-02

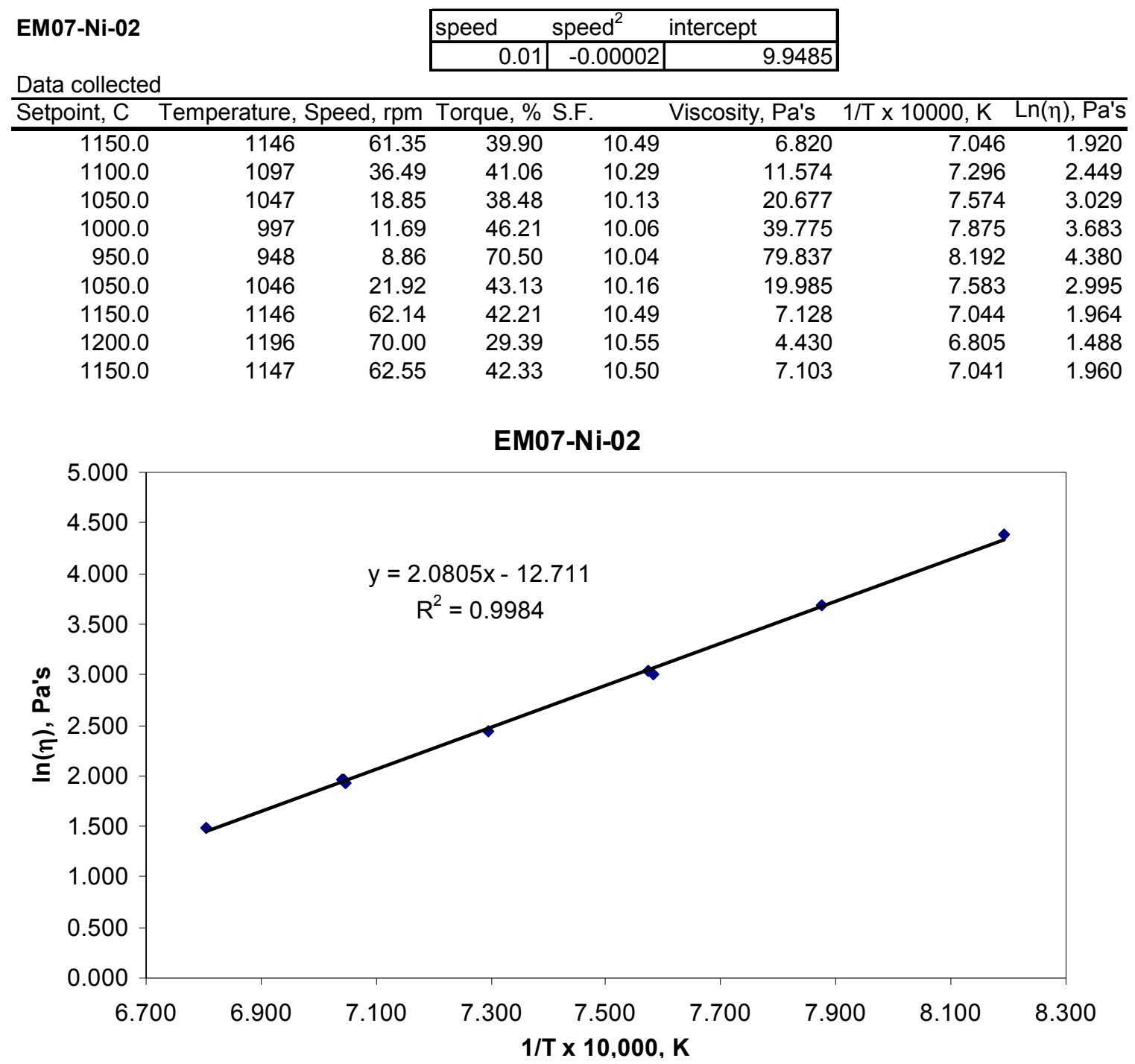

Figure A.35. Viscosity Trend for EM07-Ni-02 


\section{A.36 EM07-P-0 Viscosity Data}

Table A.36. Viscosity Data for EM07-P-0

\section{EM07-P-0}

\begin{tabular}{|r|r|r|}
\hline speed & \multicolumn{1}{c|}{ speed $^{2}$} & \multicolumn{2}{c|}{ intercept } \\
\hline 0.01 & -0.00002 & 9.9485 \\
\hline
\end{tabular}

Data collected

\begin{tabular}{rrrrrrrr}
\hline Setpoint, C & Temperature, Speed, rpm & Torque, \% S.F. & Viscosity, Pa's & $1 / \mathrm{T} \times 10000, \mathrm{~K}$ & $\operatorname{Ln}(\eta)$, Pa's \\
\hline 1150.0 & 1146 & 58.85 & 39.34 & 10.47 & 6.998 & 7.048 & 1.946 \\
1100.0 & 1096 & 36.17 & 39.60 & 10.28 & 11.258 & 7.302 & 2.421 \\
1050.0 & 1046 & 21.35 & 40.20 & 10.15 & 19.115 & 7.579 & 2.950 \\
1000.0 & 996 & 11.35 & 39.02 & 10.06 & 34.582 & 7.878 & 3.543 \\
950.0 & 946 & 11.58 & 76.94 & 10.06 & 66.849 & 8.204 & 4.202 \\
1050.0 & 1044 & 25.19 & 47.83 & 10.19 & 19.343 & 7.590 & 2.962 \\
1150.0 & 1145 & 62.34 & 42.15 & 10.49 & 7.096 & 7.053 & 1.960 \\
1200.0 & 1195 & 70.00 & 30.06 & 10.55 & 4.531 & 6.811 & 1.511 \\
1150.0 & 1146 & 63.04 & 42.15 & 10.50 & 7.019 & 7.046 & 1.949
\end{tabular}

EM07-P-0

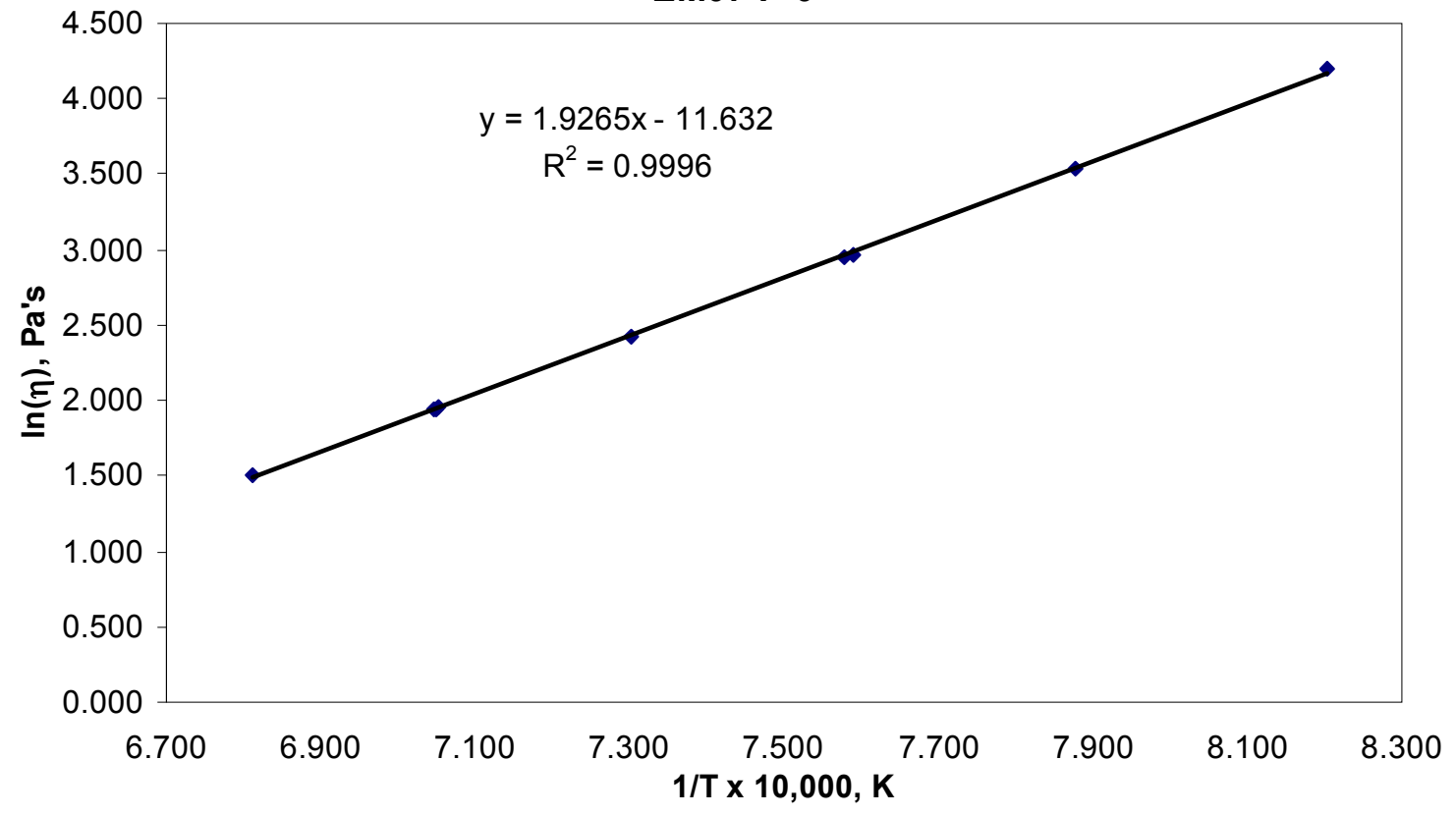

Figure A.36. Viscosity Trend for EM07-P-0 


\section{A.37 EM07-P-025 Viscosity Data}

Table A.37. Viscosity Data for EM07-P-025

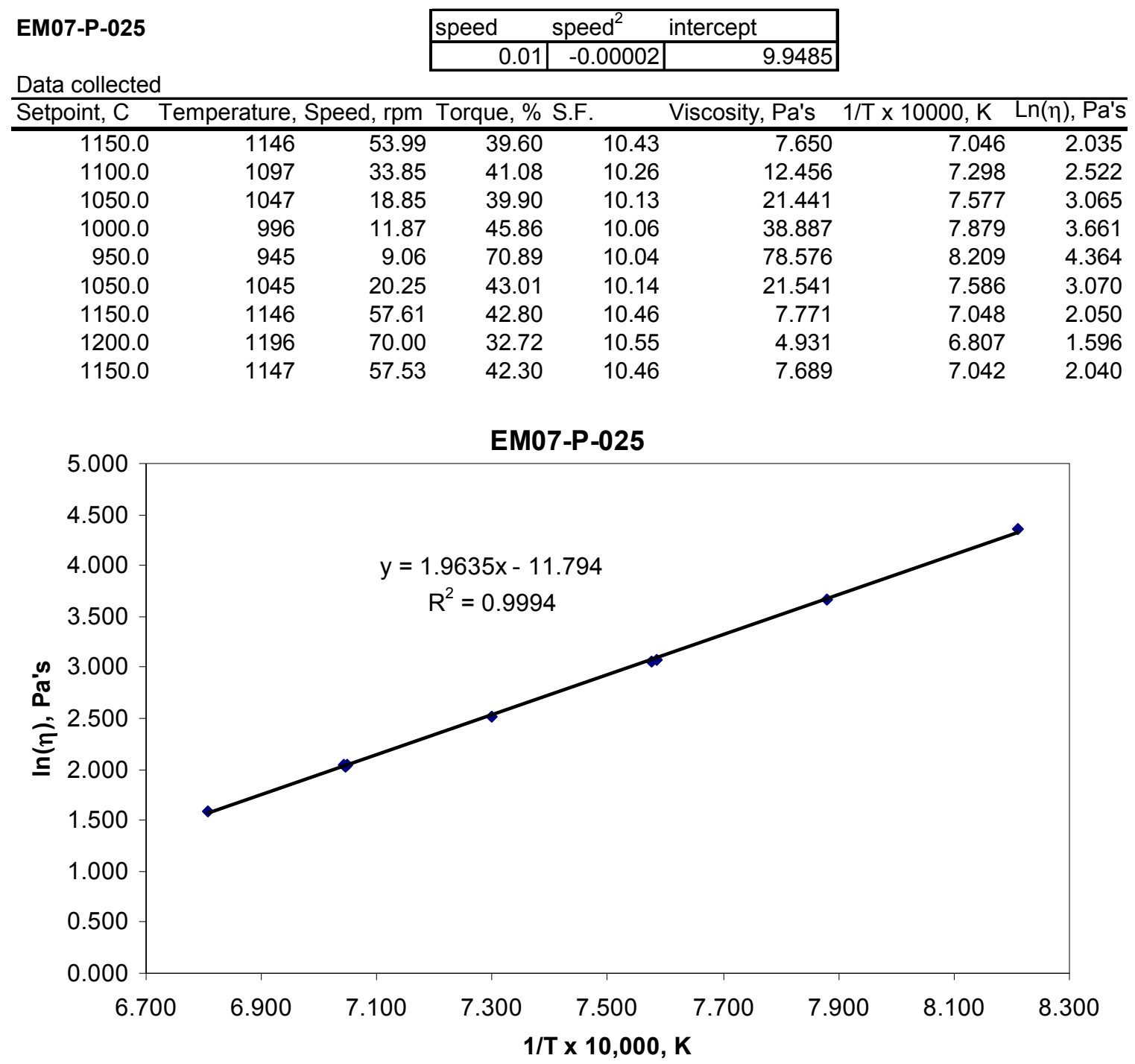

Figure A.37. Viscosity Trend for EM07-P-025 


\section{A.38 EM07-Si-30 Viscosity Data}

Table A.38. Viscosity Data for EM07-Si-30

\section{EM07-Si-30}

\begin{tabular}{|c|c|c|}
\hline speed & speed $^{2}$ & intercept \\
\hline 0.01 & -0.00002 & 9.9485 \\
\hline
\end{tabular}

Data collected

\begin{tabular}{rrrrrrrr}
\hline Setpoint, C & Temperature, Speed, rpm Torque, \% S.F. & Viscosity, Pa's & $1 / T \times 10000, \mathrm{~K}$ & $\operatorname{Ln}(\eta)$, Pa's \\
\hline 1150.0 & 1143 & 70.00 & 10.86 & 10.55 & 1.636 & 7.061 & 0.492 \\
1100.0 & 1096 & 70.00 & 17.66 & 10.55 & 2.662 & 7.306 & 0.979 \\
1050.0 & 1046 & 70.00 & 29.13 & 10.55 & 4.390 & 7.579 & 1.479 \\
1000.0 & 997 & 59.45 & 42.60 & 10.47 & 7.503 & 7.874 & 2.015 \\
950.0 & 947 & 33.70 & 45.24 & 10.26 & 13.778 & 8.194 & 2.623 \\
1050.0 & 1043 & 70.00 & 27.91 & 10.55 & 4.207 & 7.600 & 1.437 \\
1150.0 & 1144 & 70.00 & 10.72 & 10.55 & 1.616 & 7.056 & 0.480 \\
1200.0 & 1194 & 70.00 & 6.44 & 10.55 & 0.970 & 6.814 & -0.030 \\
1150.0 & 1145 & 70.00 & 10.14 & 10.55 & 1.528 & 7.050 & 0.424
\end{tabular}

EM07-Si-30

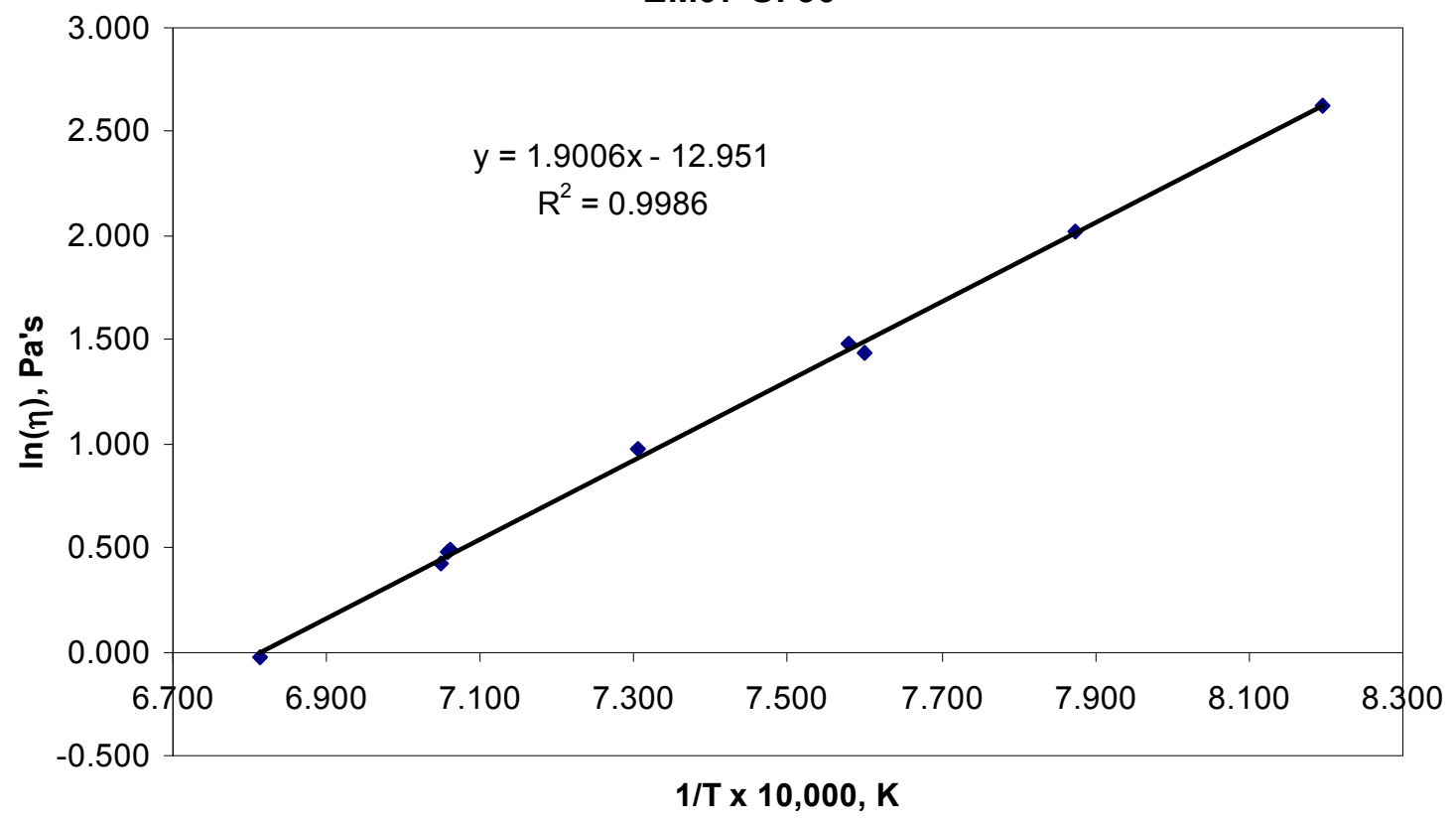

Figure A.38. Viscosity Trend for EM07-Si-30 


\section{A.39 EM07-Si-37 Viscosity Data}

Table A.39. Viscosity Data for EM07-Si-37

\begin{tabular}{|c|c|c|c|c|c|c|c|}
\hline \multirow{3}{*}{\multicolumn{3}{|c|}{ EM07-Si-37 }} & \multirow{2}{*}{\multicolumn{3}{|c|}{ speed $^{2} \quad$ intercept }} & & \\
\hline & & & & & & & \\
\hline & & & 0.01 & -0.00002 & 9.9485 & & \\
\hline Data collectec & & & & & & & \\
\hline Setpoint, C & Temperature, & $\mathrm{d}, \mathrm{rpm}$ & Torque, $\%$ & S.F. & Viscosity, Pa's & $1 / \mathrm{T} \times 10000, \mathrm{~K}$ & Ln( $(\eta), P a ' s$ \\
\hline 1150.0 & 1141 & 75.00 & 23.81 & 10.59 & 3.361 & 7.071 & 1.212 \\
\hline 1100.0 & 1092 & 75.00 & 38.84 & 10.59 & 5.482 & 7.328 & 1.701 \\
\hline 1050.0 & 1041 & 48.53 & 43.50 & 10.39 & 9.310 & 7.607 & 2.231 \\
\hline 1000.0 & 991 & 29.22 & 47.00 & 10.22 & 16.445 & 7.909 & 2.800 \\
\hline 950.0 & 940 & 14.30 & 43.77 & 10.09 & 30.874 & 8.240 & 3.430 \\
\hline 1050.0 & 1038 & 48.65 & 43.18 & 10.39 & 9.222 & 7.627 & 2.222 \\
\hline 1150.0 & 1139 & 75.00 & 24.30 & 10.59 & 3.430 & 7.083 & 1.233 \\
\hline 1200.0 & 1190 & 75.00 & 15.48 & 10.59 & 2.185 & 6.835 & 0.781 \\
\hline 1150.0 & 1141 & 75.00 & 23.89 & 10.59 & 3.372 & 7.071 & 1.215 \\
\hline
\end{tabular}

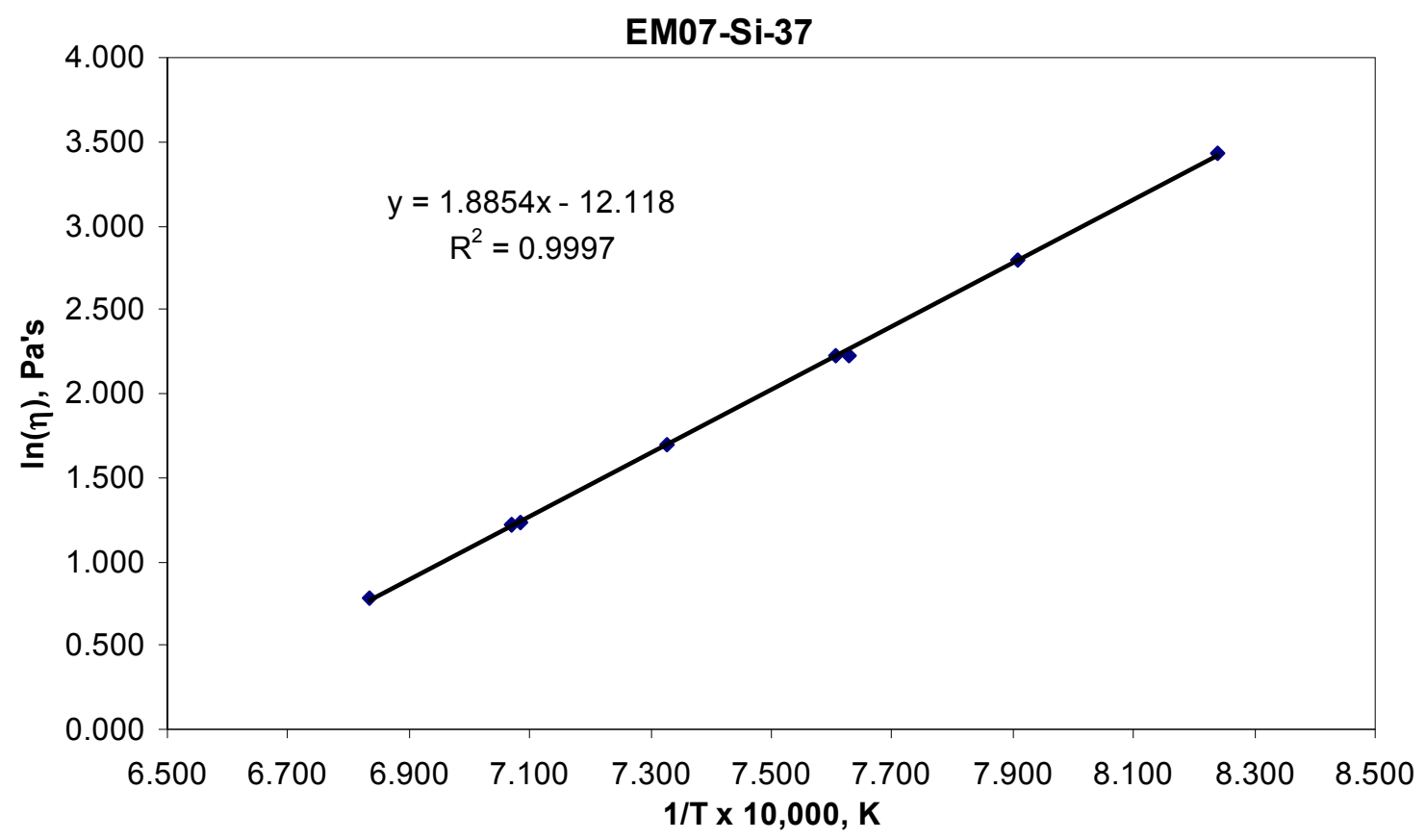

Figure A.39. Viscosity Trend for EM07-Si-37 


\section{A.40 EM07-Si-50 Viscosity Data}

Table A.40. Viscosity Data for EM07-Si-50

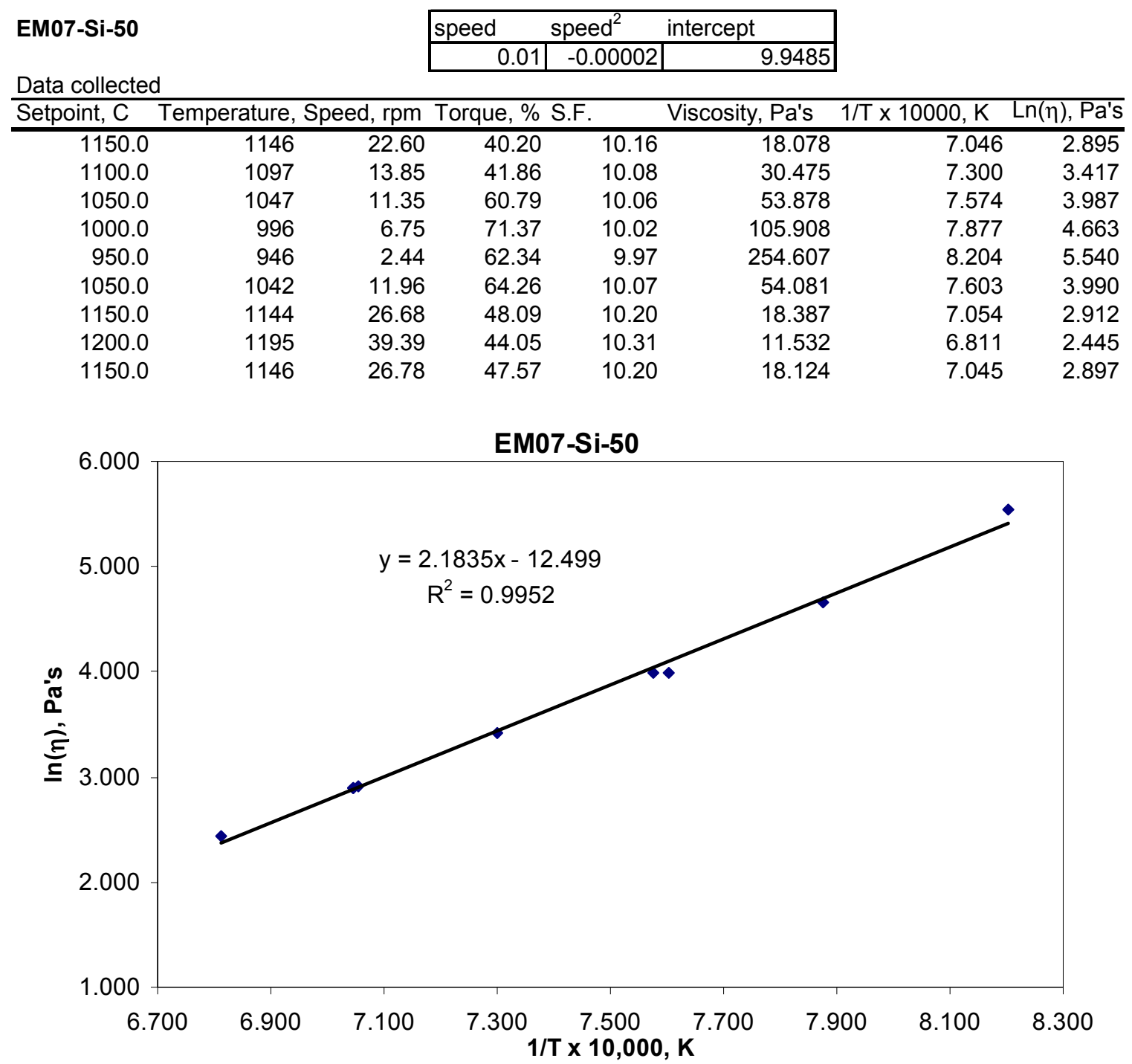

Figure A.40. Viscosity Trend for EM07-Si-50 


\section{A.41 EM07-Zr-001 Viscosity Data}

Table A.41. Viscosity Data for EM07-Zr-001

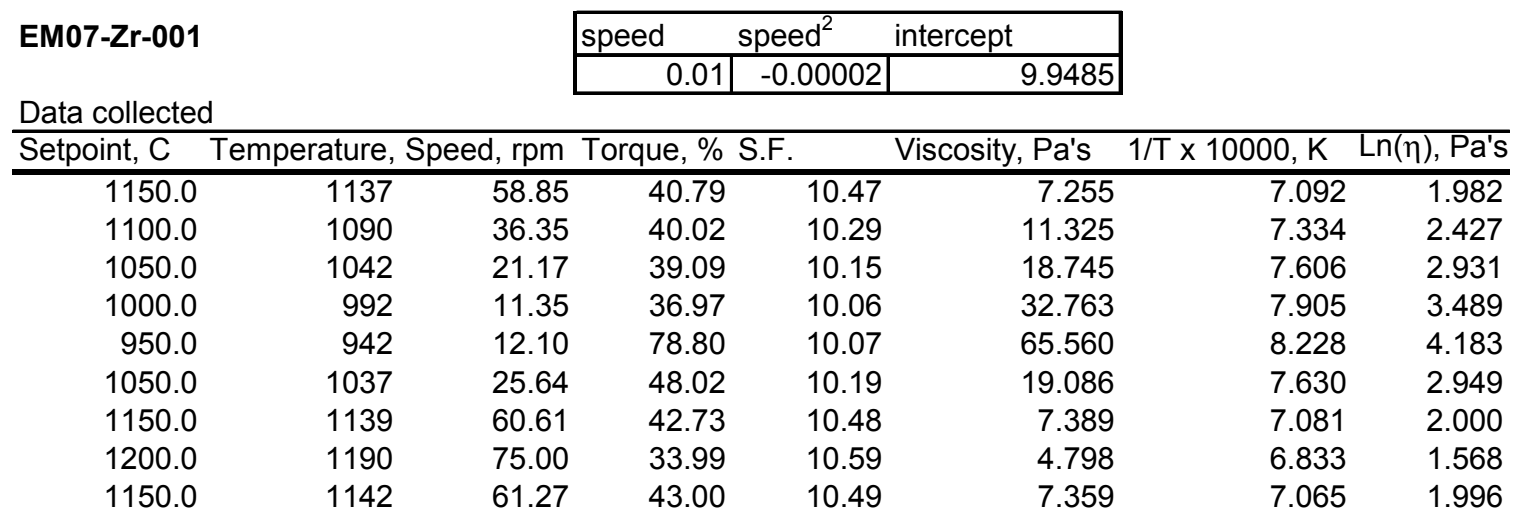

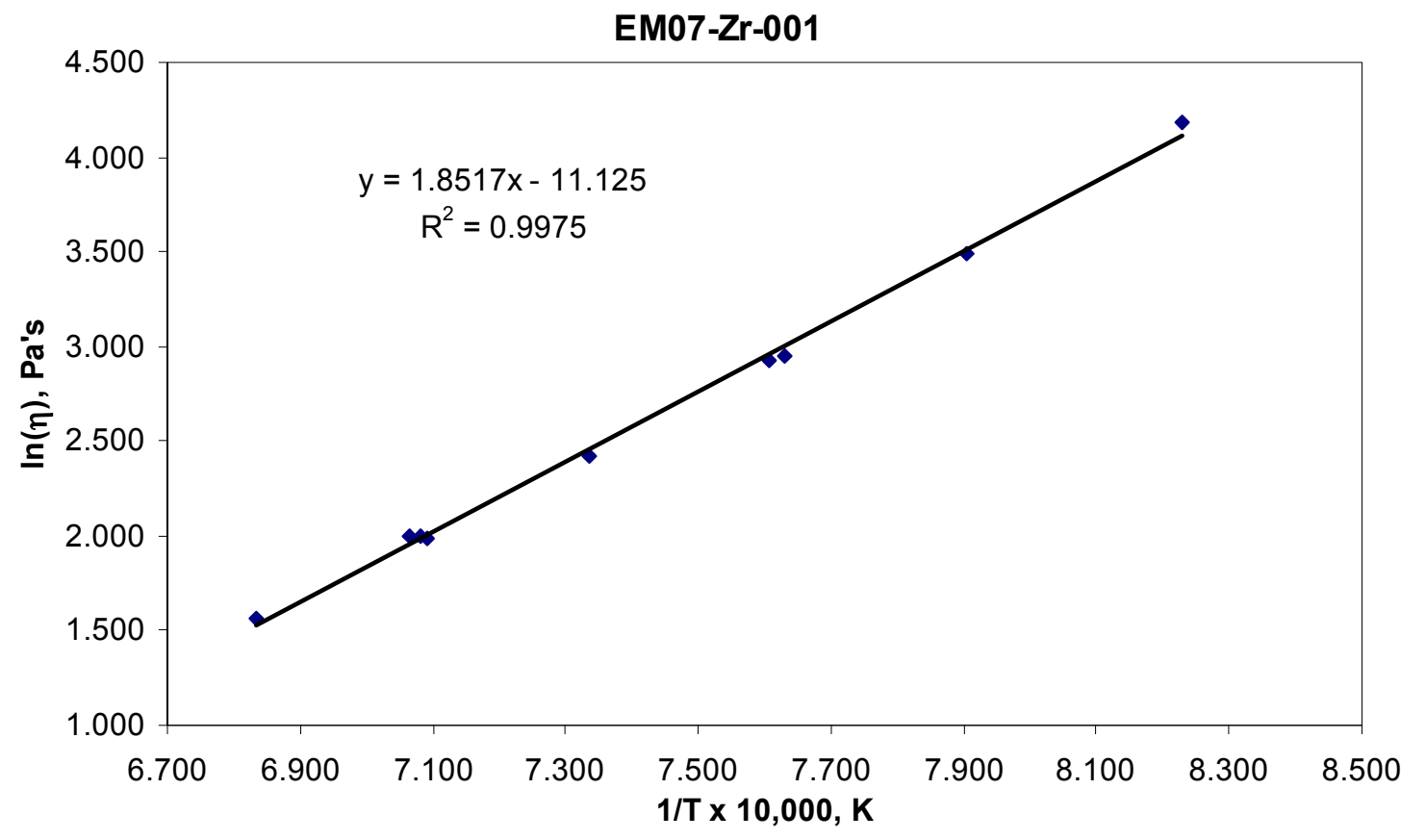

Figure A.41. Viscosity Trend for EM07-Zr-001 


\section{A.42 EM07-Zr-05 Viscosity Data}

Table A.42. Viscosity Data for EM07-Zr-05

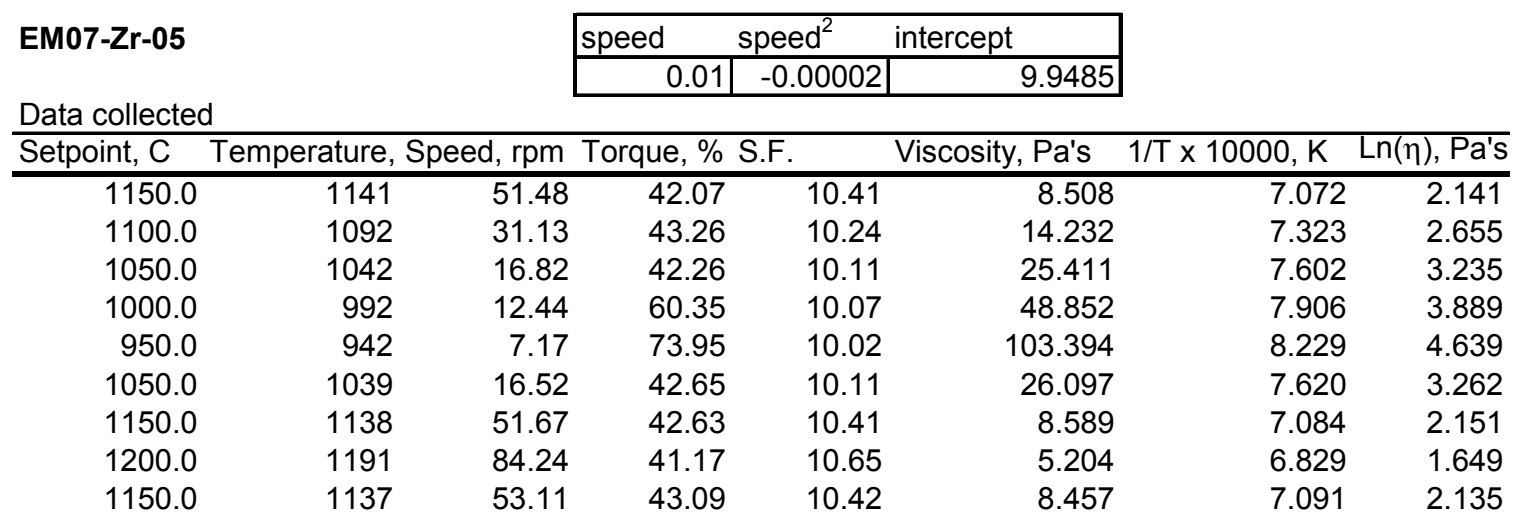

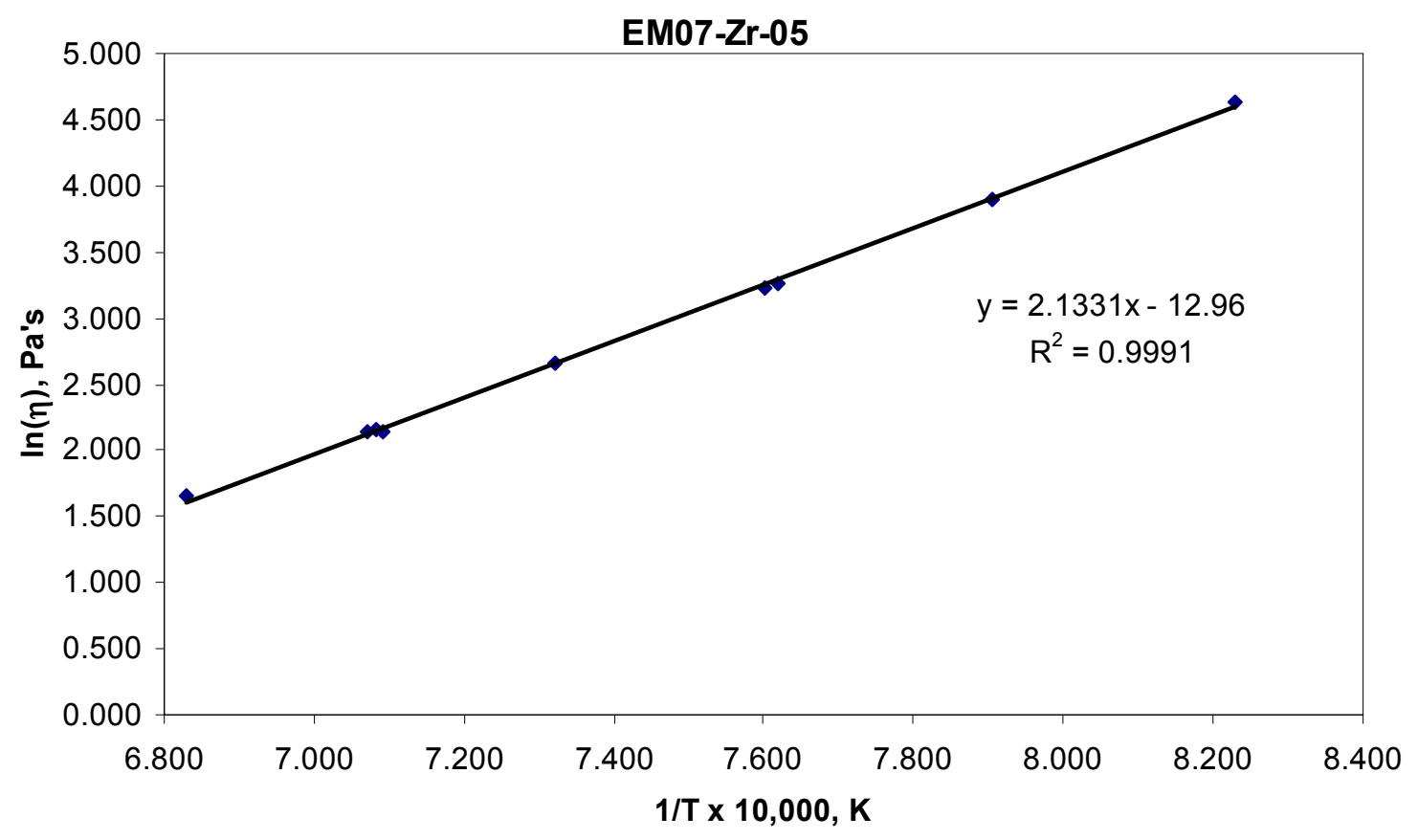

Figure A.42. Viscosity Trend for EM07-Zr-05 


\section{A.43 EM07-NM-0025 Viscosity Data}

Table A.43. Viscosity Data for EM07-NM-0025

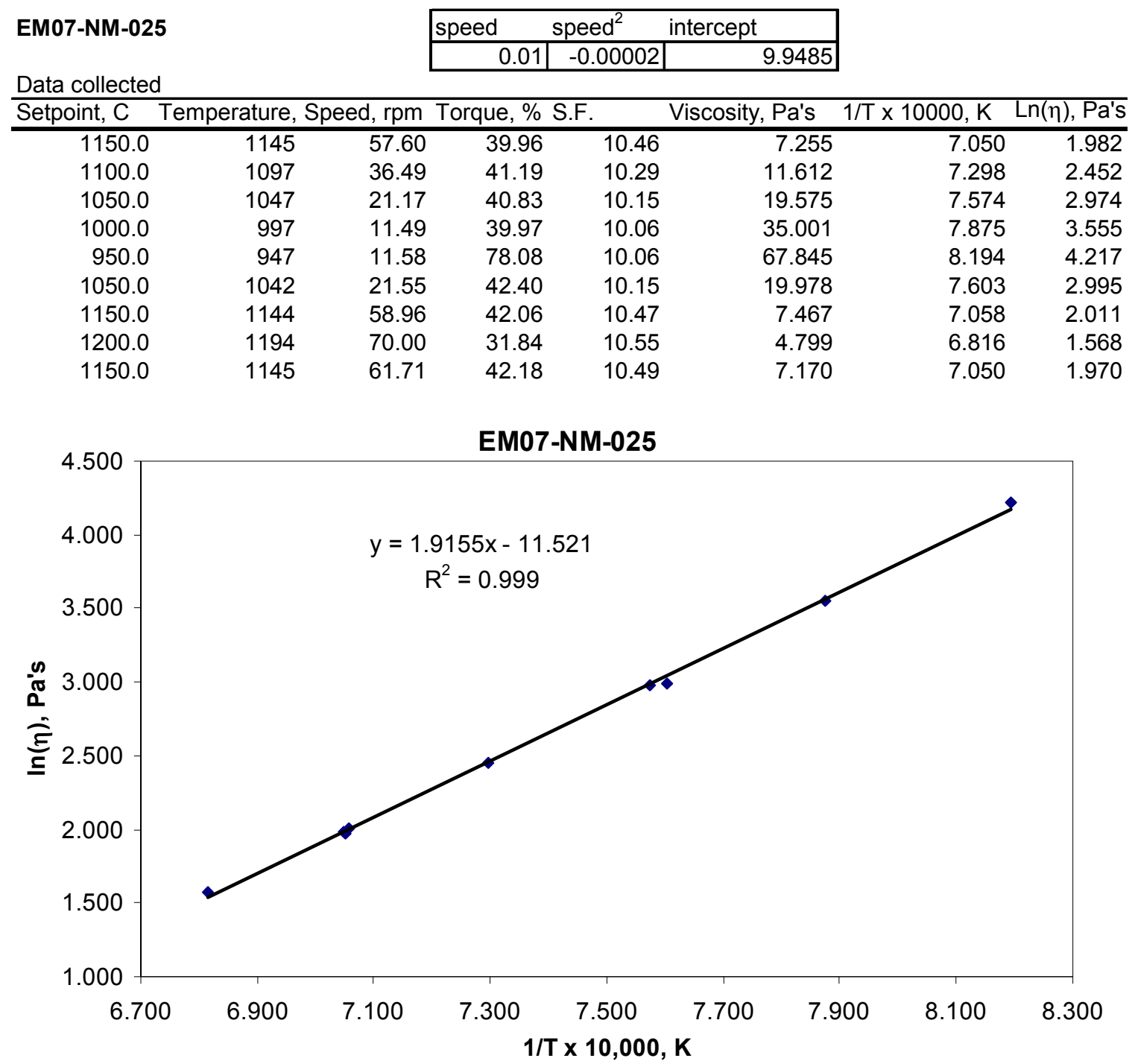

Figure A.43. Viscosity Trend for EM07-NM-0025 


\section{A.44 EM07-Val-1 Viscosity Data}

Table A.44. Viscosity Data for EM07-Val-1

\section{EM07-Val-1}

\begin{tabular}{|c|r|r|}
\hline speed & \multicolumn{1}{|c|}{ speed $^{\wedge} 2$} & intercept \\
\hline-0.1110 & 0.00170 & 13.749 \\
\hline
\end{tabular}

Data collected

\begin{tabular}{rrrrrrrr}
\multicolumn{1}{c}{ Setpoint, C } & Temperature, C & Speed, rpm & Torque, $\%$ & \multicolumn{1}{c}{ S.F. } & Viscosity, Pa's & $1 / \mathrm{T} \times 10000, \mathrm{~K}$ & $\operatorname{Ln}(\eta), \mathrm{Pa}$ 's \\
\hline 1150.0 & 1116 & 18.711 & 9.82 & 12.267 & 6.436 & 7.198 & 1.862 \\
1100.0 & 1070 & 13.433 & 10.37 & 12.565 & 9.699 & 7.447 & 2.272 \\
1050.0 & 1018 & 11.350 & 16.07 & 12.708 & 17.989 & 7.743 & 2.890 \\
1000.0 & 971 & 11.350 & 27.44 & 12.708 & 30.723 & 8.035 & 3.425 \\
950.0 & 920 & 11.489 & 55.14 & 12.698 & 60.943 & 8.380 & 4.110 \\
1050.0 & 1018 & 11.350 & 16.94 & 12.708 & 18.971 & 7.742 & 2.943 \\
1150.0 & 1117 & 19.824 & 10.73 & 12.217 & 6.612 & 7.191 & 1.889 \\
1200.0 & 1166 & 31.190 & 11.34 & 11.941 & 4.341 & 6.948 & 1.468 \\
1150.0 & 1117 & 19.274 & 10.70 & 12.241 & 6.795 & 7.193 & 1.916
\end{tabular}

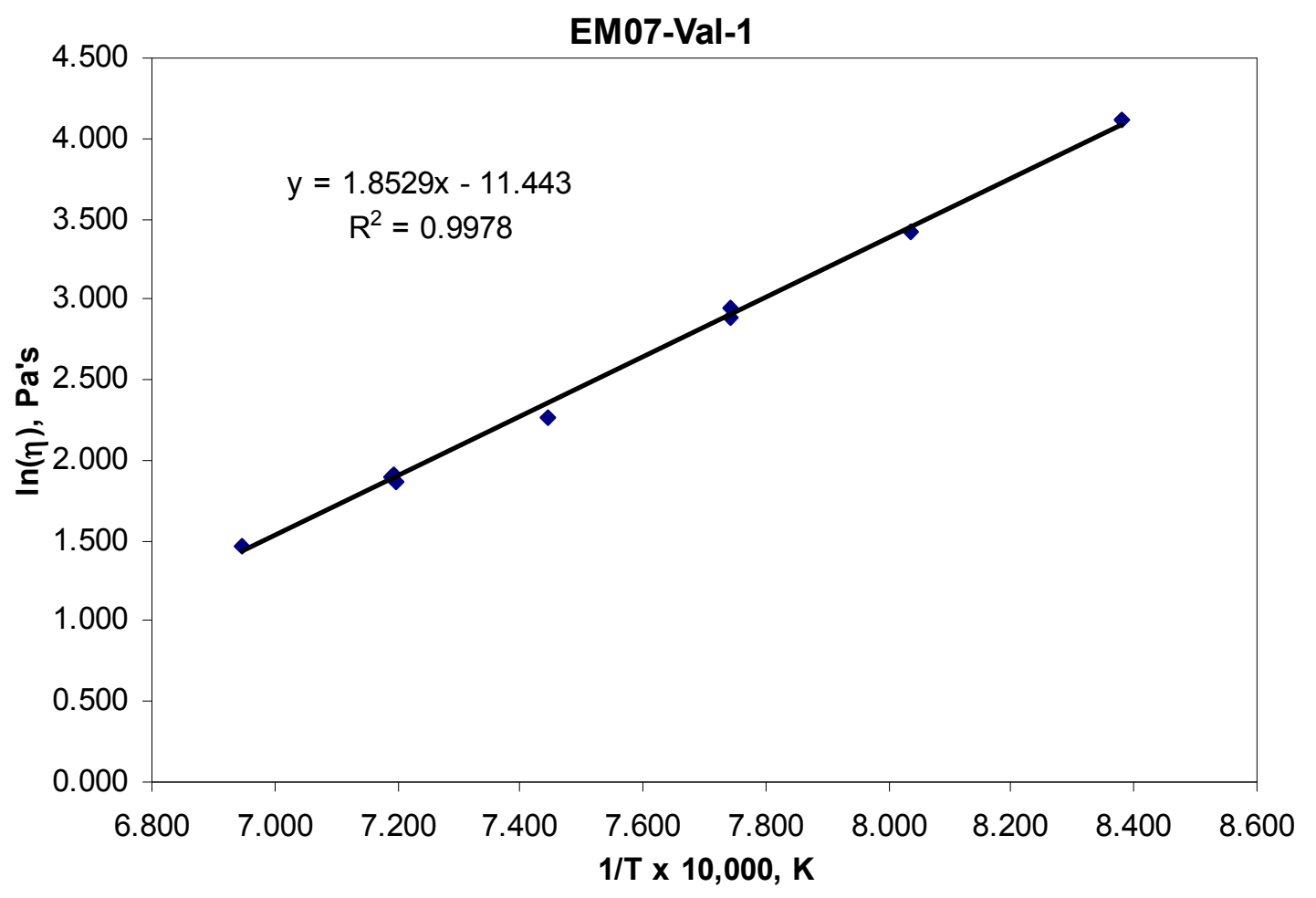

Figure A.44. Viscosity Trend for EM07-Val-1 


\section{A.45 EM07-Val-2 Viscosity Data}

Table A.45. Viscosity Data for EM07-Val-2

EM07-Val-2

\begin{tabular}{|c|r|r|}
\hline speed & speed $^{\wedge} 2$ & intercept \\
\hline-0.1110 & 0.00170 & 13.749 \\
\hline
\end{tabular}

Data collected

\begin{tabular}{|c|c|c|c|c|c|c|c|}
\hline Setpoint, C & Temperature, C & Speed, rpm & Torque, $\%$ & F. & Viscosity, Pa's & $1 / \mathrm{T} \times 10000, \mathrm{~K}$ & $\operatorname{Ln}(\eta), P a ' s$ \\
\hline 1150.0 & 1111 & 13.055 & 10.20 & 12.590 & 9.836 & 7.224 & 2.286 \\
\hline 1100.0 & 1063 & 11.590 & 15.83 & 12.691 & 17.332 & 7.481 & 2.853 \\
\hline 1050.0 & 1015 & 11.729 & 27.88 & 12.681 & 30.149 & 7.761 & 3.406 \\
\hline 1000.0 & 967 & 11.476 & 49.10 & 12.699 & 54.327 & 8.064 & 3.995 \\
\hline 950.0 & 917 & 8.590 & 72.75 & 12.921 & 109.431 & 8.403 & 4.695 \\
\hline 1050.0 & 1016 & 12.260 & 28.53 & 12.644 & 29.424 & 7.756 & 3.382 \\
\hline 1150.0 & 1112 & 14.060 & 11.15 & 12.524 & 9.929 & 7.218 & 2.295 \\
\hline 1200.0 & 1161 & 25.020 & 11.60 & 12.036 & 5.580 & 6.971 & 1.719 \\
\hline 1150.0 & 1112 & 15.950 & 11.93 & 12.411 & 9.282 & 7.219 & 2.228 \\
\hline
\end{tabular}

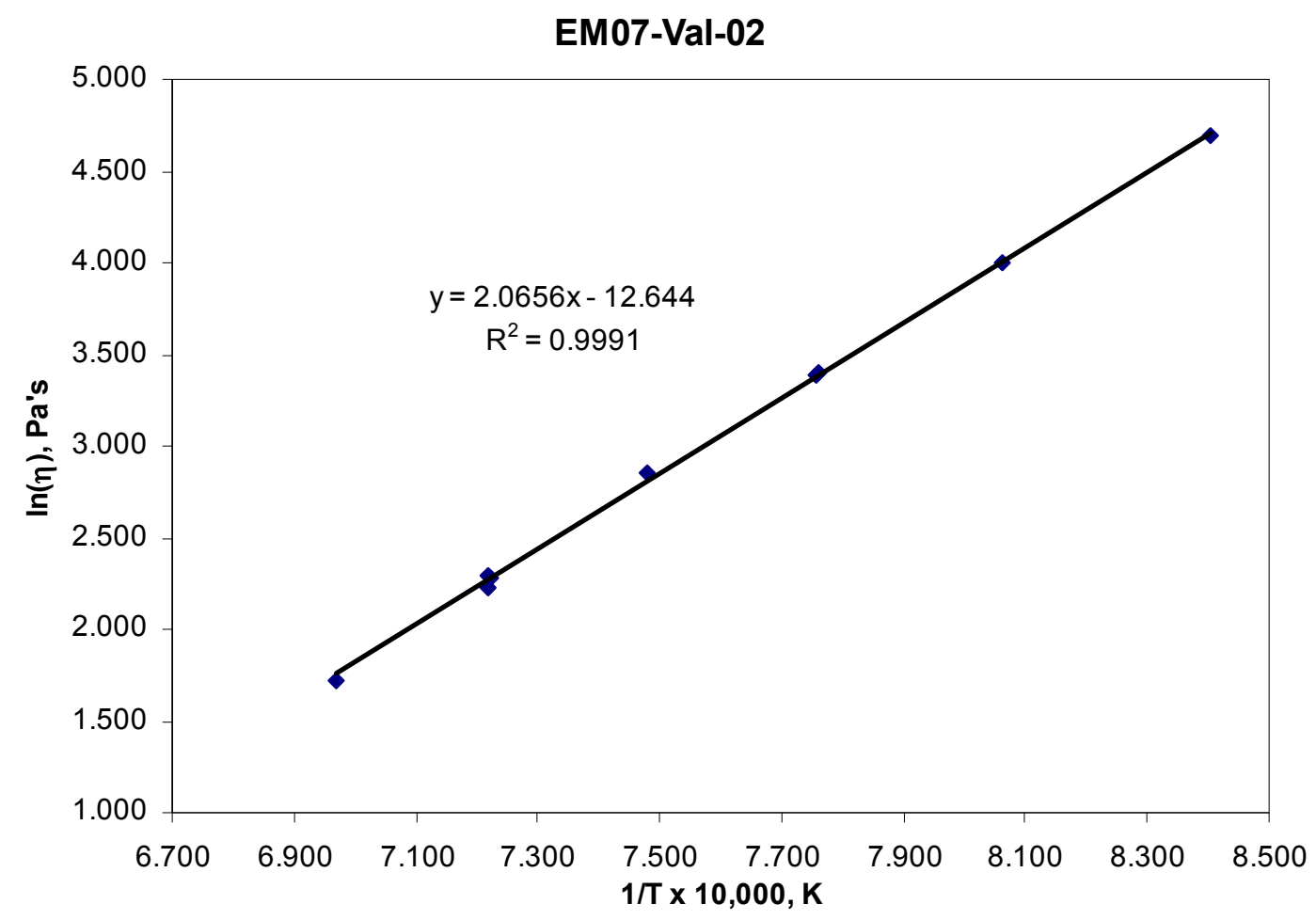

Figure A.45. Viscosity Trend for EM07-Val-2 


\section{A.46 EM07-Val-3 Viscosity Data}

Table A.46. Viscosity Data for EM07-Val-3

\section{EM07-Val-3}

\begin{tabular}{|c|r|r|}
\hline speed & speed`2 $^{\wedge}$ & intercept \\
\hline-0.1110 & 0.00170 & 13.749 \\
\hline
\end{tabular}

Data collected

\begin{tabular}{rrrrrrrr}
\multicolumn{1}{l}{ Setpoint, C } & Temperature, C & Speed, rpm & Torque, \% & S.F. & Viscosity, Pa's & 1/T x 10000, K & Ln( $\eta)$, Pa's \\
\hline 1150.0 & 1117 & 12.191 & 11.28 & 12.648 & 11.703 & 7.191 & 2.460 \\
1100.0 & 1068 & 12.370 & 19.40 & 12.636 & 19.813 & 7.456 & 2.986 \\
1050.0 & 1018 & 12.191 & 34.10 & 12.648 & 35.381 & 7.743 & 3.566 \\
1000.0 & 969 & 12.484 & 64.28 & 12.628 & 65.028 & 8.048 & 4.175 \\
950.0 & 920 & 6.269 & 70.38 & 13.120 & 147.300 & 8.379 & 4.992 \\
1050.0 & 1021 & 11.610 & 32.94 & 12.689 & 35.999 & 7.730 & 3.583 \\
1150.0 & 1119 & 11.610 & 11.84 & 12.689 & 12.946 & 7.181 & 2.561 \\
1200.0 & 1167 & 17.236 & 10.96 & 12.341 & 7.847 & 6.944 & 2.060 \\
1150.0 & 1118 & 11.930 & 11.94 & 12.667 & 12.678 & 7.188 & 2.540
\end{tabular}

\section{EM07-Val-3}

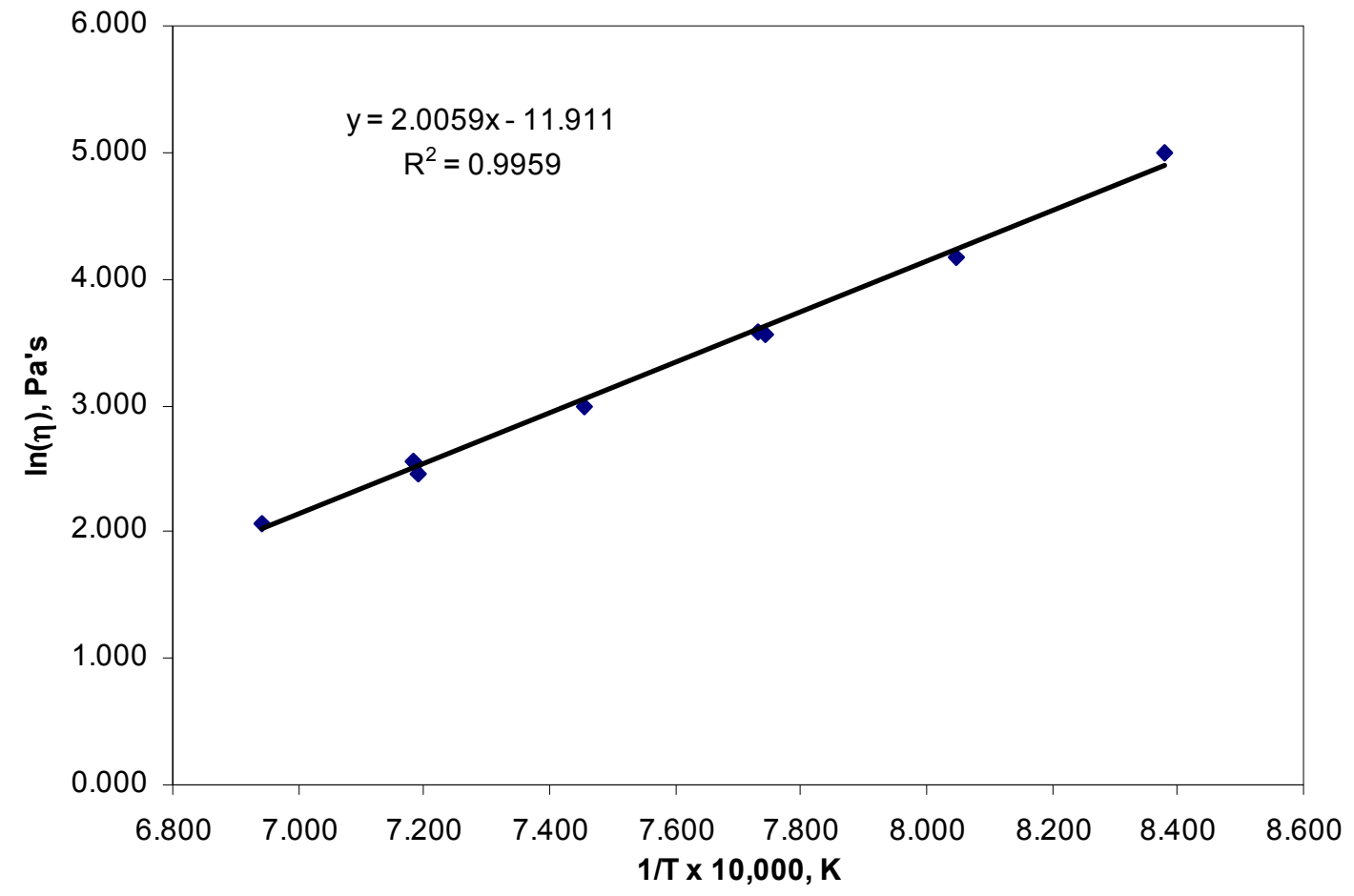

Figure A.46. Viscosity Trend for EM07-Val-3 


\section{Appendix B: XRD of Canister Centerline Cooling (CCC) Treated Glasses}

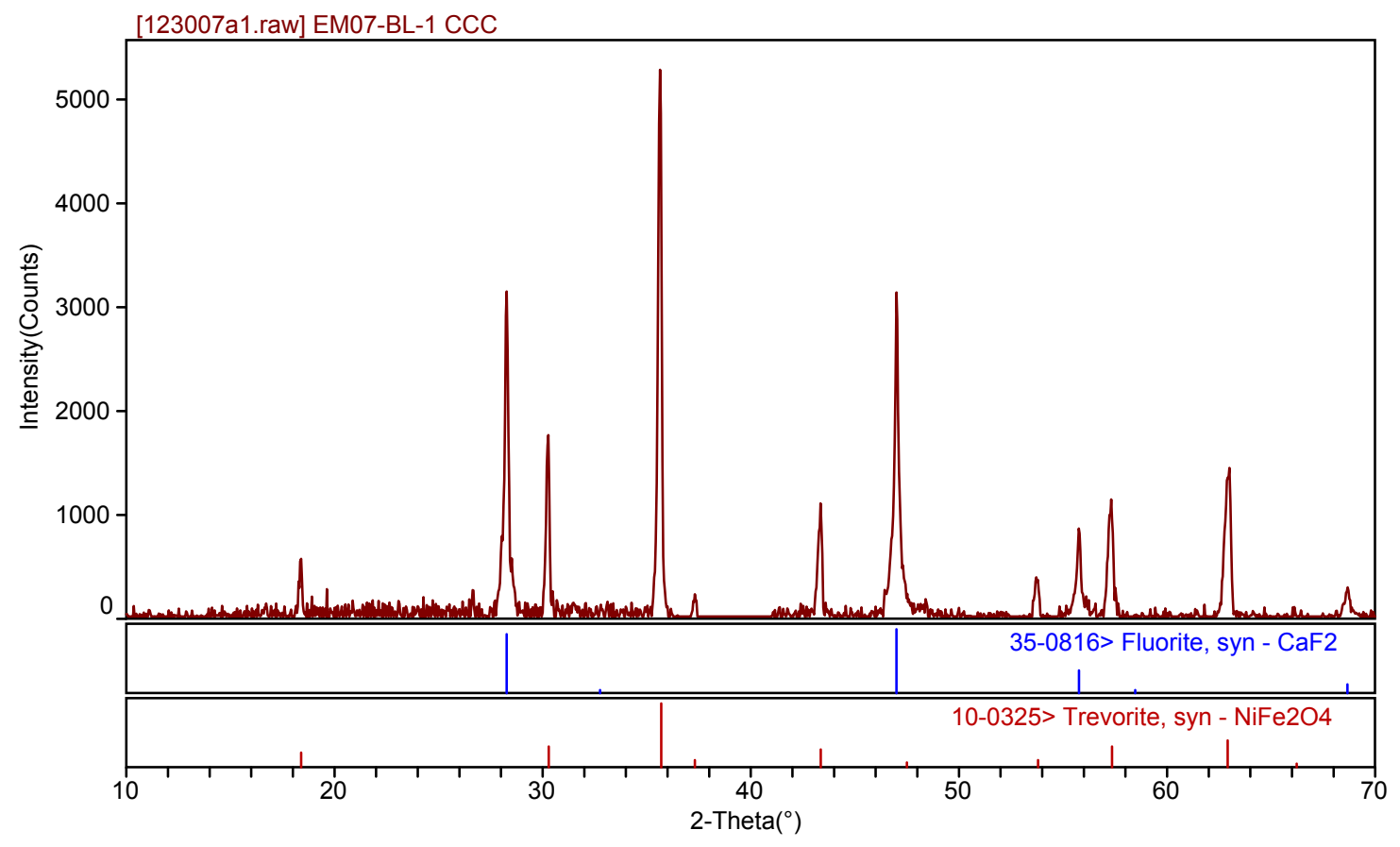

Figure B.1. XRD Spectrum of CCC Treated EM07-BL-1

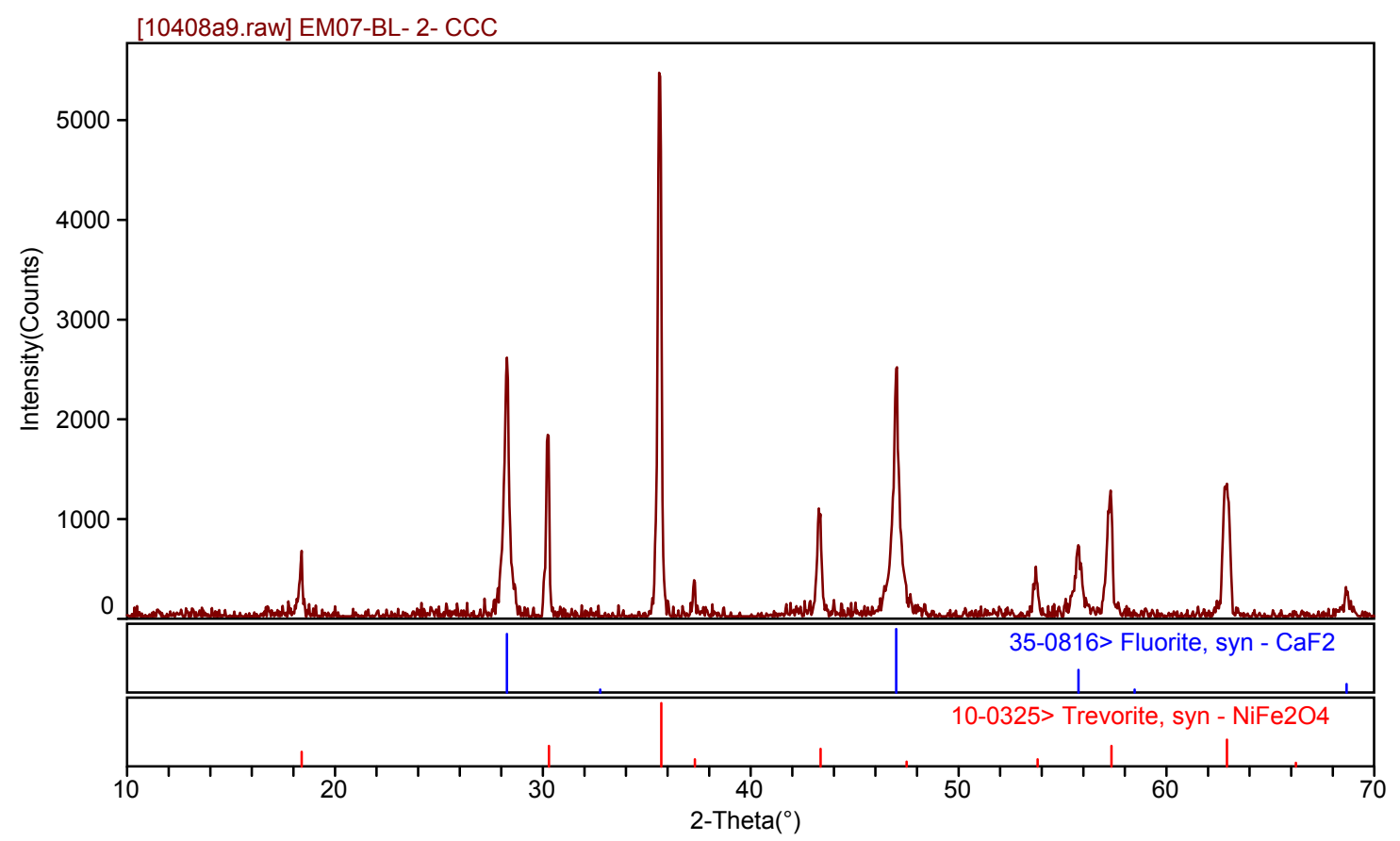

B. 1 
Figure B.2. XRD Spectrum of CCC Treated EM07-BL-2

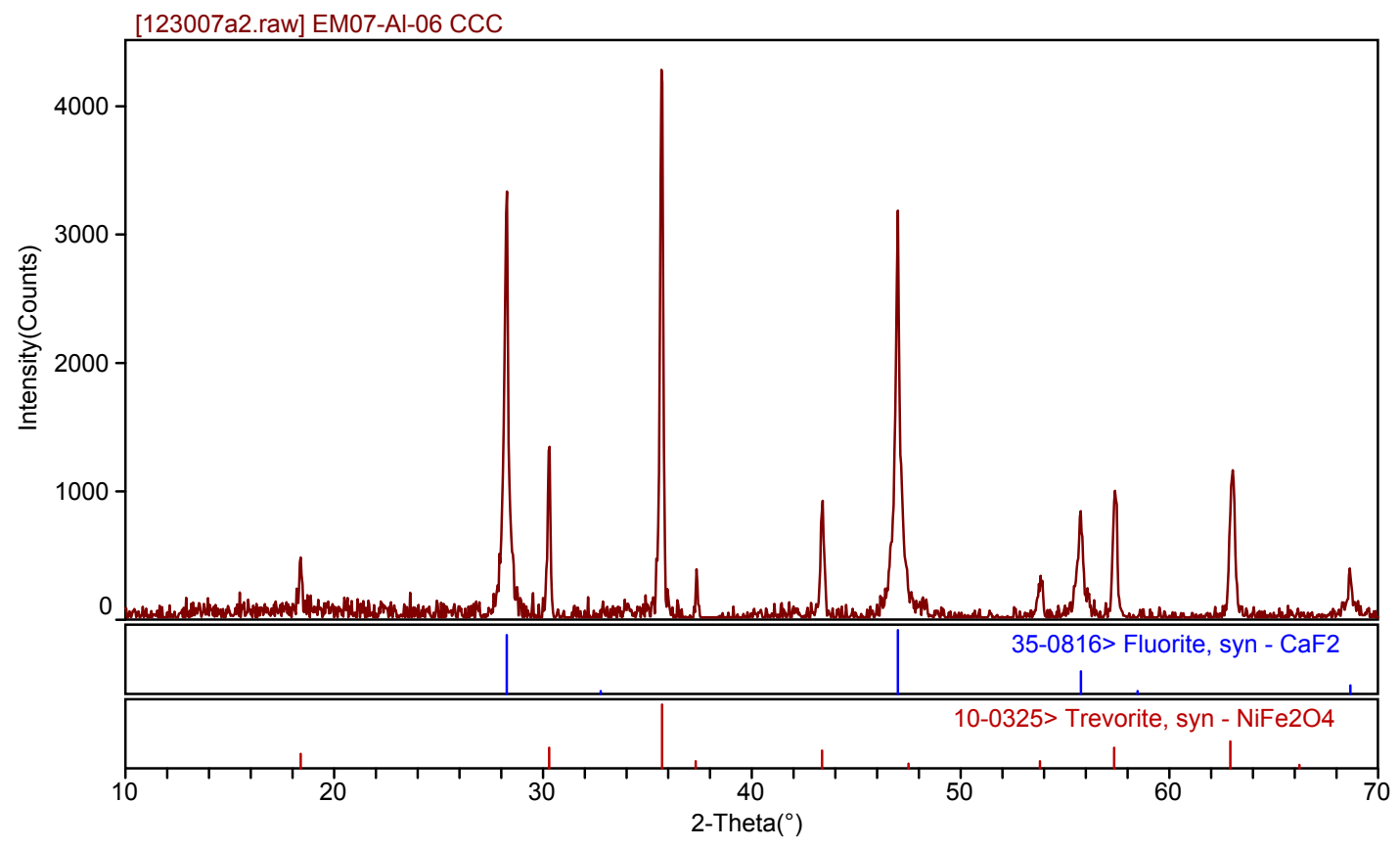

Figure B.3. XRD Spectrum of CCC Treated EM07-A1-06

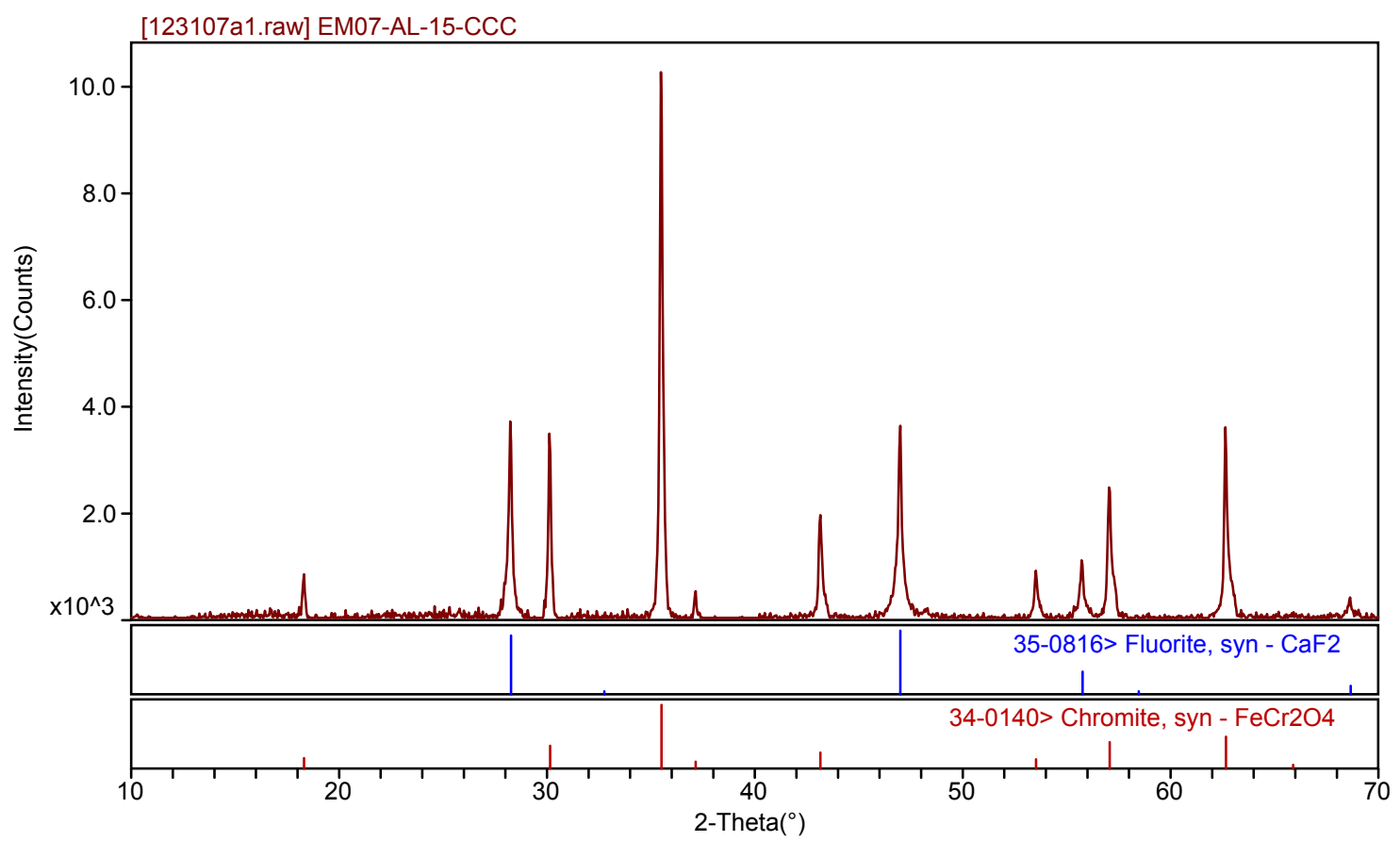

Figure B.4. XRD Spectrum of CCC Treated EM07-Al-15 


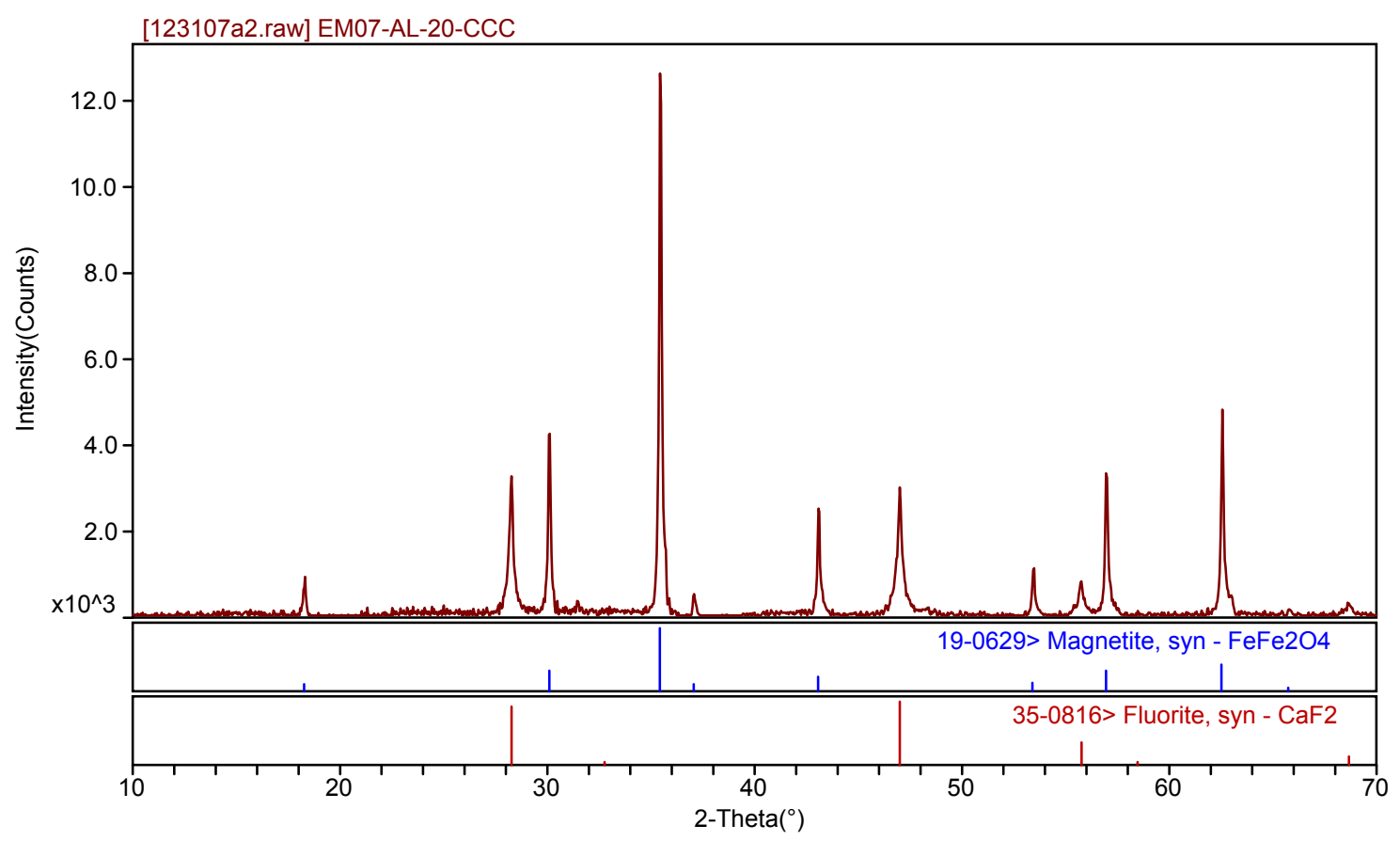

Figure B.5. XRD Spectrum of CCC Treated EM07-A1-20

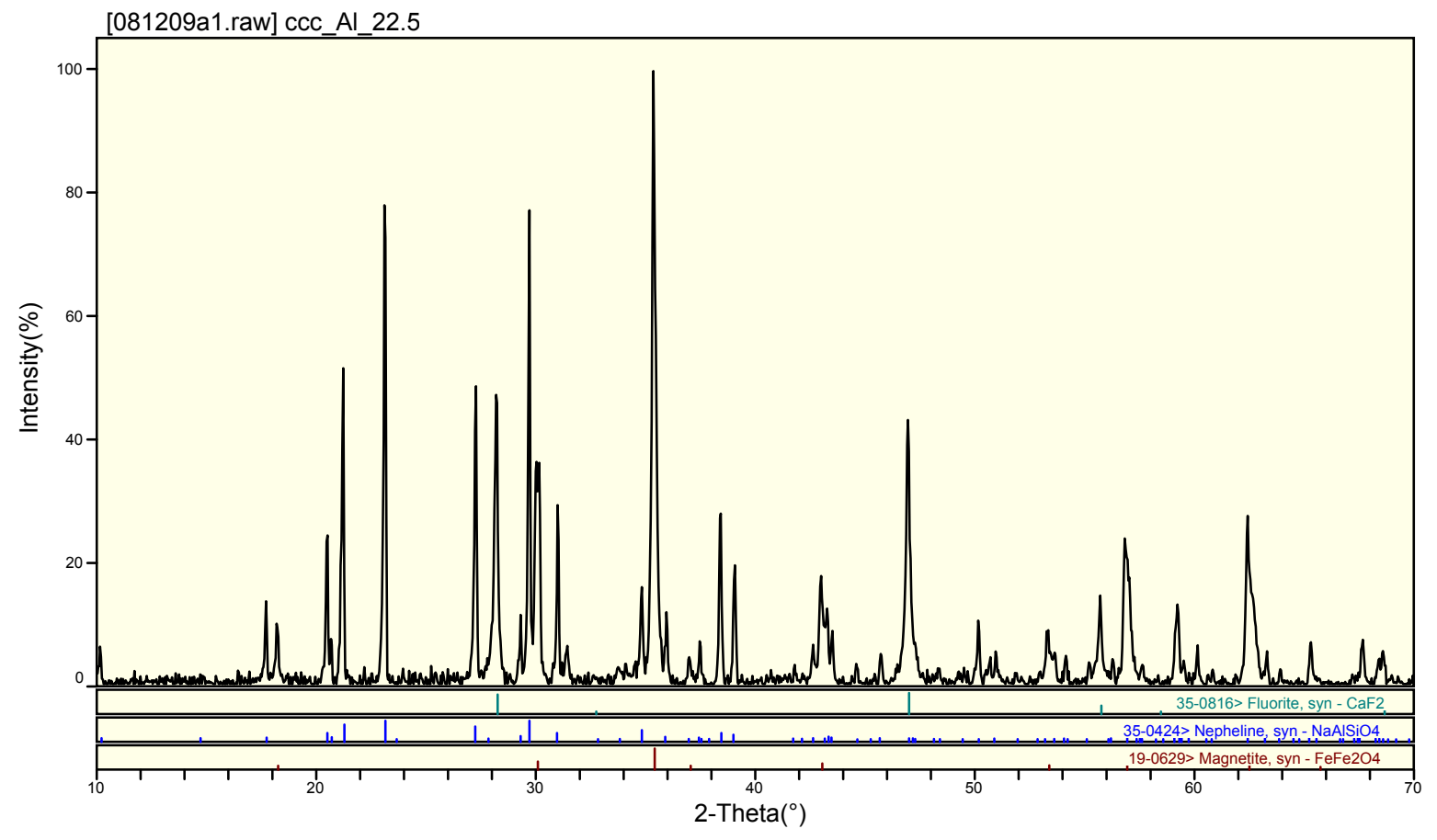

Figure B.6. XRD Spectrum of CCC Treated EM07-A1-22.5 


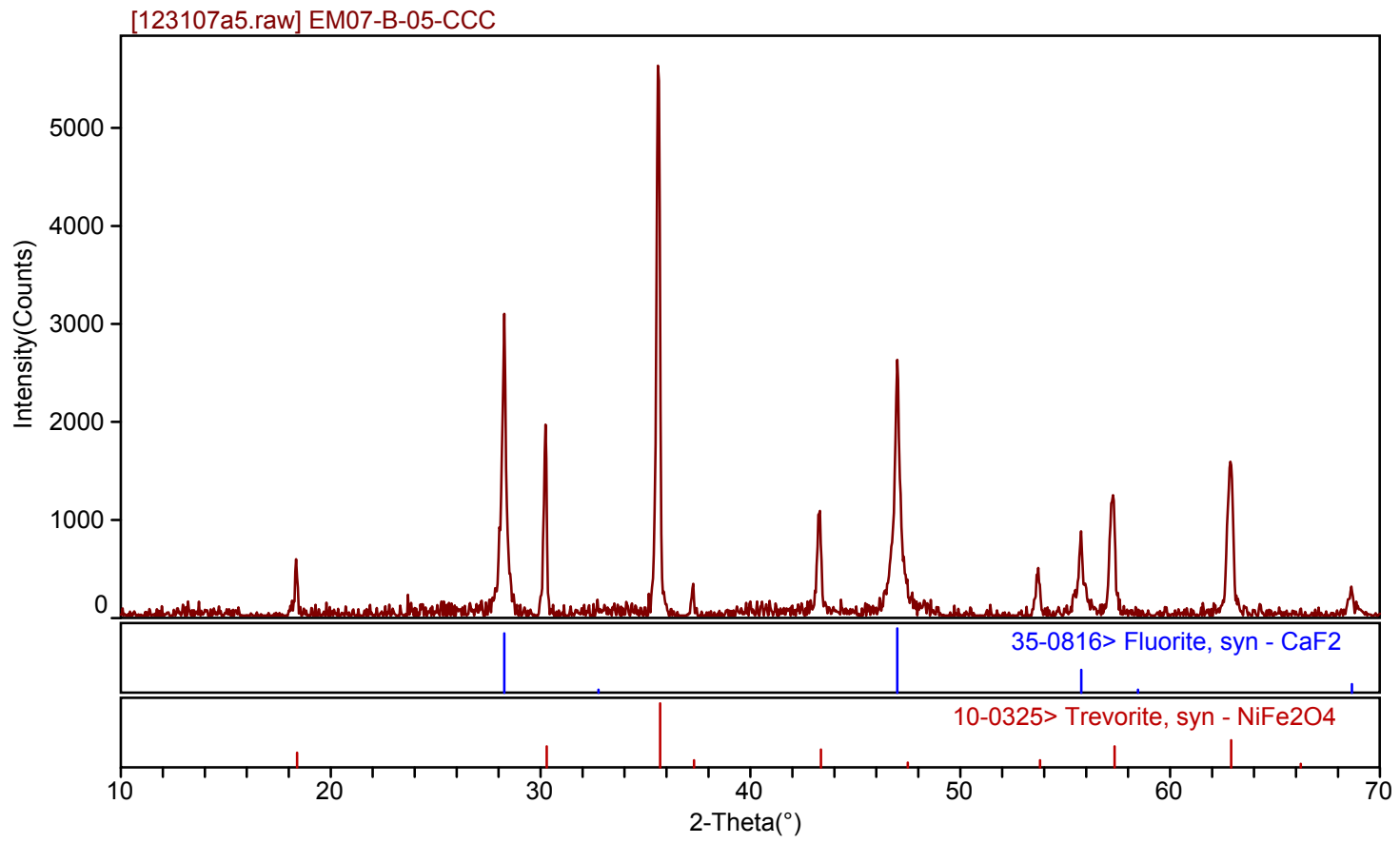

Figure B.7. XRD Spectrum of CCC Treated EM07-B-05

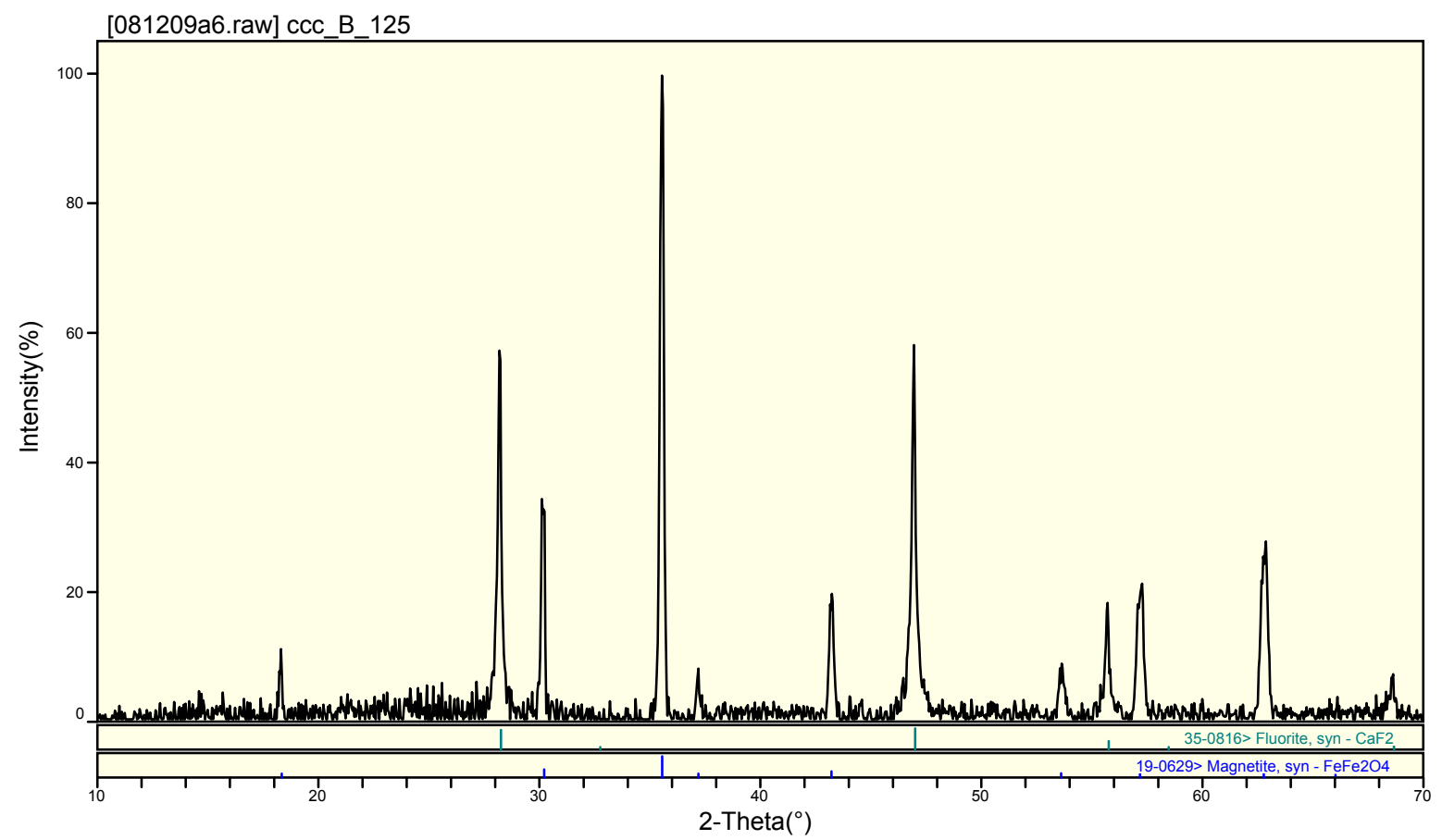

Figure B.8. XRD Spectrum of CCC Treated EM07-B-125 


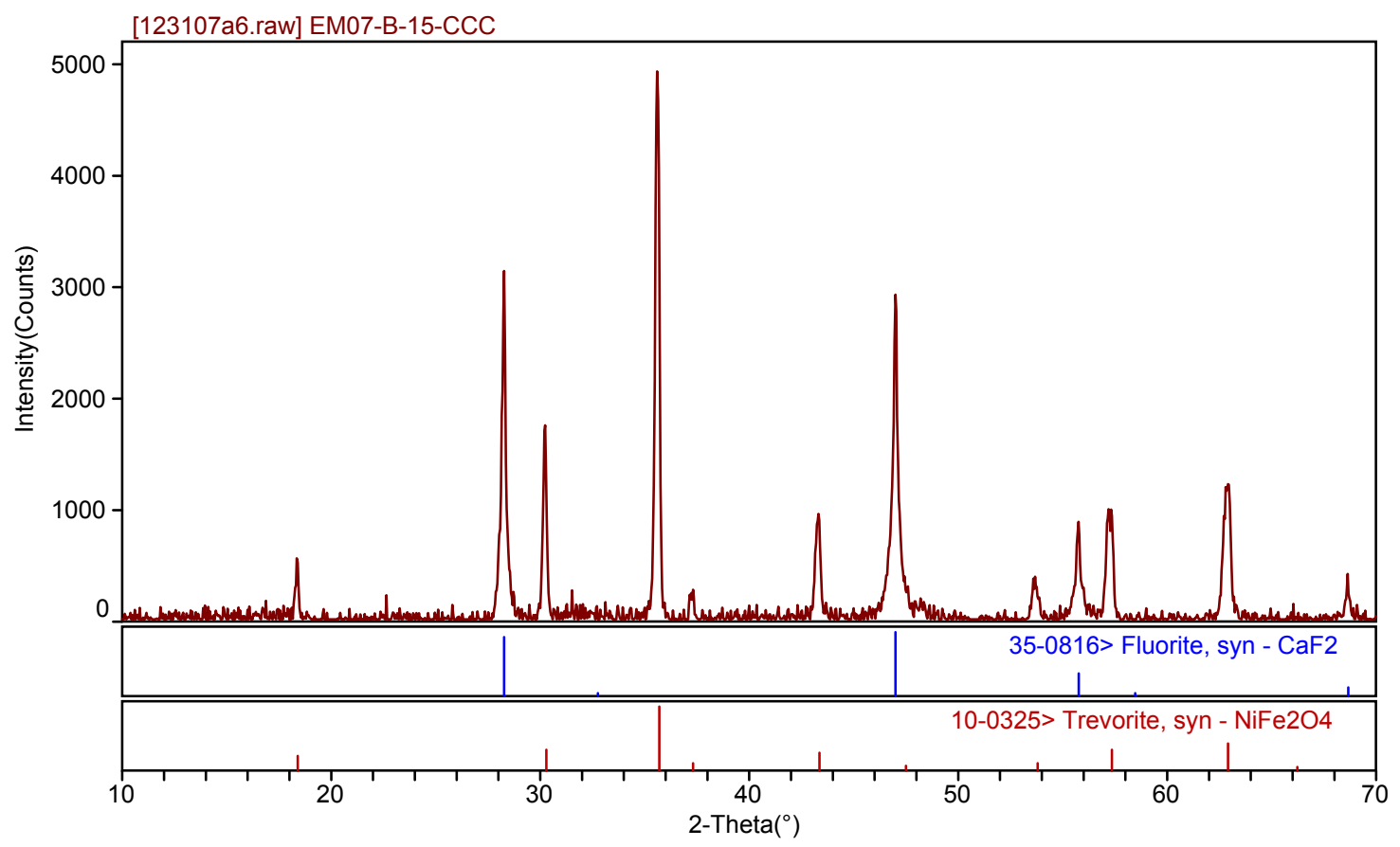

Figure B.9. XRD Spectrum of CCC Treated EM07-B-15

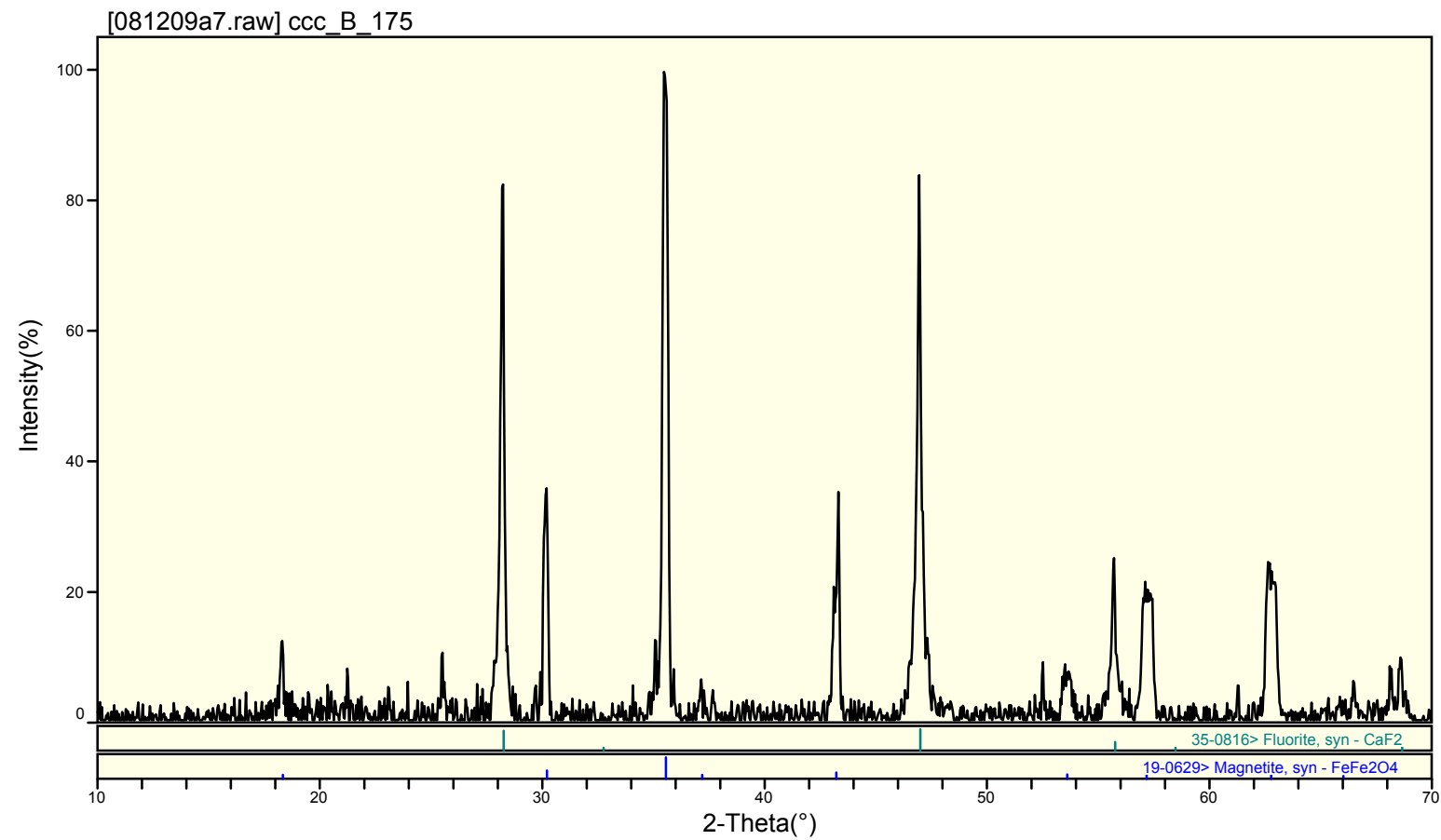

Figure B.10. XRD Spectrum of CCC Treated EM07-B-175 


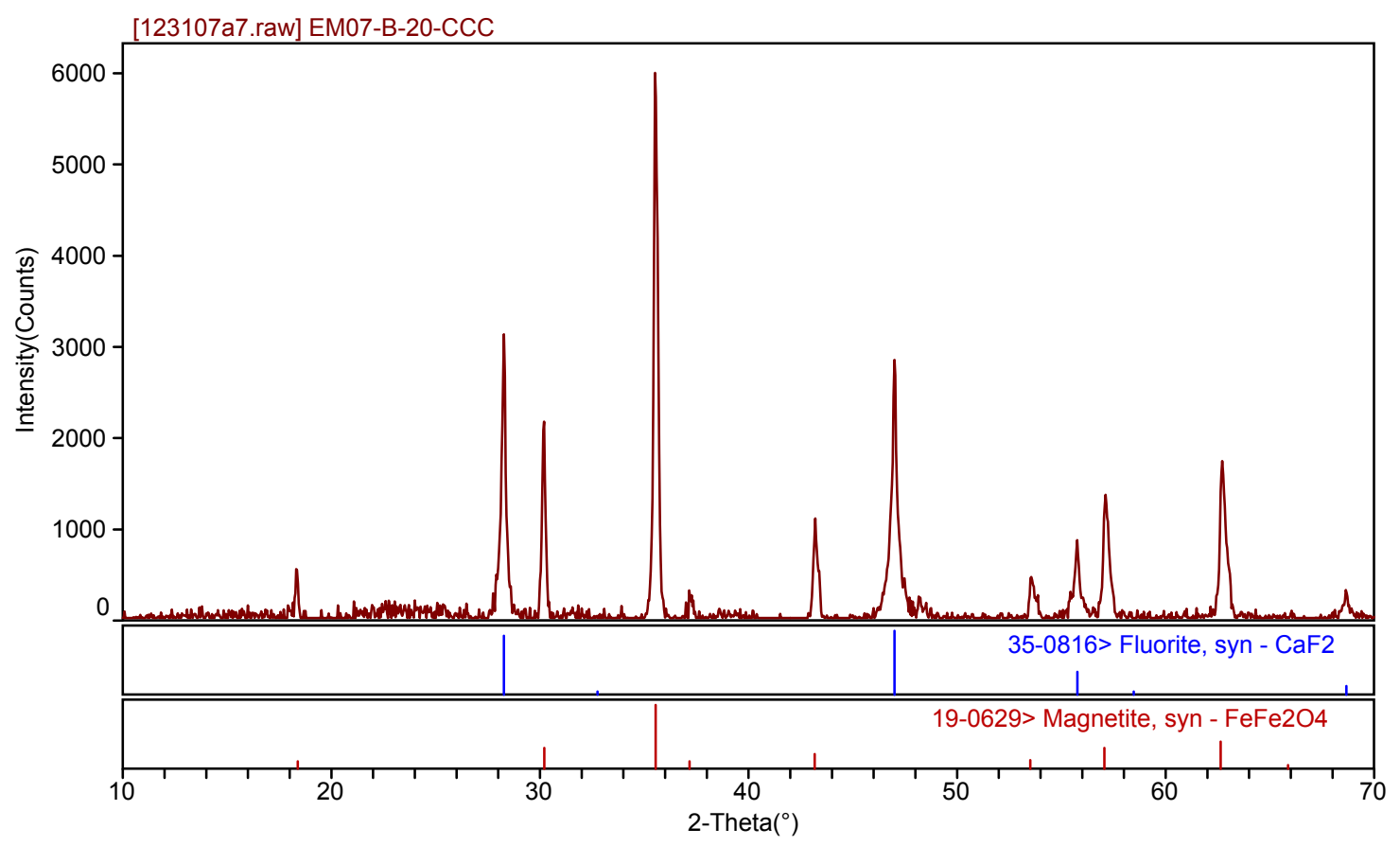

Figure B.11. XRD Spectrum of CCC Treated EM07-B-20

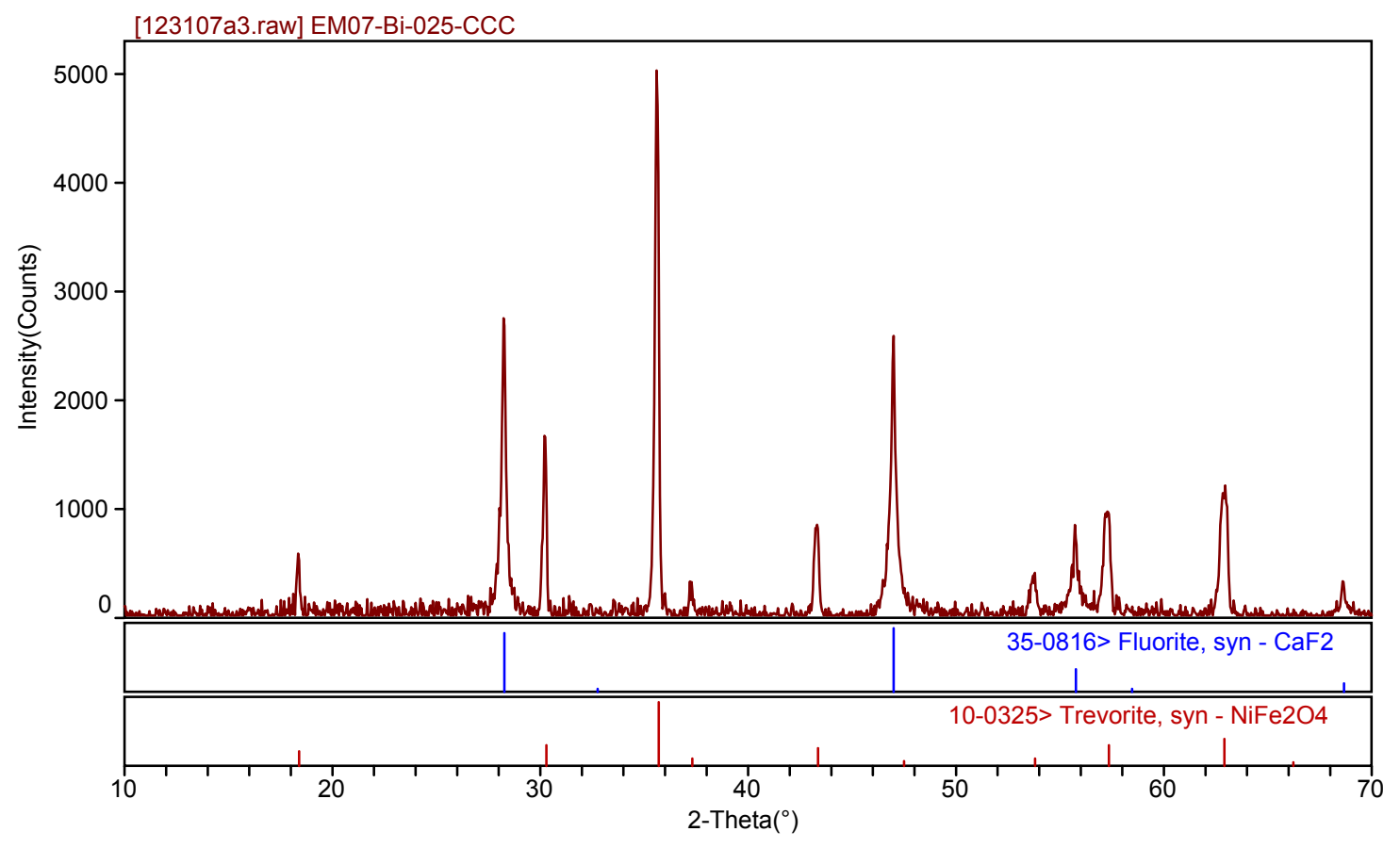

Figure B.12. XRD Spectrum of CCC Treated EM07-Bi-025 


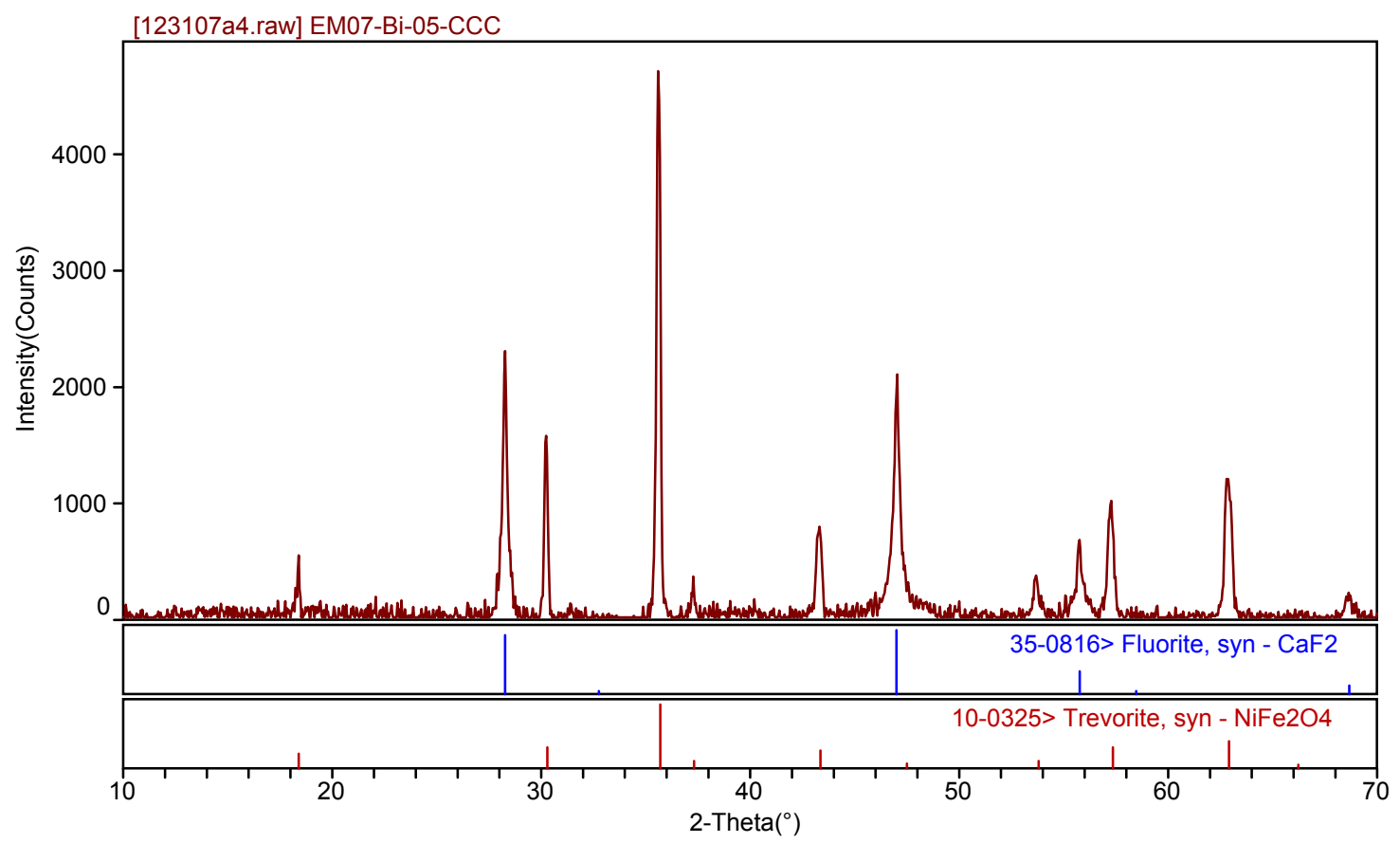

Figure B.13. XRD Spectrum of CCC Treated EM07-Bi-05

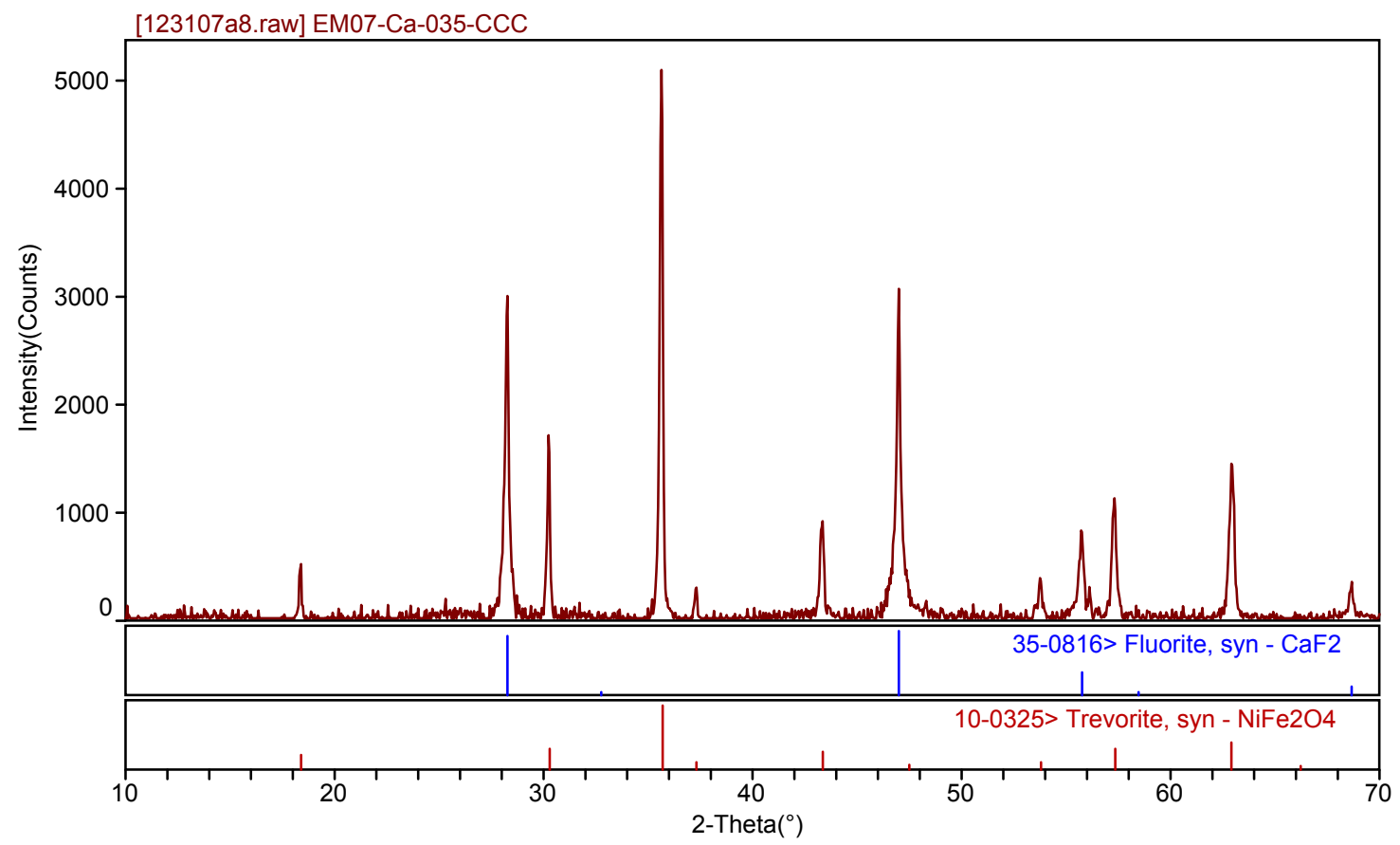

Figure B.14. XRD Spectrum of CCC Treated EM07-Ca-035 


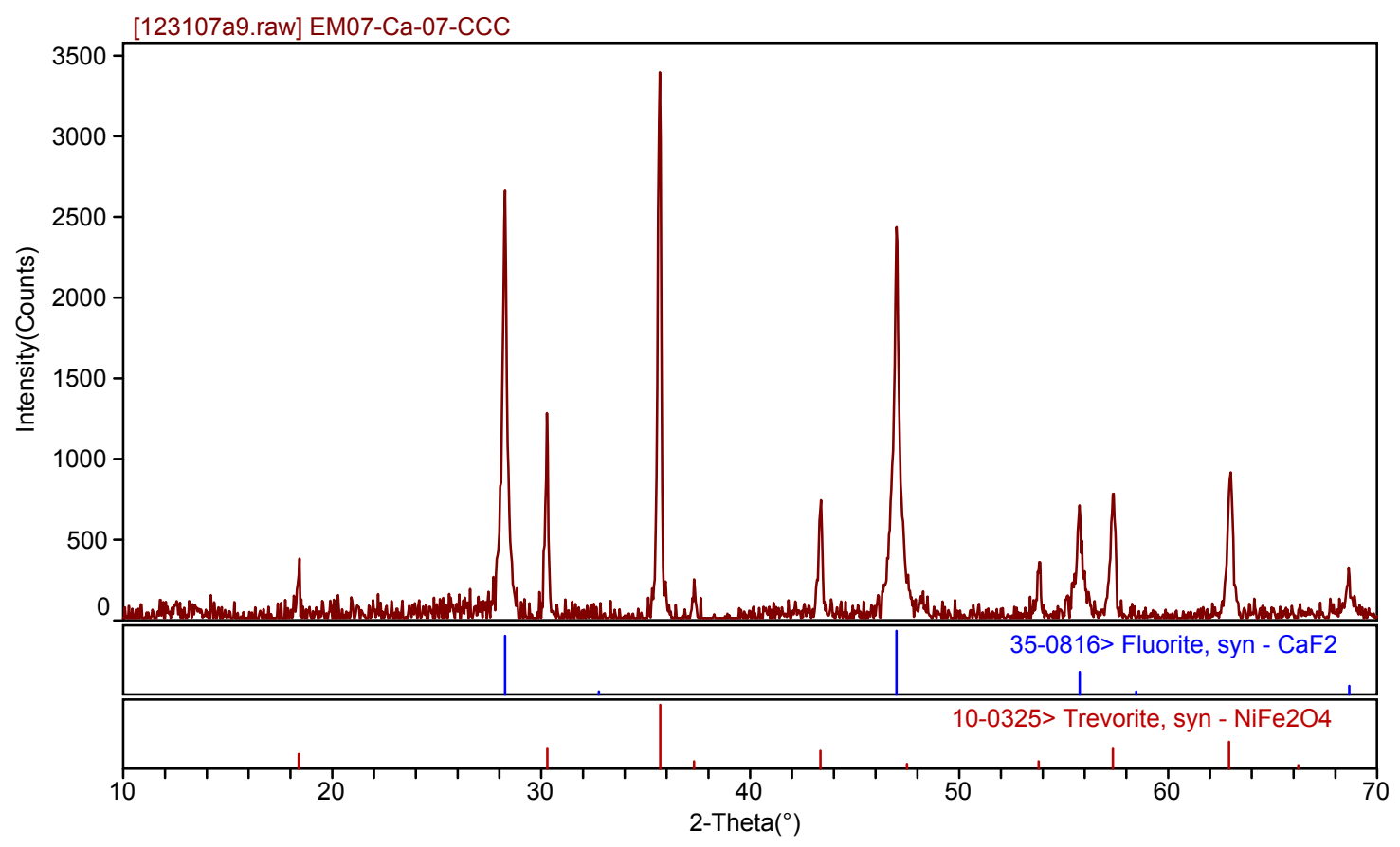

Figure B.15. XRD Spectrum of CCC Treated EM07-Ca-07

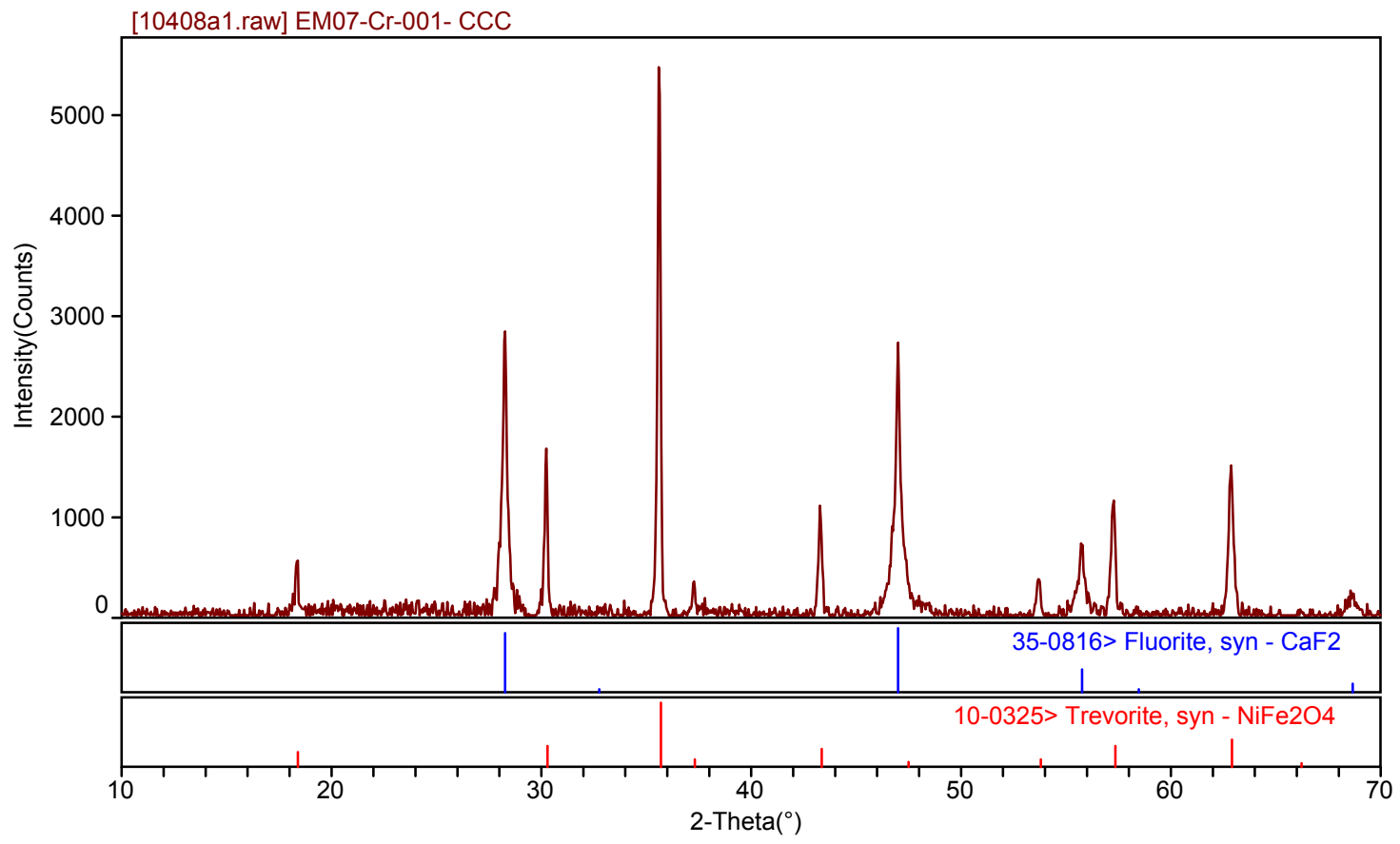

Figure B.16. XRD Spectrum of CCC Treated EM07-Cr-001 


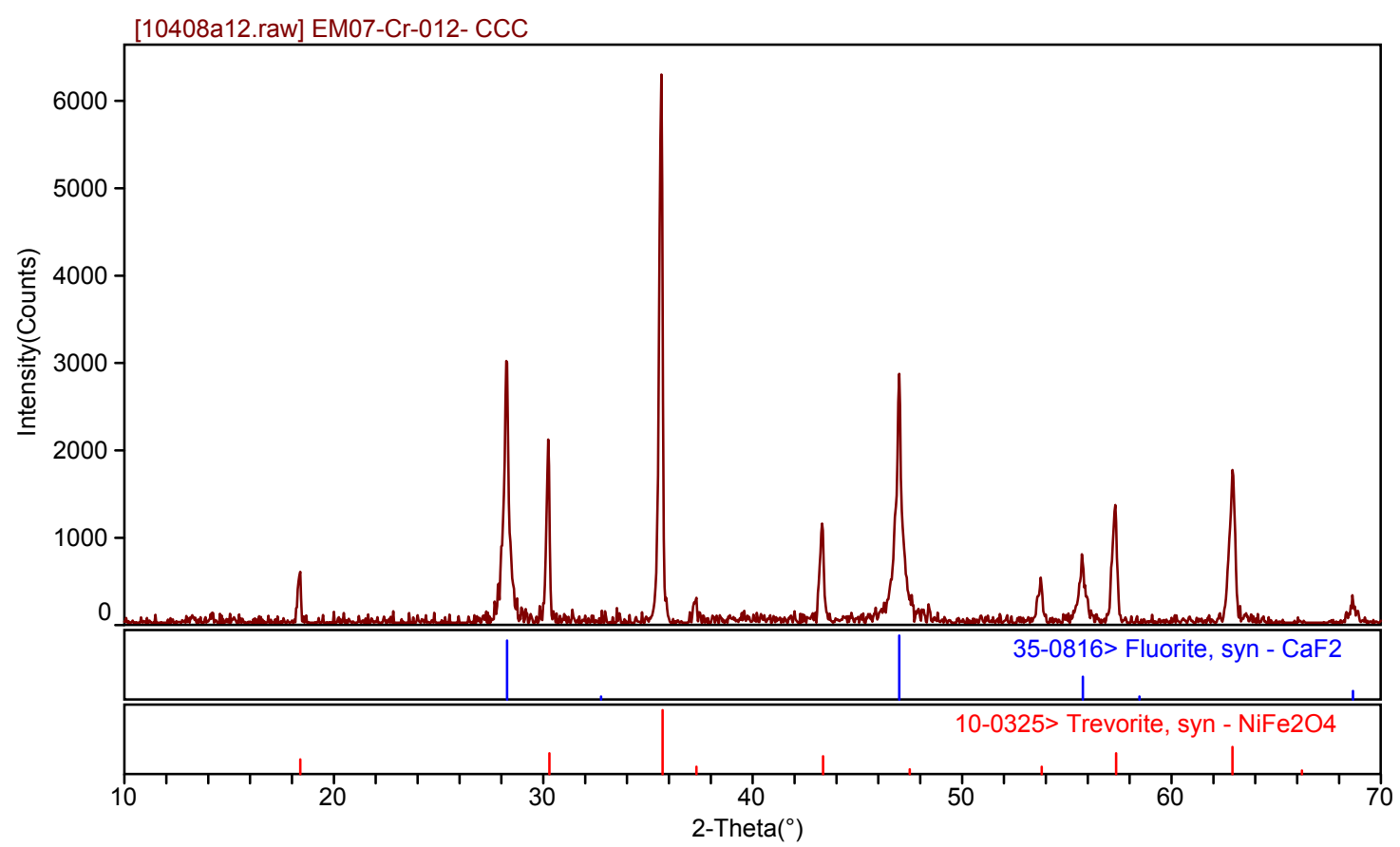

Figure B.17. XRD Spectrum of CCC Treated EM07-Cr-012

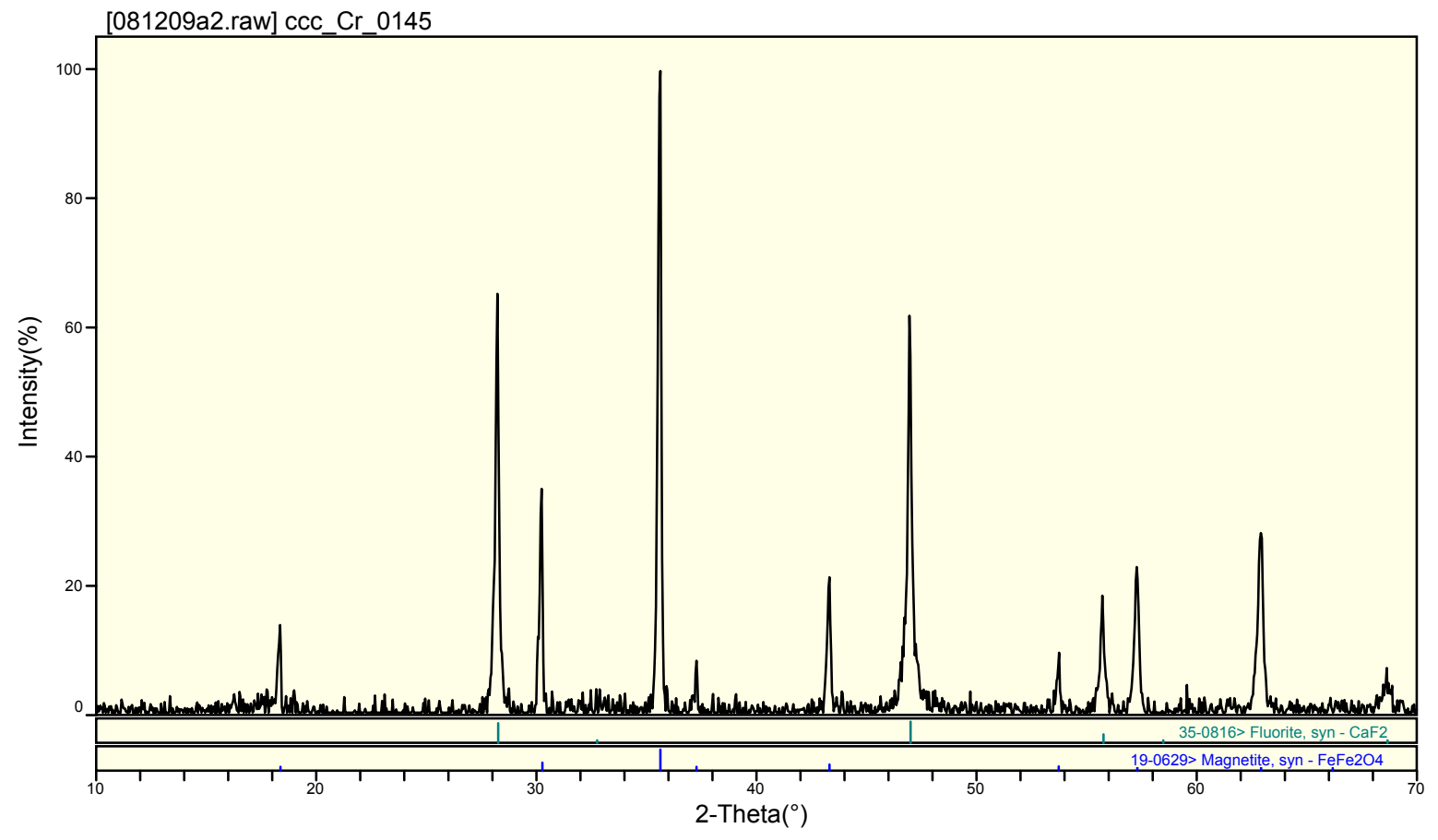

Figure B.18. XRD Spectrum of CCC Treated EM07-Cr-0145 


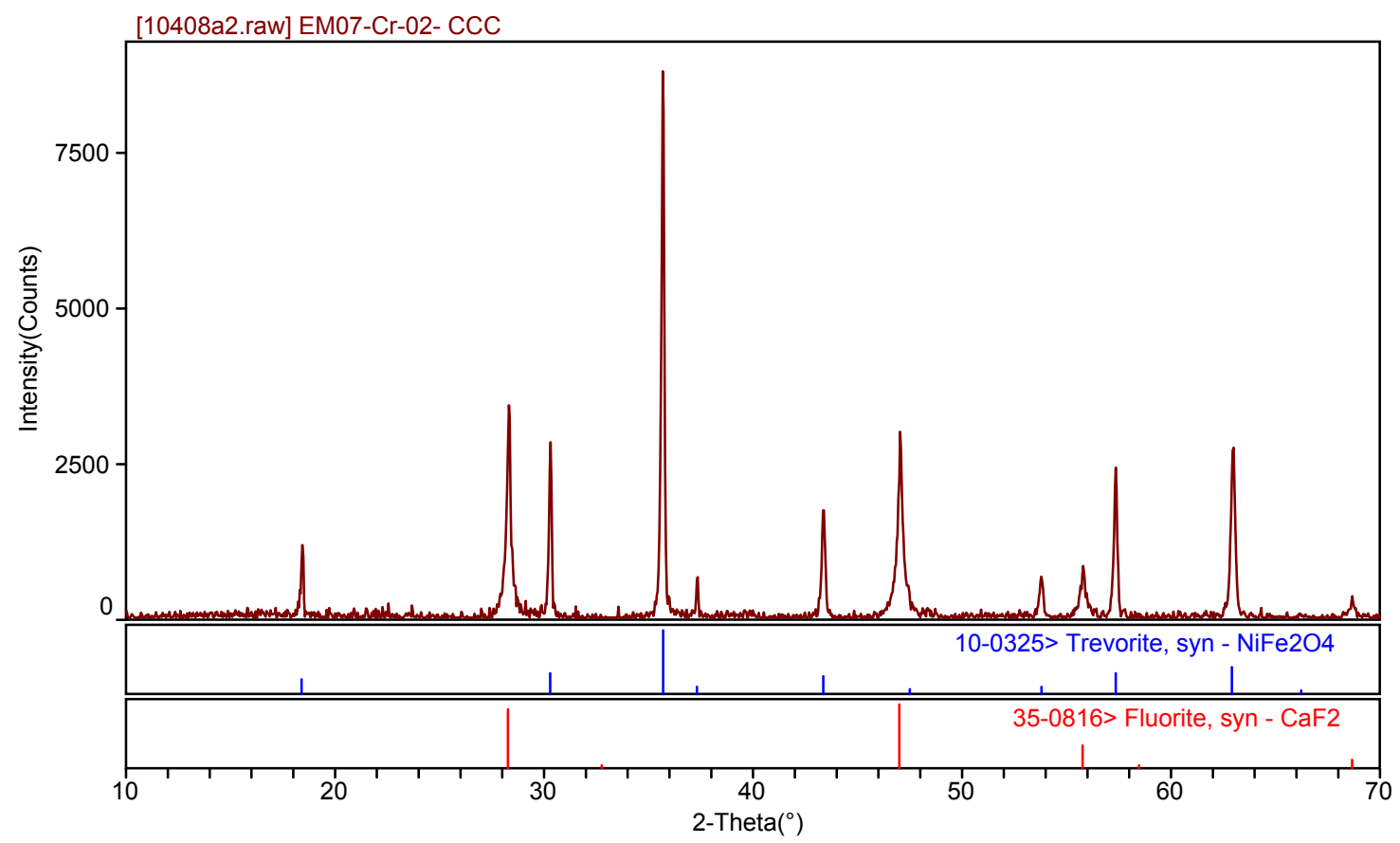

Figure B.19. XRD Spectrum of CCC Treated EM07-Cr-02

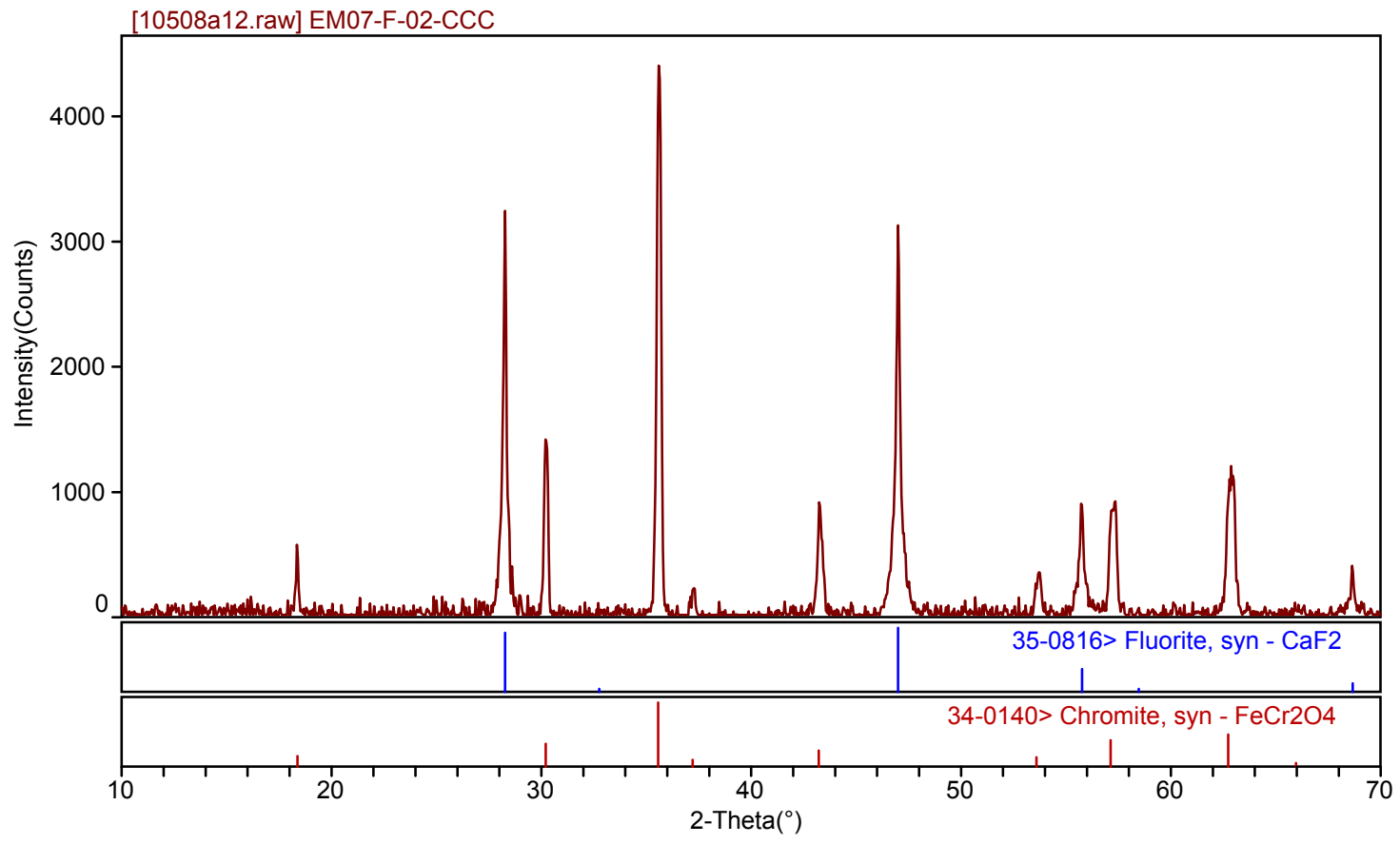

Figure B.20. XRD Spectrum of CCC Treated EM07-F-02 


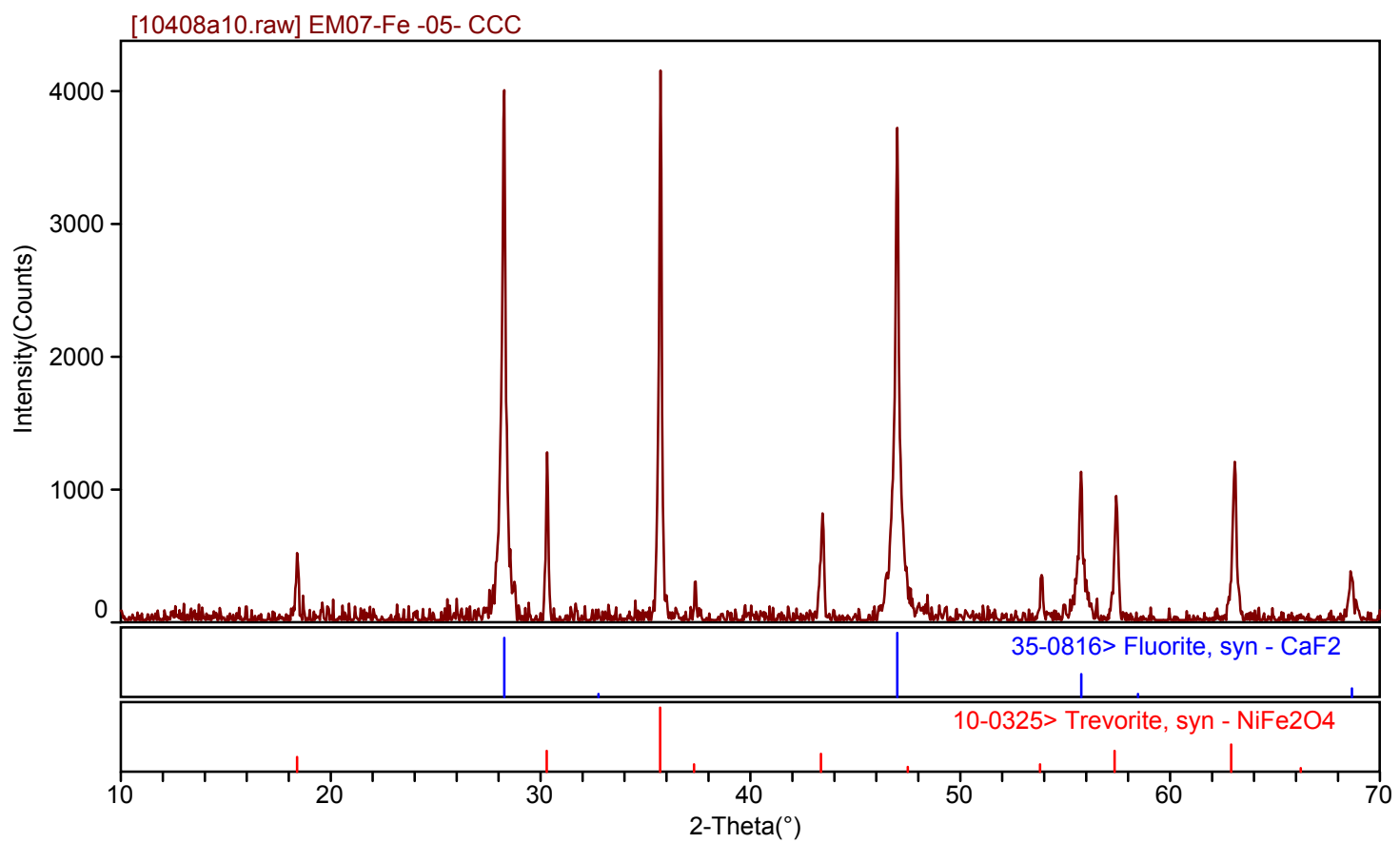

Figure B.21. XRD Spectrum of CCC Treated EM07-Fe-05

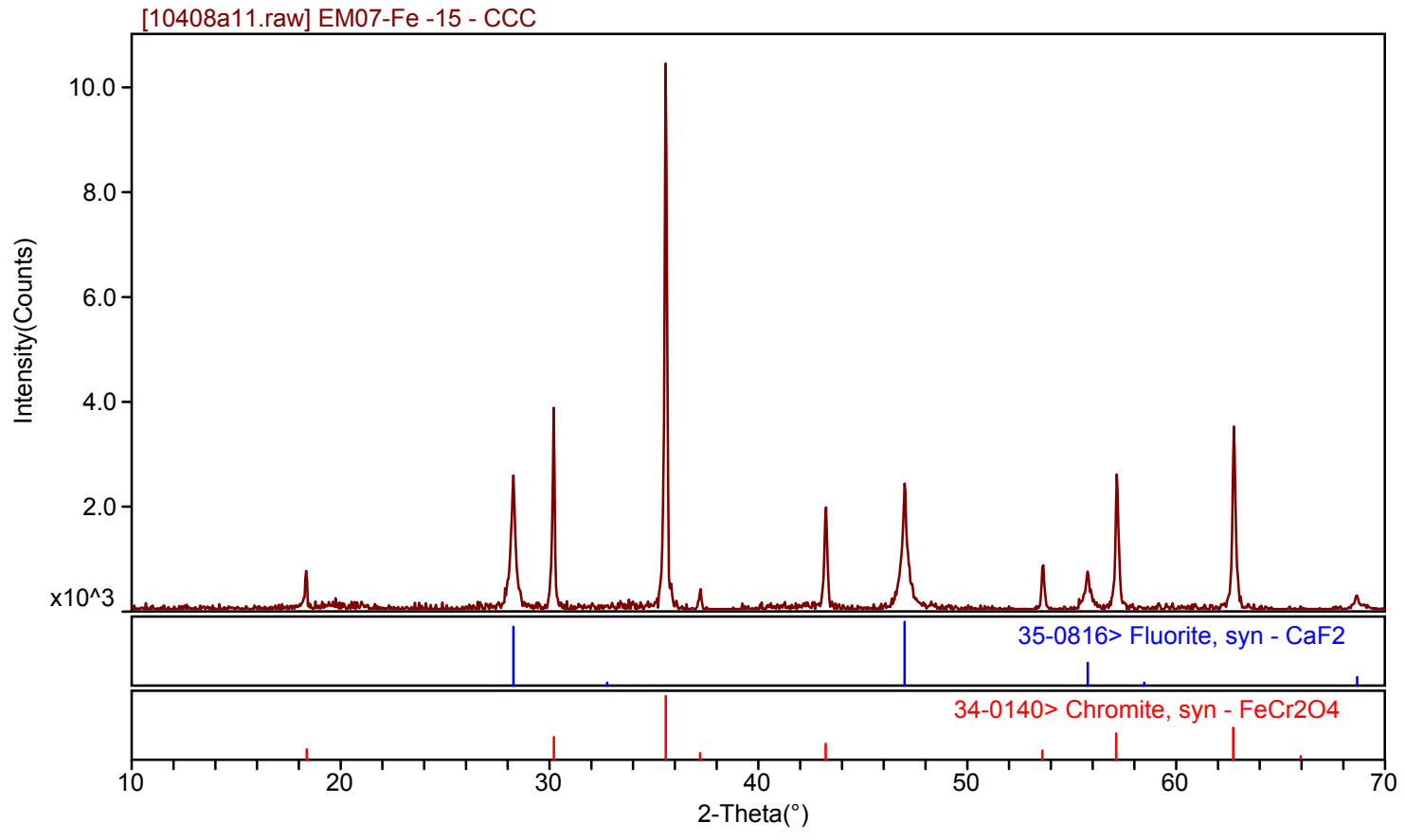

Figure B.22. XRD Spectrum of CCC Treated EM07-Fe-15 


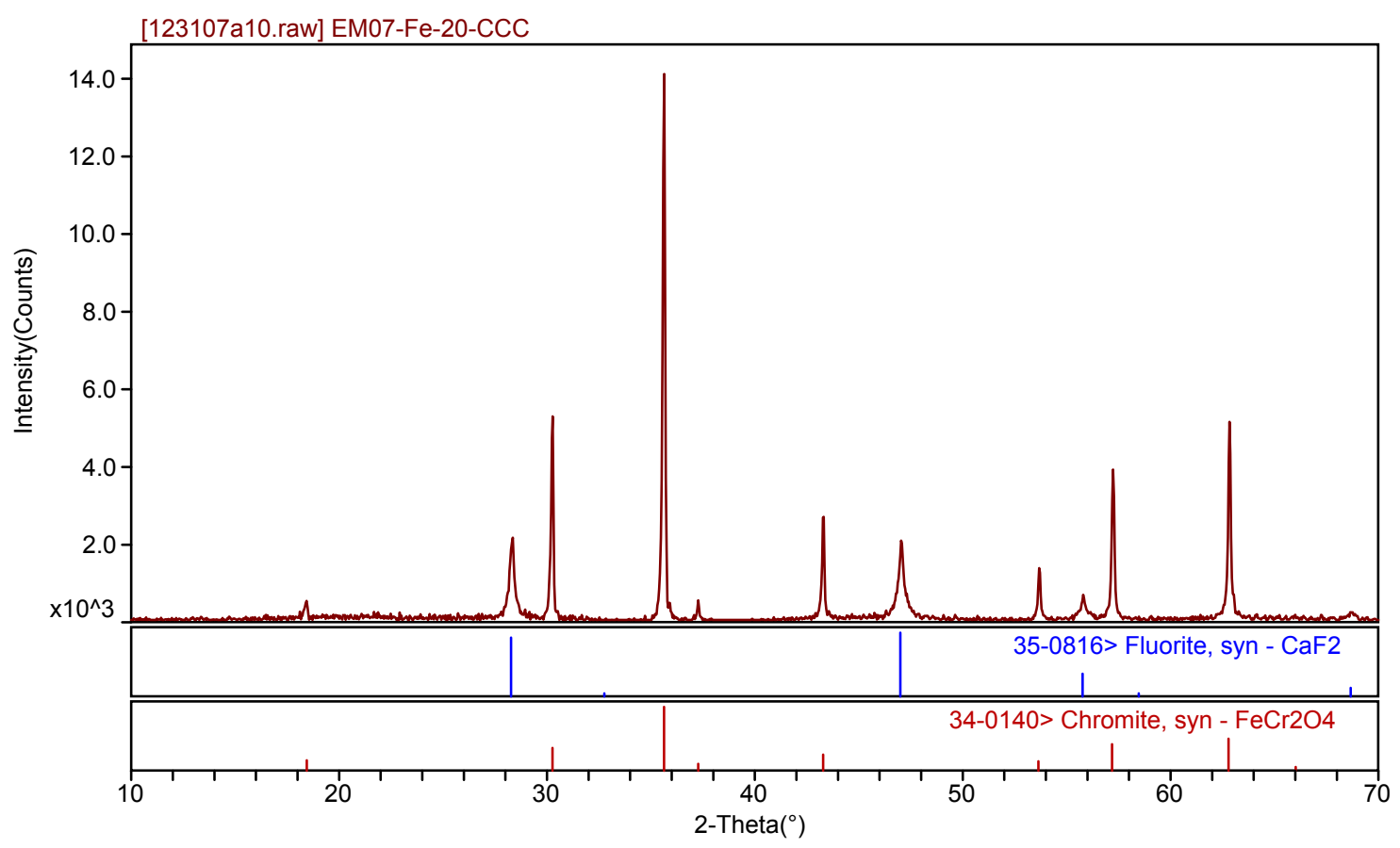

Figure B.23. XRD Spectrum of CCC Treated EM07-Fe-20

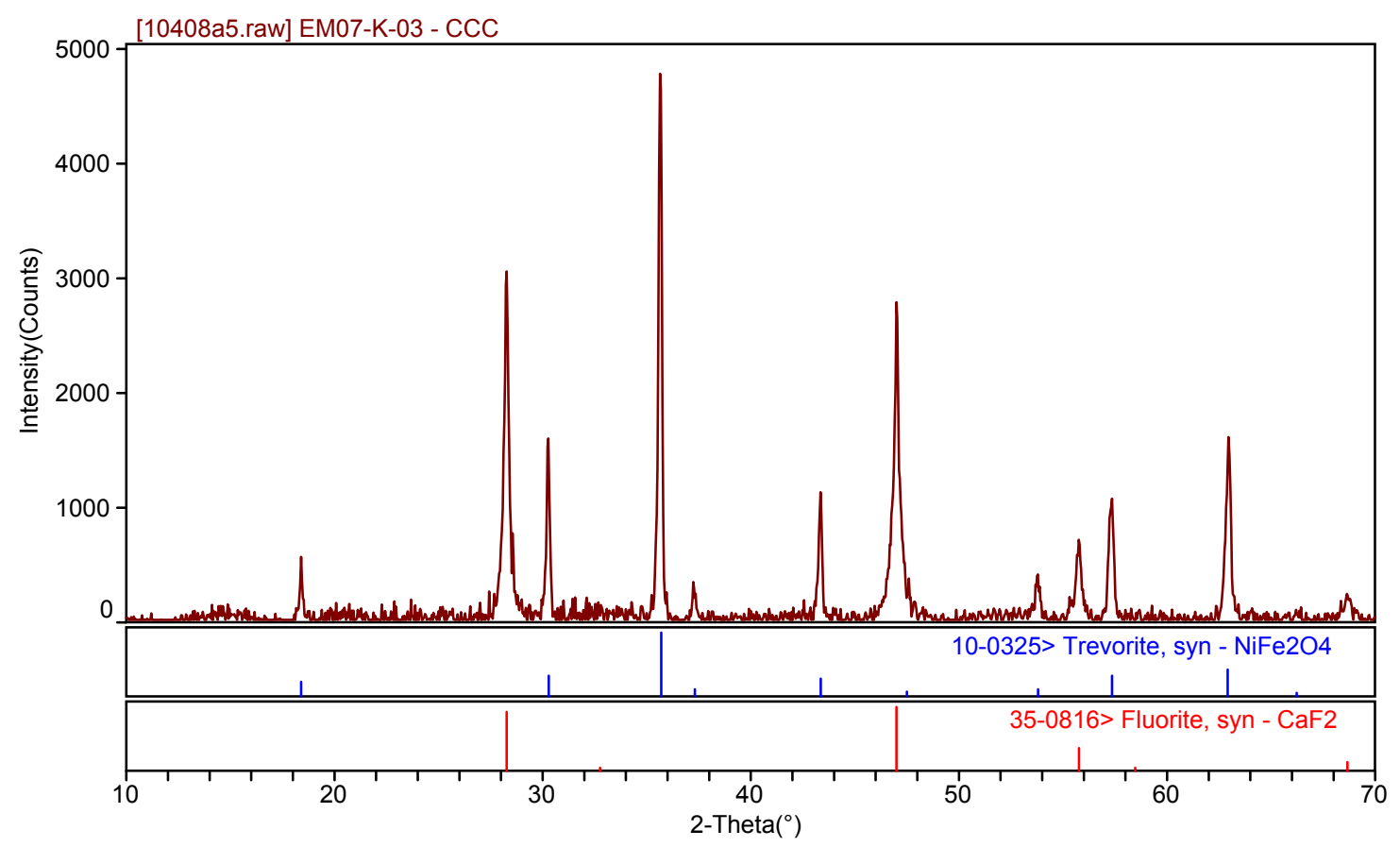

Figure B.24. XRD Spectrum of CCC Treated EM07-K-03 


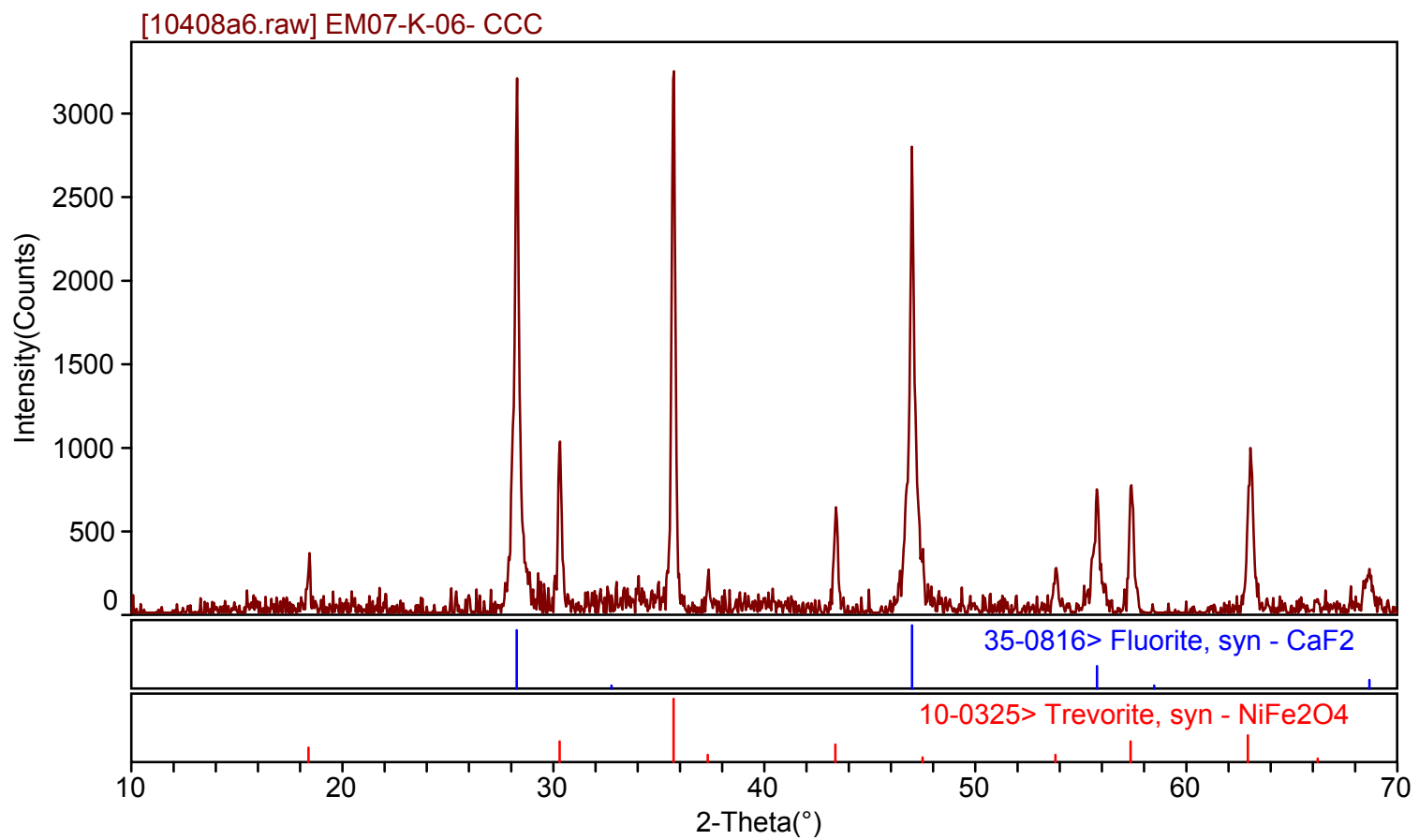

Figure B.25. XRD Spectrum of CCC Treated EM07-K-06

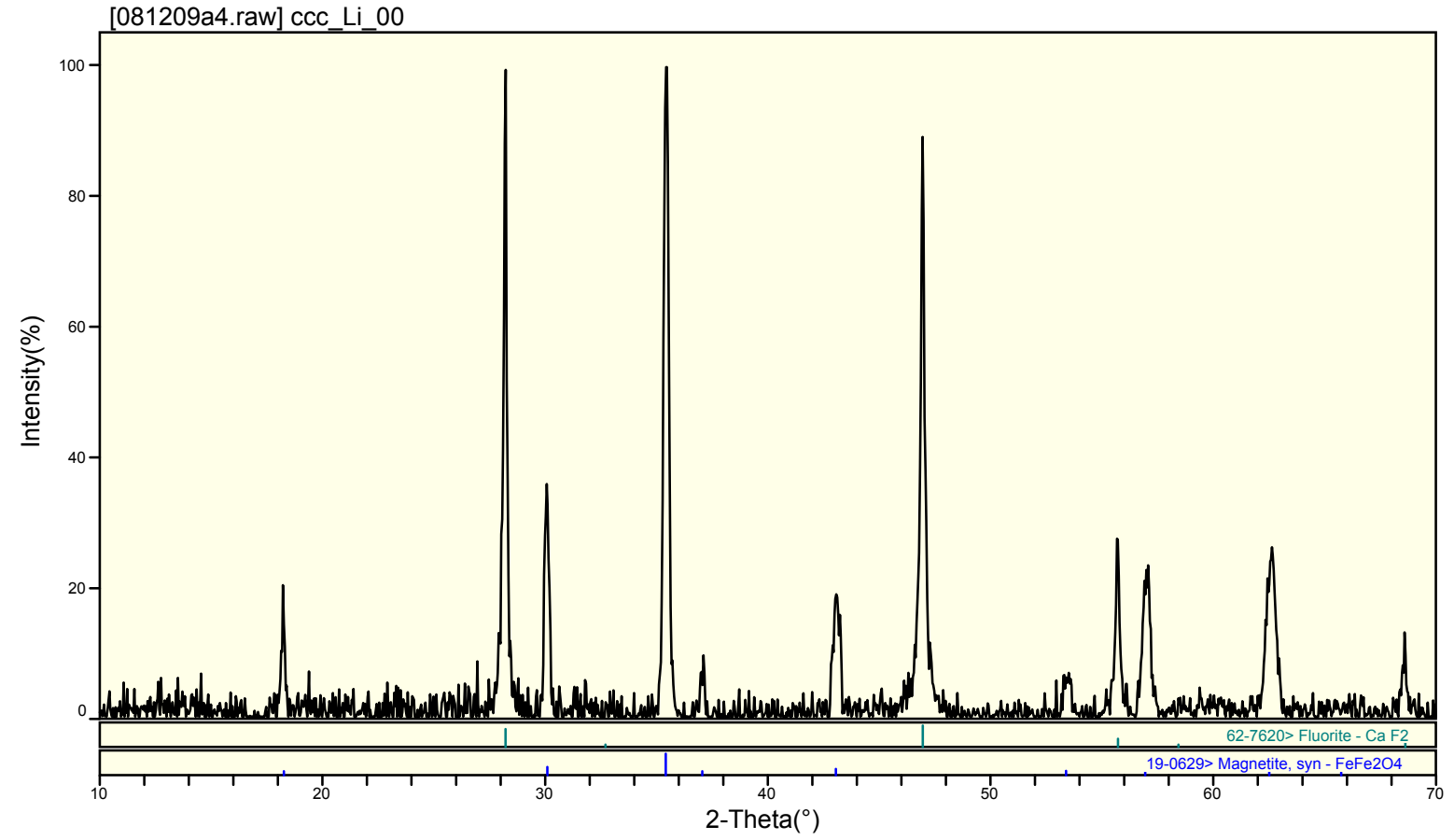

Figure B.26. XRD Spectrum of CCC Treated EM07-Li-00 


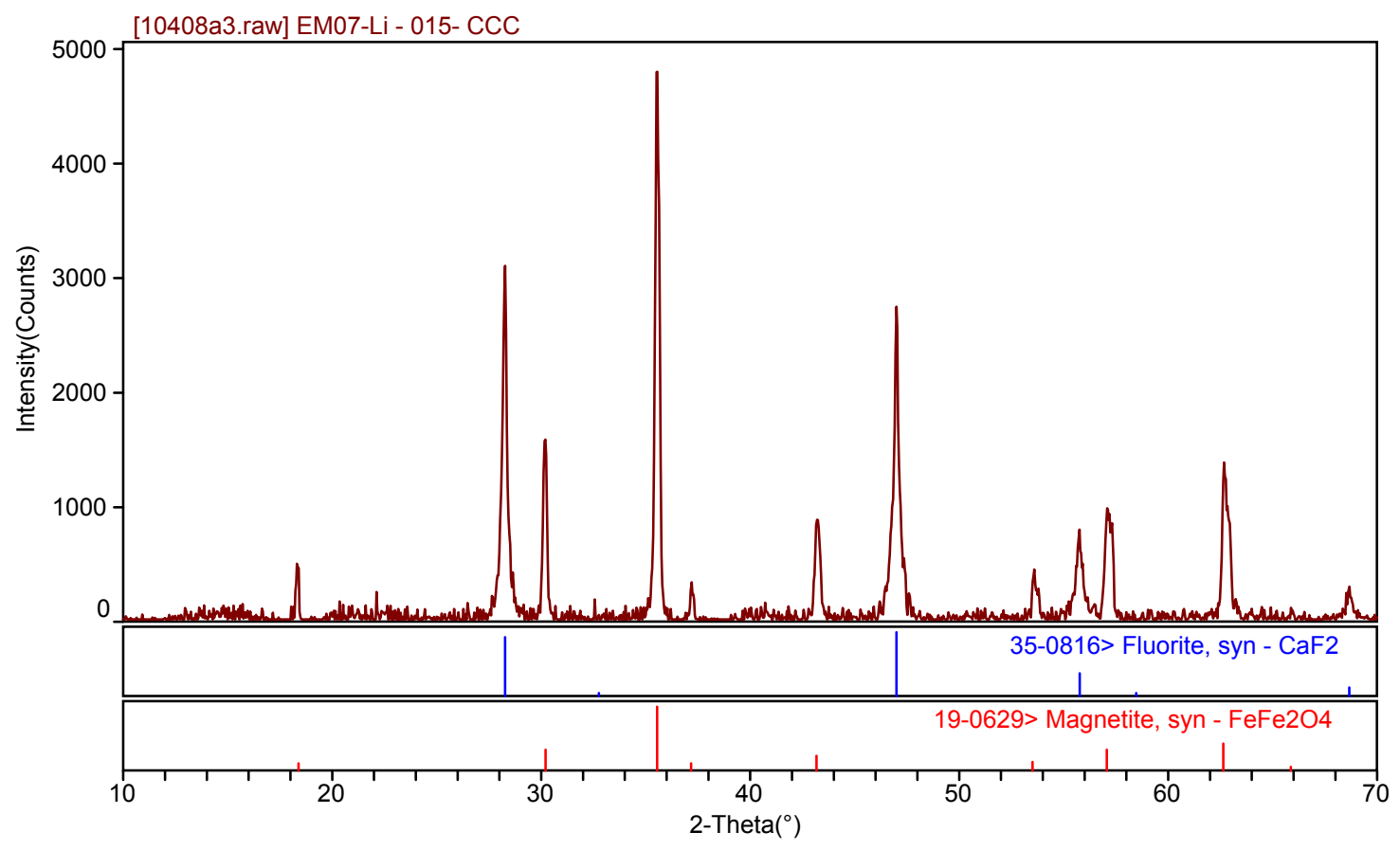

Figure B.27. XRD Spectrum of CCC Treated EM07-Li-015

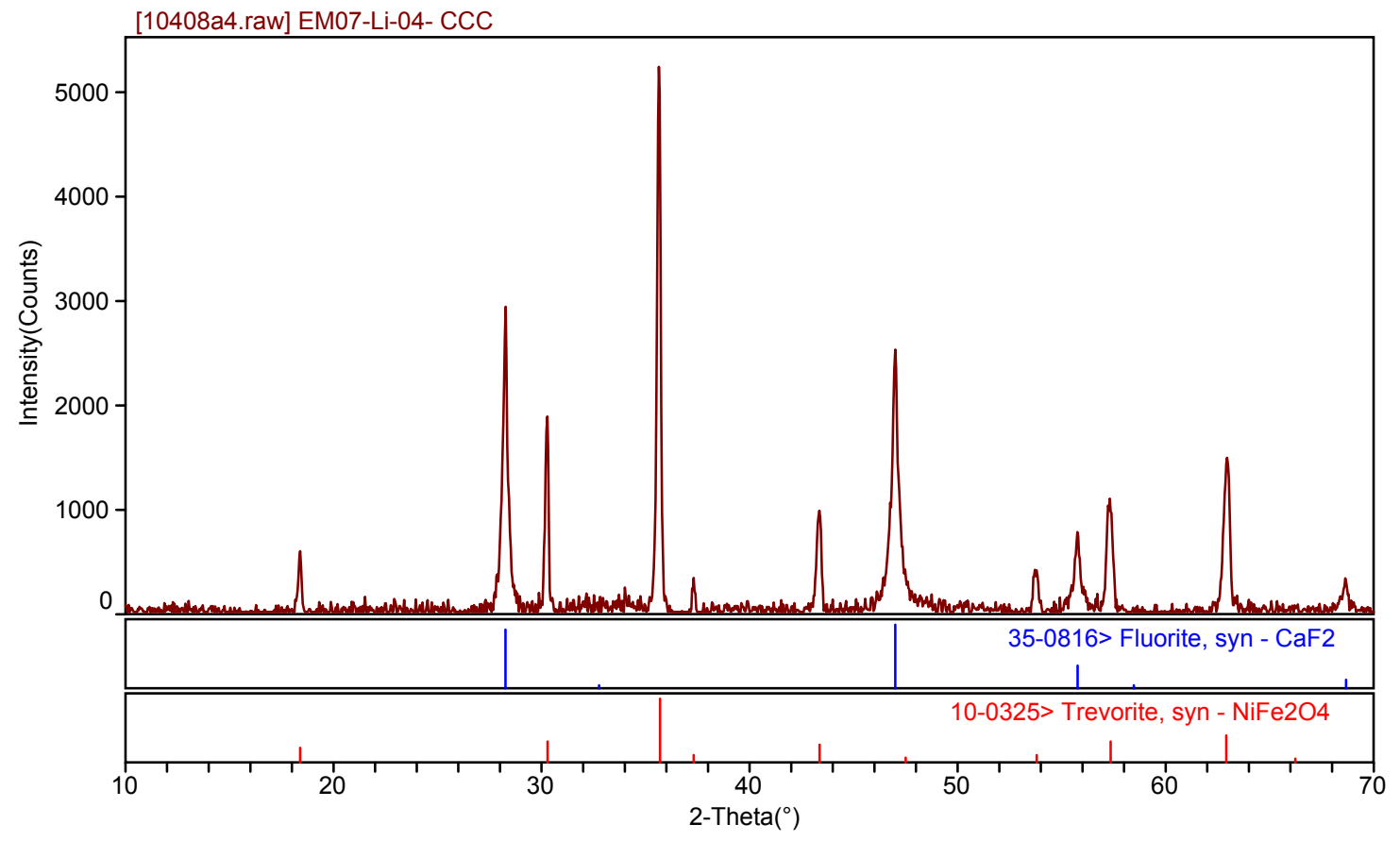

Figure B.28. XRD Spectrum of CCC Treated EM07-Li-04 


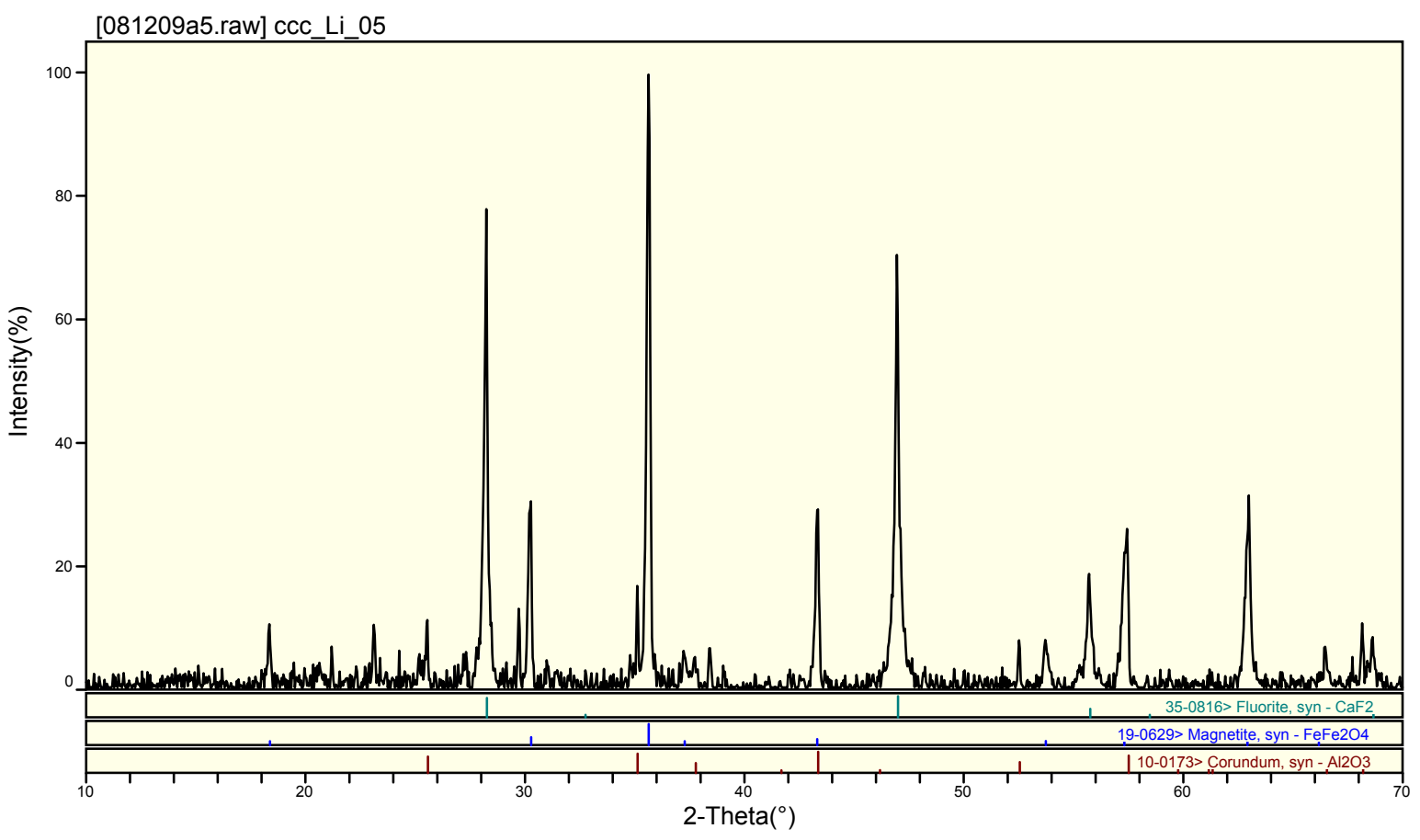

Figure B.29. XRD Spectrum of CCC Treated EM07-Li-05

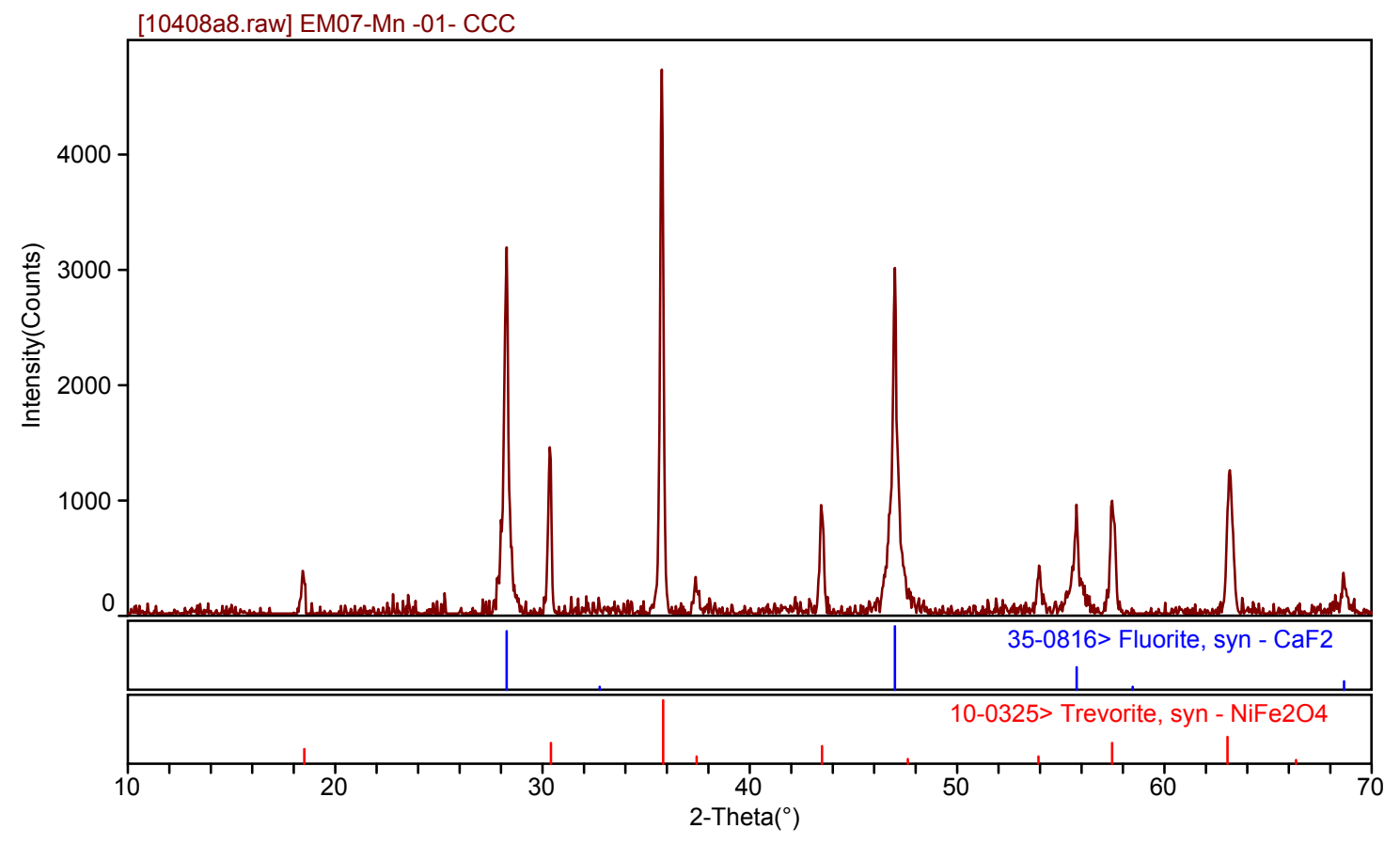

Figure B.30. XRD Spectrum of CCC Treated EM07-Mn-01 


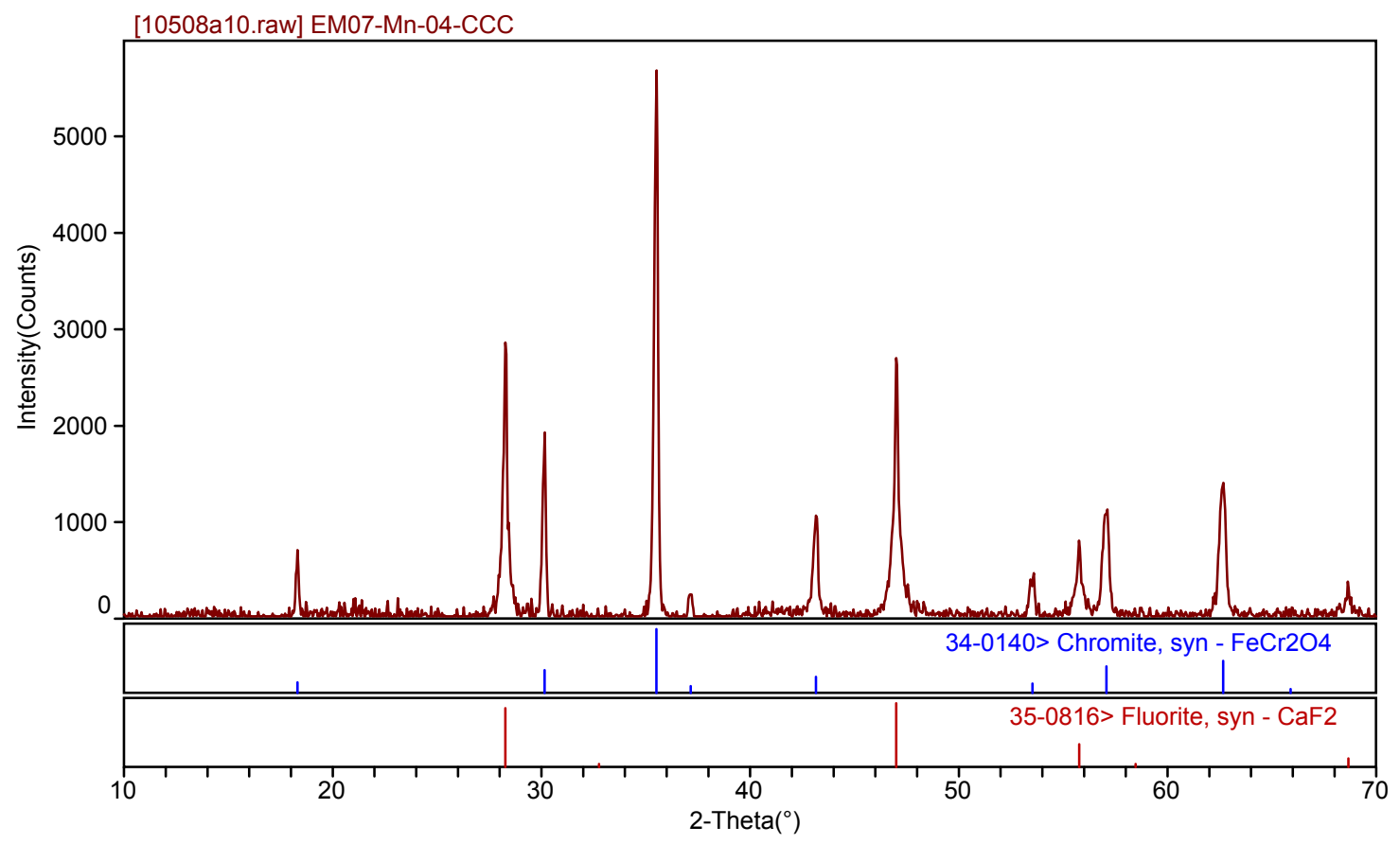

Figure B.31. XRD Spectrum of CCC Treated EM07-Mn-04

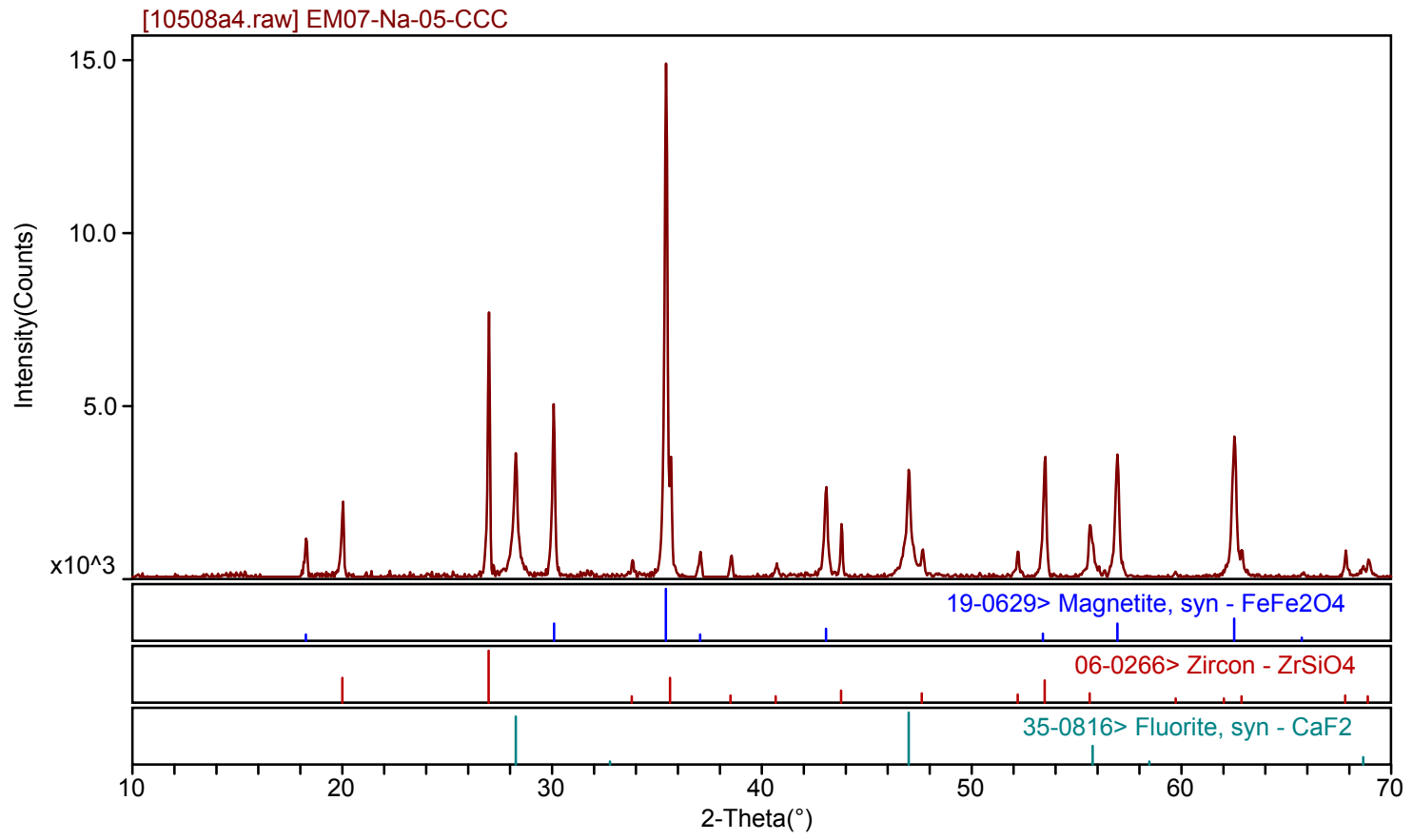

Figure B.32. XRD Spectrum of CCC Treated EM07-Na-05 


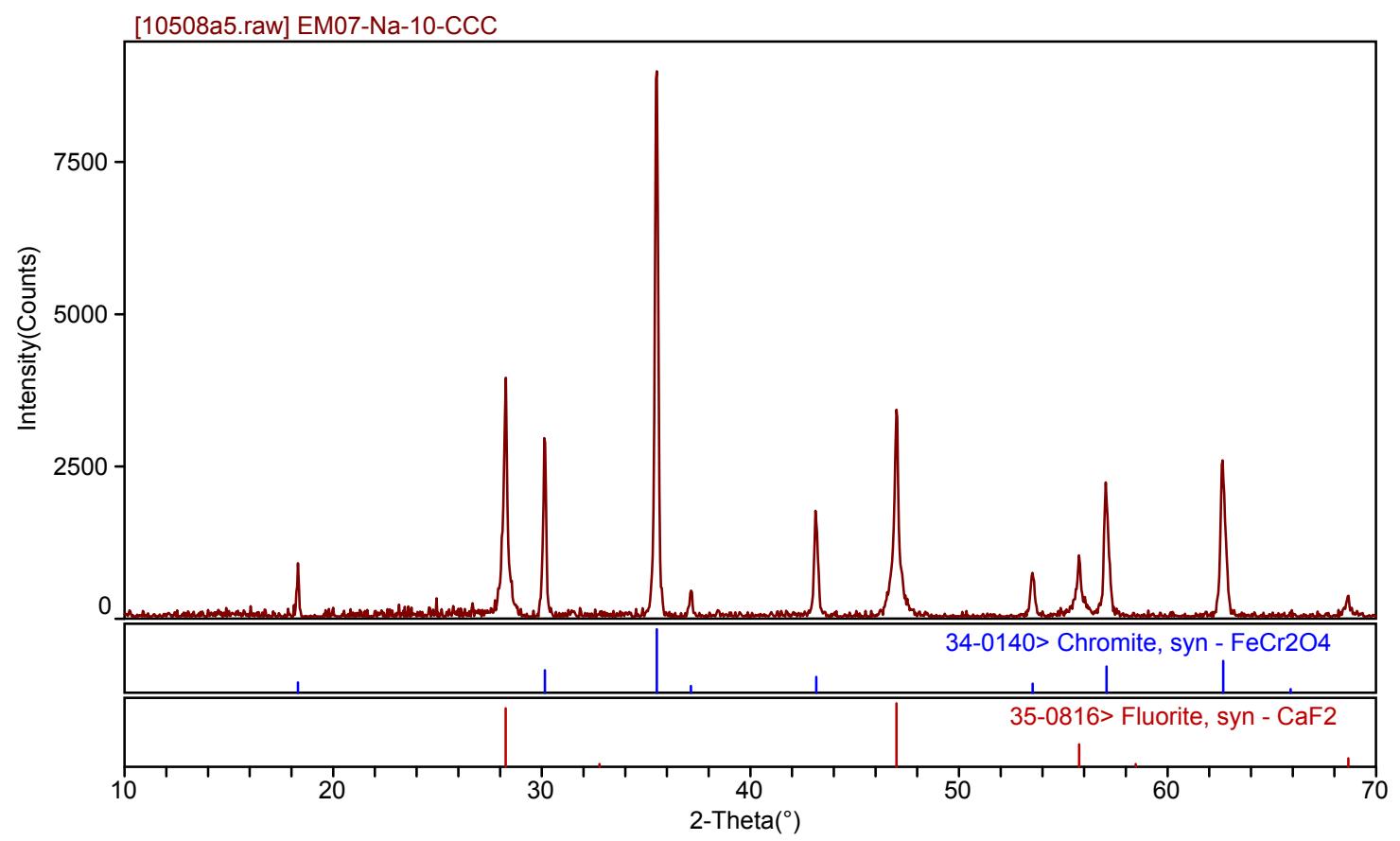

Figure B.33. XRD Spectrum of CCC Treated EM07-Na-10

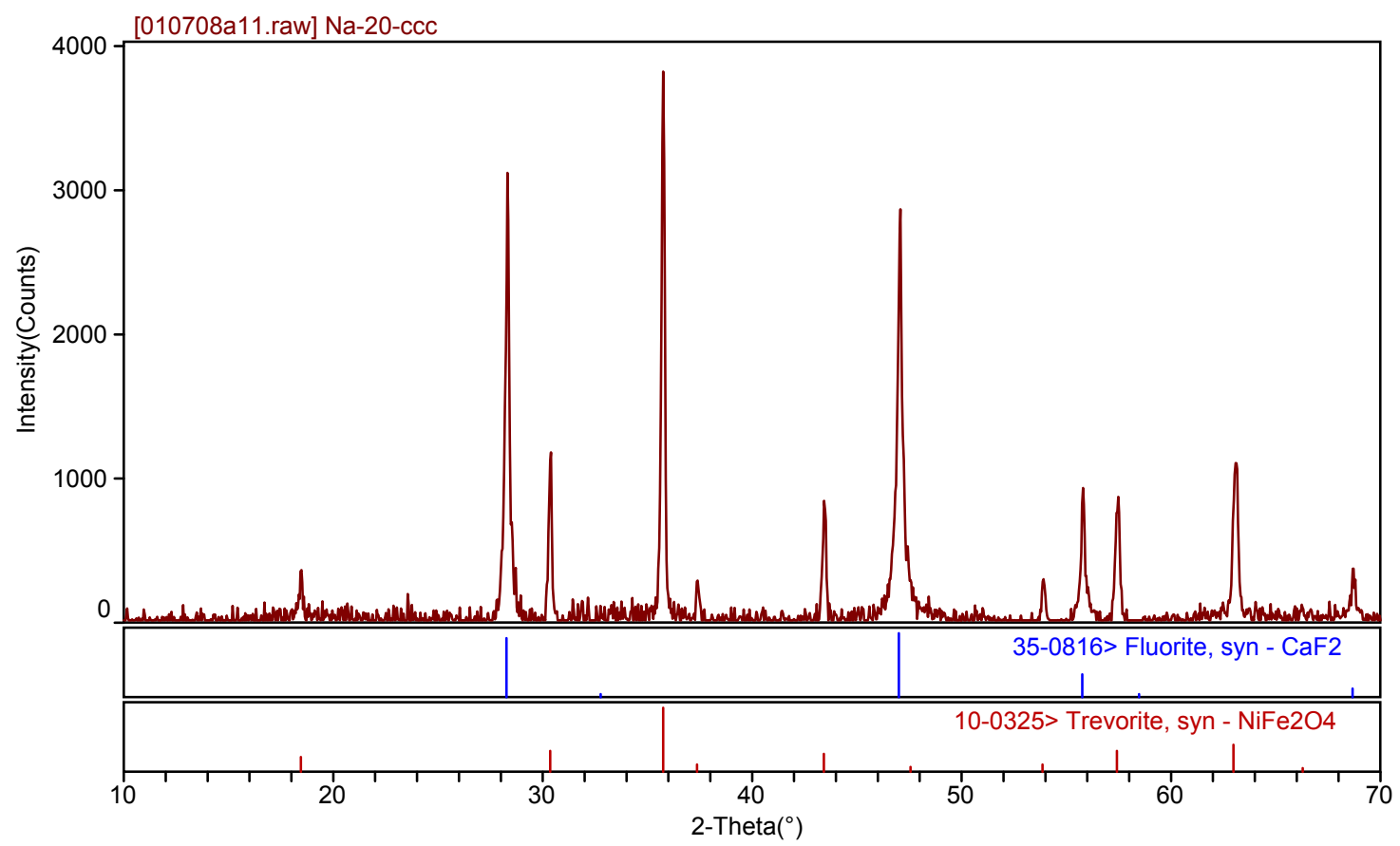

Figure B.34. XRD Spectrum of CCC Treated EM07-Na-20 


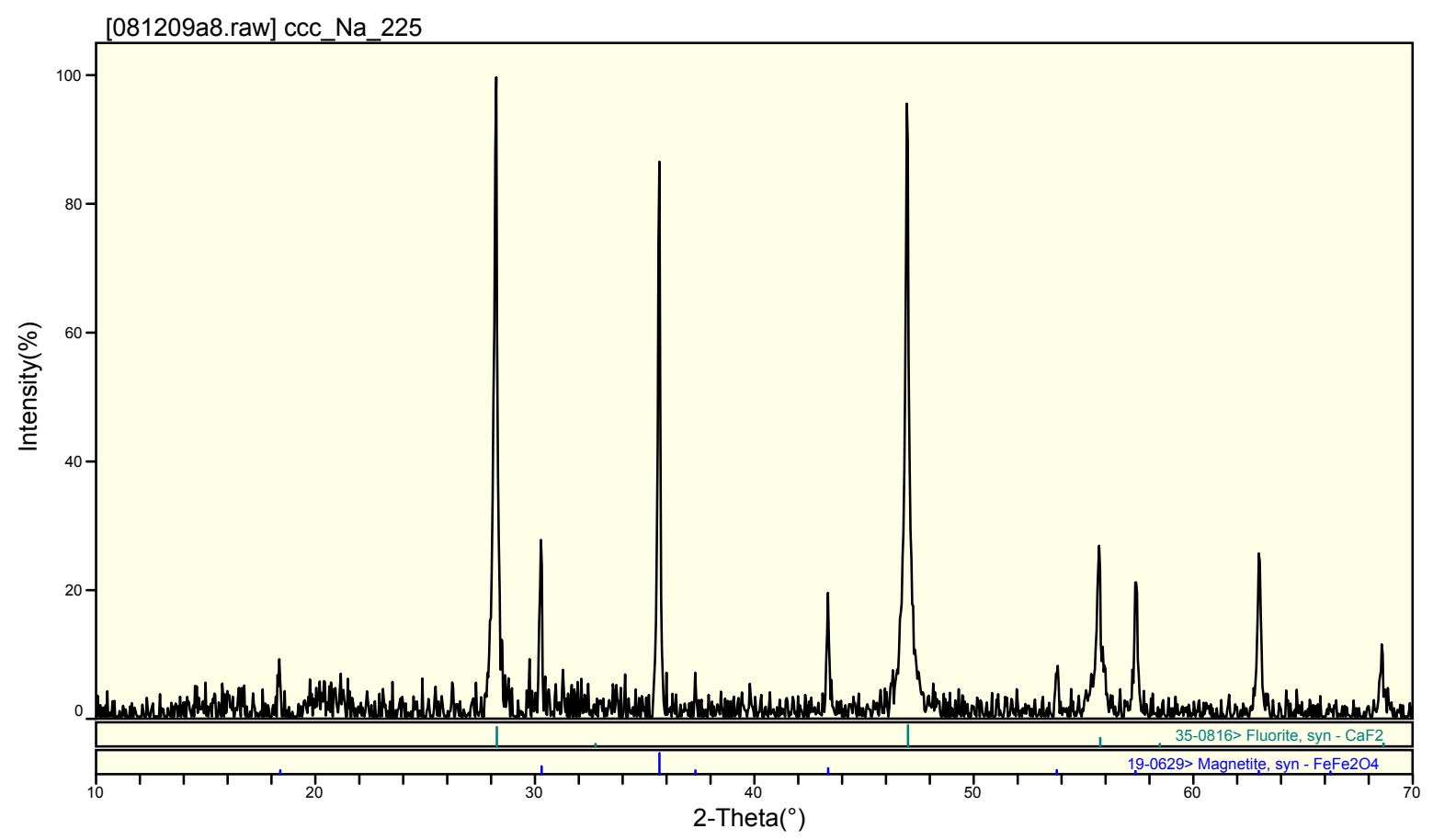

Figure B.35. XRD Spectrum of CCC Treated EM07-Na-225

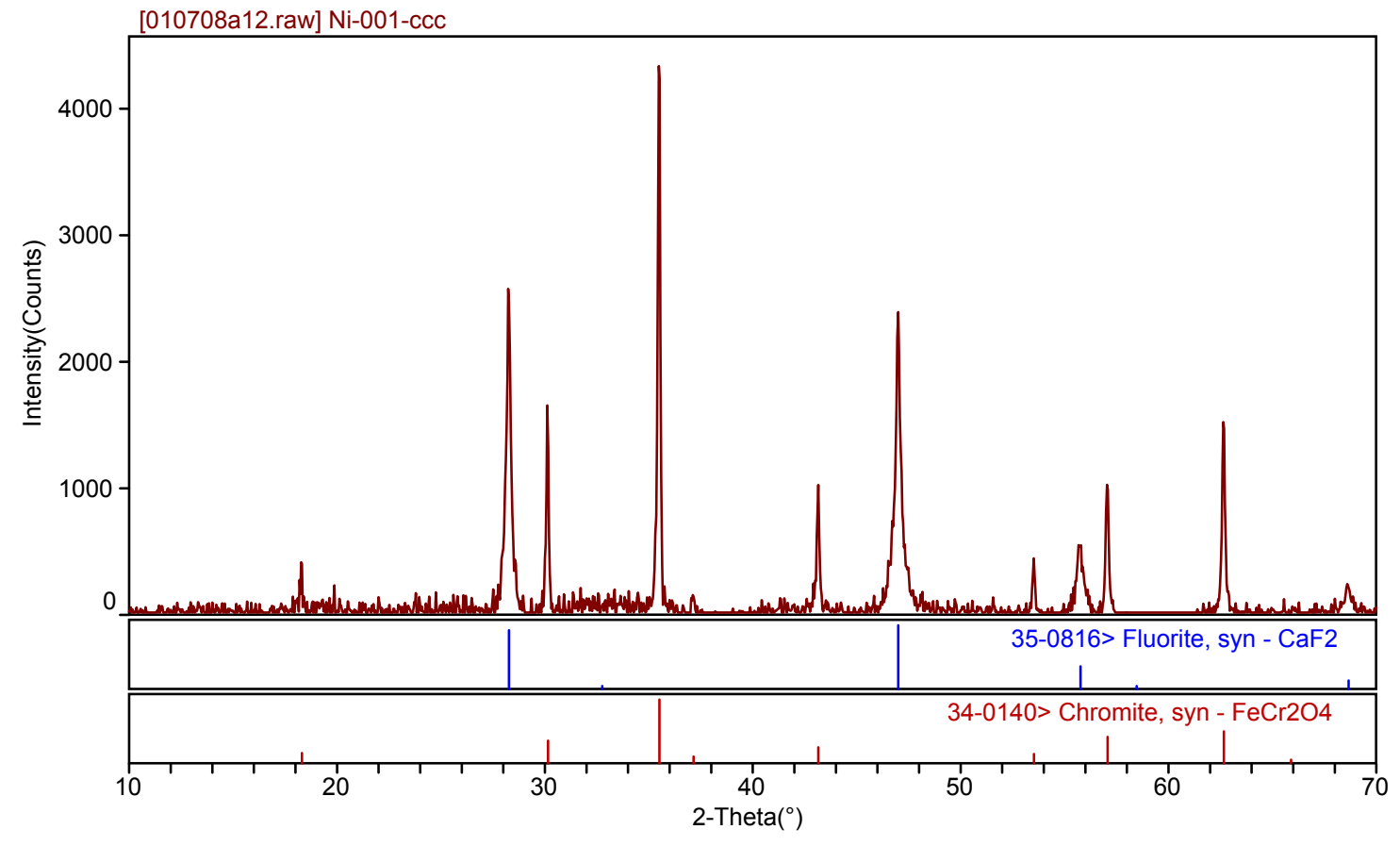

Figure B.36. XRD Spectrum of CCC Treated EM07-Ni-001 


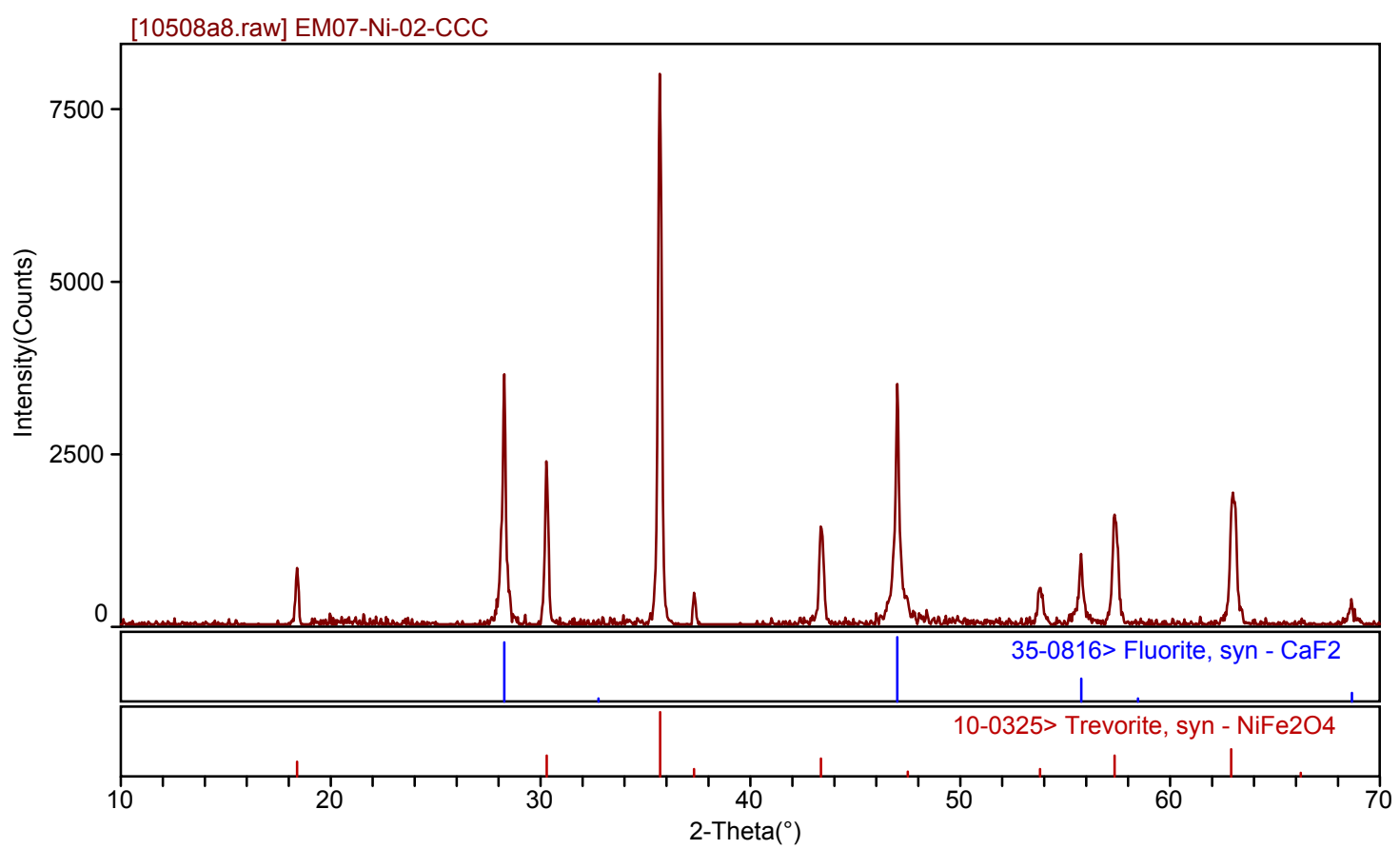

Figure B.37. XRD Spectrum of CCC Treated EM07-Ni-02

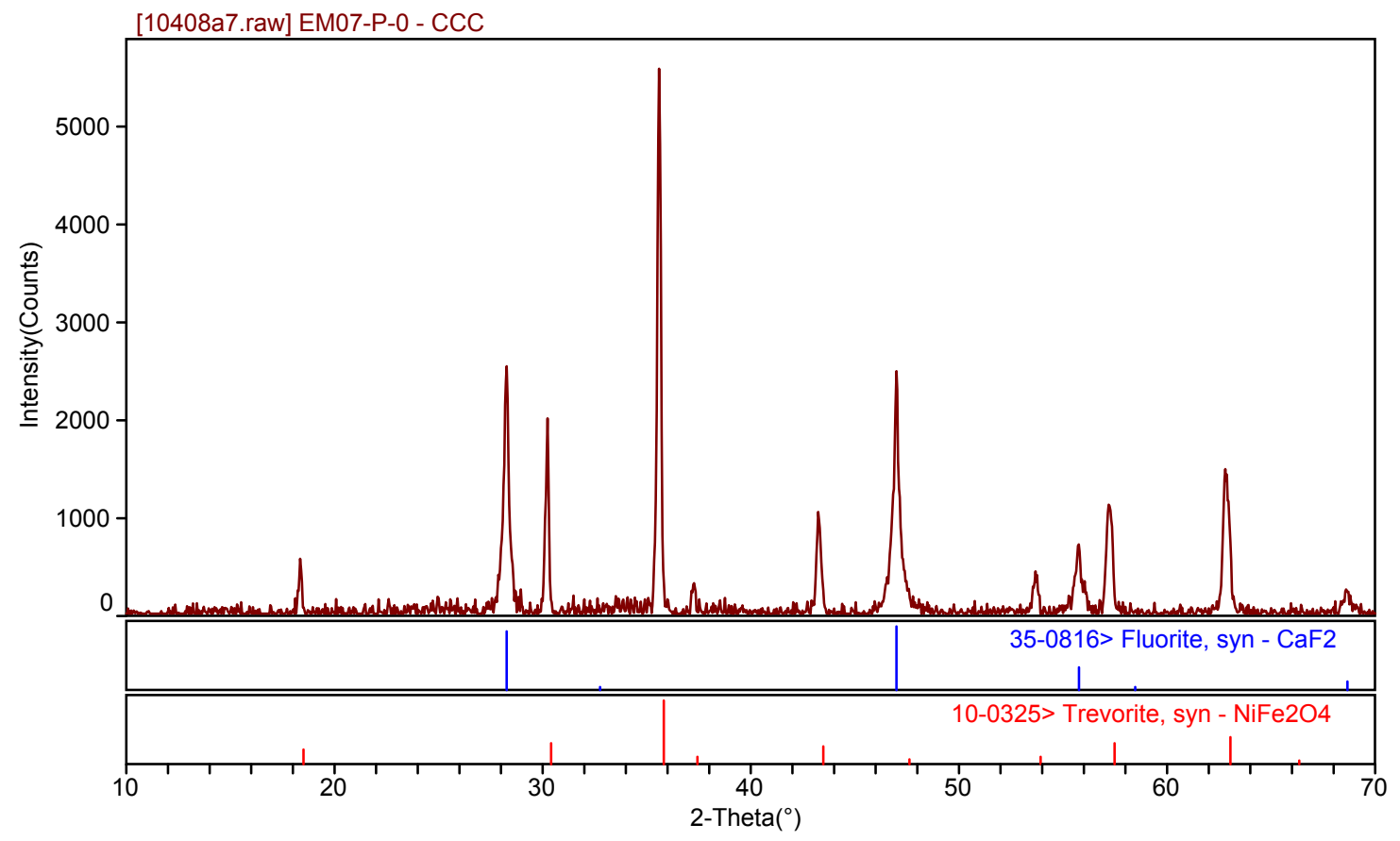

Figure B.38. XRD Spectrum of CCC Treated EM07-P-0 


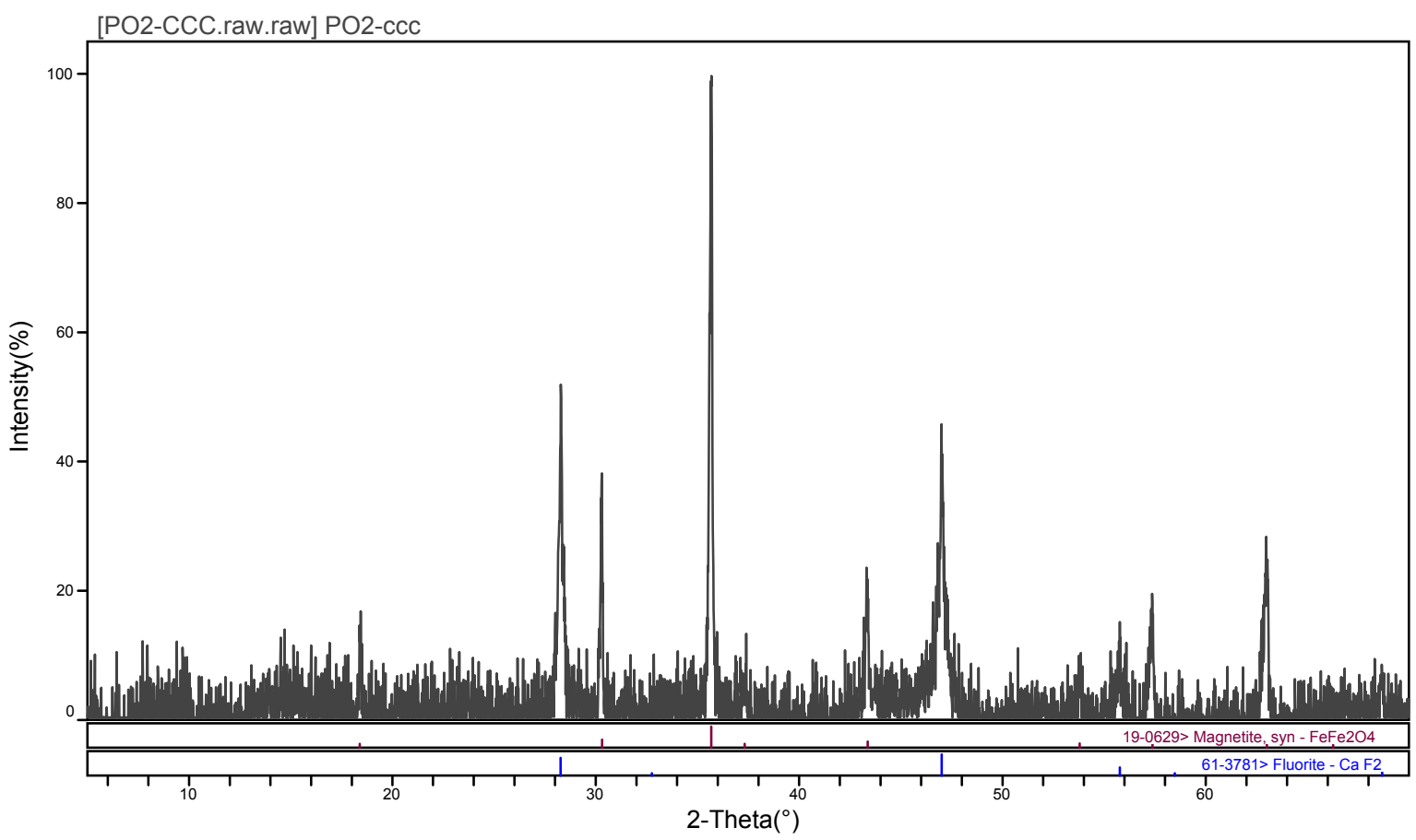

Figure B.39. XRD Spectrum of CCC Treated EM07-P-02

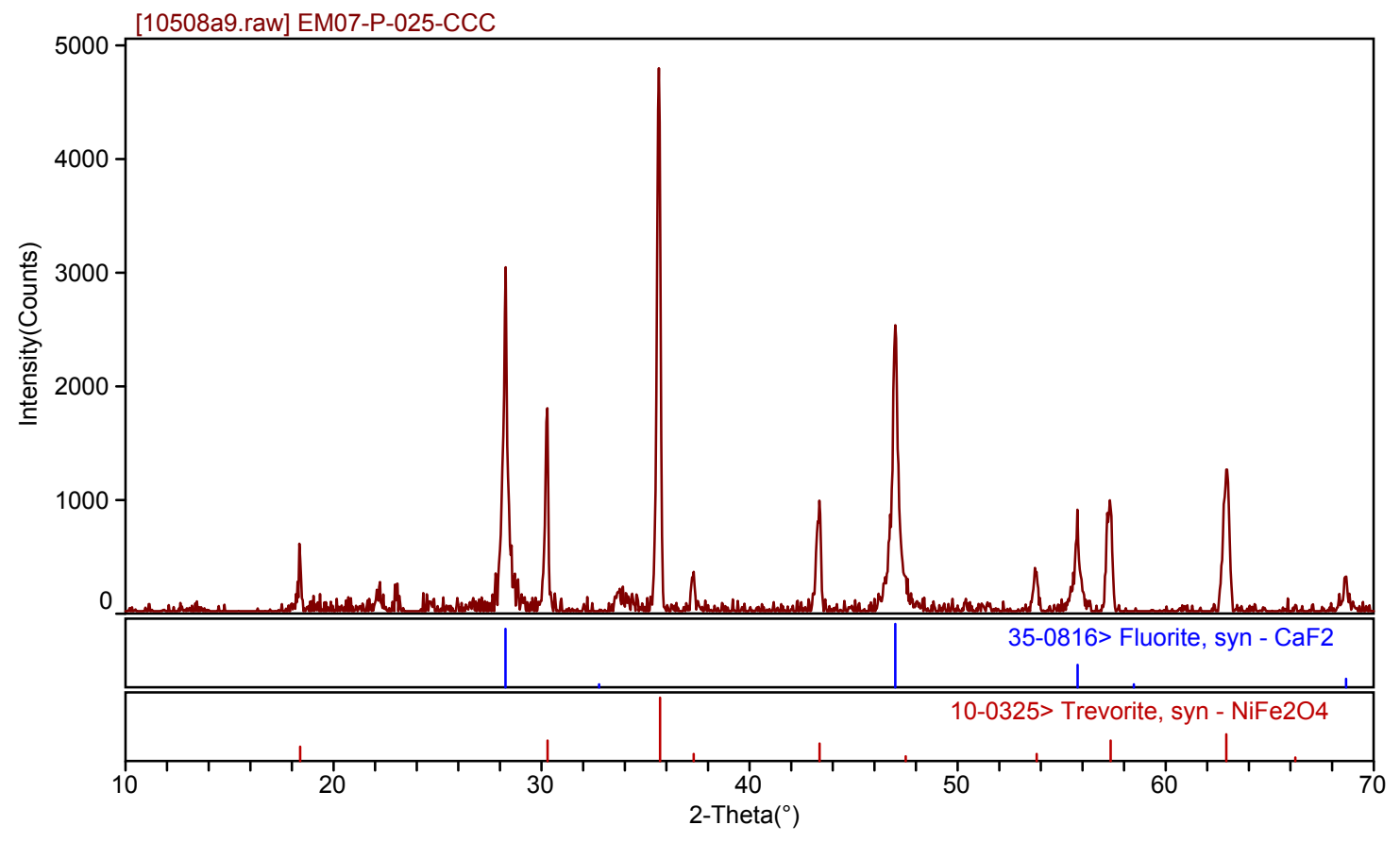

Figure B.40. XRD Spectrum of CCC Treated EM07-P-025 


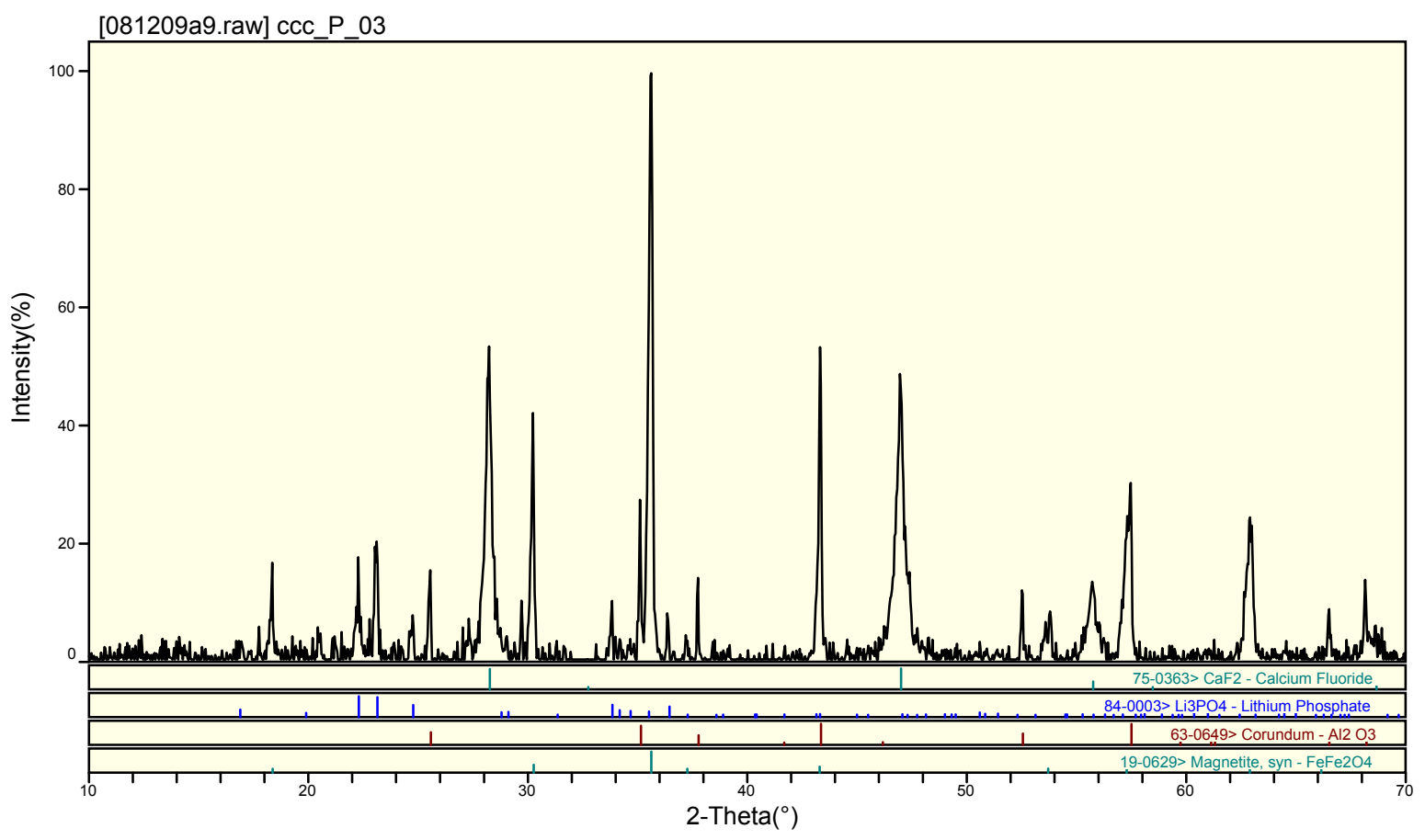

Figure B.41. XRD Spectrum of CCC Treated EM07-P-03

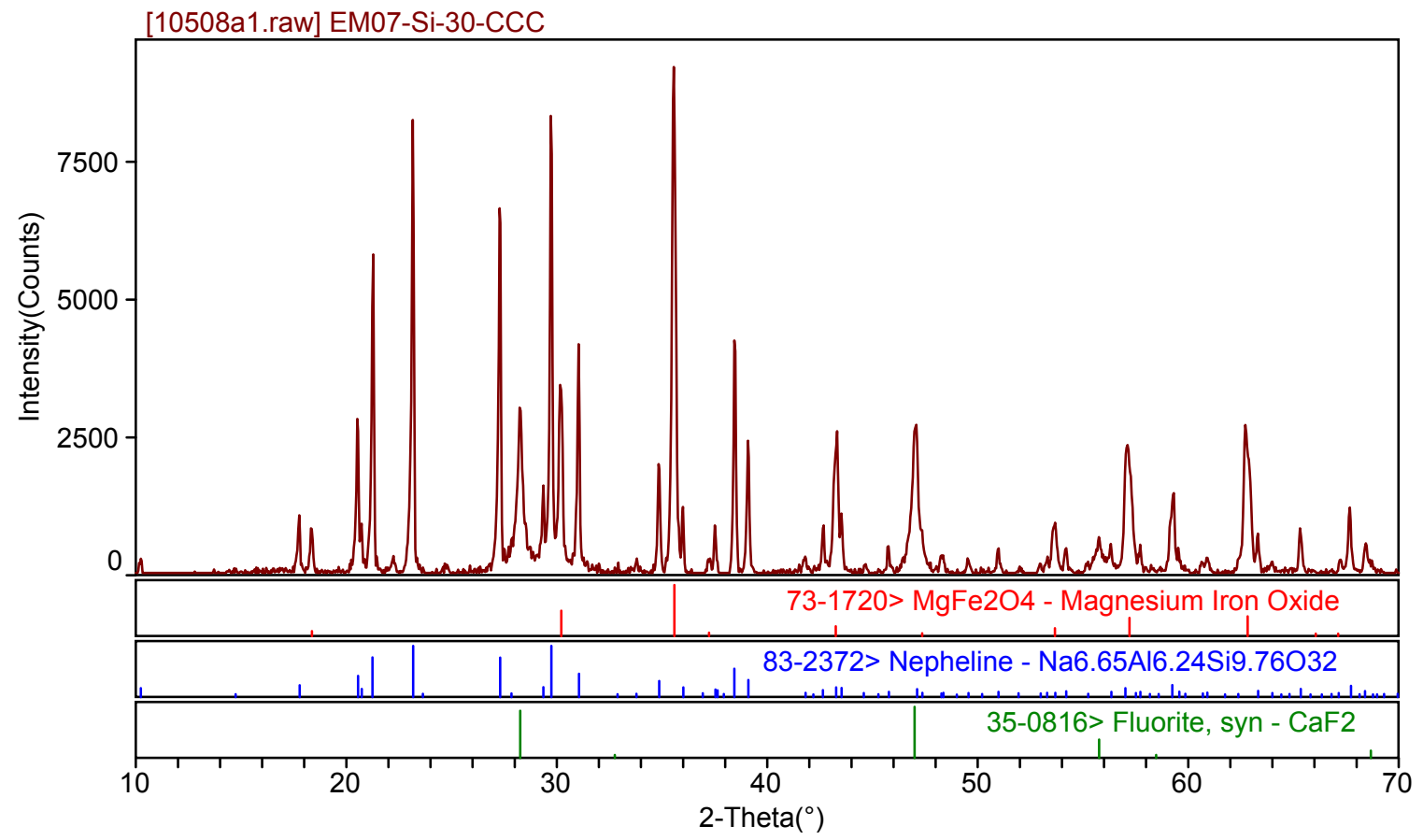

Figure B.42. XRD Spectrum of CCC Treated EM07-Si-30 


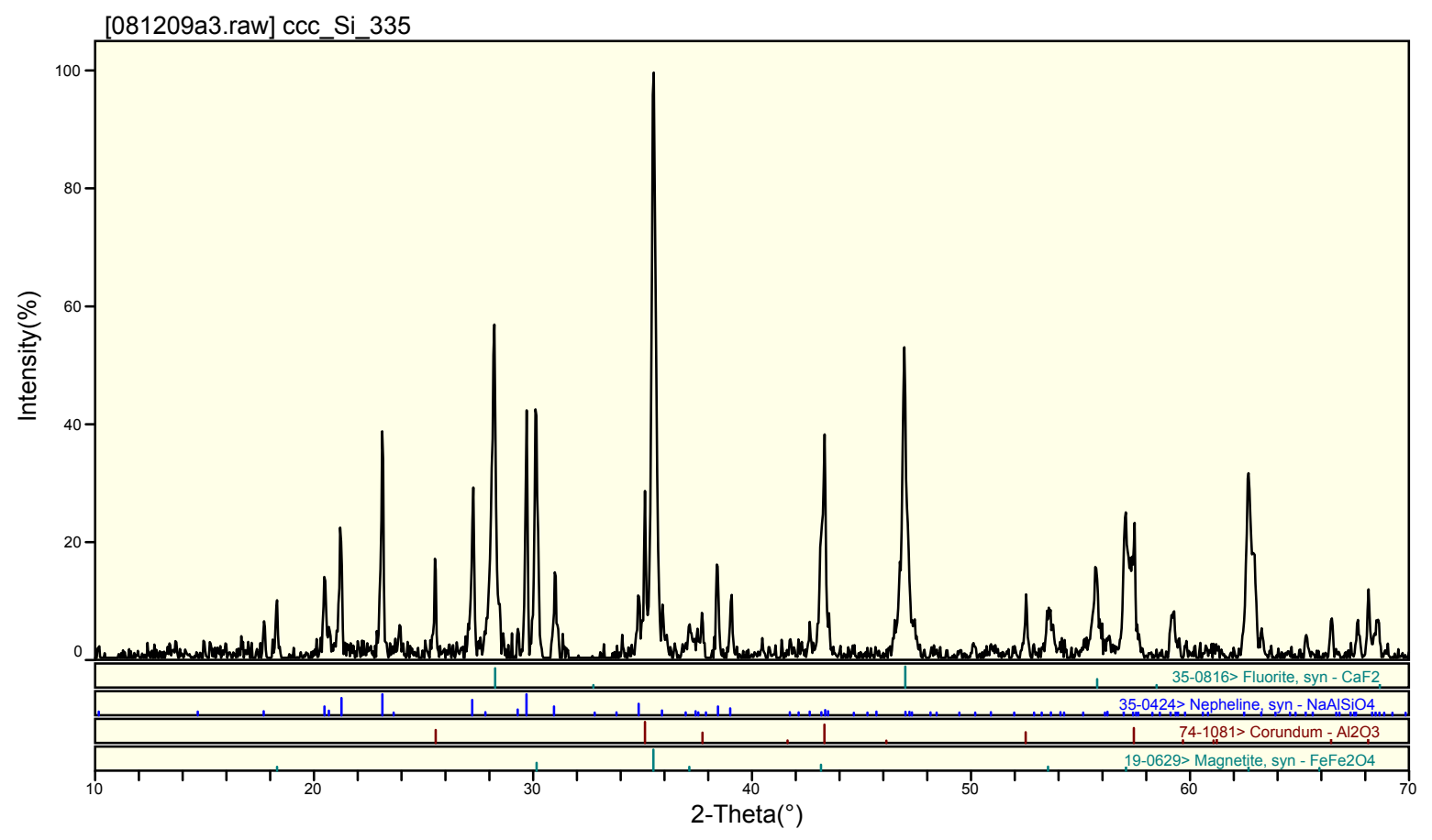

Figure B.43. XRD Spectrum of CCC Treated EM07-Si-335

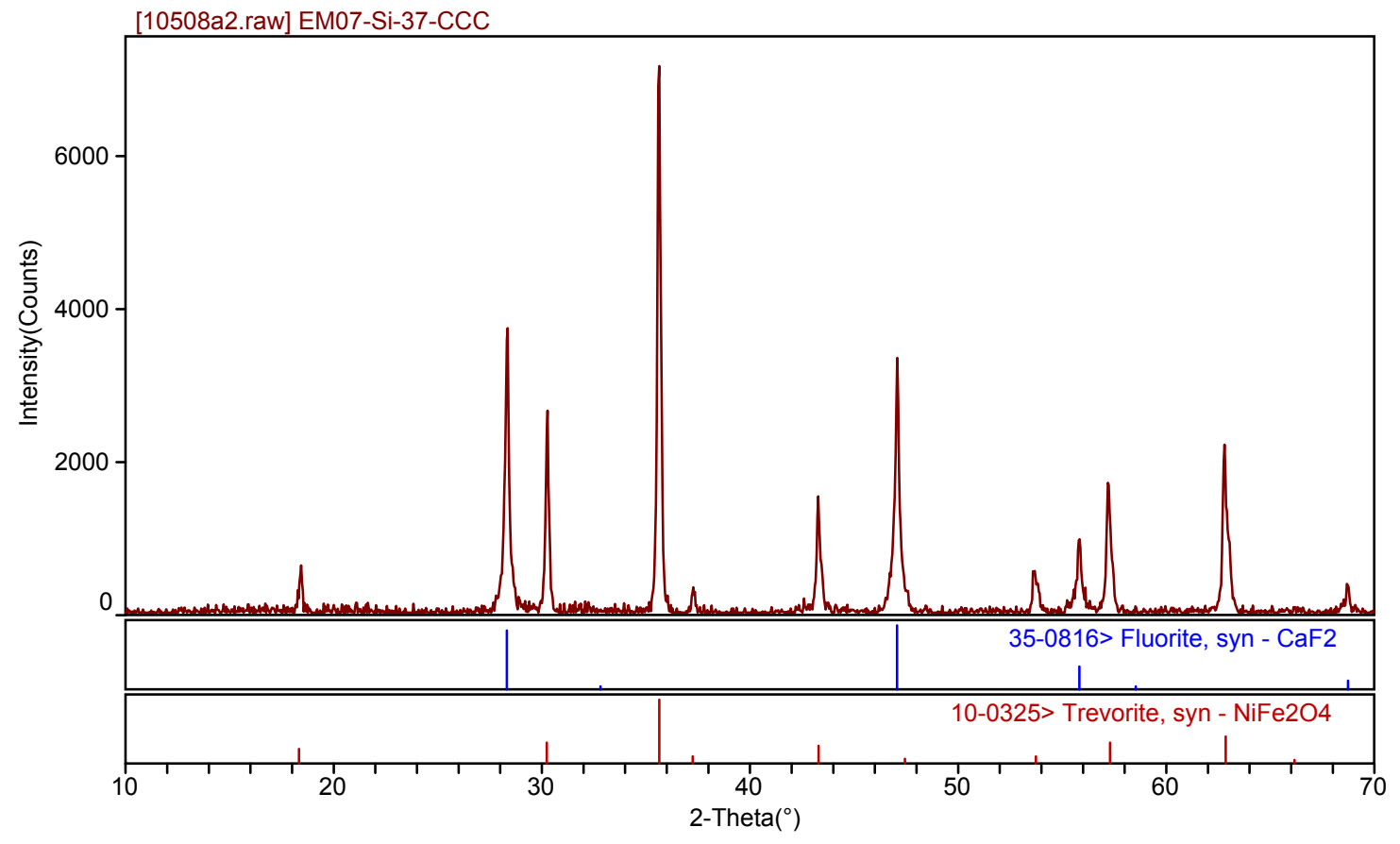

Figure B.44. XRD Spectrum of CCC Treated EM07-Si-37 


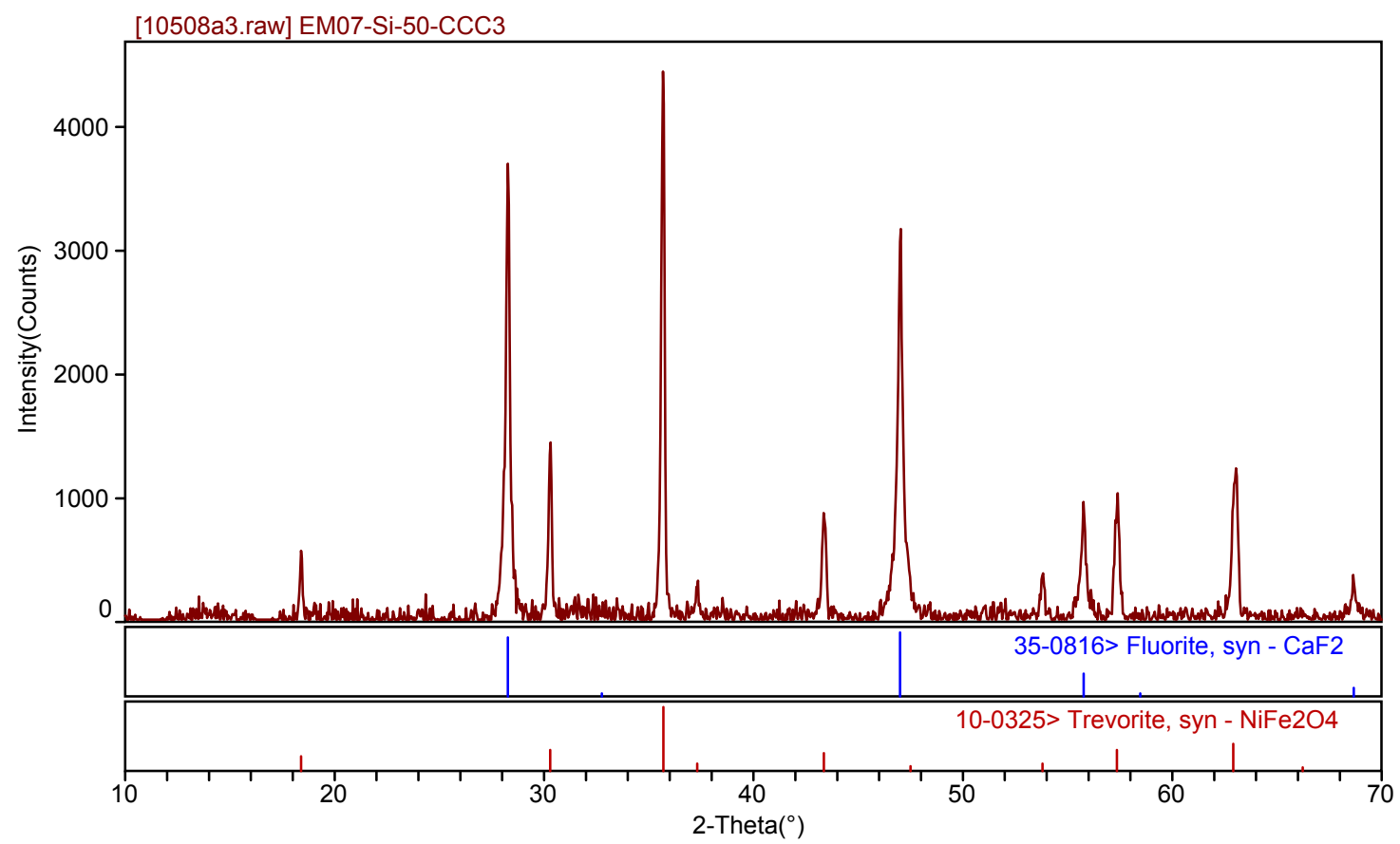

Figure B.45. XRD Spectrum of CCC Treated EM07-Si-50

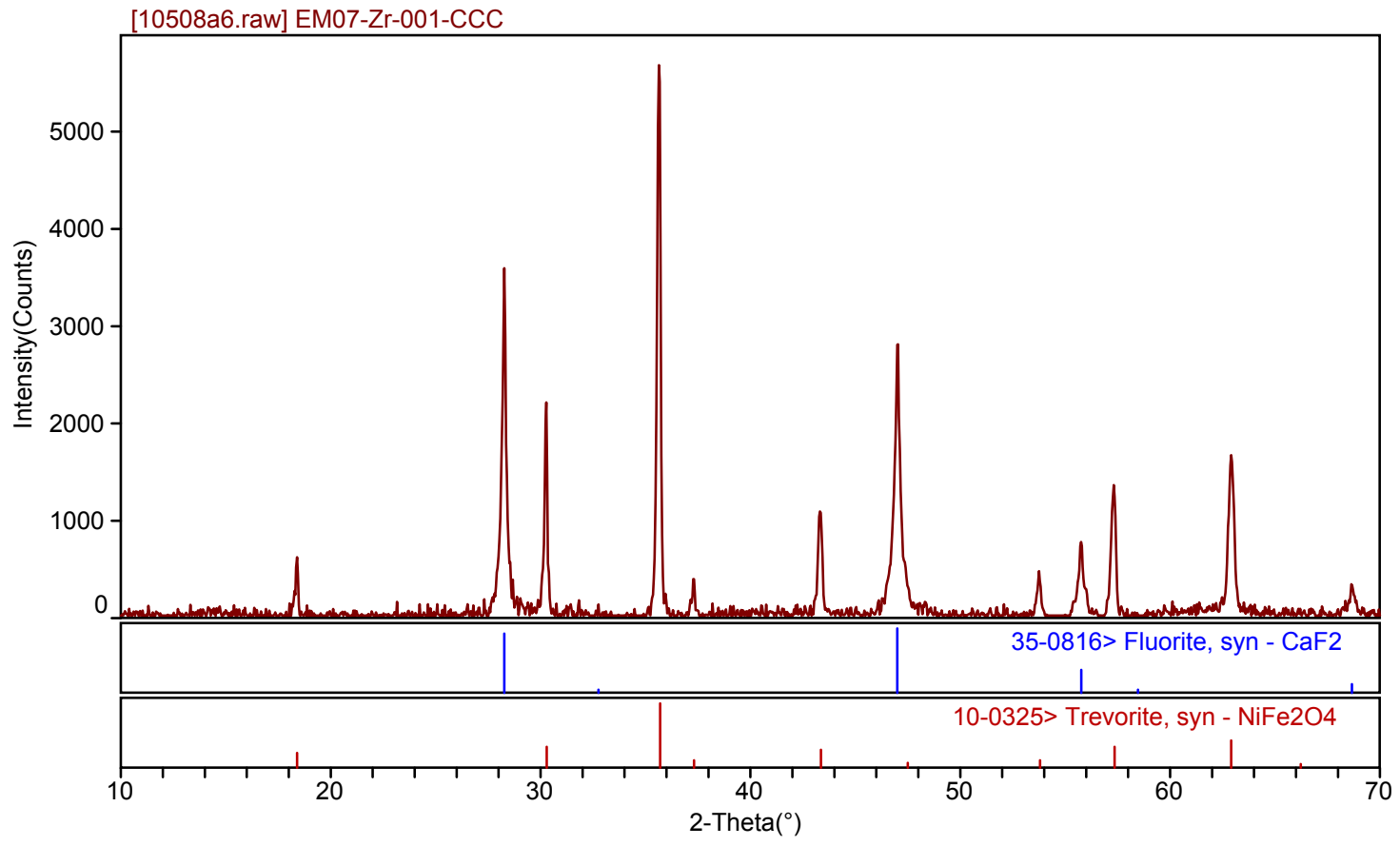

Figure B.46. XRD Spectrum of CCC Treated EM07-Zr-001 


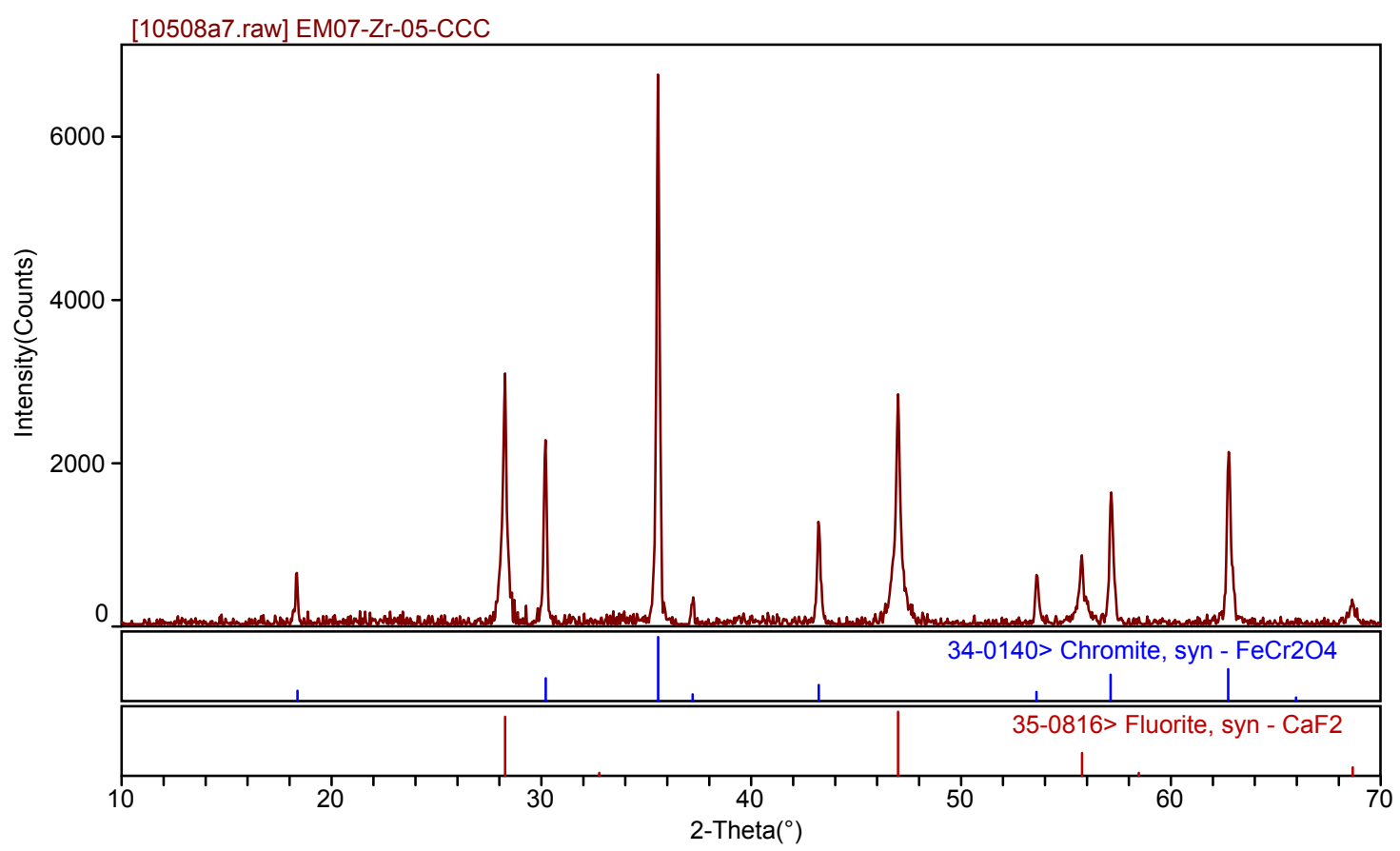

Figure B.47. XRD Spectrum of CCC Treated EM07-Zr-05

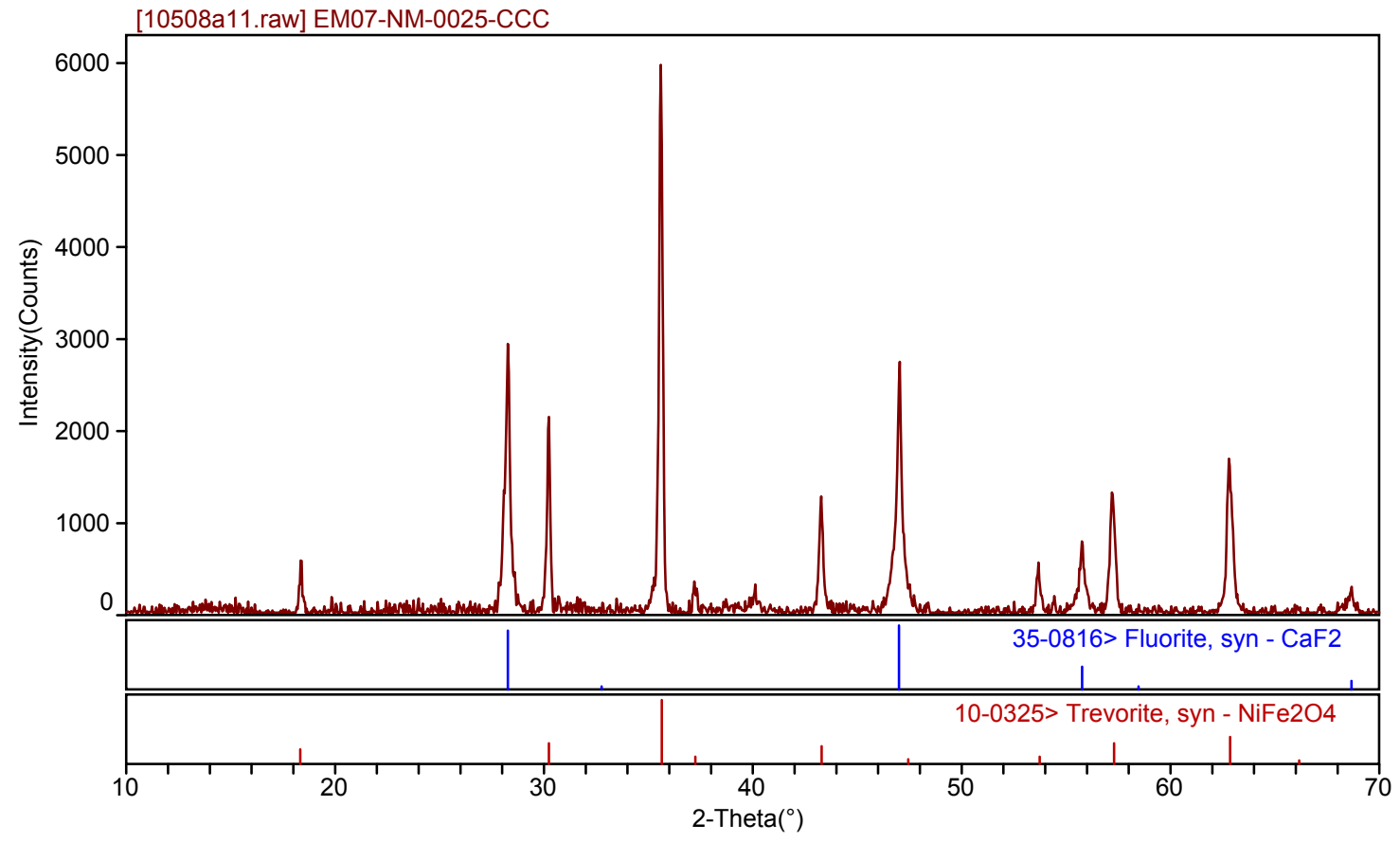

Figure B.48. XRD Spectrum of CCC Treated EM07-NM-0025 
PNNL-17950, Rev 0

* * QUANTITATIVE PHASE ANALYSis :

\section{Title: EM07-Al-225-CCC}

Range: 10 - 70, Inc: 0.04, Time: 4, NPts: 1501. Quantitative results based on all phases used in the refinement. The sum of the weight fractions is normalized to 1.0

Phases
1: Fluorite (CaF2)

Quantitative results scaled to the internal standard. The values in () are calculated excluding the internal standard. Internal std Phase: 1, wgt: 5.00\%, Wgt.Scale=4.01, K*RHO(M)/U(M): 2690.92

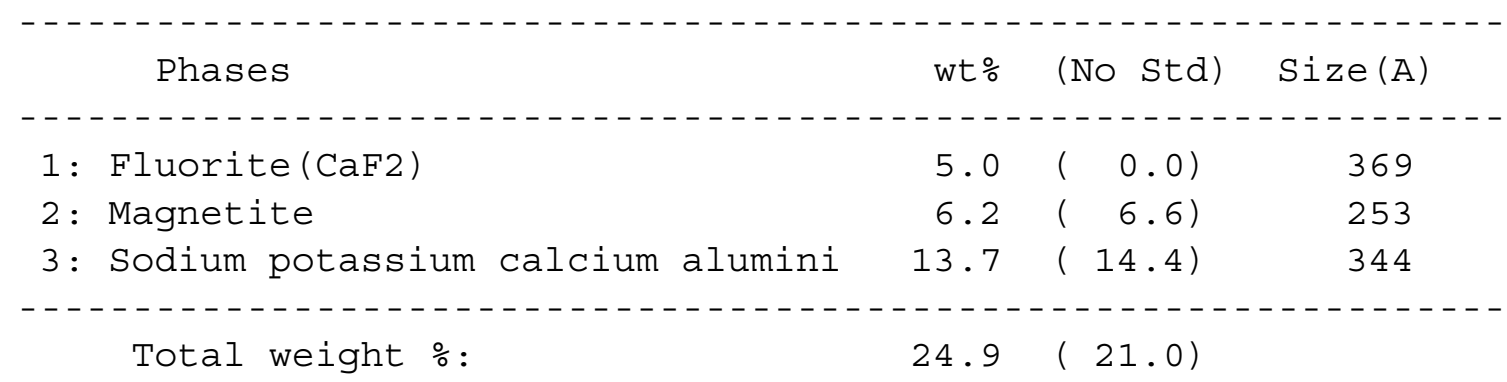

\section{Title: EM07-B-125-CCC}

Range: $10-70$, Inc: 0.04, Time: 4, NPts: 1501.

Quantitative results based on all phases used in the refinement.

The sum of the weight fractions is normalized to 1.0

Phases
1: Fl 20

Quantitative results scaled to the internal standard.

The values in () are calculated excluding the internal standard.

Internal std Phase: 1 , wgt: 5.00\%, Wgt.Scale=10.99, K*RHO(M)/U(M) : 2256.56

Phases
1: Flo




\section{Title: EM07-B-175-CCC}

Range: 10 - 70, Inc: 0.04, Time: 4, NPts: 1501.

Quantitative results based on all phases used in the refinement.

The sum of the weight fractions is normalized to 1.0

\begin{tabular}{|c|c|c|c|}
\hline Phases & wt $\%$ & $(\mathrm{ESD})$ & $\operatorname{Size}(\mathrm{A})$ \\
\hline \multicolumn{4}{|c|}{ - - - - - - - - - - - - - - - - - - - - - - - - - - - - - - - - - - - - - - - - - - - - - - - - - - - - - - - } \\
\hline 1: Fluorite (CaF2) & 57.6 & $(2.0)$ & 352 \\
\hline 2: Magnetite & 42.4 & $(0.9)$ & 233 \\
\hline
\end{tabular}

Quantitative results scaled to the internal standard.

The values in () are calculated excluding the internal standard. Internal Std Phase: 1, wgt: 5.00\%, Wgt.Scale=11.53, K*RHO(M)/U(M) : 2916.04

Phases
wtor

Total weight :

$8.7(3.9)$

\section{Title: EM07-Bi-05-CCC}

Range: 10 - 70, Inc: 0.04, Time: 4, NPts: 1501.

Quantitative results based on all phases used in the refinement.

The sum of the weight fractions is normalized to 1.0

\begin{tabular}{|c|c|c|c|}
\hline Phases & wt $\%$ & $(\mathrm{ESD})$ & $\operatorname{Size}(A)$ \\
\hline \multicolumn{4}{|c|}{ - - - - - - - - - - - - - - - - - - - - - - - - - - - - - - - - - - - - - - - - - - - - - - - - - - - - - - } \\
\hline 1: Fluorite (CaF2) & 55.8 & $(1.6)$ & 238 \\
\hline 2: Chromite & 44.2 & $(0.8)$ & 279 \\
\hline
\end{tabular}

Quantitative results scaled to the internal standard.

The values in () are calculated excluding the internal standard.

Internal Std Phase: 1, wgt: 5.00\%, Wgt.Scale=11.16, K*RHO(M)/U(M) : 4011.71

Phases
wto

Total weight :

$9.0(4.2)$ 


\section{Title: EM07-Ca-07-CCC}

Range: $10-70$, Inc: 0.04, Time: 4, NPts: 1501.

Quantitative results based on all phases used in the refinement.

The sum of the weight fractions is normalized to 1.0

Phases
1: Fluorite (CaF2)

Quantitative results scaled to the internal standard.

The values in () are calculated excluding the internal standard.

Internal Std Phase: 1, wgt: 5.00\%, Wgt.Scale=12.96, K*RHO (M)/U(M) : 3597.38

Phases
1: Fluorite (CaF2)

Title: EM07-Cr-0145-CCC

Range: $10-70$, Inc: 0.04, Time: 4, NPts: 1501.

Quantitative results based on all phases used in the refinement. The sum of the weight fractions is normalized to 1.0

Phases
wt

Quantitative results scaled to the internal standard.

The values in () are calculated excluding the internal standard.

Internal Std Phase: 1, wgt: 5.00\%, Wgt.Scale=11.78, K*RHO (M)/U(M) : 2732.78

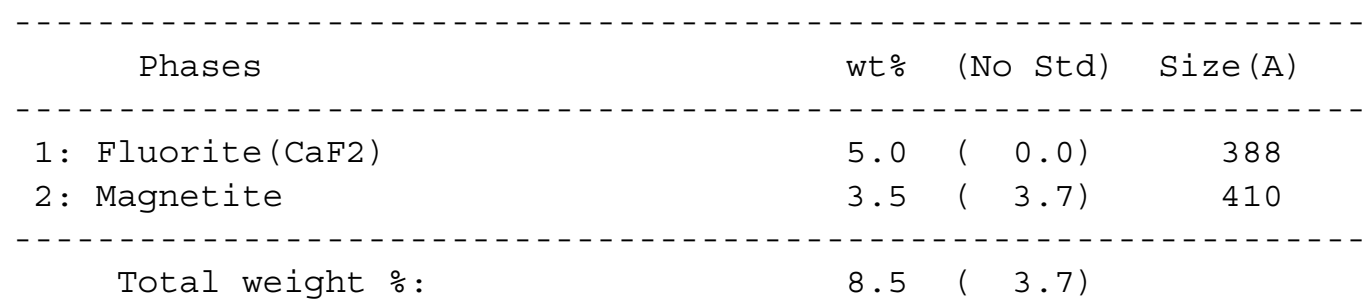




\section{Title: EM07-Fe-05-CCC}

Range: 10 - 70, Inc: 0.04, Time: 4, NPts: 1501.

Quantitative results based on all phases used in the refinement.

The sum of the weight fractions is normalized to 1.0

Phases
wto

Quantitative results scaled to the internal standard.

The values in () are calculated excluding the internal standard.

Internal Std Phase: 1, wgt: 5.00\%, Wgt.Scale=14.04, K*RHO(M)/U(M): 4727.23

Phases
wto

Total weight :

$7.1(2.2)$

\section{Title: EM07-Fe-15-CCC}

Range: 10 - 70, Inc: 0.04, Time: 4, NPts: 1501.

Quantitative results based on all phases used in the refinement.

The sum of the weight fractions is normalized to 1.0

Phases
wto

Quantitative results scaled to the internal standard.

The values in () are calculated excluding the internal standard.

Internal Std Phase: 1, wgt: 5.00\%, Wgt.Scale=8.32, K*RHO(M)/U(M) : 3410.45

\begin{tabular}{|c|c|c|c|}
\hline Phases & wt $\%$ & (No std) & $\operatorname{Size}(A)$ \\
\hline \multicolumn{4}{|c|}{ - - - - - - - - - - - - - - - - - - - - - - - - - - - - - - - - - - - - - - - - - - - - - - - - - - - - - - - - - } \\
\hline 1: Fluorite (CaF2) & 5.0 & $0.0)$ & 277 \\
\hline 2: Chromite & 7.0 & $7.4)$ & 565 \\
\hline
\end{tabular}

Total weight :

$12.0(7.4)$ 


\section{Title: EM07-Fe-20-CCC}

Range: 10 - 70, Inc: 0.04, Time: 4, NPts: 1501.

Quantitative results based on all phases used in the refinement.

The sum of the weight fractions is normalized to 1.0

Phases
wto

Quantitative results scaled to the internal standard.

The values in () are calculated excluding the internal standard.

Internal Std Phase: 1, wgt: 5.00\%, Wgt.Scale=7.77, K*RHO(M) /U (M) : 4059.83

Phases
wto

Total weight :

$12.9(8.3)$

\section{Title: EM07-K-06-CCC}

Range: 10 - 70, Inc: 0.04, Time: 4, NPts: 1501.

Quantitative results based on all phases used in the refinement. The sum of the weight fractions is normalized to 1.0

Phases
1: Fl 20

Quantitative results scaled to the internal standard.

The values in () are calculated excluding the internal standard.

Internal Std Phase:1, wgt:5.00\%,Wgt.Scale=13.11, K*RHO(M)/U(M): 4738.41

\begin{tabular}{|c|c|c|c|}
\hline Phases & wt $\%$ & (No std) & Size (A) \\
\hline 1: Fluorite (CaF2) & 5.0 & $0.0)$ & 209 \\
\hline 2: Magnetite & 2.6 & $(2.8)$ & 398 \\
\hline
\end{tabular}

Total weight :

$7.6(2.8)$


Title: EM07-Li-00-CCC

Range: $10-70$, Inc: 0.04, Time: 4, NPts: 1501.

Quantitative results based on all phases used in the refinement.

The sum of the weight fractions is normalized to 1.0

Phases
wt

Quantitative results scaled to the internal standard.

The values in () are calculated excluding the internal standard.

Internal std Phase: 1, wgt: 5.00\%, Wgt.Scale=11.19, K*RHO (M)/U (M) : 2124.97

Phases
wtor

Total weight \%: $\quad 8.9 \quad(4.1)$

Title: EM07-Li-05-CCC

Range: $10-70$, Inc: 0.04, Time: 4, NPts: 1501.

Quantitative results based on all phases used in the refinement.

The sum of the weight fractions is normalized to 1.0

Phases
1: Fluorite (CaF2)

Quantitative results scaled to the internal standard.

The values in () are calculated excluding the internal standard.

Internal Std Phase: 1, wgt: 5.00\%, Wgt.Scale=11.40, K*RHO (M)/U(M) : 3018.40

Phases
1: Fluorite (CaF2)




\section{Title: EM07-Na-05-CCC}

Range: 10 - 70, Inc: 0.04, Time: 4, NPts: 1501.

Quantitative results based on all phases used in the refinement.

The sum of the weight fractions is normalized to 1.0

Phases
1: Fluorite (CaF2)

Quantitative results scaled to the internal standard.

The values in () are calculated excluding the internal standard. Internal std Phase: 1, wgt: 5.00\%, Wgt.Scale=6.75, K*RHO(M) /U (M) : 5032.67

Phases
1: Fluorite (CaF2)

\section{Title: EM07-NM-0025-CCC}

Range: $10-70$, Inc: 0.04, Time: 4, NPts: 1501.

Quantitative results based on all phases used in the refinement. The sum of the weight fractions is normalized to 1.0

Phases
wto

Quantitative results scaled to the internal standard.

The values in () are calculated excluding the internal standard. Internal std Phase: 1 , wgt: 5.00\%, Wgt.Scale=10.48, K*RHO(M)/U(M) : 4446.20

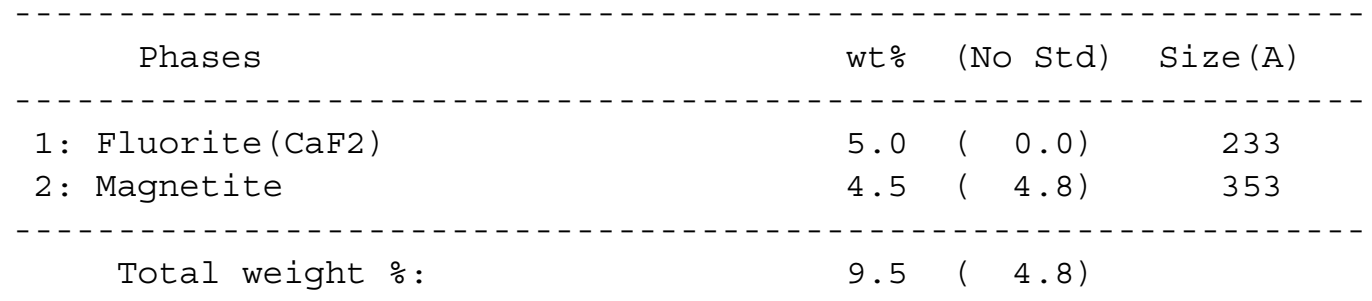


Title: EM07-P-O2-CCC

Range: 9.99249-69.9915, Inc: 0.0297172, Time: 57.6, NPts: 2020. Quantitative results based on all phases used in the refinement.

The sum of the weight fractions is normalized to 1.0

Phases
wto

Quantitative results scaled to the internal standard.

The values in () are calculated excluding the internal standard.

Internal Std Phase: 1, wgt: 5.00\%, Wgt.Scale=10.78, K*RHO (M)/U(M): 639.98

Phases
1: Flor

Total weight \%: $\quad 9.3(4.5)$

Title: EM07-P-03-CCC

Range: $10-70$, Inc: 0.04, Time: 4, NPts: 1501.

Quantitative results based on all phases used in the refinement. The sum of the weight fractions is normalized to 1.0

\begin{tabular}{|c|c|c|c|}
\hline Phases & wt $\%$ & $(\mathrm{ESD})$ & Size (A) \\
\hline----------------------------- & ----- & ------ & ---------- \\
\hline 1: Fluorite (CaF2) & 35.8 & $(1.2)$ & 191 \\
\hline 2: Magnetite & 25.3 & $(0.7)$ & 264 \\
\hline 4: Dialuminium trioxide & 17.8 & $(1.2)$ & 121 \\
\hline 5: Lithium phosphate - \$-gamma & 21.1 & $(1.7)$ & 3 \\
\hline
\end{tabular}

Quantitative results scaled to the internal standard.

The values in () are calculated excluding the internal standard.

Internal Std Phase: 1, wgt: 5.00\%, Wgt.Scale=7.15, K*RHO (M)/U(M): 2184.68

Phases
wto


Title: EM07-Si-30-CCC

Range: $10-70$, Inc: 0.04, Time: 4, NPts: 1501.

Quantitative results based on all phases used in the refinement.

The sum of the weight fractions is normalized to 1.0

Phases
1: Fluorite (CaF2)

Quantitative results scaled to the internal standard.

The values in () are calculated excluding the internal standard.

Internal Std Phase: 1, wgt: 5.00\%, Wgt.Scale=2.50, K*RHO (M)/U(M): 3936.93

Phases
1: Fluorite (CaF2)

Title: $\operatorname{ccc} \mathrm{Si} 335$

Range: $10-70$, Inc: 0.04, Time: 4, NPts: 1501.

Quantitative results based on all phases used in the refinement.

The sum of the weight fractions is normalized to 1.0

Phases
1: Fluorite(CaF2)

Quantitative results scaled to the internal standard.

The values in () are calculated excluding the internal standard.

Internal Std Phase: 1, wgt: 5.00\%, Wgt.Scale=4.96, K*RHO (M)/U (M) : 2337.98

Phases
1: Fluorite(CaF2)


Title: EM07-Si-37-CCC

Range: $10-70$, Inc: 0.04, Time: 4, NPts: 1501.

Quantitative results based on all phases used in the refinement.

The sum of the weight fractions is normalized to 1.0

Phases
1: Fl

Quantitative results scaled to the internal standard.

The values in () are calculated excluding the internal standard.

Internal Std Phase: 1, wgt: 5.00\%, Wgt.Scale=9.43, K*RHO (M)/U(M): 4492.28

Phases
1: Fluorite (CaF2)




\section{Appendix C: Electrical Conductivity Data}

\section{C.1 EM07-BL-1 Electrical Conductivity Data}

Table C.1. Electrical Conductivity Data for EM07-BL-1

\begin{tabular}{|c|c|c|c|}
\hline Temperature, ${ }^{\circ} \mathrm{C}$ & Conductivity, $\mathrm{S} / \mathrm{m}$ & $1 / \mathrm{T}, \mathrm{K}$ & $\operatorname{Ln}(\mathrm{EC}), \mathrm{S} / \mathrm{m}$ \\
\hline 1181 & 45.20 & 0.00069 & 3.81 \\
1181 & 45.89 & 0.00069 & 3.83 \\
1082 & 33.04 & 0.00074 & 3.50 \\
1082 & 33.08 & 0.00074 & 3.50 \\
983 & 21.81 & 0.00080 & 3.08 \\
983 & 21.86 & 0.00080 & 3.08 \\
883 & 12.71 & 0.00086 & 2.54 \\
883 & 12.73 & 0.00086 & 2.54 \\
\hline
\end{tabular}

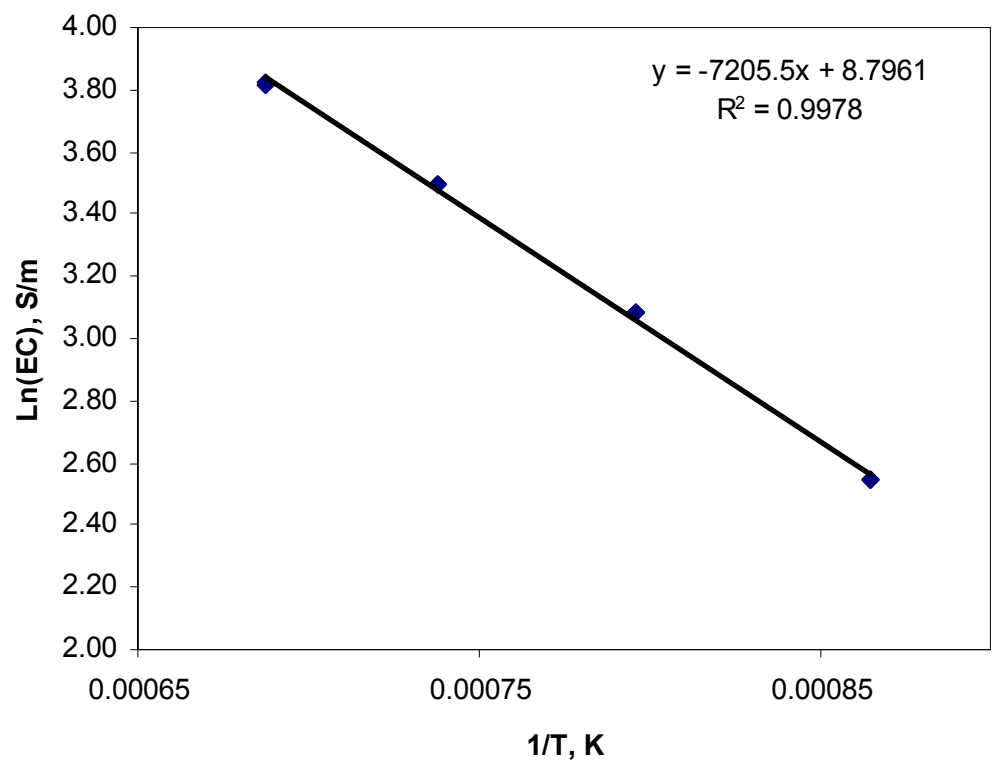

Figure C.1. Electrical Conductivity Trend for EM07-BL-1 over Inverse Temperature 


\section{C.2 EM07-BL-2 Electrical Conductivity Data}

Table C.2. Electrical Conductivity Data for EM07-BL-2

\begin{tabular}{|c|c|c|c|}
\hline Temperature,$^{\circ} \mathrm{C}$ & Conductivity, S/m & $1 / \mathrm{T}, \mathrm{K}$ & $\mathrm{Ln}(\mathrm{EC}), \mathrm{S} / \mathrm{m}$ \\
\hline 1180 & 45.45 & 0.00069 & 3.82 \\
1180 & 45.47 & 0.00069 & 3.82 \\
1081 & 33.44 & 0.00074 & 3.51 \\
1081 & 33.45 & 0.00074 & 3.51 \\
983 & 22.53 & 0.00080 & 3.11 \\
983 & 22.57 & 0.00080 & 3.12 \\
882 & 13.29 & 0.00087 & 2.59 \\
882 & 13.35 & 0.00087 & 2.59 \\
\hline
\end{tabular}

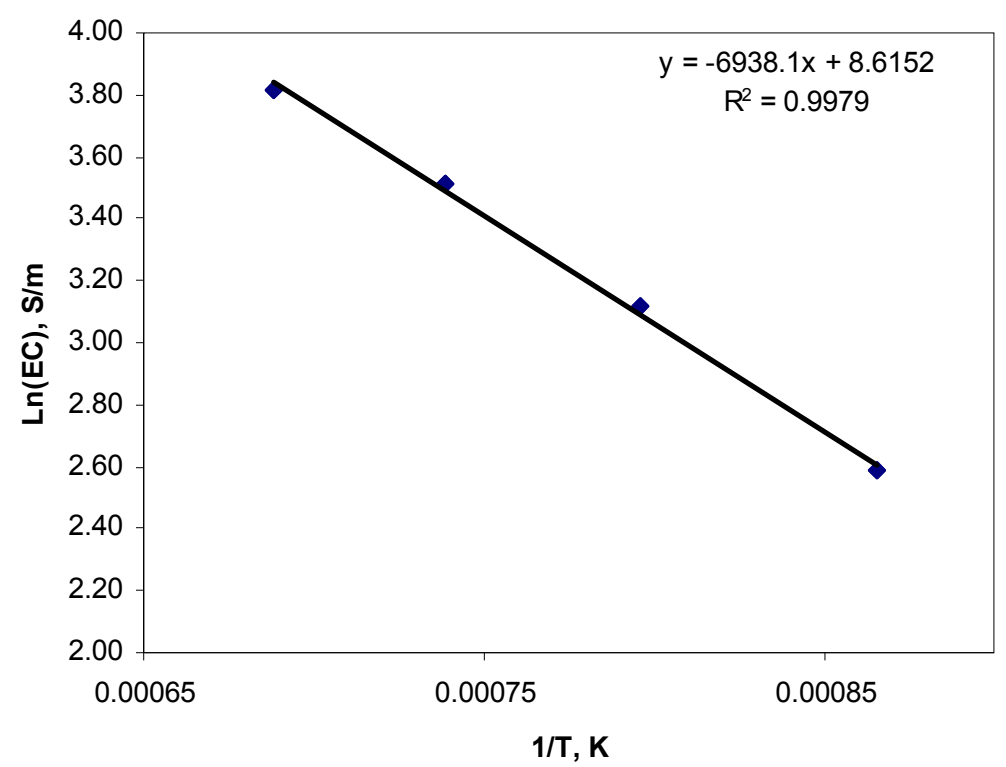

Figure C.2. Electrical Conductivity Trend for EM07-BL-2 over Inverse Temperature 


\section{C.3 EM07-Al-06 Electrical Conductivity Data}

Table C.3. Electrical Conductivity Data for EM07-Al-06

\begin{tabular}{|c|c|c|c|}
\hline Temperature,$^{\circ} \mathrm{C}$ & Conductivity, S/m & $1 / \mathrm{T}, \mathrm{K}$ & $\operatorname{Ln}(\mathrm{EC}), \mathrm{S} / \mathrm{m}$ \\
\hline 1180 & 54.47 & 0.00069 & 4.00 \\
1180 & 54.53 & 0.00069 & 4.00 \\
1081 & 40.66 & 0.00074 & 3.71 \\
1081 & 40.68 & 0.00074 & 3.71 \\
983 & 27.51 & 0.00080 & 3.31 \\
983 & 27.54 & 0.00080 & 3.32 \\
882 & 16.21 & 0.00087 & 2.79 \\
882 & 16.27 & 0.00087 & 2.79 \\
\hline
\end{tabular}

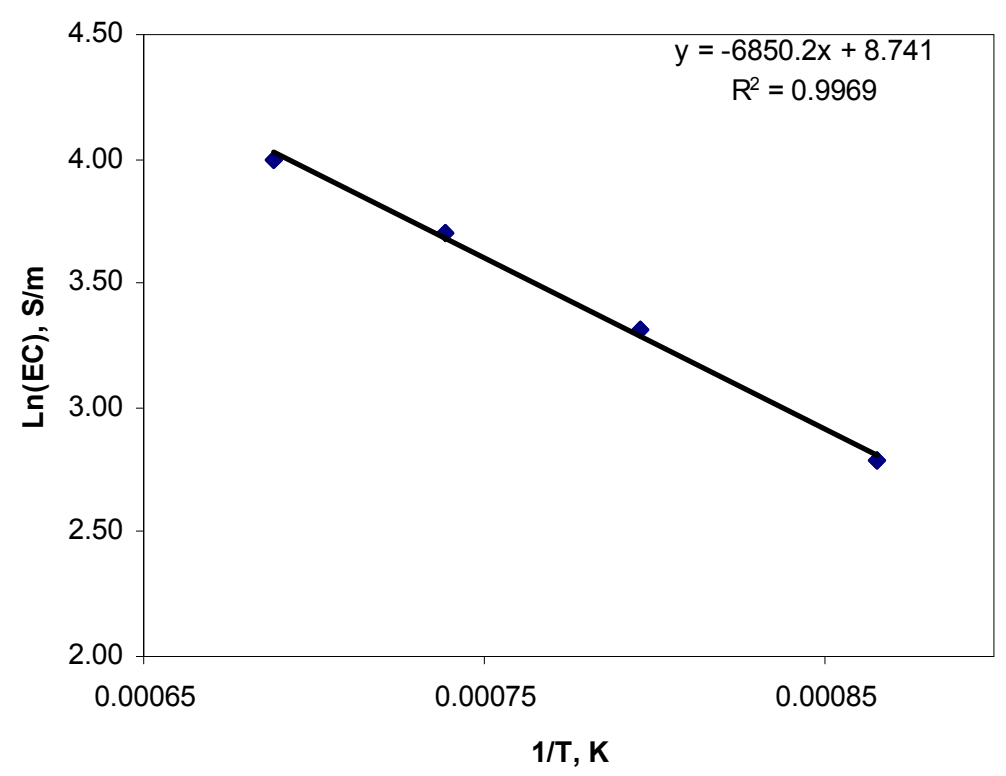

Figure C.3. Electrical Conductivity Trend for EM07-Al-06 over Inverse Temperature 


\section{C.4 EM07-Al-15 Electrical Conductivity Data}

Table C.4. Electrical Conductivity Data for EM07-Al-15

\begin{tabular}{|c|c|c|c|}
\hline Temperature ${ }^{\circ} \mathrm{C}$ & Conductivity, S/m & $1 / \mathrm{T}, \mathrm{K}$ & $\mathrm{Ln}(\mathrm{EC}), \mathrm{S} / \mathrm{m}$ \\
\hline 1180 & 36.92 & 0.00069 & 3.61 \\
1180 & 36.92 & 0.00069 & 3.61 \\
1081 & 25.36 & 0.00074 & 3.23 \\
1081 & 25.80 & 0.00074 & 3.25 \\
988 & 17.74 & 0.00079 & 2.88 \\
988 & 17.82 & 0.00079 & 2.88 \\
882 & 9.91 & 0.00087 & 2.29 \\
882 & 9.93 & 0.00087 & 2.30 \\
\hline
\end{tabular}

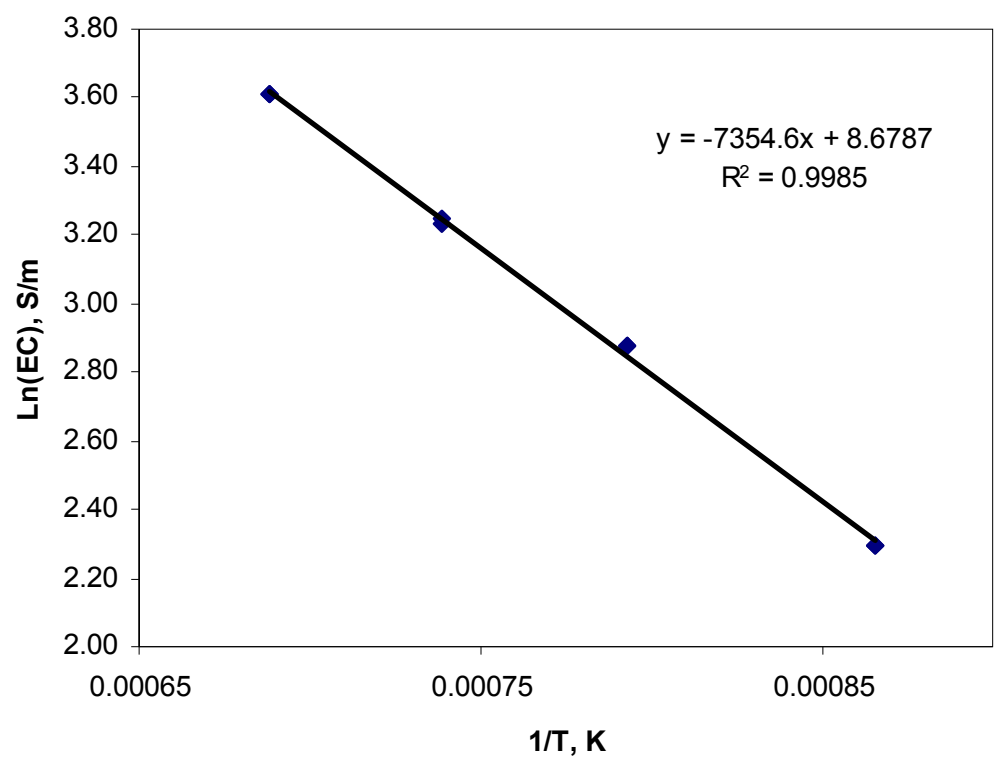

Figure C.4. Electrical Conductivity Trend for EM07-Al-15 over Inverse Temperature 


\section{C.5 EM07-Al-20 Electrical Conductivity Data}

Table C.5. Electrical Conductivity Data for EM07-A1-20

\begin{tabular}{|c|c|c|c|}
\hline Temperature $^{\circ} \mathrm{C}$ & Conductivity, S/m & $1 / \mathrm{T}, \mathrm{K}$ & $\mathrm{Ln}(\mathrm{EC}), \mathrm{S} / \mathrm{m}$ \\
\hline 1184 & 41.95 & 0.00069 & 3.74 \\
1184 & 41.97 & 0.00069 & 3.74 \\
1085 & 30.00 & 0.00074 & 3.40 \\
1085 & 30.06 & 0.00074 & 3.40 \\
986 & 19.85 & 0.00079 & 2.99 \\
986 & 19.86 & 0.00079 & 2.99 \\
887 & 11.56 & 0.00086 & 2.45 \\
887 & 11.59 & 0.00086 & 2.45 \\
\hline
\end{tabular}

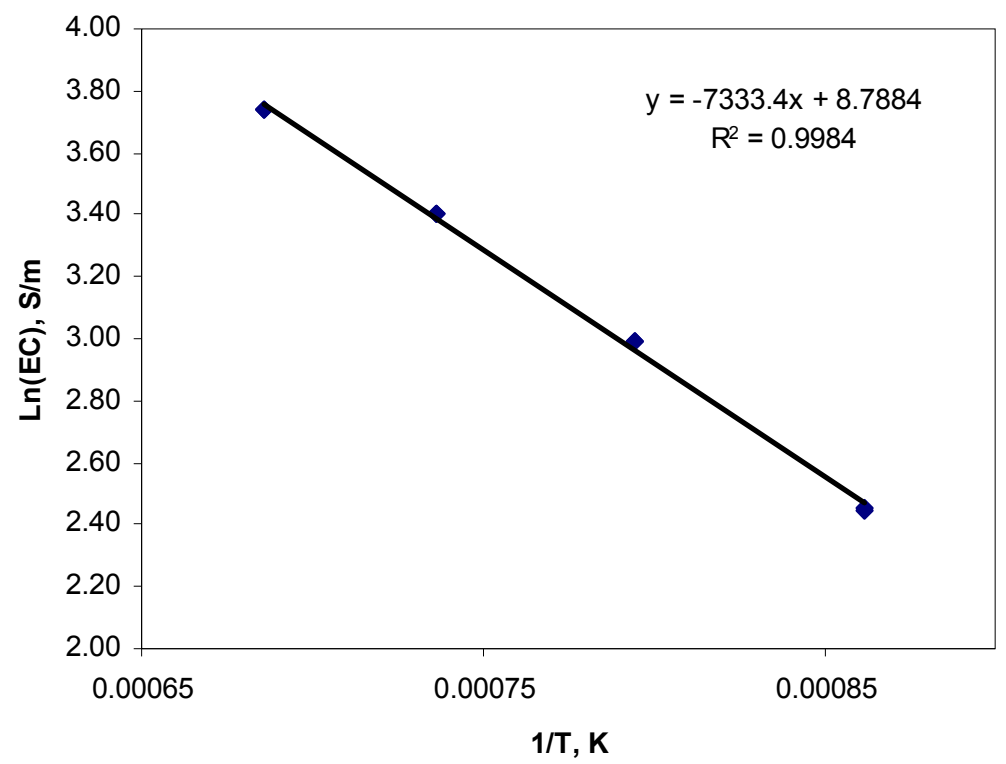

Figure C.5. Electrical Conductivity Trend for EM07-Al-20 over Inverse Temperature 


\section{C.6 EM07-B-05 Electrical Conductivity Data}

Table C.6. Electrical Conductivity Data for EM07-B-05

\begin{tabular}{|c|c|c|c|}
\hline Temperature,$^{\circ} \mathrm{C}$ & Conductivity, S/m & $1 / \mathrm{T}, \mathrm{K}$ & $\mathrm{Ln}(\mathrm{EC}), \mathrm{S} / \mathrm{m}$ \\
\hline 1186 & 43.75 & 0.00069 & 3.78 \\
1186 & 43.51 & 0.00069 & 3.77 \\
1088 & 31.38 & 0.00073 & 3.45 \\
1088 & 31.40 & 0.00073 & 3.45 \\
990 & 21.06 & 0.00079 & 3.05 \\
990 & 21.08 & 0.00079 & 3.05 \\
891 & 12.61 & 0.00086 & 2.53 \\
891 & 12.65 & 0.00086 & 2.54 \\
\hline
\end{tabular}

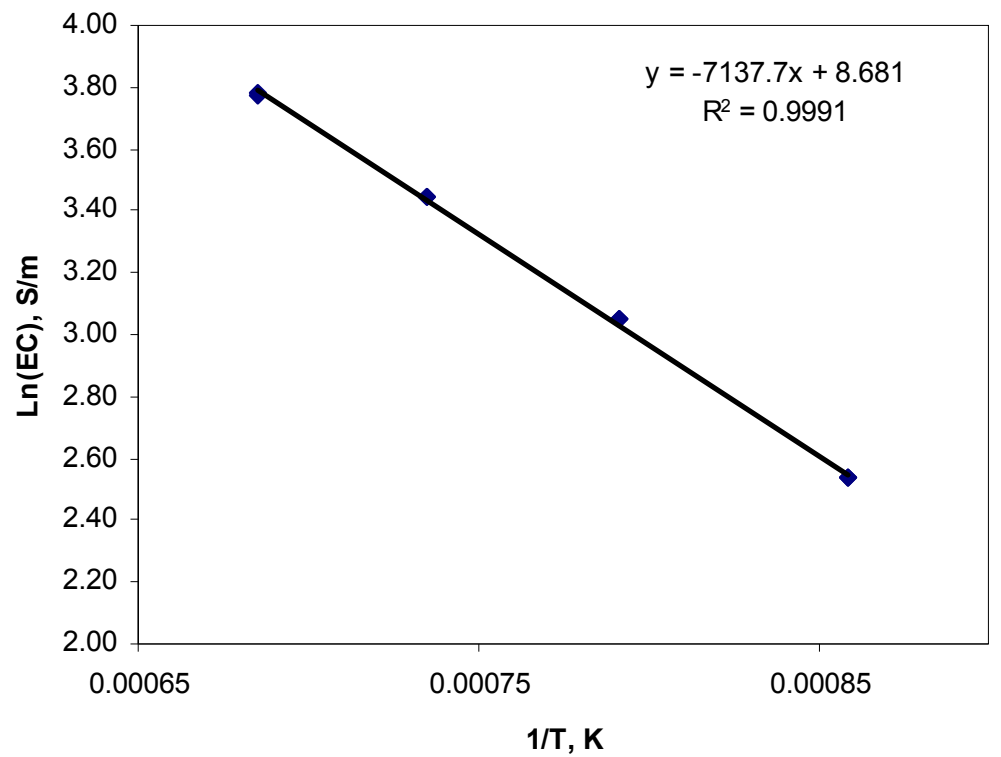

Figure C.6. Electrical Conductivity Trend for EM07-B-05 over Inverse Temperature 


\section{C.7 EM07-B-15 Electrical Conductivity Data}

Table C.7. Electrical Conductivity Data for EM07-B-15

\begin{tabular}{|c|c|c|c|}
\hline Temperature, ${ }^{\circ} \mathrm{C}$ & Conductivity, S/m & $1 / \mathrm{T}, \mathrm{K}$ & $\operatorname{Ln}(\mathrm{EC}), \mathrm{S} / \mathrm{m}$ \\
\hline 1180 & 42.30 & 0.00069 & 3.74 \\
1180 & 42.43 & 0.00069 & 3.75 \\
1081 & 31.02 & 0.00074 & 3.43 \\
1081 & 31.05 & 0.00074 & 3.44 \\
983 & 20.63 & 0.00080 & 3.03 \\
983 & 20.67 & 0.00080 & 3.03 \\
882 & 11.86 & 0.00087 & 2.47 \\
882 & 11.92 & 0.00087 & 2.48 \\
\hline
\end{tabular}

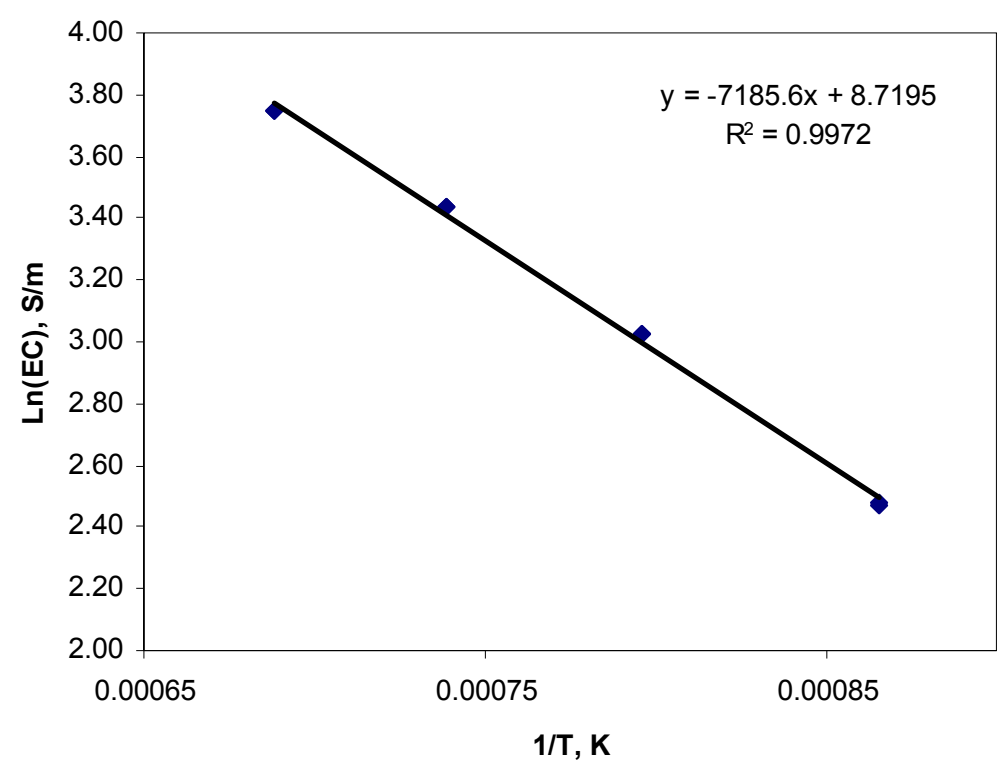

Figure C.8. Electrical Conductivity Trend for EM07-B-15 over Inverse Temperature 


\section{C.8 EM07-B-20 Electrical Conductivity Data}

Table C.8. Electrical Conductivity Data for EM07-B-20

\begin{tabular}{|c|c|c|c|}
\hline Temperature, ${ }^{\circ} \mathrm{C}$ & Conductivity, S/m & $1 / \mathrm{T}, \mathrm{K}$ & $\mathrm{Ln}(\mathrm{EC}), \mathrm{S} / \mathrm{m}$ \\
\hline 1180 & 33.00 & 0.00069 & 3.50 \\
1180 & 32.84 & 0.00069 & 3.49 \\
1081 & 22.51 & 0.00074 & 3.11 \\
1081 & 22.59 & 0.00074 & 3.12 \\
983 & 14.37 & 0.00080 & 2.67 \\
983 & 14.50 & 0.00080 & 2.67 \\
882 & 8.01 & 0.00087 & 2.08 \\
882 & 8.06 & 0.00087 & 2.09 \\
\hline
\end{tabular}

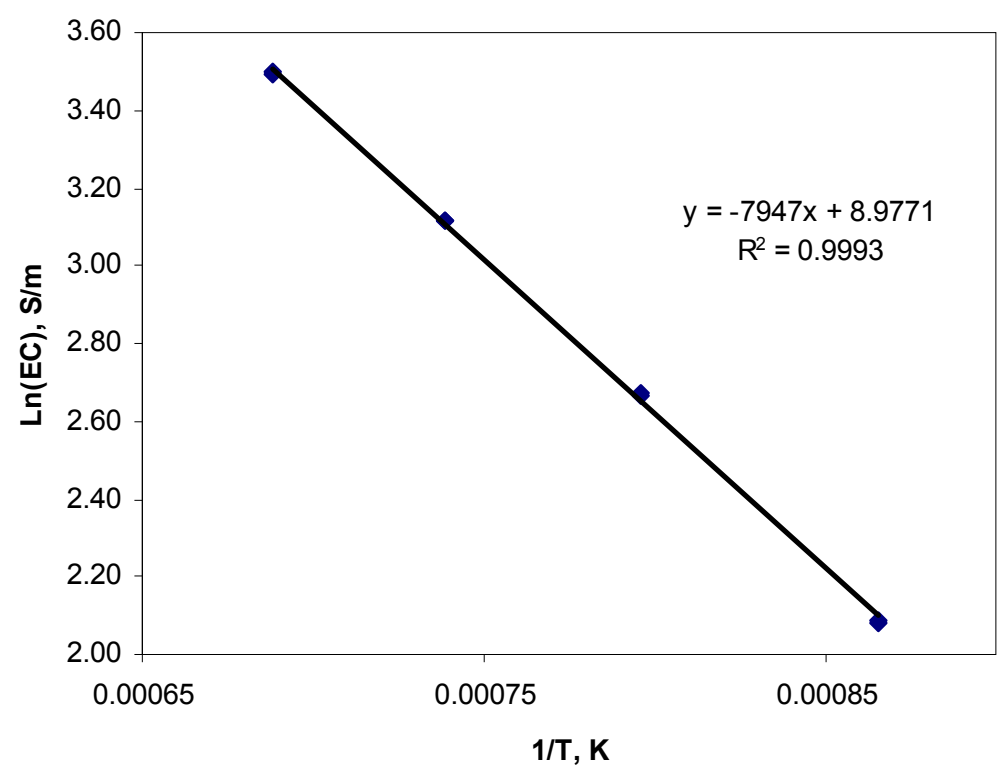

Figure C.8. Electrical Conductivity Trend for EM07-B-20 over Inverse Temperature 


\section{C.9 EM07-Bi-025 Rebatch Electrical Conductivity Data}

Table C.9. Electrical Conductivity Data for EM07-Bi-025

\begin{tabular}{|c|c|c|c|}
\hline Temperature,$^{\circ} \mathrm{C}$ & Conductivity, S/m & $1 / \mathrm{T}, \mathrm{K}$ & $\mathrm{Ln}(\mathrm{EC}), \mathrm{S} / \mathrm{m}$ \\
\hline 1180 & 43.10 & 0.00069 & 3.76 \\
1180 & 43.10 & 0.00069 & 3.76 \\
1081 & 31.77 & 0.00074 & 3.46 \\
1081 & 32.00 & 0.00074 & 3.47 \\
983 & 21.67 & 0.00080 & 3.08 \\
983 & 21.74 & 0.00080 & 3.08 \\
882 & 12.84 & 0.00087 & 2.55 \\
882 & 12.88 & 0.00087 & 2.56 \\
\hline
\end{tabular}

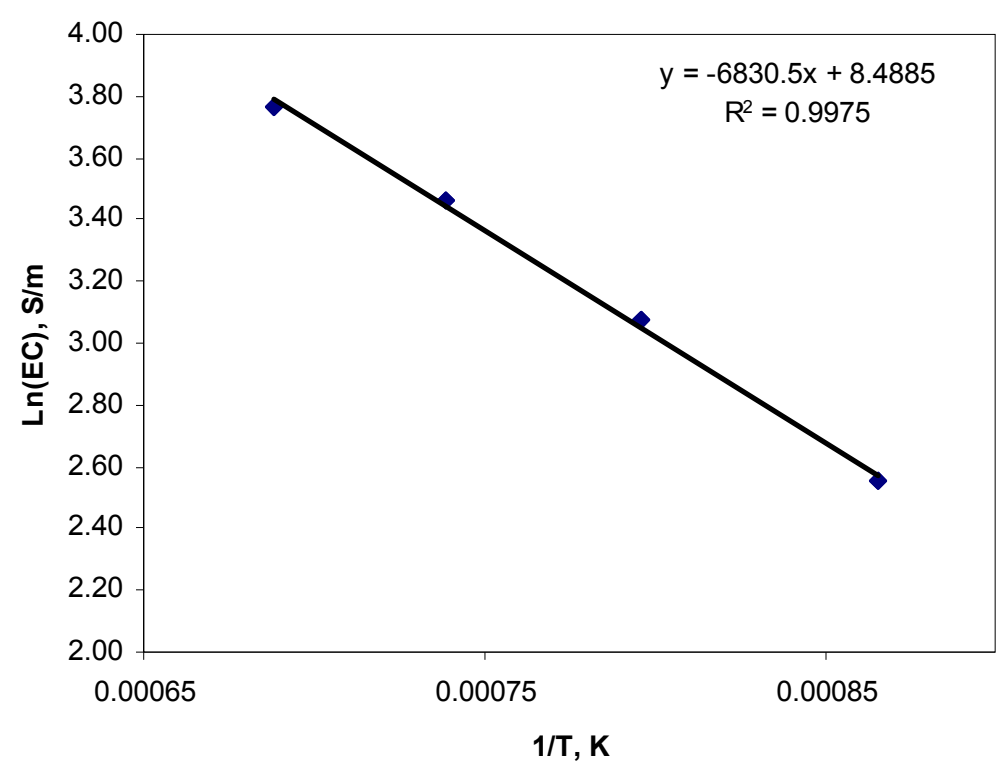

Figure C.9. Electrical Conductivity Trend for EM07-Bi-025 over Inverse Temperature 


\section{C.10 EM07-Bi-05 Rebatch Electrical Conductivity Data}

Table C.10. Electrical Conductivity Data for EM07-Bi-05

\begin{tabular}{|c|c|c|c|}
\hline Temperature ${ }^{\circ} \mathrm{C}$ & Conductivity, S/m & $1 / \mathrm{T}, \mathrm{K}$ & $\mathrm{Ln}(\mathrm{EC}), \mathrm{S} / \mathrm{m}$ \\
\hline 1180 & 44.18 & 0.00069 & 3.79 \\
1180 & 44.26 & 0.00069 & 3.79 \\
1081 & 32.63 & 0.00074 & 3.49 \\
1081 & 32.64 & 0.00074 & 3.49 \\
983 & 22.02 & 0.00080 & 3.09 \\
983 & 22.05 & 0.00080 & 3.09 \\
882 & 12.99 & 0.00087 & 2.56 \\
882 & 13.04 & 0.00087 & 2.57 \\
\hline
\end{tabular}

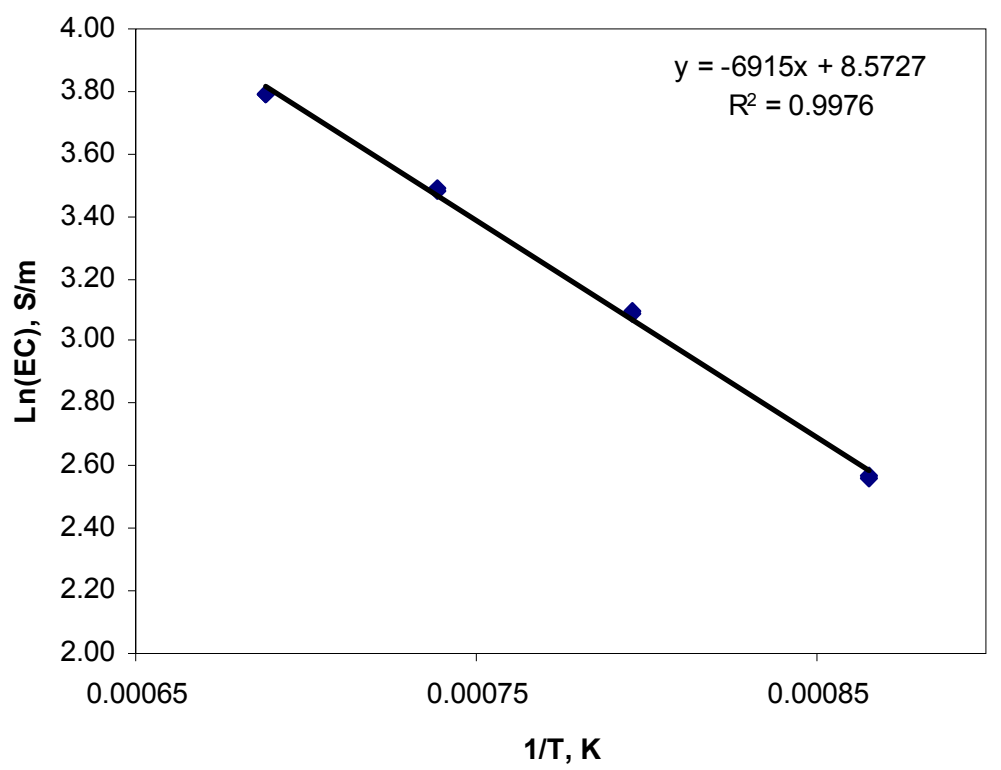

Figure C.10. Electrical Conductivity Trend for EM07-Bi-05 over Inverse Temperature 


\section{C.11 EM07-Ca-035 Electrical Conductivity Data}

Table C.11. Electrical Conductivity Data for EM07-Ca-035

\begin{tabular}{|c|c|c|c|}
\hline Temperature ${ }^{\circ} \mathrm{C}$ & Conductivity, S/m & $1 / \mathrm{T}, \mathrm{K}$ & $\mathrm{Ln}(\mathrm{EC}), \mathrm{S} / \mathrm{m}$ \\
\hline 1180 & 41.55 & 0.00069 & 3.73 \\
1180 & 41.56 & 0.00069 & 3.73 \\
1081 & 29.65 & 0.00074 & 3.39 \\
1081 & 29.66 & 0.00074 & 3.39 \\
983 & 19.17 & 0.00080 & 2.95 \\
983 & 19.20 & 0.00080 & 2.95 \\
882 & 10.69 & 0.00087 & 2.37 \\
882 & 10.74 & 0.00087 & 2.37 \\
\hline
\end{tabular}

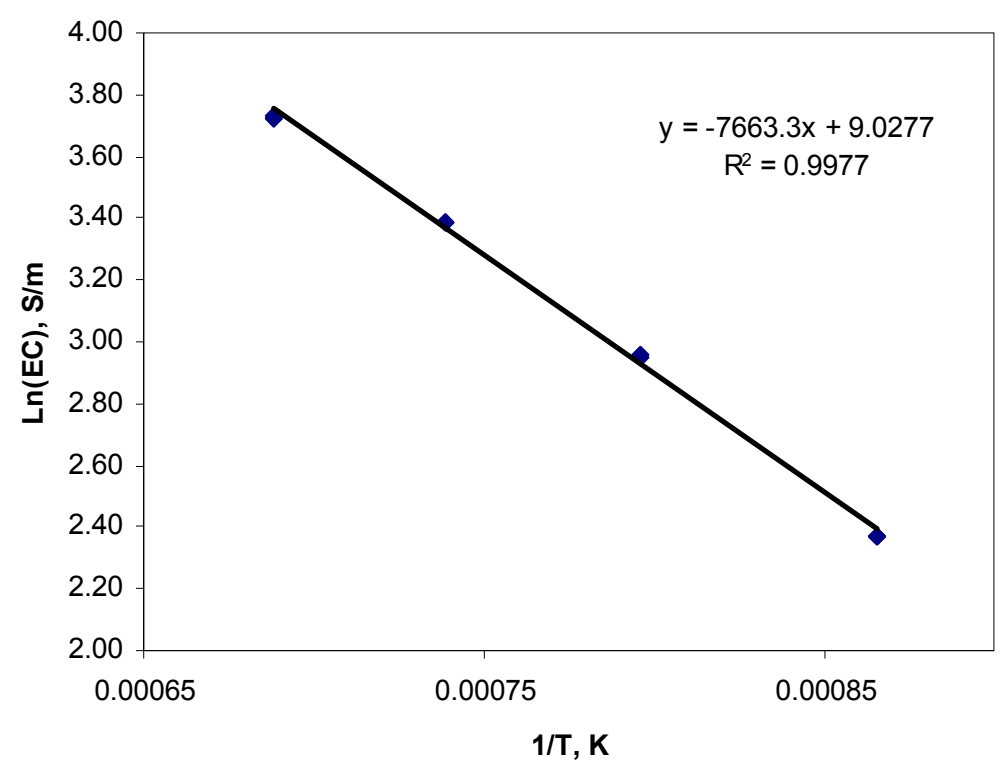

Figure C.11. Electrical Conductivity Trend for EM07-Ca-035 over Inverse Temperature 


\section{C.12 EM07-Ca-07 Electrical Conductivity Data}

Table C.12. Electrical Conductivity Data for EM07-Ca-07

\begin{tabular}{|c|c|c|c|}
\hline Temperature,$^{\circ} \mathrm{C}$ & Conductivity, S/m & $1 / \mathrm{T}, \mathrm{K}$ & $\mathrm{Ln}(\mathrm{EC}), \mathrm{S} / \mathrm{m}$ \\
\hline 1184 & 37.66 & 0.00069 & 3.63 \\
1184 & 37.69 & 0.00069 & 3.63 \\
1085 & 25.49 & 0.00074 & 3.24 \\
1085 & 25.51 & 0.00074 & 3.24 \\
987 & 15.59 & 0.00079 & 2.75 \\
987 & 15.62 & 0.00079 & 2.75 \\
885 & 7.93 & 0.00086 & 2.07 \\
885 & 7.98 & 0.00086 & 2.08 \\
\hline
\end{tabular}

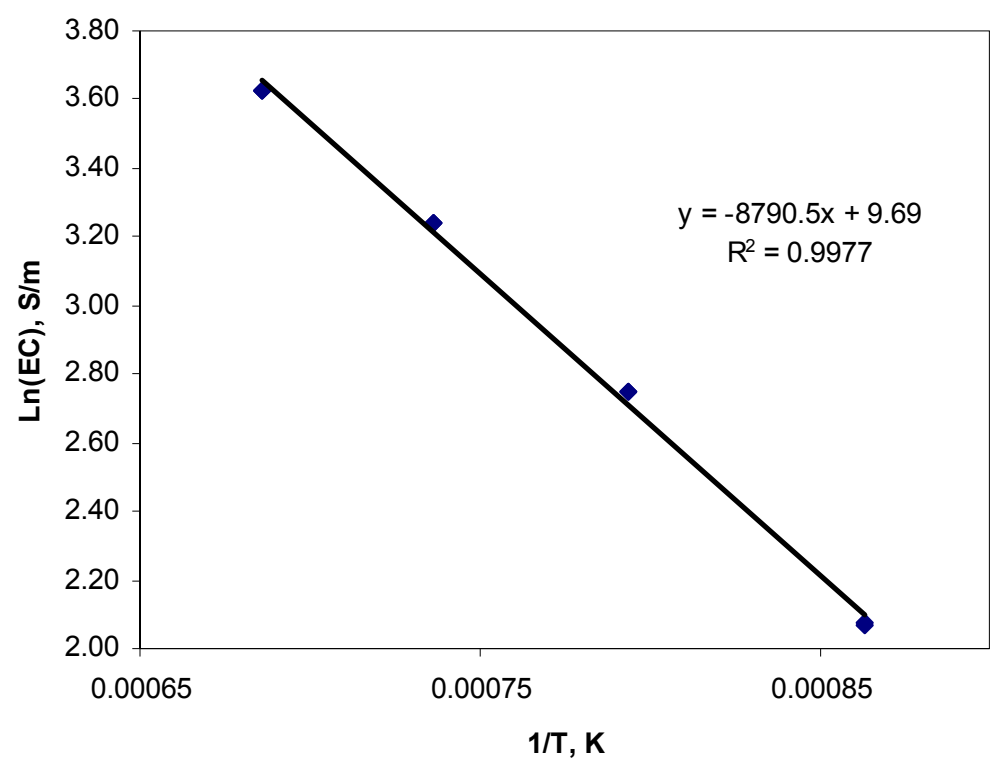

Figure C.12. Electrical Conductivity Trend for EM07-Ca-07 over Inverse Temperature 


\section{C.13 EM07-Cr-001 Electrical Conductivity Data}

Table C.13. Electrical Conductivity Data for EM07-Cr-001

\begin{tabular}{|c|c|c|c|}
\hline Temperature,$^{\circ} \mathrm{C}$ & Conductivity, S/m & $1 / \mathrm{T}, \mathrm{K}$ & $\mathrm{Ln}(\mathrm{EC}), \mathrm{S} / \mathrm{m}$ \\
\hline 1182 & 48.02 & 0.00069 & 3.87 \\
1182 & 47.99 & 0.00069 & 3.87 \\
1073 & 33.63 & 0.00074 & 3.52 \\
1073 & 33.70 & 0.00074 & 3.52 \\
974 & 22.07 & 0.00080 & 3.09 \\
974 & 22.11 & 0.00080 & 3.10 \\
871 & 12.78 & 0.00087 & 2.55 \\
871 & 12.80 & 0.00087 & 2.55 \\
\hline
\end{tabular}

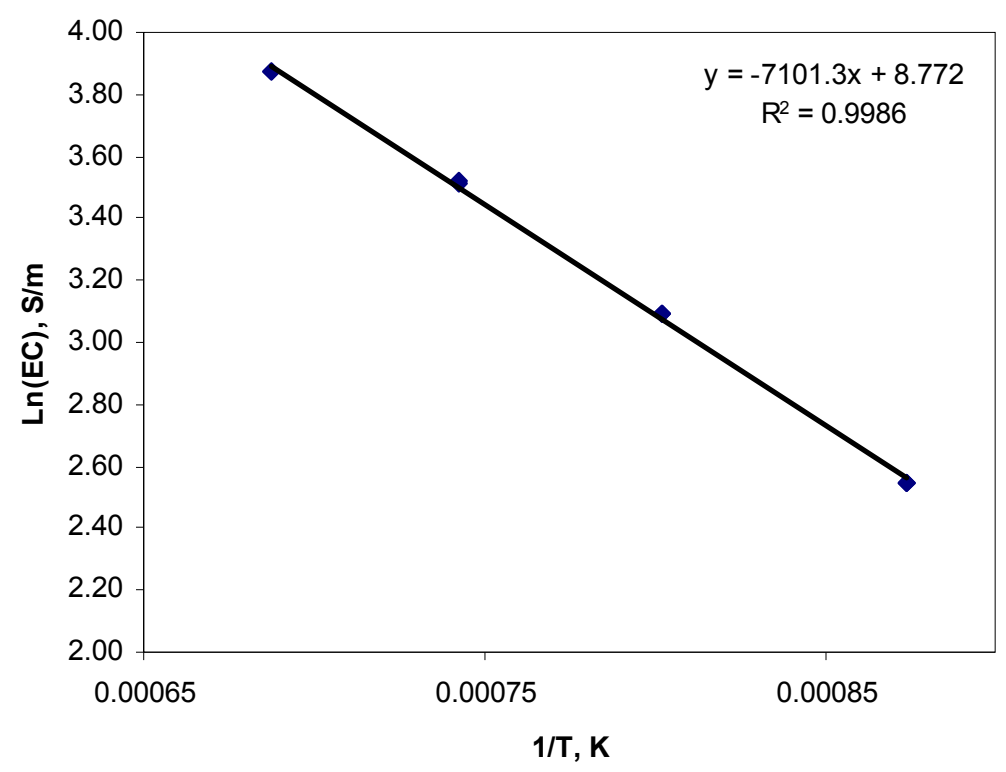

Figure C.13. Electrical Conductivity Trend for EM07-Cr-001 over Inverse Temperature 


\section{C.14 EM07-Cr-012 Electrical Conductivity Data}

Table C.14. Electrical Conductivity Data for EM07-Cr-012

\begin{tabular}{|c|c|c|c|}
\hline Temperature,$^{\circ} \mathrm{C}$ & Conductivity, S/m & $1 / \mathrm{T}, \mathrm{K}$ & $\operatorname{Ln}(\mathrm{EC}), \mathrm{S} / \mathrm{m}$ \\
\hline 1180 & 42.03 & 0.00069 & 3.74 \\
1180 & 42.03 & 0.00069 & 3.74 \\
1081 & 30.80 & 0.00074 & 3.43 \\
1081 & 30.81 & 0.00074 & 3.43 \\
983 & 20.74 & 0.00080 & 3.03 \\
983 & 20.77 & 0.00080 & 3.03 \\
882 & 12.24 & 0.00087 & 2.50 \\
882 & 12.28 & 0.00087 & 2.51 \\
\hline
\end{tabular}

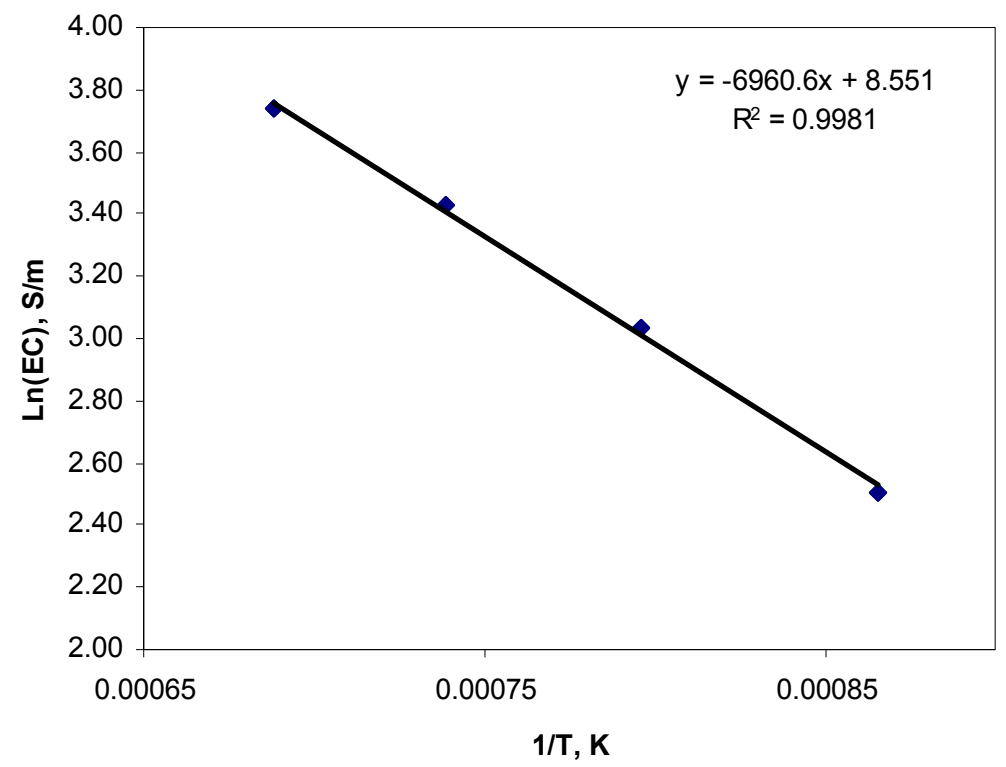

Figure C.14. Electrical Conductivity Trend for EM07-Cr-012 over Inverse Temperature 


\section{C.15 EM07-Cr-02 Electrical Conductivity Data}

Table C.15. Electrical Conductivity Data for EM07-Cr-02

\begin{tabular}{|c|c|c|c|}
\hline Temperature $^{\circ} \mathrm{C}$ & Conductivity, S/m & $1 / \mathrm{T}, \mathrm{K}$ & $\mathrm{Ln}(\mathrm{EC}), \mathrm{S} / \mathrm{m}$ \\
\hline 1183 & 41.87 & 0.00069 & 3.73 \\
1183 & 42.22 & 0.00069 & 3.74 \\
1083 & 30.60 & 0.00074 & 3.42 \\
1083 & 30.65 & 0.00074 & 3.42 \\
983 & 20.36 & 0.00080 & 3.01 \\
983 & 20.38 & 0.00080 & 3.01 \\
885 & 11.92 & 0.00086 & 2.48 \\
885 & 11.94 & 0.00086 & 2.48 \\
\hline
\end{tabular}

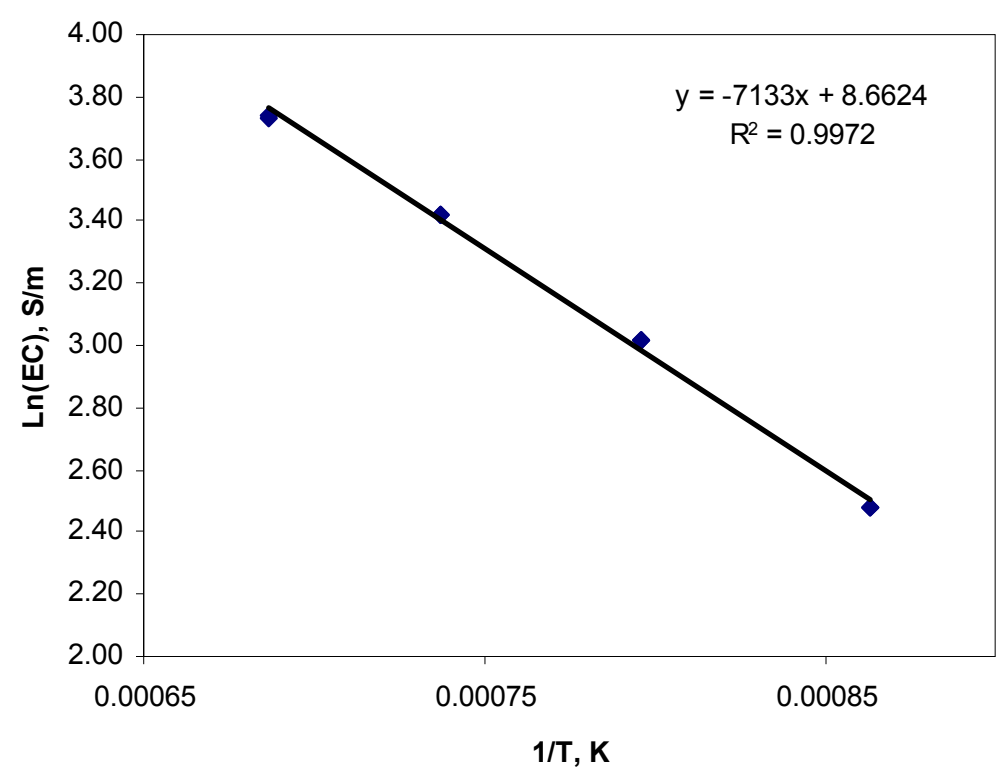

Figure C.15. Electrical Conductivity Trend for EM07-Cr-02 over Inverse Temperature 


\section{C.16 EM07-F-02 Electrical Conductivity Data}

Table C.16. Electrical Conductivity Data for EM07-F-02

\begin{tabular}{|c|c|c|c|}
\hline Temperature $^{\circ} \mathrm{C}$ & Conductivity, S/m & $1 / \mathrm{T}, \mathrm{K}$ & $\mathrm{Ln}(\mathrm{EC}), \mathrm{S} / \mathrm{m}$ \\
\hline 1180 & 57.00 & 0.00069 & 4.04 \\
1180 & 56.99 & 0.00069 & 4.04 \\
1081 & 43.57 & 0.00074 & 3.77 \\
1081 & 43.62 & 0.00074 & 3.78 \\
983 & 30.71 & 0.00080 & 3.42 \\
983 & 30.77 & 0.00080 & 3.43 \\
882 & 19.18 & 0.00087 & 2.95 \\
882 & 19.24 & 0.00087 & 2.96 \\
\hline
\end{tabular}

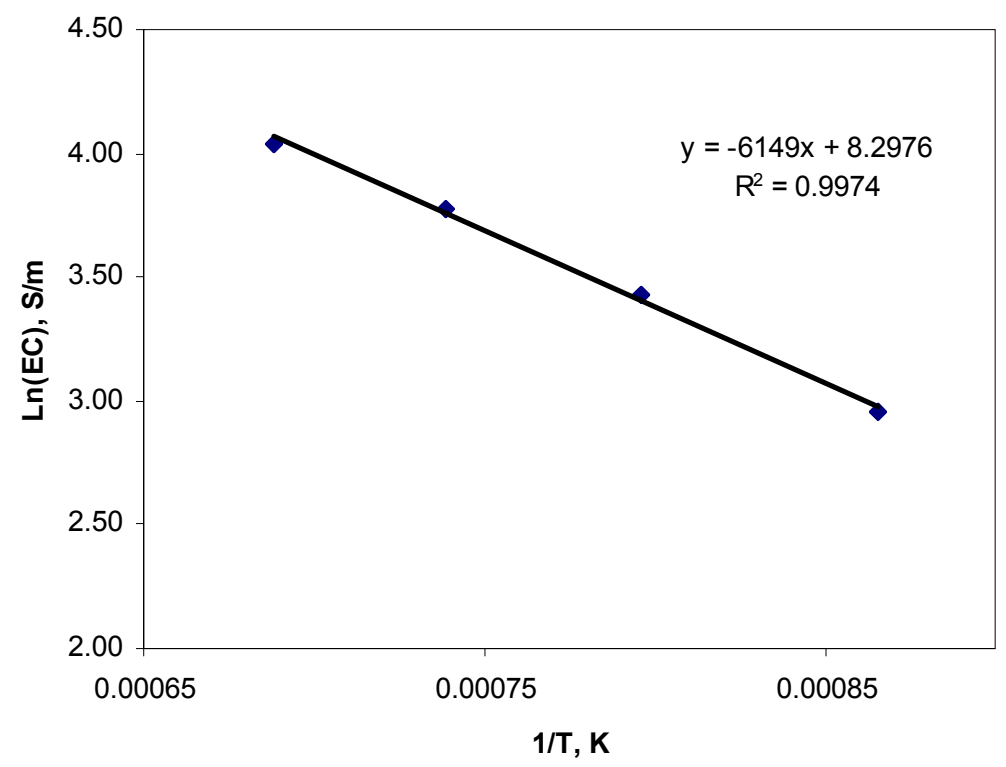

Figure C.16. Electrical Conductivity Trend for EM07-F-02 over Inverse Temperature 


\section{C.17 EM07-Fe-05 Electrical Conductivity Data}

Table C.17. Electrical Conductivity Data for EM07-Fe-05

\begin{tabular}{|c|c|c|c|}
\hline Temperature $^{\circ} \mathrm{C}$ & Conductivity, S/m & $1 / \mathrm{T}, \mathrm{K}$ & $\mathrm{Ln}(\mathrm{EC}), \mathrm{S} / \mathrm{m}$ \\
\hline 1180 & 40.97 & 0.00069 & 3.71 \\
1180 & 40.93 & 0.00069 & 3.71 \\
1081 & 30.15 & 0.00074 & 3.41 \\
1081 & 30.16 & 0.00074 & 3.41 \\
983 & 20.40 & 0.00080 & 3.02 \\
983 & 20.40 & 0.00080 & 3.02 \\
882 & 11.73 & 0.00087 & 2.46 \\
882 & 11.74 & 0.00087 & 2.46 \\
\hline
\end{tabular}

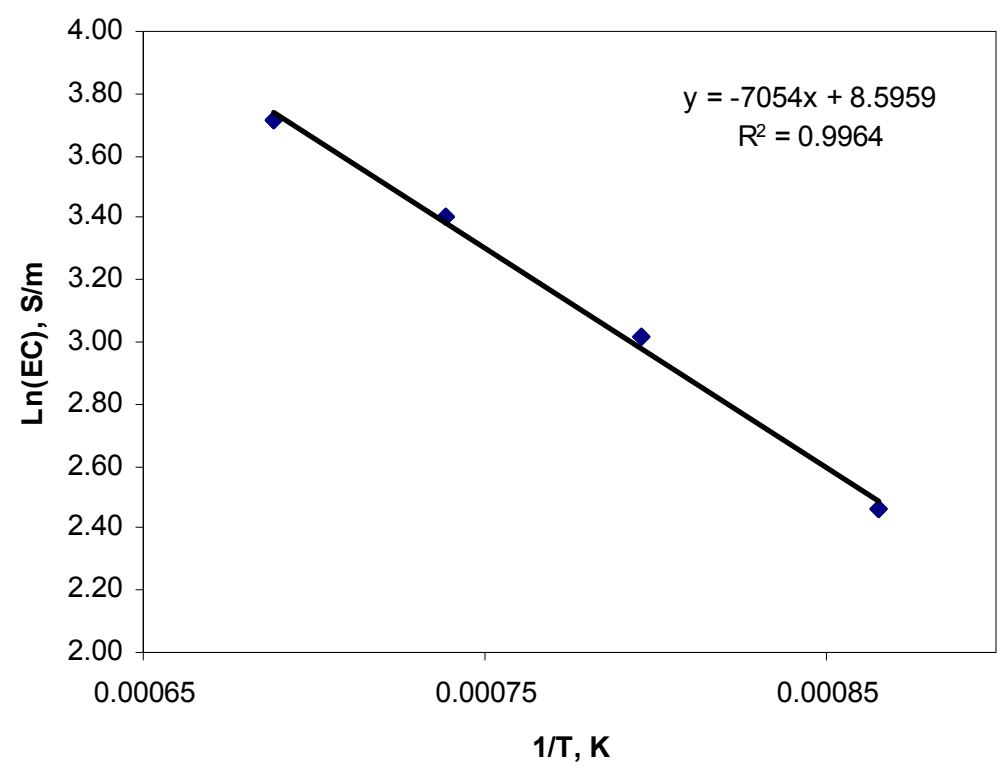

Figure C.17. Electrical Conductivity Trend for EM07-Fe-05 over Inverse Temperature 


\section{C.18 EM07-Fe-15 Electrical Conductivity Data}

Table C.18. Electrical Conductivity Data for EM07-Fe-15

\begin{tabular}{|c|c|c|c|}
\hline Temperature $^{\circ} \mathrm{C}$ & Conductivity, S/m & $1 / \mathrm{T}, \mathrm{K}$ & $\mathrm{Ln}(\mathrm{EC}), \mathrm{S} / \mathrm{m}$ \\
\hline 1180 & 38.89 & 0.00069 & 3.66 \\
1180 & 38.78 & 0.00069 & 3.66 \\
1081 & 28.52 & 0.00074 & 3.35 \\
1081 & 28.53 & 0.00074 & 3.35 \\
983 & 19.36 & 0.00080 & 2.96 \\
983 & 19.40 & 0.00080 & 2.97 \\
882 & 11.51 & 0.00087 & 2.44 \\
882 & 11.30 & 0.00087 & 2.43 \\
\hline
\end{tabular}

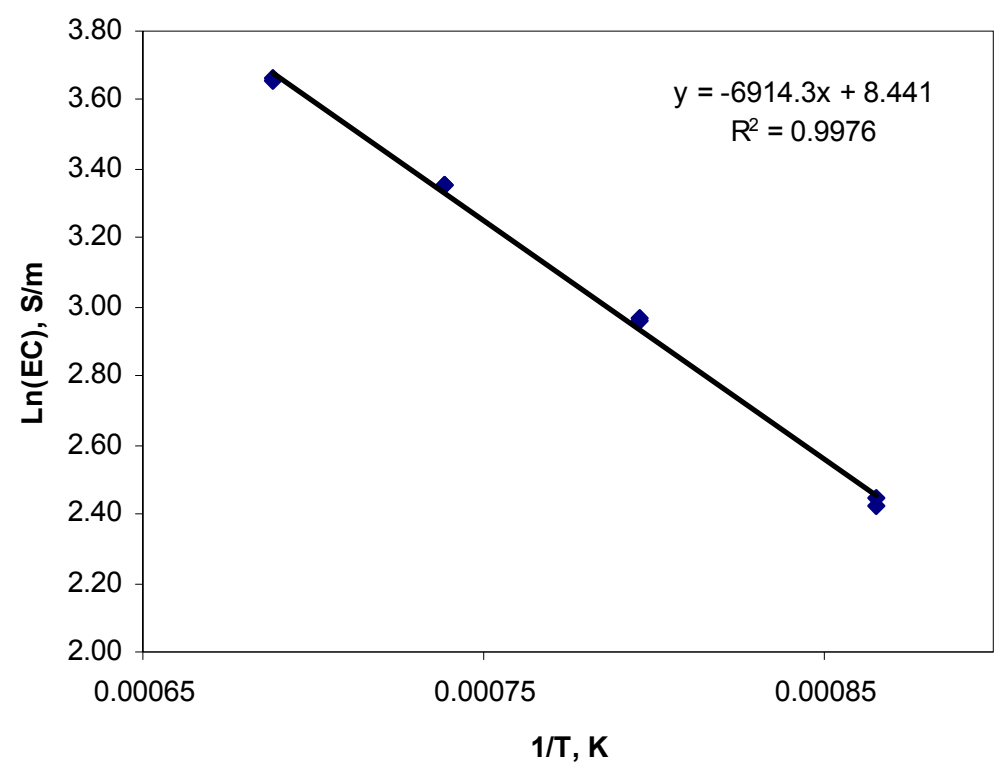

Figure C.18. Electrical Conductivity Trend for EM07-Fe-15 over Inverse Temperature 


\section{C.19 EM07-Fe-20 Electrical Conductivity Data}

Table C.19. Electrical Conductivity Data for EM07-Fe-20

\begin{tabular}{|c|c|c|c|}
\hline Temperature ${ }^{\circ} \mathrm{C}$ & Conductivity, S/m & $1 / \mathrm{T}, \mathrm{K}$ & $\mathrm{Ln}(\mathrm{EC}), \mathrm{S} / \mathrm{m}$ \\
\hline 1180 & 38.89 & 0.00069 & 3.66 \\
1180 & 38.78 & 0.00069 & 3.66 \\
1081 & 28.52 & 0.00074 & 3.35 \\
1081 & 28.53 & 0.00074 & 3.35 \\
983 & 19.36 & 0.00080 & 2.96 \\
983 & 19.40 & 0.00080 & 2.97 \\
882 & 11.51 & 0.00087 & 2.44 \\
882 & 11.30 & 0.00087 & 2.43 \\
\hline
\end{tabular}

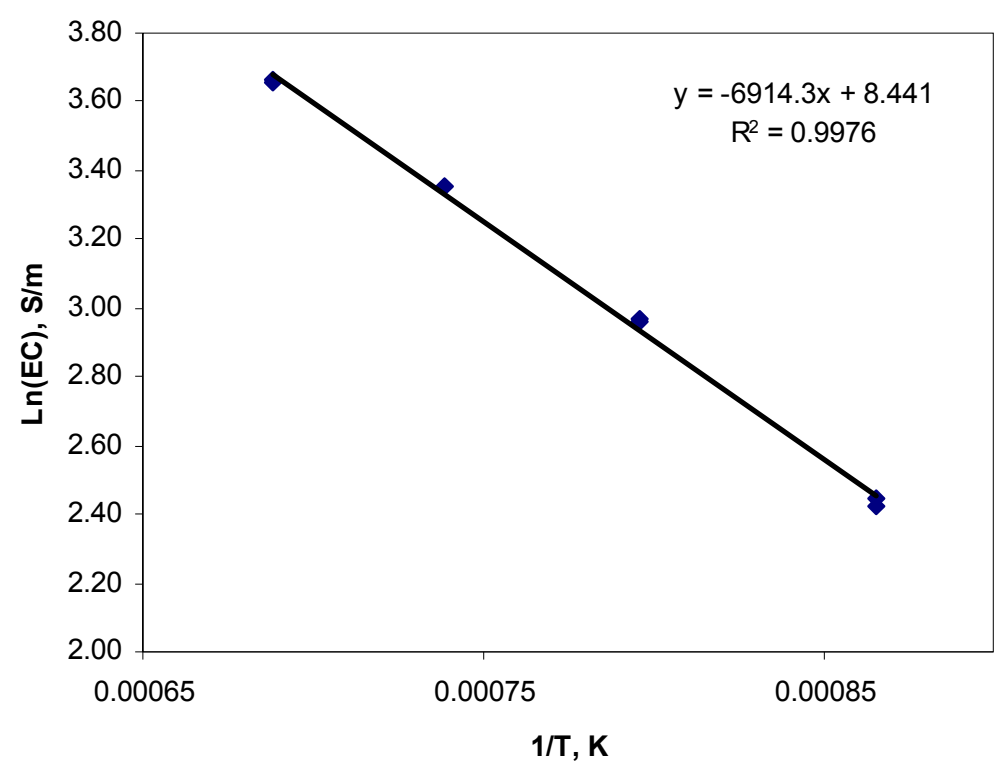

Figure C.19. Electrical Conductivity Trend for EM07-Fe-20 over Inverse Temperature 


\section{C.20 EM07-K-03 Electrical Conductivity Data}

Table C.20. Electrical Conductivity Data for EM07-K-03

\begin{tabular}{|c|c|c|c|}
\hline Temperature,$^{\circ} \mathrm{C}$ & Conductivity, S/m & $1 / \mathrm{T}, \mathrm{K}$ & $\mathrm{Ln}(\mathrm{EC}), \mathrm{S} / \mathrm{m}$ \\
\hline 1180 & 42.15 & 0.00069 & 3.74 \\
1180 & 42.25 & 0.00069 & 3.74 \\
1081 & 30.91 & 0.00074 & 3.43 \\
1081 & 30.93 & 0.00074 & 3.43 \\
983 & 20.46 & 0.00080 & 3.02 \\
983 & 20.49 & 0.00080 & 3.02 \\
882 & 11.75 & 0.00087 & 2.46 \\
882 & 11.79 & 0.00087 & 2.47 \\
\hline
\end{tabular}

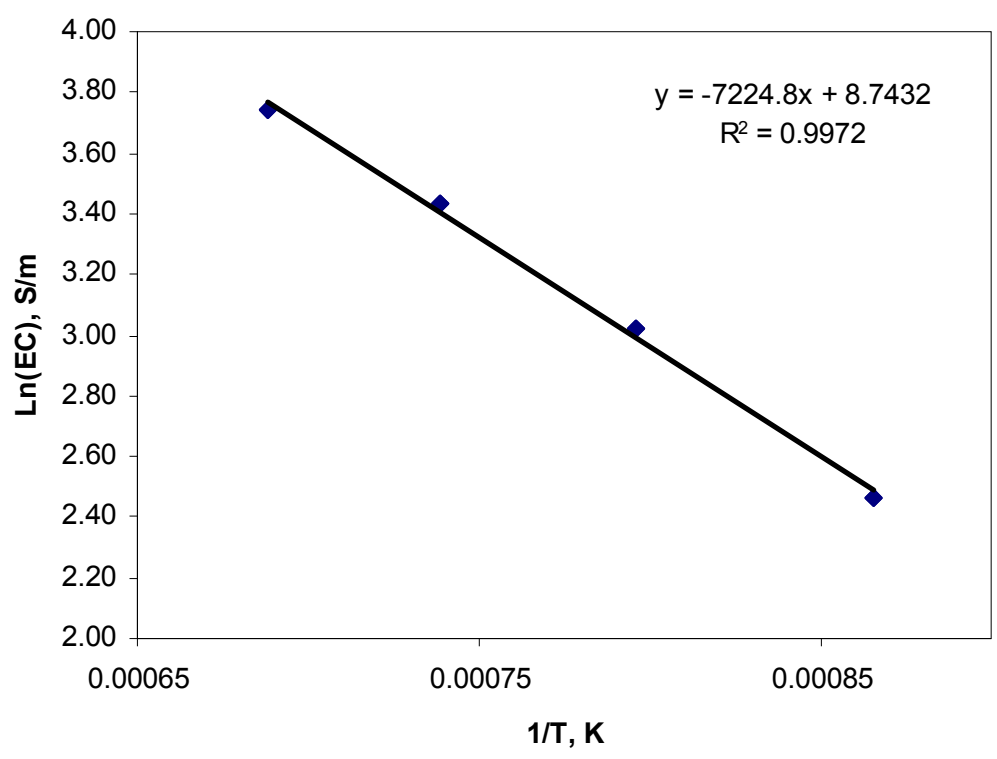

Figure C.20. Electrical Conductivity Trend for EM07-K-03 over Inverse Temperature 


\section{C.21 EM07-K-06 Electrical Conductivity Data}

Table C.21. Electrical Conductivity Data for EM07-K-06

\begin{tabular}{|c|c|c|c|}
\hline Temperature, ${ }^{\circ} \mathrm{C}$ & Conductivity, S/m & $1 / \mathrm{T}, \mathrm{K}$ & $\mathrm{Ln}(\mathrm{EC}), \mathrm{S} / \mathrm{m}$ \\
\hline 1185 & 44.00 & 0.00069 & 3.78 \\
1185 & 44.09 & 0.00069 & 3.79 \\
1086 & 31.43 & 0.00074 & 3.45 \\
1086 & 31.49 & 0.00074 & 3.45 \\
987 & 20.43 & 0.00079 & 3.02 \\
987 & 20.49 & 0.00079 & 3.02 \\
888 & 11.62 & 0.00086 & 2.45 \\
888 & 11.68 & 0.00086 & 2.46 \\
\hline
\end{tabular}

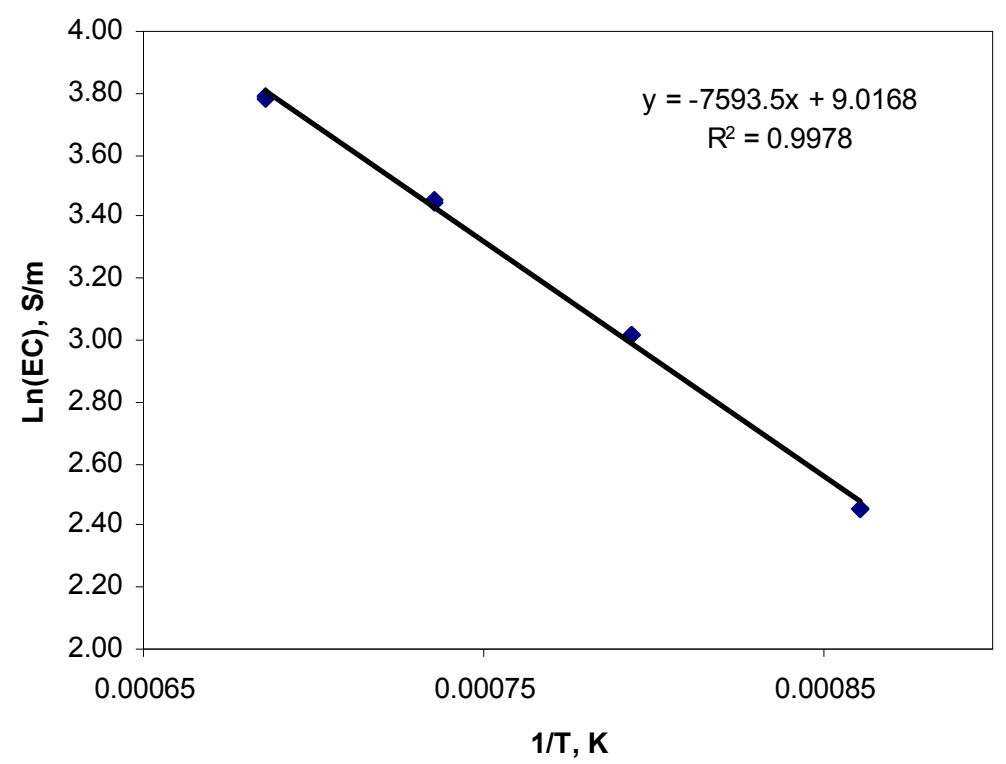

Figure C.21. Electrical Conductivity Trend for EM07-K-06 over Inverse Temperature 


\section{C.22 EM07-Li-015 Electrical Conductivity Data}

Table C.22. Electrical Conductivity Data for EM07-Li-015

\begin{tabular}{|c|c|c|c|}
\hline $\begin{array}{c}\text { Temperature, } \\
{ }^{\circ} \mathrm{C}\end{array}$ & Conductivity, S/m & $1 / \mathrm{T}, \mathrm{K}$ & $\operatorname{Ln}(\mathrm{EC}), \mathrm{S} / \mathrm{m}$ \\
\hline 1180 & 36.19 & 0.00069 & 3.59 \\
1180 & 36.18 & 0.00069 & 3.59 \\
1081 & 26.59 & 0.00074 & 3.28 \\
1081 & 26.59 & 0.00074 & 3.28 \\
983 & 17.96 & 0.00080 & 2.89 \\
983 & 17.99 & 0.00080 & 2.89 \\
882 & 10.60 & 0.00087 & 2.36 \\
882 & 10.64 & 0.00087 & 2.36 \\
\hline
\end{tabular}

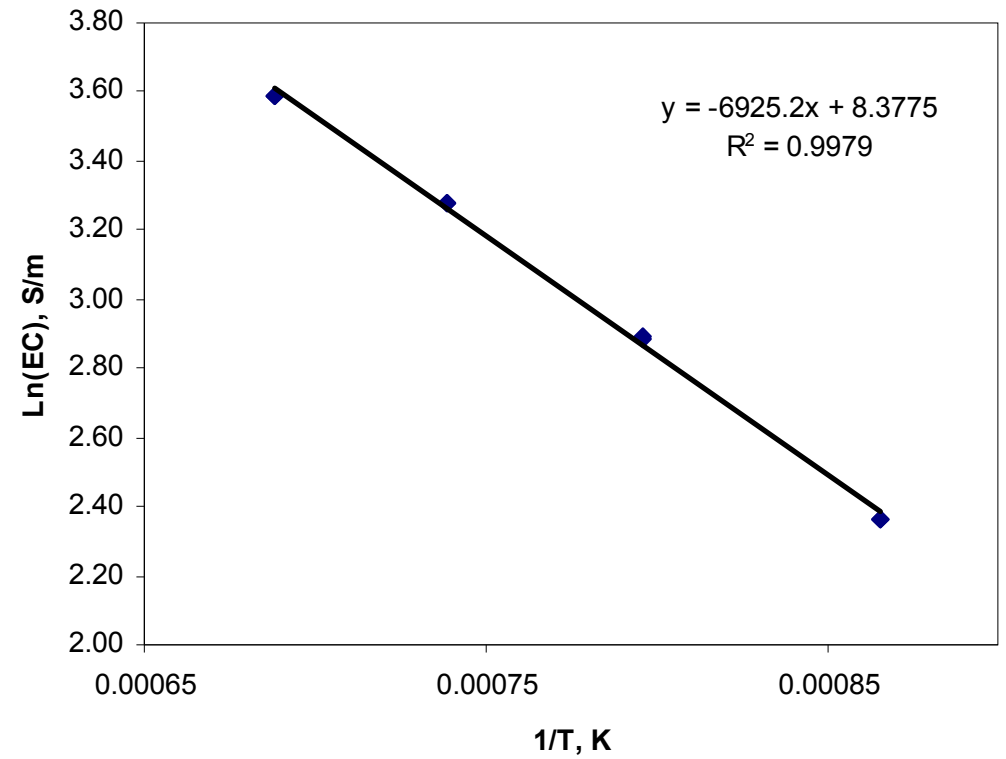

Figure C.22. Electrical Conductivity Trend for EM07-Li-015 over Inverse Temperature 


\section{C.23 EM07-Li-04 Electrical Conductivity Data}

Table C.23. Electrical Conductivity Data for EM07-Li-04

\begin{tabular}{|c|c|c|c|}
\hline Temperature ${ }^{\circ} \mathrm{C}$ & Conductivity, S/m & $1 / \mathrm{T}, \mathrm{K}$ & $\mathrm{Ln}(\mathrm{EC}), \mathrm{S} / \mathrm{m}$ \\
\hline 1180 & 45.95 & 0.00069 & 3.83 \\
1180 & 46.80 & 0.00069 & 3.85 \\
1081 & 34.36 & 0.00074 & 3.54 \\
1081 & 34.37 & 0.00074 & 3.54 \\
983 & 23.14 & 0.00080 & 3.14 \\
983 & 23.14 & 0.00080 & 3.14 \\
882 & 13.58 & 0.00087 & 2.61 \\
882 & 13.59 & 0.00087 & 2.61 \\
\hline
\end{tabular}

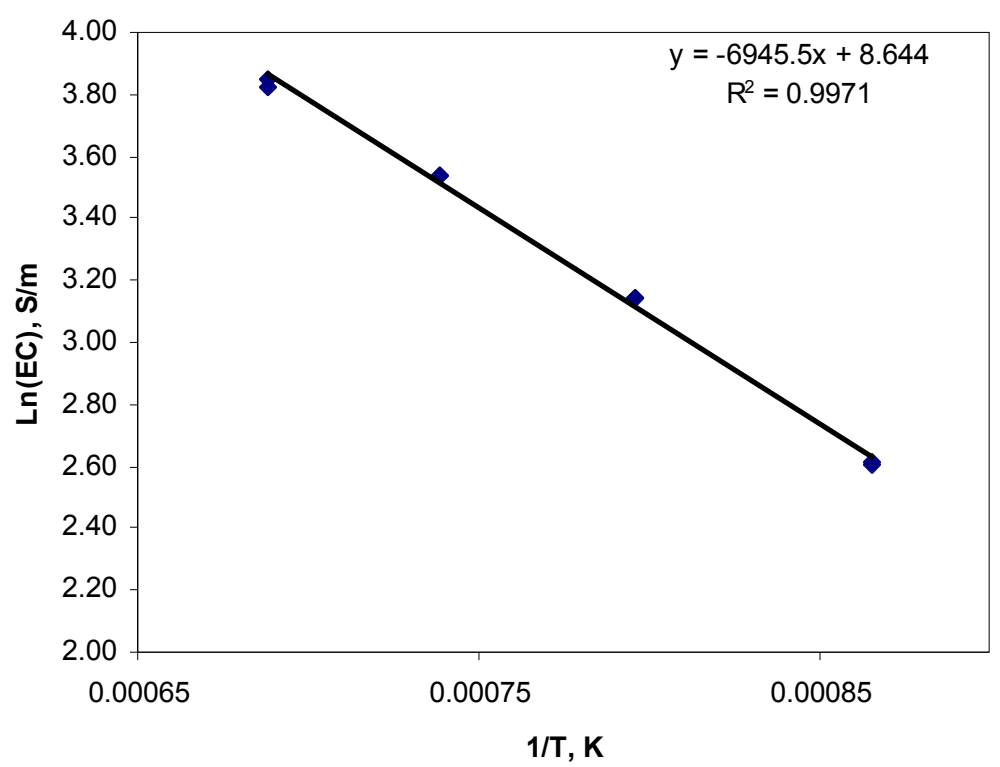

Figure C.24. Electrical Conductivity Trend for EM07-Li-04 over Inverse Temperature 


\section{C.25 EM07-Mn-01 Electrical Conductivity Data}

Table C.25. Electrical Conductivity Data for EM07-Mn-01

\begin{tabular}{|c|c|c|c|}
\hline Temperature,$^{\circ} \mathrm{C}$ & Conductivity, S/m & $1 / \mathrm{T}, \mathrm{K}$ & $\mathrm{Ln}(\mathrm{EC}), \mathrm{S} / \mathrm{m}$ \\
\hline 1184 & 52.78 & 0.00069 & 3.97 \\
1184 & 52.82 & 0.00069 & 3.97 \\
1085 & 38.17 & 0.00074 & 3.64 \\
1085 & 38.18 & 0.00074 & 3.64 \\
986 & 25.35 & 0.00079 & 3.23 \\
986 & 25.41 & 0.00079 & 3.24 \\
887 & 14.83 & 0.00086 & 2.70 \\
887 & 14.91 & 0.00086 & 2.70 \\
\hline
\end{tabular}

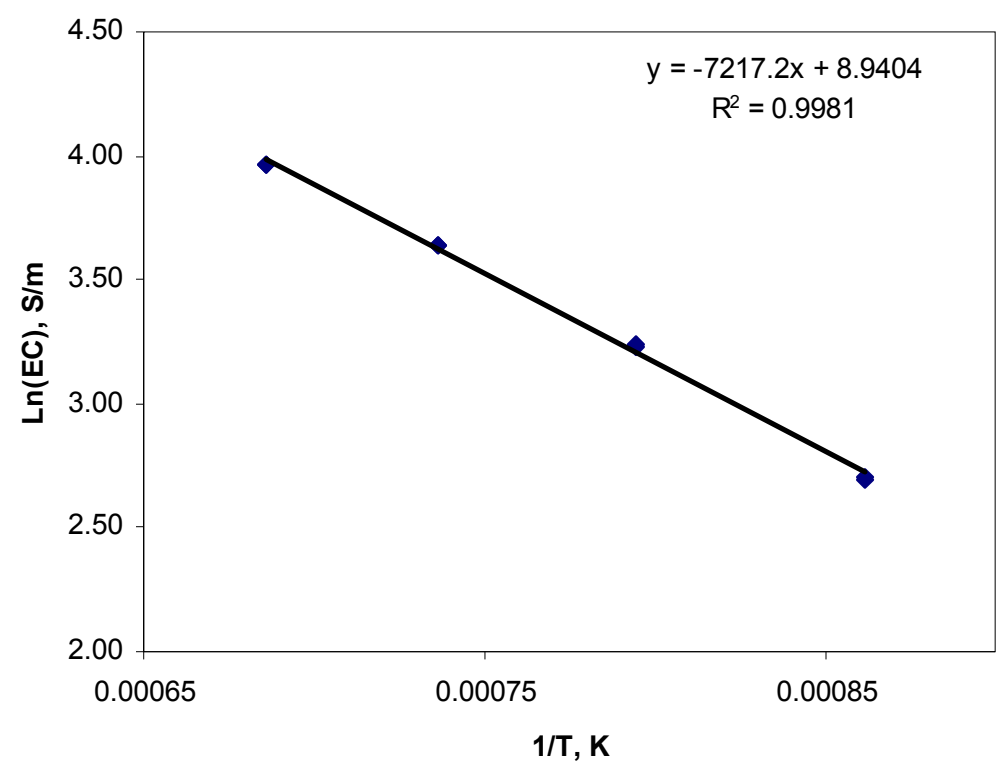

Figure C.25. Electrical Conductivity Trend for EM07-Mn-01 over Inverse Temperature 


\section{C.26 EM07-Mn-04 Electrical Conductivity Data}

Table C.26. Electrical Conductivity Data for EM07-Mn-04

\begin{tabular}{|c|c|c|c|}
\hline Temperature,$^{\circ} \mathrm{C}$ & Conductivity, S/m & $1 / \mathrm{T}, \mathrm{K}$ & $\operatorname{Ln}(\mathrm{EC}), \mathrm{S} / \mathrm{m}$ \\
\hline 1180 & 42.27 & 0.00069 & 3.74 \\
1180 & 42.35 & 0.00069 & 3.75 \\
1081 & 30.94 & 0.00074 & 3.43 \\
1081 & 30.95 & 0.00074 & 3.43 \\
983 & 20.57 & 0.00080 & 3.02 \\
983 & 20.62 & 0.00080 & 3.03 \\
882 & 12.00 & 0.00087 & 2.48 \\
882 & 12.05 & 0.00087 & 2.49 \\
\hline
\end{tabular}

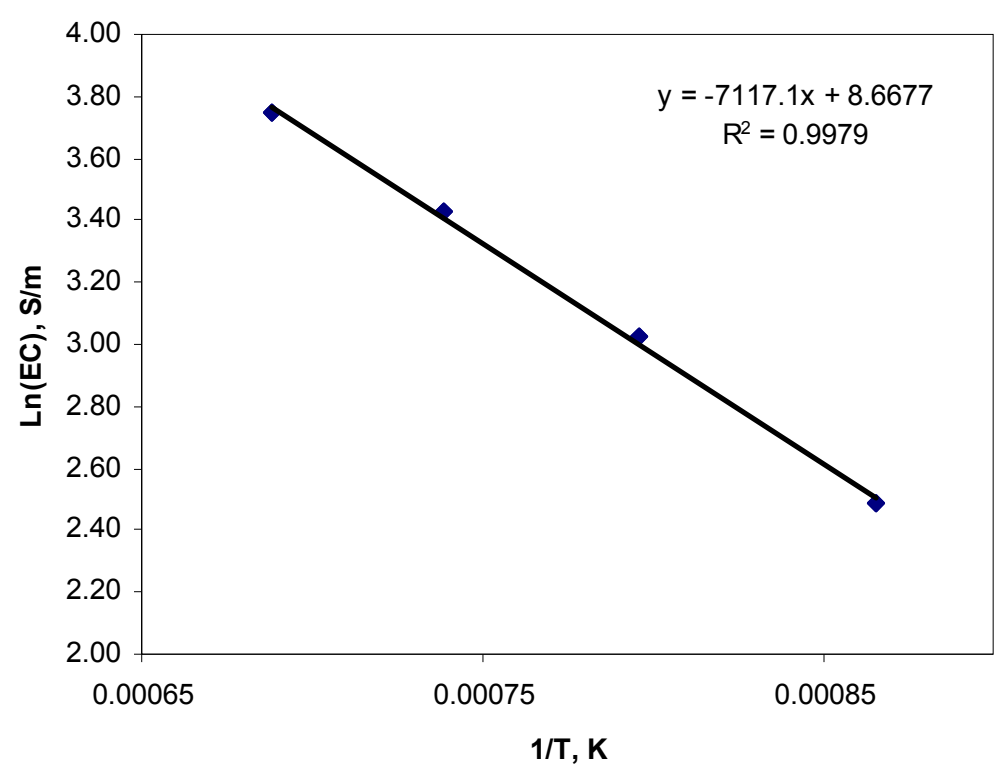

Figure C.26. Electrical Conductivity Trend for EM07-Mn-04 over Inverse Temperature 


\section{C.27 EM07-Na-05 Electrical Conductivity Data}

Table C.27. Electrical Conductivity Data for EM07-Na-05

\begin{tabular}{|c|c|c|c|}
\hline Temperature,$^{\circ} \mathrm{C}$ & Conductivity, S/m & $1 / \mathrm{T}, \mathrm{K}$ & $\mathrm{Ln}(\mathrm{EC}), \mathrm{S} / \mathrm{m}$ \\
\hline 1180 & 16.80 & 0.00069 & 2.82 \\
1180 & 16.66 & 0.00069 & 2.81 \\
1081 & 12.22 & 0.00074 & 2.50 \\
1081 & 12.22 & 0.00074 & 2.50 \\
983 & 8.04 & 0.00080 & 2.08 \\
983 & 8.06 & 0.00080 & 2.09 \\
882 & 4.71 & 0.00087 & 1.55 \\
882 & 4.72 & 0.00087 & 1.55 \\
\hline
\end{tabular}

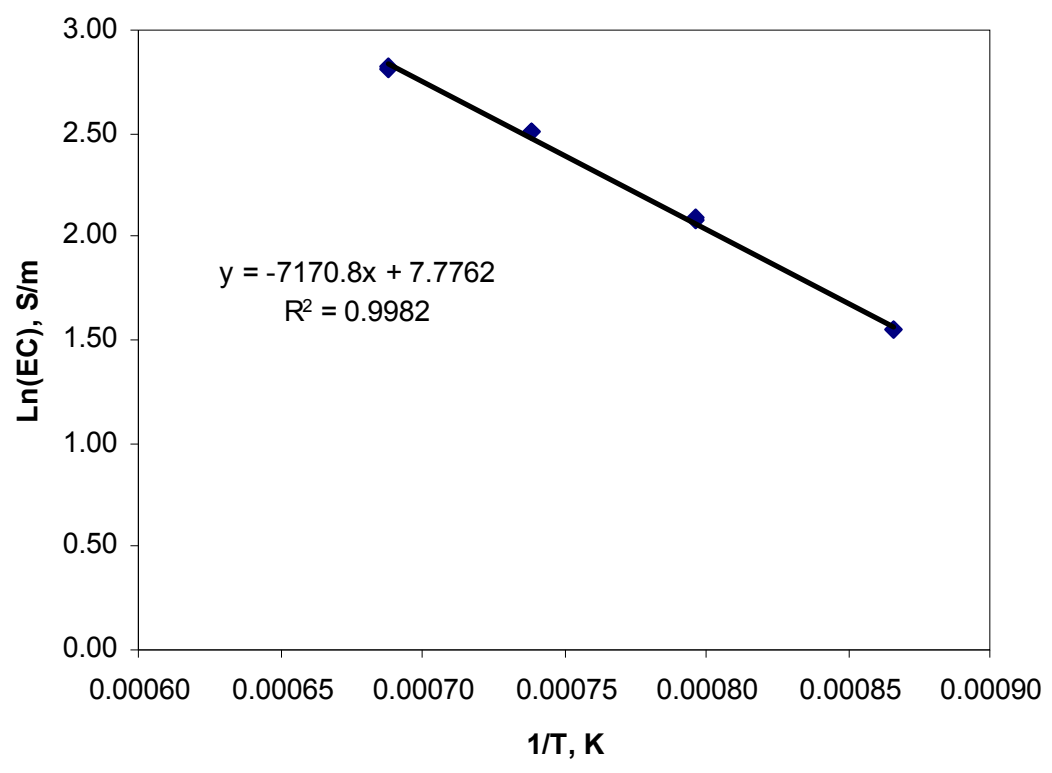

Figure C.27. Electrical Conductivity Trend for EM07-Na-05 over Inverse Temperature 


\section{C.28 EM07-Na-10 Electrical Conductivity Data}

Table C.28. Electrical Conductivity Data for EM07-Na-10

\begin{tabular}{|c|c|c|c|}
\hline Temperature,$^{\circ} \mathrm{C}$ & Conductivity, S/m & $1 / \mathrm{T}, \mathrm{K}$ & $\mathrm{Ln}(\mathrm{EC}), \mathrm{S} / \mathrm{m}$ \\
\hline 1180 & 23.20 & 0.00069 & 3.14 \\
1180 & 23.29 & 0.00069 & 3.15 \\
1081 & 16.39 & 0.00074 & 2.80 \\
1081 & 16.55 & 0.00074 & 2.81 \\
983 & 10.44 & 0.00080 & 2.35 \\
983 & 10.44 & 0.00080 & 2.35 \\
882 & 6.11 & 0.00087 & 1.81 \\
882 & 6.12 & 0.00087 & 1.81 \\
\hline
\end{tabular}

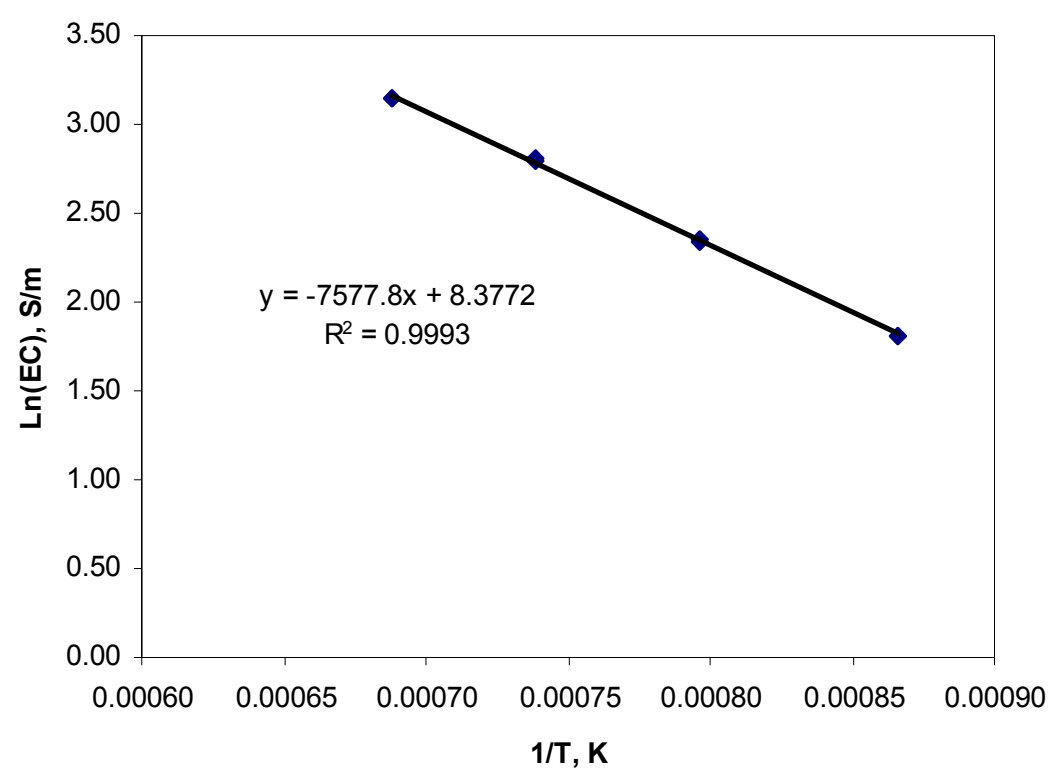

Figure C.28. Electrical Conductivity Trend for EM07-Na-10 over Inverse Temperature 


\section{C.28 EM07-Na-20 Electrical Conductivity Data}

Table C.28. Electrical Conductivity Data for EM07-Na-20

\begin{tabular}{|c|c|c|c|}
\hline Temperature,$^{\circ} \mathrm{C}$ & Conductivity, S/m & $1 / \mathrm{T}, \mathrm{K}$ & $\mathrm{Ln}(\mathrm{EC}), \mathrm{S} / \mathrm{m}$ \\
\hline 1180 & 66.40 & 0.00069 & 4.20 \\
1180 & 66.52 & 0.00069 & 4.20 \\
1081 & 51.29 & 0.00074 & 3.94 \\
1081 & 51.31 & 0.00074 & 3.94 \\
983 & 36.50 & 0.00080 & 3.60 \\
983 & 36.54 & 0.00080 & 3.60 \\
882 & 22.99 & 0.00087 & 3.13 \\
882 & 23.05 & 0.00087 & 3.14 \\
\hline
\end{tabular}

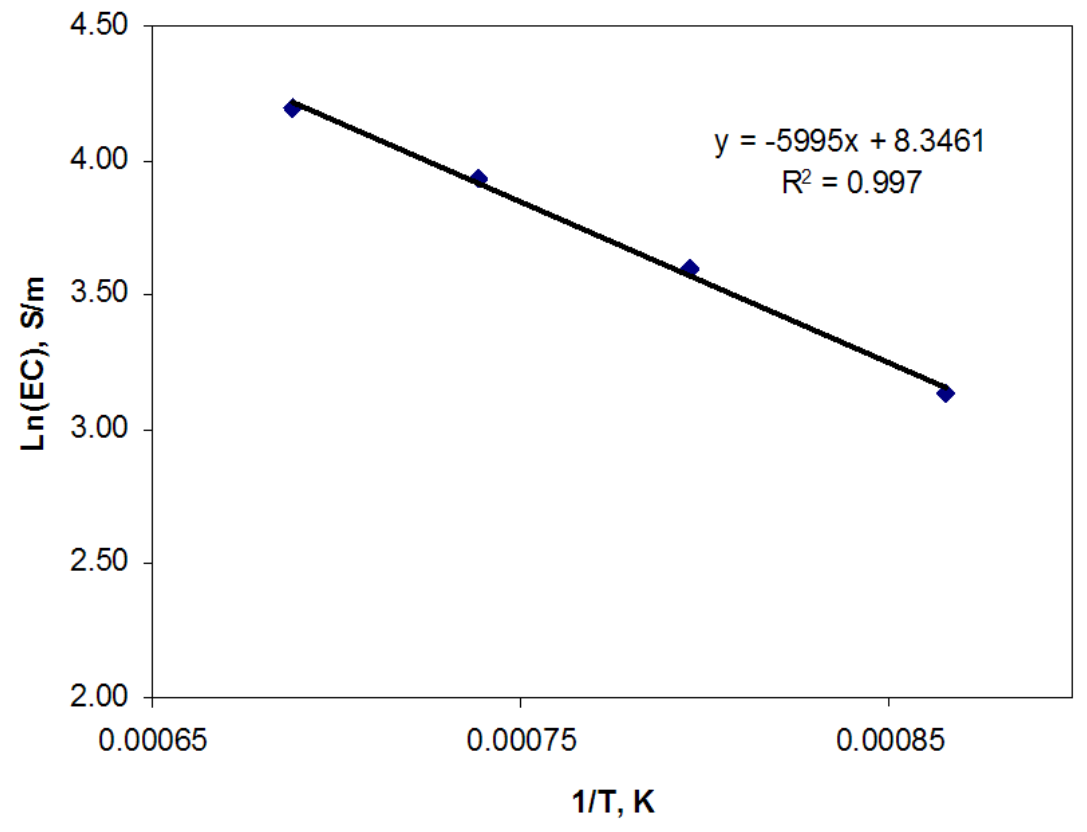

Figure C.28. Electrical Conductivity Trend for EM07-Na-20 over Inverse Temperature 


\section{C.29 EM07-Ni-001 Electrical Conductivity Data}

Table C.29. Electrical Conductivity Data for EM07-Ni-001

\begin{tabular}{|c|c|c|c|}
\hline Temperature,$^{\circ} \mathrm{C}$ & Conductivity, S/m & $1 / \mathrm{T}, \mathrm{K}$ & $\mathrm{Ln}(\mathrm{EC}), \mathrm{S} / \mathrm{m}$ \\
\hline 1184 & 51.05 & 0.00069 & 3.93 \\
1184 & 51.06 & 0.00069 & 3.93 \\
1135 & 43.43 & 0.00071 & 3.77 \\
1135 & 43.44 & 0.00071 & 3.77 \\
1036 & 29.57 & 0.00076 & 3.39 \\
1036 & 29.68 & 0.00076 & 3.39 \\
937 & 18.26 & 0.00083 & 2.90 \\
937 & 18.27 & 0.00083 & 2.91 \\
\hline
\end{tabular}

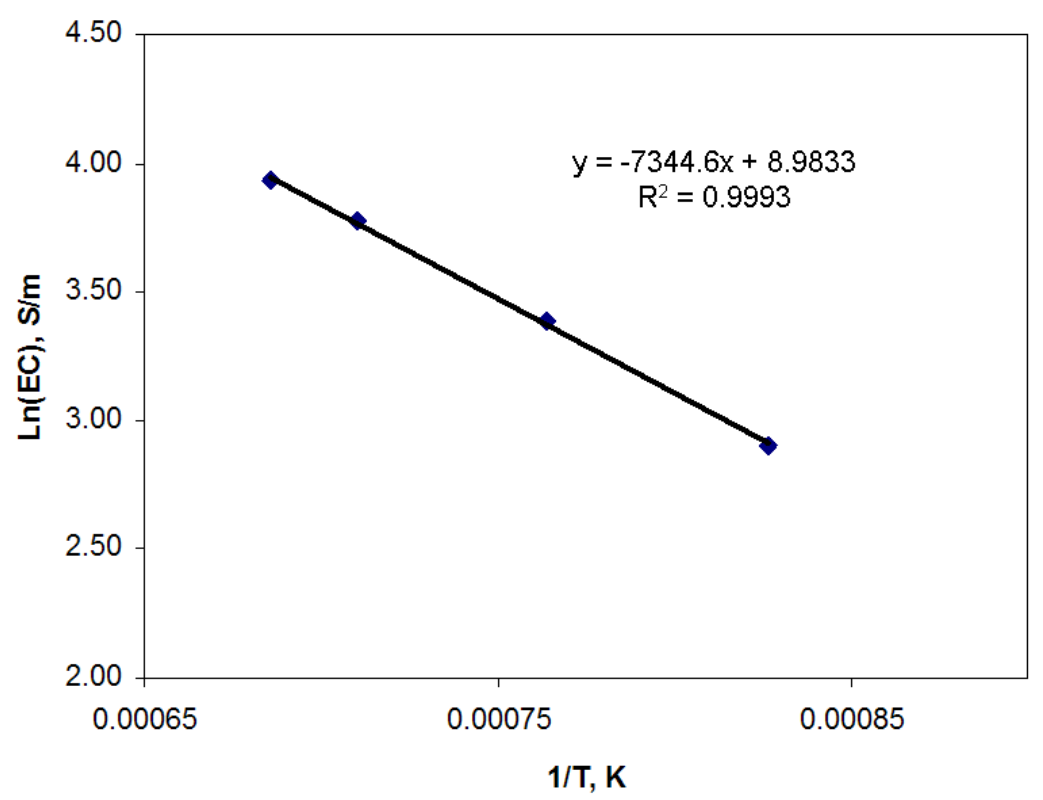

Figure C.29. Electrical conductivity trend for EM07-Ni-001 over inverse temperature 


\section{C.30 EM07-Ni-02 Electrical Conductivity Data}

Table C.30. Electrical Conductivity Data for EM07-Ni-02

\begin{tabular}{|c|c|c|c|}
\hline Temperature,$^{\circ} \mathrm{C}$ & Conductivity, S/m & $1 / \mathrm{T}, \mathrm{K}$ & $\operatorname{Ln}(\mathrm{EC}), \mathrm{S} / \mathrm{m}$ \\
\hline 1180 & 36.28 & 0.00069 & 3.59 \\
1180 & 37.78 & 0.00069 & 3.63 \\
1081 & 29.11 & 0.00074 & 3.37 \\
1081 & 26.77 & 0.00074 & 3.29 \\
1081 & 27.51 & 0.00074 & 3.31 \\
1081 & 27.57 & 0.00074 & 3.32 \\
882 & 10.81 & 0.00087 & 2.38 \\
882 & 10.83 & 0.00087 & 2.38 \\
\hline
\end{tabular}

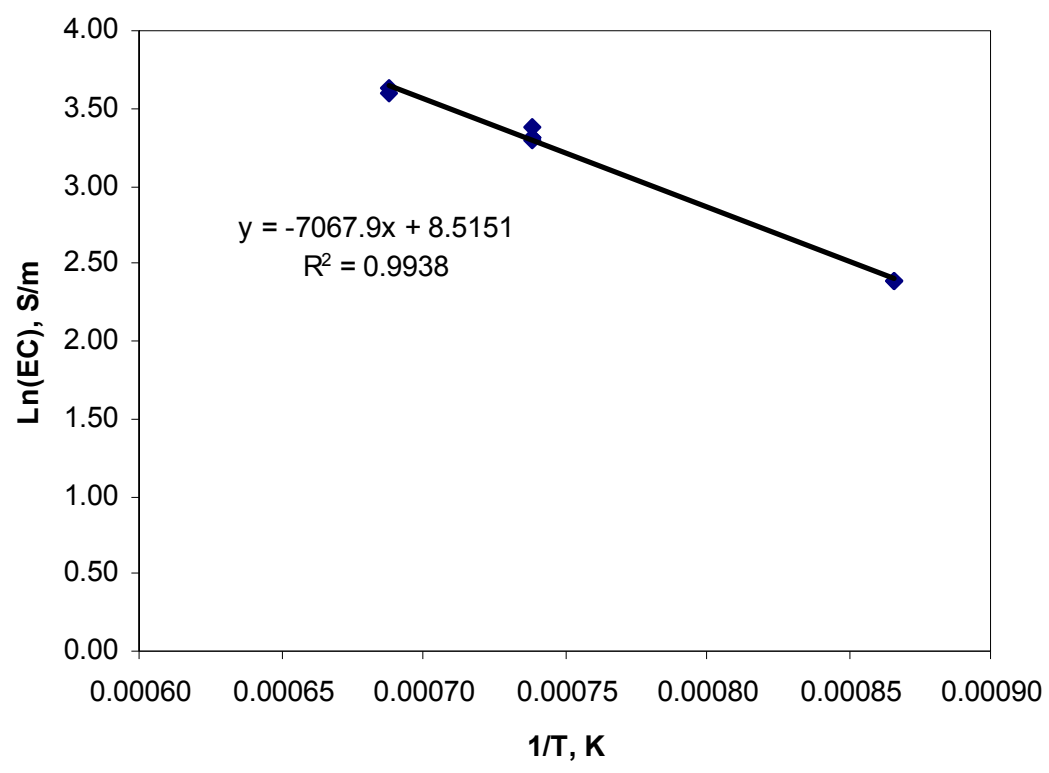

Figure C.30. Electrical Conductivity Trend for EM07-Ni-02 over Inverse Temperature 


\section{C.31 EM07-P-0 Electrical Conductivity Data}

Table C.31. Electrical conductivity data for EM07-P-0

\begin{tabular}{|c|c|c|c|}
\hline Temperature $^{\circ} \mathrm{C}$ & Conductivity, S/m & $1 / \mathrm{T}, \mathrm{K}$ & $\mathrm{Ln}(\mathrm{EC}), \mathrm{S} / \mathrm{m}$ \\
\hline 1180 & 44.15 & 0.00069 & 3.79 \\
1180 & 44.19 & 0.00069 & 3.79 \\
1081 & 32.59 & 0.00074 & 3.48 \\
1081 & 32.60 & 0.00074 & 3.48 \\
983 & 21.99 & 0.00080 & 3.09 \\
983 & 22.02 & 0.00080 & 3.09 \\
882 & 12.96 & 0.00087 & 2.56 \\
882 & 13.02 & 0.00087 & 2.57 \\
\hline
\end{tabular}

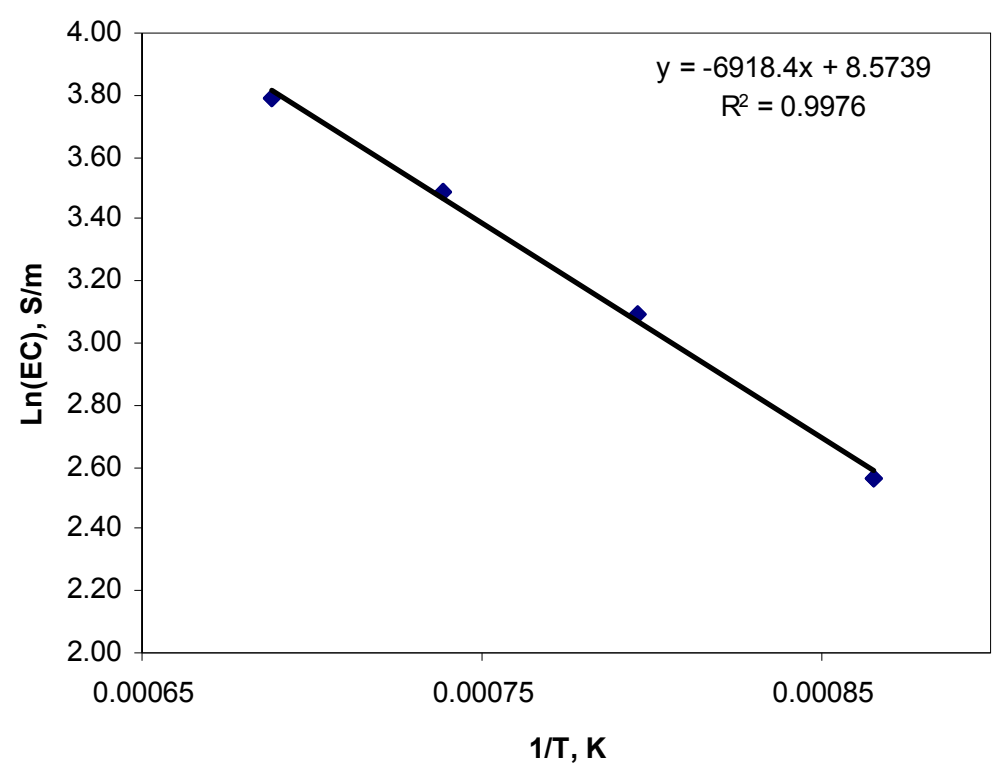

Figure C.32. Electrical Conductivity Trend for EM07-P-0 over Inverse Temperature 


\section{C.33 EM07-P-025 Electrical Conductivity Data}

Table C.33. Electrical Conductivity Data for EM07-P-025

\begin{tabular}{|c|c|c|c|}
\hline Temperature,$^{\circ} \mathrm{C}$ & Conductivity, S/m & $1 / \mathrm{T}, \mathrm{K}$ & $\mathrm{Ln}(\mathrm{EC}), \mathrm{S} / \mathrm{m}$ \\
\hline 1183 & 46.52 & 0.00069 & 3.84 \\
1183 & 46.55 & 0.00069 & 3.84 \\
1081 & 34.53 & 0.00074 & 3.54 \\
1081 & 34.57 & 0.00074 & 3.54 \\
988 & 34.55 & 0.00079 & 3.54 \\
988 & 34.58 & 0.00079 & 3.54 \\
882 & 23.60 & 0.00087 & 3.16 \\
882 & 23.68 & 0.00087 & 3.16 \\
\hline
\end{tabular}

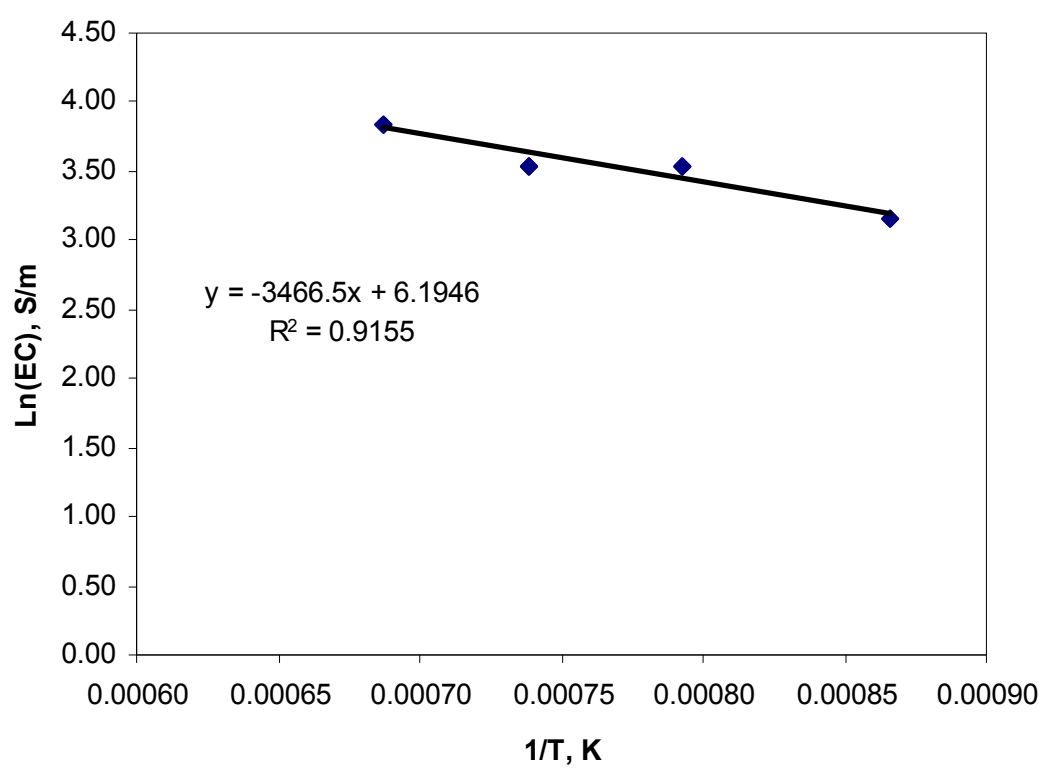

Figure C.33. Electrical Conductivity Trend for EM07-P-025 over Inverse Temperature 


\section{C.34 EM07-Si-30 Duplicate Electrical Conductivity Data}

Table C.34. Electrical Conductivity Data for EM07-Si-30 Duplicate

\begin{tabular}{|c|c|c|c|}
\hline Temperature, ${ }^{\circ} \mathrm{C}$ & Conductivity, S/m & $1 / \mathrm{T}, \mathrm{K}$ & $\mathrm{Ln}(\mathrm{EC}), \mathrm{S} / \mathrm{m}$ \\
\hline 1186 & 56.63 & 0.00069 & 4.04 \\
1186 & 56.38 & 0.00069 & 4.03 \\
1085 & 42.76 & 0.00074 & 3.76 \\
1085 & 42.79 & 0.00074 & 3.76 \\
984 & 30.13 & 0.00080 & 3.41 \\
984 & 30.17 & 0.00080 & 3.41 \\
885 & 18.94 & 0.00086 & 2.94 \\
885 & 19.02 & 0.00086 & 2.95 \\
\hline
\end{tabular}

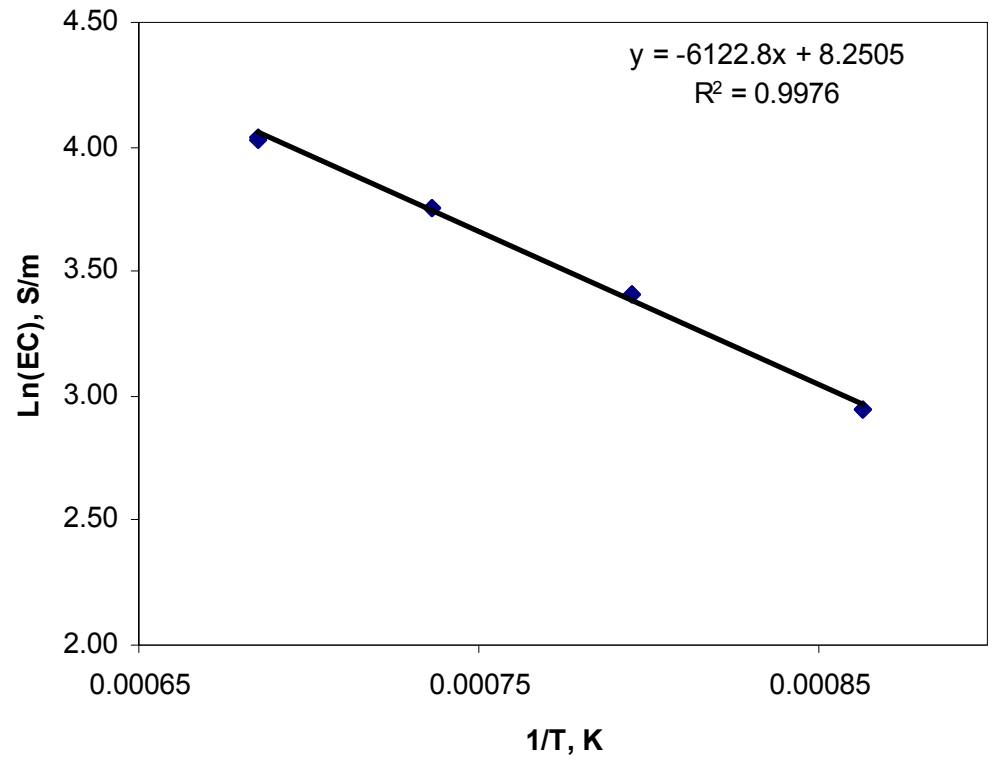

Figure C.35. Electrical Conductivity Trend for EM07-Si-30 Duplicate over Inverse Temperature 


\section{C.35 EM07-Si-37 Electrical Conductivity Data}

Table C.35. Electrical Conductivity Data for EM07-Si-37

\begin{tabular}{|c|c|c|c|}
\hline Temperature, ${ }^{\circ} \mathrm{C}$ & Conductivity, S/m & $1 / \mathrm{T}, \mathrm{K}$ & $\mathrm{Ln}(\mathrm{EC}), \mathrm{S} / \mathrm{m}$ \\
\hline 1183 & 54.57 & 0.00069 & 4.00 \\
1183 & 54.59 & 0.00069 & 4.00 \\
1081 & 40.14 & 0.00074 & 3.69 \\
1081 & 40.17 & 0.00074 & 3.69 \\
988 & 27.58 & 0.00079 & 3.32 \\
988 & 27.64 & 0.00079 & 3.32 \\
882 & 16.92 & 0.00087 & 2.83 \\
882 & 16.95 & 0.00087 & 2.83 \\
\hline
\end{tabular}

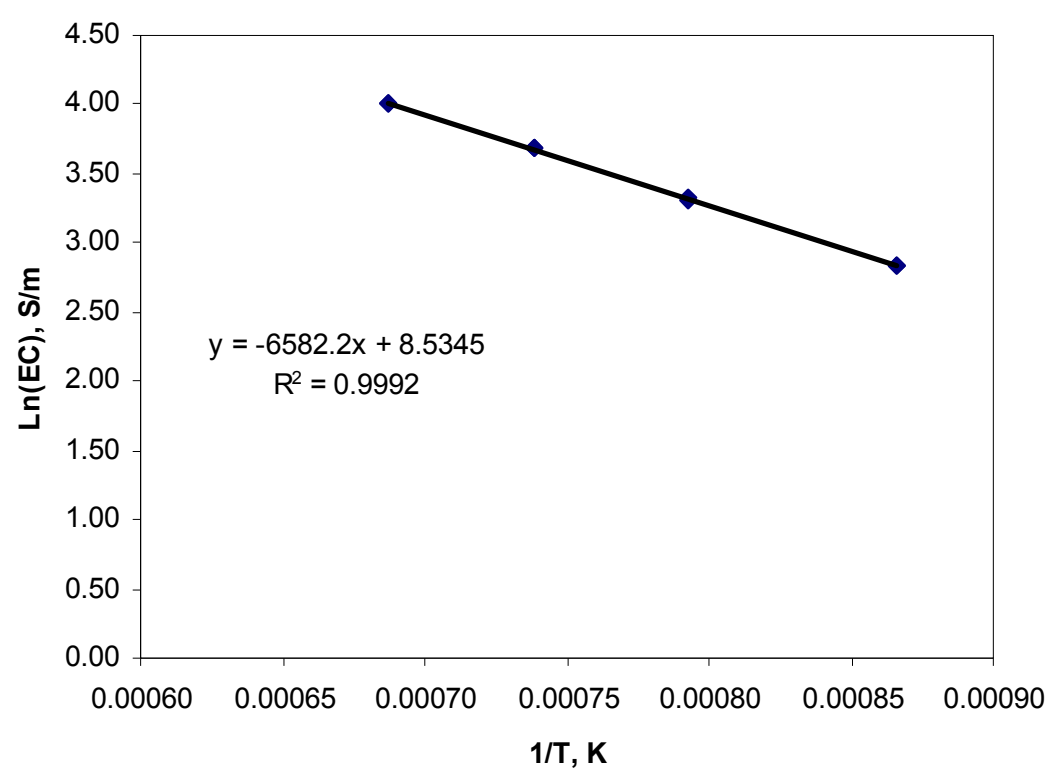

Figure C.35. Electrical Conductivity Trend for EM07-Si-37 over Inverse Temperature 


\section{C.36 EM07-Si-37 Duplicate Electrical Conductivity Data}

Table C.36. Electrical Conductivity Data for EM07-Si-37 Duplicate

\begin{tabular}{|c|c|c|c|}
\hline Temperature, ${ }^{\circ} \mathrm{C}$ & Conductivity, S/m & $1 / \mathrm{T}, \mathrm{K}$ & $\mathrm{Ln}(\mathrm{EC}), \mathrm{S} / \mathrm{m}$ \\
\hline 1186 & 51.36 & 0.00069 & 3.94 \\
1186 & 51.77 & 0.00069 & 3.95 \\
1085 & 39.04 & 0.00074 & 3.66 \\
1085 & 39.00 & 0.00074 & 3.66 \\
984 & 26.68 & 0.00080 & 3.28 \\
984 & 26.74 & 0.00080 & 3.29 \\
885 & 16.24 & 0.00086 & 2.79 \\
885 & 16.28 & 0.00086 & 2.79 \\
\hline
\end{tabular}

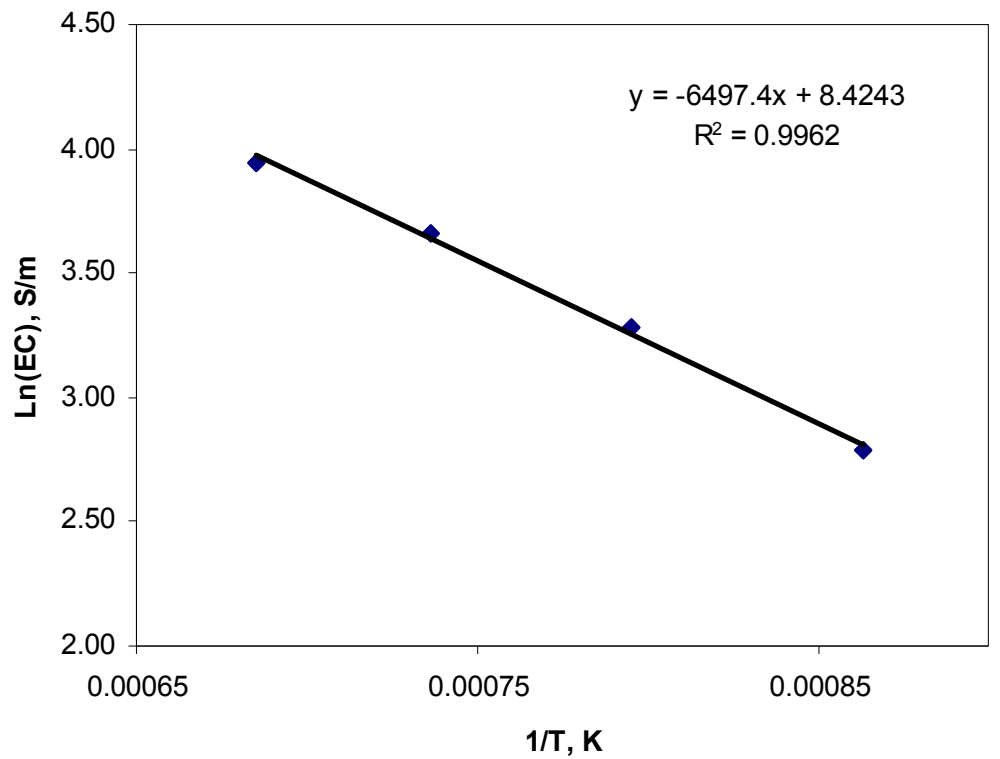

Figure C.36. Electrical Conductivity Trend for EM07-Si-37 Duplicate over Inverse Temperature 


\section{C.37 EM07-Si-50 Electrical Conductivity Data}

Table C.37. Electrical Conductivity Data for EM07-Si-50

\begin{tabular}{|c|c|c|c|}
\hline Temperature, ${ }^{\circ} \mathrm{C}$ & Conductivity, S/m & $1 / \mathrm{T}, \mathrm{K}$ & $\mathrm{Ln}(\mathrm{EC}), \mathrm{S} / \mathrm{m}$ \\
\hline 1180 & 35.51 & 0.00069 & 3.57 \\
1180 & 35.61 & 0.00069 & 3.57 \\
1081 & 25.48 & 0.00074 & 3.24 \\
1081 & 25.51 & 0.00074 & 3.24 \\
980 & 16.64 & 0.00080 & 2.81 \\
980 & 16.66 & 0.00080 & 2.81 \\
882 & 8.83 & 0.00087 & 2.18 \\
882 & 8.83 & 0.00087 & 2.18 \\
\hline
\end{tabular}

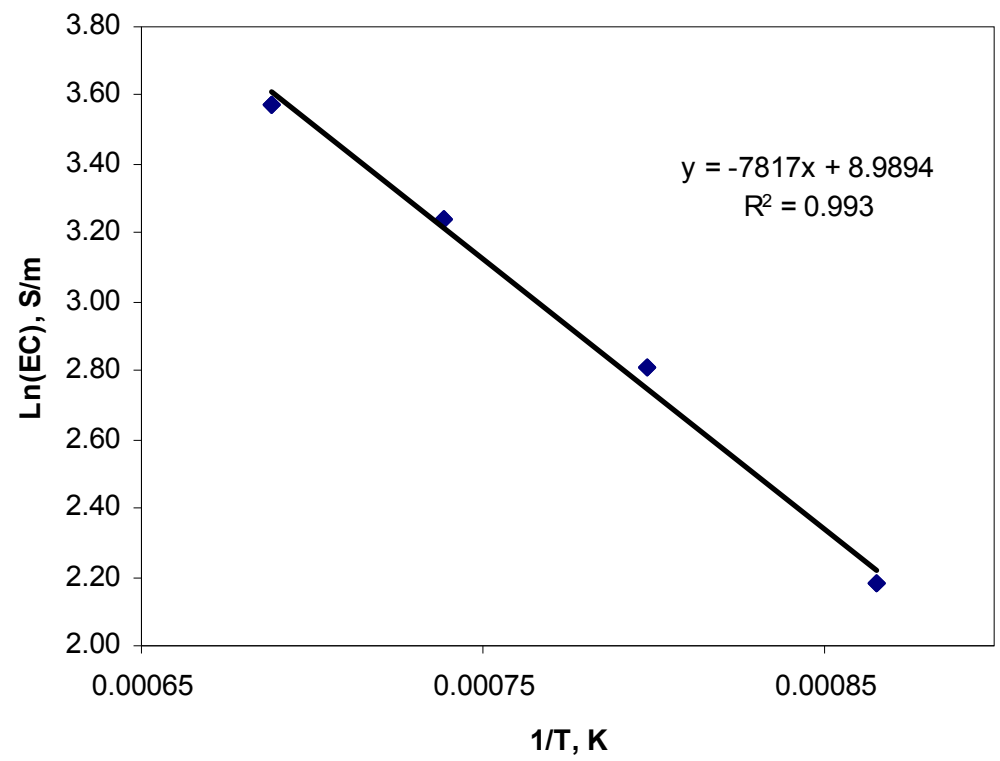

Figure C.37. Electrical Conductivity Trend for EM07-Si-50 over Inverse Temperature 


\section{C.38 EM07-Si-50 Duplicate Electrical Conductivity Data}

Table C.38. Electrical Conductivity Data for EM07-Si-50 Duplicate

\begin{tabular}{|c|c|c|c|}
\hline Temperature, ${ }^{\circ} \mathrm{C}$ & Conductivity, S/m & $1 / \mathrm{T}, \mathrm{K}$ & $\mathrm{Ln}(\mathrm{EC}), \mathrm{S} / \mathrm{m}$ \\
\hline 1186 & 25.81 & 0.00069 & 3.25 \\
1186 & 25.69 & 0.00069 & 3.25 \\
1085 & 18.25 & 0.00074 & 2.90 \\
1085 & 18.24 & 0.00074 & 2.90 \\
984 & 11.92 & 0.00080 & 2.48 \\
984 & 11.94 & 0.00080 & 2.48 \\
885 & 6.81 & 0.00086 & 1.92 \\
885 & 6.83 & 0.00086 & 1.92 \\
\hline
\end{tabular}

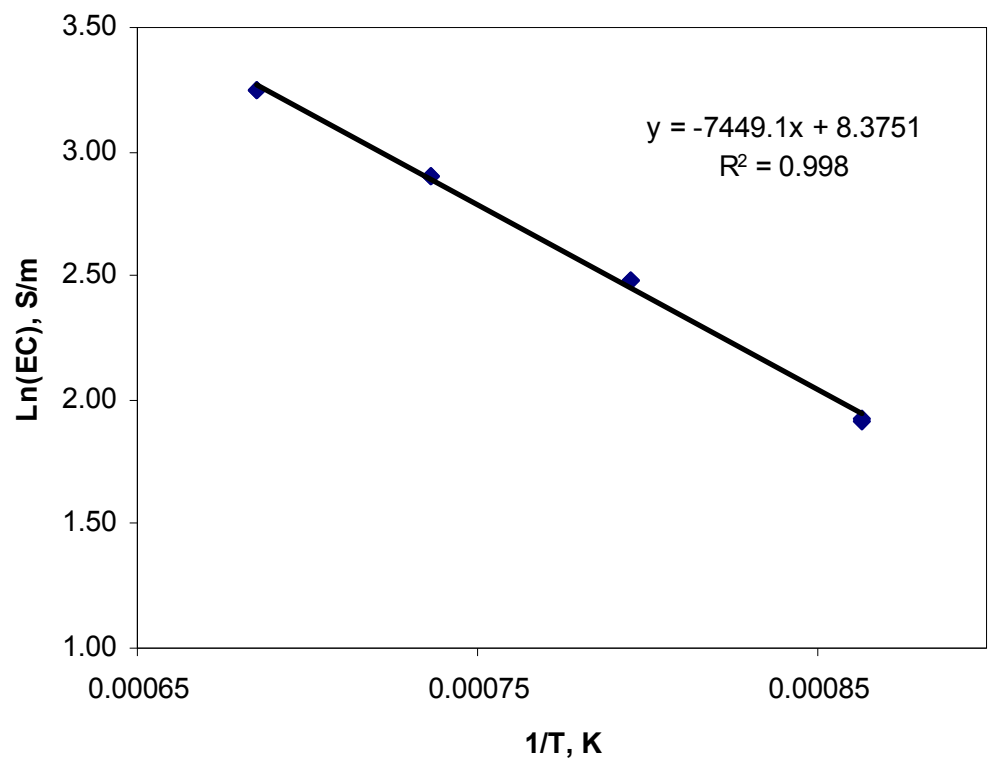

Figure C.38. Electrical Conductivity Trend for EM07-Si-50 Duplicate over Inverse Temperature 


\section{C.39 EM07-Zr-001 Electrical Conductivity Data}

Table C.39. Electrical Conductivity Data for EM07-Zr-001

\begin{tabular}{|c|c|c|c|}
\hline Temperature $^{\circ} \mathrm{C}$ & Conductivity, S/m & $1 / \mathrm{T}, \mathrm{K}$ & $\mathrm{Ln}(\mathrm{EC}), \mathrm{S} / \mathrm{m}$ \\
\hline 1180 & 38.14 & 0.00069 & 3.64 \\
1180 & 39.32 & 0.00069 & 3.67 \\
1081 & 28.94 & 0.00074 & 3.37 \\
1081 & 28.95 & 0.00074 & 3.37 \\
983 & 19.26 & 0.00080 & 2.96 \\
983 & 19.49 & 0.00080 & 2.97 \\
882 & 11.66 & 0.00087 & 2.46 \\
882 & 11.67 & 0.00087 & 2.46 \\
\hline
\end{tabular}

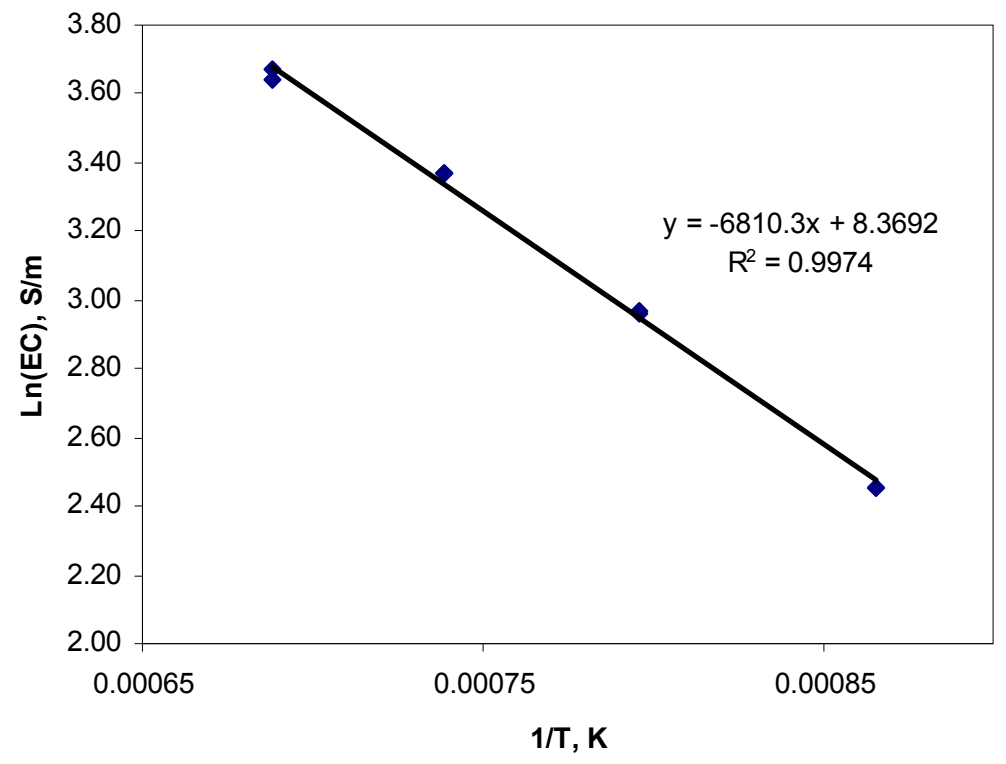

Figure C.39. Electrical Conductivity Trend for EM07-Zr-001 over Inverse Temperature 


\section{C.40 EM07-Zr-05 Electrical Conductivity Data}

Table C.40. Electrical Conductivity Data for EM07-Zr-05

\begin{tabular}{|c|c|c|c|}
\hline Temperature,$^{\circ} \mathrm{C}$ & Conductivity, S/m & $1 / \mathrm{T}, \mathrm{K}$ & $\operatorname{Ln}(\mathrm{EC}), \mathrm{S} / \mathrm{m}$ \\
\hline 1183 & 39.60 & 0.00069 & 3.68 \\
1183 & 39.46 & 0.00069 & 3.68 \\
1081 & 27.91 & 0.00074 & 3.33 \\
1081 & 27.93 & 0.00074 & 3.33 \\
988 & 18.45 & 0.00079 & 2.92 \\
988 & 18.54 & 0.00079 & 2.92 \\
882 & 10.82 & 0.00087 & 2.38 \\
882 & 10.84 & 0.00087 & 2.38 \\
\hline
\end{tabular}

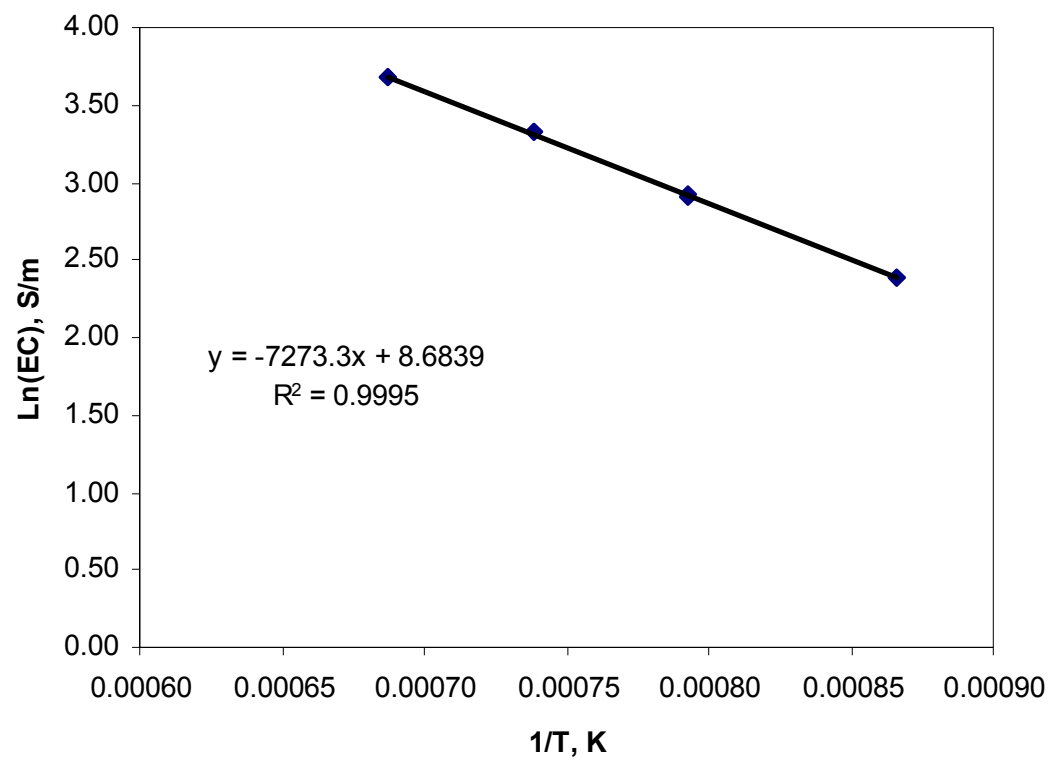

Figure C.40. Electrical Conductivity Trend for EM07-Zr-05 over Inverse Temperature 


\section{C.41 EM07-NM-0025 Electrical Conductivity Data}

Table C.41. Electrical Conductivity Data for EM07-NM-0025

\begin{tabular}{|c|c|c|c|}
\hline Temperature ${ }^{\circ} \mathrm{C}$ & Conductivity, S/m & $1 / \mathrm{T}, \mathrm{K}$ & $\mathrm{Ln}(\mathrm{EC}), \mathrm{S} / \mathrm{m}$ \\
\hline 1183 & 28.36 & 0.00069 & 3.34 \\
1183 & 28.55 & 0.00069 & 3.35 \\
1080 & 19.07 & 0.00074 & 2.95 \\
1080 & 18.49 & 0.00074 & 2.92 \\
980 & 11.62 & 0.00080 & 2.45 \\
980 & 11.62 & 0.00080 & 2.45 \\
888 & 8.76 & 0.00086 & 2.17 \\
888 & 8.79 & 0.00086 & 2.17 \\
\hline
\end{tabular}

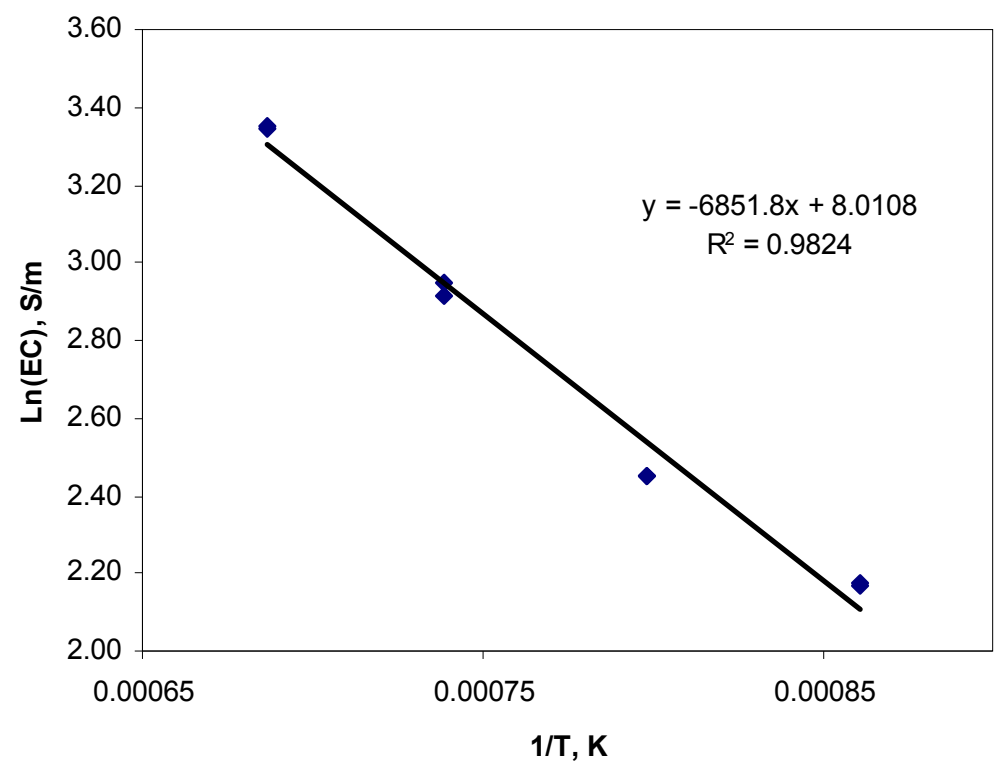

Figure C.41. Electrical Conductivity Trend for EM07-NM-0025 over Inverse Temperature 


\section{Appendix D: Optical $T_{L}$ Experiments and Observations}

This section summarizes the results of heat treatments and optical observations performed on the EM07 and HWL glasses They are sorted alphabetically by sample ID (Content column) and then by the date/time that the heat treatment was initiated.

Table D.1. Summary of Liquidus Temperature $\left(T_{L}\right)$ Heat Treatments and Optical Observations for EM07 and HWL Glasses as well as the Reference Material. ("Fur" refers the furnace used for that a particular heat treatment, "TS" means that a thin section as made of this specimen, and the strikethrough entries represent specimens that were only made for XRD purposes - no optical data collected).

\begin{tabular}{|c|c|c|c|c|c|c|c|c|}
\hline Content & Fur & IN/H.T.START & TEMP & OUT/H.T. FINISH & Dur. (hrs) & Undis. $N M$ & Spinel & Total Observations \\
\hline EM07-Al-06 & 10 & 10/25/2007 16:44 & 1201 & 10/26/2007 15:40 & 22.9 & NO & NO & Sample clear \\
\hline EM07-Al-06 & 8 & 10/25/2007 16:44 & 996 & 10/26/2007 15:40 & 22.9 & YES & YES & Lots of spinel, some undissolved material \\
\hline EM07-Al-06 & 5 & 10/25/2007 16:44 & 1099 & 10/26/2007 15:40 & 22.9 & NO & NO & Sample clear \\
\hline EM07-Al-06 & 10 & 10/29/2007 17:36 & 1173 & 10/30/2007 16:25 & 22.8 & NO & NO & Sample clear \\
\hline EM07-Al-06 & 5 & 10/30/2007 17:36 & 1051 & 10/31/2007 16:50 & 23.2 & YES & YES & Lots of spinel, Ru present \\
\hline EM07 A1-06 & 5 & 11/2/200716:30 & 1078 & $11 / 3 / 200715: 29$ & 23.0 & - & - & Volatilized out of encible \\
\hline EM07-Al-06 & 10 & 11/4/2007 16:30 & 1074 & $11 / 5 / 200714: 20$ & 21.8 & YES & YES & Very few spinel, some other undissolved material present \\
\hline EM07-Al-06 & 10 & 11/12/2007 16:41 & 1085 & 11/13/2007 15:07 & 22.4 & YES & NO & Sample clear of spinel, some Ru \\
\hline EM07-Al-06 & 10 & 11/14/2007 15:26 & 1077 & 11/15/2007 15:37 & 24.2 & YES & NO & No spinel, some other undissolved materials \\
\hline EM07 A1-06 & 10 & 1/16/200811:05 & 947 & $1 / 17 / 20089: 20$ & 22.3 & $\overline{-}$ & $\overline{-}$ & XRDsample only \\
\hline EM07A1-06 & 5 & 1/28/200814:40 & 1026 & 1/29/2008 14:40 & 24.0 & - & - & XRD sample only \\
\hline EM07-Al-15 & 10 & 10/30/2007 17:36 & 1190 & $10 / 31 / 200716: 50$ & 23.2 & YES & YES & Lots of spinel and other undissolved material \\
\hline EM07-Al-15 & 8 & $10 / 30 / 200717: 36$ & 1151 & $10 / 31 / 200716: 50$ & 23.2 & YES & YES & Lots of spinel, some undissolved material \\
\hline EM07-Al-15 & 5 & 10/30/2007 17:36 & 1051 & 10/31/2007 16:50 & 23.2 & YES & YES & Highly crystallized, Ru present \\
\hline EM07-Al-15 & 5 & 10/31/2007 18:33 & 1262 & $11 / 1 / 200716: 35$ & 22.0 & YES & YES & Redox, several spinel, some other undissolved materials \\
\hline EM07-Al-15 & 5 & $11 / 1 / 200717: 45$ & 1281 & $11 / 2 / 200715: 55$ & 22.2 & $?$ & YES & Brown in color, redox, very dark, some large spinel \\
\hline EM07-Al-15 & 5 & 11/8/2007 17:12 & 1301 & 11/9/2007 15:40 & 22.5 & $?$ & YES & Very dark, redox, few spinel, other undissolved mat'ls at base \\
\hline EM07 $A 115$ & 5 & $+2 / 20 / 200713: 22$ & +322 & $12 / 20 / 200717: 15$ & 3.9 & - & $=$ & Volatilize out of erueible \\
\hline EM07-Al-15 & 5 & $12 / 22 / 200711: 30$ & 1343 & 12/22/2007 15:50 & 4.3 & NO & NO & Redox visible (appears to be a glass/ceramic) no spinel visible \\
\hline EM07-Al-15 & 5 & $1 / 3 / 200814: 00$ & 1330 & $1 / 3 / 200818: 14$ & 4.2 & $?$ & $?$ & Redox visible, sample clear of spinel as observed \\
\hline EM07-Al-15 & 8 & $1 / 9 / 200814: 35$ & 1314 & 1/9/2008 18:20 & 3.8 & $?$ & $?$ & Redox visible, sample appears clear of spinel or other undissolved materials \\
\hline EM07-Al-15 & 5 & 1/17/2008 14:05 & 1308 & 1/17/2008 17:40 & 3.6 & ? & YES & Redox visible, few crystals throughout bulk (star-like but geometric) \\
\hline EM07-Al-15 & 5 & $1 / 24 / 200814: 24$ & 1318 & $1 / 24 / 200818: 21$ & 3.9 & Und. mat'l & Crys. & Redox, one crystal, one agglomerate (noble metals) \\
\hline EM07-Al-15 & 5 & 2/1/20089:30 & 1327 & $2 / 1 / 200814: 20$ & 4.8 & NO & NO & Sample clear of large spinels - lots of small particles (due to air quench) \\
\hline EM07-Al-15 & 5 & $2 / 7 / 200810: 00$ & 1326 & 2/7/200814:00 & 4.0 & 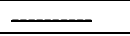 & 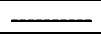 & XRD sample only \\
\hline EM07-Al-20 & 10 & 11/6/2007 15:55 & 1227 & 11/7/2007 15:30 & 23.6 & YES & YES & Several spinel and other undissolved materials \\
\hline EM07-Al-20 & 8 & 11/6/2007 15:55 & 1140 & 11/7/2007 15:30 & 23.6 & YES & YES & Extremely crystallized with spinel, undissolved material \\
\hline EM07-Al-20 & 5 & 11/6/2007 15:55 & 1065 & 11/7/2007 15:30 & 23.6 & YES & YES & Extremely crystallized with spinel, undissolved material \\
\hline EM07-Al-20 & 8 & 11/8/2007 17:12 & 1327 & 11/9/2007 15:40 & 22.5 & ? & YES & Redox, dark brown, volatilized components, spinel, some other undissolved materials \\
\hline EM07-Al-20 & 5 & 11/8/2007 17:12 & 1301 & 11/9/2007 15:40 & 22.5 & $?$ & YES & Very dark, redox, spinel, other undissolved materials \\
\hline EM07-Al-20 & 5 & 12/20/2007 13:22 & 1322 & 12/20/2007 17:15 & 3.9 & YES & YES & Redox visible, very few spinel \\
\hline EM07-A1-20 & 5 & $12 / 28 / 200711: 14$ & 1361 & $12 / 28 / 200715: 30$ & 4.3 & $\overline{-}$ & 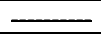 & Volatilized out of crucible \\
\hline EM07-Al-20 & 5 & 1/2/2008 14:34 & 1364 & 1/2/2008 18:34 & 4.0 & ? & ? & Redox visible, sample appears to be clear \\
\hline EM07-Al-20 & 5 & 1/8/2008 12:01 & 1350 & $1 / 8 / 200816: 30$ & 4.5 & $?$ & YES & Redox visible, one spinel surrounded by light halo \\
\hline EM07-Al-20 & 5 & 1/15/2008 12:02 & 1360 & 1/15/2008 17:05 & 5.1 & $?$ & YES & Redox visible, some spinel at base surrounded by like halo \\
\hline
\end{tabular}




\begin{tabular}{|c|c|c|c|c|c|c|c|c|}
\hline Content & Fur & IN/H.T.START & TEMP & OUT/H.T. FINISH & Dur. (hrs) & Undis. $N M$ & Spinel & Total Observations \\
\hline EM07-Al-225 & 5 & 7/21/2009 15:00 & 1152 & 7/21/2009 15:00 & 0.0 & 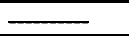 & YES & Bad glass \\
\hline EM07-Al-225 & 8 & $7 / 23 / 200915: 00$ & 1275 & $7 / 24 / 200913: 35$ & 22.6 & - & YES & Crystals throughout \\
\hline EM07-Al-225 & 5 & 9/2/2009 11:30 & 1320 & 9/3/2009 9:30 & 22.0 & 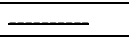 & YES & Crystals \\
\hline EM07-Al-225 & 5 & $\begin{array}{l}\text { 9/14/2009 10:45 } \\
\end{array}$ & 1340 & 9/15/2009 9:00 & 22.3 & 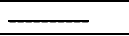 & YES & Crystals \\
\hline EM07-Al-225 & 5 & 9/22/2009 11:40 & 1380 & 9/22/2009 15:40 & 4.0 & $\overline{-}$ & YES & Crystals \\
\hline EM07-Al-225 & 5 & 10/5/2009 9:20 & 1400 & 10/5/2009 13:20 & 4.0 & $\longrightarrow$ & YES & Spinel \\
\hline EM07-Al-225 & 5 & 10/9/2009 9:30 & 1438 & 10/9/2009 13:40 & 4.2 & 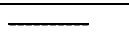 & YES & Sponel \\
\hline EM07-Al-225 & 5 & 10/9/2009 15:15 & 1428 & 10/9/2009 19:35 & 4.3 & - & YES & Spinel \\
\hline EM07-Al-225 & 5 & $10 / 13 / 2009$ 10:30 & 1446 & 10/13/2009 14:30 & 4.0 & - & YES & Spinel \\
\hline EM07-Al-225 & 5 & $10 / 13 / 200914: 45$ & 1456 & $10 / 13 / 200918: 50$ & 4.1 & 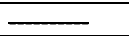 & YES & Spinel peppered \\
\hline EM07-Al-225 & 5 & 10/14/2009 12:00 & 1465 & 10/14/2009 17:00 & 5.0 & - & YES & Spinel peppered \\
\hline EM07-Al-225 & 5 & 10/16/2009 9:00 & 1476 & 10/16/2009 13:40 & 4.7 & 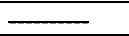 & YES & Spinel peppered \\
\hline EM07-B-05 & 10 & $10 / 7 / 200714: 37$ & 942 & 10/8/2007 15:30 & 24.9 & YES & YES & Surface crystallization, undissolved materials, spinel everywhere \\
\hline EM07-B-05 & 8 & 10/7/2007 14:38 & 1042 & 10/8/2007 15:30 & 24.9 & YES & YES & Surface crystallization, undissolved materials, spinel everywhere \\
\hline EM07-B-05 & 10 & 10/10/2007 15:15 & 1157 & 10/11/2007 13:40 & 22.4 & YES & YES & Several spinel, some undissolved material \\
\hline EM07-B-05 & 10 & 10/11/2007 16:23 & 1247 & 10/12/2007 14:30 & 22.1 & NO & NO & Sample clear \\
\hline EM07-B-05 & 10 & 10/17/2007 17:28 & 1202 & 10/18/2007 15:31 & 22.0 & YES & YES & Lots of spinel, some undissolved material \\
\hline EM07-B-05 & 5 & 10/29/2007 17:36 & 1238 & 10/30/2007 16:25 & 22.8 & NO & NO & Sample clear \\
\hline EM07-B-05 & 10 & 11/3/2007 16:30 & 1220 & 11/4/2007 15:18 & 22.8 & YES & YES & Few small spinel throughout bulk, some Ru \\
\hline EM07-B-05 & 10 & $11 / 6 / 200715: 55$ & 1227 & 11/7/2007 15:30 & 23.6 & $?$ & $?$ & Sample clear aside from a strange undissolved agglomerate at base \\
\hline EM07-B-05 & 10 & 11/9/2007 16:42 & 1230 & 11/10/2007 14:56 & 22.2 & YES & YES & Very few large spinels, some other undissolved materials \\
\hline EM07-B-05 & 10 & 11/13/2007 15:55 & 1239 & 11/14/2007 14:18 & 22.4 & NO & NO & Sample clear \\
\hline EM07 B-05 & 10 & 1/17/2008 13:40 & 1054 & 1/18/200812:00 & 22.3 & $\overline{-}$ & 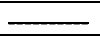 & XRD sample only \\
\hline EM07-B-05 & 10 & 1/23/2008 17:49 & 1103 & $1 / 24 / 200817: 45$ & 23.9 & $\overline{-1}$ & $\overline{-}$ & XRD sample only \\
\hline EM07-B-125 & 5 & 7/21/2009 15:00 & 1152 & 7/21/2009 15:00 & 0.0 & $\overline{-}$ & YES & Crystals throughout \\
\hline EM07-B-125 & 5 & $7 / 30 / 200914: 35$ & 1234 & $7 / 31 / 200914: 50$ & 24.2 & $\overline{-}$ & YES & Lightly peppered \\
\hline EM07-B-125 & 5 & $10 / 1 / 200914: 25$ & 1243 & $10 / 1 / 200914: 25$ & 0.0 & $\ldots$ & YES & Bottom layer and peppered throughout \\
\hline EM07-B-125 & 8 & $10 / 13 / 200914: 45$ & 1252 & 10/14/2009 11:45 & 21.0 & - & YES & Spinel bottom layer \\
\hline EM07-B-125 & 8 & 10/14/2009 12:00 & 1261 & 10/15/2009 10:15 & 22.3 & $\overline{-}$ & YES & 1 spinel glob on bottom \\
\hline EM07-B-125 & 8 & 10/15/2009 13:35 & 1271 & 10/16/2009 11:35 & 22.0 & $\overline{-}$ & YES & 1 spinel glob on bottom \\
\hline EM07-B-125 & 8 & 10/16/2009 12:00 & 1281 & 10/17/2009 13:10 & 25.2 & - & YES & Tiny spinel \\
\hline EM07-B-125 & 8 & 10/17/2009 13:20 & 1291 & 10/18/2009 13:35 & 24.2 & $\overline{-}$ & YES & Tiny spinel \\
\hline EM07-B-15 & 10 & 10/7/2007 14:37 & 942 & 10/8/2007 15:30 & 24.9 & YES & YES & Undissolved materials, spinel everywhere \\
\hline EM07-B-15 & 8 & 10/7/2007 14:38 & 1042 & 10/8/2007 15:30 & 24.9 & YES & YES & Undissolved materials, spinel everywhere \\
\hline EM07-B-15 & 10 & 10/10/2007 15:15 & 1157 & 10/11/2007 13:40 & 22.4 & YES & YES & Very small quantity of spinel, few undissolved Ru agglomerates \\
\hline EM07B-15 & 10 & $10 / 11 / 200716: 23$ & 1247 & $10 / 12 / 200714: 30$ & 22.1 & $\overline{-}$ & $\overline{-}$ & $\overline{-}$ \\
\hline EM07-B-15 & 10 & 10/15/2007 17:17 & 1254 & 10/16/2007 17:50 & 24.6 & NO & NO & Sample clear \\
\hline EM07-B-15 & 5 & 10/18/2007 17:13 & 1220 & 10/19/2007 15:15 & 22.0 & NO & NO & Sample is clear \\
\hline EM07-B-15 & 10 & 10/22/2007 16:20 & 1187 & $10 / 23 / 200716: 53$ & 24.6 & NO & NO & Sample clear \\
\hline EM07-B-15 & 10 & 10/26/2007 17:08 & 1184 & 10/27/2007 15:20 & 22.2 & YES & $\mathrm{NO}$ & Sample clear of spinel, some $\mathrm{Ru}$ \\
\hline EM07-B-15 & 10 & 10/29/2007 17:36 & 1173 & 10/30/2007 16:25 & 22.8 & NO & NO & Sample clear \\
\hline EM07-B-15 & 10 & $11 / 1 / 200717: 45$ & 1165 & $11 / 2 / 200715: 55$ & 22.2 & YES & YES & Very few spinel, some undissolved agglomerates \\
\hline EM07B-15 & 10 & 1/17/2008 13:40 & 1054 & 1/18/200812:00 & 22.3 & $\overline{-}$ & $\overline{-}$ & XRD sample only \\
\hline EM07-B-15 & 10 & $1 / 23 / 200817: 49$ & 1103 & $1 / 24 / 200817: 45$ & 23.9 & - & 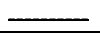 & XRD sample only \\
\hline EM07 B 15 & 8 & $1 / 28 / 200814: 40$ & 999 & $1 / 29 / 200814: 40$ & 24.0 & $\overline{-}$ & $\overline{-}$ & XRD sample only \\
\hline EM07-B-15 & 10 & $1 / 31 / 200817: 15$ & 974 & 2/1/2008 15:54 & 22.6 & 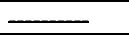 & 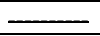 & XRD sample only \\
\hline EM07-B-175 & 5 & 7/21/2009 15:00 & 1152 & $7 / 21 / 200915: 00$ & 0.0 & 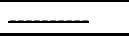 & YES & Peppered throughout \\
\hline
\end{tabular}




\begin{tabular}{|c|c|c|c|c|c|c|c|c|}
\hline Content & Fur & IN/H.T.START & TEMP & OUT/H.T. FINISH & Dur. (hrs) & Undis. $N M$ & Spinel & Total Observations \\
\hline EM07-B-175 & 5 & $7 / 30 / 200914: 35$ & 1234 & $7 / 31 / 200914: 50$ & 24.2 & $=$ & YES & 1 small blob on bottom \\
\hline EM07-B-175 & 5 & $8 / 24 / 200915: 55$ & 1190 & $8 / 25 / 200914: 00$ & 22.1 & 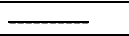 & YES & Small crystal blobs on bottom \\
\hline EM07-B-175 & 5 & 10/2/2009 16:00 & 1201 & 10/2/2009 16:00 & 0.0 & $\overline{-}$ & YES & Spinel glob \\
\hline EM07-B-175 & 5 & $10 / 3 / 200916: 30$ & 1211 & 10/4/2009 14:30 & 22.0 & $\overline{-}$ & YES & Spinel glob \\
\hline EM07-B-175 & 5 & 10/6/2009 15:30 & 1221 & 10/7/2009 14:40 & 23.2 & $\overline{-}$ & $\mathrm{N} / \mathrm{A}$ & Boiled out \\
\hline EM07-B-175 & 5 & 10/10/2009 15:10 & 1245 & 10/11/2009 14:00 & 22.8 & $\overline{-}$ & NO & Glass \\
\hline EM07-B-175 & 5 & $10 / 17 / 200916: 00$ & 1221 & $10 / 8 / 200915: 00$ & -217.0 & - & YES & 1 spinel glob on bottom \\
\hline EM07-B-20 & 10 & 10/7/2007 14:37 & 942 & $10 / 8 / 200715: 30$ & 24.9 & YES & YES & Undissolved materials, spinel everywhere \\
\hline EM07-B-20 & 8 & 10/7/2007 14:38 & 1042 & 10/8/2007 15:30 & 24.9 & YES & YES & Lots of undissolved material, spinel everywhere \\
\hline EM07-B-20 & 10 & 10/10/2007 15:15 & 1157 & 10/11/2007 13:40 & 22.4 & YES & YES & Sample clear of spinel, one small undissolved Ru agglomerate \\
\hline EM07-B-20 & 10 & 10/11/2007 16:23 & 1247 & 10/12/2007 14:30 & 22.1 & NO & NO & Sample clear \\
\hline EM07-B-20 & 5 & $10 / 16 / 200718: 12$ & 1102 & $10 / 17 / 200716: 45$ & 22.5 & YES & YES & Several spinel, some undissolved material \\
\hline EM07-B-20 & 8 & 10/18/2007 17:13 & 1123 & 10/19/2007 15:15 & 22.0 & YES & YES & Lots of little spinel, some undissolved material \\
\hline EM07-B-20 & 8 & 10/22/2007 16:20 & 1141 & 10/23/2007 16:53 & 24.6 & 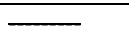 & 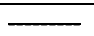 & $\ldots$ \\
\hline EM07-B-20 & 8 & 10/24/2007 16:20 & 1160 & 10/25/2007 15:52 & 23.5 & YES & NO & Sample clear of spinel, some Ru \\
\hline EM07-B-20 & 8 & 10/26/2007 17:08 & 1134 & 10/27/2007 15:20 & 22.2 & YES & YES & Several spinel, some undissolved material \\
\hline EM07-B-20 & 8 & 10/30/2007 17:36 & 1151 & 10/31/2007 16:50 & 23.2 & YES & YES & Lots of spinel, some undissolved material \\
\hline EM07-B-20 & 8 & 11/1/2007 17:45 & 1149 & $11 / 2 / 200715: 55$ & 22.2 & YES & NO & Sample clear \\
\hline EM07-B-20 & 8 & $11 / 6 / 200715: 55$ & 1140 & 11/7/2007 15:30 & 23.6 & YES & NO & Sample clear of spinel, a few undissolved agglomerates \\
\hline EM07-Bi-025 & 10 & 10/7/2007 14:37 & 942 & 10/8/2007 15:30 & 24.9 & YES & YES & Undissolved materials, spinel everywhere \\
\hline EM07-Bi-025 & 8 & 10/7/2007 14:38 & 1042 & 10/8/2007 15:30 & 24.9 & YES & YES & Undissolved materials, spinel everywhere \\
\hline EM07-Bi-025 & 10 & 10/10/2007 15:15 & 1157 & 10/11/2007 13:40 & 22.4 & YES & YES & Few spinel, very small quantity of undissolved material \\
\hline EM07-Bi-025 & 10 & 10/11/2007 16:23 & 1247 & 10/12/2007 14:30 & 22.1 & NO & NO & Sample clear \\
\hline EM07-Bi-025 & 10 & 10/17/2007 17:28 & 1202 & 10/18/2007 15:31 & 22.0 & NO & NO & Sample clear \\
\hline EM07-Bi-025 & 10 & 10/21/2007 14:45 & 1177 & 10/22/2007 15:10 & 24.4 & YES & YES & Very few spinel, some $\mathrm{RuO} 2$ agglomerates \\
\hline EM07-Bi-025 & 10 & 10/23/2007 17:37 & 1190 & 10/24/2007 15:57 & 22.3 & YES & NO & Sample clear of spinel, a few small undissolved agglomerates \\
\hline EM07-Bi-025 & 10 & 10/24/2007 16:20 & 1194 & 10/25/2007 15:52 & 23.5 & YES & NO & Sample clear of spinel, one small undissolved Ru agglomerate \\
\hline EM07-Bi-025 & 10 & 10/26/2007 17:08 & 1184 & 10/27/2007 15:20 & 22.2 & NO & NO & Sample clear \\
\hline EM07 Bi 025 & 10 & 1/23/2008 17:49 & 1103 & $1 / 24 / 200817: 45$ & 23.9 & - & - & XRD sample only \\
\hline EM07-Bi-05 & 10 & 10/7/2007 14:37 & 942 & 10/8/2007 15:30 & 24.9 & YES & YES & Surface crystallization, undissolved materials, spinel everywhere \\
\hline EM07-Bi-05 & 8 & 10/7/2007 14:38 & 1042 & 10/8/2007 15:30 & 24.9 & YES & YES & Undissolved materials, spinel everywhere \\
\hline EM07-Bi-05 & 10 & 10/10/2007 15:15 & 1157 & 10/11/2007 13:40 & 22.4 & YES & YES & Few spinel, very small quantity of undissolved material \\
\hline EM07-Bi-05 & 10 & 10/11/2007 16:23 & 1247 & 10/12/2007 14:30 & 22.1 & NO & NO & Sample clear \\
\hline EM07-Bi-05 & 10 & 10/17/2007 17:28 & 1202 & 10/18/2007 15:31 & 22.0 & NO & NO & Sample clear \\
\hline EM07-Bi-05 & 10 & $10 / 21 / 200714: 45$ & 1177 & 10/22/2007 15:10 & 24.4 & YES & NO & Sample clear of spinel, a few small undissolved Ru agglomerates \\
\hline EM07-Bi-05 & 10 & 10/27/2007 16:24 & 1167 & 10/28/2007 14:27 & 22.0 & YES & YES & Very few spinel \\
\hline EM07-Bi-05 & 10 & 11/1/2007 17:45 & 1165 & 11/2/2007 15:55 & 22.2 & YES & YES & Very few spinel, some undissolved agglomerates \\
\hline EM07-Bi-05 & 10 & 11/8/2007 17:12 & 1170 & $11 / 9 / 200715: 40$ & 22.5 & YES & YES & Very few spinel, some undissolved agglomerates \\
\hline EM07 Bi-05 & 10 & $1 / 23 / 200817: 49$ & 1103 & $1 / 24 / 200817: 45$ & 23.9 & - & $\overline{-}$ & XRD sample only \\
\hline EM07 BL 4 & 10 & $9 / 26 / 200715: 30$ & E & $9 / 27 / 200715: 52$ & 24.4 & NO & NO & Sample clear \\
\hline EM07-BL-1 & 10 & 9/27/2007 16:07 & $\ldots$ & $9 / 28 / 200714: 15$ & 22.1 & YES & YES & Ruthenium throughout, several small spinel \\
\hline EM07-BL-1 & 8 & 10/4/2007 16:06 & 1048 & $10 / 5 / 200715: 00$ & 22.9 & YES & YES & Undissolved materials, spinel everywhere \\
\hline EM07-BL-1 & 10 & 10/4/2007 16:06 & 944 & 10/5/2007 15:00 & 22.9 & YES & YES & Surface pigmentation, extremely crystallized w/ spinel \\
\hline EM07-BL-1 & 8 & 10/10/2007 15:15 & 1148 & 10/11/2007 13:40 & 22.4 & YES & YES & Lots of spinel, some undissolved material \\
\hline EM07-BL-1 & 8 & $10 / 11 / 200716: 23$ & 1251 & $10 / 12 / 200714: 30$ & 22.1 & NO & NO & Sample clear \\
\hline EM07-BL-1 & 10 & 10/17/2007 17:28 & 1202 & 10/18/2007 15:31 & 22.0 & $\mathrm{NO}$ & $\mathrm{NO}$ & Sample clear \\
\hline EM07-BL-1 & 10 & $10 / 21 / 200714: 45$ & 1177 & 10/22/2007 15:10 & 24.4 & YES & YES & Several spinel, some undissolved material \\
\hline
\end{tabular}




\begin{tabular}{|c|c|c|c|c|c|c|c|c|}
\hline Content & Fur & IN /H.T. START & TEMP & OUT/H.T. FINISH & Dur. (hrs) & Undis. $N M$ & Spinel & Total Observations \\
\hline EM07-BL-1 & 10 & 10/23/2007 17:37 & 1190 & 10/24/2007 15:57 & 22.3 & YES & $\mathrm{NO}$ & Sample clear of spinel, a few small undissolved agglomerates \\
\hline EM07-BL-1 & 10 & $10 / 24 / 200716: 20$ & 1194 & 10/25/2007 15:52 & 23.5 & NO & YES & One small cluster of spinel \\
\hline EM07-BL-1 & 10 & 10/26/2007 17:08 & 1184 & 10/27/2007 15:20 & 22.2 & YES & $\mathrm{NO}$ & No spinel observed, some Ru \\
\hline EM07-BL-1 & 10 & $10 / 28 / 200714: 45$ & 1200 & 10/29/2007 15:35 & 24.8 & YES & YES & Very few spinel near bubble on side \\
\hline EM07-BL-1 & 10 & $10 / 31 / 200718: 33$ & 1208 & 11/1/2007 16:35 & 22.0 & NO & NO & Sample clear \\
\hline EM07-BL-1 & 10 & $1 / 17 / 200813: 40$ & 1051 & 1/18/2008 12:00 & 22.3 & $\longrightarrow$ & $\overline{-}$ & XRD sample only \\
\hline EM07-BL-1 & 10 & $1 / 23 / 200817: 49$ & 1103 & $1 / 24 / 200817: 45$ & 23.9 & 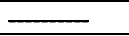 & - & XRD sample only \\
\hline EM07-BL-2 & 10 & $10 / 25 / 200716: 44$ & 1201 & 10/26/2007 15:40 & 22.9 & NO & YES & Very small clusters of spinel $(\mathrm{TL} \sim+5-10)$ \\
\hline EM07-BL-2 & 8 & 10/25/2007 16:44 & 996 & 10/26/2007 15:40 & 22.9 & YES & YES & Highly crysallized, Ru present \\
\hline EM07-BL-2 & 5 & $10 / 25 / 200716: 44$ & 1099 & $10 / 26 / 200715: 40$ & 22.9 & YES & YES & Lots of spinel and other undissolved material \\
\hline EM07-BL-2 & 10 & $10 / 29 / 200717: 36$ & 1173 & $10 / 30 / 200716: 25$ & 22.8 & $\overline{-}$ & $\overline{-\infty}$ & $\overline{-}$ \\
\hline EM07-BL-2 & 10 & $10 / 30 / 200717: 36$ & 1190 & 10/31/2007 16:50 & 23.2 & NO & $\mathrm{NO}$ & Sample clear \\
\hline EM07-BL-2 & 10 & $10 / 31 / 200718: 33$ & 1208 & $11 / 1 / 200716: 35$ & 22.0 & NO & NO & Sample clear \\
\hline EM07-BL-2 & 10 & 1/17/2008 13:40 & 1051 & 1/18/2008 12:00 & 22.3 & $\overline{-n}$ & $\overline{-m}$ & XRD sample only \\
\hline EM07-BL-2 & 10 & 1/23/2008 17:49 & 1103 & 1/24/2008 17:45 & 23.9 & $\overline{-}$ & $\overline{-}$ & XRD sample only \\
\hline EM07-Ca-035 & 10 & 10/14/2007 12:20 & 1251 & $10 / 15 / 200711: 50$ & 23.5 & 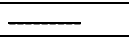 & $\overline{-}$ & 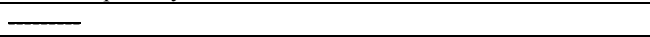 \\
\hline EM07-Ca-035 & 8 & 10/14/2007 12:20 & 1150 & 10/15/2007 11:50 & 23.5 & NO & NO & Sample clear \\
\hline EM07-Ca-035 & 5 & 10/14/2007 12:20 & 1053 & $10 / 15 / 200711: 50$ & 23.5 & YES & YES & Lots of spinel, some undissolved material \\
\hline EM07-Ca-035 & 10 & 10/15/2007 17:17 & 1254 & $10 / 16 / 200717: 50$ & 24.6 & $\overline{-1}$ & $\overline{-1}$ & $\overline{-}$ \\
\hline EM07-Ca-035 & 8 & 10/18/2007 17:13 & 1123 & 10/19/2007 15:15 & 22.0 & YES & YES & A few spinel, some undissolved material \\
\hline EM07-Ca-035 & 8 & $10 / 22 / 200716: 20$ & 1141 & $10 / 23 / 200716: 53$ & 24.6 & NO & $\mathrm{NO}$ & Sample clear \\
\hline EM07-Ca-035 & 8 & 10/26/2007 17:08 & 1134 & 10/27/2007 15:20 & 22.2 & YES & YES & Very small quantity of spinel, some Ru \\
\hline EM07Ca 035 & 10 & 1/8/2008 18:14 & 915 & 1/10/200816:08 & 45.9 & $\overline{-}$ & 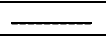 & XRD sample only \\
\hline EM07-Ca-035 & 10 & 1/16/2008 11:05 & 947 & 1/17/20089:20 & 22.3 & $\overline{-}$ & 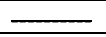 & XRD sample only \\
\hline EM07-Ca-035 & 10 & 1/23/2008 17:49 & 1103 & 1/24/2008 17:45 & 23.9 & $\overline{-}$ & $\overline{-}$ & XRD sample only \\
\hline EM07Ca-035 & 8 & 1/28/2008 14:40 & 999 & 1/29/2008 14:40 & 24.0 & $\overline{-}$ & $\underline{-}$ & XRDsample only \\
\hline EM07-Ca-07 & 10 & 10/7/2007 14:37 & 942 & 10/8/2007 15:30 & 24.9 & YES & YES & Surface crystallization, undissolved materials, spinel everywhere \\
\hline EM07-Ca-07 & 8 & 10/7/2007 14:38 & 1042 & 10/8/2007 15:30 & 24.9 & YES & YES & Few spinel, very small quantity of undissolved material \\
\hline EM07-Ca-07 & 10 & 10/10/2007 15:15 & 1157 & 10/11/2007 13:40 & 22.4 & YES & YES & Sample clear \\
\hline EM07-Ca-07 & 10 & 10/11/2007 16:23 & 1247 & 10/12/2007 14:30 & 22.1 & $\overline{-}$ & $\bar{\ldots}$ & $\overline{-}$ \\
\hline EM07Ca 07 & 10 & 10/15/2007 17:17 & 1254 & 10/16/200717:50 & 24.6 & - & - & - \\
\hline EM07-Ca-07 & 5 & 10/16/2007 18:12 & 1102 & $10 / 17 / 200716: 45$ & 22.5 & NO & NO & Sample clear \\
\hline EM07-Ca-07 & 5 & $10 / 21 / 200714: 45$ & 1073 & 10/22/2007 15:10 & 24.4 & YES & YES & Lots of little spinel, some undissolved material \\
\hline EM07-Ca-07 & 5 & 10/23/2007 17:37 & 1089 & 10/24/2007 15:57 & 22.3 & YES & YES & Few spinel agglomerates at base, some Ru agglomerates at base \\
\hline EM07-Ca-07 & 5 & $10 / 25 / 200716: 44$ & 1099 & 10/26/2007 15:40 & 22.9 & YES & YES & Many spinel clusters at base of sample along with $\mathrm{Ru}$ \\
\hline EM07Ca-07 & 8 & $12 / 27 / 200717: 20$ & 1003 & $12 / 28 / 200715: 45$ & 22.4 & - & - & XRD-sample only \\
\hline EM07-Cr-001 & 10 & $10 / 25 / 200716: 44$ & 1201 & 10/26/2007 15:40 & 22.9 & $\overline{-}$ & $\overline{-}$ & Sample volatilized out of conical crucible \\
\hline EM07-Cr-001 & 8 & $10 / 25 / 200716: 44$ & 996 & 10/26/2007 15:40 & 22.9 & YES & YES & Highly crystallized, Ru present \\
\hline EM07-Cr-001 & 5 & 10/25/2007 16:44 & 1099 & $10 / 26 / 200715: 40$ & 22.9 & YES & YES & Small clusters at the base of the sample \\
\hline EM07-Cr-001 & 5 & $10 / 30 / 200717: 36$ & 1051 & 10/31/2007 16:50 & 23.2 & YES & YES & Lots of small spinel, Ru present \\
\hline EM07-Cr-001 & 10 & 11/1/2007 17:45 & 1165 & $11 / 2 / 200715: 55$ & 22.2 & NO & NO & Sample clear \\
\hline EM07-Cr-001 & 8 & 11/1/2007 17:45 & 1149 & $11 / 2 / 200715: 55$ & 22.2 & NO & NO & Sample clear \\
\hline EM07-Cr-001 & 8 & 11/5/2007 15:00 & 1224 & 11/6/2007 15:54 & 24.9 & YES & NO & No spinel, a few $\mathrm{Ru}$ \\
\hline EM07-Cr-001 & 8 & 11/7/2007 16:42 & 1112 & $11 / 8 / 200715: 40$ & 23.0 & YES & NO & No spinel, some undissolved materials present \\
\hline EM07-Cr-001 & 8 & 11/9/2007 16:42 & 1105 & $11 / 10 / 200714: 56$ & 22.2 & NO & NO & Sample clear \\
\hline EM07-Cr-001 & 8 & 11/28/2007 16:04 & 1098 & 11/29/2007 14:21 & 22.3 & $\mathrm{NO}$ & $\mathrm{NO}$ & Sample clear \\
\hline EM07-Cr-001 & 8 & 12/2/2007 14:57 & 1091 & $12 / 3 / 200714: 30$ & 23.5 & YES & NO & Sample clear of spinel, several undissolved agglomerates \\
\hline
\end{tabular}




\begin{tabular}{|c|c|c|c|c|c|c|c|c|}
\hline Content & Fur & IN/H.T. START & TEMP & OUT / H.T. FINISH & Dur. (hrs) & Undis. $N M$ & Spinel & Total Observations \\
\hline EM07-Cr-001 & 8 & 12/4/2007 17:22 & 1082 & 12/5/2007 16:27 & 23.1 & YES & NO & Sample clear of spinel, lots of other undissolved mat'ls \\
\hline EM07-Cr-001 & 8 & 12/9/2007 14:17 & 1074 & 12/10/2007 15:00 & 24.7 & YES & NO & Several undissolved agglomerates, no spinel \\
\hline EM07-Cr-001 & 8 & 12/14/2007 16:11 & 1065 & $12 / 15 / 200714: 20$ & 22.1 & YES & $?$ & Lots of small cubic crystals (appear to be spinel) some other undissolved mat'ls \\
\hline EM07-Cr-001 & 10 & 1/8/2008 18:14 & 915 & 1/10/2008 16:08 & 45.9 & $\overline{-}$ & 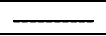 & XRD sample only \\
\hline EM07-Cr-001 & 5 & 1/28/2008 14:40 & 1026 & 1/29/2008 14:40 & 24.0 & $\overline{-}$ & $\overline{-}$ & XRD sample only \\
\hline EM07-Cr-001 & 10 & $1 / 31 / 200817: 15$ & 974 & $2 / 1 / 200815: 54$ & 22.6 & $\overline{-}$ & $\ldots$ & XRD sample only \\
\hline EM07-Cr-012 & 10 & $10 / 25 / 200716: 44$ & 1201 & 10/26/2007 15:40 & 22.9 & YES & YES & Surface crystallization, spinel everywhere, undissolved Ru \\
\hline EM07-Cr-012 & 8 & 10/25/2007 16:44 & 996 & 10/26/2007 15:40 & 22.9 & YES & YES & Highly crystallized, Ru present \\
\hline EM07-Cr-012 & 5 & $10 / 25 / 200716: 44$ & 1099 & 10/26/2007 15:40 & 22.9 & 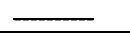 & 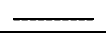 & 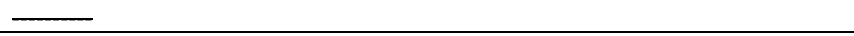 \\
\hline EM07-Cr-012 & 5 & 10/29/2007 17:36 & 1238 & $10 / 30 / 200716: 25$ & 22.8 & YES & YES & Surface crystallization, severely crystallized, some Ru \\
\hline EM07-Cr-012 & 5 & 11/1/2007 17:45 & 1281 & $11 / 2 / 200715: 55$ & 22.2 & YES & YES & Brown in color, redox, lots of spinel and other undissolved material \\
\hline EM07-Cr-012 & 8 & 11/8/2007 17:12 & 1327 & 11/9/2007 15:40 & 22.5 & ? & YES & Redox, dark brown, volatilized components, spinel, some other undissolved materials \\
\hline EM07-Cr-012 & 5 & 12/20/2007 13:22 & 1322 & $12 / 20 / 200717: 15$ & 3.9 & YES & YES & Redox visible, few spinel throughout bulk, some other undissolved mat'ls (Cr?) \\
\hline EM07-Cr-012 & 5 & 12/22/2007 11:30 & 1343 & $12 / 22 / 200715: 50$ & 4.3 & YES & YES & Redox visible (appears to be a glass/ceramic), very few spinel visible \\
\hline EM07-Cr-012 & 5 & $12 / 28 / 200711: 14$ & 1361 & $12 / 28 / 200715: 30$ & 4.3 & $\overline{-}$ & $\overline{-}$ & Volatilized out of crucible \\
\hline EM07-Cr-012 & 5 & 1/2/2008 14:34 & 1364 & $1 / 2 / 200818: 34$ & 4.0 & ? & ? & Redox visible, sample appears to be clear \\
\hline EM07-Cr-012 & 5 & 1/8/2008 12:01 & 1350 & 1/8/2008 16:30 & 4.5 & $?$ & YES & Redox visible, few spinel throughout bulk surrounded by light halo \\
\hline EM07-Cr-012 & 5 & 1/15/2008 12:02 & 1360 & 1/15/2008 17:05 & 5.1 & ? & YES & $\begin{array}{l}\text { Redox visible, very few crystal (multi-faceted geometric shapes) w/ light halo } \\
\text { surrounding }\end{array}$ \\
\hline EM07-Cr-0145 & 5 & 6/17/2009 10:30 & 1110 & $6 / 18 / 20098: 45$ & 22.3 & $\overline{-}$ & YES & Full of crystals \\
\hline EM07-Cr-0145 & 8 & $6 / 17 / 200910: 30$ & 970 & 6/18/2009 8:45 & 22.3 & $\overline{-}$ & YES & \\
\hline EM07-Cr-0145 & 8 & 7/8/2009 15:25 & 1140 & 7/9/2009 13:35 & 22.2 & $\overline{-}$ & YES & Crystals throughout \\
\hline EM07-Cr-0145 & 8 & 7/14/2009 11:45 & 1180 & 7/15/2009 10:10 & 22.4 & - & YES & Crystals throughout \\
\hline EM07-Cr-0145 & 8 & $7 / 16 / 200912: 15$ & 1215 & 7/17/2009 13:20 & 25.1 & 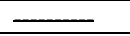 & YES & Crystals throughout \\
\hline EM07-Cr-0145 & 8 & 7/23/2009 15:00 & 1275 & 7/24/2009 13:35 & 22.6 & 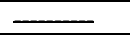 & YES & Crystals throughout \\
\hline EM07-Cr-0145 & 5 & 9/2/2009 11:30 & 1320 & 9/3/2009 9:30 & 22.0 & 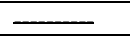 & YES & Crystals scattered throughout \\
\hline EM07-Cr-0145 & 5 & 9/14/2009 10:45 & 1340 & 9/15/20099:00 & 22.3 & 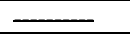 & YES & Crystals \\
\hline EM07-Cr-0145 & 5 & 9/22/2009 11:40 & 1380 & 9/22/2009 15:40 & 4.0 & $\overline{-}$ & YES & Crystals \\
\hline EM07-Cr-0145 & 5 & 10/5/20099:20 & 1400 & 10/5/2009 13:20 & 4.0 & 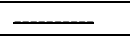 & YES & Spinel \\
\hline EM07-Cr-0145 & 5 & 10/9/20099:30 & 1438 & 10/9/2009 13:40 & 4.2 & - & YES & Spinel \\
\hline EM07-Cr-0145 & 5 & 10/9/2009 15:15 & 1428 & 10/9/2009 19:35 & 4.3 & 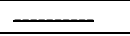 & YES & Spinel \\
\hline EM07-Cr-0145 & 5 & 10/13/2009 10:30 & 1446 & 10/13/2009 14:30 & 4.0 & - & YES & Spinel \\
\hline EM07-Cr-0145 & 5 & $10 / 13 / 200914: 45$ & 1456 & 10/13/2009 18:50 & 4.1 & - & YES & Spinel peppered \\
\hline EM07-Cr-0145 & 5 & 10/14/2009 12:00 & 1465 & 10/14/2009 17:00 & 5.0 & $\overline{-}$ & YES & Spinel peppered \\
\hline EM07-Cr-0145 & 5 & 10/16/2009 9:00 & 1476 & $10 / 16 / 200913: 40$ & 4.7 & $\overline{-}$ & YES & Spinel peppered \\
\hline EM07-Cr-02 & 10 & $10 / 25 / 200716: 44$ & 1201 & $10 / 26 / 200715: 40$ & 22.9 & YES & YES & Surface crystallization, spinel everywhere, undissolved $\mathrm{Ru}$ \\
\hline EM07-Cr-02 & 8 & 10/25/2007 16:44 & 996 & 10/26/2007 15:40 & 22.9 & YES & YES & Surface crystallized, highly crystallized, Ru present \\
\hline EM07-Cr-02 & 5 & $10 / 25 / 200716: 44$ & 1099 & 10/26/2007 15:40 & 22.9 & $\overline{-}$ & $\ldots$ & Surface crystallization \\
\hline EM07-Cr-02 & 5 & 10/29/2007 17:36 & 1238 & $10 / 30 / 200716: 25$ & 22.8 & YES & YES & Surface crystallization, severely crystallized, some Ru \\
\hline EM07-Cr-02 & 5 & $11 / 1 / 200717: 45$ & 1281 & 11/2/2007 15:55 & 22.2 & YES & YES & Surface crystallization, large quantity of spinel and other undissolved materials \\
\hline EM07-Cr-02 & 8 & 11/8/2007 17:12 & 1327 & 11/9/2007 15:40 & 22.5 & $?$ & YES & Redox, dark brown, volatilized components, spinel, some other undissolved materials \\
\hline EM07-Cr-02 & 5 & 12/20/2007 13:22 & 1322 & $12 / 20 / 200717: 15$ & 3.9 & YES & YES & Redox visible, spinel throughout bulk, some other undissolved mat'ls (Cr?) \\
\hline EM07-Cr-02 & 5 & $12 / 22 / 200711: 30$ & 1343 & $12 / 22 / 200715: 50$ & 4.3 & YES & YES & Redox visible, sample packed with spinel, some other undissolved mat'ls (Cr?) \\
\hline EM07-Cr-02 & 5 & 12/28/2007 11:14 & 1361 & $12 / 28 / 200715: 30$ & 4.3 & YES & YES & Redox visible, lots of spinel, other undissolved mat'ls (Cr) \\
\hline EM07-Cr-02 & 5 & 1/10/2008 11:06 & 1418 & $1 / 10 / 200814: 15$ & 3.1 & YES & YES & Redox visible, several large spinel everywhere, other undissolved mat'ls \\
\hline EM07-Cr-02 & 5 & $2 / 12 / 200810: 03$ & 1457 & $2 / 12 / 200814: 42$ & 4.7 & Und. mat'l & Crys. & Long brown crystals, geometric opaque cryst's (spinel?), light brown round crystals \\
\hline EM07-Cr-02 & 5 & $2 / 13 / 200811: 40$ & 1495 & $2 / 13 / 200816: 05$ & 4.4 & Und. mat'l & Crys. & Long brown crystals (light transparent brown) \\
\hline EM07-F-02 & 8 & 10/4/2007 16:06 & 1048 & 10/5/2007 15:00 & 22.9 & YES & YES & Undissolved materials, spinel everywhere \\
\hline
\end{tabular}




\begin{tabular}{|c|c|c|c|c|c|c|c|c|}
\hline Content & Fur & IN/H.T. START & TEMP & OUT/H.T. FINISH & Dur. (hrs) & Undis. $N M$ & Spinel & Total Observations \\
\hline EM07-F-02 & 10 & 10/4/2007 16:06 & 944 & 10/5/2007 15:00 & 22.9 & YES & YES & Undissolved materials, spinel everywhere \\
\hline EM07-F-02 & 8 & 10/10/2007 15:15 & 1148 & 10/11/2007 13:40 & 22.4 & YES & YES & Several spinel, some undissolved material \\
\hline EM07-F-02 & 8 & 10/11/2007 16:23 & 1251 & 10/12/2007 14:30 & 22.1 & NO & NO & Sample clear \\
\hline EM07-F-02 & 10 & 10/17/2007 17:28 & 1202 & 10/18/2007 15:31 & 22.0 & NO & NO & Sample clear \\
\hline EM07-F-02 & 8 & $10 / 21 / 200714: 45$ & 1176 & 10/22/2007 15:10 & 24.4 & YES & YES & Few spinel, very small quantity of undissolved material \\
\hline EM07-F-02 & 8 & 10/23/2007 17:37 & 1191 & 10/24/2007 15:57 & 22.3 & YES & YES & Very few spinel in cluster at base, some undissolved Ru \\
\hline EM07-F-02 & 8 & 11/4/2007 16:30 & 1198 & 11/5/2007 14:20 & 21.8 & YES & NO & No spinel, some undissolved materials present \\
\hline EM07-F-02 & 10 & 1/28/2008 14:40 & 1101 & 1/29/2008 14:40 & 24.0 & $\overline{-}$ & $\overline{-}$ & XRD sample only \\
\hline EM07-Fe-05 & 10 & 10/25/2007 16:44 & 1201 & $10 / 26 / 200715: 40$ & 22.9 & NO & NO & Sample clear \\
\hline EM07-Fe-05 & 8 & 10/25/2007 16:44 & 996 & $10 / 26 / 200715: 40$ & 22.9 & YES & YES & Lots of spinel in sample, some Ru \\
\hline EM07-Fe-05 & 5 & 10/25/2007 16:44 & 1099 & $10 / 26 / 200715: 40$ & 22.9 & NO & NO & Sample clear \\
\hline EM07-Fe-05 & 10 & 10/29/2007 17:36 & 1173 & $10 / 30 / 200716: 25$ & 22.8 & 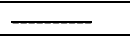 & - & 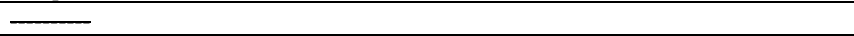 \\
\hline EM07-Fe-05 & 5 & $10 / 30 / 200717: 36$ & 1051 & 10/31/2007 16:50 & 23.2 & YES & YES & Highly crystallized, Ru present \\
\hline EM07-Fe-05 & 5 & 11/2/2007 16:30 & 1078 & 11/3/2007 15:29 & 23.0 & YES & NO & No spinel, some Ru present \\
\hline EM07-Fe-05 & 5 & 11/6/2007 15:55 & 1065 & 11/7/2007 15:30 & 23.6 & YES & YES & Many little spinel throughout in convective patterns, some other undissolved mat'l \\
\hline EM07-Fe-05 & 5 & 11/9/2007 16:42 & 1079 & 11/10/2007 14:56 & 22.2 & YES & YES & Several little spinel, some other undissolved materials \\
\hline EM07-Fe-05 & 5 & 11/13/2007 15:55 & 1090 & 11/14/2007 14:18 & 22.4 & YES & YES & Very few spinel and some other undissolved materials \\
\hline EM07-Fe-05 & 5 & 11/15/2007 16:48 & 1099 & $11 / 16 / 200716: 42$ & 23.9 & YES & NO & No spinel, some other undissolved materials \\
\hline EM07-Fe-05 & 8 & 12/28/2007 17:08 & 844 & $12 / 30 / 200717: 29$ & 48.4 & $\overline{-}$ & $\overline{-1}$ & XRD sample only \\
\hline EM07-Fe-05 & 10 & 1/8/2008 18:14 & 915 & 1/10/2008 16:08 & 45.9 & $\overline{-m}$ & $\overline{-2}$ & XRD sample only \\
\hline EM07-Fe-05 & 10 & 1/16/2008 11:05 & 947 & 1/17/20089:20 & 22.3 & $\ldots$ & $\overline{-m}$ & XRD sample only \\
\hline EM07-Fe-15 & 10 & 10/30/2007 17:36 & 1190 & 10/31/2007 16:50 & 23.2 & YES & YES & Lots of spinel, some undissolved material \\
\hline EM07-Fe-15 & 8 & 10/30/2007 17:36 & 1151 & 10/31/2007 16:50 & 23.2 & YES & YES & Lots of spinel, some undissolved material \\
\hline EM07-Fe-15 & 5 & 10/30/2007 17:36 & 1051 & 10/31/2007 16:50 & 23.2 & YES & YES & Highly crystallized, Ru present \\
\hline EM07-Fe-15 & 5 & 11/1/2007 17:45 & 1281 & 11/2/2007 15:55 & 22.2 & $?$ & YES & Some spinel, redox, very dark, lost some glass formers \\
\hline EM07-Fe-15 & 5 & 11/8/2007 17:12 & 1301 & 11/9/2007 15:40 & 22.5 & - & - & Sample ambiguous with Fe-20 at same run \\
\hline EM07-Fe-15 & 5 & 12/20/2007 13:22 & 1322 & $12 / 20 / 200717: 15$ & 3.9 & $?$ & $?$ & Redox visible, seems to be a glass/ceramic, no spinel seen \\
\hline EM07 Fe 15 & 10 & $12 / 27 / 200717: 20$ & 1214 & $12 / 28 / 200715: 45$ & 22.4 & - & - & XRD sample only \\
\hline EM07-Fe-15 & 5 & 12/31/2007 11:47 & 1301 & $12 / 31 / 200715: 51$ & 4.1 & NO & NO & Redox visible, sample clear \\
\hline EM07-Fe-15 & 5 & 1/7/2008 13:30 & 1214 & 1/7/2008 17:48 & 4.3 & $\overline{-}$ & 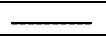 & Volatilized out of crucible \\
\hline EM07-Fe-15 & 5 & 1/9/2008 14:35 & 1292 & 1/9/2008 18:20 & 3.8 & $?$ & $?$ & Redox visible, sample appears clear of spinel or other undissolved materials \\
\hline EM07-Fe-20 & 10 & 10/30/2007 17:36 & 1190 & $10 / 31 / 200716: 50$ & 23.2 & YES & YES & Surface crystallization, lots of spinel and other undissolved material \\
\hline EM07-Fe-20 & 8 & 10/30/2007 17:36 & 1151 & 10/31/2007 16:50 & 23.2 & YES & YES & Lots of spinel, some undissolved material \\
\hline EM07-Fe-20 & 5 & 10/30/2007 17:36 & 1051 & 10/31/2007 16:50 & 23.2 & YES & YES & Surface crystallization, highly crystallized, Ru present \\
\hline EM07-Fe-20 & 5 & 10/31/2007 18:33 & 1262 & $11 / 1 / 200716: 35$ & 22.0 & YES & YES & Volatilized out of crucible, redox, several spinel, other undissolved materials \\
\hline EM07-Fe-20 & 5 & $11 / 1 / 200717: 45$ & 1281 & $11 / 2 / 200715: 55$ & 22.2 & $?$ & YES & Sample volatilized out mostly - lost some glass formers, lots of spinel, redox \\
\hline EM07-Fe-20 & 5 & 11/8/2007 17:12 & 1301 & 11/9/2007 15:40 & 22.5 & $\underline{-}$ & $\overline{-}$ & Sample ambiguous with $\mathrm{Fe}-15$ at same run \\
\hline EM07-Fe-20 & 5 & $12 / 20 / 200713: 22$ & 1322 & $12 / 20 / 200717: 15$ & 3.9 & YES & YES & Redox visible, several spinel in bulk \\
\hline EM07-Fe-20 & 5 & $12 / 22 / 200711: 30$ & 1343 & $12 / 22 / 200715: 50$ & 4.3 & YES & YES & Redox visible (appears to be a glass/ceramic), very few spinel visible \\
\hline EM07-Fe-20 & 5 & $12 / 28 / 200711: 14$ & 1361 & $12 / 28 / 200715: 30$ & 4.3 & NO & NO & Redox visible, no spinel visible, glass-ceramic \\
\hline EM07-Fe-20 & 5 & 1/8/2008 12:01 & 1350 & 1/8/2008 16:30 & 4.5 & ? & ? & $\begin{array}{l}\text { Redox visible, sample appears to be clear of spinel, lots of reflective specks in sample } \\
\text { (Fe?) }\end{array}$ \\
\hline EM07-K -03 & 10 & $10 / 14 / 200712: 20$ & 1251 & $10 / 15 / 200711: 50$ & 23.5 & 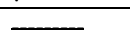 & 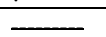 & 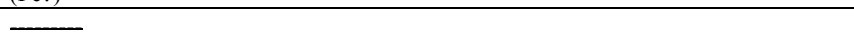 \\
\hline EM07-K-03 & 8 & 10/14/2007 12:20 & 1150 & 10/15/2007 11:50 & 23.5 & YES & YES & Sample clear \\
\hline EM07-K-03 & 5 & $10 / 14 / 200712: 20$ & 1053 & 10/15/2007 11:50 & 23.5 & YES & YES & Lots of undissolved material, spinel everywhere \\
\hline EM07-K-03 & 5 & 10/16/2007 18:12 & 1102 & 10/17/2007 16:45 & 22.5 & YES & NO & Sample clear of spinel \\
\hline EM07-K-03 & 5 & $10 / 21 / 200714: 45$ & 1073 & 10/22/2007 15:10 & 24.4 & YES & YES & Lots of little spinel, some undissolved material \\
\hline EM07-K-03 & 5 & 10/23/2007 17:37 & 1089 & $10 / 24 / 200715: 57$ & 22.3 & YES & YES & Lots of spinel throughout, some Ru agglomerates \\
\hline
\end{tabular}




\begin{tabular}{|c|c|c|c|c|c|c|c|c|}
\hline Content & Fur & IN/H.T.START & TEMP & OUT/H.T. FINISH & Dur. (hrs) & Undis. $N M$ & Spinel & Total Observations \\
\hline EM07-K-03 & 5 & $10 / 25 / 200716: 44$ & 1099 & 10/26/2007 15:40 & 22.9 & NO & $\mathrm{NO}$ & Sample clear \\
\hline EM07-K-03 & 5 & 11/3/2007 16:30 & 1093 & 11/4/2007 15:18 & 22.8 & YES & YES & Small spinels throughout, some undissolved agglomerates \\
\hline EM07-K-03 & 5 & 1/28/2008 14:40 & 1026 & 1/29/2008 14:40 & 24.0 & 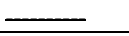 & 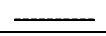 & XRD sample only \\
\hline EM07-K-03 & 8 & 1/28/2008 14:40 & 999 & 1/29/2008 14:40 & 24.0 & 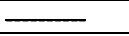 & $\ldots$ & XRD sample only \\
\hline EM07-K-03 & 10 & 1/31/2008 17:15 & 974 & 2/1/2008 15:54 & 22.6 & 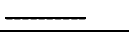 & $\overline{-}$ & XRD sample only \\
\hline EM07-K-06 & 10 & $10 / 14 / 200712: 20$ & 1251 & 10/15/2007 11:50 & 23.5 & NO & $\mathrm{NO}$ & Sample clear of spinel \\
\hline EM07-K-06 & 8 & 10/14/2007 12:20 & 1150 & 10/15/2007 11:50 & 23.5 & NO & NO & Sample clear \\
\hline EM07-K-06 & 5 & 10/14/2007 12:20 & 1053 & 10/15/2007 11:50 & 23.5 & YES & NO & No spinel, one Ru agglomerate \\
\hline EM07-K-06 & 8 & $10 / 27 / 200716: 24$ & 1024 & $10 / 28 / 200714: 27$ & 22.0 & YES & NO & Sample clear \\
\hline EM07-K-06 & 8 & $10 / 31 / 200718: 33$ & 1009 & 11/1/2007 16:35 & 22.0 & YES & YES & Several spinel, some other undissolved material present \\
\hline EM07-K-06 & 8 & 11/3/2007 16:30 & 1018 & 11/4/2007 15:18 & 22.8 & YES & YES & Very few spinel, some $\mathrm{RuO} 2$ agglomerates \\
\hline EM07-K-06 & 10 & 1/31/2008 17:15 & 974 & 2/1/2008 15:54 & 22.6 & 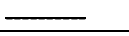 & 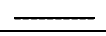 & XRD sample only \\
\hline EM07-K-06 & 10 & 2/1/2008 17:00 & 849 & 2/5/2008 14:20 & 93.3 & $\overline{-}$ & $\overline{-}$ & XRD sample only \\
\hline EM07-K-06 & 10 & 2/7/2008 10:00 & 903 & 2/8/2008 11:40 & 25.7 & $\bar{m}$ & $\ldots$ & XRD sample only \\
\hline EM07-Li-00 & 5 & 6/16/2009 11:20 & 1092 & 6/17/20099:30 & 22.2 & 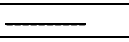 & YES & Small crystals throughout \\
\hline EM07-Li-00 & 8 & 6/16/2009 11:20 & 992 & 6/17/2009 9:30 & 22.2 & 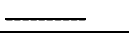 & YES & \\
\hline EM07-Li-00 & 5 & 7/13/2009 11:30 & 1121 & 7/14/2009 9:35 & 22.1 & - & YES & Crystals throughout \\
\hline EM07-Li-00 & 5 & $7 / 14 / 200911: 45$ & 1165 & 7/15/2009 10:10 & 22.4 & $\ldots$ & YES & Crystals throughout \\
\hline EM07-Li-00 & 5 & 7/22/2009 16:00 & 1185 & 7/22/2009 16:00 & 0.0 & - & YES & Crystals throughout \\
\hline EM07-Li-00 & 5 & $7 / 30 / 200914: 35$ & 1234 & 7/31/2009 14:50 & 24.2 & $\overline{-}$ & YES & Full of crystals \\
\hline EM07-Li-00 & 5 & 9/2/2009 11:30 & 1320 & 9/3/2009 9:30 & 22.0 & $\bar{m}$ & YES & Cystals peppered \\
\hline EM07-Li-00 & 5 & 9/14/2009 10:45 & 1340 & 9/15/20099:00 & 22.3 & $\overline{-}$ & YES & Crystals \\
\hline EM07-Li-00 & 5 & 9/22/2009 11:40 & 1380 & 9/22/2009 15:40 & 4.0 & - & YES & Crystals \\
\hline EM07-Li-00 & 5 & 10/5/20099:20 & 1400 & 10/5/2009 13:20 & 4.0 & $=$ & N/A & Boiled out \\
\hline EM07-Li-00 & 5 & 10/12/2009 10:10 & 1382 & 10/12/2009 14:10 & 4.0 & $\bar{m}$ & YES & Tiny spinel \\
\hline EM07-Li-00 & 5 & 10/12/2009 15:25 & 1390 & $10 / 12 / 200920: 15$ & 4.8 & - & YES & Tiny spinel \\
\hline EM07-Li-00 & 5 & 10/21/2009 14:00 & 1360 & 10/21/2009 18:05 & 4.1 & - & YES & Tiny spinel \\
\hline EM07-Li-00 & 5 & 10/22/2009 8:05 & 1370 & 10/22/2009 12:05 & 4.0 & - & YES & Tiny spinel \\
\hline EM07-Li-00 & 5 & 10/22/2009 12:10 & 1349 & 10/22/200916:10 & 4.0 & $\overline{-}$ & YES & Tiny spinel \\
\hline EM07-Li-015 & 10 & 10/14/2007 12:20 & 1251 & 10/15/2007 11:50 & 23.5 & NO & YES & Very small amount of spinel \\
\hline EM07-Li-015 & 8 & $10 / 14 / 200712: 20$ & 1150 & 10/15/2007 11:50 & 23.5 & YES & YES & Lots of spinel, some undissolved material \\
\hline EM07-Li-015 & 5 & 10/14/2007 12:20 & 1053 & 10/15/2007 11:50 & 23.5 & YES & YES & Lots of spinel, some undissolved material \\
\hline EM07-Li-015 & 5 & 10/17/2007 17:28 & 1300 & 10/18/2007 15:31 & 22.0 & NO & NO & Sample is brown in color, no crystals or other undissolved material \\
\hline EM07-Li-015 & 5 & 10/27/2007 16:24 & 1275 & $10 / 28 / 200714: 27$ & 22.0 & NO & NO & Sample clear \\
\hline EM07-Li-015 & 5 & $10 / 31 / 200718: 33$ & 1262 & $11 / 1 / 200716: 35$ & 22.0 & YES & YES & Volatilized out of crucible, Redox, some spinel, possibly other undissolved materials \\
\hline EM07-Li-015 & 5 & 11/4/2007 16:30 & 1268 & 11/5/2007 14:20 & 21.8 & NO & NO & Sample clear \\
\hline EM07Li 015 & 8 & 1/118/20089:40 & $\$ 219$ & $1 / 19 / 200810: 22$ & 24.7 & $\overline{-}$ & $\overline{-}$ & XRD-sample only \\
\hline EM07 Li 015 & 8 & 1/21/200813:19 & 1313 & $1 / 21 / 200817: 30$ & 4.2 & $\overline{-}$ & $\overline{-}$ & XRDsample only \\
\hline EM07-Li-04 & 10 & 10/14/2007 12:20 & 1251 & 10/15/2007 11:50 & 23.5 & NO & $\mathrm{NO}$ & Sample clear of spinel \\
\hline EM07-Li-04 & 8 & 10/14/2007 12:20 & 1150 & 10/15/2007 11:50 & 23.5 & NO & NO & Sample Clear \\
\hline EM07-Li-04 & 5 & 10/14/2007 12:20 & 1053 & 10/15/2007 11:50 & 23.5 & YES & YES & Lots of spinel, some undissolved material \\
\hline EM07-Li-04 & 8 & 10/18/2007 17:13 & 1123 & 10/19/2007 15:15 & 22.0 & YES & YES & Lots of little spinel, some undissolved material \\
\hline EM07-Li-04 & 8 & 10/22/2007 16:20 & 1141 & $10 / 23 / 200716: 53$ & 24.6 & NO & NO & Sample clear \\
\hline EM07-Li-04 & 8 & 10/26/2007 17:08 & 1134 & 10/27/2007 15:20 & 22.2 & NO & $\mathrm{NO}$ & Sample clear of spinel \\
\hline EM07-Li-04 & 8 & 11/2/2007 16:30 & 1129 & 11/3/2007 15:29 & 23.0 & YES & YES & Very few spinel, some undissolved agglomerates \\
\hline EM07-Li-04 & 8 & $12 / 27 / 200717: 20$ & 1003 & $12 / 28 / 200715: 45$ & 22.4 & 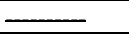 & $\ldots$ & XRD sample only \\
\hline EM07-Li-04 & 10 & 1/8/2008 18:14 & 915 & 1/10/2008 16:08 & 45.9 & 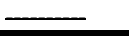 & $\overline{-}$ & XRD sample only \\
\hline
\end{tabular}




\begin{tabular}{|c|c|c|c|c|c|c|c|c|}
\hline Content & Fur & IN /H.T. START & TEMP & OUT/H.T. FINISH & Dur. (hrs) & Undis. $N M$ & Spinel & Total Observations \\
\hline EM07-Li-04 & 10 & 1/28/2008 14:40 & 1101 & 1/29/2008 14:40 & 24.0 & $\overline{-}$ & 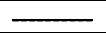 & XRD sample only \\
\hline EM07-Li-04 & 10 & 1/31/2008 17:15 & 974 & $2 / 1 / 200815: 54$ & 22.6 & - & - & XRD sample only \\
\hline EM07-Li-05 & 5 & $6 / 16 / 200911: 20$ & 1092 & 6/17/20099:30 & 22.2 & 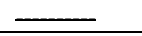 & YES & Crystal lightly peppered \\
\hline EM07-Li-05 & 8 & 6/16/2009 11:20 & 992 & 6/17/20099:30 & 22.2 & 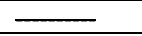 & YES & \\
\hline EM07-Li-05 & 5 & 7/13/2009 11:30 & 1121 & 7/14/20099:35 & 22.1 & $\overline{-}$ & YES & Clusters on bottom \\
\hline EM07-Li-05 & 5 & $7 / 14 / 200911: 45$ & 1165 & $7 / 15 / 2009$ 10:10 & 22.4 & 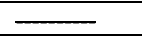 & NO & Glass \\
\hline EM07-Li-05 & 5 & $7 / 21 / 200915: 00$ & 1152 & 7/21/2009 15:00 & 0.0 & + & $\mathrm{NO}$ & Glass \\
\hline EM07-Li-05 & 5 & 9/26/2009 12:40 & 1140 & 9/27/2009 14:00 & 25.3 & - & NO & Glass \\
\hline EM07-Li-05 & 5 & 9/30/2009 13:00 & 1131 & 10/1/2009 13:00 & 24.0 & - & $\mathrm{NO}$ & Glass \\
\hline EM07-Mn-01 & 10 & 9/27/2007 16:07 & - & $9 / 28 / 200714: 15$ & 22.1 & YES & $?$ & Few RuO2, possibly some spinel \\
\hline EM07-Mn-01 & 8 & 10/4/2007 16:06 & 1048 & 10/5/2007 15:00 & 22.9 & YES & YES & Undissolved materials, spinel everywhere \\
\hline EM07-Mn-01 & 10 & 10/4/2007 16:06 & 944 & 10/5/2007 15:00 & 22.9 & YES & YES & Surface pigmentation, undissolved materials, spinel everywhere \\
\hline EM07-Mn-01 & 8 & $10 / 10 / 200715: 15$ & 1148 & 10/11/2007 13:40 & 22.4 & $\mathrm{NO}$ & YES & Very small quantity of spinel \\
\hline EM07-Mn-01 & 8 & 10/11/2007 16:23 & 1251 & $10 / 12 / 200714: 30$ & 22.1 & $\mathrm{NO}$ & NO & Sample clear \\
\hline EM07-Mn-01 & 8 & 10/17/2007 17:28 & 1202 & 10/18/2007 15:31 & 22.0 & NO & NO & Sample clear \\
\hline EM07-Mn-01 & 8 & $10 / 21 / 200714: 45$ & 1176 & 10/22/2007 15:10 & 24.4 & NO & NO & Sample clear \\
\hline EM07-Mn-01 & 8 & $10 / 24 / 200716: 20$ & 1160 & 10/25/2007 15:52 & 23.5 & YES & NO & Sample clear of spinel, one small undissolved Ru agglomerate \\
\hline EM07-Mn-01 & 8 & 10/30/2007 17:36 & 1151 & 10/31/2007 16:50 & 23.2 & YES & NO & No spinel, some Ru present \\
\hline EM07-Mn-01 & 8 & $12 / 27 / 200717: 20$ & 1003 & $12 / 28 / 200715: 45$ & 22.4 & $\overline{-}$ & $\overline{-}$ & XRD sample only \\
\hline Em07-Mn-01 & 10 & 1/28/2008 14:40 & 1101 & 1/29/2008 14:40 & 24.0 & - & $\overline{-1}$ & XRD sample only \\
\hline EM07-Mn-04 & 10 & 10/7/2007 14:37 & 942 & 10/8/2007 15:30 & 24.9 & 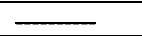 & $\overline{-m}$ & Surface crystallization \\
\hline EM07-Mn-04 & 8 & 10/7/2007 14:38 & 1042 & 10/8/2007 15:30 & 24.9 & YES & YES & Undissolved materials, spinel everywhere \\
\hline EM07-Mn-04 & 8 & 10/10/2007 15:15 & 1148 & 10/11/2007 13:40 & 22.4 & YES & YES & Several spinel, some undissolved material \\
\hline EM07-Mn-04 & 8 & 10/11/2007 16:23 & 1251 & 10/12/2007 14:30 & 22.1 & YES & YES & Sample clear \\
\hline EM07-Mn-04 & 10 & 10/16/2007 18:12 & 1201 & 10/17/2007 16:45 & 22.5 & YES & YES & Very few spinel, some $\mathrm{RuO} 2$ agglomerates \\
\hline EM07-Mn-04 & 5 & 10/18/2007 17:13 & 1220 & 10/19/2007 15:15 & 22.0 & YES & NO & Sample is clear of spinel, one ruthenium agglomerate at base of sample \\
\hline EM07-Mn-04 & 5 & $10 / 22 / 200716: 20$ & 1210 & 10/23/2007 16:53 & 24.6 & Maybe & YES & Three or four small spinel clusters in bulk and at base \\
\hline EM07-Mn-04 & 5 & 10/26/2007 17:08 & 1213 & 10/27/2007 15:20 & 22.2 & NO & YES & Very few spinel \\
\hline EM07 Mn-04 & 5 & 1/16/200811:05 & 1175 & $1 / 17 / 20089: 20$ & 22.3 & $\overline{-}$ & $\overline{-}$ & Volatilized of enteible \\
\hline EM07-Mn-04 & 10 & 1/28/2008 14:40 & 1101 & 1/29/2008 14:40 & 24.0 & $\overline{-}$ & $\overline{-2}$ & XRD sample only \\
\hline EM07-Na-05 & 8 & 10/4/2007 16:06 & 1048 & 10/5/2007 15:00 & 22.9 & YES & YES & Surface crystallization, spinel everywhere, other crystals present \\
\hline EM07-Na-05 & 10 & 10/4/2007 16:06 & 944 & 10/5/2007 15:00 & 22.9 & YES & YES & Surface crystallization, extremely crystallized w/ spinel \\
\hline EM07-Na-05 & 8 & 10/10/2007 15:15 & 1148 & 10/11/2007 13:40 & 22.4 & YES & YES & Extremely crystallized with spinel, undissolved material \\
\hline EM07-Na-05 & 8 & 10/11/2007 16:23 & 1251 & 10/12/2007 14:30 & 22.1 & YES & YES & Lots of spinel, some undissolved material \\
\hline EM07-Na-05 & 5 & 10/17/2007 17:28 & 1300 & 10/18/2007 15:31 & 22.0 & YES & YES & Lots of spinel, some undissolved material \\
\hline EM07-Na-05 & 5 & $10 / 24 / 200716: 20$ & 1349 & 10/25/2007 15:52 & 23.5 & YES & YES & Sample volatilized out of conical crucible, very dark, several spinel \\
\hline EM07-Na-05 & 5 & $12 / 20 / 200713: 22$ & 1322 & $12 / 20 / 200717: 15$ & 3.9 & YES & YES & Redox visible, several spinel in bulk, some other undissolved mat'ls \\
\hline EM07-Na-05 & 5 & $12 / 22 / 200711: 30$ & 1343 & 12/22/2007 15:50 & 4.3 & YES & YES & Redox visible, some spinel appear to have spines on corners \\
\hline EM07-Na-05 & 5 & $12 / 28 / 200711: 14$ & 1361 & 12/28/2007 15:30 & 4.3 & YES & YES & Redox visible, several spinel, other undissolved mat'ls \\
\hline EM07 Na-05 & 5 & 1/4/2008 14:05 & 1378 & 1/4/200818:05 & 4.0 & $\overline{-}$ & $\overline{-}$ & Volatilized ont of encible \\
\hline EM07-Na-10 & 8 & 10/4/2007 16:06 & 1048 & 10/5/2007 15:00 & 22.9 & YES & YES & Surface crystallization, undissolved materials, spinel everywhere \\
\hline EM07-Na-10 & 10 & 10/4/2007 16:06 & 944 & 10/5/2007 15:00 & 22.9 & YES & YES & Surface crystallization, extremely crystallized $\mathrm{w} /$ spinel \\
\hline EM07-Na-10 & 5 & $10 / 10 / 200715: 15$ & 1150 & 10/11/2007 13:40 & 22.4 & YES & YES & Several spinel, some undissolved material \\
\hline EM07-Na-10 & 5 & 10/11/2007 16:23 & 1250 & 10/12/2007 14:30 & 22.1 & YES & YES & Lots of spinel, some undissolved material \\
\hline EM07-Na-10 & 5 & 10/17/2007 17:28 & 1300 & 10/18/2007 15:31 & 22.0 & YES & YES & Sample is brown in color, lots of spinel in bulk \\
\hline EM07-Na-10 & 5 & $10 / 24 / 200716: 20$ & 1349 & 10/25/2007 15:52 & 23.5 & $\mathrm{NO}$ & NO & Sample volatilized out of conical crucible, very dark, seems to be amorphous \\
\hline EM07-Na-10 & 8 & 11/8/2007 17:12 & 1327 & 11/9/2007 15:40 & 22.5 & $?$ & YES & Redox, dark brown, few spinel, some other undissolved materials \\
\hline
\end{tabular}




\begin{tabular}{|c|c|c|c|c|c|c|c|c|}
\hline Content & Fur & IN/H.T. START & TEMP & OUT/H.T. FINISH & Dur. (hrs) & Undis. $N M$ & Spinel & Total Observations \\
\hline EM07-Na-10 & 5 & $12 / 20 / 200713: 22$ & 1322 & $12 / 20 / 200717: 15$ & 3.9 & $\overline{-}$ & $\overline{-}$ & Volatilized out of crueible \\
\hline EM07-Na-10 & 5 & 12/22/2007 11:30 & 1343 & 12/22/2007 15:50 & 4.3 & YES & YES & $\begin{array}{l}\text { Redox visible, section of sample viewable is packed with spinel, some other } \\
\text { undissolved mat'ls present }\end{array}$ \\
\hline EM07-Na-10 & 5 & 12/28/2007 11:14 & 1361 & $12 / 28 / 200715: 30$ & 4.3 & YES & YES & Redox visible, some spinel present, glass-ceramic \\
\hline EM07 Na 10 & 5 & 144/200814:05 & 1378 & 1/4/2008 18:05 & 4.0 & 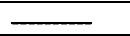 & $\overline{-}$ & Volatilized ont entuible \\
\hline EM07 Na 10 & 8 & $1 / 8 / 200815: 25$ & 1403 & $1 / 8 / 200817: 30$ & 2.1 & $\overline{-}$ & $\overline{-}$ & Volatilized out of erucible \\
\hline EM07-Na-10 & 5 & 1/10/2008 11:06 & 1418 & $1 / 10 / 200814: 15$ & 3.1 & $?$ & $?$ & Redox visible, appears to be clear \\
\hline EM07-Na-10 & 5 & 1/21/2008 13:19 & 1400 & 1/21/2008 17:30 & 4.2 & $?$ & Crys. & Few crystals throughout bulk (star-like) \\
\hline EM07-Na-10 & 5 & 2/8/2008 12:23 & 1410 & 2/8/2008 16:36 & 4.2 & Und. mat'l & Crys. & 5-10 star-like crystals throughout bulk \\
\hline EM07-Na-20 & 8 & 10/9/2007 14:30 & 1047 & 10/10/2007 14:12 & 23.7 & NO & NO & Sample clear \\
\hline EM07-Na-20 & 10 & 10/9/2007 14:30 & 949 & 10/10/2007 14:12 & 23.7 & YES & YES & Several spinel, some undissolved material \\
\hline EM07-Na-20 & 5 & 10/10/2007 15:15 & 1150 & 10/11/2007 13:40 & 22.4 & NO & NO & Sample clear \\
\hline EM07-Na-20 & 5 & 10/11/2007 16:23 & 1250 & $10 / 12 / 200714: 30$ & 22.1 & YES & YES & Sample clear of spinel \\
\hline EM07-Na-20 & 8 & $10 / 16 / 200718: 12$ & 999 & $10 / 17 / 200716: 45$ & 22.5 & YES & YES & Few pools of spinel, some undissolved $\mathrm{RuO} 2$ \\
\hline EM07-Na-20 & 8 & 10/27/2007 16:24 & 1024 & 10/28/2007 14:27 & 22.0 & YES & NO & Sample clear of spinel, few Ru agglomerates \\
\hline EM07-Na-20 & 8 & 10/31/2007 18:33 & 1009 & 11/1/2007 16:35 & 22.0 & YES & YES & Very few spinel, some other undissolved material present \\
\hline EM07-Na-20 & 8 & $11 / 3 / 200716: 30$ & 1018 & 11/4/2007 15:18 & 22.8 & YES & YES & Very few spinel, some $\mathrm{RuO} 2$ agglomerates \\
\hline EM07-Na-20 & 8 & $12 / 28 / 200717: 08$ & 844 & $12 / 30 / 200717: 29$ & 48.4 & $\overline{-}$ & $\overline{-}$ & XRD sample only \\
\hline EM07 Na 20 & 8 & 1/28/2008 14:40 & 999 & 1/29/2008 14:40 & 24.0 & $\overline{-}$ & 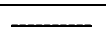 & XRD sample only \\
\hline EM07-Na-20 & 10 & 2/7/2008 10:00 & 903 & 2/8/2008 11:40 & 25.7 & 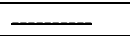 & 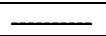 & XRD sample only \\
\hline EM07-Na-225 & 5 & $6 / 16 / 200911: 20$ & 1092 & 6/17/20099:30 & 22.2 & - & NO & Glass \\
\hline EM07-Na-225 & 8 & 6/16/2009 11:20 & 992 & 6/17/20099:30 & 22.2 & $\overline{-}$ & NO & Glass \\
\hline EM07-Na-225 & 8 & $7 / 13 / 2009$ 11:30 & 891 & 7/14/2009 9:35 & 22.1 & - & YES & Crystals throughout \\
\hline EM07-Na-225 & 8 & $7 / 30 / 200914: 35$ & 942 & $7 / 31 / 200914: 50$ & 24.2 & - & YES & Crystal swirls \\
\hline EM07-Na-225 & 8 & 9/8/2009 15:50 & 916 & 9/9/2009 13:45 & 21.9 & - & YES & Crystal swirls \\
\hline EM07-Na-225 & 8 & $10 / 3 / 200916: 30$ & 927 & 10/4/2009 14:30 & 22.0 & 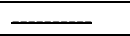 & YES & Spinel swirls \\
\hline EM07-Na-225 & 8 & 10/6/2009 13:50 & 935 & 10/7/2009 13:00 & 23.2 & 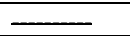 & N/A & In furnace too long \\
\hline EM07-Na-225 & 8 & 10/7/2009 14:30 & 935 & 10/8/2009 13:30 & 23.0 & 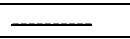 & YES & Spinel swirls \\
\hline EM07-Na-225 & 8 & $10 / 20 / 200910: 45$ & 952 & 10/21/20099:00 & 22.3 & 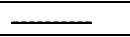 & YES & Spinel swirls \\
\hline EM07-Na-225 & 8 & 10/21/2009 11:15 & 962 & 10/22/20099:20 & 22.1 & 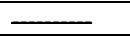 & YES & Broken spinel clumps \\
\hline EM07-Na-225 & 8 & $10 / 22 / 200911: 30$ & 972 & 10/23/2009 11:15 & 23.8 & ב-ב & YES & Broken spinel \\
\hline EM07-Ni-001 & 10 & 10/28/2007 14:45 & 1200 & 10/29/2007 15:35 & 24.8 & YES & NO & No spinel, several small Ru agglomerates throughout bulk \\
\hline EM07-Ni-001 & 5 & $10 / 28 / 200714: 45$ & 1151 & $10 / 29 / 200715: 35$ & 24.8 & YES & NO & Sample clear of spinel, some Ru \\
\hline EM07-Ni-001 & 5 & $10 / 30 / 200717: 36$ & 1051 & $10 / 31 / 200716: 50$ & 23.2 & YES & YES & Lots of small spinel, Ru present \\
\hline EM07-Ni-001 & 8 & 10/31/2007 18:33 & 1009 & 11/1/2007 16:35 & 22.0 & YES & YES & Lots of spinel, some other undissolved material present \\
\hline EM07-Ni-001 & 8 & 11/2/2007 16:30 & 1129 & $11 / 3 / 200715: 29$ & 23.0 & YES & NO & No spinel, some Ru present \\
\hline EM07-Ni-001 & 5 & $11 / 2 / 200716: 30$ & 1078 & 11/3/2007 15:29 & 23.0 & YES & YES & Several small spinel, some undissolved Ru materials \\
\hline EM07-Ni-001 & 5 & $11 / 3 / 200716: 30$ & 1093 & 11/4/2007 15:18 & 22.8 & YES & YES & Very few small spinel in bulk, some undissolved agglomerates \\
\hline EM07-Ni-001 & 5 & 11/5/2007 15:00 & 1111 & $11 / 6 / 200715: 54$ & 24.9 & YES & NO & No spinel, a few $\mathrm{Ru}$ \\
\hline EM07-Ni-001 & 5 & 11/7/2007 16:42 & 1105 & 11/8/2007 15:40 & 23.0 & NO & NO & Sample clear \\
\hline EM07-Ni-001 & 5 & $11 / 12 / 200716: 41$ & 1099 & 11/13/2007 15:07 & 22.4 & YES & YES & Lots of small undissolved particles, some appear cubic \\
\hline EM07-Ni-001 & 10 & 1/16/2008 11:05 & 947 & 1/17/20089:20 & 22.3 & $\overline{-}$ & $\overline{-}$ & XRD sample only \\
\hline EM07-Ni-001 & 10 & 2/7/2008 10:00 & 903 & 2/8/2008 11:40 & 25.7 & 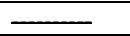 & 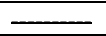 & XRD sample only \\
\hline EM07-Ni-02 & 10 & $10 / 28 / 200714: 45$ & 1200 & $10 / 29 / 200715: 35$ & 24.8 & YES & YES & Several spinel, some undissolved material \\
\hline EM07-Ni-02 & 5 & $10 / 28 / 200714: 45$ & 1151 & $10 / 29 / 200715: 35$ & 24.8 & YES & YES & Spinel everywhere, undissolved $\mathrm{Ru}$ \\
\hline EM07-Ni-02 & 5 & 10/31/2007 18:33 & 1262 & 11/1/2007 16:35 & 22.0 & 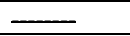 & 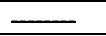 & Volatilized out of erueible \\
\hline EM07-Ni-02 & 5 & 11/1/2007 17:45 & 1281 & 11/2/2007 15:55 & 22.2 & NO & NO & Sample clear \\
\hline EM07-Ni-02 & 10 & 11/5/2007 15:00 & 1240 & $11 / 6 / 200715: 54$ & 24.9 & YES & YES & Several spinel at the base of the sample \\
\hline
\end{tabular}




\begin{tabular}{|c|c|c|c|c|c|c|c|c|}
\hline Content & Fur & IN/H.T. START & $T E M P$ & OUT/H.T. FINISH & Dur. (hrs) & Undis. $N M$ & Spinel & Total Observations \\
\hline EM07-Ni-02 & 10 & 11/7/2007 16:42 & 1249 & 11/8/2007 15:40 & 23.0 & $\mathrm{NO}$ & YES & Very few large spinel, no Ru \\
\hline EM07-Ni-02 & 8 & 11/12/2007 16:41 & 1270 & 11/13/2007 15:07 & 22.4 & YES & $\mathrm{NO}$ & Sample clear of spinel, some Ru \\
\hline EM07-Ni-02 & 8 & $11 / 14 / 200715: 26$ & 1257 & $11 / 15 / 200715: 37$ & 24.2 & YES & YES & One spinel, some small undissolved agglomerates \\
\hline EM07-Ni-02 & 8 & $11 / 15 / 200716: 48$ & 1250 & $11 / 16 / 200716: 42$ & 23.9 & YES & YES & Few large spinel throughout bulk, some small clusters of $\mathrm{Ru}$ \\
\hline EM07-Ni-02 & 8 & 11/19/2007 13:30 & 1264 & $11 / 20 / 200711: 30$ & 22.0 & $\mathrm{NO}$ & $\mathrm{NO}$ & Sample clear \\
\hline EM07-Ni-02 & 10 & $1 / 22 / 200812: 05$ & 1060 & 1/23/2008 10:47 & 22.7 & 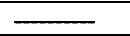 & - & XRD sample only \\
\hline EM07-Ni-02 & 10 & 1/28/2008 14:40 & 1101 & 1/29/2008 14:40 & 24.0 & $\overline{-}$ & - & XRD sample only \\
\hline EM07-NM-0025 & 10 & 11/6/2007 15:55 & 1227 & 11/7/2007 15:30 & 23.6 & YES & $?$ & Several very small undissolved particles (maybe spinel) \\
\hline EM07-NM-0025 & 8 & 11/6/2007 15:55 & 1140 & 11/7/2007 15:30 & 23.6 & YES & YES & Large quantities of spinel and other undissolved materials \\
\hline EM07-NM-0025 & 5 & $11 / 6 / 200715: 55$ & 1065 & 11/7/2007 15:30 & 23.6 & YES & YES & Large quantities of spinel and other undissolved materials \\
\hline EM07-NM-0025 & 10 & 11/8/2007 17:12 & 1170 & 11/9/2007 15:40 & 22.5 & YES & YES & Several spinel, some undissolved material \\
\hline EM07-NM-0025 & 10 & 11/9/2007 16:42 & 1230 & 11/10/2007 14:56 & 22.2 & $?$ & ? & Several very small undissolved particles (maybe spinel) \\
\hline EM07-NM-0025 & 8 & 11/12/2007 16:41 & 1270 & 11/13/2007 15:07 & 22.4 & $?$ & ? & Lots of very small undissolved particles \\
\hline EM07-NM-0025 & 10 & 12/11/2007 16:25 & 1229 & 12/12/2007 14:35 & 22.2 & $\overline{-}$ & $\overline{-}$ & $\ldots$ \\
\hline EM07-NM-0025 & 10 & $12 / 14 / 200716: 11$ & 1221 & $12 / 15 / 200714: 20$ & 22.1 & YES & $?$ & Lots of small undissolved particles, some larger \\
\hline EM07-NM-0025 & 10 & 12/20/2007 17:18 & 1193 & $12 / 21 / 200716: 30$ & 23.2 & YES & YES & Several spinel throughout bulk, lots of small undissolved particles \\
\hline EM07-NM-0025 & 10 & $12 / 27 / 200717: 20$ & 1214 & $12 / 28 / 200715: 45$ & 22.4 & YES & NO & No spinel observed, some small undissolved particles (Ru?) \\
\hline EM07-NM-0025 & 10 & 1/2/2008 14:12 & 1203 & 1/3/2008 13:45 & 23.5 & YES & YES & One spinel (very near TL), lots of small undissolved particles, few agglom. at base \\
\hline EM07-P-0 & 8 & 10/9/2007 14:30 & 1047 & 10/10/2007 14:12 & 23.7 & YES & YES & Undissolved materials, spinel everywhere \\
\hline EM07-P-0 & 10 & 10/9/2007 14:30 & 949 & 10/10/2007 14:12 & 23.7 & YES & YES & Undissolved materials, spinel everywhere \\
\hline EM07-P-0 & 5 & 10/10/2007 15:15 & 1150 & 10/11/2007 13:40 & 22.4 & YES & YES & Several spinel, some undissolved material \\
\hline EM07-P-0 & 5 & $10 / 11 / 200716: 23$ & 1250 & $10 / 12 / 200714: 30$ & 22.1 & YES & YES & Sample clear of spinel \\
\hline EM07-P-0 & 8 & 10/17/2007 17:28 & 1202 & 10/18/2007 15:31 & 22.0 & $\mathrm{NO}$ & NO & Sample clear \\
\hline EM07-P-0 & 8 & $10 / 21 / 200714: 45$ & 1176 & 10/22/2007 15:10 & 24.4 & YES & YES & Very few spinel, some Ru agglomerates \\
\hline EM07-P-0 & 8 & 10/23/2007 17:37 & 1191 & $10 / 24 / 200715: 57$ & 22.3 & YES & NO & Sample clear of spinel, a few Ru agglomerates \\
\hline EM07-P-0 & 8 & $10 / 28 / 200714: 45$ & 1184 & 10/29/2007 15:35 & 24.8 & YES & YES & Very few spinel \\
\hline EM07-P-0 & 10 & 1/28/2008 14:40 & 1101 & 1/29/2008 14:40 & 24.0 & 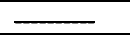 & $\ldots$ & XRD sample only \\
\hline EM07P 0 & 8 & 1/29/2008 14:40 & 999 & 1/30/2008 14:30 & 23.8 & - & - & XRDsample only \\
\hline EM07-P-02 & 8 & 6/16/2009 11:20 & 992 & 6/17/20099:30 & 22.2 & - & YES & \\
\hline EM07-P-02 & 5 & $6 / 17 / 200910: 30$ & 1110 & 6/18/20098:45 & 22.3 & $\overline{-}$ & YES & Small crystals throughout \\
\hline EM07-P-02 & 5 & 7/14/2009 11:45 & 1165 & 7/15/2009 10:10 & 22.4 & - & YES & Crystals throughout \\
\hline EM07-P-02 & 8 & 7/16/2009 12:15 & 1215 & 7/17/2009 13:20 & 25.1 & $\overline{-}$ & YES & Clusters on bottom \\
\hline EM07-P-02 & 8 & 7/22/2009 16:00 & 1224 & 7/22/2009 16:00 & 0.0 & - & $\mathrm{NO}$ & Glass \\
\hline EM07-P-025 & 8 & 10/4/2007 16:06 & 1048 & 10/5/2007 15:00 & 22.9 & YES & YES & Undissolved materials, spinel everywhere \\
\hline EM07-P-025 & 10 & 10/4/2007 16:06 & 944 & 10/5/2007 15:00 & 22.9 & YES & YES & Surface pigmentation, undissolved materials, spinel everywhere \\
\hline EM07-P-025 & 8 & $10 / 10 / 200715: 15$ & 1148 & 10/11/2007 13:40 & 22.4 & YES & YES & Few spinel, very small quantity of undissolved material \\
\hline EM07P025 & 8 & $10 / 11 / 200716: 23$ & 1251 & 10/12/200714:30 & 22.1 & $\overline{-}$ & - & 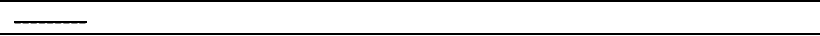 \\
\hline EM07-P-025 & 10 & 10/15/2007 17:17 & 1254 & $10 / 16 / 200717: 50$ & 24.6 & $\mathrm{NO}$ & NO & Sample clear \\
\hline EM07-P-025 & 10 & 10/16/2007 18:12 & 1201 & $10 / 17 / 200716: 45$ & 22.5 & $\mathrm{NO}$ & $\mathrm{NO}$ & Sample clear \\
\hline EM07-P-025 & 10 & 10/18/2007 17:13 & 1161 & 10/19/2007 15:15 & 22.0 & YES & YES & Lots of spinel, some undissolved material \\
\hline EM07-P-025 & 10 & $10 / 21 / 200714: 45$ & 1177 & 10/22/2007 15:10 & 24.4 & YES & YES & Very few spinel, some Ru agglomerates \\
\hline EM07-P-025 & 10 & $10 / 22 / 200716: 20$ & 1187 & $10 / 23 / 200716: 53$ & 24.6 & YES & YES & One cluster of spinel, some ruthenium oxide \\
\hline EM07-P-025 & 10 & $10 / 24 / 200716: 20$ & 1194 & $10 / 25 / 200715: 52$ & 23.5 & YES & $\mathrm{NO}$ & Sample clear of spinel, one small undissolved Ru agglomerate \\
\hline EM07-P-03 & 5 & $6 / 17 / 200910: 30$ & 1110 & 6/18/20098:45 & 22.3 & $\overline{-}$ & YES & Crystals throughout \\
\hline EM07-P-03 & 8 & $\begin{array}{ll}6 / 17 / 2009 & 10: 30 \\
\end{array}$ & 970 & $6 / 18 / 20098: 45$ & 22.3 & - & YES & - \\
\hline EM07-P-03 & 5 & $7 / 14 / 200911: 45$ & 1165 & 7/15/2009 10:10 & 22.4 & 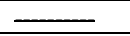 & YES & Crystals throughout \\
\hline EM07-P-03 & 8 & 7/16/2009 12:15 & 1215 & $\begin{array}{l}7 / 17 / 2009 \\
\end{array}$ & 25.1 & 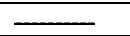 & YES & Clusters on bottom \\
\hline
\end{tabular}




\begin{tabular}{|c|c|c|c|c|c|c|c|c|}
\hline Content & Fur & IN /H.T. START & TEMP & OUT/H.T. FINISH & Dur. (hrs) & Undis. $N M$ & Spinel & Total Observations \\
\hline EM07-P-03 & 8 & $7 / 22 / 200916: 00$ & 1224 & 7/22/2009 16:00 & 0.0 & $\underline{\square}$ & YES & Globs at bottom \\
\hline EM07-P-03 & 5 & $7 / 30 / 200914: 35$ & 1234 & 7/31/2009 14:50 & 24.2 & 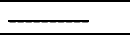 & $\mathrm{NO}$ & Glass \\
\hline EM07-Si-30 & 10 & 10/28/2007 14:45 & 1200 & $10 / 29 / 200715: 35$ & 24.8 & 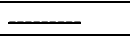 & 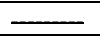 & Volatilized out of erueible \\
\hline EM07-Si-30 & 5 & $10 / 28 / 200714: 45$ & 1151 & 10/29/2007 15:35 & 24.8 & YES & YES & Several spinel, some undissolved material \\
\hline EM07-Si-30 & 5 & 10/29/2007 17:36 & 1238 & $10 / 30 / 200716: 25$ & 22.8 & $?$ & ? & Sample looks very strange, temperature was too high for this glass, altered chemistry \\
\hline EM07-Si-30 & 10 & 10/30/2007 17:36 & 1190 & 10/31/2007 16:50 & 23.2 & YES & YES & Lost glass formers - some spinel in reduced glass at bottom \\
\hline EM07-Si-30 & 10 & 1/4/2008 14:05 & 1194 & 1/4/2008 18:05 & 4.0 & $?$ & YES & Redox visible, several multi-pronged star-like crystals present throughout bulk \\
\hline EM07-Si-30 & 10 & 1/7/2008 13:30 & 1214 & 1/7/2008 17:48 & 4.3 & $\overline{-2}$ & $\overline{-2}$ & Volatilized out of crucible \\
\hline EM07-Si-30 & 8 & 1/10/2008 11:06 & 1205 & 1/10/2008 14:15 & 3.1 & ? & YES & $\begin{array}{l}\text { Several multi-pronged star-like crystals throughout bulk, maybe other undissolved } \\
\text { mat'ls }\end{array}$ \\
\hline EM07-Si-30 & 8 & $1 / 18 / 200810: 15$ & 1218 & 1/18/2008 15:11 & 4.9 & Und. mat'l & Crys. & A few crystals - look like cubes with spines on the corners \\
\hline EM07-Si-30 & 8 & 1/21/2008 13:19 & 1313 & $1 / 21 / 200817: 30$ & 4.2 & 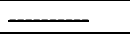 & 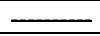 & - \\
\hline EM07-Si-30 & 10 & 1/21/2008 18:21 & 1213 & 1/22/2008 17:45 & 23.4 & $\overline{-}$ & $\overline{-}$ & $\overline{-}$ \\
\hline EM07 Si 30 & 10 & $1 / 22 / 200812: 05$ & 1060 & 1/23/200810:47 & 22.7 & $\overline{-}$ & $\overline{-}$ & XRDsample only \\
\hline EM07-Si-30 & 8 & 1/24/2008 14:24 & 1229 & $1 / 24 / 200818: 21$ & 3.9 & Und. mat'l & Crys. & Lots of star-like crystals throughout sample \\
\hline EM07-Si-30 & 5 & 1/25/2008 9:20 & 1335 & $1 / 25 / 200815: 20$ & 6.0 & Und. mat'l & Crys. & Lots of tiny specs (small spinel?) \\
\hline EM07-Si-30 & 8 & 2/7/2008 10:00 & 1250 & 2/7/2008 14:00 & 4.0 & Und. mat'l & Crys. & Redox present, several star-like crystals throughout bulk \\
\hline EM07-Si-30 & 8 & 2/12/2008 10:03 & 1267 & $2 / 12 / 200814: 42$ & 4.7 & Und. mat'l & Crys. & Crystals apparent (just a few) \\
\hline EM07-Si-30 & 8 & 2/13/2008 11:40 & 1288 & $2 / 13 / 200816: 05$ & 4.4 & $?$ & $?$ & Sample appears clear \\
\hline EM07-Si-30 & 8 & 2/14/2008 12:14 & 1280 & $2 / 14 / 200816: 18$ & 4.1 & Und. mat'l & Crys. & Some star-like crystals throughout sample \\
\hline EM07-Si-335 & 5 & 6/17/2009 10:30 & 1110 & 6/18/2009 8:45 & 22.3 & - & YES & Crystals throughout \\
\hline EM07-Si-335 & 8 & $6 / 17 / 200910: 30$ & 970 & 6/18/2009 8:45 & 22.3 & 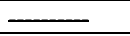 & YES & 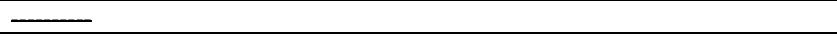 \\
\hline EM07-Si-335 & 8 & $7 / 8 / 200915: 25$ & 1140 & 7/9/2009 13:35 & 22.2 & 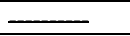 & YES & Bottom layer of crystals \\
\hline EM07-Si-335 & 8 & $7 / 14 / 200911: 45$ & 1180 & 7/15/2009 10:10 & 22.4 & 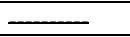 & YES & Bottom layer of crystals \\
\hline EM07-Si-335 & 8 & 7/16/2009 12:15 & 1215 & 7/17/2009 13:20 & 25.1 & 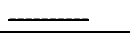 & YES & Glass with boil off \\
\hline EM07-Si-335 & 5 & $8 / 24 / 200915: 55$ & 1190 & $8 / 25 / 200914: 00$ & 22.1 & - & YES & Spinel clusters on bottom \\
\hline EM07-Si-335 & 5 & 9/28/2009 11:15 & 1208 & 9/29/2009 9:25 & 22.2 & - & YES & Giant spinel peppered \\
\hline EM07-Si-37 & 10 & 10/16/2007 18:12 & 1201 & $10 / 17 / 200716: 45$ & 22.5 & NO & NO & Sample clear \\
\hline EM07-Si-37 & 8 & $10 / 16 / 200718: 12$ & 999 & 10/17/2007 16:45 & 22.5 & YES & YES & Lots of spinel, some undissolved material \\
\hline EM07-Si-37 & 5 & 10/16/2007 18:12 & 1102 & 10/17/2007 16:45 & 22.5 & YES & YES & Lots of spinel, some undissolved material \\
\hline EM07-Si-37 & 10 & 10/18/2007 17:13 & 1161 & 10/19/2007 15:15 & 22.0 & YES & YES & Lots of spinel, some undissolved material \\
\hline EM07-Si-37 & 10 & $10 / 21 / 200714: 45$ & 1177 & $10 / 22 / 200715: 10$ & 24.4 & YES & YES & Lots of spinel, some undissolved material \\
\hline EM07-Si-37 & 10 & $10 / 22 / 200716: 20$ & 1187 & $10 / 23 / 200716: 53$ & 24.6 & YES & YES & Few large spinel throughout bulk, some small clusters of Ru at base \\
\hline EM07-Si-37 & 10 & $10 / 24 / 200716: 20$ & 1194 & 10/25/2007 15:52 & 23.5 & YES & YES & Few small spinel agglomerates at base, some Ru \\
\hline EM07-Si-37 & 5 & 1/16/2008 11:05 & 1175 & 1/17/20089:20 & 22.3 & 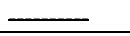 & 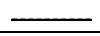 & Volatilized out of erueible \\
\hline EM07-Si-37 & 10 & 1/22/2008 12:05 & 1060 & $1 / 23 / 200810: 47$ & 22.7 & 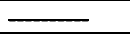 & 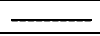 & XRD sample only \\
\hline EM07-Si-50 & 10 & 10/16/2007 18:12 & 1201 & 10/17/2007 16:45 & 22.5 & YES & NO & Sample clear of spinel, a few small undissolved Ru agglomerates \\
\hline EM07-Si-50 & 8 & 10/16/2007 18:12 & 999 & $10 / 17 / 200716: 45$ & 22.5 & YES & YES & Extremely crystallized with spinel, undissolved material \\
\hline EM07-Si-50 & 5 & 10/16/2007 18:12 & 1102 & 10/17/2007 16:45 & 22.5 & YES & YES & Lots of spinel, some undissolved material \\
\hline EM07-Si-50 & 10 & 10/18/2007 17:13 & 1161 & 10/19/2007 15:15 & 22.0 & YES & YES & Lots of tiny spinel, some undissolved material at base \\
\hline EM07-Si-50 & 10 & $10 / 21 / 200714: 45$ & 1177 & 10/22/2007 15:10 & 24.4 & YES & YES & Lots of little spinel, some undissolved material \\
\hline EM07-Si-50 & 10 & $10 / 22 / 200716: 20$ & 1187 & $10 / 23 / 200716: 53$ & 24.6 & YES & NO & Sample clear of spinel, few Ru agglomerates \\
\hline EM07-Si-50 & 10 & 10/26/2007 17:08 & 1184 & 10/27/2007 15:20 & 22.2 & YES & YES & Lots of little spinel throughout, some Ru agglomerates \\
\hline EM07-Si-50 & 10 & $10 / 30 / 200717: 36$ & 1190 & 10/31/2007 16:50 & 23.2 & YES & YES & Very few spinel (TL probably +10$)$ \\
\hline EM07-Si-50 & 10 & $11 / 2 / 200716: 30$ & 1197 & $11 / 3 / 200715: 29$ & 23.0 & YES & NO & Sample clear of spinel, some Ru \\
\hline EM07-Si-50 & 10 & $11 / 29 / 200715: 47$ & 1202 & 11/30/2007 15:40 & 23.9 & YES & $\mathrm{NO}$ & Sample clear of spinel, some other undissolved mat'ls \\
\hline EM07-Si-50 & 10 & 1/22/2008 12:05 & 1060 & 1/23/2008 10:47 & 22.7 & 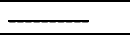 & 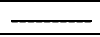 & XRD sample only \\
\hline EM07-Zr-001 & 10 & 10/14/2007 12:20 & 1251 & 10/15/2007 11:50 & 23.5 & NO & $\mathrm{NO}$ & Sample clear of spinel \\
\hline
\end{tabular}




\begin{tabular}{|c|c|c|c|c|c|c|c|c|}
\hline Content & Fur & IN/H.T. START & TEMP & OUT/H.T. FINISH & Dur. (hrs) & Undis. $N M$ & Spinel & Total Observations \\
\hline EM07-Zr-001 & 8 & 10/14/2007 12:20 & 1150 & 10/15/2007 11:50 & 23.5 & YES & YES & Lots of spinel, some undissolved material \\
\hline EM07-Zr-001 & 5 & 10/14/2007 12:20 & 1053 & 10/15/2007 11:50 & 23.5 & YES & YES & Lots of undissolved material, spinel everywhere \\
\hline EM07-Zr-001 & 8 & 10/17/2007 17:28 & 1202 & 10/18/2007 15:31 & 22.0 & YES & $\mathrm{NO}$ & Sample clear of spinel, one small undissolved Ru agglomerate \\
\hline EM07-Zr-001 & 8 & $10 / 21 / 200714: 45$ & 1176 & $10 / 22 / 200715: 10$ & 24.4 & $\mathrm{NO}$ & $\mathrm{NO}$ & Sample clear \\
\hline EM07-Zr-001 & 8 & 10/24/2007 16:20 & 1160 & 10/25/2007 15:52 & 23.5 & YES & YES & Several spinel, some undissolved material \\
\hline EM07-Zr-001 & 8 & 10/29/2007 17:36 & 1166 & $10 / 30 / 200716: 25$ & 22.8 & YES & YES & Very few spinel \\
\hline EM07-Zr-001 & 10 & 1/29/2008 14:40 & 1101 & 1/30/2008 14:30 & 23.8 & $\overline{-}$ & - & XRD sample only \\
\hline EM07-Zr-001 & 8 & 1/29/2008 14:40 & 999 & 1/30/2008 14:30 & 23.8 & $\ldots$ & + & XRD sample only \\
\hline EM07-Zr-05 & 8 & 10/4/2007 16:06 & 1048 & 10/5/2007 15:00 & 22.9 & YES & YES & Undissolved materials, spinel everywhere \\
\hline EM07-Zr-05 & 10 & 10/4/2007 16:06 & 944 & 10/5/2007 15:00 & 22.9 & YES & YES & Surface crystallization, undissolved materials, spinel everywhere \\
\hline EM07-Zr-05 & 5 & 10/10/2007 15:15 & 1150 & 10/11/2007 13:40 & 22.4 & YES & YES & Several spinel, some undissolved material \\
\hline EM07-Zr-05 & 5 & 10/11/2007 16:23 & 1250 & $10 / 12 / 200714: 30$ & 22.1 & $\mathrm{NO}$ & $\mathrm{NO}$ & Sample clear \\
\hline EM07-Zr-05 & 8 & 10/17/2007 17:28 & 1202 & 10/18/2007 15:31 & 22.0 & YES & NO & Sample clear of spinel, a few small undissolved Ru agglomerates \\
\hline EM07-Zr-05 & 8 & $10 / 21 / 200714: 45$ & 1176 & $10 / 22 / 200715: 10$ & 24.4 & YES & YES & Very few spinel, some $\mathrm{RuO} 2$ agglomerates \\
\hline EM07-Zr-05 & 8 & 10/23/2007 17:37 & 1191 & 10/24/2007 15:57 & 22.3 & $\mathrm{NO}$ & NO & Sample clear of spinel \\
\hline EM07-Zr-05 & 8 & $10 / 28 / 200714: 45$ & 1184 & 10/29/2007 15:35 & 24.8 & YES & YES & Very few spinel \\
\hline EM07-Zr-05 & 10 & 1/22/2008 12:05 & 1060 & 1/23/2008 10:47 & 22.7 & - & $\ldots$ & XRD sample only \\
\hline HAL-17 & 10 & 11/12/2007 16:41 & 1085 & 11/13/2007 15:07 & 22.4 & $?$ & YES & Extremely crystallized with spinel, undissolved material \\
\hline HAL-17 & 8 & 11/12/2007 16:41 & 1270 & 11/13/2007 15:07 & 22.4 & ? & YES & Redox, brown, lots of spinel visible on bottom/side edges \\
\hline HAL-17 & 5 & 11/12/2007 16:41 & 1099 & 11/13/2007 15:07 & 22.4 & ? & YES & Extremely crystallized with spinel, undissolved material \\
\hline HAL-17 & 5 & $12 / 31 / 200711: 47$ & 1301 & $12 / 31 / 200715: 51$ & 4.1 & YES & YES & Redox visible, spinel in corner visible, glass-ceramic \\
\hline HAL-17 & 5 & 1/3/2008 14:00 & 1330 & 1/3/2008 18:14 & 4.2 & $?$ & $?$ & Redox visible, sample clear of spinel \\
\hline HAL-17 & 8 & 1/9/2008 14:35 & 1314 & 1/9/2008 18:20 & 3.8 & YES & YES & Redox visible, a few spinel in polished corner \\
\hline HAL-17 & 8 & 2/22/2008 11:42 & 1325 & 2/22/2008 15:20 & 3.6 & Und. mat'l & YES & Few spinel in bulk and base with halos around them \\
\hline HAL-17 & 5 & 2/22/2008 11:42 & 1322 & 2/22/2008 15:20 & 3.6 & Und. mat'l & YES & Few spinel in bulk and base with halos around them \\
\hline HWL $01 Q$ & 10 & $11 / 13 / 200715.55$ & 1239 & $11 / 14 / 200714: 18$ & 22.4 & $\overline{-}$ & - & Sample volatilized out of erueible \\
\hline HWL-01Q & 8 & $11 / 13 / 200715: 55$ & 994 & $11 / 14 / 200714: 18$ & 22.4 & YES & YES & Lots of spinels and some other undissolved materials \\
\hline HWL-01Q & 5 & $11 / 13 / 200715: 55$ & 1090 & $11 / 14 / 200714: 18$ & 22.4 & YES & YES & Lots of spinels and some other undissolved materials \\
\hline HWL-01Q & 10 & $11 / 15 / 200716: 48$ & 1152 & $11 / 16 / 200716: 42$ & 23.9 & YES & $\mathrm{NO}$ & No spinel, some other undissolved materials \\
\hline HWL-01Q & 5 & 11/19/2007 13:30 & 1121 & 11/20/2007 11:30 & 22.0 & ? & YES & Several spinel throughout sample, some undissolved materials \\
\hline HWL-01Q & 5 & 11/28/2007 16:04 & 1138 & $11 / 29 / 200714: 21$ & 22.3 & YES & YES & Several spinel, some other undissolved material present \\
\hline HWL-01Q & 5 & $12 / 2 / 200714: 57$ & 1148 & $12 / 3 / 200714: 30$ & 23.5 & YES & YES & Very few spinel, some other small undissolved mat'ls \\
\hline HWL-01Q & 5 & $12 / 6 / 200716: 28$ & 1162 & 12/7/2007 15:10 & 22.7 & NO & NO & Sample clear of spinel \\
\hline HWL-01Q & 8 & $12 / 10 / 200715: 50$ & 1157 & 12/11/2007 15:57 & 24.1 & YES & NO & Sample clear of spinel, some other undissolved mat'ls \\
\hline HWL-02Q & 10 & $12 / 2 / 200714: 57$ & 1168 & $12 / 3 / 200714: 30$ & 23.5 & YES & YES & Lots of spinel throughout bulk, other undissolved mat'ls (some star-like spinel) \\
\hline HWL-02Q & 8 & 12/2/2007 14:57 & 1091 & $12 / 3 / 200714: 30$ & 23.5 & YES & YES & Several large spinel everywhere, other undissolved mat'ls \\
\hline HWL-02Q & 10 & 12/4/2007 17:22 & 1191 & 12/5/2007 16:27 & 23.1 & YES & YES & Spinel present, some star-like crystals (baddeleyite?), and other undissolved mat'ls \\
\hline HWL-02Q & 10 & 12/9/2007 14:17 & 1198 & $12 / 10 / 200715: 00$ & 24.7 & YES & YES & Lots of large spinel at base, some throughout bulk, strange pentagonal crystals \\
\hline HWL-02Q & 10 & $12 / 12 / 200716: 13$ & 1208 & $12 / 13 / 200716: 13$ & 24.0 & YES & $\mathrm{NO}$ & Sample clear of spinel, some undissolved materials \\
\hline HWL-03Q & 10 & $11 / 13 / 200715: 55$ & 1239 & $11 / 14 / 200714: 18$ & 22.4 & $\mathrm{NO}$ & $\mathrm{NO}$ & Sample clear \\
\hline HWL-03Q & 8 & $11 / 13 / 200715: 55$ & 994 & $11 / 14 / 200714: 18$ & 22.4 & YES & YES & Lots of spinels and some other undissolved materials \\
\hline HWL-03Q & 5 & 11/13/2007 15:55 & 1090 & $11 / 14 / 200714: 18$ & 22.4 & YES & YES & Lots of spinels and some other undissolved materials \\
\hline HWL-03Q & 10 & $11 / 15 / 200716: 48$ & 1152 & $11 / 16 / 200716: 42$ & 23.9 & $\mathrm{NO}$ & $\mathrm{NO}$ & Sample clear \\
\hline HWL-03Q & 5 & 11/19/2007 13:30 & 1121 & 11/20/2007 11:30 & 22.0 & ? & YES & Several spinel throughout sample, some undissolved materials \\
\hline HWL-03Q & 5 & 11/28/2007 16:04 & 1138 & $11 / 29 / 200714: 21$ & 22.3 & YES & YES & Several spinel, some other undissolved material present \\
\hline HWL-03Q & 5 & 12/2/2007 14:57 & 1148 & $12 / 3 / 200714: 30$ & 23.5 & YES & $\mathrm{NO}$ & Clear of spinel, one small undissolved agglomerate \\
\hline HWL-04Q & 10 & $12 / 2 / 200714: 57$ & 1168 & $12 / 3 / 200714: 30$ & 23.5 & YES & YES & Lots of spinel throughout bulk, other undissolved mat'ls (some star-like spinel) \\
\hline
\end{tabular}




\begin{tabular}{|c|c|c|c|c|c|c|c|c|}
\hline Content & Fur & IN/H.T.START & $T E M P$ & OUT/H.T. FINISH & Dur. (hrs) & Undis. $N M$ & Spinel & Total Observations \\
\hline HWL-04Q & 8 & $12 / 2 / 200714: 57$ & 1091 & $12 / 3 / 200714: 30$ & 23.5 & YES & YES & Several large spinel everywhere, other undissolved mat'ls \\
\hline HWL $04 \mathrm{Q}$ & 10 & $12 / 4 / 200717: 22$ & 1191 & 12/5/2007 16:27 & 23.1 & 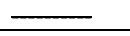 & 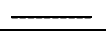 & Sample volatilized out of erueible \\
\hline HWL-04Q & 10 & 12/9/2007 14:17 & 1198 & 12/10/2007 15:00 & 24.7 & YES & NO & One small cluster of undissolved material, no spinel \\
\hline HWL-04Q & 8 & 12/10/2007 15:50 & 1157 & 12/11/2007 15:57 & 24.1 & YES & YES & Several spinel and other undissolved materials \\
\hline HWL-04Q & 8 & 12/11/2007 16:25 & 1098 & 12/12/2007 14:35 & 22.2 & $\cdots$ & $\overline{-}$ & $\cdots$ \\
\hline HWL-04Q & 10 & 12/13/2007 15:35 & 1187 & $12 / 14 / 200715: 50$ & 24.2 & YES & YES & Various shapes/sizes of crystals (cubes, stars, pentagons, etc.) \\
\hline HWL-04Q & 10 & $12 / 20 / 200717: 18$ & 1193 & $12 / 21 / 200716: 30$ & 23.2 & - & $\ldots$ & Volatilized out of erueible \\
\hline HWL-05Q & 10 & 11/13/2007 15:55 & 1239 & 11/14/2007 14:18 & 22.4 & - & - & Sample volatilized out of crucible \\
\hline HWL-05Q & 8 & 11/13/2007 15:55 & 994 & 11/14/2007 14:18 & 22.4 & YES & YES & Lots of spinels and some other undissolved materials \\
\hline HWL-05Q & 5 & 11/13/2007 15:55 & 1090 & 11/14/2007 14:18 & 22.4 & YES & YES & Several little spinels and some other undissolved materials \\
\hline HWL-05Q & 10 & 11/15/2007 16:48 & 1152 & 11/16/2007 16:42 & 23.9 & NO & NO & Sample clear \\
\hline HWL-05Q & 5 & 11/19/2007 13:30 & 1121 & 11/20/2007 11:30 & 22.0 & ? & YES & Very few spinel, some rather large undissolved agglomerates \\
\hline HWL-05Q & 5 & 11/28/2007 16:04 & 1138 & 11/29/2007 14:21 & 22.3 & YES & YES & Several spinel, some other undissolved material present \\
\hline HWL-05Q & 5 & $12 / 2 / 200714: 57$ & 1148 & 12/3/2007 14:30 & 23.5 & YES & NO & Sample clear of spinel, some other undissolved mat'ls \\
\hline HWL-06Q & 10 & 11/13/2007 15:55 & 1239 & 11/14/2007 14:18 & 22.4 & NO & NO & Sample clear \\
\hline HWL-06Q & 8 & 11/13/2007 15:55 & 994 & 11/14/2007 14:18 & 22.4 & YES & YES & Lots of spinels and some other undissolved materials \\
\hline HWL-06Q & 5 & 11/13/2007 15:55 & 1090 & 11/14/2007 14:18 & 22.4 & YES & YES & Lots of spinels and some other undissolved materials \\
\hline HWL-06Q & 10 & 11/15/2007 16:48 & 1152 & 11/16/2007 16:42 & 23.9 & YES & YES & Moderate amount of spinel, some other undissolved materials \\
\hline HWL-06Q & 10 & 11/19/2007 13:30 & 1161 & 11/20/2007 11:30 & 22.0 & $?$ & YES & Few cubic structures amongst base of sample, other undissolved materials? \\
\hline HWL-06Q & 10 & 11/28/2007 16:04 & 1172 & 11/29/2007 14:21 & 22.3 & NO & NO & Sample clear \\
\hline HWL-06Q & 10 & $12 / 2 / 200714: 57$ & 1168 & $12 / 3 / 200714: 30$ & 23.5 & YES & NO & Sample clear of spinel, some other undissolved mat'ls \\
\hline HWL-07Q & 10 & 11/20/2007 16:05 & 1050 & 11/21/2007 14:22 & 22.3 & YES & YES & Lots of spinel equally distributed throughout bulk, some undissolved mat'ls at base \\
\hline HWL-07Q & 8 & 11/20/2007 16:05 & 1148 & 11/21/2007 14:22 & 22.3 & NO & NO & Sample clear \\
\hline HWL-07Q & 5 & 11/20/2007 16:05 & 1250 & 11/21/2007 14:22 & 22.3 & NO & NO & Sample clear, sample volatilized out of crucible \\
\hline HWL-07Q & 8 & 11/28/2007 16:04 & 1098 & 11/29/2007 14:21 & 22.3 & YES & YES & Several spinel and some other undissolved mat'ls (mainly at base) \\
\hline HWL-07Q & 8 & 12/6/2007 16:28 & 1126 & 12/7/2007 15:10 & 22.7 & NO & NO & Sample clear of spinel \\
\hline HWL-07Q & 8 & 12/13/2007 15:35 & 1108 & 12/14/2007 15:50 & 24.2 & YES & YES & Several spinel and other undissolved materials at base on one side \\
\hline HWL-07Q & 8 & 12/20/2007 17:18 & 1118 & $12 / 21 / 200716: 30$ & 23.2 & NO & NO & Sample clear \\
\hline HWL-08Q & 10 & 11/20/2007 16:05 & 1050 & $11 / 21 / 200714: 22$ & 22.3 & YES & YES & Lots of spinel equally distributed throughout bulk, some undissolved mat'ls at base \\
\hline HWL-08Q & 8 & 11/20/2007 16:05 & 1148 & 11/21/2007 14:22 & 22.3 & ? & YES & Few somewhat cubic structures throughout bottom half of sample \\
\hline HWL-08Q & 5 & 11/20/2007 16:05 & 1250 & 11/21/2007 14:22 & 22.3 & NO & NO & Sample clear \\
\hline HWL-08Q & 10 & 11/28/2007 16:04 & 1172 & 11/29/2007 14:21 & 22.3 & NO & NO & Sample clear \\
\hline HWL-08Q & 10 & 12/2/2007 14:57 & 1168 & $12 / 3 / 200714: 30$ & 23.5 & NO & NO & Sample clear of spinel, some other undissolved mat'ls \\
\hline HWL-08Q & 8 & 12/10/2007 15:50 & 1157 & 12/11/2007 15:57 & 24.1 & NO & NO & Sample clear \\
\hline HWL-09Q & 10 & 11/20/2007 16:05 & 1050 & $11 / 21 / 200714: 22$ & 22.3 & YES & NO & Sample clear of spinel, some other undissolved mat'ls \\
\hline HWL-09Q & 8 & 11/20/2007 16:05 & 1148 & $11 / 21 / 200714: 22$ & 22.3 & NO & NO & Sample clear \\
\hline HWL-09Q & 5 & 11/20/2007 16:05 & 1250 & 11/21/2007 14:22 & 22.3 & NO & NO & Sample clear, sample volatilized out of crucible \\
\hline HWL-09Q & 10 & 11/27/2007 16:00 & 994 & 11/28/2007 16:00 & 24.0 & NO & NO & Sample clear \\
\hline HWL-09Q & 5 & 11/29/2007 15:47 & 954 & 11/30/2007 15:40 & 23.9 & YES & YES & Sample full of spinel throughout bulk, some other undissolved mat'ls \\
\hline HWL-09Q & 5 & $12 / 4 / 200717: 22$ & 974 & $12 / 5 / 200716: 27$ & 23.1 & YES & YES & $\begin{array}{l}\text { Some cubic crystals at base and side, other undissolved mat'ls (some appear } \\
\text { pentagonal) }\end{array}$ \\
\hline HWL-09Q & 5 & 12/12/2007 16:13 & 983 & 12/13/2007 16:13 & 24.0 & YES & YES & Few spinel in trails from base, some other undissolved materials \\
\hline HWL-09Q & 5 & $12 / 26 / 200716: 47$ & 991 & $12 / 27 / 200716: 20$ & 23.5 & YES & NO & Sample clear of spinel, some other undissolved mat'ls \\
\hline HWL-10Q & 10 & 11/20/2007 16:05 & 1050 & $11 / 21 / 200714: 22$ & 22.3 & YES & NO & Sample clear of spinel, one large undissolved agglomerate at top of sample \\
\hline HWL-10Q & 8 & 11/20/2007 16:05 & 1148 & 11/21/2007 14:22 & 22.3 & YES & NO & Sample clear of spinel, one small undissolved Ru agglomerate \\
\hline HWL-10Q & 5 & 11/20/2007 16:05 & 1250 & $11 / 21 / 200714: 22$ & 22.3 & NO & NO & Sample clear, sample volatilized out of crucible \\
\hline HWL-10Q & 10 & 11/27/2007 16:00 & 994 & 11/28/2007 16:00 & 24.0 & YES & YES & Lots of spinel, some other undissolved material present \\
\hline HWL-10Q & 8 & 11/29/2007 15:47 & 1019 & 11/30/2007 15:40 & 23.9 & YES & YES & Several spinel (mostly at base and sides), some other undissolved mat'ls \\
\hline
\end{tabular}




\begin{tabular}{|c|c|c|c|c|c|c|c|c|}
\hline Content & Fur & IN/H.T. START & TEMP & OUT/H.T. FINISH & Dur. (hrs) & Undis. $N M$ & Spinel & Total Observations \\
\hline HWL-10Q & 8 & 12/3/2007 17:37 & 1033 & 12/4/2007 16:58 & 23.3 & YES & NO & No spinel, some other undissolved mat'ls \\
\hline HWL-10Q & 8 & 12/5/2007 17:06 & 1026 & 12/6/2007 16:30 & 23.4 & YES & YES & Very few spinels, some other undissolved agglomerates and particles \\
\hline HWL-11Q & 10 & 11/20/2007 16:05 & 1050 & $11 / 21 / 200714: 22$ & 22.3 & YES & YES & Lots of spinel (mainly at base/sides) one large undissolved agglomerate \\
\hline HWL-11Q & 8 & 11/20/2007 16:05 & 1148 & $11 / 21 / 200714: 22$ & 22.3 & $\mathrm{NO}$ & NO & Sample clear \\
\hline HWL-11Q & 5 & 11/20/2007 16:05 & 1250 & 11/21/2007 14:22 & 22.3 & NO & NO & Sample clear, sample volatilized out of crucible \\
\hline HWL-11Q & 8 & 11/28/2007 16:04 & 1098 & $11 / 29 / 200714: 21$ & 22.3 & $\mathrm{NO}$ & NO & Sample clear \\
\hline HWL-11Q & 10 & 12/3/2007 17:37 & 1072 & 12/4/2007 16:58 & 23.3 & YES & YES & Modest amount of spinel, other undissolved mat'ls \\
\hline HWL-11Q & 10 & 12/5/2007 17:06 & 1187 & $12 / 6 / 200716: 30$ & 23.4 & $\overline{-}$ & $\ldots$ & Sample volatilized out of crucible \\
\hline HWL-11Q & 10 & 12/6/2007 16:28 & 1082 & 12/7/2007 15:10 & 22.7 & YES & YES & Few spinel, other materials \\
\hline HWL-11Q & 10 & 12/10/2007 15:50 & 1090 & 12/11/2007 15:57 & 24.1 & NO & NO & Sample clear \\
\hline HWL-12Q & 10 & $11 / 23 / 200712: 25$ & 1045 & $11 / 24 / 200712: 13$ & 23.8 & $\mathrm{NO}$ & NO & Sample clear \\
\hline HWL-12Q & 8 & $11 / 23 / 200712: 25$ & 1149 & $11 / 24 / 200712: 13$ & 23.8 & $\mathrm{NO}$ & NO & Sample clear \\
\hline HWL-12Q & 5 & $11 / 23 / 200712: 25$ & 1249 & 11/24/2007 12:13 & 23.8 & $\mathrm{NO}$ & NO & Sample clear \\
\hline HWL-12Q & 10 & 11/27/2007 16:00 & 994 & 11/28/2007 16:00 & 24.0 & YES & NO & Sample clear of spinel, some other undissolved mat'ls \\
\hline HWL-12Q & 5 & 11/29/2007 15:47 & 954 & $11 / 30 / 200715: 40$ & 23.9 & YES & YES & Few patches of spinel at base, some other undissolved mat'ls \\
\hline HWL-12Q & 5 & 12/4/2007 17:22 & 974 & 12/5/2007 16:27 & 23.1 & NO & NO & Sample clear \\
\hline HWL-12Q & 5 & 12/10/2007 15:50 & 965 & 12/11/2007 15:57 & 24.1 & $\mathrm{NO}$ & NO & Sample clear \\
\hline HWL-12Q & 5 & 12/14/2007 16:11 & 960 & $12 / 15 / 200714: 20$ & 22.1 & NO & NO & Sample clear \\
\hline HWL-13Q & 10 & 11/23/2007 12:25 & 1045 & 11/24/2007 12:13 & 23.8 & NO & NO & Sample clear \\
\hline HWL-13Q & 8 & $11 / 23 / 200712: 25$ & 1149 & 11/24/2007 12:13 & 23.8 & $\mathrm{NO}$ & NO & Sample clear \\
\hline HWL-13Q & 5 & $11 / 23 / 200712: 25$ & 1249 & 11/24/2007 12:13 & 23.8 & $\mathrm{NO}$ & NO & Sample clear \\
\hline HWL-13Q & 10 & 11/27/2007 16:00 & 994 & 11/28/2007 16:00 & 24.0 & YES & YES & A few sets of spinel (mainly at base and sides) \\
\hline HWL-13Q & 8 & 11/29/2007 15:47 & 1019 & 11/30/2007 15:40 & 23.9 & YES & YES & Very few spinel (mostly at base and sides), some other undissolved mat'ls \\
\hline HWL-13Q & 8 & 12/3/2007 17:37 & 1033 & 12/4/2007 16:58 & 23.3 & YES & NO & No spinel, some other undissolved mat'ls \\
\hline HWL-13Q & 8 & 12/5/2007 17:06 & 1026 & 12/6/2007 16:30 & 23.4 & NO & NO & Sample clear \\
\hline HWL-14Q & 10 & 11/23/2007 12:25 & 1045 & 11/24/2007 12:13 & 23.8 & YES & YES & Lots of spinel, mostly at base and sides, some large agglomerates of undissolved mat'ls \\
\hline HWL-14Q & 8 & $11 / 23 / 200712: 25$ & 1149 & 11/24/2007 12:13 & 23.8 & NO & NO & Sample clear \\
\hline HWL-14Q & 5 & $11 / 23 / 200712: 25$ & 1249 & $11 / 24 / 200712: 13$ & 23.8 & $\mathrm{NO}$ & NO & Sample clear \\
\hline HWL-14Q & 8 & 11/28/2007 16:04 & 1098 & 11/29/2007 14:21 & 22.3 & $\mathrm{NO}$ & NO & Sample clear \\
\hline HWL-14Q & 10 & 12/3/2007 17:37 & 1072 & 12/4/2007 16:58 & 23.3 & YES & YES & Modest amount of spinel, other undissolved mat'ls \\
\hline HWL $14 Q$ & 10 & 12/5/200717:06 & 1187 & $12 / 6 / 200716: 30$ & 23.4 & 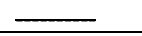 & $\overline{-}$ & Sample volatilized out of erueible \\
\hline HWL-14Q & 10 & 12/6/2007 16:28 & 1082 & 12/7/2007 15:10 & 22.7 & YES & YES & Few spinel, other materials \\
\hline HWL-14Q & 10 & 12/10/2007 15:50 & 1090 & 12/11/2007 15:57 & 24.1 & NO & NO & Sample clear \\
\hline HWL-15Q & 10 & $11 / 23 / 200712: 25$ & 1045 & 11/24/2007 12:13 & 23.8 & YES & NO & Sample clear of spinel, one undissolved agglomerate at base \\
\hline HWL $15 Q$ & 8 & $11 / 23 / 200712: 25$ & 1149 & $11 / 24 / 200712: 13$ & 23.8 & - & - & Volatilized of encible \\
\hline HWL-15Q & 5 & 11/23/2007 12:25 & 1249 & 11/24/2007 12:13 & 23.8 & NO & NO & Sample clear \\
\hline HWL-15Q & 10 & 11/27/2007 16:00 & 994 & 11/28/2007 16:00 & 24.0 & YES & YES & Two agglomerates at base, appear to contain some cubic structures (spinel?) \\
\hline HWL-15Q & 8 & 11/29/2007 15:47 & 1019 & $11 / 30 / 200715: 40$ & 23.9 & NO & NO & Sample clear of spinel, some other undissolved mat'ls \\
\hline HWL-15Q & 5 & 11/29/2007 15:47 & 954 & 11/30/2007 15:40 & 23.9 & YES & YES & Very few spinel (verify w/ lower temperature run), some other undissolved mat'ls \\
\hline HWL-15Q & 5 & $12 / 3 / 200717: 37$ & 903 & 12/4/2007 16:58 & 23.3 & YES & YES & Several streaks of spinel, other undissolved mat'ls \\
\hline HWL-15Q & 5 & 12/5/2007 17:06 & 924 & 12/6/2007 16:30 & 23.4 & YES & YES & Lots of spinel throughout bulk, other undissolved mat'ls \\
\hline HWL-15Q & 5 & 12/9/2007 14:17 & 941 & 12/10/2007 15:00 & 24.7 & YES & YES & Some possibly crystalline materials \\
\hline HWL-15Q & 5 & $12 / 11 / 200716: 25$ & 948 & $12 / 12 / 200714: 35$ & 22.2 & YES & YES & Few spinel on sides, lots of other undissolved materials \\
\hline HWL-16Q & 10 & $11 / 23 / 200712: 25$ & 1045 & 11/24/2007 12:13 & 23.8 & NO & NO & Sample clear \\
\hline HWL-16Q & 8 & $11 / 23 / 200712: 25$ & 1149 & $11 / 24 / 200712: 13$ & 23.8 & $\mathrm{NO}$ & NO & Sample clear \\
\hline HWL-16Q & 5 & $11 / 23 / 200712: 25$ & 1249 & 11/24/2007 12:13 & 23.8 & $\mathrm{NO}$ & NO & Sample clear \\
\hline HWL-16Q & 10 & 11/27/2007 16:00 & 994 & 11/28/2007 16:00 & 24.0 & YES & YES & Few agglomerates, some spinel in trails from sides and at base \\
\hline
\end{tabular}




\begin{tabular}{|c|c|c|c|c|c|c|c|c|}
\hline Content & Fur & IN/H.T.START & TEMP & OUT/H.T. FINISH & Dur. (hrs) & Undis. $N M$ & Spinel & Total Observations \\
\hline HWL-16Q & 8 & 11/29/2007 15:47 & 1019 & $11 / 30 / 200715: 40$ & 23.9 & $\mathrm{NO}$ & NO & Sample clear \\
\hline HWL-16Q & 5 & $11 / 29 / 200715: 47$ & 954 & $11 / 30 / 200715: 40$ & 23.9 & $\mathrm{NO}$ & $\mathrm{NO}$ & Sample clear \\
\hline HWL-16Q & 5 & 12/3/2007 17:37 & 903 & $12 / 4 / 200716: 58$ & 23.3 & YES & YES & Several streaks of spinel, other undissolved mat'ls \\
\hline HWL-16Q & 5 & 12/5/2007 17:06 & 924 & 12/6/2007 16:30 & 23.4 & YES & YES & Lots of crystals- cubic, rectangular, pentagonal, hematite?, others irregularly shaped \\
\hline HWL-16Q & 5 & 12/9/2007 14:17 & 941 & 12/10/2007 15:00 & 24.7 & YES & YES & Spinel and other undissolved materials throughout bulk of sample \\
\hline HWL-16Q & 5 & $12 / 11 / 200716: 25$ & 948 & $12 / 12 / 200714: 35$ & 22.2 & YES & YES & Lots of spinel and other undissolved material \\
\hline HWL-16Q & 5 & 12/13/2007 15:35 & 1003 & $12 / 14 / 200715: 50$ & 24.2 & YES & NO & Sample clear of spinel, some other undissolved mat'ls \\
\hline HWL-16Q & 10 & $12 / 21 / 200716: 57$ & 1000 & $12 / 22 / 200715: 50$ & 22.9 & YES & NO & Sample clear of spinel, some other undissolved mat'ls \\
\hline HWL-17Q & 10 & $11 / 26 / 200716: 30$ & 995 & 11/27/2007 15:08 & 22.6 & $?$ & Yes & Several spinel throughout sample, some undissolved materials \\
\hline HWL-17Q & 8 & 11/26/2007 16:30 & 1063 & 11/27/2007 15:08 & 22.6 & $\mathrm{NO}$ & $\mathrm{NO}$ & Sample clear \\
\hline HWL-17Q & 5 & 11/26/2007 16:30 & 1152 & 11/27/2007 15:08 & 22.6 & $\mathrm{NO}$ & NO & Sample clear \\
\hline HWL-17Q & 8 & 11/29/2007 15:47 & 1019 & $11 / 30 / 200715: 40$ & 23.9 & YES & YES & Very few spinel (mostly at sides), some other undissolved mat'ls \\
\hline HWL-17Q & 8 & 12/3/2007 17:37 & 1033 & $12 / 4 / 200716: 58$ & 23.3 & YES & NO & No spinel, some other undissolved mat'ls \\
\hline HWL-17Q & 8 & 12/5/2007 17:06 & 1026 & $12 / 6 / 200716: 30$ & 23.4 & YES & YES & Few spinel (base and sides), some other undissolved materials \\
\hline HWL-18Q & 10 & 11/26/2007 16:30 & 995 & 11/27/2007 15:08 & 22.6 & $\mathrm{NO}$ & NO & Sample clear \\
\hline HWL-18Q & 8 & 11/26/2007 16:30 & 1063 & 11/27/2007 15:08 & 22.6 & $\mathrm{NO}$ & NO & Sample clear \\
\hline HWL-18Q & 5 & 11/26/2007 16:30 & 1152 & 11/27/2007 15:08 & 22.6 & $\mathrm{NO}$ & $\mathrm{NO}$ & Sample clear \\
\hline HWL-18Q & 5 & 11/29/2007 15:47 & 954 & $11 / 30 / 200715: 40$ & 23.9 & $\mathrm{NO}$ & NO & Sample clear \\
\hline HWL-18Q & 5 & 12/3/2007 17:37 & 903 & 12/4/2007 16:58 & 23.3 & YES & NO & Sample clear \\
\hline HWL-18Q & 10 & 12/17/2007 11:53 & 861 & $12 / 18 / 200713: 38$ & 25.8 & YES & $\mathrm{NO}$ & Sample clear of spinel, a few undissolved agglomerates \\
\hline HWL-18Q & 10 & 12/19/2007 17:52 & 869 & $12 / 20 / 200716: 30$ & 22.6 & $\mathrm{NO}$ & NO & Sample clear \\
\hline HWL-18Q & 8 & $12 / 21 / 200716: 57$ & 802 & $12 / 23 / 200714: 45$ & 45.8 & YES & YES & Very few spinel \\
\hline HWL-18Q & 8 & $12 / 26 / 200716: 47$ & 816 & $12 / 27 / 200716: 20$ & 23.5 & YES & YES & Several spinel through bulk \\
\hline HWL-18Q & 8 & 12/28/2007 17:08 & 844 & $12 / 30 / 200717: 29$ & 48.4 & YES & NO & No spinel present, some other undissolved mat'ls \\
\hline HWL-18Q & 8 & $12 / 31 / 200716: 30$ & 820 & 1/2/2008 13:51 & 45.3 & YES & YES & Lots of spinel trails visible throughout bulk, other undissolved mat'ls \\
\hline HWL-18Q & 8 & 1/2/2008 14:12 & 833 & 1/4/2008 13:20 & 47.1 & YES & YES & One small trail of spinel in bulk, other undissolved materials present \\
\hline HWL-18Q & 8 & 1/4/2008 13:55 & 825 & 1/6/2008 14:50 & 48.9 & YES & YES & Several spinel and other undissolved materials in streams through bulk \\
\hline HWL-19Q & 10 & $11 / 26 / 200716: 30$ & 995 & 11/27/2007 15:08 & 22.6 & $\mathrm{NO}$ & NO & Sample clear \\
\hline HWL-19Q & 8 & 11/26/2007 16:30 & 1063 & 11/27/2007 15:08 & 22.6 & $\mathrm{NO}$ & NO & Sample clear \\
\hline HWL-19Q & 5 & 11/26/2007 16:30 & 1152 & 11/27/2007 15:08 & 22.6 & $\mathrm{NO}$ & $\mathrm{NO}$ & Sample clear \\
\hline HWL-19Q & 5 & 11/29/2007 15:47 & 954 & $11 / 30 / 200715: 40$ & 23.9 & $\mathrm{NO}$ & $\mathrm{NO}$ & Sample clear \\
\hline HWL-19Q & 8 & 12/17/2007 11:53 & 895 & $12 / 18 / 200713: 38$ & 25.8 & - & $\overline{-}$ & $\overline{-}$ \\
\hline HWL-19Q & 10 & 12/19/2007 17:52 & 869 & $12 / 20 / 200716: 30$ & 22.6 & YES & YES & Sample full of spinel throughout bulk, some other undissolved mat'ls \\
\hline HWL-19Q & 10 & $12 / 26 / 200716: 47$ & 899 & $12 / 27 / 200716: 20$ & 23.5 & YES & YES & Lots of undissolved materials (some appear cubic, others round) \\
\hline HWL-19Q & 10 & 1/3/2008 14:46 & 924 & 1/4/2008 13:20 & 22.6 & YES & NO & Sample clear of spinel, possibly some undissolved materials \\
\hline HWL-19Q & 10 & 1/7/2008 17:50 & 909 & 1/8/2008 15:55 & 22.1 & YES & YES & Some spinel and other materials in streams \\
\hline HWL-19Q & 10 & 1/8/2008 17:20 & 915 & 1/10/2008 16:08 & 46.8 & $?$ & $\mathrm{NO}$ & Sample clear of spinel, possibly some undissolved materials \\
\hline HWL-20Q & 10 & 11/26/2007 16:30 & 995 & 11/27/2007 15:08 & 22.6 & YES & YES & Several little spinel (at base and sides), some other undissolved mat'ls \\
\hline HWL-20Q & 8 & 11/26/2007 16:30 & 1063 & 11/27/2007 15:08 & 22.6 & $\mathrm{NO}$ & NO & Sample clear \\
\hline HWL-20Q & 5 & 11/26/2007 16:30 & 1152 & 11/27/2007 15:08 & 22.6 & $\mathrm{NO}$ & NO & Sample clear \\
\hline HWL-20Q & 8 & $11 / 29 / 200715: 47$ & 1019 & $11 / 30 / 200715: 40$ & 23.9 & YES & YES & Very few spinel (mostly at sides and base), some other undissolved mat'ls \\
\hline HWL-20Q & 8 & 12/3/2007 17:37 & 1033 & 12/4/2007 16:58 & 23.3 & YES & NO & No spinel, some other undissolved mat'ls \\
\hline HWL-20Q & 8 & 12/5/2007 17:06 & 1026 & 12/6/2007 16:30 & 23.4 & YES & YES & Very few spinel at base, some other undissolved materials \\
\hline HWL-21Q & 10 & $11 / 26 / 200716: 30$ & 995 & 11/27/2007 15:08 & 22.6 & YES & YES & Lots of spinel throughout, some other undissolved materials \\
\hline HWL-21Q & 8 & $11 / 26 / 200716: 30$ & 1063 & 11/27/2007 15:08 & 22.6 & $\mathrm{NO}$ & NO & Sample clear \\
\hline HWL-21Q & 5 & $11 / 26 / 200716: 30$ & 1152 & 11/27/2007 15:08 & 22.6 & $\mathrm{NO}$ & NO & Sample clear \\
\hline HWL-21Q & 8 & 11/29/2007 15:47 & 1019 & $11 / 30 / 200715: 40$ & 23.9 & YES & YES & Very few spinel (mostly at sides and base), some other undissolved mat'ls \\
\hline
\end{tabular}




\begin{tabular}{|c|c|c|c|c|c|c|c|c|}
\hline Content & Fur & IN/H.T.START & TEMP & OUT/H.T. FINISH & Dur. (hrs) & Undis. $N M$ & Spinel & Total Observations \\
\hline HWL-21Q & 8 & 12/3/2007 17:37 & 1033 & 12/4/2007 16:58 & 23.3 & YES & YES & Very few spinel \\
\hline HWL-21Q & 8 & 12/12/2007 16:13 & 1043 & 12/13/2007 16:13 & 24.0 & NO & $\mathrm{NO}$ & Sample clear \\
\hline HWL-22Q & 10 & $12 / 2 / 200714: 57$ & 1168 & $12 / 3 / 200714: 30$ & 23.5 & $\mathrm{NO}$ & $\mathrm{NO}$ & Sample clear of spinel, some other undissolved mat'ls \\
\hline HWL-22Q & 8 & $12 / 2 / 200714: 57$ & 1091 & $12 / 3 / 200714: 30$ & 23.5 & YES & YES & Few spinel at base, what appears to be hematite, some other undissolved mat'ls \\
\hline HWL-22Q & 5 & $12 / 6 / 200716: 28$ & 1162 & 12/7/2007 15:10 & 22.7 & $\overline{-}$ & $\overline{-}$ & Sample volatilized out of crucible \\
\hline HWL-22Q & 8 & 12/9/2007 14:17 & 1074 & 12/10/2007 15:00 & 24.7 & YES & YES & Several streak of spinel, some other undissolved materials \\
\hline HWL-22Q & 8 & $12 / 11 / 200716: 25$ & 1098 & $12 / 12 / 200714: 35$ & 22.2 & NO & $\mathrm{NO}$ & Sample clear \\
\hline SRM-773 & 10 & 10/1/2007 13:22 & - & 10/2/2007 14:08 & 24.8 & $\mathrm{~N} / \mathrm{A}$ & $\mathrm{N} / \mathrm{A}$ & Crystals \\
\hline SRM-773 & 8 & 10/1/2007 13:22 & 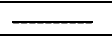 & $10 / 2 / 200714: 08$ & 24.8 & $\mathrm{~N} / \mathrm{A}$ & $\mathrm{N} / \mathrm{A}$ & Crystals \\
\hline SRM-773 & 5 & $10 / 1 / 200713: 22$ & $\overline{-1}$ & $10 / 2 / 200714: 08$ & 24.8 & $\mathrm{~N} / \mathrm{A}$ & $\mathrm{N} / \mathrm{A}$ & Crystals \\
\hline SRM-773 & 10 & 10/2/2007 16:08 & $\overline{-1}$ & 10/3/2007 15:12 & 23.1 & $\mathrm{~N} / \mathrm{A}$ & N/A & Crystals \\
\hline SRM-773 & 8 & $10 / 2 / 200716: 08$ & - & $10 / 3 / 200715: 12$ & 23.1 & N/A & N/A & No crystals \\
\hline SRM-773 & 5 & $\begin{array}{l}10 / 2 / 200716: 08 \\
\end{array}$ & - & $10 / 3 / 2007 \quad 15: 12$ & 23.1 & $\mathrm{~N} / \mathrm{A}$ & $\mathrm{N} / \mathrm{A}$ & Crystals \\
\hline SRM-773 & 10 & $10 / 3 / 2007 \quad 15: 49$ & - & 10/4/2007 15:00 & 23.2 & $\mathrm{~N} / \mathrm{A}$ & $\mathrm{N} / \mathrm{A}$ & No crystals \\
\hline SRM-773 & 8 & 10/3/2007 15:49 & $\overline{-}$ & 10/4/2007 15:00 & 23.2 & $\mathrm{~N} / \mathrm{A}$ & $\mathrm{N} / \mathrm{A}$ & Crystals \\
\hline SRM-773 & 5 & 10/3/2007 15:49 & 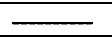 & 10/4/2007 15:00 & 23.2 & $\mathrm{~N} / \mathrm{A}$ & $\mathrm{N} / \mathrm{A}$ & Crystals \\
\hline SRM-773 & 5 & 10/4/2007 16:06 & 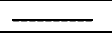 & 10/5/2007 15:00 & 22.9 & N/A & N/A & No crystals \\
\hline SRM-773 & 5 & 10/7/2007 14:36 & $=$ & 10/8/2007 15:30 & 24.9 & N/A & $\mathrm{N} / \mathrm{A}$ & Crystals \\
\hline
\end{tabular}


PNNL-17950, Rev 0

\section{Appendix E: Crystal Fraction Data}

This section presents the data obtained by performing XRD on heat treated specimens from each glass sample run at different temperatures. The temperature $(T)$ versus crystal mass fraction $\left(C_{F}\right)$ data was plotted and the resulting trend line was presented in Table E. 1 as $T=$ Slope $\times C_{F}+Y$-Int and thus $Y$-Int is equal to the $T_{L}$ value obtained using the crystal fraction method.

Table E.1. Summary of Crystal Mass Fraction Data for EM07 Glasses. The slope, $Y$-Int, and correlation coefficient $\left(R^{2}\right)$ are statistical parameters fit to the temperature as a function of the concentration of trevorite (spinel). The $Y$-Int listed in the table corresponds to the calculated data for $T_{L}$ using the crystal fraction method.

\begin{tabular}{|l|r|r|r|r|r|r|r|c|}
\hline Glass ID & Trevorite & Zircon & Total Crys. & Glass & Temp. $\left({ }^{\circ} \mathbf{C}\right)$ & Slope & Y-Int. & $\boldsymbol{R}^{2}$ \\
\hline EM07-BL-1 & 2.0 & & 2.0 & 98.0 & 944 & -116.18 & 1181 & 0.985 \\
\hline EM07-BL-1 & 0.2 & & 0.2 & 99.8 & 1148 & & & \\
\hline EM07-BL-1 & 0.8 & & 0.8 & 99.2 & 1103 & & & \\
\hline EM07-BL-1 & 1.1 & & 1.1 & 98.9 & 1051 & & & \\
\hline EM07-Al-06 & 1.6 & & 1.6 & 98.4 & 947 & -84.62 & 1086 & 0.991 \\
\hline EM07-Al-06 & 0.8 & & 0.8 & 99.2 & 1026 & & & \\
\hline EM07-Al-06 & 0.4 & & 0.4 & 99.6 & 1051 & & & \\
\hline EM07-A1-06 & 0.1 & & 0.1 & 99.9 & 1074 & & & \\
\hline EM07-Al-15 & 1.0 & & 1.0 & 99.0 & 1190 & -135.14 & 1297 & 0.864 \\
\hline EM07-Al-15 & 1.8 & & 1.8 & 98.2 & 1051 & & & \\
\hline EM07-Al-15 & 0.9 & & 0.9 & 99.1 & 1151 & & & \\
\hline EM07-Al-20 & 2.4 & & 2.4 & 97.6 & 1140 & -101.04 & 1380 & 0.999 \\
\hline EM07-Al-20 & 3.1 & & 3.1 & 96.9 & 1065 & & & \\
\hline EM07-Al-20 & 1.5 & & 1.5 & 98.5 & 1227 & & & \\
\hline EM07-B-05 & 0.5 & & 0.5 & 99.5 & 1157 & -107.71 & 1228 & 0.923 \\
\hline EM07-B-05 & 1.5 & & 1.5 & 98.5 & 1103 & & & \\
\hline EM07-B-05 & 1.6 & & 1.6 & 98.4 & 1051 & & & \\
\hline EM07-B-05 & 2.5 & & 2.5 & 97.5 & 942 & & & \\
\hline EM07-B-15 & 1.0 & & 1.0 & 99.0 & 1051 & -66.13 & 1107 & 0.879 \\
\hline EM07-B-15 & 1.4 & & 1.4 & 98.6 & 999 & & & \\
\hline EM07-B-15 & 2.1 & & 2.1 & 97.9 & 974 & & & \\
\hline EM07-B-20 & 1.1 & & 1.1 & 98.9 & 942 & -172.08 & 1143 & 0.971 \\
\hline EM07-B-20 & 0.7 & & 0.7 & 99.3 & 1042 & & & \\
\hline EM07-B-20 & 0.2 & & 0.2 & 99.8 & 1102 & & & \\
\hline EM07-B-20 & 0.1 & & 0.1 & 99.9 & 1123 & & & \\
\hline EM07-Bi-025 & 1.9 & & 1.9 & 98.1 & 942 & -121.40 & 1173 & 0.952 \\
\hline EM07-Bi-025 & 0.9 & & 0.9 & 99.1 & 1042 & & & \\
\hline EM07-Bi-025 & 0.8 & & 0.8 & 99.2 & 1103 & & & \\
\hline EM07-Bi-025 & 0.1 & & 0.1 & 99.9 & 1157 & & & \\
\hline EM07-Bi-05 & 1.6 & & 1.6 & 98.4 & 942 & -146.30 & 1174 & 0.980 \\
\hline EM07-Bi-05 & 0.8 & & 0.8 & 99.2 & 1042 & & & \\
\hline EM07-Bi-05 & 0.6 & & 0.6 & 99.4 & 1103 & & & \\
\hline
\end{tabular}


PNNL-17950, Rev 0

\begin{tabular}{|c|c|c|c|c|c|c|c|}
\hline EM07-Bi-05 & 0.1 & 0.1 & 99.9 & 1157 & & & \\
\hline EM07-Ca-035 & 2.3 & 2.3 & 97.7 & 915 & -92.52 & 1126 & 0.976 \\
\hline EM07-Ca-035 & 2.0 & 2.0 & 98.0 & 947 & & & \\
\hline EM07-Ca-035 & 1.2 & 1.2 & 98.8 & 999 & & & \\
\hline EM07-Ca-035 & 0.7 & 0.7 & 999.3 & 1053 & & & \\
\hline EM07-Ca-035 & 0.4 & 0.4 & 99.6 & 1103 & & & \\
\hline EM07-Ca-07 & 1.6 & 1.6 & 98.4 & 942 & -104.44 & 1112 & 0.989 \\
\hline EM07-Ca-07 & 0.4 & 0.4 & 99.6 & 1073 & & & \\
\hline EM07-Ca-07 & 0.6 & 0.6 & 99.4 & 1042 & & & \\
\hline EM07-Ca-07 & 1.1 & 1.1 & 98.9 & 1003 & & & \\
\hline EM07-Cr-001 & 1.6 & 1.6 & 98.4 & 915 & -86.68 & 1067 & 0.904 \\
\hline EM07-Cr-001 & 1.3 & 1.3 & 98.7 & 974 & & & \\
\hline EM07-Cr-001 & 0.6 & 0.6 & 99.4 & 996 & & & \\
\hline EM07-Cr-001 & 0.6 & 0.6 & 99.4 & 1026 & & & \\
\hline EM07-Cr-001 & 0.2 & 0.2 & 99.8 & 1051 & & & \\
\hline EM07-Cr-012 & 1.9 & 1.9 & 98.1 & 1099 & -125.46 & 1320 & 0.979 \\
\hline EM07-Cr-012 & 2.5 & 2.5 & 97.5 & 996 & & & \\
\hline EM07-Cr-012 & 0.9 & 0.9 & 99.1 & 1201 & & & \\
\hline EM07-F-02 & 0.2 & 0.2 & 99.8 & 1148 & -158.34 & 1182 & 0.994 \\
\hline EM07-F-02 & 0.9 & 0.9 & 99.1 & 1048 & & & \\
\hline EM07-F-02 & 1.1 & 1.1 & 98.9 & 1001 & & & \\
\hline EM07-F-02 & 1.5 & 1.5 & 98.5 & 944 & & & \\
\hline EM07-Fe-05 & 1.4 & 1.4 & 98.6 & 844 & -154.57 & 1065 & 0.995 \\
\hline EM07-Fe-05 & 1.0 & 1.0 & 99.0 & 915 & & & \\
\hline EM07-Fe-05 & 0.8 & 0.8 & 99.2 & 947 & & & \\
\hline EM07-Fe-05 & 0.4 & 0.4 & 99.6 & 996 & & & \\
\hline EM07-Fe-05 & 0.1 & 0.1 & 99.9 & 1051 & & & \\
\hline EM07-Fe-15 & 2.0 & 2.0 & 98.0 & 1051 & -110.02 & 1270 & 0.991 \\
\hline EM07-Fe-15 & 1.0 & 1.0 & 99.0 & 1151 & & & \\
\hline EM07-Fe-15 & 0.8 & 0.8 & 99.2 & 1190 & & & \\
\hline EM07-Fe-15 & 0.5 & 0.5 & 99.5 & 1214 & & & \\
\hline EM07-Fe-20 & 3.9 & 3.9 & 96.1 & 1051 & -88.21 & 1398 & 0.989 \\
\hline EM07-Fe-20 & 2.9 & 2.9 & 97.1 & 1151 & & & \\
\hline EM07-Fe-20 & 2.3 & 2.3 & 97.7 & 1190 & & & \\
\hline EM07-K-03 & 0.2 & 0.2 & 99.8 & 1089 & -101.06 & 1105 & 0.985 \\
\hline EM07-K-03 & 0.7 & 0.7 & 99.3 & 1026 & & & \\
\hline EM07-K-03 & 1.1 & 1.1 & 98.9 & 999 & & & \\
\hline EM07-K-03 & 1.3 & 1.3 & 98.7 & 974 & & & \\
\hline EM07-K-06 & 1.8 & 1.8 & 98.2 & 849 & -112.86 & 1048 & 0.983 \\
\hline EM07-K-06 & 0.7 & 0.7 & 99.3 & 974 & & & \\
\hline EM07-K-06 & 1.2 & 1.2 & 98.8 & 903 & & & \\
\hline EM07-Li-015 & 1.4 & 1.4 & 98.6 & 1053 & -165.00 & 1283 & 1.000 \\
\hline EM07-Li-015 & 0.8 & 0.8 & 99.2 & 1150 & & & \\
\hline EM07-Li-015 & 0.2 & 0.2 & 99.8 & 1251 & & & \\
\hline EM07-Li-04 & 0.6 & 0.6 & 99.4 & 1053 & -60.13 & 1075 & 0.902 \\
\hline EM07-Li-04 & 2.0 & 2.0 & 98.0 & 974 & & & \\
\hline EM07-Li-04 & 2.5 & 2.5 & 97.5 & 915 & & & \\
\hline
\end{tabular}


PNNL-17950, Rev 0

\begin{tabular}{|c|c|c|c|c|c|c|c|}
\hline EM07-Li-04 & 1.0 & 1.0 & 99.0 & 1003 & & & \\
\hline EM07-Li-04 & 1.0 & 1.0 & 99.0 & 1001 & & & \\
\hline EM07-Mn-01 & 1.7 & 1.7 & 98.3 & 944 & -113.10 & 1134 & 0.987 \\
\hline EM07-Mn-01 & 0.8 & 0.8 & 99.2 & 1048 & & & \\
\hline EM07-Mn-01 & 1.1 & 1.1 & 98.9 & 1003 & & & \\
\hline EM07-Mn-04 & 1.0 & 1.0 & 99.0 & 1042 & -91.87 & 1143 & 0.859 \\
\hline EM07-Mn-04 & 0.3 & 0.3 & 99.7 & 1148 & & & \\
\hline EM07-Mn-04 & 1.1 & 1.1 & 98.9 & 1001 & & & \\
\hline EM07-Mn-04 & 2.4 & 2.4 & 97.6 & 942 & & & \\
\hline EM07-Na-20 & 0.1 & 0.1 & 99.9 & 999 & -90.95 & 1006 & 0.998 \\
\hline EM07-Na-20 & 0.6 & 0.6 & 99.4 & 949 & & & \\
\hline EM07-Na-20 & 1.1 & 1.1 & 98.9 & 903 & & & \\
\hline EM07-Na-20 & 1.8 & 1.8 & 98.2 & 844 & & & \\
\hline EM07-Ni-001 & 1.1 & 1.1 & 98.9 & 903 & -156.46 & 1076 & 0.990 \\
\hline EM07-Ni-001 & 1.0 & 1.0 & 99.0 & 915 & & & \\
\hline EM07-Ni-001 & 0.5 & 0.5 & 99.5 & 1009 & & & \\
\hline EM07-Ni-001 & 0.1 & 0.1 & 99.9 & 1051 & & & \\
\hline EM07-Ni-001 & 0.0 & 0.0 & 100.0 & 1078 & & & \\
\hline EM07-Ni-02 & 0.9 & 0.9 & 99.1 & 1151 & -115.00 & 1264 & 0.982 \\
\hline EM07-Ni-02 & 0.6 & 0.6 & 99.4 & 1200 & & & \\
\hline EM07-Ni-02 & 1.9 & 1.9 & 98.1 & 1060 & & & \\
\hline EM07-Ni-02 & 2.2 & 2.2 & 97.8 & 1001 & & & \\
\hline EM07-P-0 & 2.2 & 2.2 & 97.8 & 949 & -104.87 & 1174 & 0.987 \\
\hline EM07-P-0 & 1.6 & 1.6 & 98.4 & 999 & & & \\
\hline EM07-P-0 & 1.7 & 1.7 & 98.3 & 1001 & & & \\
\hline EM07-P-0 & 1.1 & 1.1 & 98.9 & 1047 & & & \\
\hline EM07-P-0 & 0.3 & 0.3 & 99.7 & 1150 & & & \\
\hline EM07-P-025 & 2.2 & 2.2 & 97.8 & 944 & -121.00 & 1202 & 0.980 \\
\hline EM07-P-025 & 1.1 & 1.1 & 98.9 & 1048 & & & \\
\hline EM07-P-025 & 0.4 & 0.4 & 99.6 & 1161 & & & \\
\hline EM07-P-025 & 0.5 & 0.5 & 99.5 & 1148 & & & \\
\hline EM07-Si-37 & 2.8 & 2.8 & 97.2 & 999 & -73.17 & 1198 & 0.879 \\
\hline EM07-Si-37 & 1.5 & 1.5 & 98.5 & 1102 & & & \\
\hline EM07-Si-37 & 0.7 & 0.7 & 99.3 & 1161 & & & \\
\hline EM07-Si-37 & 1.4 & 1.4 & 98.6 & 1060 & & & \\
\hline EM07-Si-50 & 1.3 & 1.3 & 98.7 & 999 & -147.26 & 1184 & 0.960 \\
\hline EM07-Si-50 & 0.6 & 0.6 & 99.4 & 1102 & & & \\
\hline EM07-Si-50 & 0.2 & 0.2 & 99.8 & 1161 & & & \\
\hline EM07-Si-50 & 0.7 & 0.7 & 99.3 & 1060 & & & \\
\hline EM07-Zr-001 & 0.2 & 0.2 & 99.8 & 1150 & -93.50 & 1160 & 0.975 \\
\hline EM07-Zr-001 & 0.6 & 0.6 & 99.4 & 1101 & & & \\
\hline EM07-Zr-001 & 1.0 & 1.0 & 99.0 & 1053 & & & \\
\hline EM07-Zr-001 & 1.8 & 1.8 & 98.2 & 999 & & & \\
\hline EM07-Zr-05 & 2.1 & 2.1 & 97.9 & 944 & -108.24 & 1170 & 1.000 \\
\hline EM07-Zr-05 & 0.2 & 0.2 & 99.8 & 1150 & & & \\
\hline EM07-Zr-05 & 1.0 & 1.0 & 99.0 & 1060 & & & \\
\hline EM07-NM- & 0.4 & 0.4 & 99.6 & 1170 & -129.81 & 1220 & 0.999 \\
\hline
\end{tabular}


PNNL-17950, Rev 0

\begin{tabular}{|l|r|r|r|r|r|r|r|r|}
\hline 0025 & & & & & & & & \\
\hline EM07-NM- & 0.6 & & 0.6 & 99.4 & 1140 & & & \\
\hline $\begin{array}{l}\text { EM07-NM- } \\
\text { 0025 }\end{array}$ & 1.2 & & 1.2 & 98.8 & 1065 & & & \\
\hline EM07-BL-2 & 1.9 & & 1.9 & 98.1 & 996.0 & -96.92 & 1182 & 0.995 \\
\hline EM07-BL-2 & 1.4 & & 1.4 & 98.6 & 1051.0 & & & \\
\hline EM07-BL-2 & 0.8 & & 0.8 & 99.2 & 1103.0 & & & \\
\hline EM07-Cr-02 & 2.7 & & 2.7 & 97.3 & 1099 & -189.35 & 1603 & 0.958 \\
\hline EM07-Cr-02 & 3.1 & & 3.1 & 96.9 & 996 & & & \\
\hline EM07-Cr-02 & 2.3 & & 2.3 & 97.7 & 1201 & & & \\
\hline EM07-Cr-02 & 1.6 & & 1.6 & 98.4 & 1281 & & & \\
\hline EM07-Na-05 & 5.5 & 2.0 & 7.5 & 92.5 & 1048 & & & \\
\hline EM07-Na-05 & 4.5 & 1.6 & 6.1 & 93.9 & 1148 & & & \\
\hline EM07-Na-10 & 2.5 & & 2.5 & 97.5 & 1048 & -150.00 & 1424 & 0.999 \\
\hline EM07-Na-10 & 1.2 & & 1.2 & 98.8 & 1250 & & & \\
\hline EM07-Na-10 & 0.8 & & 0.8 & 99.2 & 1300 & & & \\
\hline EM07-Si-30 & 2.1 & & 2.1 & 97.9 & 1060 & -101.93 & 1284 & 0.940 \\
\hline EM07-Si-30 & 1.5 & & 1.5 & 98.5 & 1151 & & & \\
\hline EM07-Si-30 & 0.8 & & 0.8 & 99.2 & 1194 & & & \\
\hline
\end{tabular}




\section{Appendix F: Toxicity Characteristic Leach Procedure (TCLP) Results}

\section{F.1 TCLP Metals Results for Quenched Glasses}

Table F.1. TCLP Metals Results for Quenched EM07-BL-1

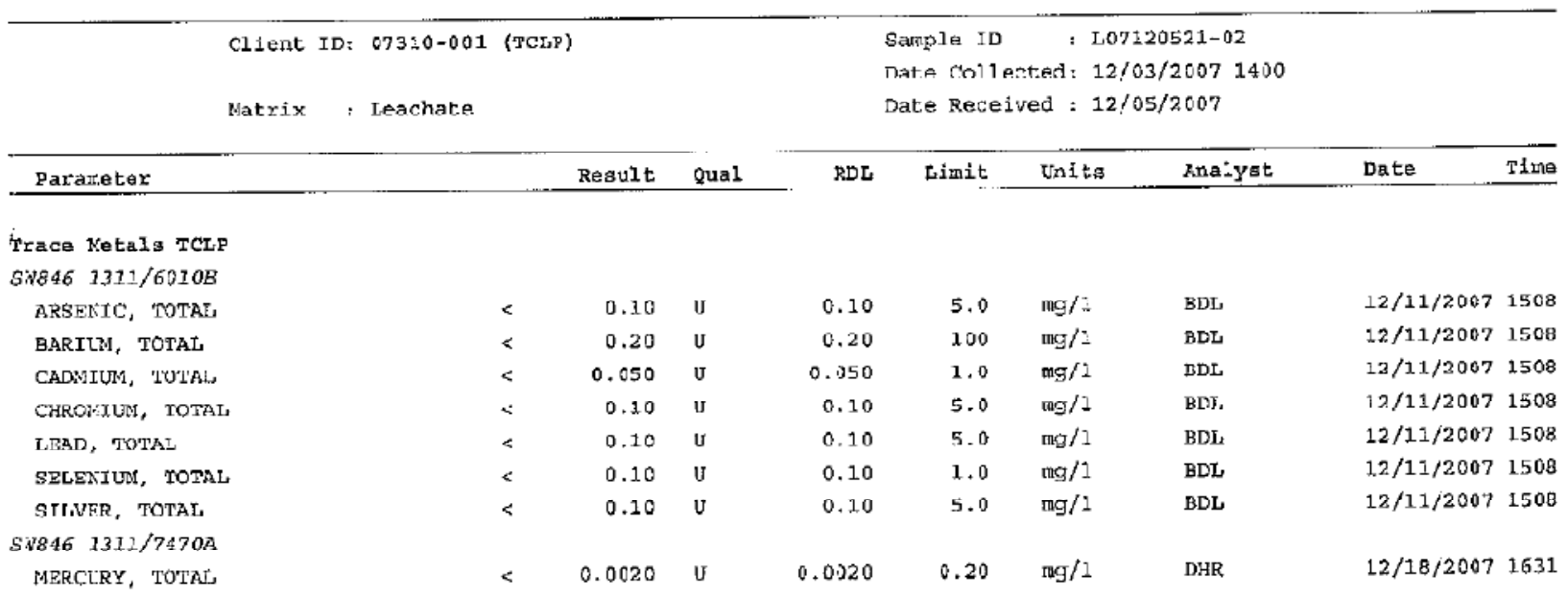

Table F.2. TCLP Metals Results for Quenched EM07-A1-06

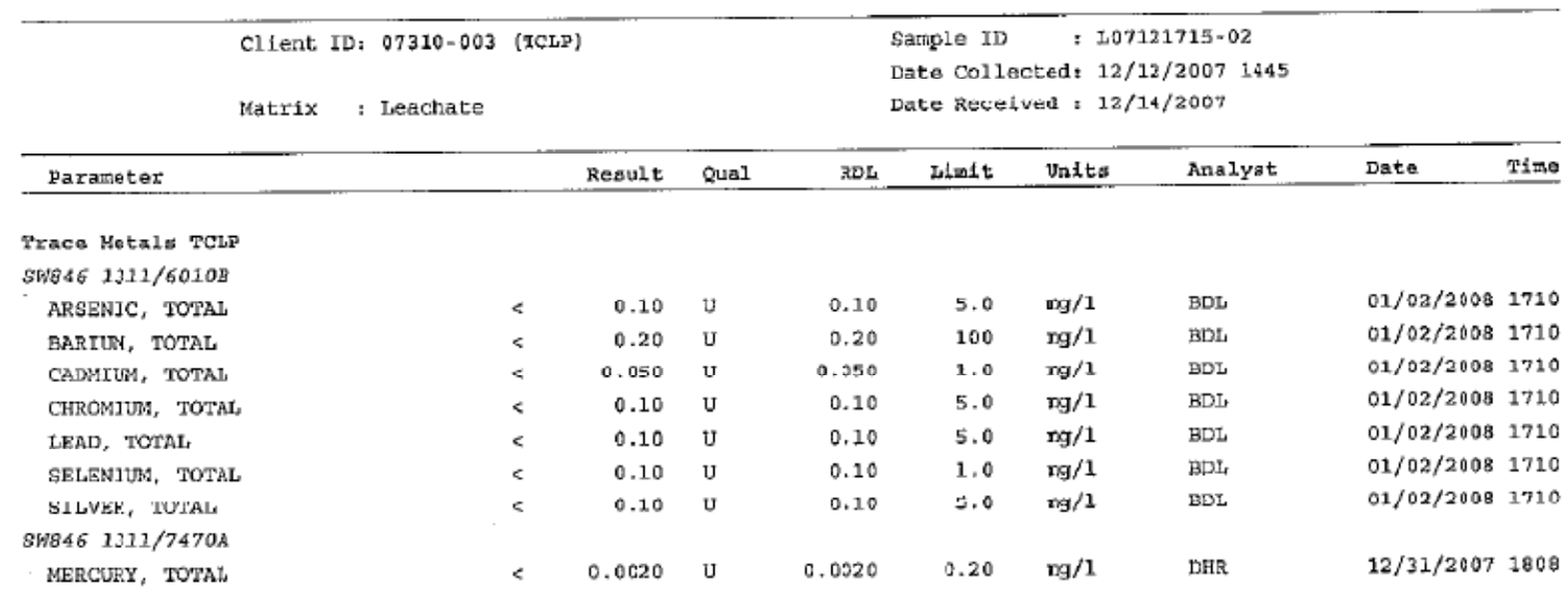


Table F.3. TCLP Metals Results for Quenched EM07-Al-15

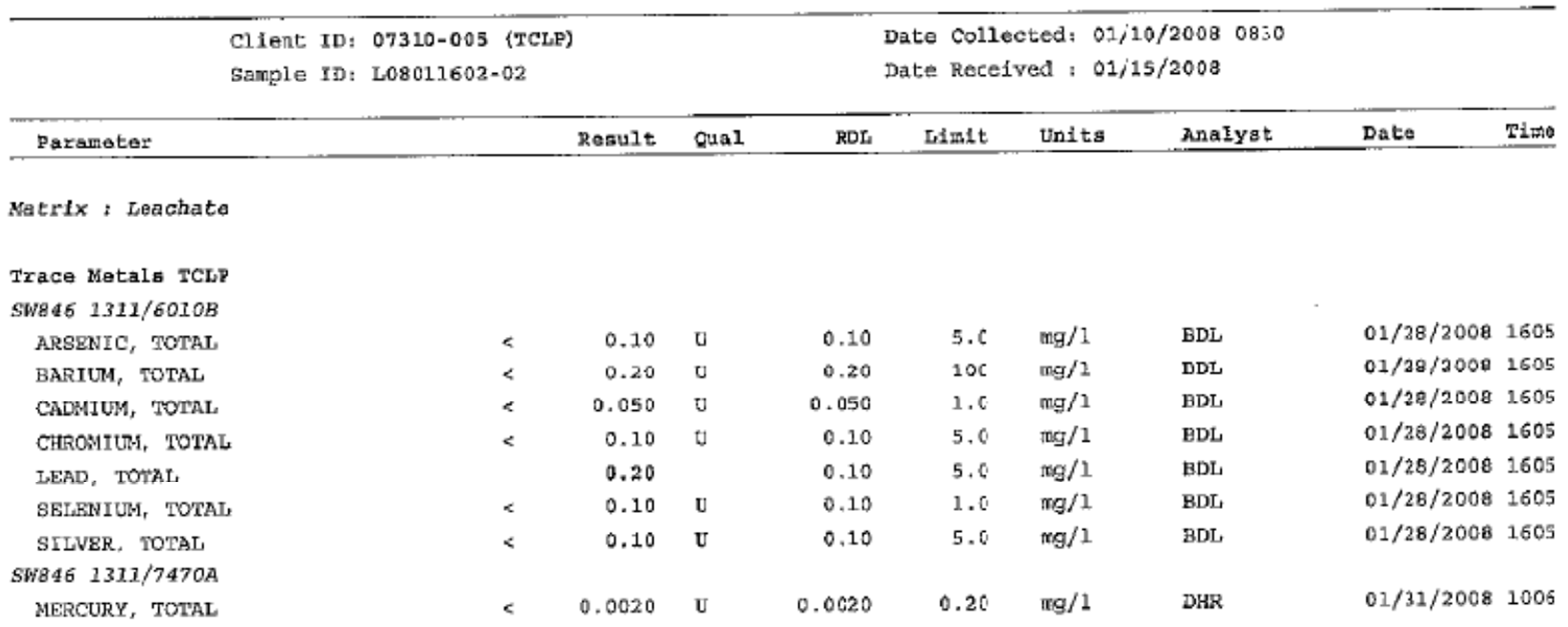

Table F.4. TCLP Metals Results for Quenched EM07-A1-20

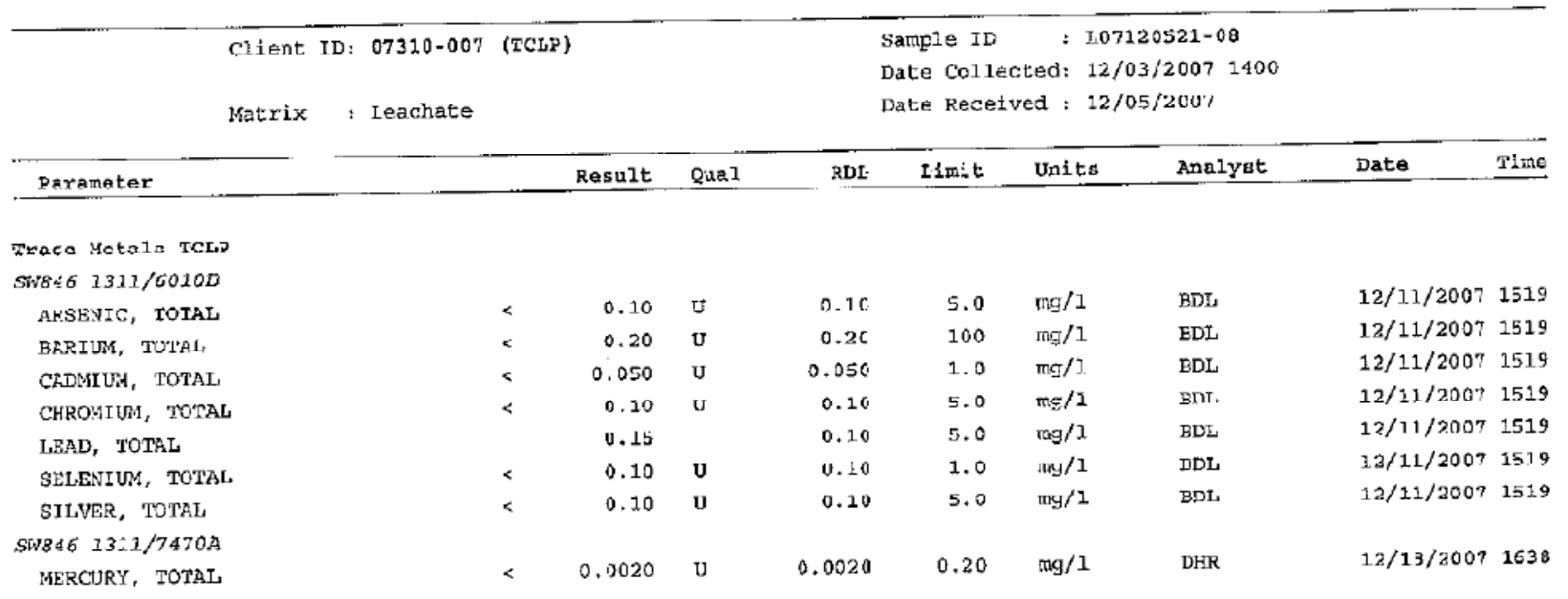


Table F.5. TCLP Metals Results for Quenched EM07-B-05

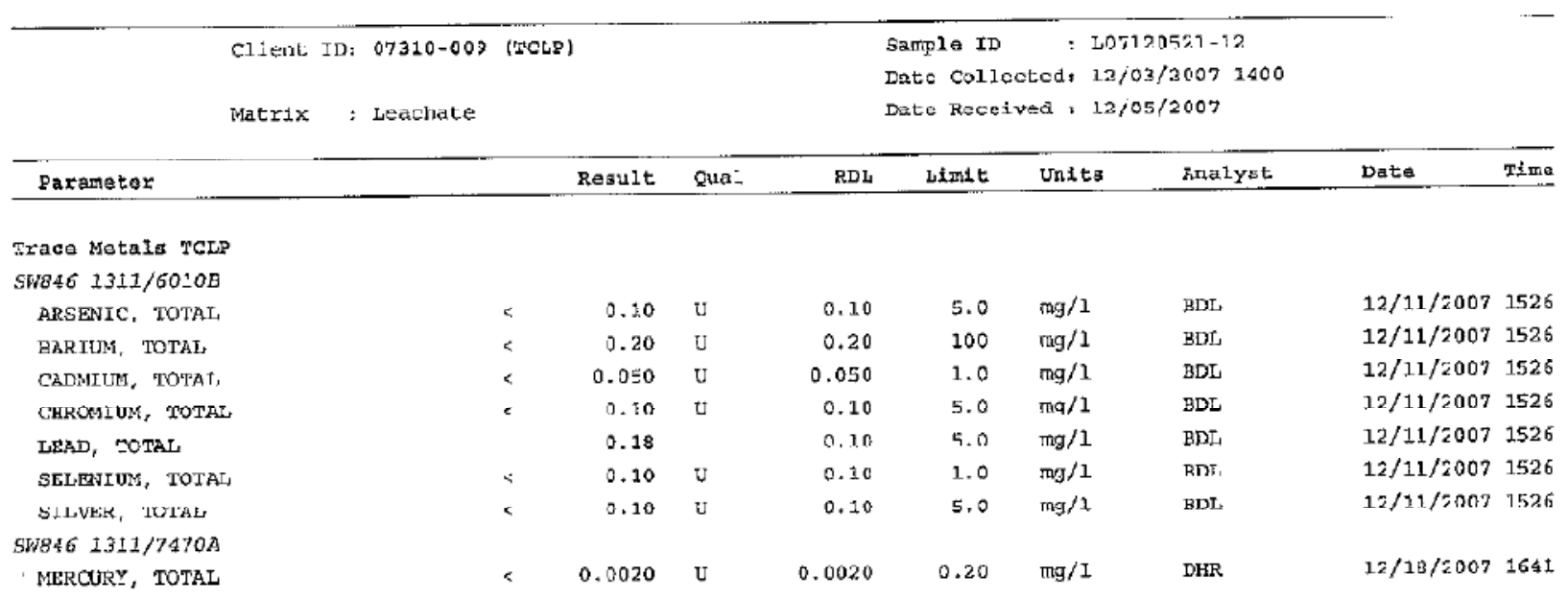

Table F.6. TCLP Metals Results for Quenched EM07-B-15

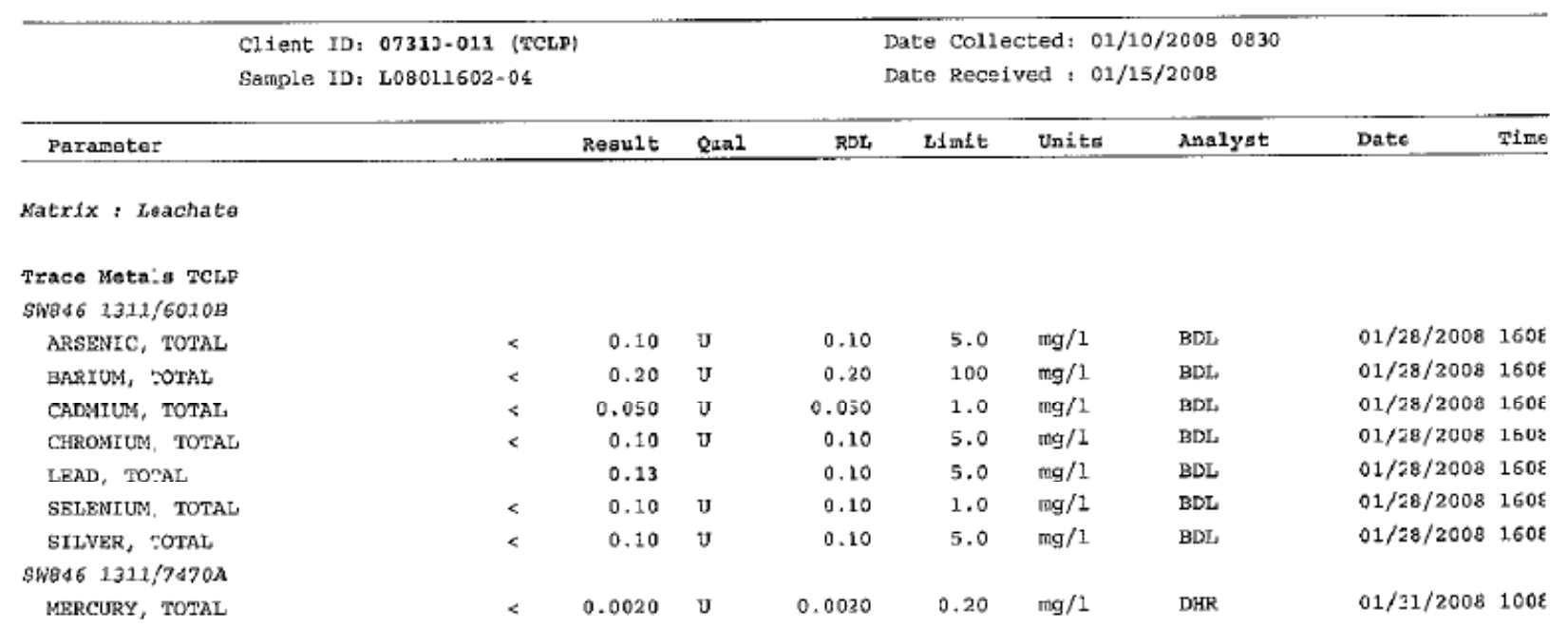


Table F.7. TCLP Metals Results for Quenched EM07-B-20

\begin{tabular}{|c|c|c|c|c|c|c|c|c|c|}
\hline \multicolumn{3}{|c|}{$\begin{array}{l}\text { Client ID; 07310-013 (TCLP) } \\
\text { Sample ID L L }\end{array}$} & \multirow[b]{2}{*}{ Qual } & \multirow[b]{2}{*}{ RDL } & \multicolumn{3}{|c|}{$\begin{array}{l}\text { Date Collected: } 01 / 10 / 20080330 \\
\text { Date Received : } 01 / 15 / 3008\end{array}$} & \multirow[b]{2}{*}{ Date } & \multirow[b]{2}{*}{ rime } \\
\hline Parameter & & Reevit & & & Limit & Unita & Analyst & & \\
\hline \multicolumn{10}{|l|}{ Natrix : Leachaice } \\
\hline \multicolumn{10}{|l|}{ Trace Xetais TCLP } \\
\hline \multicolumn{10}{|l|}{ SW846 1311/60103 } \\
\hline ARSBN:C, 'TOTAL & $<$ & 0.10 & $\mathrm{U}$ & 0.10 & 5.0 & $\mathrm{ng} / 1$ & BDE & $01 / 28 / 2 c 08$ & 1612 \\
\hline BARIUA, TOTAH & द & 0.20 & $\mathrm{U}$ & 0.20 & 100 & $\mathrm{rg} / \mathrm{l}$ & BDt & $01 / 28 / 2 \mathrm{COB}$ & 1612 \\
\hline CAINIUM, TOTAL & & 0.672 & & 0.050 & 1.0 & $\mathrm{Dg} / 1$ & BDL & $01 / 28 / 2 C 08$ & 2612 \\
\hline CHROKITM, TOTKA. & $<$ & 0.10 & $\mathrm{U}$ & 0.10 & 5.0 & $n q / 2$ & BDL & $01 / 28 / 2 \mathrm{cos}$ & 1612 \\
\hline IEAD, TOTAL & & 0.13 & & 0.10 & 5.0 & $\mathrm{rg} / 1$ & BDL & $01 / 28 / 2 \mathrm{cog}$ & 1612 \\
\hline SELENIUM， TOTAL & $<$ & 0.10 & $\mathrm{U}$ & 0.10 & 1.0 & $\mathrm{rg} / 1$ & BDL & $01 / 28 / 2 \cos$ & 1612 \\
\hline SILVER, TOTAL & $<$ & 0.10 & $\mathrm{U}$ & 0.10 & 5.0 & $\mathrm{Dg} / 1$ & BDL & $01 / 28 / 2 \mathrm{COB}$ & 1612 \\
\hline \multicolumn{10}{|l|}{ SW846 $1311 / 74702$} \\
\hline SERCURY, TOTAL & $<$ & $0.0<20$ & $\mathrm{U}$ & 0.0020 & 0.20 & $\mathrm{ng} / \mathrm{3}$ & DHR & $01 / 31 / 2 \cos$ & 1009 \\
\hline
\end{tabular}

Table F.8. TCLP Metals Results for Quenched EM07-Bi-025

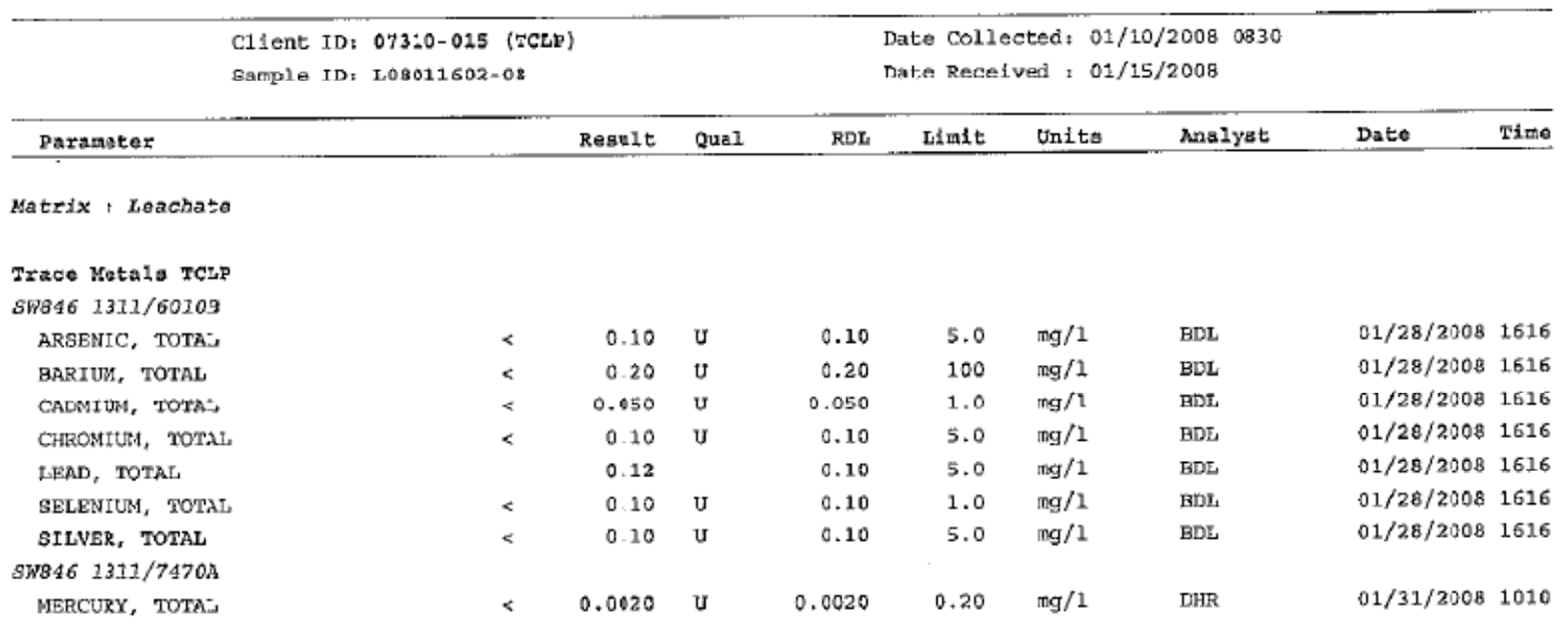


Table F.9. TCLP Metals Results for Quenched EM07-Bi-05

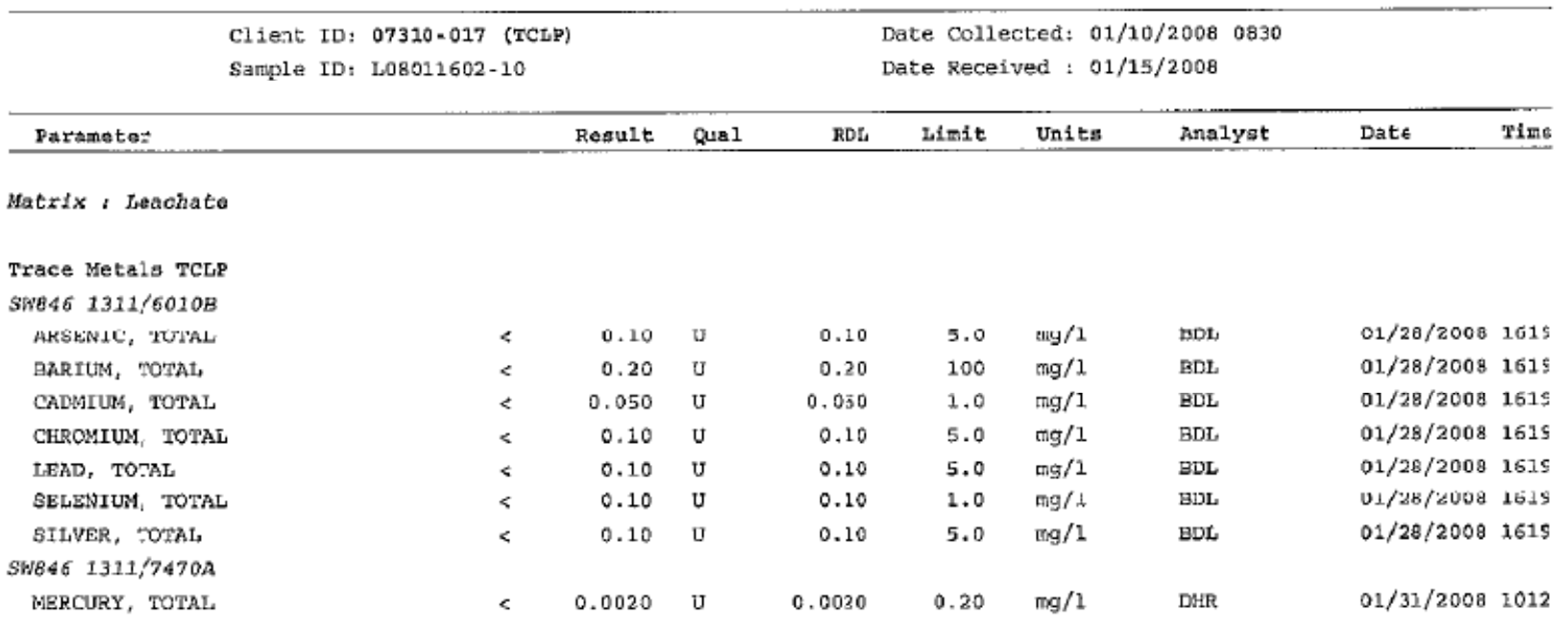

Table F.10. TCLP Metals Results for Quenched EM07-Ca-035

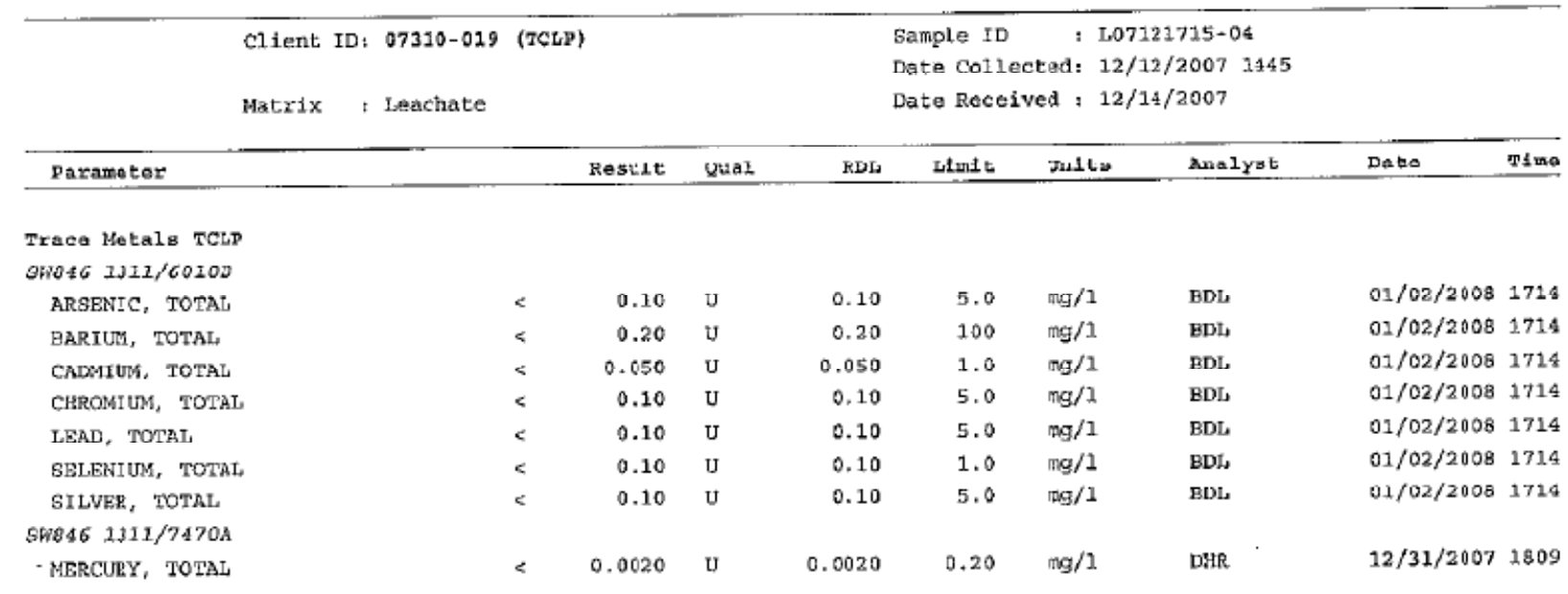

Table F.11. TCLP Metals Results for Quenched EM07-Ca-07

\begin{tabular}{|c|c|c|c|c|c|c|c|c|c|c|c|}
\hline \multirow{3}{*}{ Parameter } & \multirow{3}{*}{\multicolumn{2}{|c|}{$\begin{array}{l}\text { Client ID: } 07310-021 \\
\text { Metrix : Icachate }\end{array}$}} & \multirow{2}{*}{\multicolumn{3}{|c|}{ (TCLP) }} & \multirow{2}{*}{\multicolumn{4}{|c|}{$\begin{array}{l}\text { Sanple iD : Lo7120521-14 } \\
\text { Date Collenten: } 12 / 03 / 20071400 \\
\text { Datee Received : } 12 / 05 / 2007\end{array}$}} & \multirow[b]{3}{*}{ Date } & \multirow[b]{3}{*}{ Time } \\
\hline & & & & & & & & & & & \\
\hline & & & \multicolumn{2}{|r|}{ Result } & Qual & RDL & Linit & Units & Analyst & & \\
\hline \multicolumn{12}{|l|}{ Trace Metala TCLP } \\
\hline \multicolumn{12}{|l|}{ SW846 1311/601OR } \\
\hline ARSENIC, TOTAT. & & & $<$ & 0.10 & U & 0.10 & 5.0 & $m g / 1$ & $\mathrm{BDL}$ & $12 / 11 / 20 \mathrm{C} 7$ & -530 \\
\hline BARIUM, TOTAI. & & & $<$ & 0.20 & $\mathrm{u}$ & 0.20 & 100 & $\pi \mathrm{g} / 1$ & gDL & $12 / 11 / 2007$ & -530 \\
\hline ГATMTIMM, TOTAL & & & & 0.079 & & 0.050 & 1.0 & $\mathrm{mg} / \mathrm{l}$ & gDL & $12 / 11 / 20 C 7$ & -530 \\
\hline CHROMIUN, TOTAL & & & $<$ & 0.10 & v & 0.10 & 5.0 & $\mathrm{mg} / 1$ & EDE & $12 / 11 / 2007$ & -530 \\
\hline LEAD, TOTAL & & & & 0.13 & & 0.10 & 5.0 & $\operatorname{mog} / 1$ & EDL & $=2 / 11 / 2007$ & $\therefore 530$ \\
\hline SELENIUM, TOTAL & & & $<$ & 0.10 & $\mathrm{u}$ & 0.10 & 1.0 & $\mathrm{mg} / 1$ & EDL & $-2 / 11 / 2007$ & $\therefore 530$ \\
\hline SILVER, TOTAL & & & $<$ & 0.10 & u & 0.10 & 5.0 & $\mathrm{mg} / 1$ & EDL & $\therefore 2 / 11 / 2007$ & 1530 \\
\hline \multicolumn{12}{|l|}{ SWO46 $1311 / 7470 \mathrm{~A}$} \\
\hline MERCURY, TOTAL & & & $<$ & 0.0020 & U & 0.0020 & C. .20 & $\mathrm{mg} / 1$ & DHR & $22 / 18 / 2007$ & 1642 \\
\hline
\end{tabular}


PNNL-17950, Rev 0

Table F.12. TCLP Metals Results for Quenched EM07-Cr-001

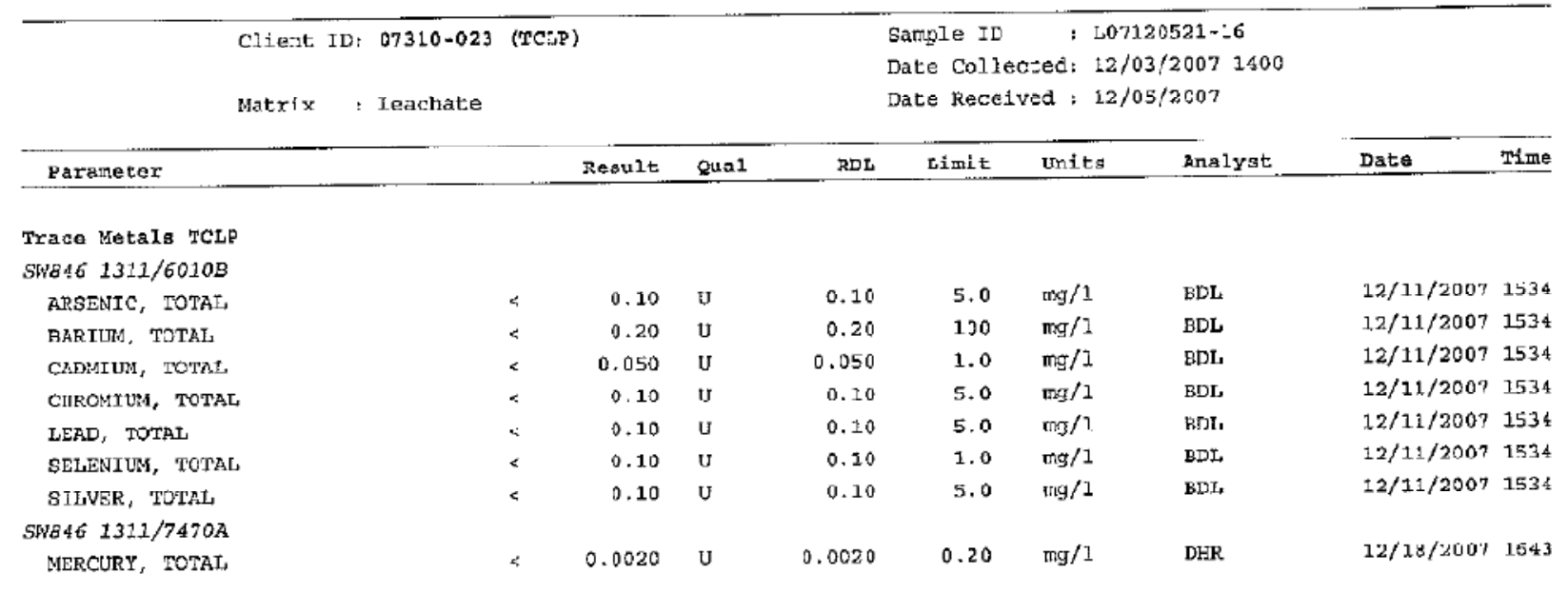

Table F.13. TCLP Metals Results for Quenched EM07-Cr-012

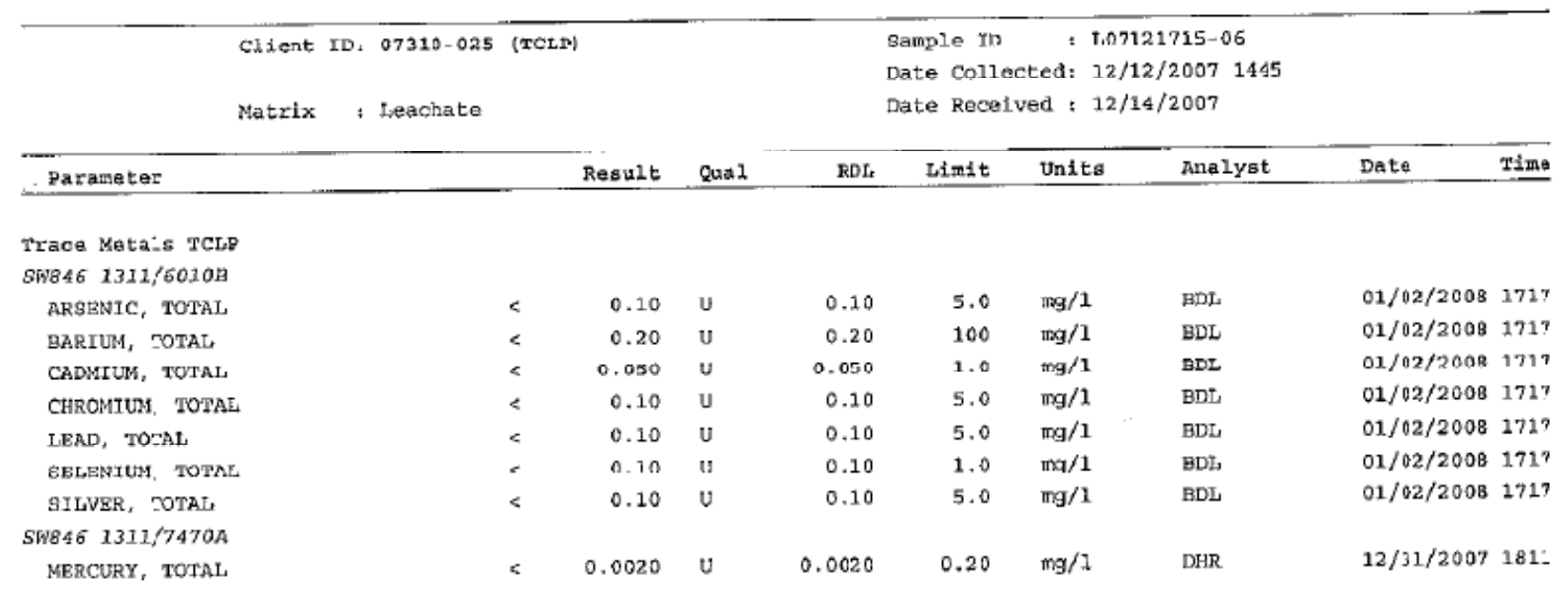


Table F.14. TCLP Metals Results for Quenched EM07-Cr-02

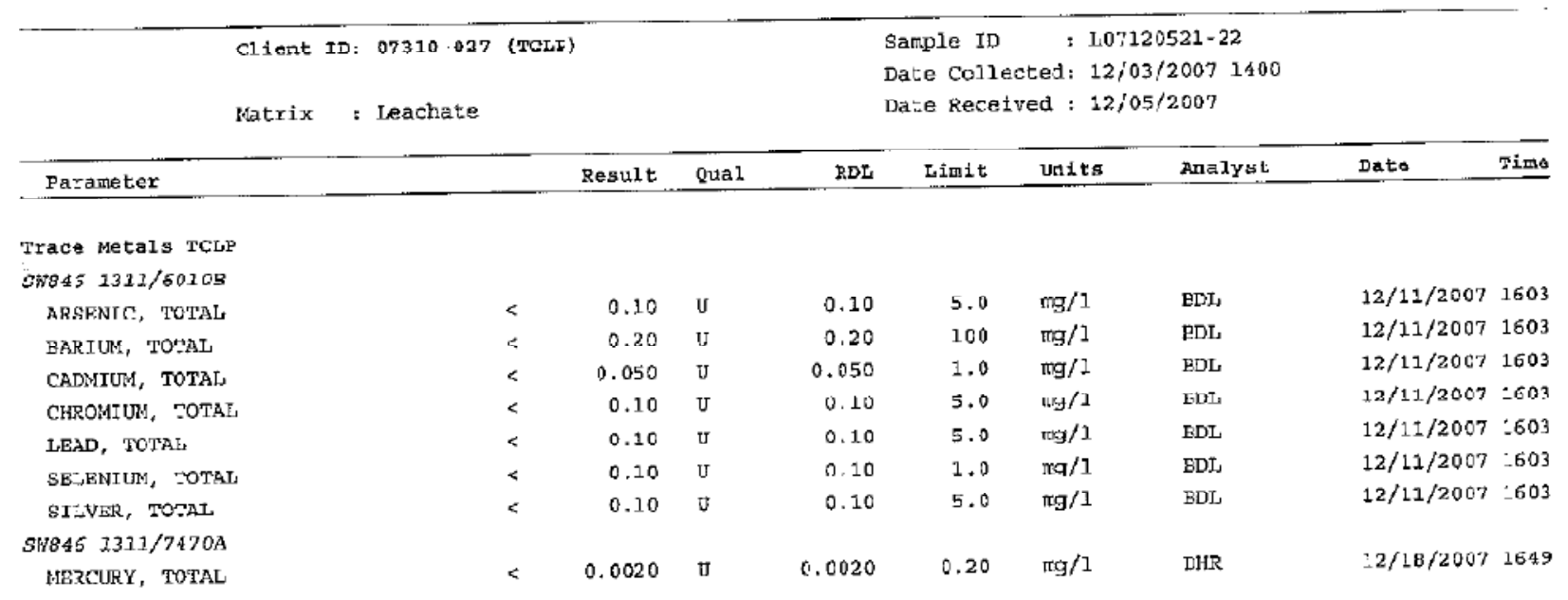

Table F.15. TCLP Metals Results for Quenched EM07-F-02

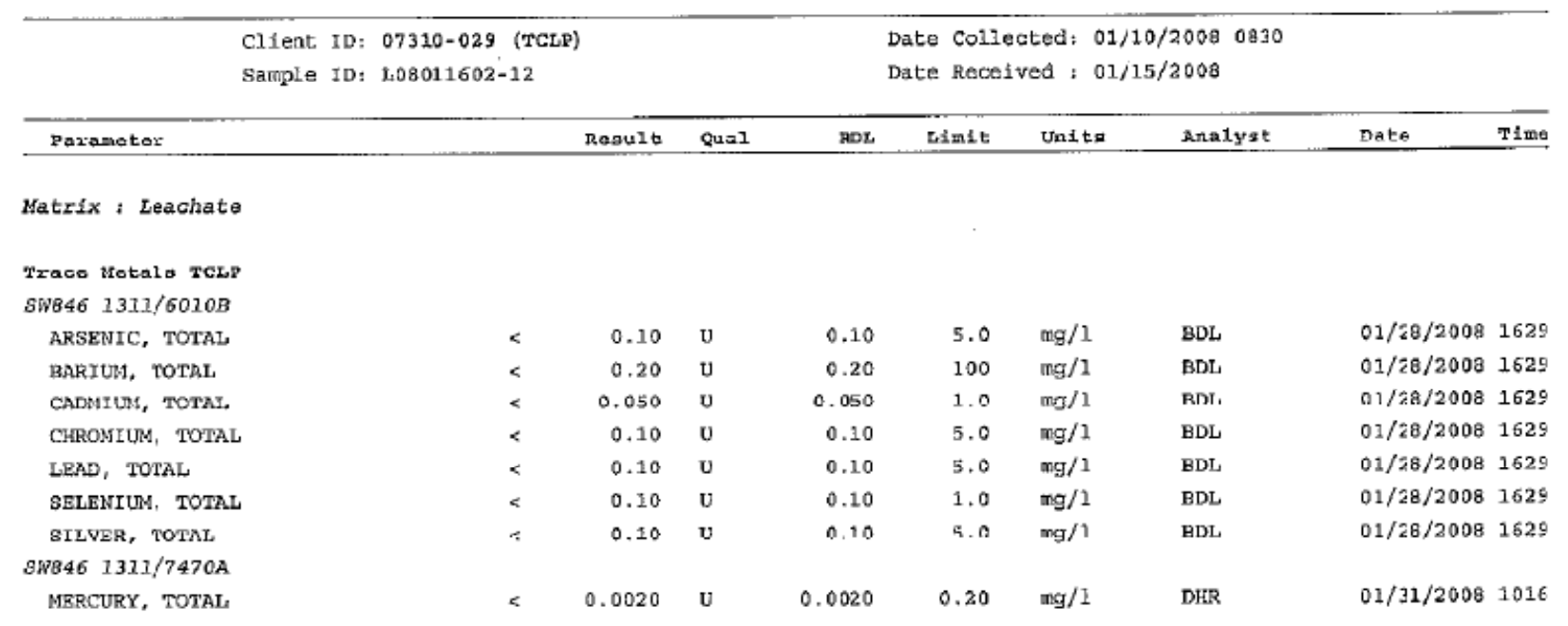


Table F.16. TCLP Metals Results for Quenched EM07-Fe-05

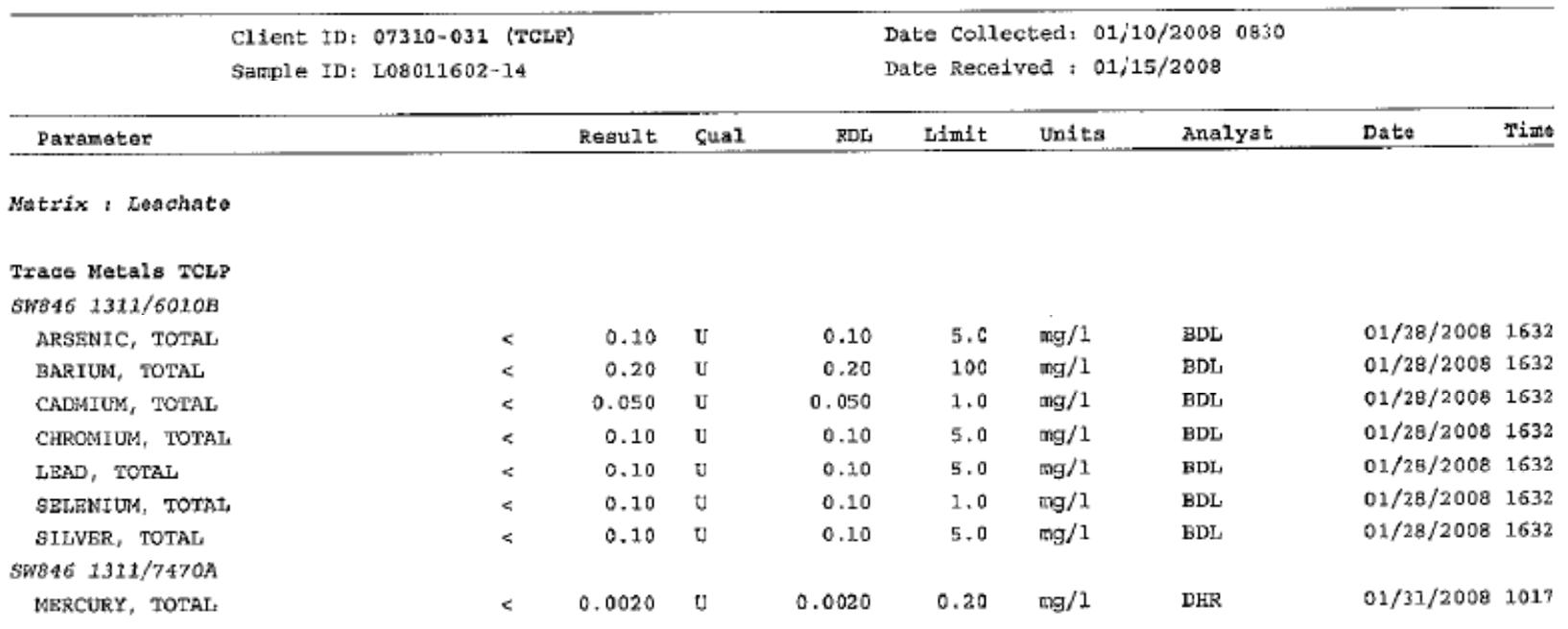

Table F.17. TCLP Metals Results for Quenched EM07-Fe-15

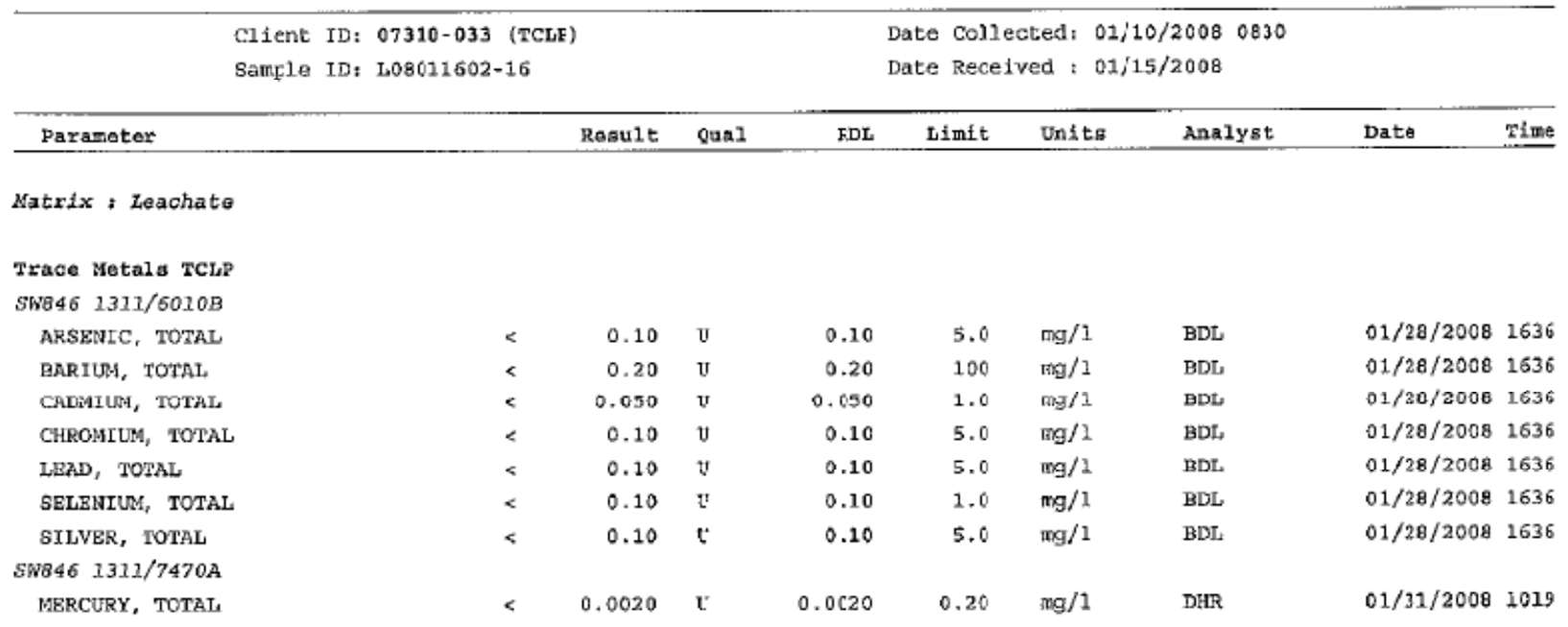


Table F.18. TCLP Metals Results for Quenched EM07-Fe-20

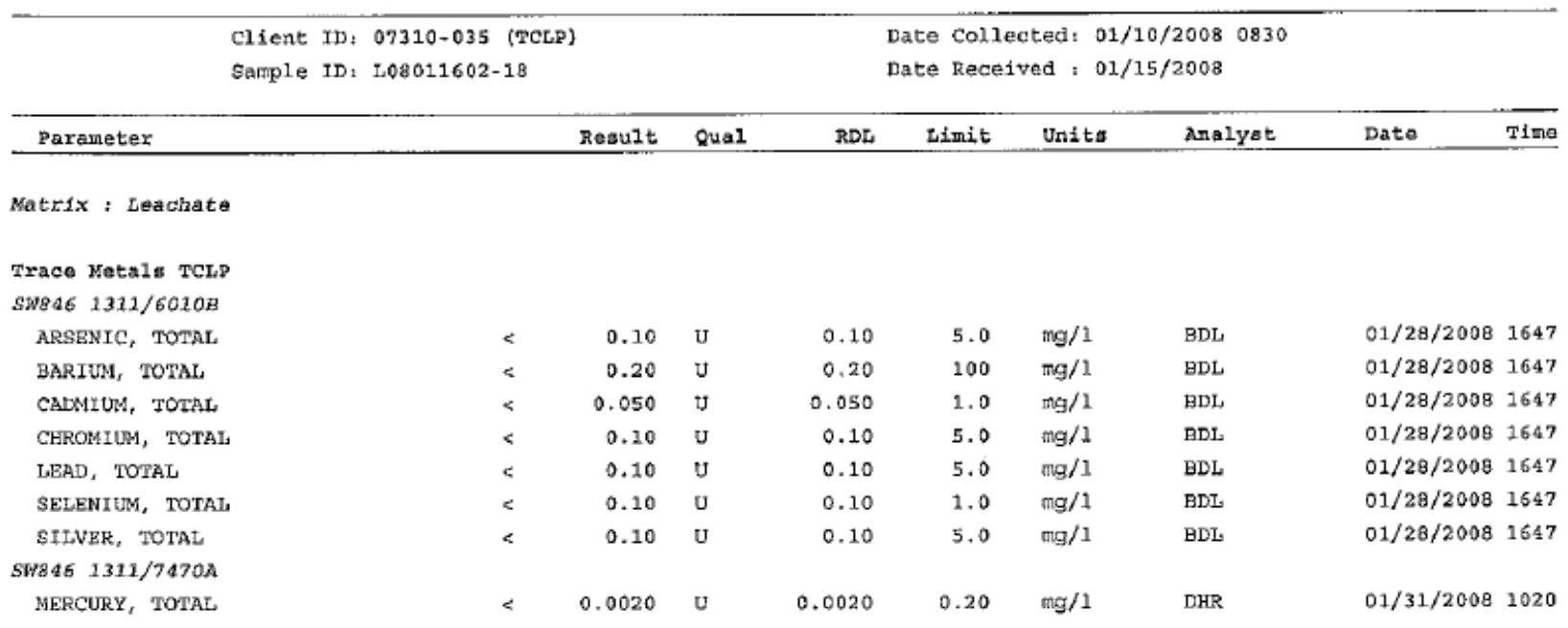

Table F.19. TCLP Metals Results for Quenched EM07-K-03

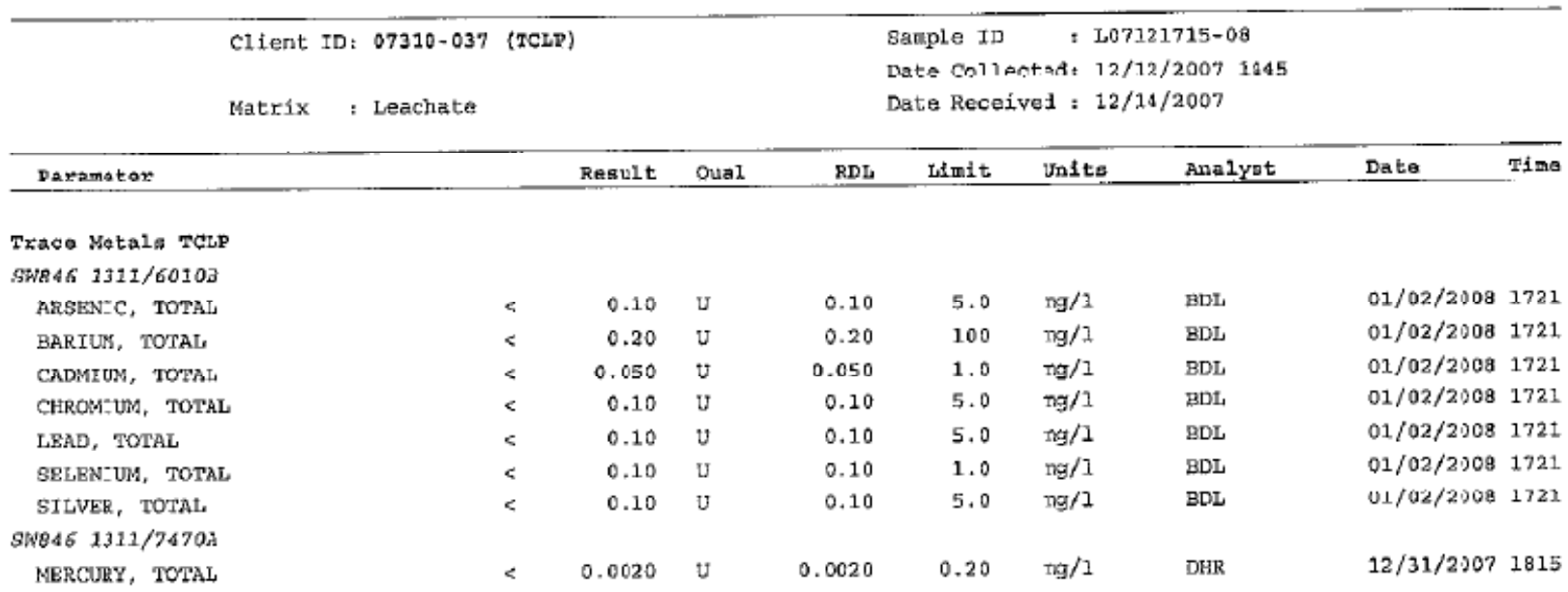


Table F.20. TCLP Metals Results for Quenched EM07-K-06

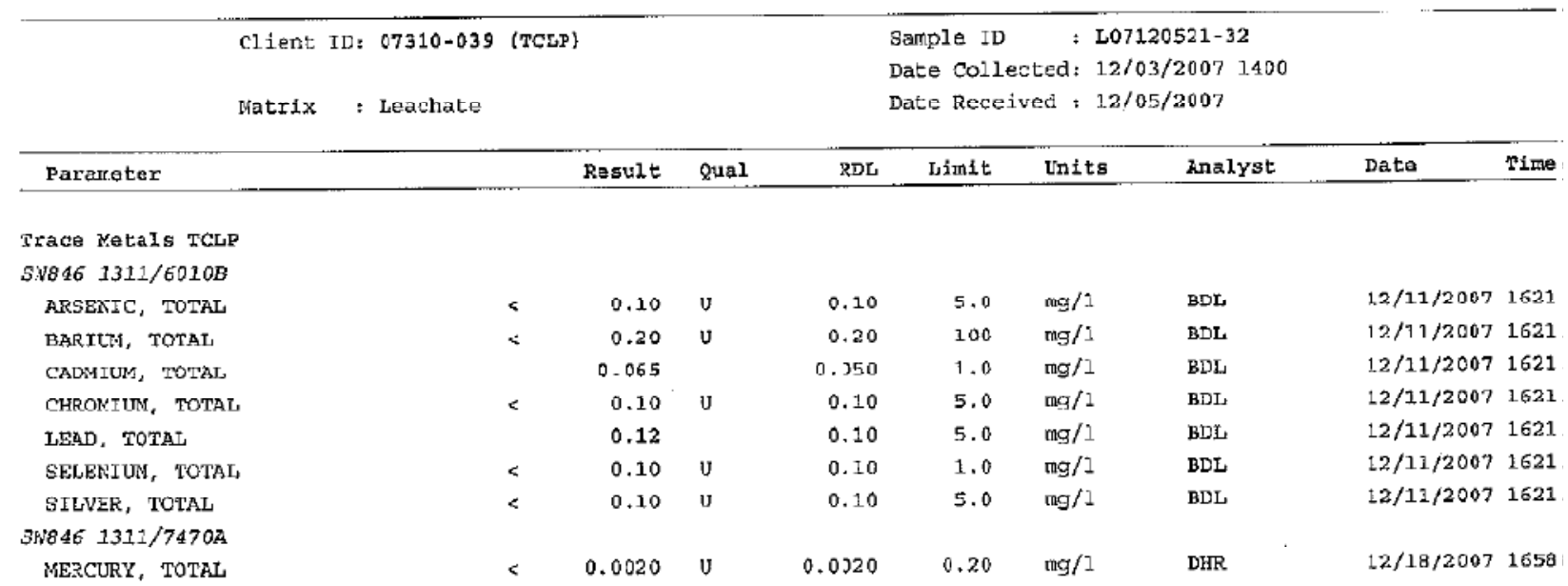

Table F.21. TCLP Metals Results for Quenched EM07-Li-015

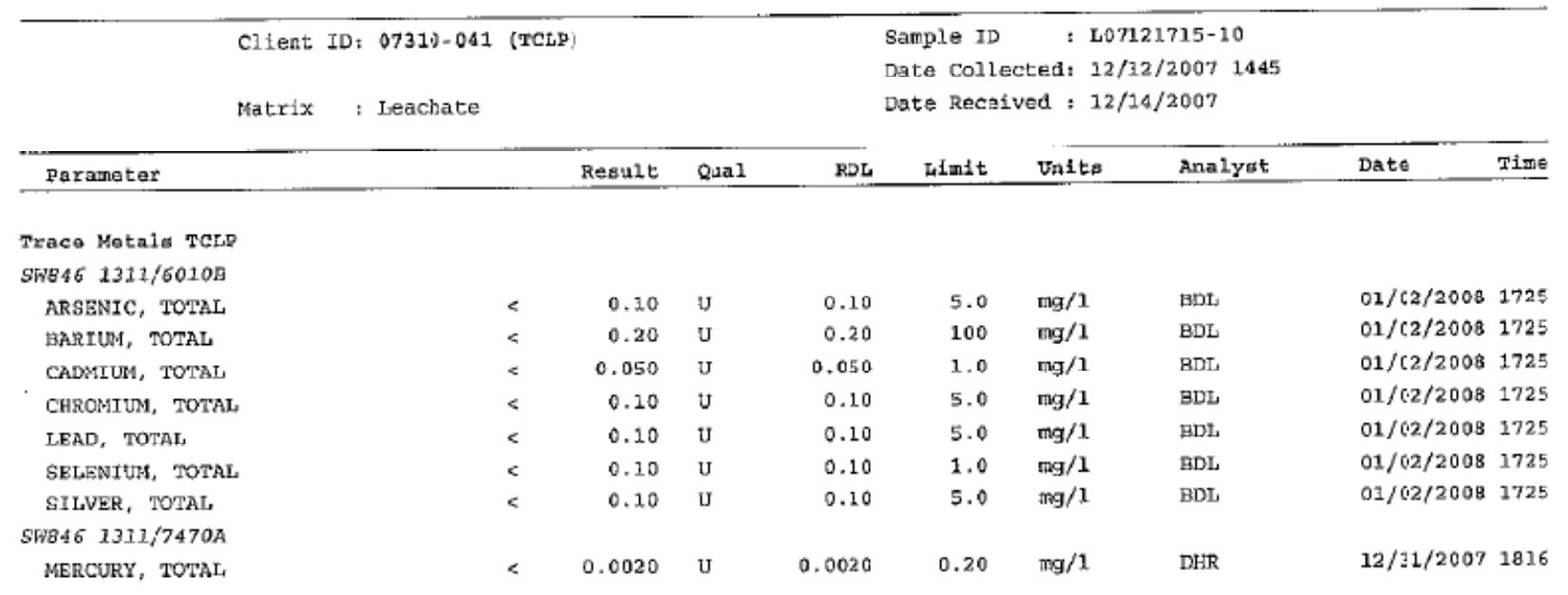


Table F.22. TCLP Metals Results for Quenched EM07-Li-04

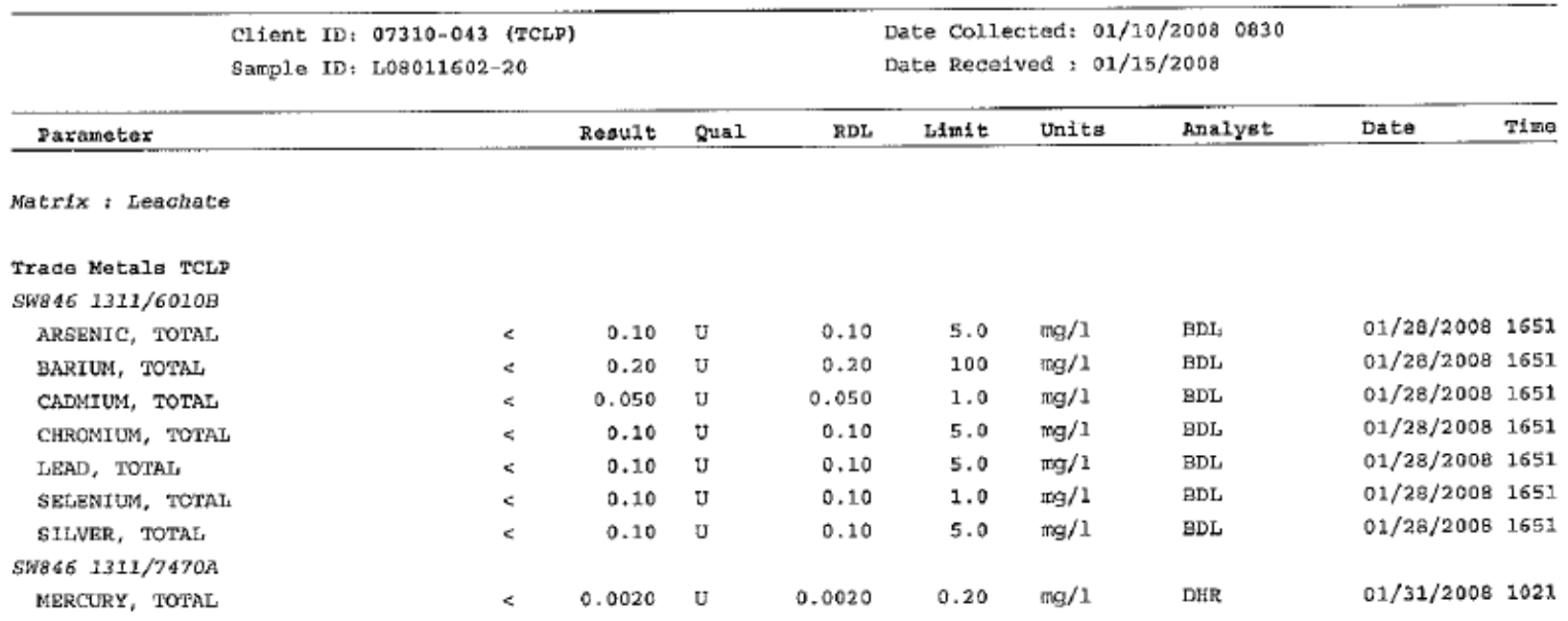

Table F.23. TCLP Metals Results for Quenched EM07-Mn-01

\begin{tabular}{|c|c|c|c|c|c|c|c|c|c|c|}
\hline \multirow{3}{*}{$\begin{array}{r}\text { Paranetur } \\
\text { Pand }\end{array}$} & \multirow{3}{*}{$\begin{array}{l}\text { V: } 07310-045 \\
\text { : Legcthate }\end{array}$} & \multirow[t]{3}{*}{ (TCLP) } & \multirow{2}{*}{\multicolumn{6}{|c|}{$\begin{array}{l}\text { Sample ID : L07120521-31 } \\
\text { Date Collected; } 12 / 03 / 20071120 \\
\text { Datc Received : } 12 / 05 / 2007\end{array}$}} & \multirow[b]{3}{*}{ Dato } & \multirow[b]{3}{*}{ Time } \\
\hline & & & & & & & & & & \\
\hline & & & Raoult & Quai & SEL & Limit & Lntts & inalyst & & \\
\hline \multicolumn{11}{|l|}{ Trace Netals ToLP } \\
\hline \multicolumn{11}{|l|}{ SW816 $131 \pm / 6010 D$} \\
\hline ARSENIC, TOTAI & & $e$ & 0.10 & $\mathrm{U}$ & 0.10 & 5.0 & $\mathrm{mg} / 1$ & $\mathrm{BDL}_{2}$ & $12 / 11 / 2007$ & 1632 \\
\hline BARIIM, TOTAL & & $<$ & 0.20 & १ & 0.20 & 200 & $\mathrm{ng} / 1$ & BDL & $12 / 11 / 2007$ & 1632 \\
\hline CADMIUM, TOTAT, & & $<$ & 0.050 & U & 0.050 & 1.0 & $\mathrm{mg} / 1$ & $\mathrm{BDL}$ & $12 / 11 / 2007$ & 1632 \\
\hline CHRONIUM, TOTAL & & $<$ & 0.10 & $\mathrm{U}$ & 0.10 & 5.0 & $\operatorname{lng} / 2$ & BDL & $12 / 11 / 2007$ & 1632 \\
\hline LEAD, TOTAL & & $<$ & 0.10 & U & 0.10 & 5.0 & $\operatorname{Ing} / 1$ & BDL & $12 / 11 / 2007$ & 1632 \\
\hline SELENIUM, TOTAL & & $<$ & 0.10 & $\mathrm{u}$ & 0.10 & 1.0 & $\operatorname{mog} / 1$ & BDL & $12 / 11 / 2007$ & 1632 \\
\hline SILVER, TOTAE & & $<$ & 0.10 & $\mathrm{u}$ & 0.10 & 5.0 & $\mathrm{mg} / 1$ & BDL & $12 / 11 / 2007$ & 1632 \\
\hline \multicolumn{11}{|l|}{ ST846 $131 \pm / 7470 A$} \\
\hline MERCLRY, TOTAL & & $<$ & 0.0620 & 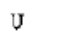 & 0.0020 & 0.20 & $\mathrm{Lg} / 1$ & DHR & $12 / 18 / 2007$ & 1659 \\
\hline
\end{tabular}


Table F.24. TCLP Metals Results for Quenched EM07-Mn-04

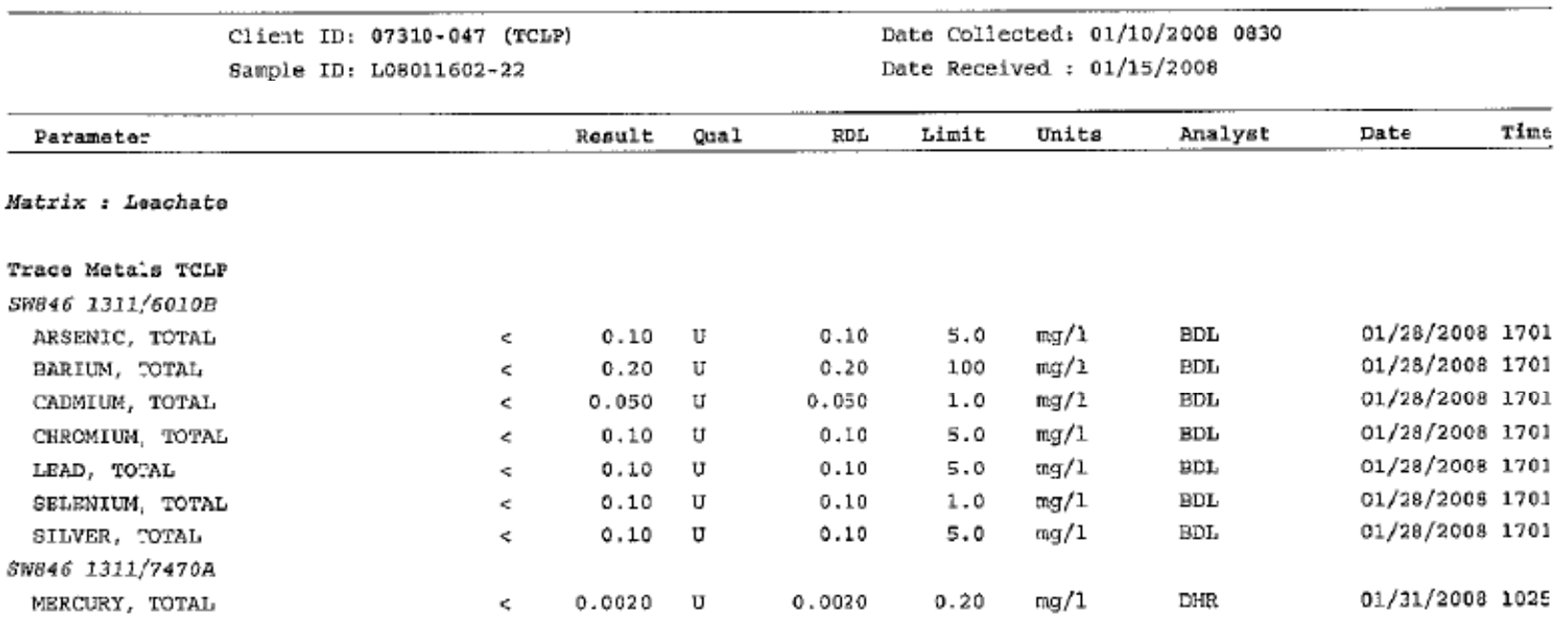

Table F.25. TCLP Metals Results for Quenched EM07-Na-05

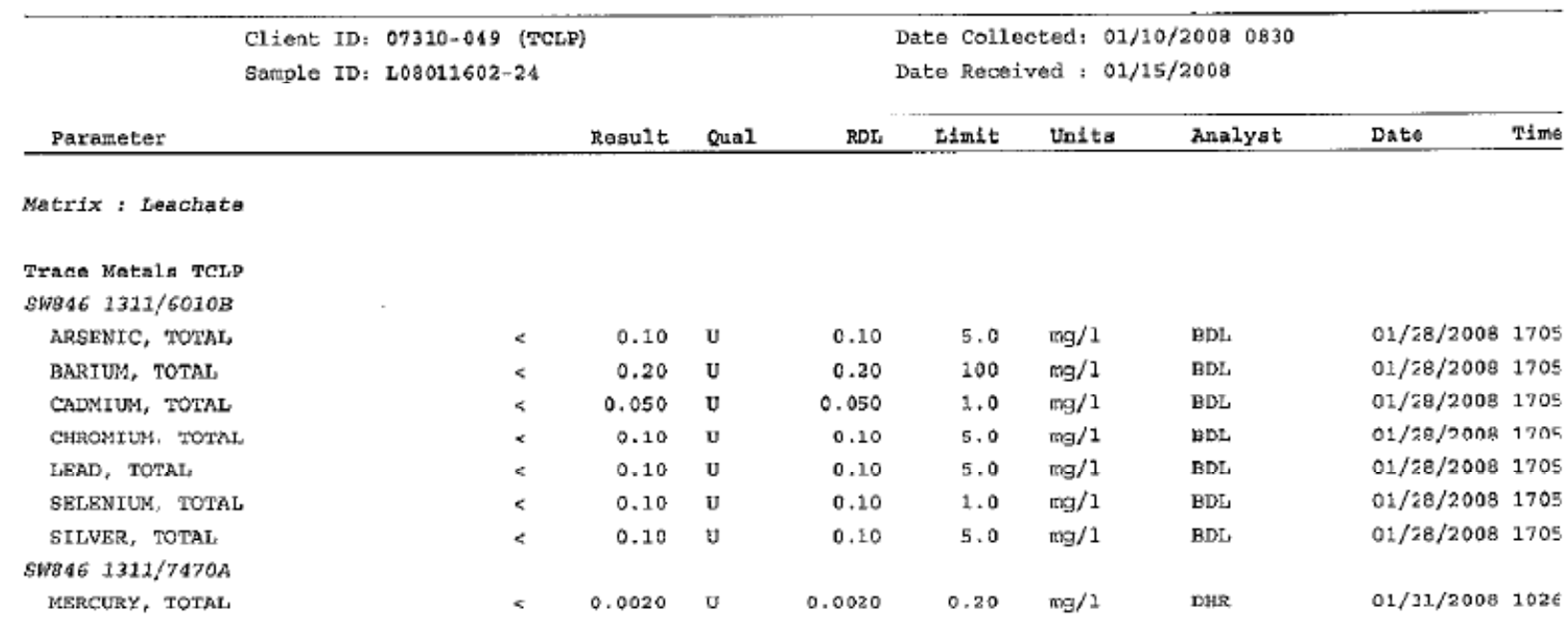


Table F.26. TCLP Metals Results for Quenched EM07-Na-10

\begin{tabular}{|c|c|c|c|c|c|c|c|c|c|}
\hline \multirow[b]{2}{*}{ Paxametex } & \multicolumn{4}{|c|}{$\begin{array}{l}\text { CIient ID: } 07310-051 \text { (TCLP) } \\
\text { Sample ID: L08011602-26 }\end{array}$} & \multicolumn{3}{|c|}{$\begin{array}{l}\text { Date collected: } 01 / 10 / 20080830 \\
\text { pate Received: } 01 / 15 / 2008\end{array}$} & \multirow[b]{2}{*}{ Date } & \multirow[b]{2}{*}{ Time } \\
\hline & & Result & Qual. & $R D L$ & Lixit & Units & Analyat & & \\
\hline \multicolumn{10}{|l|}{ Natrix : Leschate } \\
\hline \multicolumn{10}{|l|}{ Trace Natala TCLP } \\
\hline \multicolumn{10}{|l|}{ SW846 $13 \pi \lambda / 6010 B$} \\
\hline ARSENIC, TOTAL & $<$ & 0.10 & $\mathrm{U}$ & 0.10 & 5.0 & $\mathrm{mg} / 1$ & $\mathrm{BDL}$ & $01 / 28 / 2008$ & 1709 \\
\hline BARIUM, TOTAL & $<$ & 0.20 & $\mathrm{U}$ & 0.20 & 100 & $\mathrm{mg} / 1$ & BDL & $01 / 28 / 2008$ & 1709 \\
\hline CADMIUM, TOTAL & $<$ & 0.050 & $\theta$ & 0.050 & 1.0 & $\mathrm{mg} / 1$ & $\mathrm{BDL}$ & $01 / 28 / 2008$ & 1709 \\
\hline CHROMIUM, TOTAL & $<$ & 0.10 & $\mathrm{U}$ & 0.10 & 5.0 & $\mathrm{mgg} / 1$ & Bpt & $01 / 28 / 2008$ & 1709 \\
\hline LEAD, TOTAL & $<$ & 0.10 & $\mathrm{U}$ & 0.10 & 5.0 & $\mathrm{mg} / 1$ & BDL & $01 / 28 / 2008$ & 1709 \\
\hline SEEENIUM, TOTAL. & $<$ & 0.10 & $t$ & 0.10 & 1.0 & $\mathrm{mg} / 1$ & $\mathrm{BDL}$ & $01 / 28 / 2008$ & 1709 \\
\hline SILVER, TOTAL & $\Leftrightarrow$ & 0.10 & 0 & 0.10 & 5.0 & $\mathrm{mg} / 1$ & BDL & $01 / 28 / 2008$ & 1709 \\
\hline \multicolumn{10}{|l|}{ SW866 $1311 / 7470 \mathrm{~A}$} \\
\hline MERCURY, TOTAL & $<$ & 0.0020 & $\mathrm{U}$ & 0.0020 & 0.20 & $\mathrm{mg} / 1$ & DHR & $01 / 31 / 2008$ & 1028 \\
\hline
\end{tabular}

Table F.27. TCLP Metals Results for Quenched EM07-Na-20

\begin{tabular}{|c|c|c|c|c|c|c|c|c|c|c|c|}
\hline \multirow{2}{*}{ Paramater } & \multicolumn{2}{|c|}{ Cilent ID: $07310-053$} & \multicolumn{2}{|c|}{ (TCLP) } & \multicolumn{5}{|c|}{ 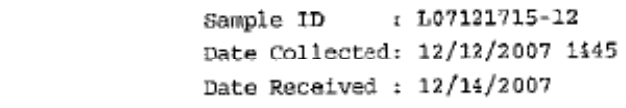 } & \multirow[b]{2}{*}{ Date } & \multirow[b]{2}{*}{ Time } \\
\hline & & & \multicolumn{2}{|r|}{ Restlt } & Q:20I & \multirow[t]{2}{*}{$\mathrm{RDL}$} & \multirow[t]{2}{*}{ Limit } & \multirow[t]{2}{*}{ Units } & \multirow[t]{2}{*}{ Analyst } & & \\
\hline & & 1 & & & & & & & & & \\
\hline \multirow{2}{*}{\multicolumn{11}{|c|}{ SW846 1311/60103 }} & \\
\hline & & & & & $\mathrm{u}$ & 0.10 & 5.0 & & & & \\
\hline BARIUM，TOTAL & & & $<$ & 0.20 & u & 0.20 & 100 & $\begin{array}{l}\mathrm{mg} / 1 \\
\mathrm{mg} / \mathrm{I}\end{array}$ & BDL & $01 / 02 / 2008$ & 1728 \\
\hline CADUYUM, TOTAL & & & & 0.157 & & 0.050 & 1.0 & $\mathrm{mg} / 1$ & $B D L$ & $01 / 02 / 2,008$ & 2728 \\
\hline CHROMIOA, TOTAL & & & $<$ & 0.10 & u & 0.10 & 5.0 & $\mathrm{mg} / 1$ & BDE & $01 / 02 / 2.308$ & 1728 \\
\hline LEAD, TOTAL & & & $<$ & 0.10 & $\mathrm{U}$ & 0.10 & 5.0 & $\mathrm{mg} / 1$ & BDI & $01 / 02 / 2308$ & 1728 \\
\hline SELENIUT, TOTAL & & & $<$ & 0.10 & $\mathrm{u}$ & 0.10 & 1.0 & $\mathrm{mg} / 1$ & BDI & $01 / 02 / 2308$ & 1728 \\
\hline SILVER, TOTAL & & & $<$ & 0.10 & U & 0.10 & 5.0 & $\mathrm{mg} / 1$ & BDI & $01 / 02 / 2308$ & 1728 \\
\hline \multicolumn{12}{|l|}{ 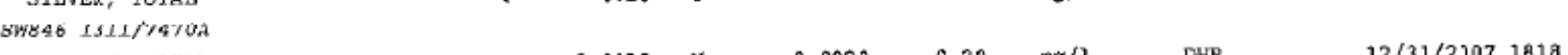 } \\
\hline MERCURY, TOTAL & & & $<$ & 0.0020 & u & 0.0020 & 0.20 & $\operatorname{thg} / 1$ & DHR & $12 / 31 / 2307$ & $181 \mathrm{a}$ \\
\hline
\end{tabular}


Table F.28. TCLP Metals Results for Quenched EM07-Ni-001

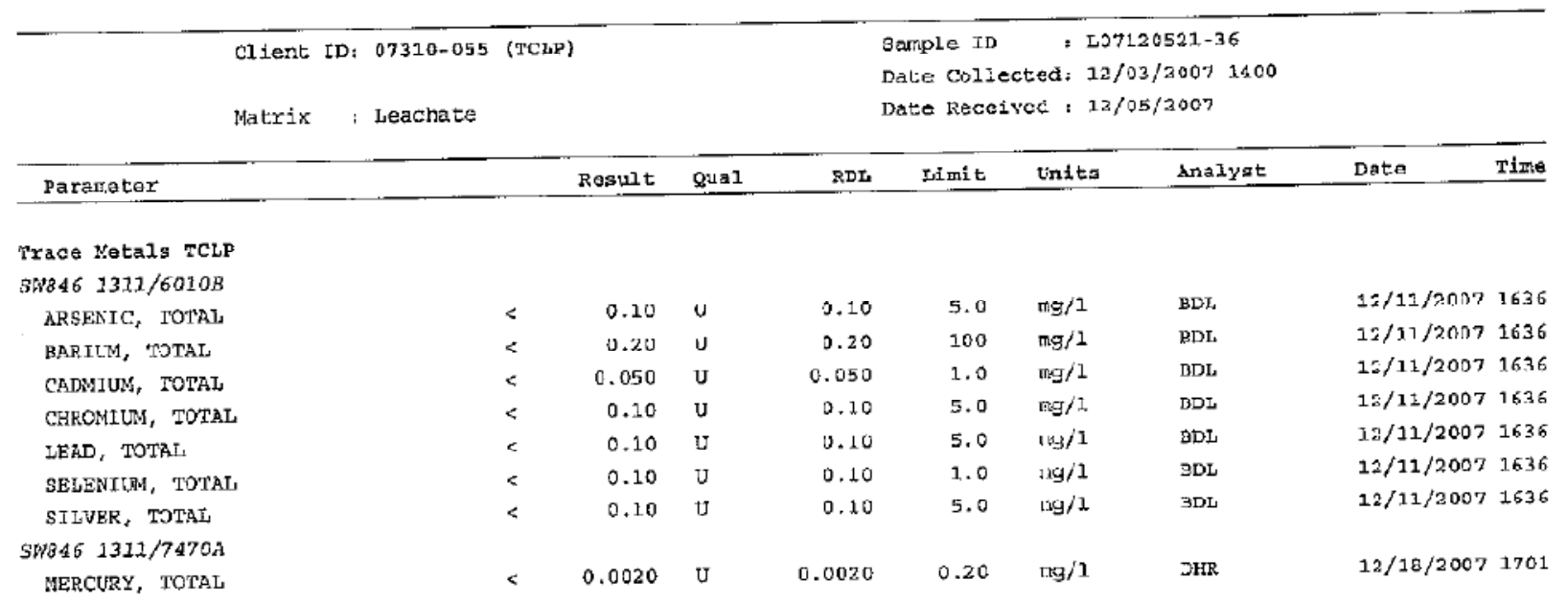

Table F.29. TCLP Metals Results for Quenched EM07-Ni-02

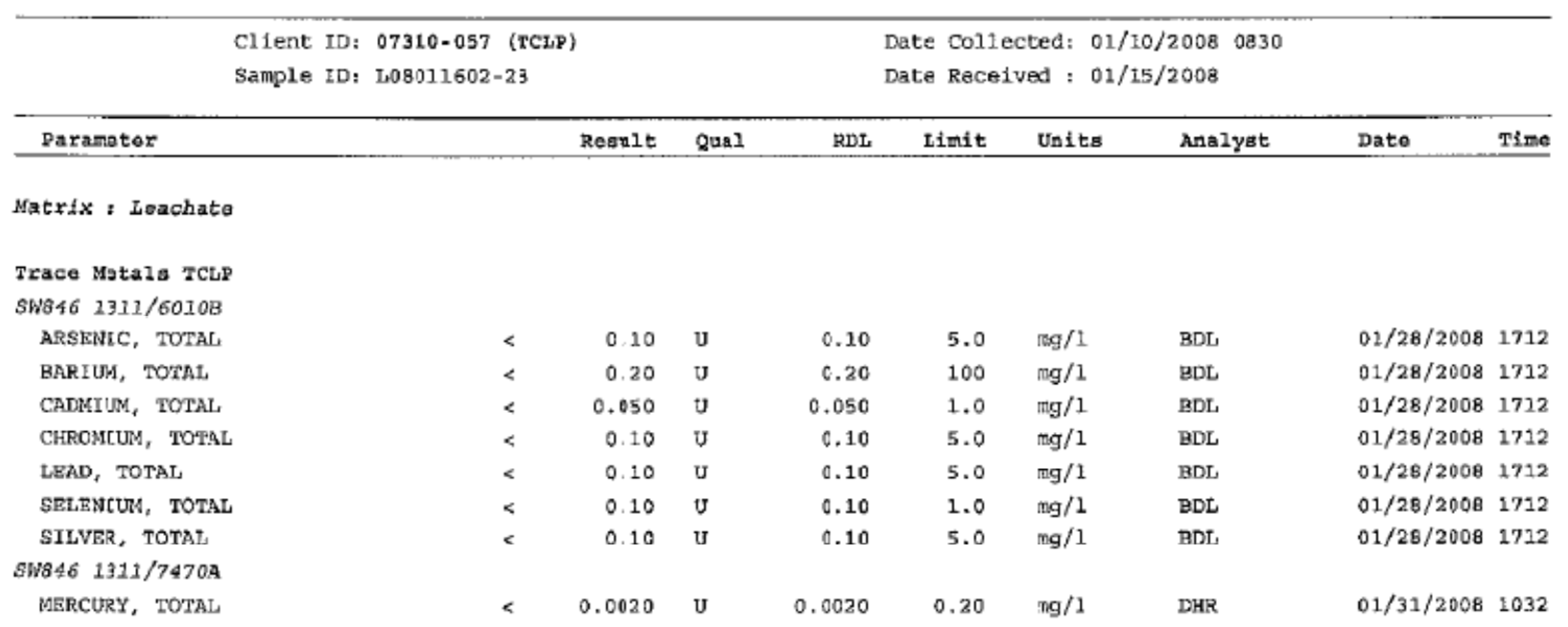


Table F.30. TCLP Metals Results for Quenched EM07-P-0

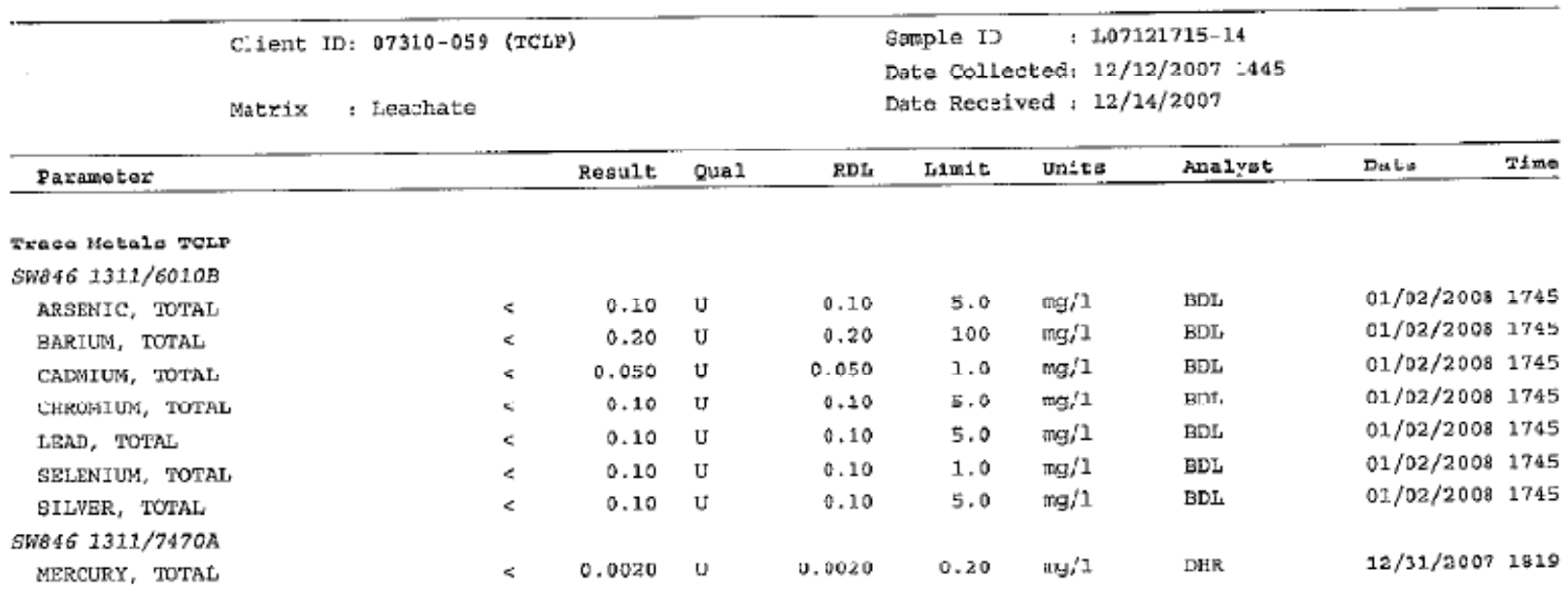

Table F.31. TCLP Metals Results for Quenched EM07-P-025

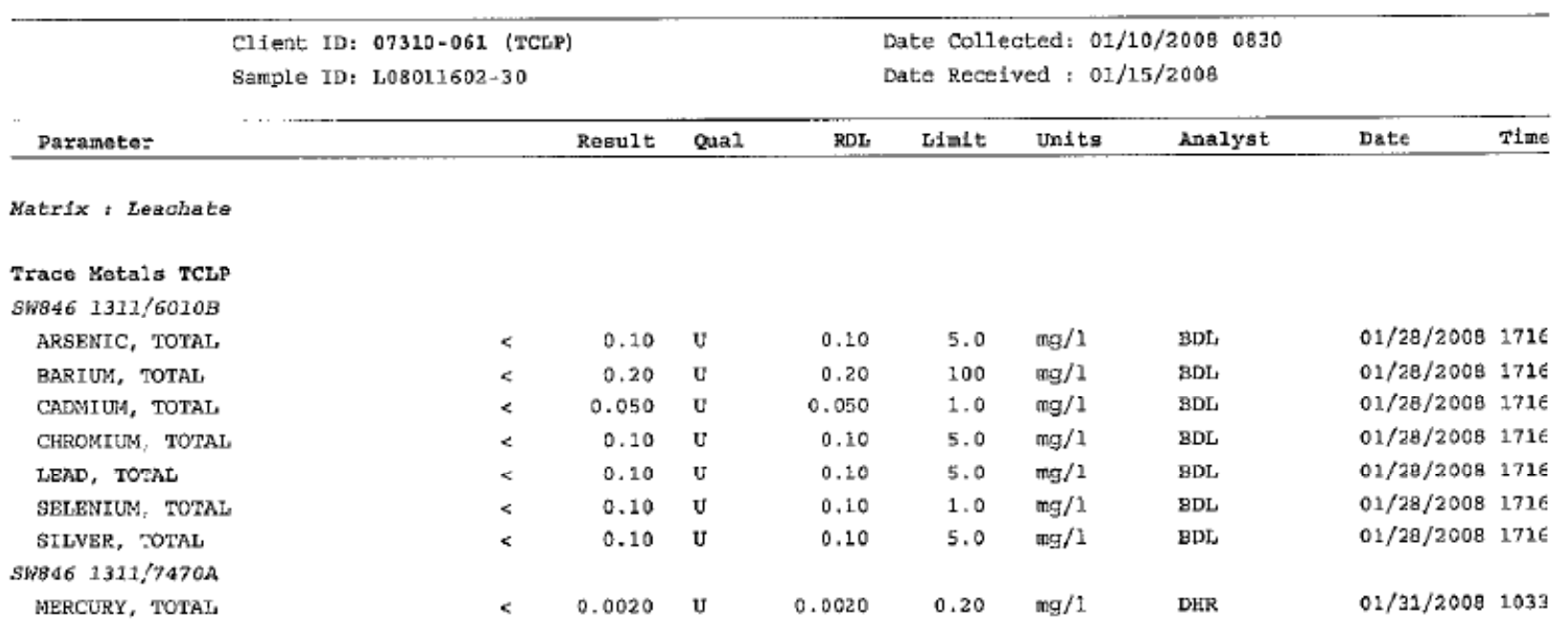


Table F.32. TCLP Metals Results for Quenched EM07-Si-30

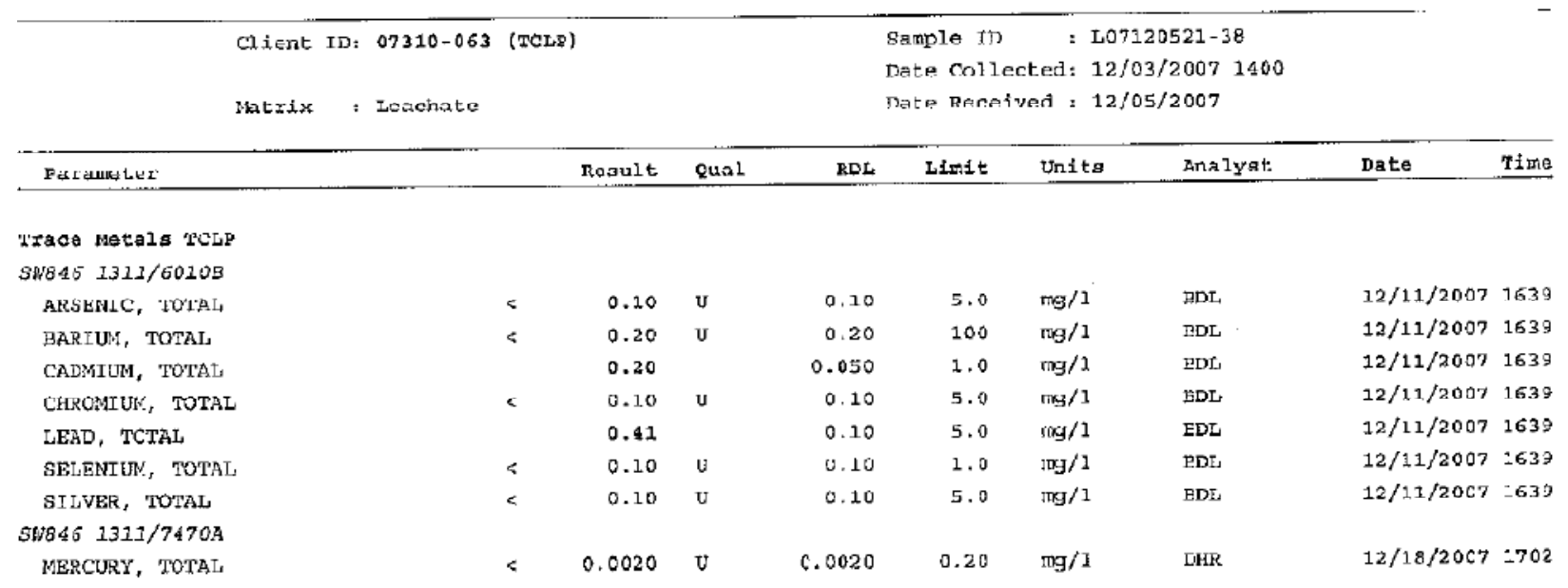

Table F.33. TCLP Metals Results for Quenched EM07-Si-37

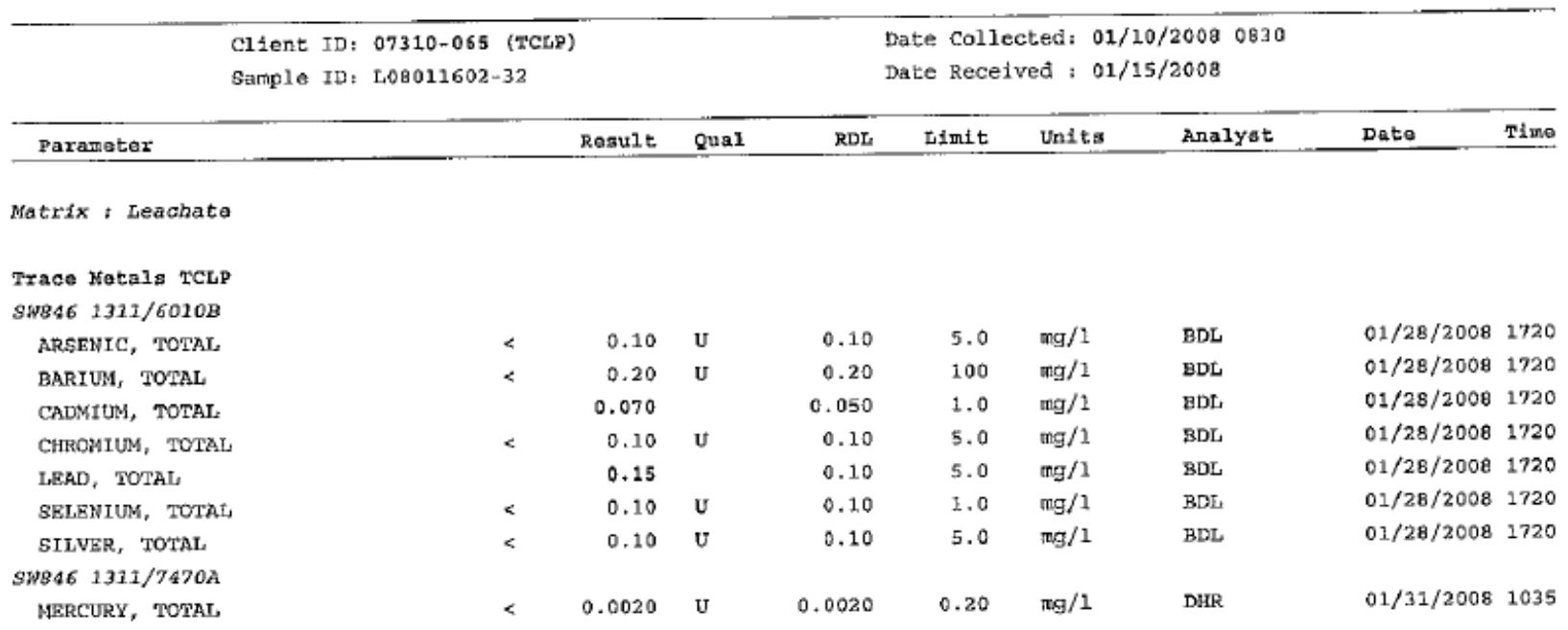


Table F.34. TCLP Metals Results for Quenched EM07-Si-50

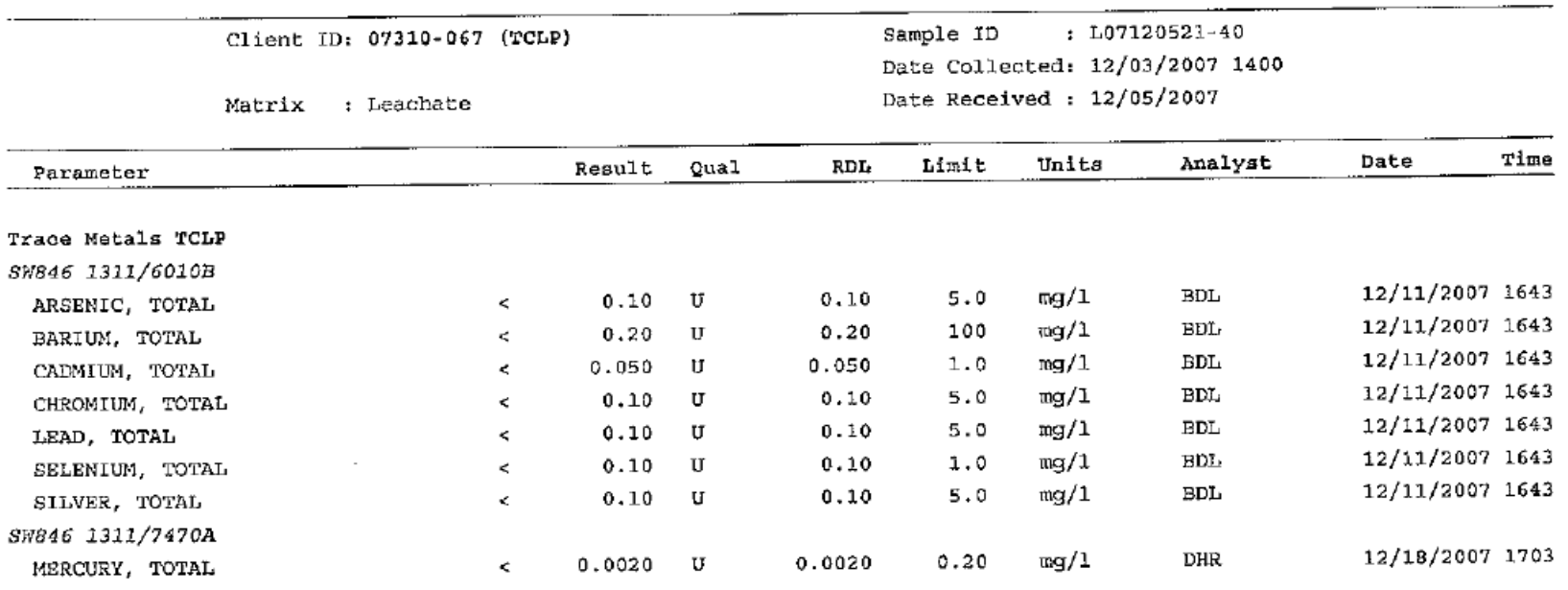

Table F.35. TCLP Metals Results for Quenched EM07-Zr-001

\begin{tabular}{|c|c|c|c|c|c|c|c|c|c|}
\hline \multirow[b]{2}{*}{ Parameter } & \multicolumn{3}{|c|}{$\begin{array}{l}\text { Client ID: } 07310-069 \text { (TCLP) } \\
\text { Sample ID: L08011602-34 }\end{array}$} & \multicolumn{4}{|c|}{$\begin{array}{l}\text { Date Collected: } 01 / 10 / 20080830 \\
\text { Date Received : } 01 / 15 / 2008\end{array}$} & \multirow[b]{2}{*}{ Date } & \multirow[b]{2}{*}{ Tine } \\
\hline & & ResuIt & Qual & RDL & Limit & Units & Analygt & & \\
\hline \multicolumn{10}{|l|}{ Matrix : Leachate } \\
\hline \multicolumn{10}{|l|}{ Trace Motals TCLP } \\
\hline \multicolumn{10}{|l|}{ SW846 1311/6010B } \\
\hline ARSENIC, TOTAL & $<$ & 0.10 & $\mathrm{v}$ & 0.10 & 5.0 & $\mathrm{ng} / 1$ & $\mathrm{BDL}$ & $01 / 28 / 2008$ & 1731 \\
\hline BARIUN, TOTAL & $<$ & 0.20 & $\mathrm{u}$ & 0.20 & 100 & $\mathrm{mg} / 1$ & BDL & $01 / 28 / 2008$ & 1731 \\
\hline CADMIUM, TOTAL & $<$ & 0.050 & $\mathrm{u}$ & 0.050 & 1.0 & $\mathrm{mg} / \mathrm{I}$ & BDI & $01 / 28 / 2008$ & 1731 \\
\hline CHROMIUM, TOTAL, & $<$ & 0.10 & $v$ & 0.10 & 5.0 & $\mathrm{mg} / \mathrm{I}$ & BDI & $01 / 28 / 2008$ & 1731 \\
\hline LEAD, TOTAL & $<$ & 0.10 & $\mathrm{u}$ & 0.10 & 5.0 & $\mathrm{mg} / 1$ & BDI & $01 / 28 / 2008$ & 1731 \\
\hline SBLENIUM， TOTAL & $<$ & 0.10 & $\mathrm{u}$ & 0.10 & 1.0 & ng/1 & BDI & $01 / 28 / 2008$ & 1731 \\
\hline SILVER, TOTAL & $<$ & 0.10 & $\mathrm{u}$ & 0.10 & 5.0 & $\mathrm{mg} / 1$ & BDI & $01 / 28 / 2008$ & 1731 \\
\hline \multicolumn{10}{|l|}{ SWS46 1311/747OA } \\
\hline MERCURY, TOTAL, & $<$ & 0.0020 & u & 0.0020 & 0.20 & $\mathrm{mg} / \mathrm{I}$ & DHR & $01 / 31 / 2008$ & 1036 \\
\hline
\end{tabular}


Table F.36. TCLP Metals Results for Quenched EM07-Zr-05

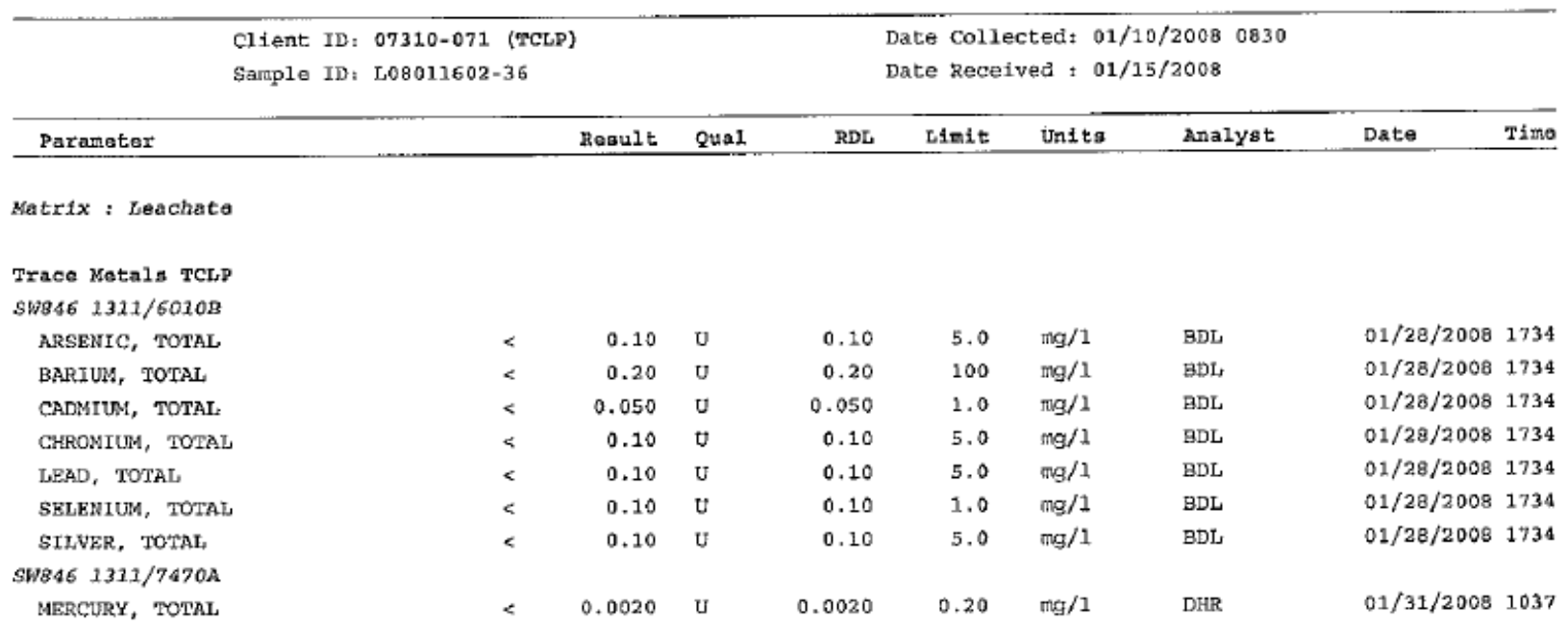

Table F.37. TCLP Metals Results for Quenched EM07-NM-0025

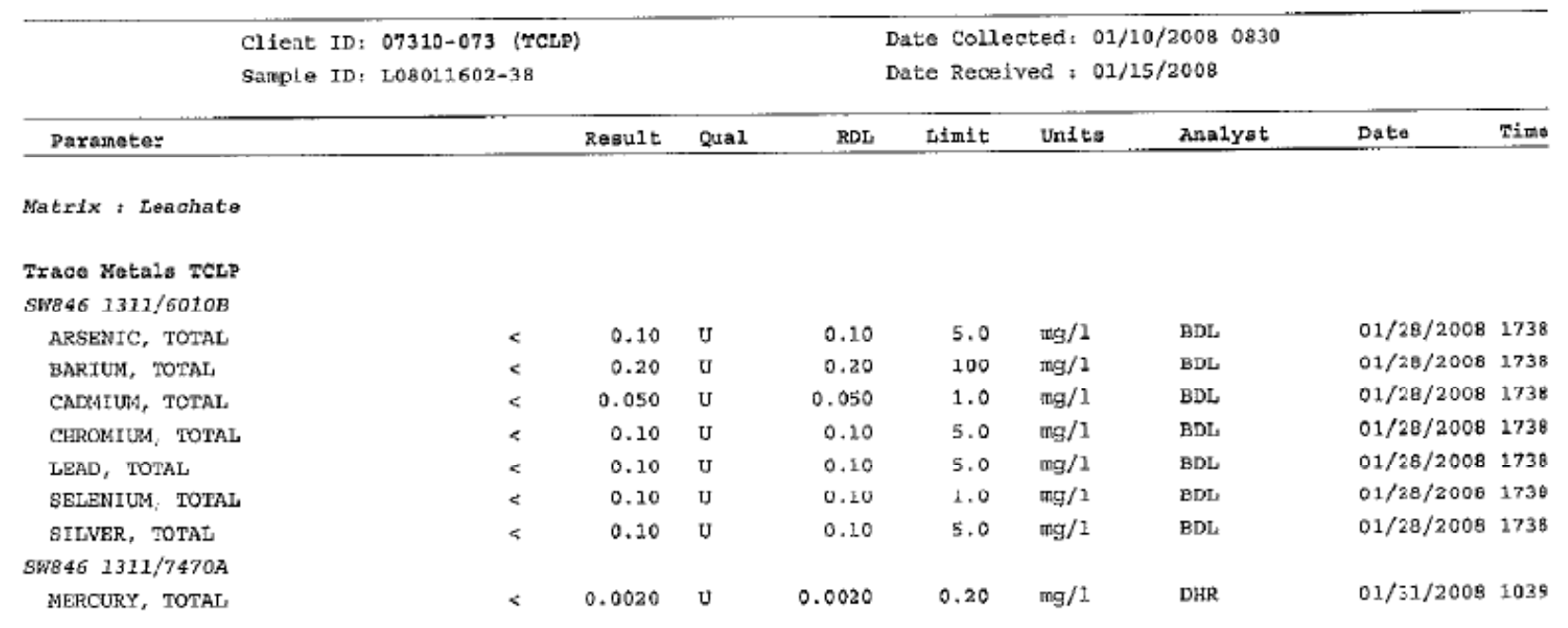


PNNL-17950, Rev 0

Table F.38. TCLP Metals Results for Quenched EM07-BL-2

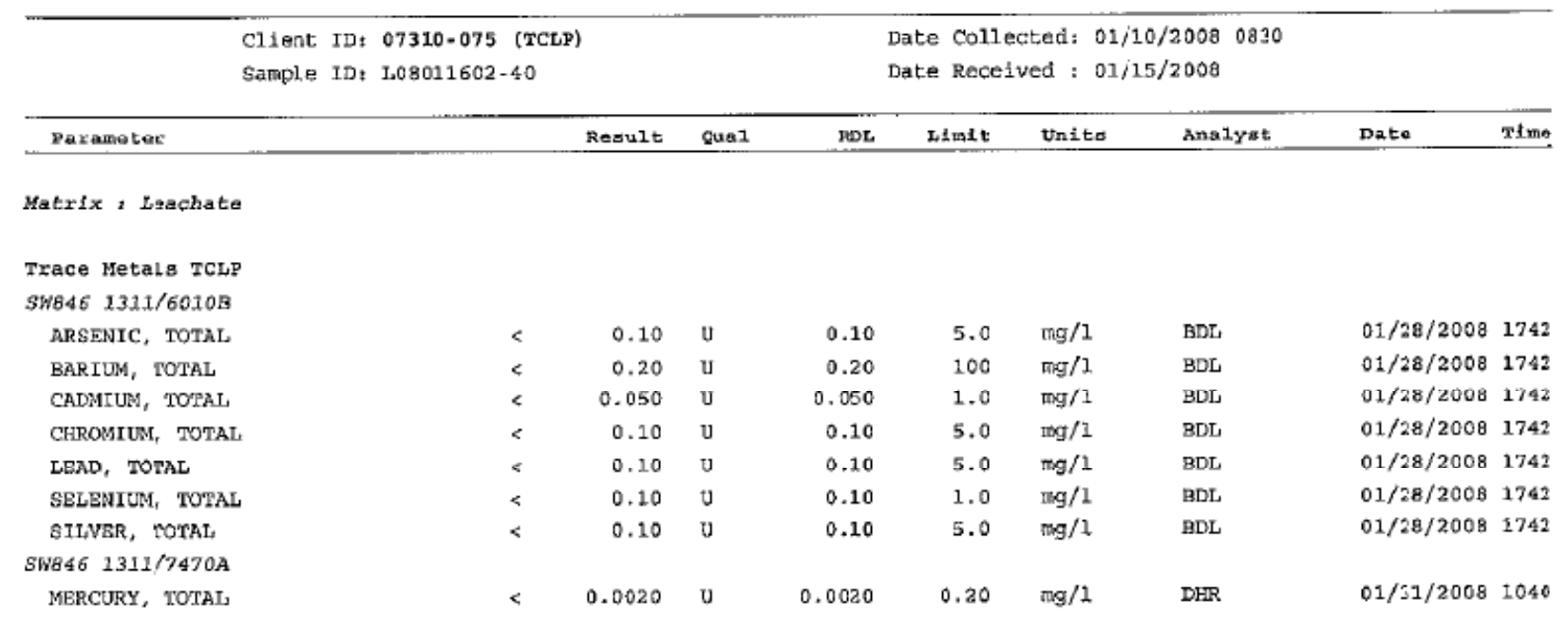


PNNL-17950, Rev 0

\section{F.2 TCLP Metals Results for CCC-Treated Glasses}

Table F.39. TCLP Metals Results for CCC-Treated EM07-BL-1

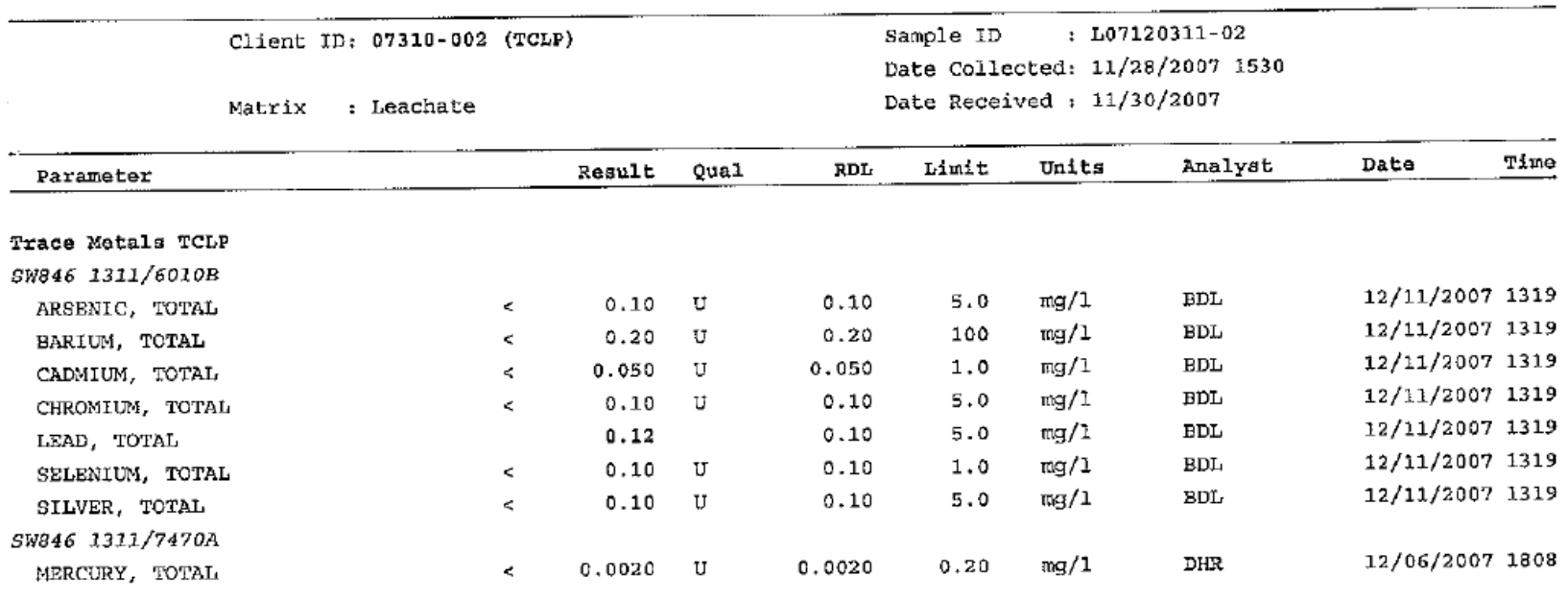

Table F.40. TCLP Metals Results for CCC-Treated EM07-A1-06

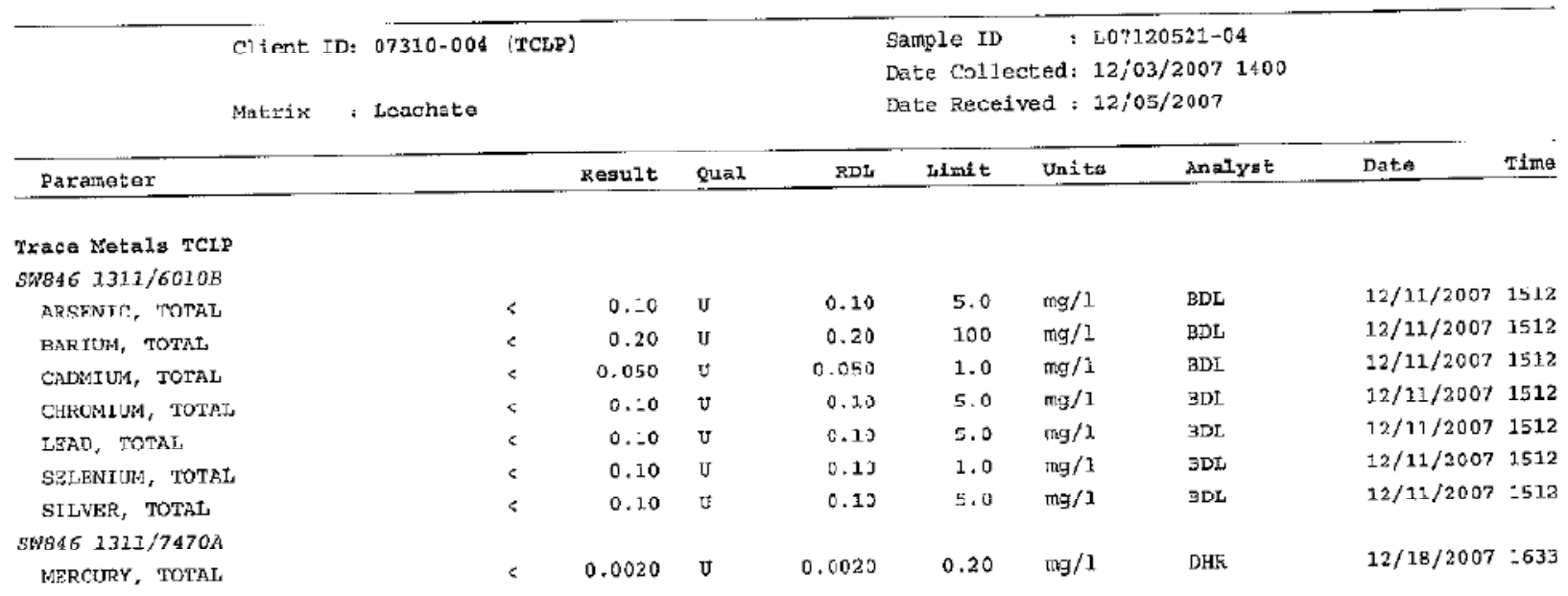


Table F.41. TCLP Metals Results for CCC-Treated EM07-Al-15

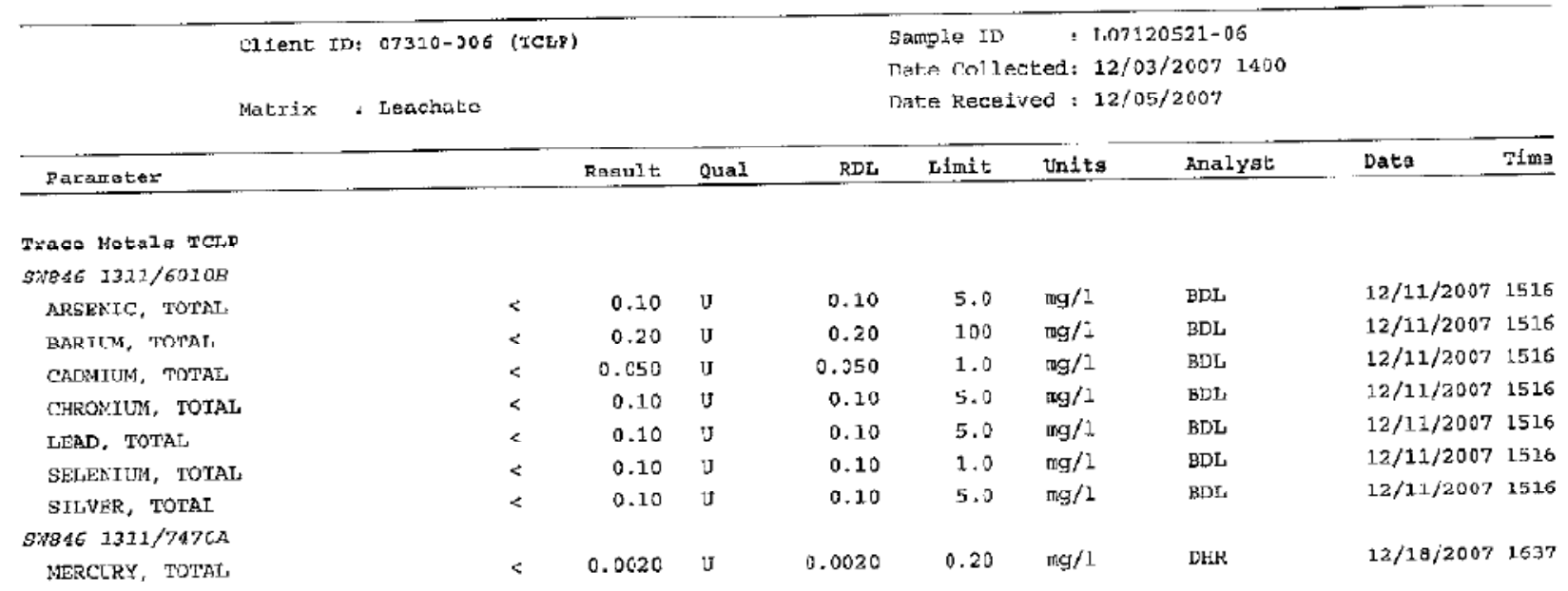

Table F.42. TCLP Metals Results for CCC-Treated EM07-Al-20

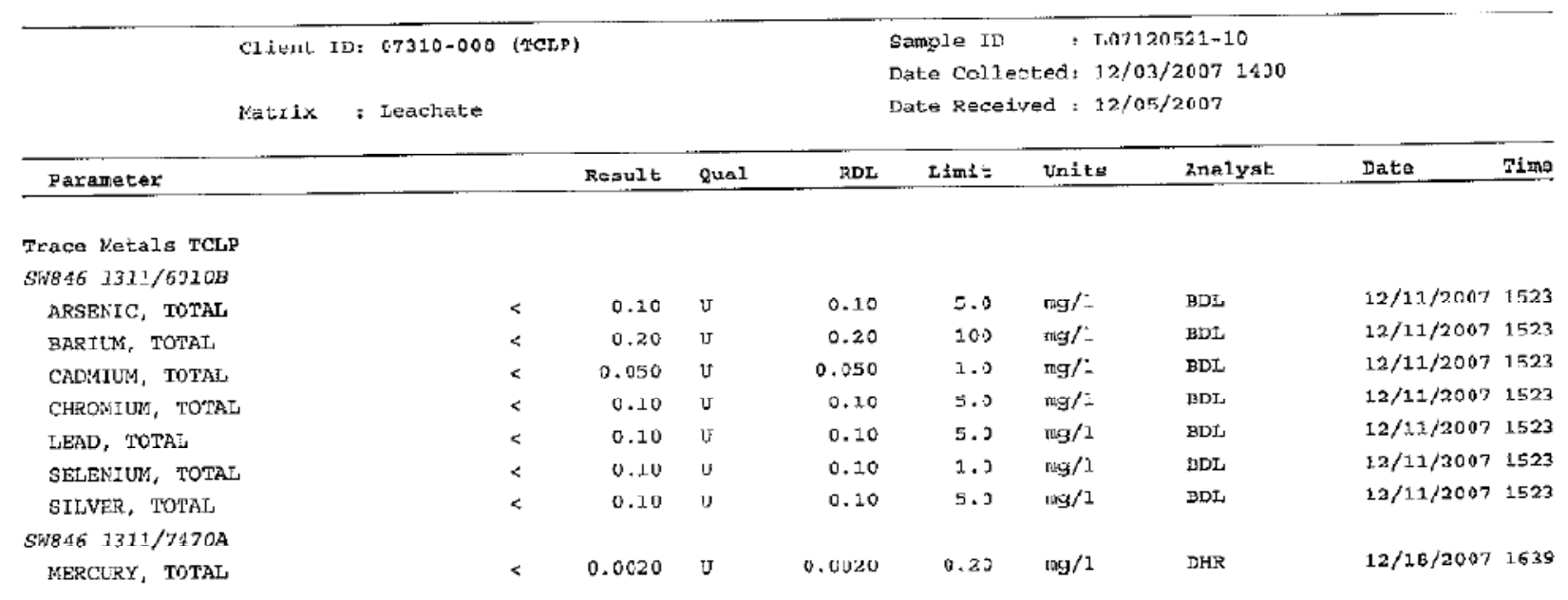

Table F.43. TCLP Metals Results for CCC-Treated EM07-B-05

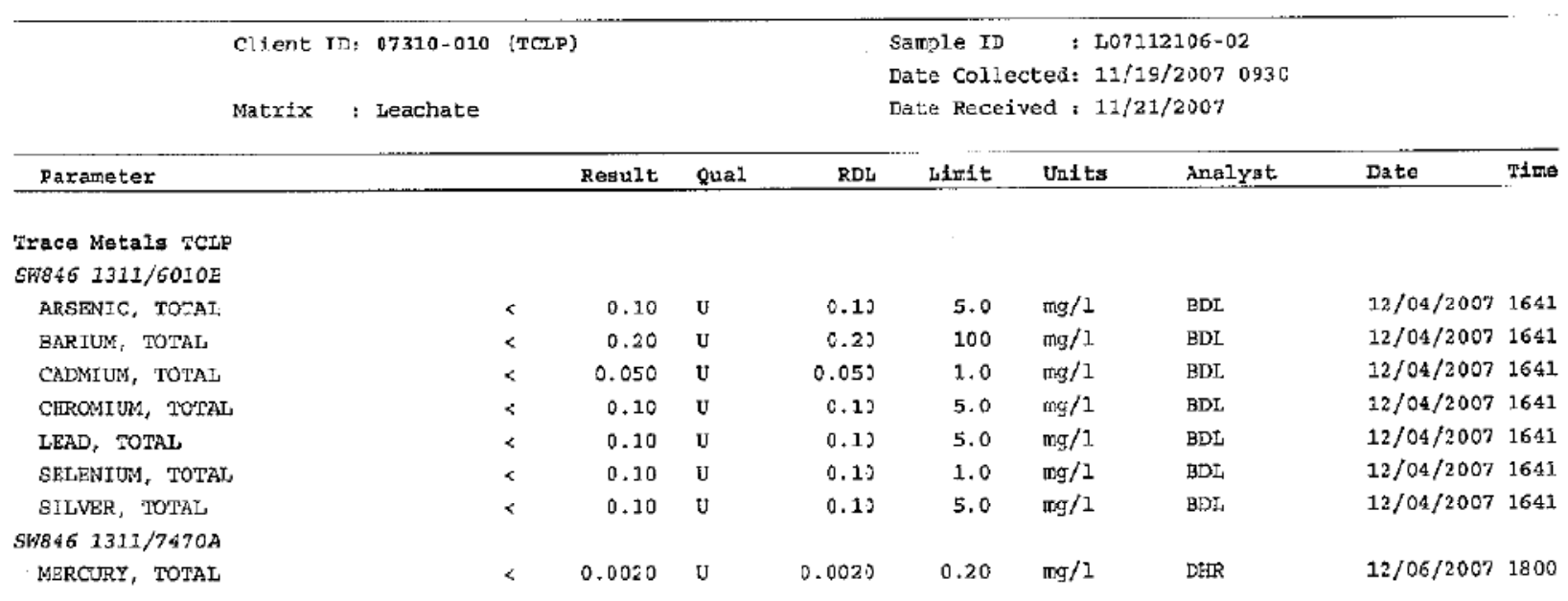


Table F.44. TCLP Metals Results for CCC-Treated EM07-B-15

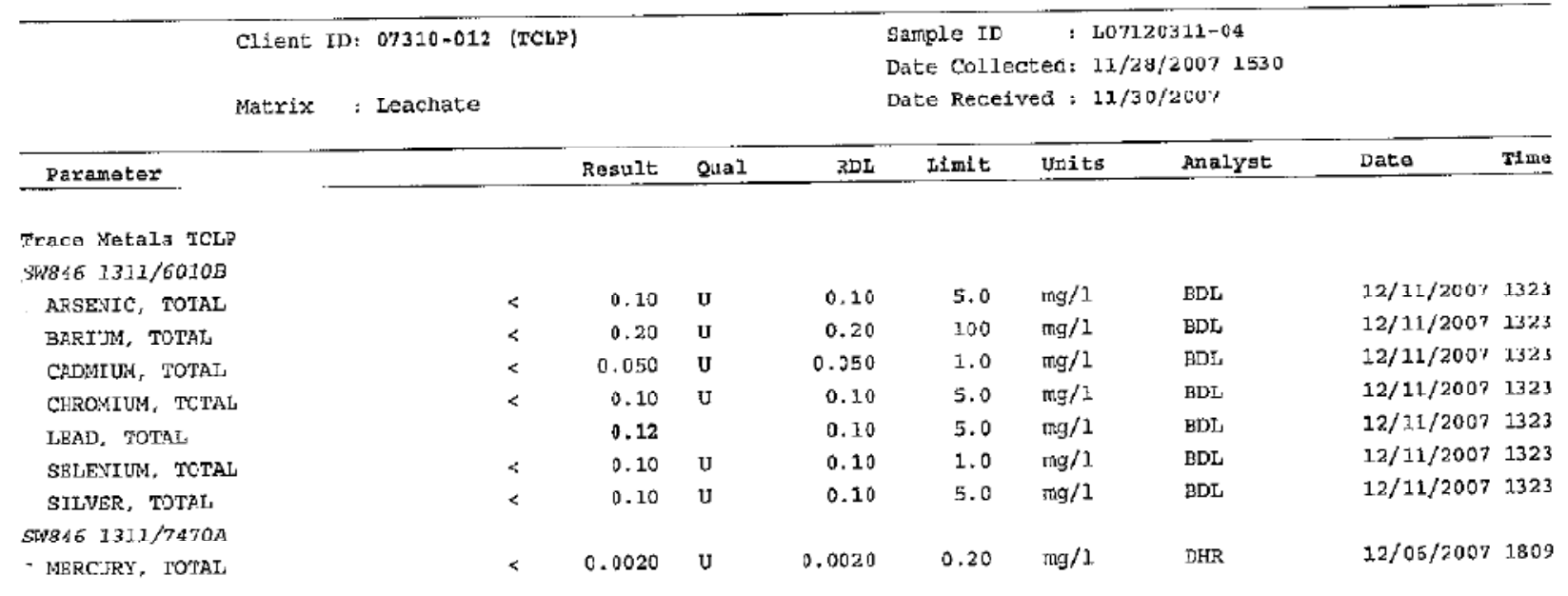

Table F.45. TCLP Metals Results for CCC-Treated EM07-B-20

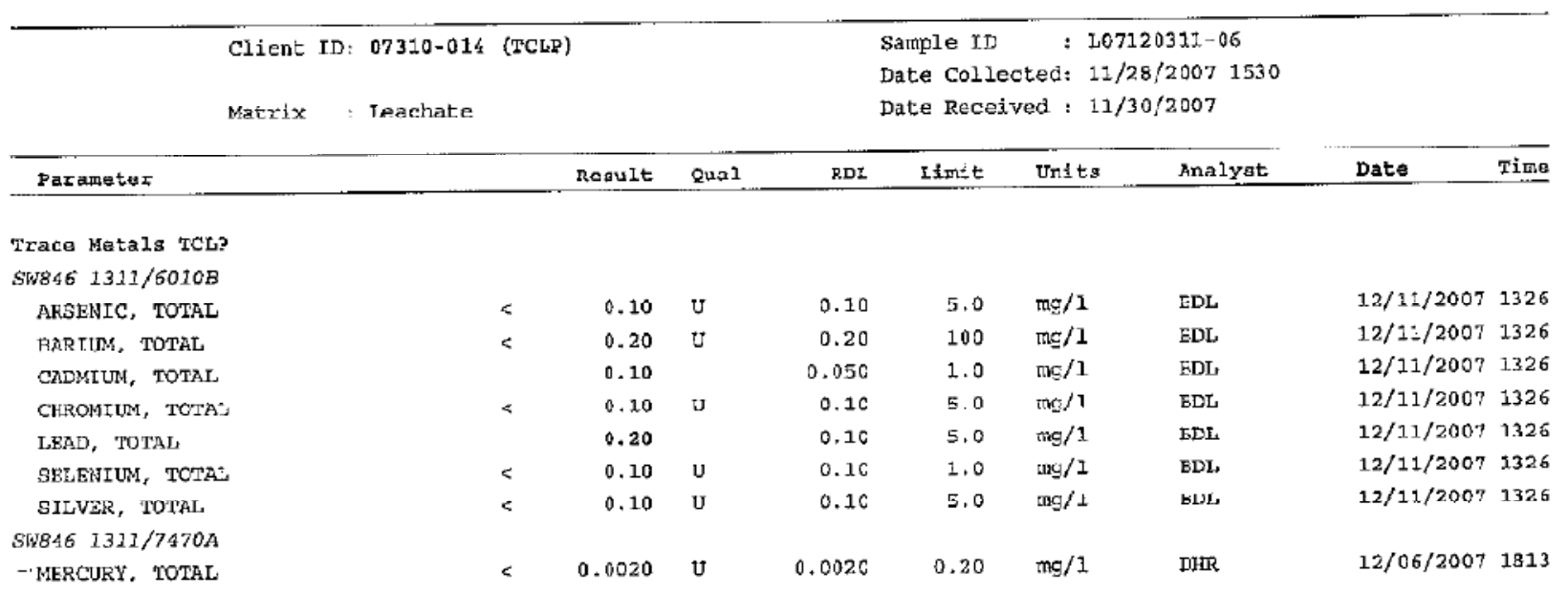


Table F.46. TCLP Metals Results for CCC-Treated EM07-Bi-025

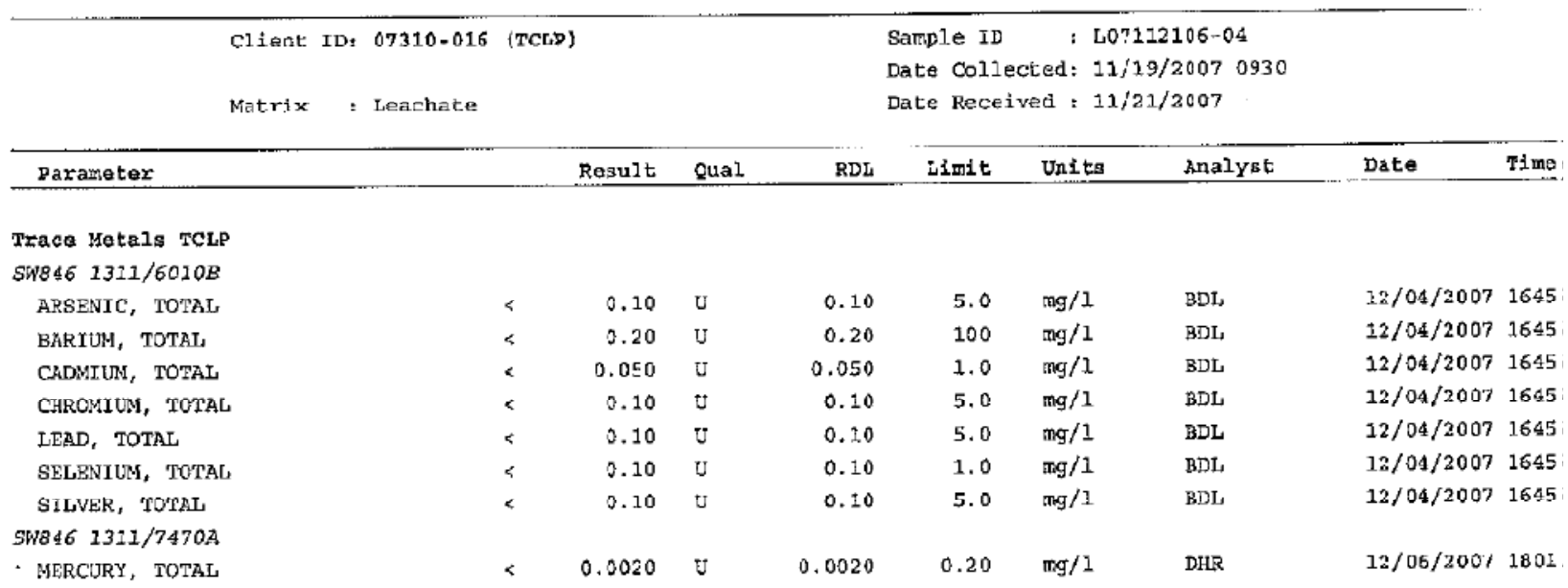

Table F.47. TCLP Metals Results for CCC-Treated EM07-Bi-05

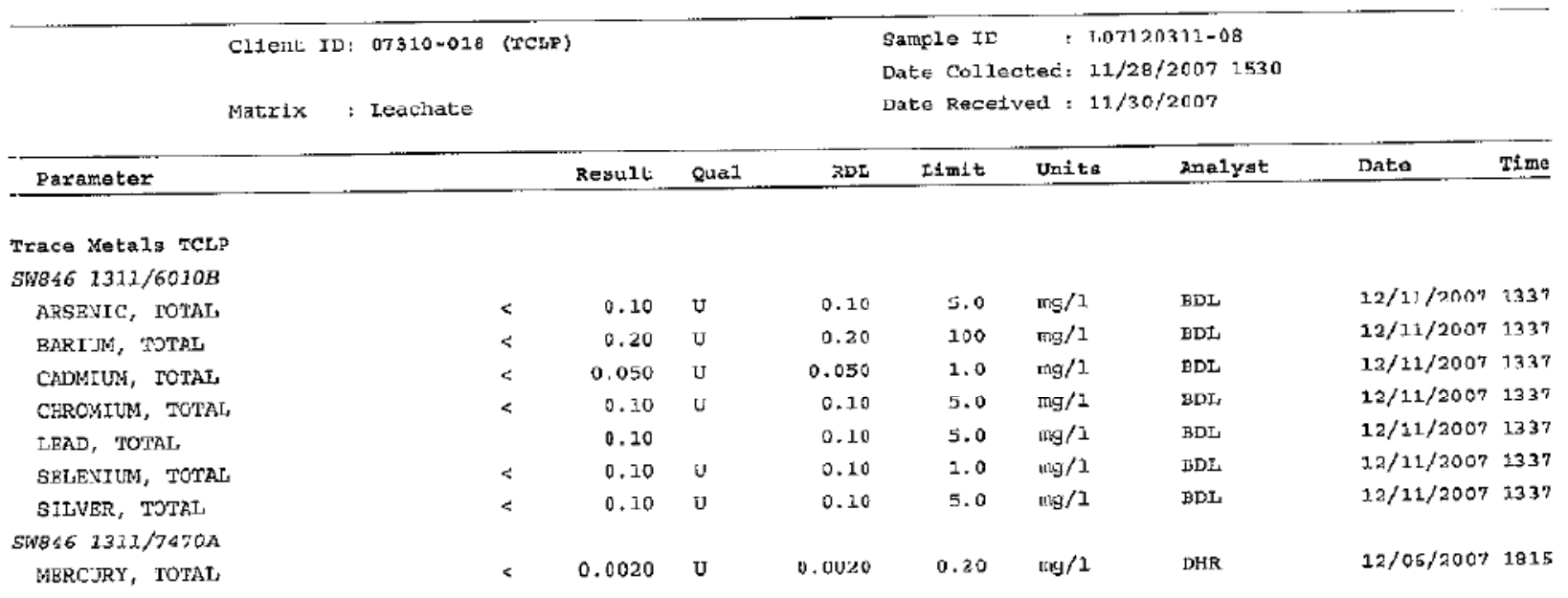

Table F.48. TCLP Metals Results for CCC-Treated EM07-Ca-035

\begin{tabular}{|c|c|c|c|c|c|c|c|c|c|c|}
\hline \multirow[b]{2}{*}{ Paraneter } & \multirow[t]{2}{*}{$\begin{array}{l}\text { Client ID, } \$ 7310-020 \\
\text { Matrix : Jeachatc }\end{array}$} & \multirow[t]{2}{*}{ ('PCLP) } & \multicolumn{6}{|c|}{$\begin{array}{l}\text { Sample fD : Lo7120311-10 } \\
\text { Date Collected: } 11 / 28 / 20071530 \\
\text { Date Received : } 11 / 30 / 2007\end{array}$} & \multirow[b]{2}{*}{ Date } & \multirow[b]{2}{*}{ Time } \\
\hline & & & Rogult & Qual & RDL & Limit & Units & Analyat: & & \\
\hline \multicolumn{11}{|l|}{ Trace Vetals TCL? } \\
\hline \multicolumn{11}{|l|}{$S N 8461311 / 6010 B$} \\
\hline AKSEN 1C, LUTAL & & $<$ & 0.10 & $\mathrm{~J}$ & 0.10 & 5.3 & $\mathrm{ng} / 1$ & GDL & $12 / 71 / 2007$ & 1.341 \\
\hline BARILM, TOTAL & & $<$ & 0.20 & כ' & 0.20 & 100 & $\mathrm{ng} / \mathrm{i}$ & EDL & $12 / 11 / 2007$ & 1341 \\
\hline CALMLUM, TOTAL & & $<$ & 0.050 & $\mathrm{~J}$ & 0.050 & 1.0 & $\mathrm{ng} / 1$ & BDL & $12 / 11 / 2007$ & 1341 \\
\hline CHROYIUM, TOTAL & & $<$ & 0.10 & $\mathrm{~J}$ & 0.10 & 5.0 & $\mathrm{ng} / 1$ & BDL & $12 / 11 / 2007$ & 1341 \\
\hline LEAD, TOTAL & & & 0.10 & & 0.10 & 5.3 & $\mathrm{ng} / 1$ & BDL & $12 / 11 / 2007$ & 1341 \\
\hline SELEAIUM, TOTAI & & $<$ & 0.10 & 于 & 0.10 & 1.3 & $\pi g / 1$ & DDL. & $13 / 11 / 3007$ & $\pm 3 \Delta 1$ \\
\hline SILVER, TOTAL & & $<$ & 0.10 & $\mathrm{~J}$ & 0.10 & 5.0 & $\mathrm{mg} / 1$ & BDL & $13 / 11 / 2007$ & 1341 \\
\hline \multicolumn{11}{|l|}{ SW846 $13.11 / 7470 \mathrm{~A}$} \\
\hline MERCLRY, TOTAL & & $<$ & 0.0020 & 小 & 0.0020 & 0.20 & ing $/ 1$ & $\mathrm{DHR}$ & $12 / 06 / 2007$ & 1816 \\
\hline
\end{tabular}


PNNL-17950, Rev 0

Table F.49. TCLP Metals Results for CCC-Treated EM07-Ca-07

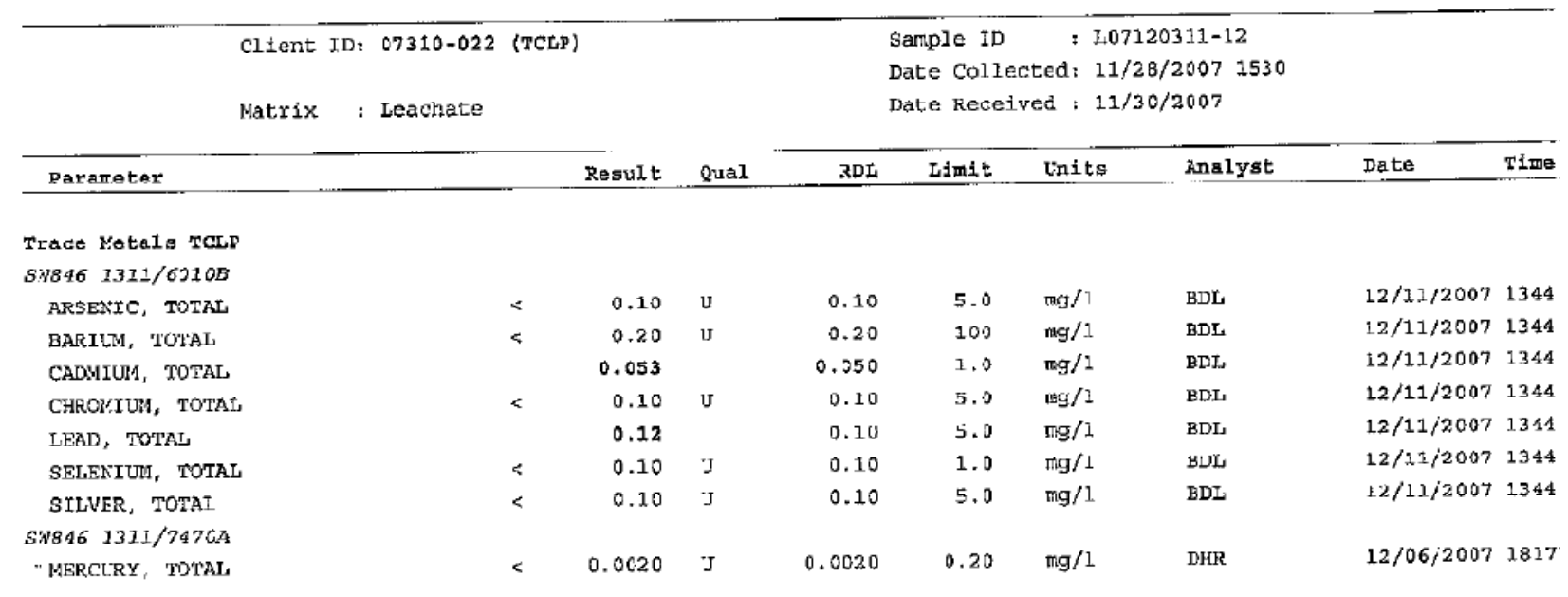

Table F.50. TCLP Metals Results for CCC-Treated EM07-Cr-001

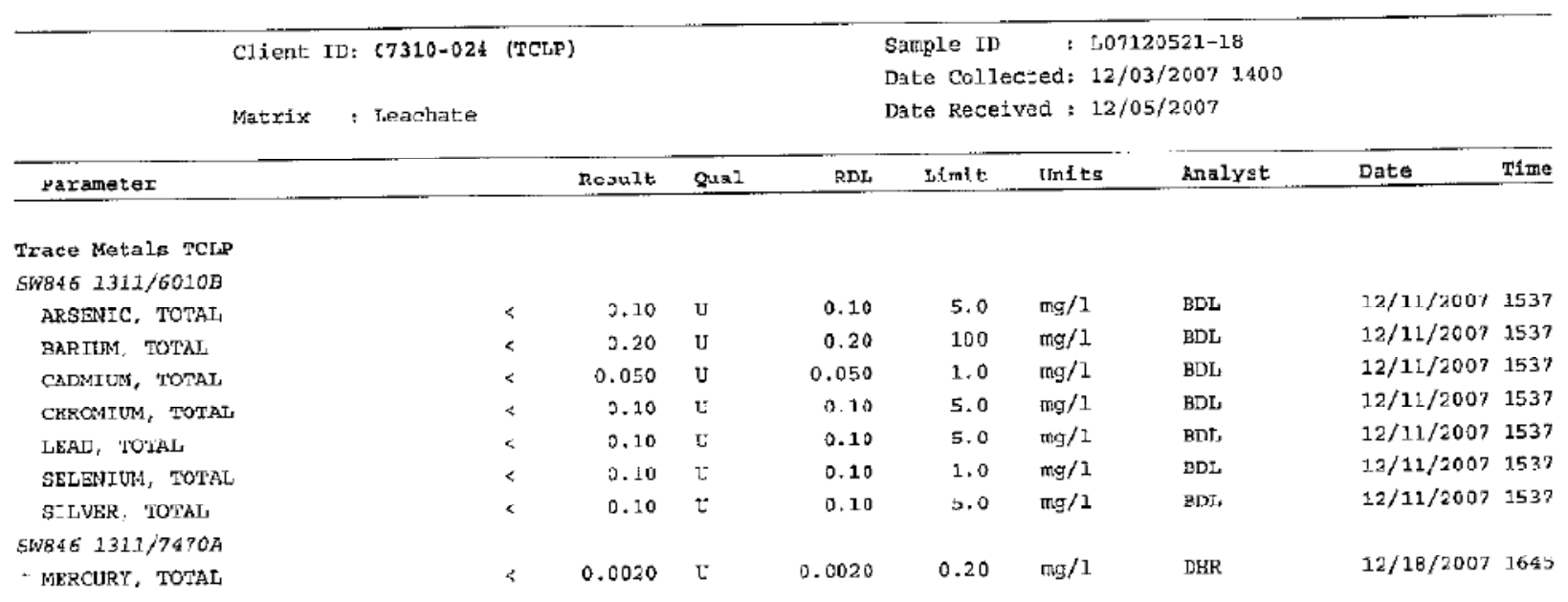


Table F.51. TCLP Metals Results for CCC-Treated EM07-Cr-012

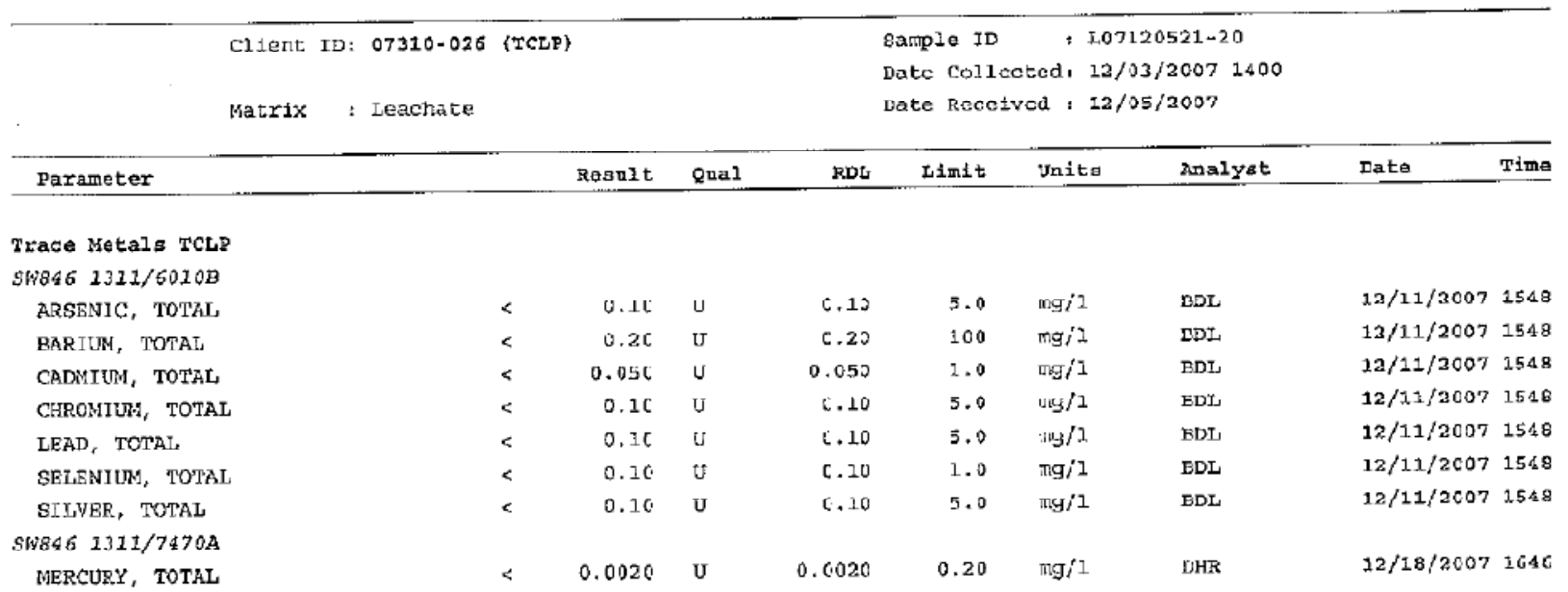

Table F.52. TCLP Metals Results for CCC-Treated EM07-Cr-02

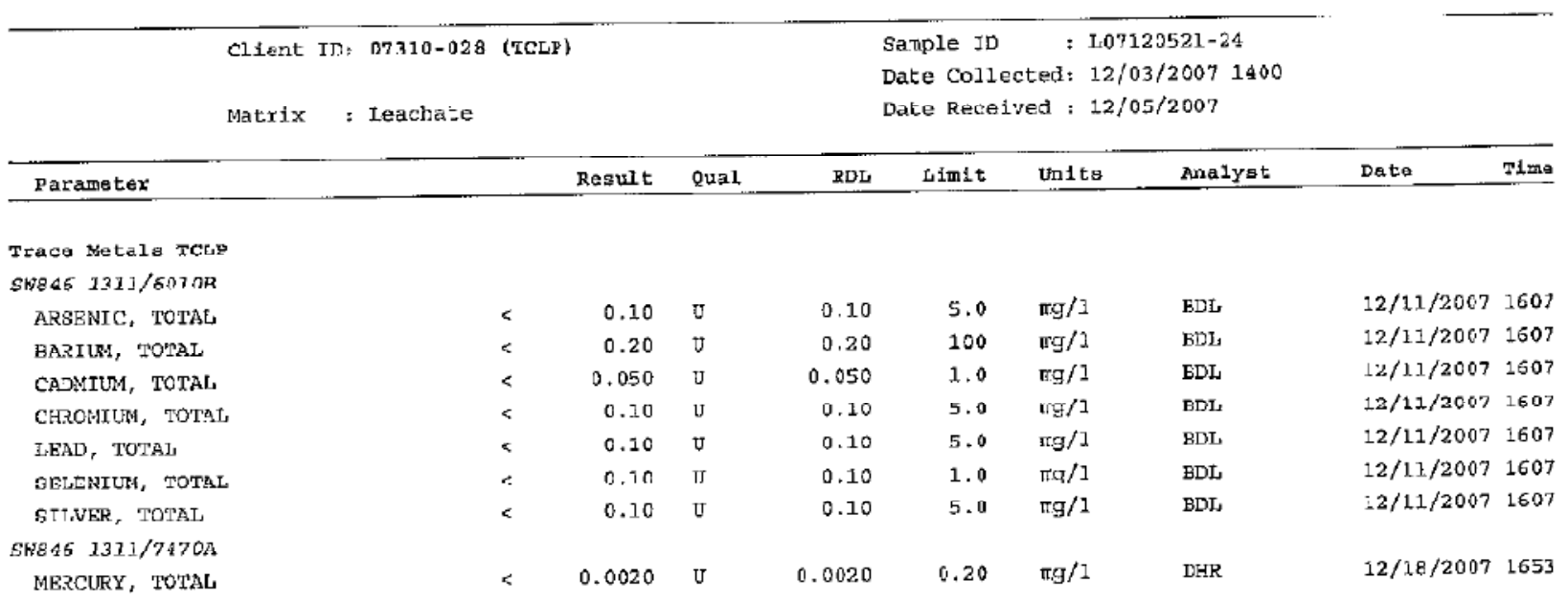

Table F.53. TCLP Metals Results for CCC-Treated EM07-F-02

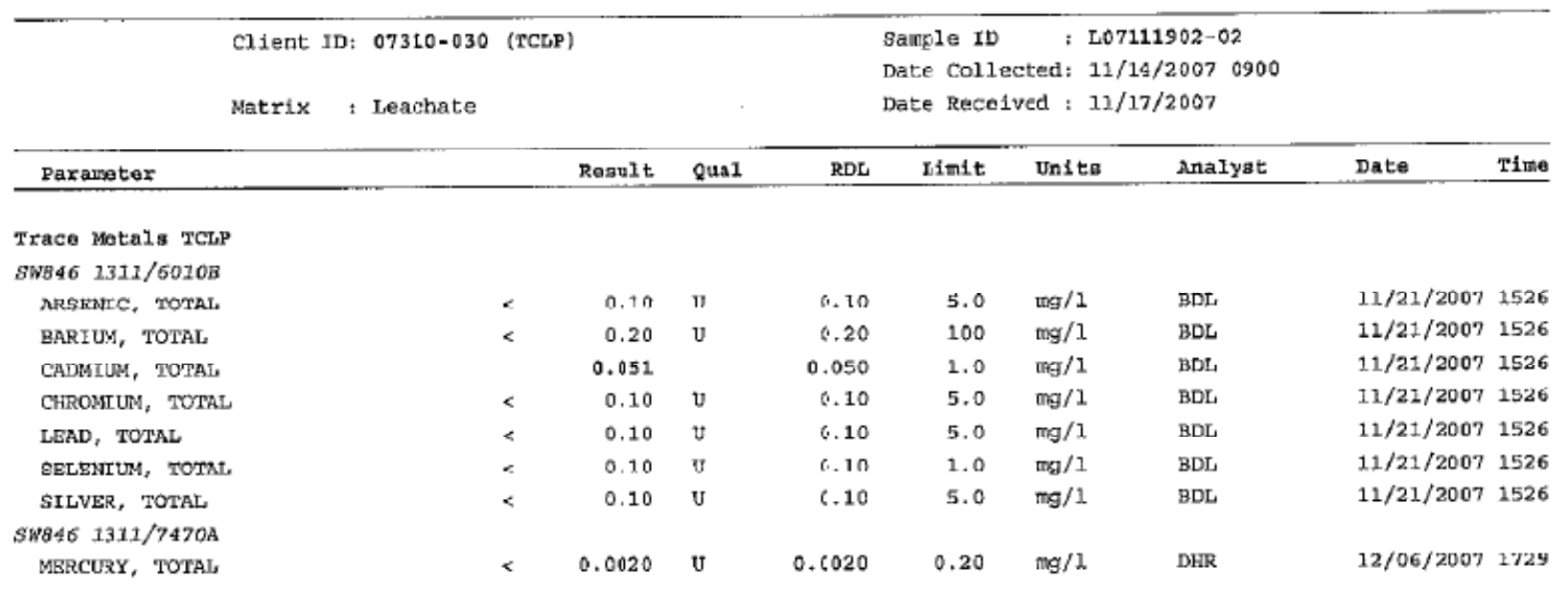


PNNL-17950, Rev 0

Table F.54. TCLP Metals Results for CCC-Treated EM07-Fe-05

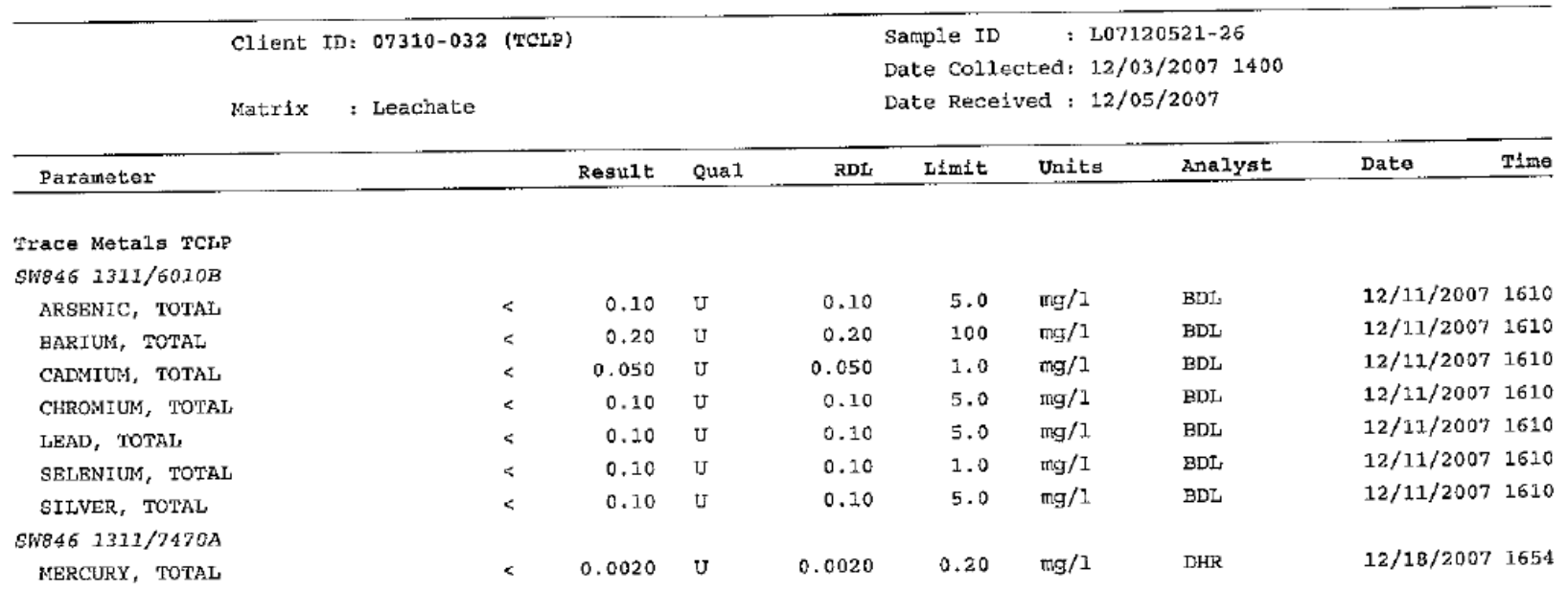

Table F.55. TCLP Metals Results for CCC-Treated EM07-Fe-15

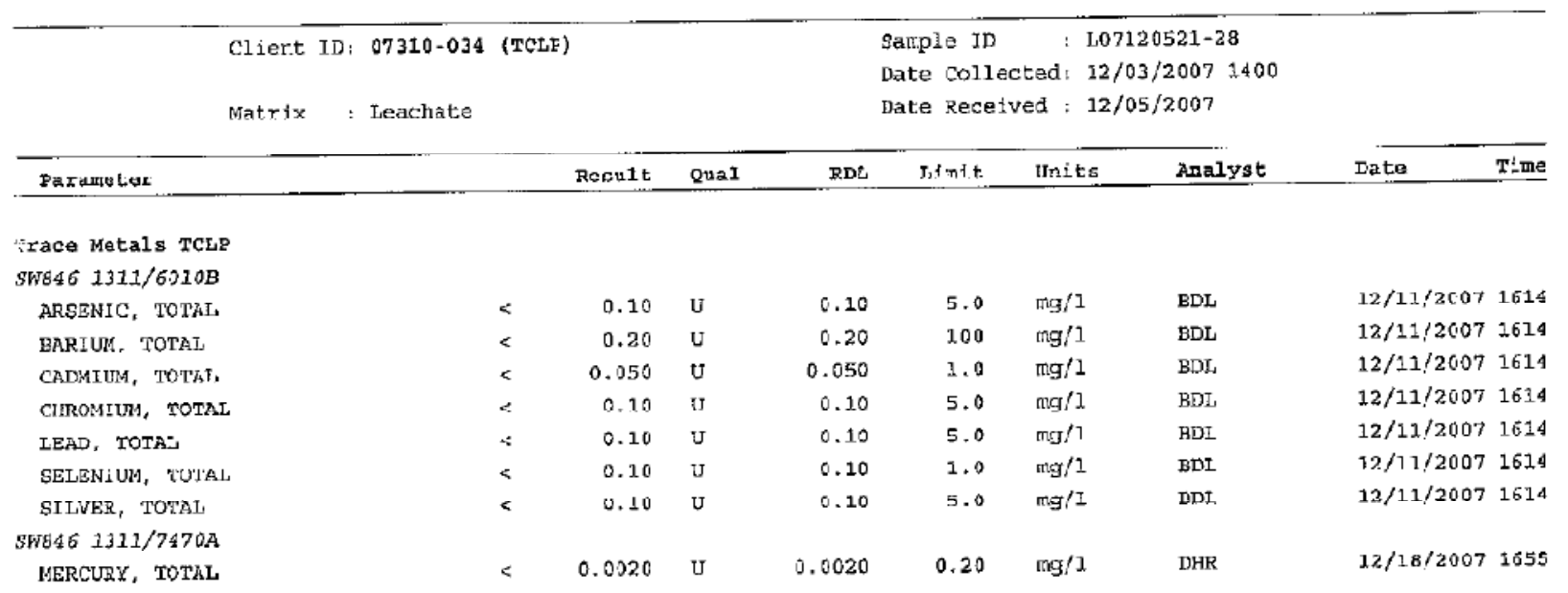


Table F.56. TCLP Metals Results for CCC-Treated EM07-Fe-20

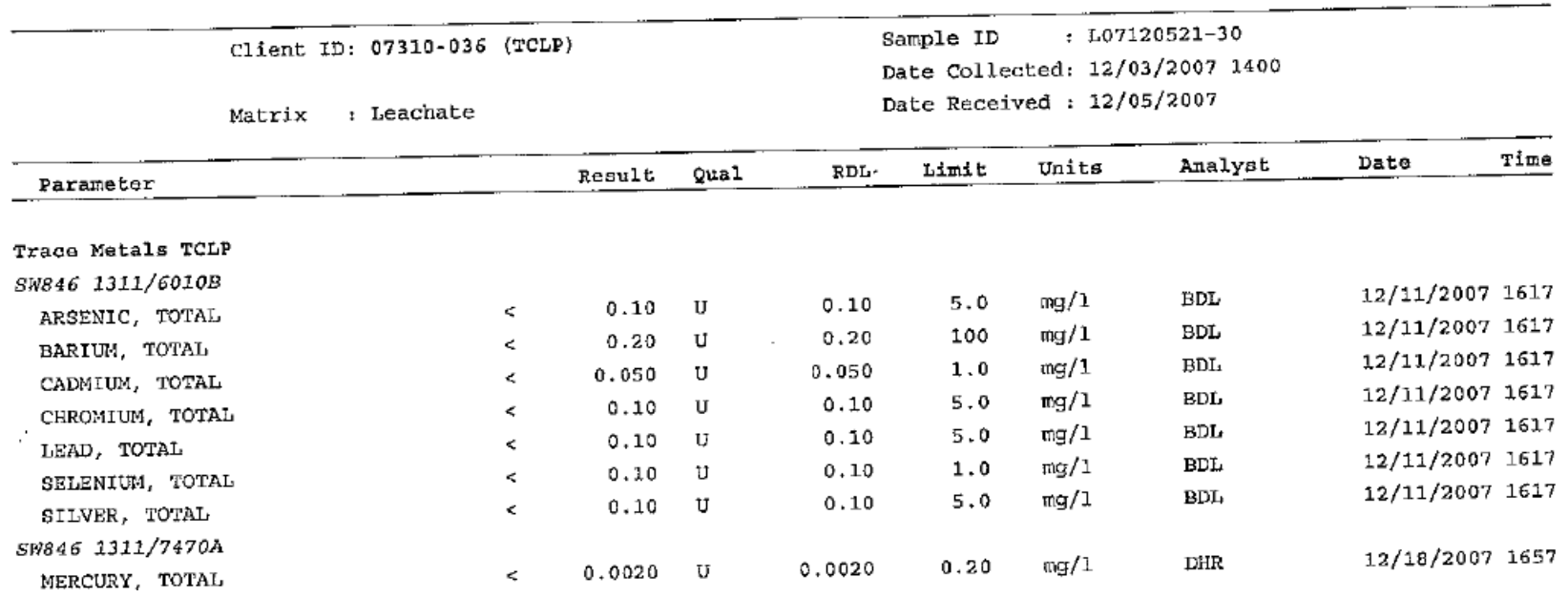

Table F.57. TCLP Metals Results for CCC-Treated EM07-K-03

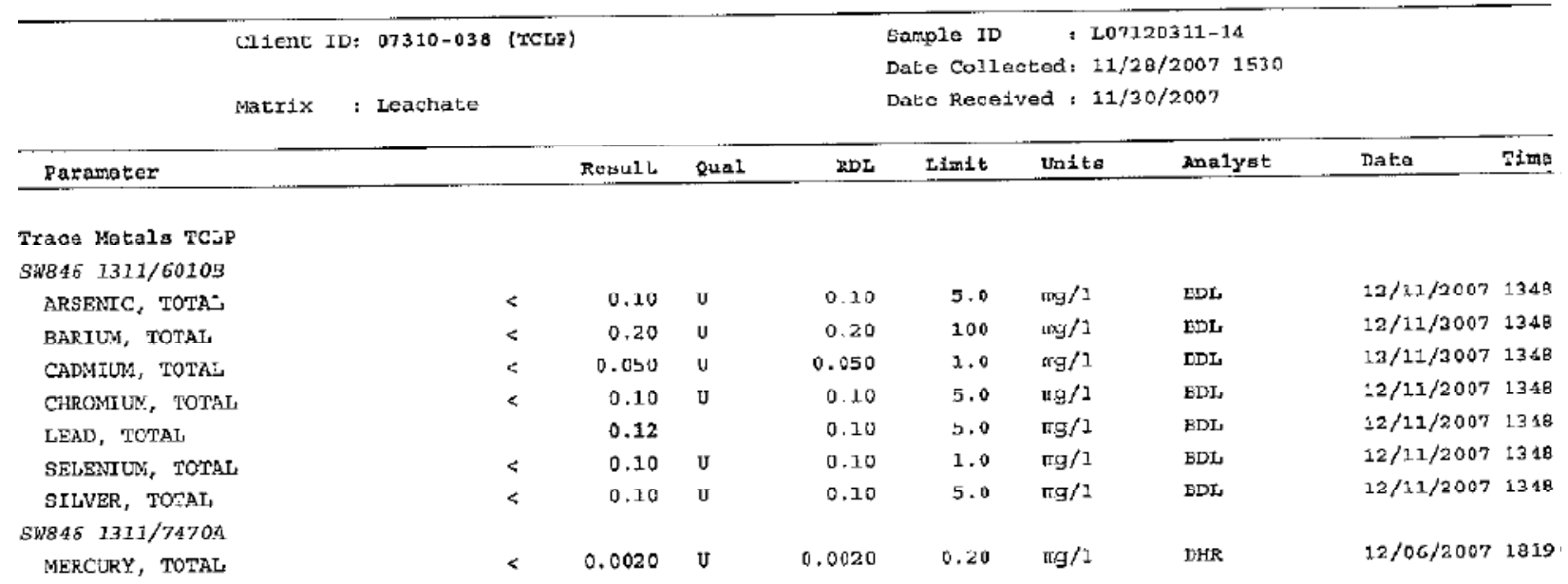

Table F.58. TCLP Metals Results for CCC-Treated EM07-K-06

\begin{tabular}{|c|c|c|c|c|c|c|c|c|c|c|}
\hline \multirow[b]{2}{*}{ Paxamoter. } & \multirow[t]{2}{*}{$\begin{array}{l}\text { Client ID: } 07310-040 \\
\text { Matrix : Leachate }\end{array}$} & \multicolumn{3}{|c|}{ (TCLP) } & \multicolumn{4}{|c|}{$\begin{array}{l}\text { Sample ID } \quad \text { : L07120311-16 } \\
\text { Date Collected: } 11 / 28 / 20071530 \\
\text { Date Received : } 11 / 30 / 2007\end{array}$} & \multirow[b]{2}{*}{ Date } & \multirow[b]{2}{*}{ Time } \\
\hline & & & ResuIt & Qual & RDL & Limit & Units & Analyst & & \\
\hline \multicolumn{11}{|l|}{ Trace Meta1s TCuP } \\
\hline \multicolumn{11}{|l|}{$\begin{array}{l}\text { Trace Metals TCLP } \\
\text { SW846 } 1311 / 6010 B\end{array}$} \\
\hline ARSENIC, TOTAL & & $<$ & 0.10 & $\mathrm{u}$ & 0.10 & 5.0 & $\mathrm{mg} / 1$ & BDL & $12 / 11 / 2007$ & 1352 \\
\hline BARIUM, TOTAL & & $<$ & 0.20 & $\mathrm{u}$ & 0.20 & 100 & $\mathrm{mg} / 1$ & $\mathrm{BDL}$ & $12 / 11 / 2007$ & 1352 \\
\hline CADMIUM, TOTAL & & $<$ & 0.050 & $\mathrm{u}$ & 0.050 & 1.0 & $\mathrm{mg} / 1$ & RDL & $12 / 11 / 2007$ & 1352 \\
\hline CHROMIUM, TOTAL & & $<$ & 0.10 & u & 0.10 & 5.0 & $\mathrm{mg} / 1$ & BDL & $12 / 11 / 2007$ & 1352 \\
\hline LEAD, TOTAL & & & 0.11 & & 0.10 & 5.0 & $\mathrm{mg} / 1$ & $\mathrm{BDE}$ & $12 / 11 / 2007$ & 1352 \\
\hline SELENIUM, TOTAL & & $<$ & 0.10 & $\mathrm{u}$ & 0.10 & 1.0 & $\operatorname{mg} / 1$ & BDE & $12 / 11 / 2007$ & 1352 \\
\hline SILVER, TOTAL & & $<$ & 0.10 & v & 0.10 & 5.0 & $\mathrm{mg} / \mathrm{I}$ & BDL & $12 / 11 / 2007$ & 1352 \\
\hline \multicolumn{11}{|l|}{ SW846 1311/7470A } \\
\hline MERCURY, TOTAL & & $<$ & 0.0020 & $\mathrm{u}$ & 0.0020 & 0.20 & $\mathrm{mg} / 1$ & DHR & $12 / 06 / 2007$ & 1820 \\
\hline
\end{tabular}


PNNL-17950, Rev 0

Table F.59. TCLP Metals Results for CCC-Treated EM07-Li-015

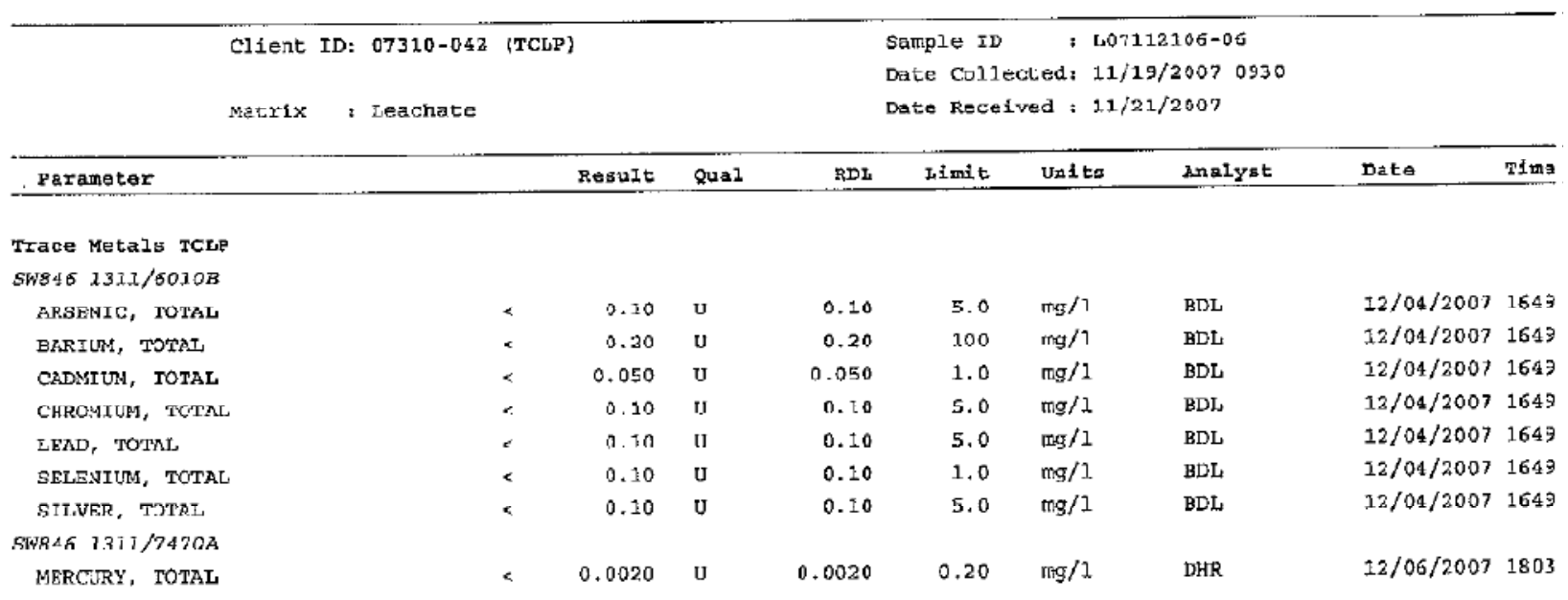

Table F.60. TCLP Metals Results for CCC-Treated EM07-Li-04

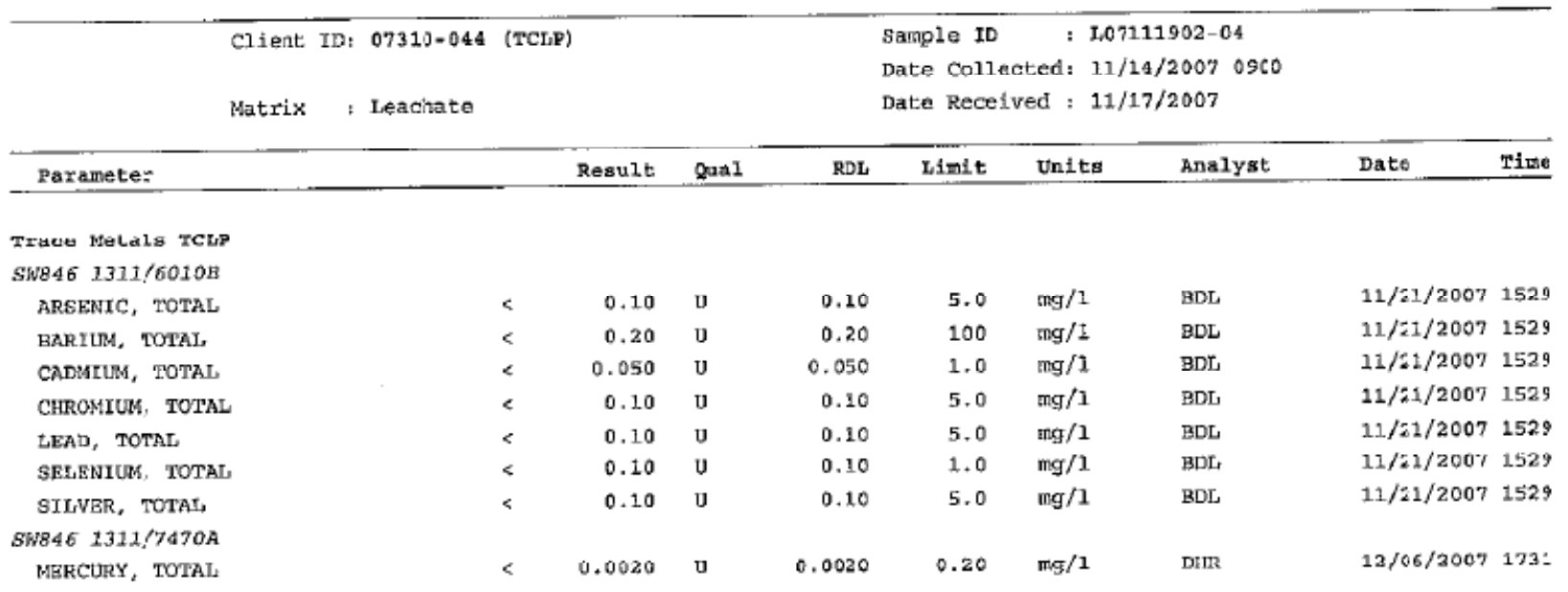


Table F.61. TCLP Metals Results for CCC-Treated EM07-Mn-01

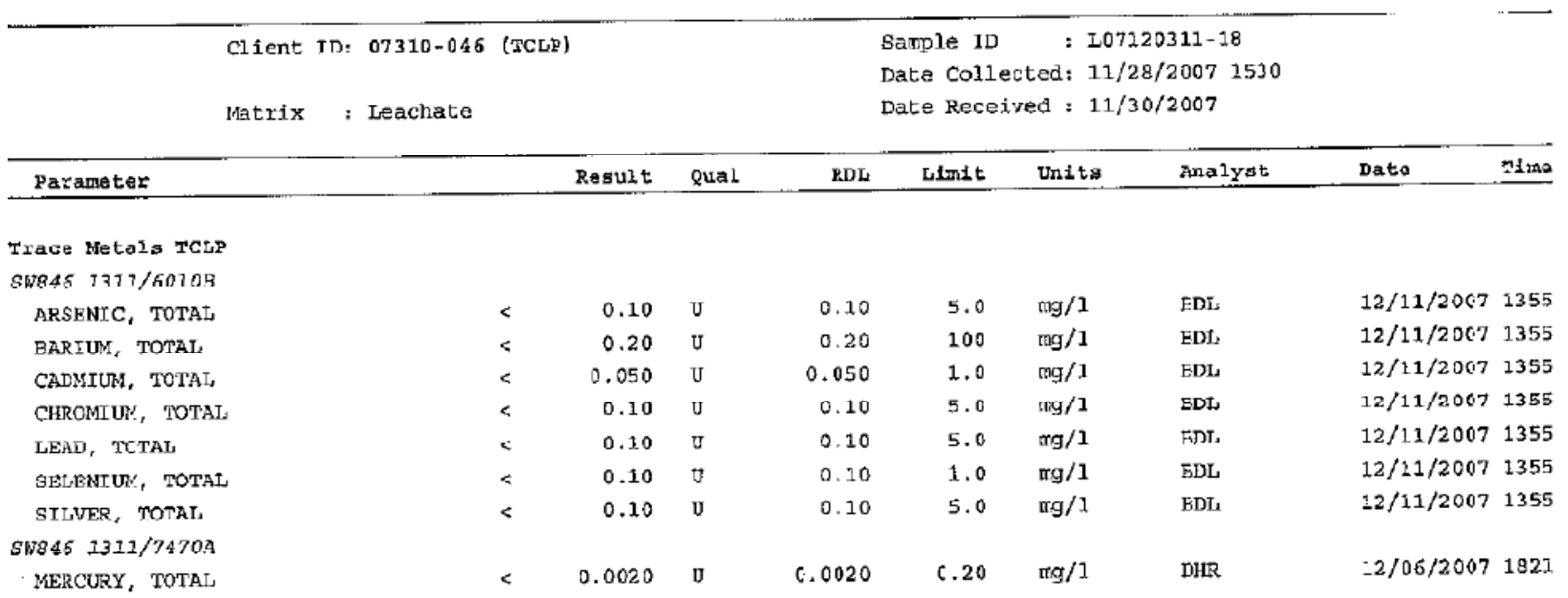

Table F.62. TCLP Metals Results for CCC-Treated EM07-Mn-04

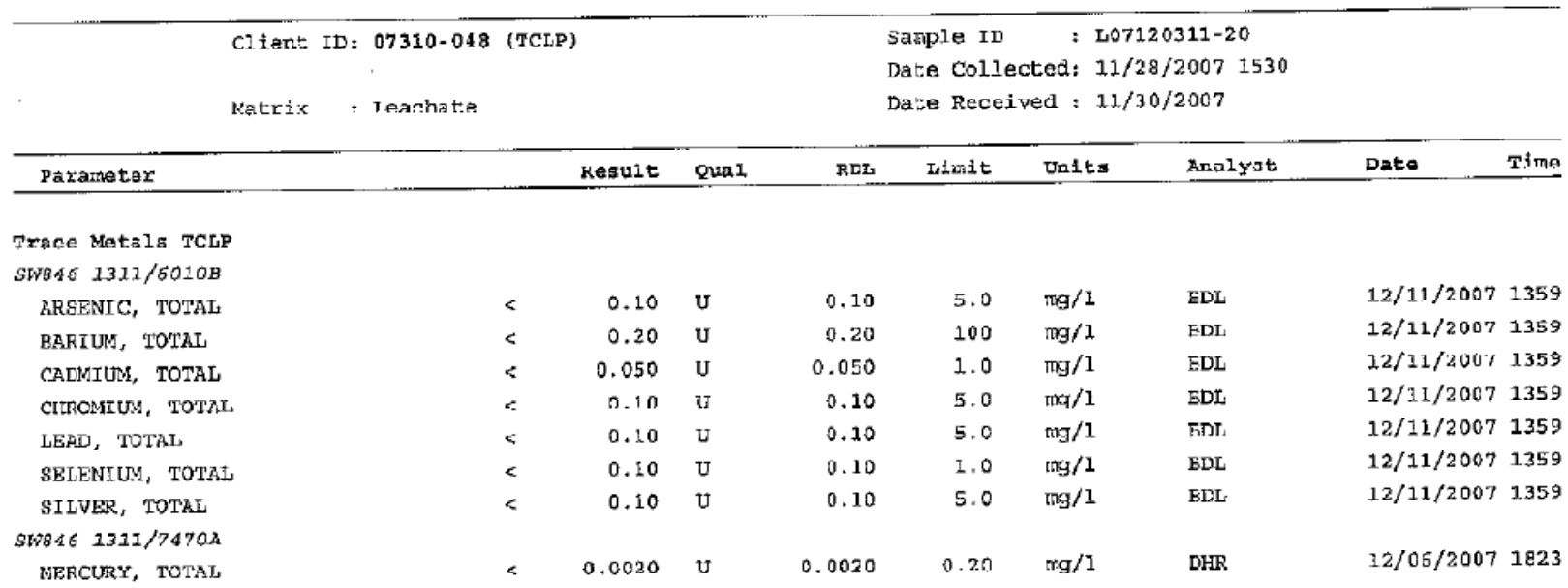

Table F.63. TCLP Metals Results for CCC-Treated EM07-Na-05

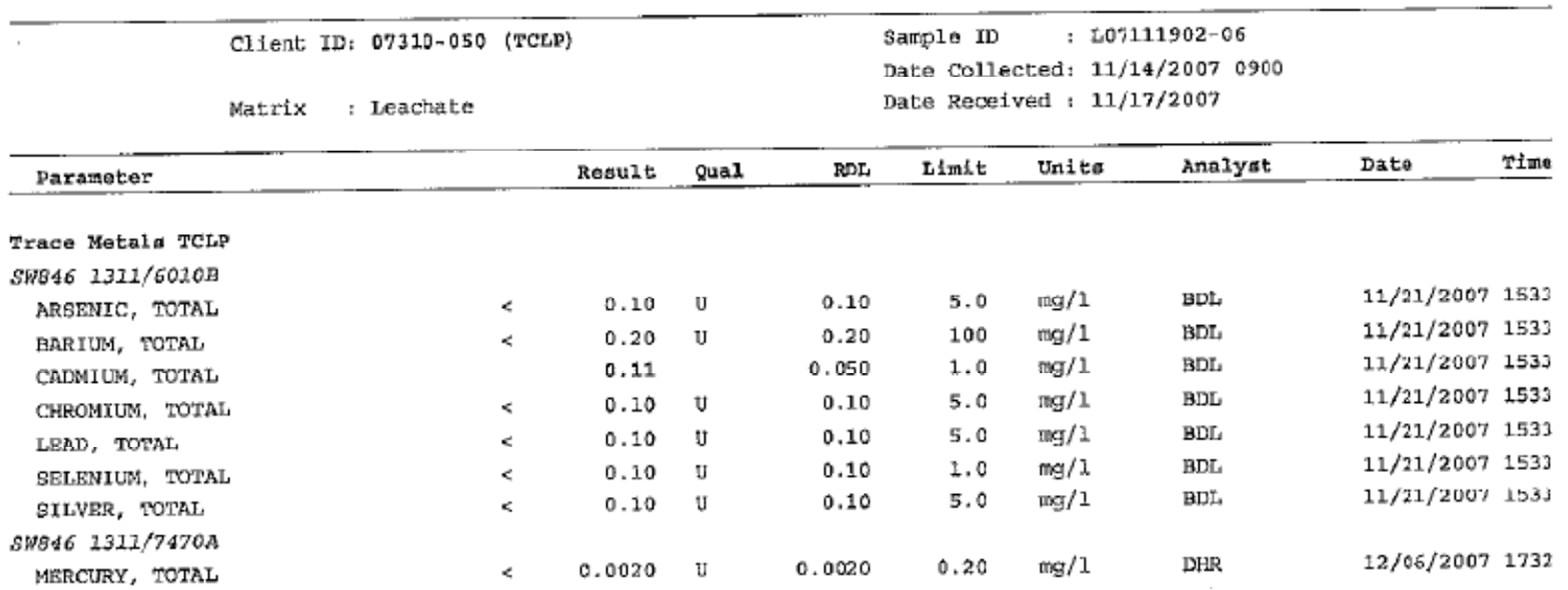


PNNL-17950, Rev 0

Table F.64. TCLP Metals Results for CCC-Treated EM07-Na-10

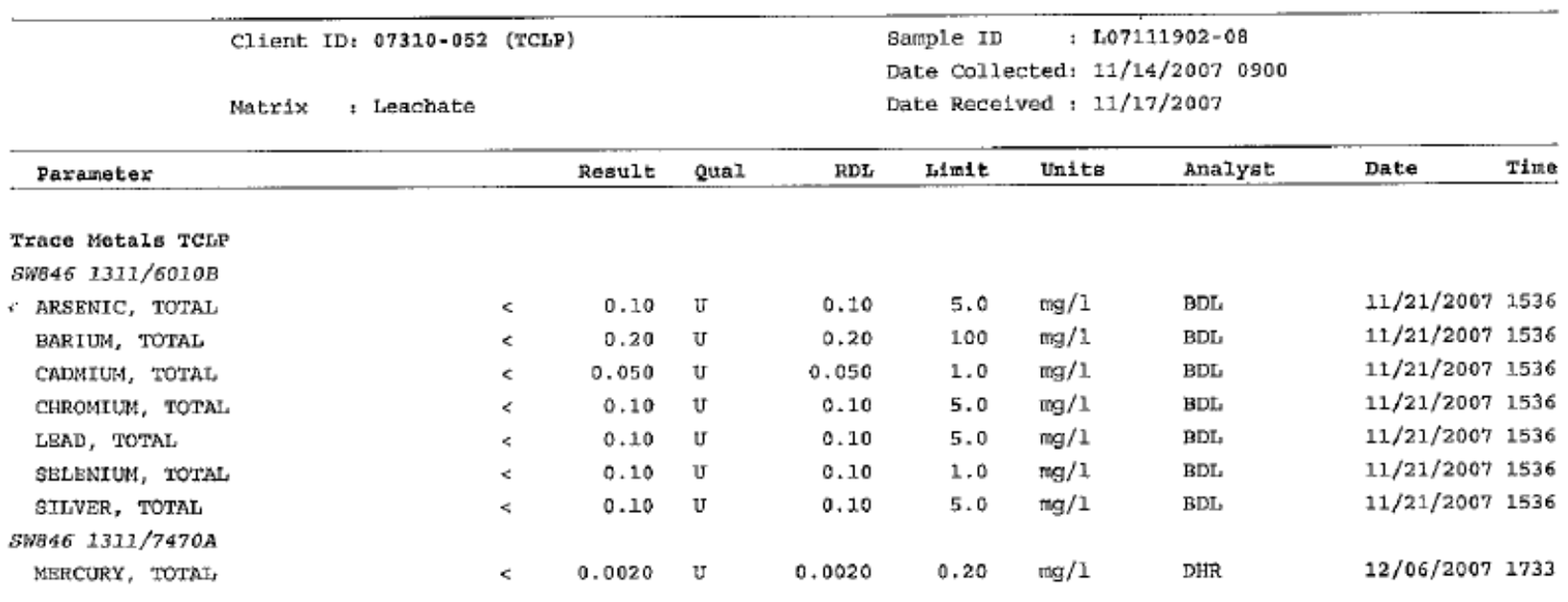

Table F.65. TCLP Metals Results for CCC-Treated EM07-Na-20

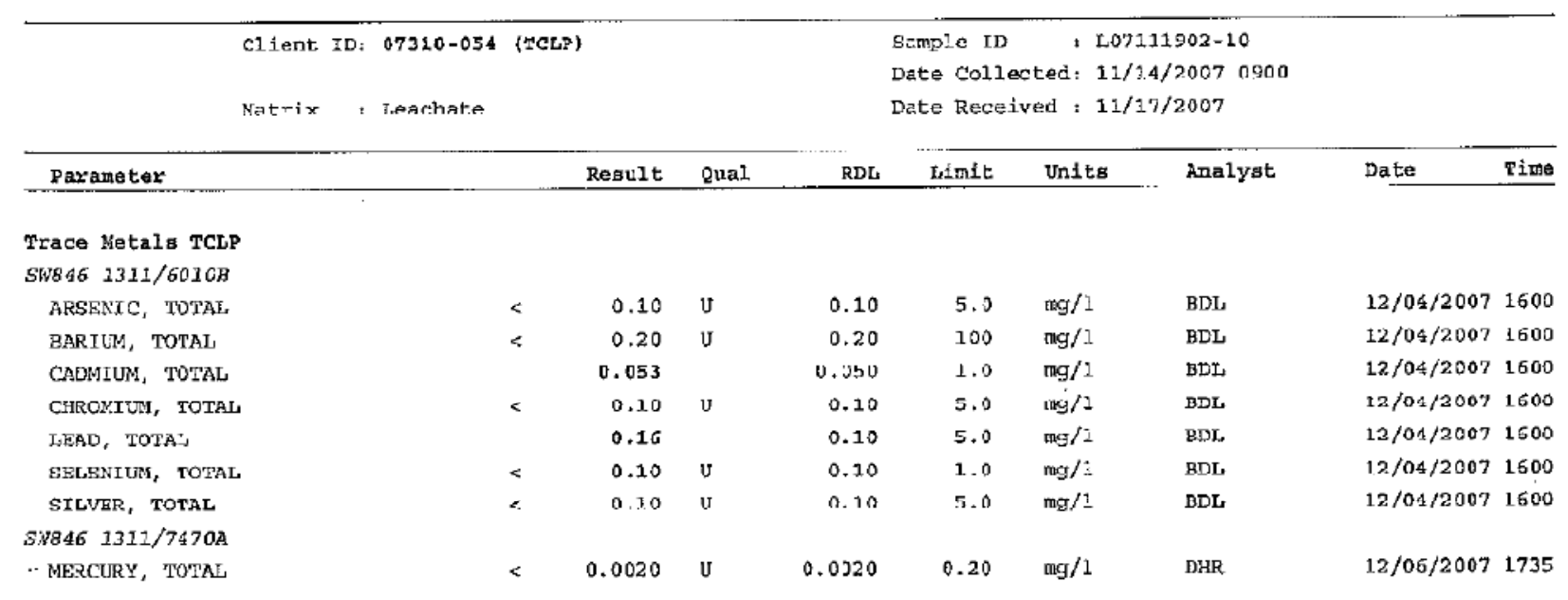


Table F.66. TCLP Metals Results for CCC-Treated EM07-Ni-001

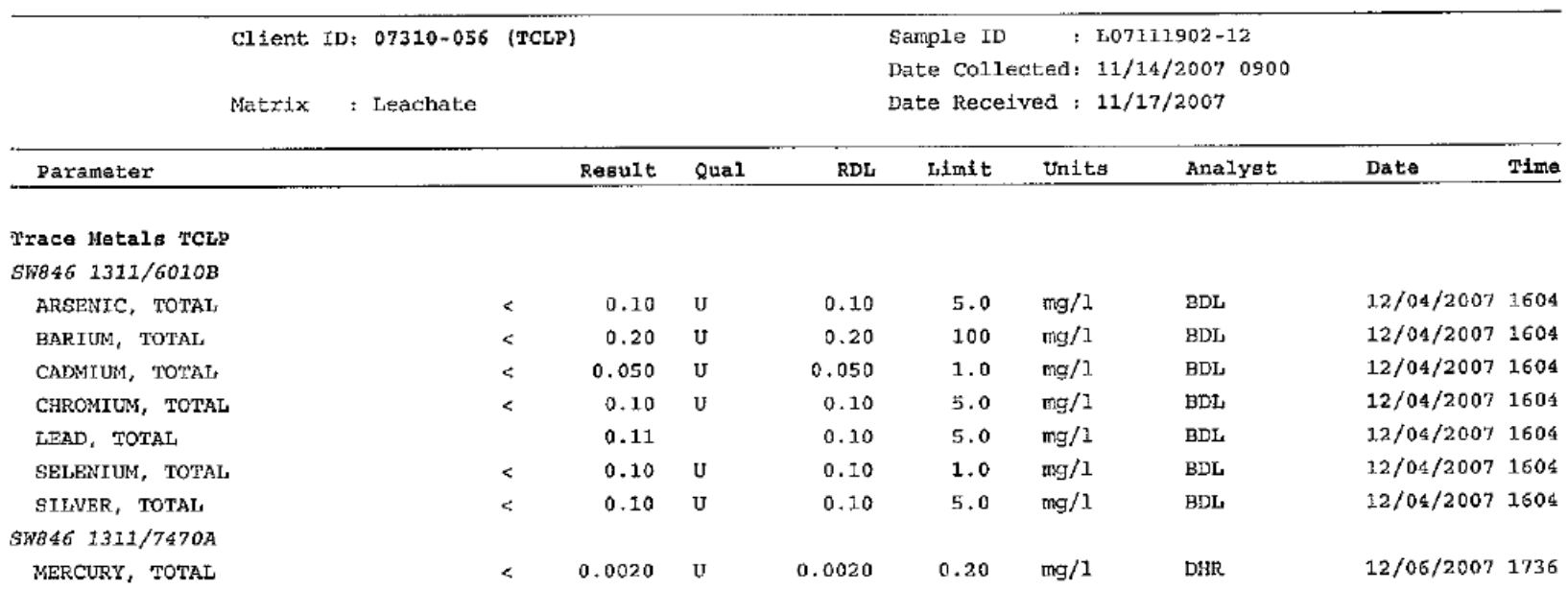

Table F.67. TCLP Metals Results for CCC-Treated EM07-Ni-02

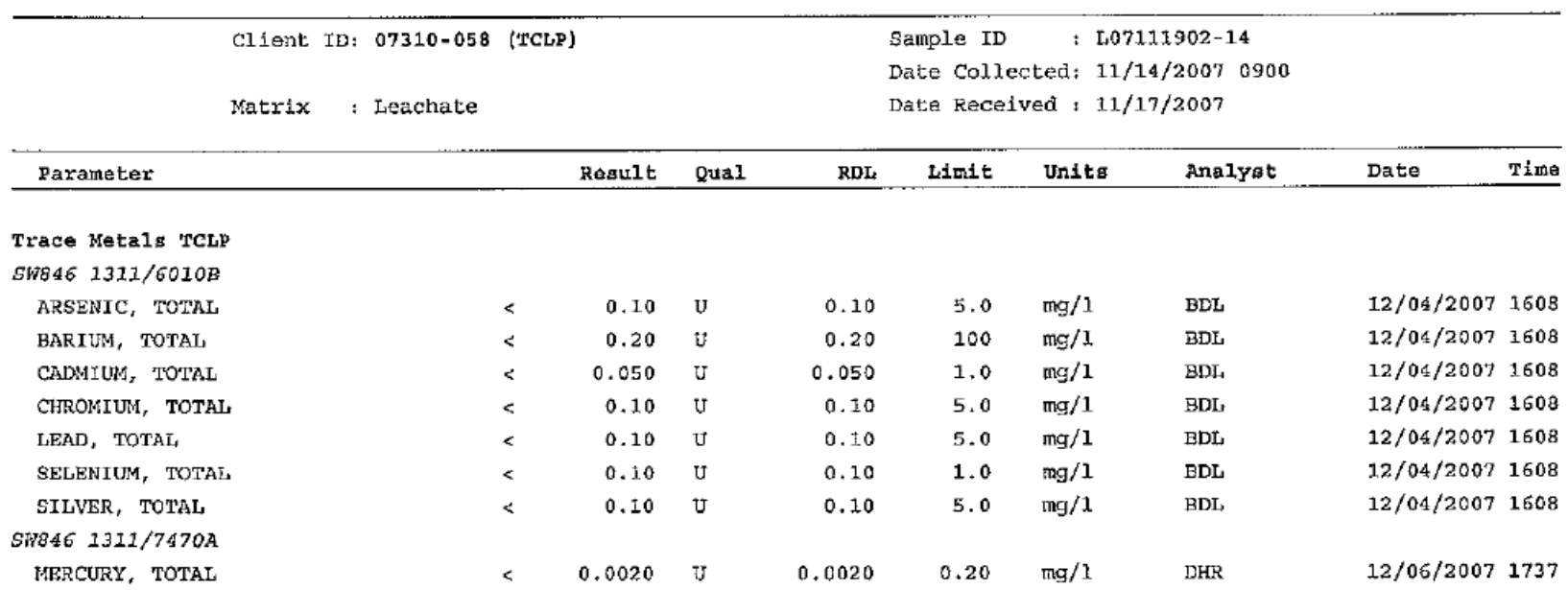

Table F.68. TCLP Metals Results for CCC-Treated EM07-P-0

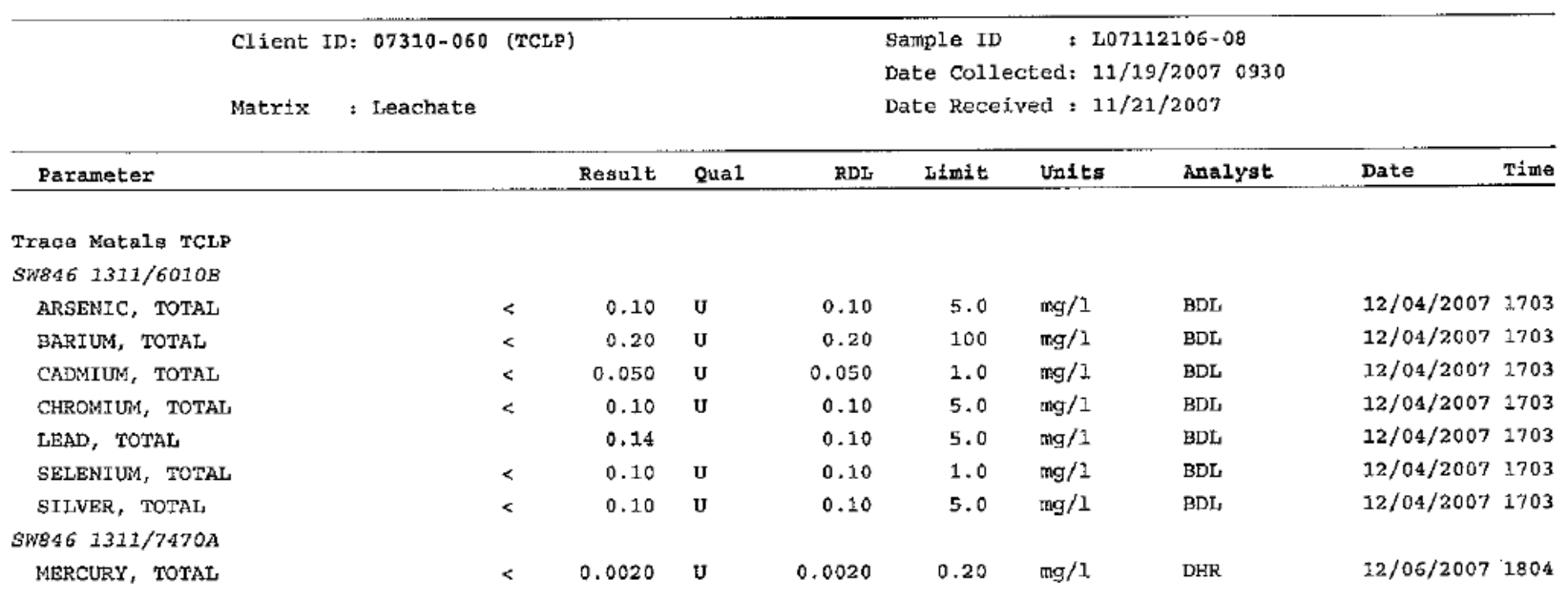


PNNL-17950, Rev 0

Table F.69. TCLP Metals Results for CCC-Treated EM07-P-025

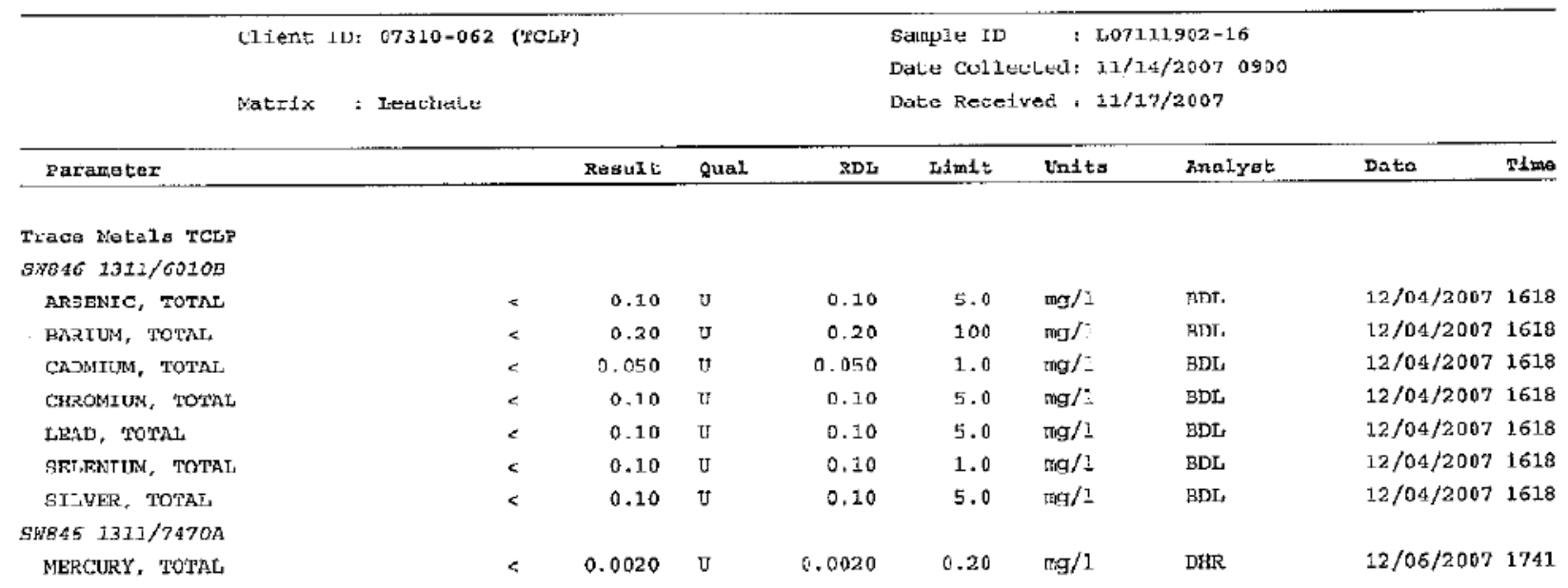

Table F.60. TCLP Metals Results for CCC-Treated EM07-Si-30

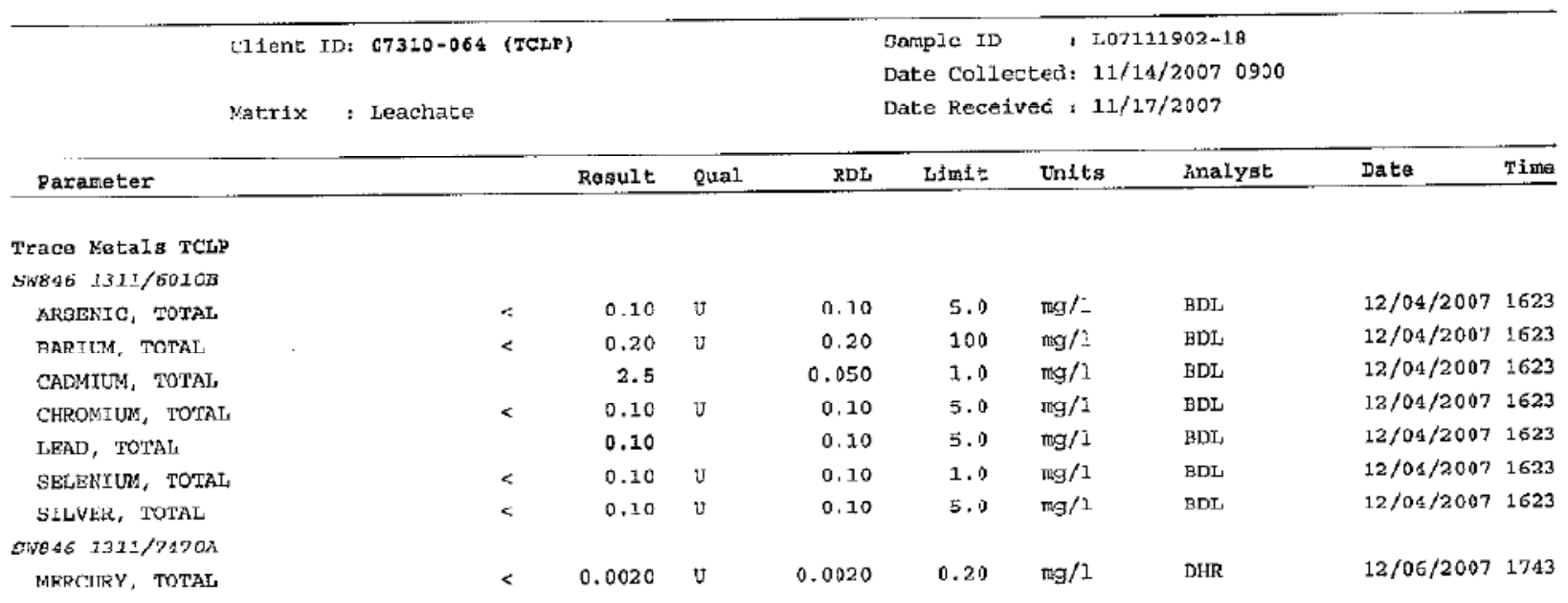


Table F.61. TCLP Metals Results for CCC-Treated EM07-Si-37

\begin{tabular}{|c|c|c|c|c|c|c|c|c|c|c|c|}
\hline \multirow{2}{*}{ Paraneter } & \multirow{2}{*}{\multicolumn{3}{|c|}{$\begin{array}{l}\text { Client ID: 07310-066 (TCLP) } \\
\text { Natrix : Tieachate }\end{array}$}} & \multicolumn{8}{|c|}{$\begin{array}{l}\text { Sample ID : L07111902-20 } \\
\text { Date Collected: } 11 / 14 / 20070900 \\
\text { Date Recelved : } 11 / 17 / 2007\end{array}$} \\
\hline & & & & Regult & Qual & $R D L$ & Limi= & \multirow[t]{2}{*}{ Units } & Analyst & Date & Time \\
\hline \multicolumn{11}{|l|}{ Trace Metals TchP } & \\
\hline \multicolumn{12}{|l|}{ SN846 $13 \lambda I / 6010 B$} \\
\hline ARSRNIC, TOTAIS & & & 5 & 0.10 & $\mathrm{v}$ & 0.10 & 5.0 & $\mathrm{mg} /=$ & BDL & $12 / 04 / 2007$ & 1627 \\
\hline BARIUK, TOTAL & & & $<$ & 0.20 & U & 0.20 & 100 & $\mathrm{mg} /=$ & BDL & $12 / 04 / 2007$ & 1627 \\
\hline CADMIUM, TOTAL & & & & 0.093 & & 0.050 & 1.0 & $\mathrm{ng} /=$ & BDL & $12 / 04 / 2007$ & 1627 \\
\hline CHRONIUM, TOTAE & & & $<$ & 0.10 & u & 0.10 & 5.0 & ng $/=$ & BDI & $12 / 01 / 2007$ & 1627 \\
\hline LEAD, TOTAL & & & & 0.15 & & 0.10 & 5.0 & $\mathrm{ng} /=$ & BDI & $12 / 04 / 2007$ & 1627 \\
\hline SELENIUM, TOTAL & & & $<$ & 0.10 & $\mathrm{v}$ & 0.10 & 1.0 & $\mathrm{mg} /=$ & $\mathrm{BDI}$ & $12 / 04 / 2007$ & 1627 \\
\hline SILVER，TOTAL & & & $<$ & 0.10 & v & 0.10 & 5.0 & $\mathrm{mg} /=$ & BDL & $12 / 04 / 2007$ & 1527 \\
\hline \multicolumn{12}{|l|}{$5 N 846 \quad 131 \pm / 747 O A$} \\
\hline MERCURY, TOTAL & & & $<$ & 0.0020 & $v$ & 0.0020 & 0.20 & ng/: & DHR & $12 / 06 / 2007$ & 1744 \\
\hline
\end{tabular}

Table F.62. TCLP Metals Results for CCC-Treated EM07-Si-50

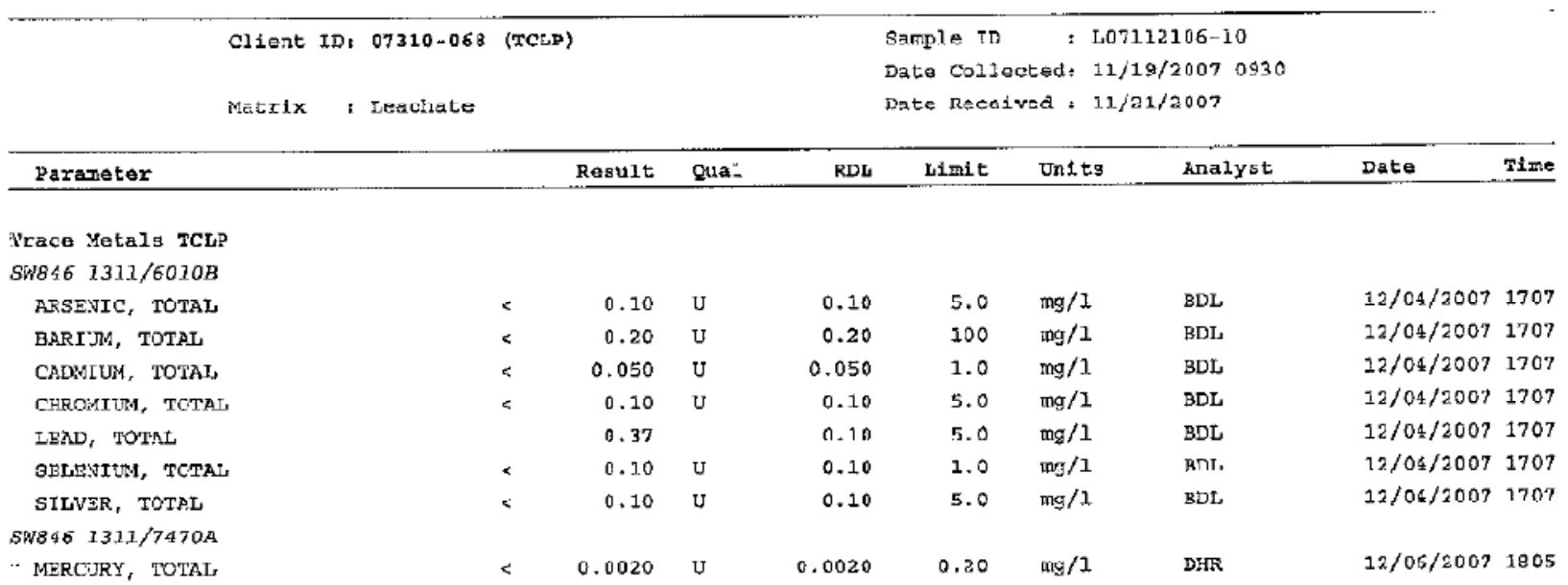

Table F.63. TCLP Metals Results for CCC-Treated EM07-Zr-001

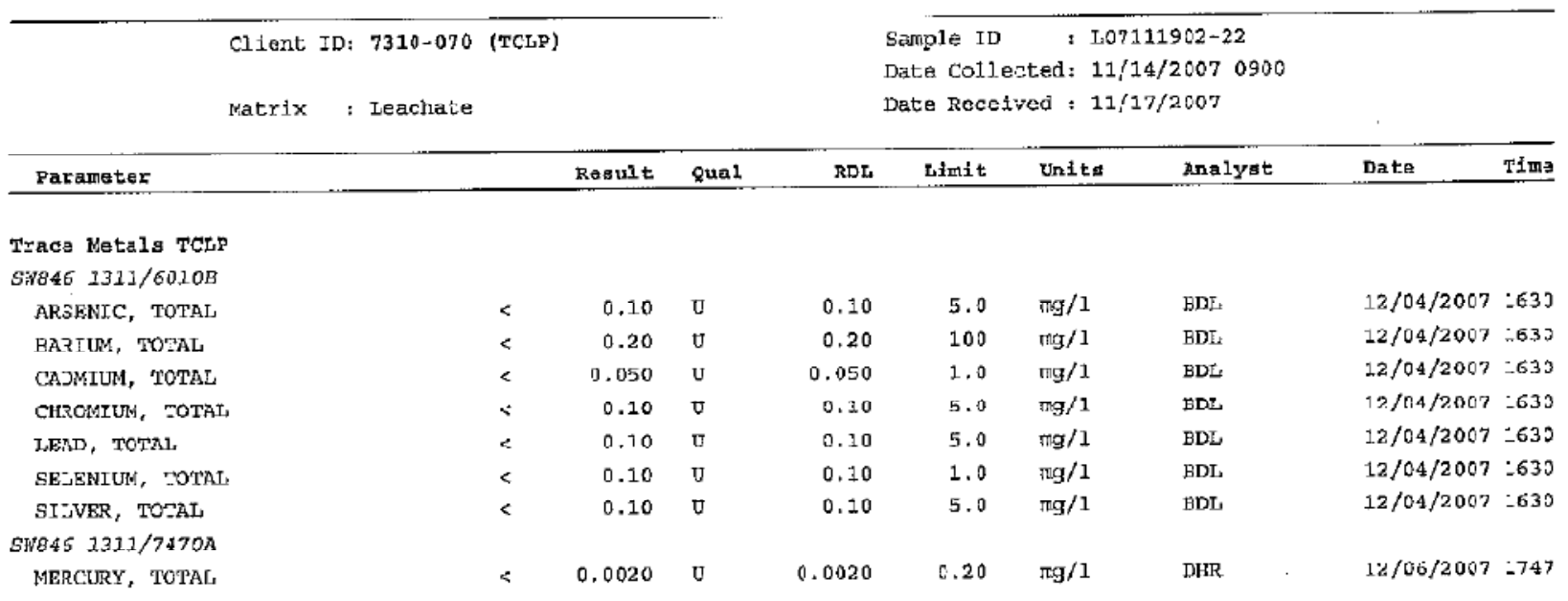


PNNL-17950, Rev 0

Table F.64. TCLP Metals Results for CCC-Treated EM07-Zr-05

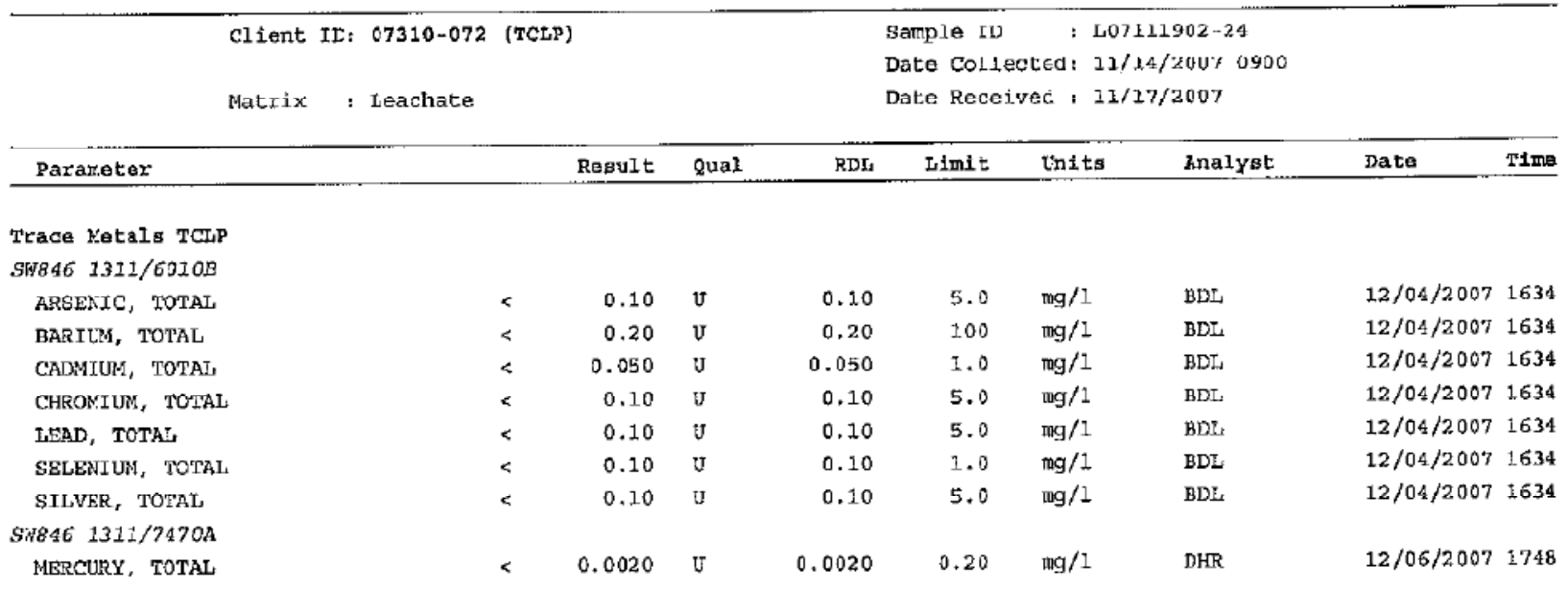

Table F.65. TCLP Metals Results for CCC-Treated EM07-NM-0025

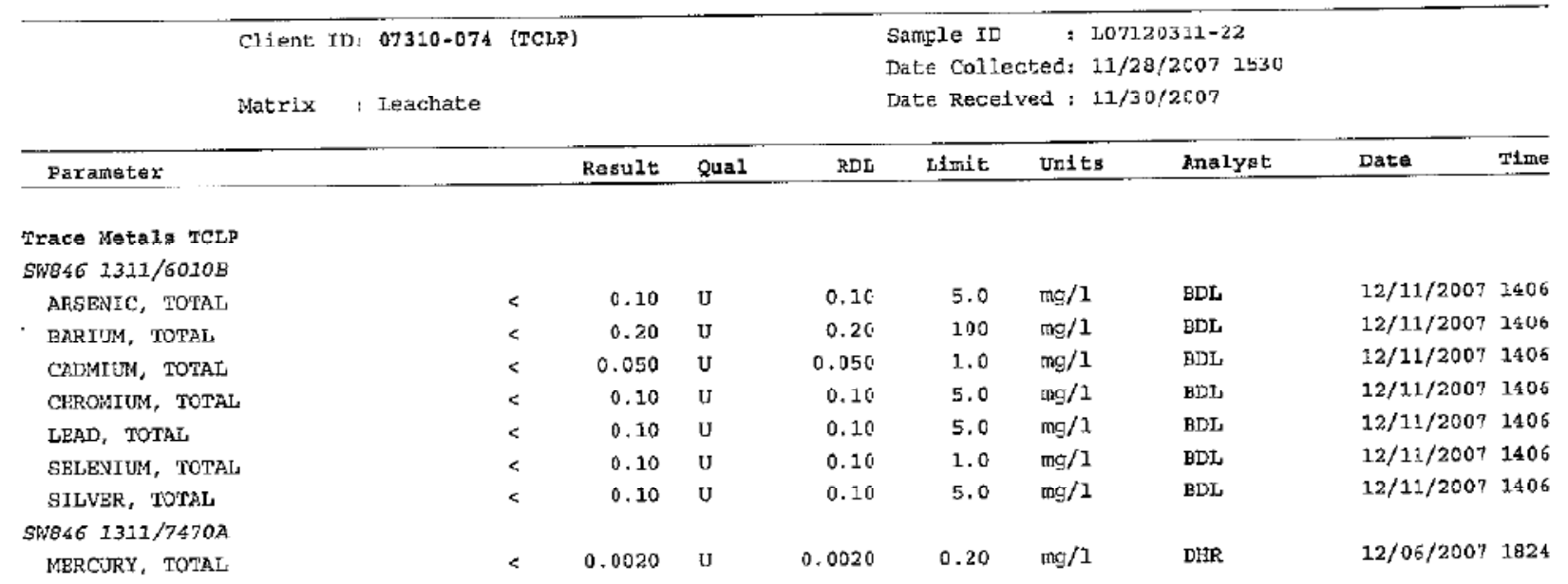


Table F.66. TCLP Metals Results for CCC-Treated EM07-BL-2

\begin{tabular}{|c|c|c|c|c|c|c|c|c|c|c|}
\hline \multirow[b]{2}{*}{ Parametex } & \multirow[t]{2}{*}{$\begin{array}{l}\text { Client ID: 07310-076 } \\
\text { Matrix : Leachate }\end{array}$} & \multicolumn{2}{|c|}{ (TCLP) } & \multicolumn{5}{|c|}{$\begin{array}{l}\text { Sampie ID : } 107120311-24 \\
\text { Date Collected: } 11 / 28 / 20071530 \\
\text { Date Received : } 11 / 30 / 2007\end{array}$} & \multirow[b]{2}{*}{ Date } & \multirow[b]{2}{*}{ Time } \\
\hline & & & Result & QuaI & RDL & Limit & Unita & Analyst & & \\
\hline \multicolumn{11}{|l|}{ Trace Metals TCuP } \\
\hline \multicolumn{11}{|l|}{ SWB45 $1311 / 6010 B$} \\
\hline ARSENIC, TOTAL & & $<$ & 0.10 & $\mathrm{U}$ & 0.10 & 5.0 & $\mathrm{mg} / \mathrm{I}$ & BDL & $12 / 11 / 2007$ & 141.0 \\
\hline BARIIM, TOTAL & & $<$ & 0.20 & U & 0.20 & 100 & $\mathrm{mg} / \mathrm{i}$ & BDL & $12 / 11 / 2007$ & 1410 \\
\hline CADNIUM, TOTAL & & $<$ & 0.050 & $\mathrm{U}$ & 0.050 & 1.0 & $\mathrm{mg} / 1$ & BDL & $1.2 / 11 / 2007$ & 1410 \\
\hline CHROMIUM, TOTAI & & $<$ & 0.10 & $U$ & 0.10 & 5.0 & $\mathrm{mg} / \mathrm{I}$ & BDL & $12 / 11 / 2007$ & 1410 \\
\hline LRAD, TOTAL & & $<$ & 0.10 & U & 0.10 & 5.0 & $m g / 1$ & EDL & $12 / 11 / 2007$ & 1410 \\
\hline SELENITM, TOTAL & & $<$ & 0.10 & U & 0.10 & 1.0 & $\mathrm{rng} / 1$ & BDL & $12 / 11 / 2007$ & 1410 \\
\hline SILVER, TOTAI & & $<$ & 0.10 & $\mathrm{U}$ & 0.10 & 5.0 & $\mathrm{mg} / 1$ & BDL & $12 / 11 / 2007$ & 1410 \\
\hline \multicolumn{11}{|l|}{ SW846 $1311 / 7470 \mathrm{~A}$} \\
\hline MERCURY, TOTAL & & $<$ & 0.0020 & $\mathrm{U}$ & 0.0020 & 0.20 & $\mathrm{mg} / 1$ & DHR & $12 / 06 / 2007$ & 1825 \\
\hline
\end{tabular}


PNNL-17950, Rev 0

\section{Appendix G: Formulation and Testing of HAL Glasses}

This section documents the data on Hanford High-Aluminum (HAL) glasses formulated and tested as a part of DOE EM-31 (previously EM-21) Office of Waste Processing International Glass Program to develop high waste loaded glasses for Hanford high-alumina high-level wastes. Selected results from this study have been published by Kim, et al. (2008) and Marra et al. (2010). This section provides all the data including those not published in these papers.

Table G.1 summarizes the target glass composition and the results of crystal identification and product consistency test (PCT) of all HAL glasses. Two glasses were selected and processed using the Steklo Metallicheskie Konstruktsii (SMK) melter system and the Electricheskaya Pech-5 (EP-5) melter system at Khlopin Radium Institute (KRI) in Saint Petersburg, Russia. Table G.2 summarizes the viscosity and electrical conductivity data as a function of temperature for these two selected glasses.

\section{References}

D. Kim and J.D. Vienna, D.K. Peeler and K.M. Fox, A. Aloy, A.V. Trofimenko, and K.D. Gerdes. 2008. "Improved Alumina Loading in High-Level Waste Glasses," in WM'08 proceedings, $H L W, T R U$, LLW/ILW, Mixed, Hazardous Wastes \& Environmental Management, February 24-28, 2008, Phoenix, AZ.

J.C Marra, K.M. Fox, G.T. Jannik, E.B. Farfan, D. Kim, J.D. Vienna, J.A. Roach, A.S. Aloy, S.V. Stefanovsky, D.P. Lopukh, M.D. Bondarkov, K.D. Gerdes and A.M. Han. 2010. "The DOE Office of Environmental Management International Cooperative Program: Overview of Technical Tasks and Results,' in WM'10 proceedings, HLW, TRU, LLW/ILW, Mixed, Hazardous Wastes \& Environmental Management, March 7-11, 2010, Phoenix, AZ. 
PNNL-17950, Rev 0

Table G.1. Target Glass Composition and Results of Crystal Identification and Product Consistency Tests on HAL Glasses

Target Composition (in mass fraction)

\begin{tabular}{|c|c|c|c|c|c|c|c|c|c|}
\hline$\#$ & Hal-01 & Hal-02 & Hal-03 & Hal-04 & Hal-05 & Hal-06 & Hal-07 & Hal-08 & Hal-09 \\
\hline $\mathrm{Al}_{2} \mathrm{O}_{3}$ & 0.26633 & 0.26633 & 0.26633 & 0.26633 & 0.25570 & 0.23972 & 0.24995 & 0.24995 & 0.24995 \\
\hline $\mathrm{B}_{2} \mathrm{O}_{3}$ & 0.20212 & 0.25213 & 0.20212 & 0.25213 & 0.15800 & 0.15192 & 0.14667 & 0.14997 & 0.14997 \\
\hline $\mathrm{BaO}$ & 0.00060 & 0.00060 & 0.00060 & 0.00060 & 0.00060 & 0.00050 & 0.00060 & 0.00060 & 0.00060 \\
\hline $\mathrm{Bi}_{2} \mathrm{O}_{3}$ & 0.01270 & 0.01270 & 0.01270 & 0.01270 & 0.01220 & 0.01140 & 0.01190 & 0.01190 & 0.01190 \\
\hline $\mathrm{CaO}$ & 0.03200 & 0.01200 & 0.03700 & 0.05201 & 0.03230 & 0.06081 & 0.05949 & 0.05999 & 0.09998 \\
\hline $\mathrm{CdO}$ & 0.00030 & 0.00030 & 0.00030 & 0.00030 & 0.00030 & 0.00020 & 0.00030 & 0.00030 & 0.00030 \\
\hline $\mathrm{Cr}_{2} \mathrm{O}_{3}$ & 0.00580 & 0.00580 & 0.00580 & 0.00580 & 0.00560 & 0.00520 & 0.00540 & 0.00540 & 0.00540 \\
\hline $\bar{F}$ & 0.01240 & 0.00740 & 0.00740 & 0.01740 & 0.00710 & 0.00670 & 0.00700 & 0.00700 & 0.00700 \\
\hline $\mathrm{Fe}_{2} \mathrm{O}_{3}$ & 0.06551 & 0.06551 & 0.06551 & 0.06551 & 0.06290 & 0.05901 & 0.06149 & 0.06149 & 0.06149 \\
\hline $\mathrm{K}_{2} \mathrm{O}$ & 0.00160 & 0.00160 & 0.00160 & 0.00160 & 0.01710 & 0.00140 & 0.00150 & 0.00150 & 0.00150 \\
\hline $\mathrm{La}_{2} \mathrm{O}_{3}$ & 0.00000 & 0.02500 & 0.00000 & 0.00000 & 0.01560 & 0.00000 & 0.00000 & 0.00000 & 0.00000 \\
\hline $\mathrm{Li}_{2} \mathrm{O}$ & 0.00190 & 0.02690 & 0.00190 & 0.00190 & 0.00180 & 0.03570 & 0.03459 & 0.03499 & 0.04999 \\
\hline $\mathrm{MgO}$ & 0.00130 & 0.00130 & 0.00130 & 0.00130 & 0.00120 & 0.00120 & 0.00120 & 0.00120 & 0.00120 \\
\hline $\mathrm{Na}_{2} \mathrm{O}$ & 0.06481 & 0.03980 & 0.06481 & 0.06481 & 0.03820 & 0.09581 & 0.09518 & 0.09498 & 0.04999 \\
\hline $\mathrm{NiO}$ & 0.00440 & 0.00440 & 0.00440 & 0.00440 & 0.00430 & 0.00400 & 0.00420 & 0.00420 & 0.00420 \\
\hline $\mathrm{P}_{2} \mathrm{O}_{5}$ & 0.06171 & 0.01170 & 0.08671 & 0.01170 & 0.11520 & 0.01050 & 0.01100 & 0.01100 & 0.01100 \\
\hline $\mathrm{PbO}$ & 0.00450 & 0.00450 & 0.00450 & 0.00450 & 0.00440 & 0.00410 & 0.00430 & 0.00430 & 0.00430 \\
\hline $\mathrm{SiO}_{2}$ & 0.25443 & 0.25443 & 0.22942 & 0.22942 & 0.26020 & 0.30503 & 0.29804 & 0.29404 & 0.28404 \\
\hline $\mathrm{SO}_{3}$ & 0.00220 & 0.00220 & 0.00220 & 0.00220 & 0.00210 & 0.00200 & 0.00210 & 0.00210 & 0.00210 \\
\hline $\mathrm{TiO}_{2}$ & 0.00010 & 0.00010 & 0.00010 & 0.00010 & 0.00010 & 0.00010 & 0.00010 & 0.00010 & 0.00010 \\
\hline $\mathrm{ZnO}$ & 0.00090 & 0.00090 & 0.00090 & 0.00090 & 0.00090 & 0.00080 & 0.00090 & 0.00090 & 0.00090 \\
\hline $\mathrm{ZrO}_{2}$ & 0.00440 & 0.00440 & 0.00440 & 0.00440 & 0.00420 & 0.00390 & 0.00410 & 0.00410 & 0.00410 \\
\hline Total & 1.00000 & 1.00000 & 1.00000 & 1.00000 & 1.00000 & 1.00000 & 1.00000 & 1.00000 & 1.00000 \\
\hline
\end{tabular}

\section{Crystal Identification}

\begin{tabular}{|c|l|l|l|l|l|l|l|l|}
\hline $\begin{array}{c}\text { Visual observation } \\
\text { of quenched volass }\end{array}$ crystals & $\begin{array}{l}\text { High vol\% } \\
\text { crystals }\end{array}$ & $\begin{array}{l}\text { High vol\% } \\
\text { crystals }\end{array}$ & $\begin{array}{l}\text { High vol\% } \\
\text { crystals }\end{array}$ & $\begin{array}{l}\text { Undissolved } \\
\text { materials }\end{array}$ & Clear & Clear & N/A & Clear \\
\hline $\begin{array}{c}\text { XRD after CCC N/A } \\
\text { treatment, vol\% }\end{array}$ & N/A & N/A & N/A & N/A & Sp 0.73 & Sp, Quartz & $\begin{array}{l}\text { Visual, } \\
\text { Some } \\
\text { crystals on } \\
\text { surface and } \\
\text { bulk }\end{array}$ & Sp \\
\hline $\begin{array}{r}\text { XRD after } 950^{\circ} \mathrm{C} \text { N/A } \\
\text { heat treatment } \\
\text { vol\% }\end{array}$ & N/A & N/A & N/A & N/A & Sp 0.73 & $\begin{array}{l}\text { Sp 1.12, } \\
\text { Wuestetite }\end{array}$ & N/A & Sp 0.98 \\
\hline
\end{tabular}

N/A: not analyzed

PCT normalized releases, $\mathrm{g} / \mathrm{L}$

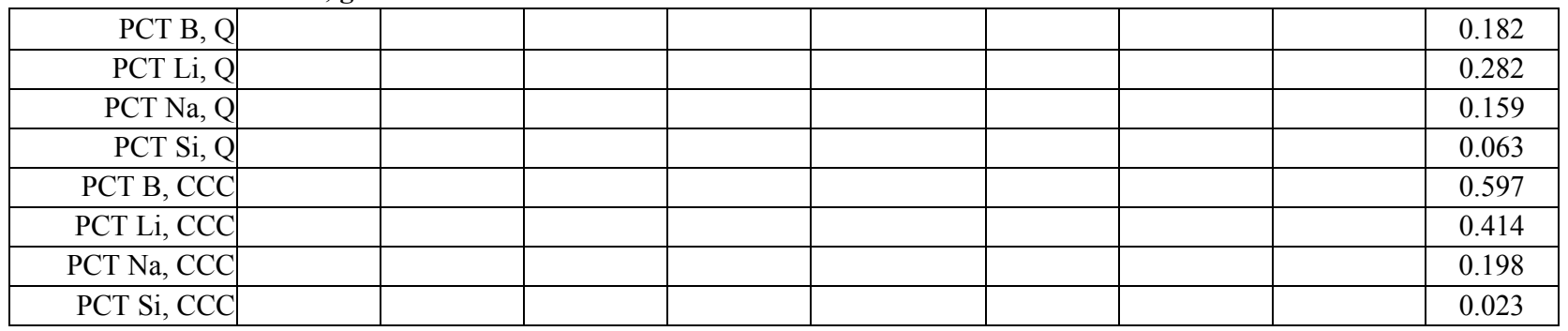

XRD: X-ray diffraction; Q: quenched; CCC: canister centerline cooling; Sp: Spinel 
Table G.1. Target Glass Composition and Results of Crystal Identification and Product Consistency Tests on HAL Glasses (Continued)

Target Composition (in mass fraction)

\begin{tabular}{|c|c|c|c|c|c|c|c|c|c|}
\hline$\#$ & Hal-10 & Hal-11 & Hal-12 & Hal-13 & Hal-14 & Hal-15 & HAL-16 & HAL-17 & $\begin{array}{c}\text { HAL- } \\
17 \mathrm{M}\end{array}$ \\
\hline $\mathrm{Al}_{2} \mathrm{O}_{3}$ & 0.24995 & 0.24995 & 0.24995 & 0.24995 & 0.26637 & 0.26635 & 0.25889 & 0.25889 & 0.25889 \\
\hline $\mathrm{B}_{2} \mathrm{O}_{3}$ & 0.14997 & 0.14997 & 0.17996 & 0.17996 & 0.17998 & 0.15211 & 0.16139 & 0.16139 & 0.16139 \\
\hline $\mathrm{BaO}$ & 0.00060 & 0.00060 & 0.00060 & 0.00060 & 0.00060 & 0.00060 & 0.00058 & 0.00058 & 0.00058 \\
\hline $\mathrm{Bi}_{2} \mathrm{O}_{3}$ & 0.01190 & 0.01190 & 0.01190 & 0.01190 & 0.01190 & 0.01272 & 0.01236 & 0.01236 & 0.01236 \\
\hline $\mathrm{CaO}$ & 0.05999 & 0.05999 & 0.07998 & 0.07998 & 0.13999 & 0.06496 & 0.07331 & 0.07331 & 0.07331 \\
\hline $\mathrm{CdO}$ & 0.00030 & 0.00030 & 0.00030 & 0.00030 & 0.00030 & 0.00027 & 0.00026 & 0.00026 & 0.00026 \\
\hline $\mathrm{Cr}_{2} \mathrm{O}_{3}$ & 0.00540 & 0.00540 & 0.00540 & 0.00540 & 0.00540 & 0.00579 & 0.00563 & 0.00563 & 0.00563 \\
\hline $\mathrm{F}$ & 0.00700 & 0.00700 & 0.00700 & 0.00700 & 0.00700 & 0.00742 & 0.00721 & 0.00721 & 0.00721 \\
\hline $\mathrm{Fe}_{2} \mathrm{O}_{3}$ & 0.06149 & 0.06149 & 0.06149 & 0.06149 & 0.06149 & 0.06555 & 0.06371 & 0.06371 & 0.06371 \\
\hline $\mathrm{K}_{2} \mathrm{O}$ & 0.03999 & 0.03999 & 0.00150 & 0.00150 & 0.03700 & 0.00157 & 0.02723 & 0.02723 & 0.02723 \\
\hline $\mathrm{La}_{2} \mathrm{O}_{3}$ & 0.00000 & 0.00000 & 0.00000 & 0.00000 & 0.00000 & 0.00000 & 0.00000 & 0.00000 & 0.00000 \\
\hline $\mathrm{Li}_{2} \mathrm{O}$ & 0.03499 & 0.04999 & 0.03499 & 0.03499 & 0.00000 & 0.04040 & 0.02754 & 0.04000 & 0.04000 \\
\hline $\mathrm{MgO}$ & 0.00120 & 0.00120 & 0.00120 & 0.00120 & 0.00120 & 0.00130 & 0.00126 & 0.00126 & 0.00126 \\
\hline $\mathrm{Na}_{2} \mathrm{O}$ & 0.07259 & 0.05749 & 0.09498 & 0.06499 & 0.05000 & 0.08978 & 0.08750 & 0.06067 & 0.06067 \\
\hline $\mathrm{NiO}$ & 0.00420 & 0.00420 & 0.00420 & 0.00420 & 0.00420 & 0.00444 & 0.00432 & 0.00432 & 0.00432 \\
\hline $\mathrm{P}_{2} \mathrm{O}_{5}$ & 0.01100 & 0.01100 & 0.01100 & 0.01100 & 0.01100 & 0.01169 & 0.01136 & 0.01136 & 0.01136 \\
\hline $\mathrm{PbO}$ & 0.00430 & 0.00430 & 0.00430 & 0.00430 & 0.00430 & 0.00455 & 0.00442 & 0.00442 & 0.00442 \\
\hline $\mathrm{SiO}_{2}$ & 0.27794 & 0.27804 & 0.24405 & 0.27405 & 0.21208 & 0.26290 & 0.24562 & 0.25999 & 0.25999 \\
\hline $\mathrm{SO}_{3}$ & 0.00210 & 0.00210 & 0.00210 & 0.00210 & 0.00210 & 0.00222 & 0.00216 & 0.00216 & 0.00216 \\
\hline $\mathrm{TiO}_{2}$ & 0.00010 & 0.00010 & 0.00010 & 0.00010 & 0.00010 & 0.00011 & 0.00011 & 0.00011 & 0.00011 \\
\hline $\mathrm{ZnO}$ & 0.00090 & 0.00090 & 0.00090 & 0.00090 & 0.00090 & 0.00092 & 0.00089 & 0.00089 & 0.00089 \\
\hline $\mathrm{ZrO}_{2}$ & 0.00410 & 0.00410 & 0.00410 & 0.00410 & 0.00410 & 0.00439 & 0.00426 & 0.00426 & 0.00426 \\
\hline Total & 1.00000 & 1.00000 & 1.00000 & 1.00000 & 1.00000 & 1.00000 & 1.00000 & 1.00000 & 1.00000 \\
\hline
\end{tabular}

HAL-17M: HAL_17 glass from melter tests

\section{Crystal Identification}

\begin{tabular}{|c|c|c|c|c|c|c|c|c|c|}
\hline $\begin{array}{l}\text { Visual observation } \\
\text { of quenched glass }\end{array}$ & $\begin{array}{l}\text { Clear, possible } \\
\text { crystals in bulk }\end{array}$ & $\begin{array}{l}\text { Silver swirl } \\
\text { on surface, } \\
\text { bulk clear }\end{array}$ & Clear & Clear & \begin{tabular}{|l} 
Spots of \\
undissolved in \\
crucible, possible \\
crystals in bulk
\end{tabular} & $\begin{array}{l}\text { A few } \\
\text { small } \\
\text { crystals on } \\
\text { surface }\end{array}$ & $\begin{array}{l}\text { Low vol\% } \\
\text { crystals }\end{array}$ & $\begin{array}{l}\text { Low vol\% } \\
\text { crystals }\end{array}$ & $\mathrm{N} / \mathrm{A}$ \\
\hline $\begin{array}{l}\text { XRD after CCC } \\
\text { treatment, vol\% }\end{array}$ & $\begin{array}{l}\text { Sp, } \\
\text { Hydroxylapatite }\end{array}$ & $\mathrm{Sp}$ & $\begin{array}{l}\text { Sp, } \\
\text { Quartz }\end{array}$ & $\mathrm{Sp}$ & Sp, Hema & $\mathrm{Sp}, \mathrm{Np}$ & $\begin{array}{l}\text { Sp 2.3, } \\
\text { Flapa 1.6, } \\
\text { Np } 2.6\end{array}$ & $\begin{array}{l}\text { Sp 2.4, } \\
\text { Flapa } 1.2\end{array}$ & N/A \\
\hline $\begin{array}{r}\text { XRD after } 950^{\circ} \mathrm{C} \\
\text { heat treatment, } \\
\mathrm{vol} \%\end{array}$ & Sp 0.88 & Sp 1.07 & $\begin{array}{l}\text { LiFe5O8 } \\
0.73\end{array}$ & $\operatorname{Sp} 0.83$ & $\begin{array}{l}\text { Hema } 1.26, \mathrm{Sp} \\
0.58, \text { Flapa } 1.03\end{array}$ & Sp 1.22 & \begin{tabular}{|l|} 
Sp 1.2, \\
Flapa 0.6
\end{tabular} & Sp 0.99 & $\mathrm{~N} / \mathrm{A}$ \\
\hline
\end{tabular}

N/A: not analyzed

PCT normalized releases, $\mathrm{g} / \mathrm{L}$

\begin{tabular}{|c|c|c|c|c|c|}
\hline PCT B, Q & 0.387 & 0.532 & 0.342 & 0.118 & 0.222 \\
\hline PCT Li, Q & 0.498 & 0.675 & 0.555 & 0.333 & 0.358 \\
\hline PCT Na, Q & 0.379 & 0.605 & 0.490 & 0.260 & 0.329 \\
\hline PCT Si, Q & 0.128 & 0.110 & 0.089 & 0.068 & 0.116 \\
\hline PCT B, CCC & 0.215 & 0.604 & 0.083 & 1.618 & 0.168 \\
\hline PCT Li, CCC & 0.311 & 0.554 & 0.395 & 0.767 & 0.302 \\
\hline PCT Na, CCC & 0.205 & 0.372 & 0.159 & 0.986 & 0.269 \\
\hline PCT Si, CCC & 0.100 & 0.024 & 0.069 & 0.001 & 0.106 \\
\hline
\end{tabular}

XRD: X-ray diffraction; Q: quenched; CCC: canister centerline cooling; Sp: Spinel; Np: Nepheline; Hema: Hematite; Flapa: Fluorapatite 
PNNL-17950, Rev 0

Table G.1. Target Glass Composition and Results of Crystal Identification and Product Consistency Tests on HAL Glasses (Continued)

Target Composition (in mass fraction)

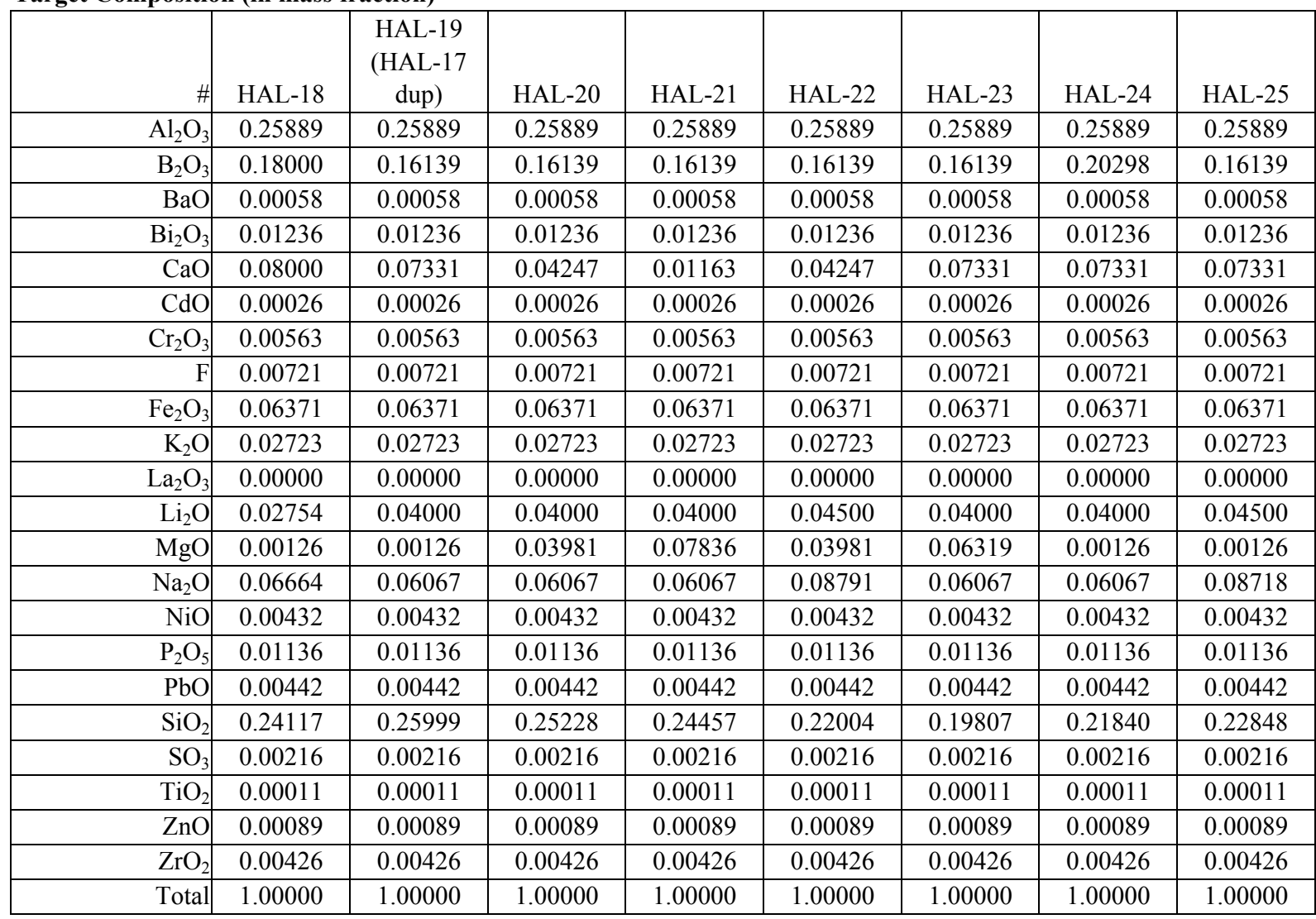

\section{Crystal Identification}

\begin{tabular}{|c|c|c|c|c|c|c|c|}
\hline \begin{tabular}{l|l}
$\begin{array}{l}\text { Visual observation } \\
\text { of quenched glass }\end{array}$ & Low vol\% $\%$ \\
crystals
\end{tabular} & \multicolumn{7}{|c|}{ A very thin slightly dark layer on the melt surface while pouring. } \\
\hline \begin{tabular}{l|l} 
XRD after CCC & Sp 2.4, \\
treatment, vol $\%$ & Flapa 1.5, \\
Hema 0.4
\end{tabular} & Sp 2.1 & $\operatorname{Sp} 3.2$ & $\mathrm{Sp} 3.4$ & $\begin{array}{l}\text { Sp 3.6, } \\
\text { Np 5.1 }\end{array}$ & Sp 5.0 & Sp 1.9 & $\begin{array}{l}\text { Sp 1.9 } \\
\text { Np 10.2 }\end{array}$ \\
\hline \begin{tabular}{r|r}
$\mathrm{XRD}$ after $950^{\circ} \mathrm{C}$ & $\mathrm{Sp} 1.2$ \\
heat treatment, & Flapa 0.6 \\
vol $\%$ &
\end{tabular} & Sp 1.1 & Sp 2.1 & Sp 1.8 & $\operatorname{Sp} 2.2$ & Sp 2.2 & Sp 1.1 & Sp 1.3 \\
\hline
\end{tabular}

N/A: not analyzed

PCT normalized releases, $\mathrm{g} / \mathrm{L}$

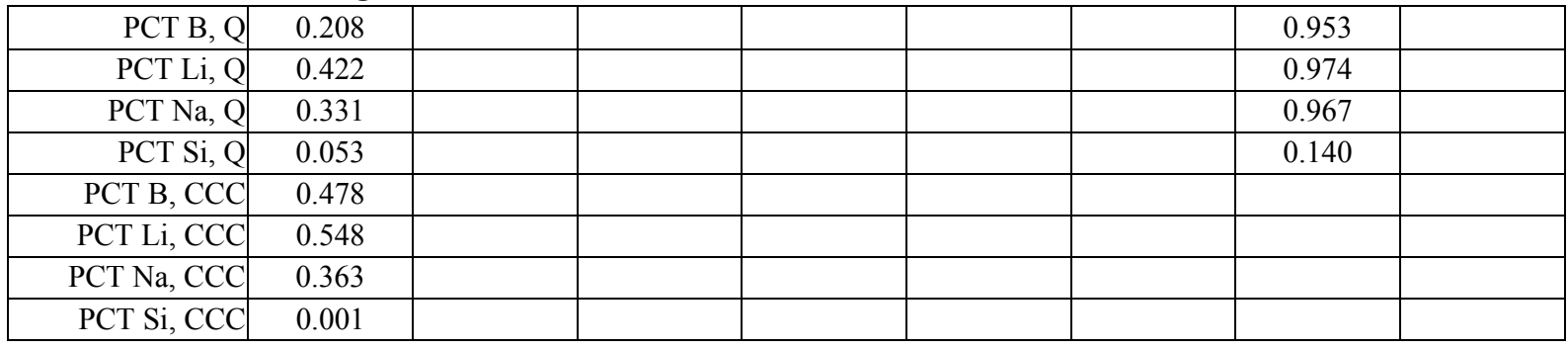

Q: quenched; CCC: canister centerline cooling; Sp: Spinel; Np: Nepheline; Hema: Hematite; Flapa: Fluorapatite 
Table G.2. Viscosity and Electrical Conductivity of Selected HAL Glasses

Viscosity

\begin{tabular}{|c|c|}
\hline \multicolumn{2}{|c|}{ HAL-17 } \\
\hline $\mathbf{T},{ }^{\circ} \mathbf{C}$ & Pa·s \\
\hline 991 & 51.79 \\
\hline 1041 & 24.17 \\
\hline 1090 & 11.80 \\
\hline 1140 & 6.38 \\
\hline 1189 & 3.68 \\
\hline 1240 & 2.10 \\
\hline
\end{tabular}

\begin{tabular}{|c|c|}
\hline \multicolumn{2}{|c|}{ HAL-24 } \\
\hline $\mathbf{T},{ }^{\circ} \mathbf{C}$ & Pa·s \\
\hline 912 & 38.39 \\
\hline 963 & 19.64 \\
\hline 1010 & 10.76 \\
\hline 1057 & 6.09 \\
\hline 1104 & 3.47 \\
\hline 1151 & 2.09 \\
\hline 1189 & 1.40 \\
\hline
\end{tabular}

Electrical Conductivity

\begin{tabular}{|c|c|c|c|}
\hline \multicolumn{5}{|c|}{ HAL-17 } \\
\hline $\mathbf{T},{ }^{\circ} \mathbf{C}$ & $\begin{array}{c}\text { at } \mathbf{1 0 0} \mathbf{~ H z}, \\
\mathbf{S} / \mathbf{m}\end{array}$ & $\begin{array}{c}\text { at } \mathbf{1} \mathbf{~ k H z}, \\
\mathbf{S} / \mathbf{m}\end{array}$ & $\begin{array}{c}\text { at } \mathbf{1 0} \mathbf{~ k H z} \\
\mathbf{S} / \mathbf{m}\end{array}$ \\
\hline 942 & 4.33 & 4.51 & 4.56 \\
\hline 992 & 6.09 & 6.40 & 6.48 \\
\hline 1043 & 8.16 & 8.63 & 8.77 \\
\hline 1094 & 10.32 & 10.94 & 11.12 \\
\hline 1143 & 13.22 & 14.03 & 14.26 \\
\hline 1193 & 16.45 & 17.36 & 17.72 \\
\hline 1242 & 19.99 & 21.10 & 21.49 \\
\hline
\end{tabular}

\begin{tabular}{|c|c|c|c|}
\hline \multicolumn{4}{|c|}{ HAL-24 } \\
\hline $\mathbf{T},{ }^{\circ} \mathbf{C}$ & $\begin{array}{c}\text { at } \mathbf{1 0 0} \mathbf{~ H z}, \\
\mathbf{S} / \mathbf{m}\end{array}$ & $\begin{array}{c}\text { at } \mathbf{1 ~ k H z}, \\
\mathbf{S} / \mathbf{m}\end{array}$ & $\begin{array}{c}\text { at } \mathbf{1 0} \mathbf{~ k H z}, \\
\mathbf{S} / \mathbf{m}\end{array}$ \\
\hline 937 & 4.07 & 4.25 & 4.29 \\
\hline 1011 & 6.52 & 6.88 & 6.95 \\
\hline 1085 & 9.68 & 10.25 & 10.38 \\
\hline 1158 & 13.55 & 14.32 & 14.52 \\
\hline 1234 & 17.87 & 18.79 & 19.04 \\
\hline
\end{tabular}




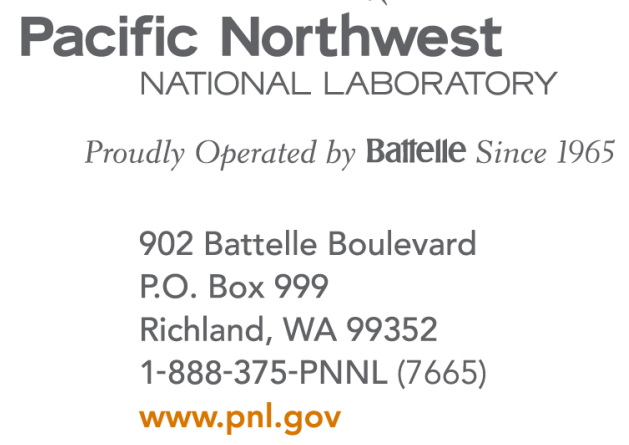

Pacific Northwest

NATIONAL LABORATORY

Proudly Operated by Battelle Since 1965

902 Battelle Boulevard

P.O. Box 999

Richland, WA 99352

1-888-375-PNNL (7665)

www.pnl.gov 